\title{
Environmental Regulation of the Heart: The Role of Non-Coding RNA and Epigenetics in Influencing Mitochondrial and Cellular Health
}

Quincy Alexander Hathaway

West Virginia University, qahathaway@mix.wvu.edu

Follow this and additional works at: https://researchrepository.wvu.edu/etd

Part of the Cardiovascular Diseases Commons, Endocrine System Diseases Commons, Genetic

Processes Commons, Medical Cell Biology Commons, and the Medical Molecular Biology Commons

\section{Recommended Citation}

Hathaway, Quincy Alexander, "Environmental Regulation of the Heart: The Role of Non-Coding RNA and Epigenetics in Influencing Mitochondrial and Cellular Health" (2019). Graduate Theses, Dissertations, and Problem Reports. 4052.

https://researchrepository.wvu.edu/etd/4052

This Dissertation is protected by copyright and/or related rights. It has been brought to you by the The Research Repository @ WVU with permission from the rights-holder(s). You are free to use this Dissertation in any way that is permitted by the copyright and related rights legislation that applies to your use. For other uses you must obtain permission from the rights-holder(s) directly, unless additional rights are indicated by a Creative Commons license in the record and/ or on the work itself. This Dissertation has been accepted for inclusion in WVU Graduate Theses, Dissertations, and Problem Reports collection by an authorized administrator of The Research Repository @ WVU.

For more information, please contact researchrepository@mail.wvu.edu. 


\title{
Environmental Regulation of the Heart: The Role of Non- Coding RNA and Epigenetics in Influencing Mitochondrial and Cellular Health
}

\author{
Quincy Alexander Hathaway \\ Dissertation submitted to the School of Medicine at West Virginia University \\ in partial fulfillment of the requirements for the degree of \\ Doctor of Philosophy in \\ Exercise Physiology
}

John M. Hollander, Ph.D, Chair

Stephen E. Alway, Ph.D.

Peter H. Mathers, Ph.D.

Timothy R. Nurkiewicz, Ph.D.

Emidio E. Pistilli, Ph.D.

Aaron R. Robart, Ph.D.
Department of Human Performance, Division of Exercise Physiology,
West Virginia University, Morgantown, WV, USA - 2019

Keywords: Mitochondria, Epigenetics, Non-coding RNA, Heart, Diabetes, Nanotoxicology, Machine Learning, Bioinformatics

Copyright 2019 Quincy Alexander Hathaway 


\title{
ABSTRACT \\ Environmental Regulation of the Heart: The Role of Non-Coding RNA and Epigenetics in Influencing Mitochondrial and Cellular Health
}

\author{
Quincy Alexander Hathaway
}

Introduction: The mitochondrion, a small but ubiquitously distributed organelle in the cell, continues to be the focus of many disease pathogeneses, tissue and organ dysfunctions, and other morbidities that occur throughout the body. By controlling major aspects of metabolism, energy homeostasis, ion concentrations, apoptosis, and inflammation, the mitochondrion serves as a multifunctional organelle that has the capacity to impact many crucial aspects of cellular homeostasis. While mitochondrial function has been broadly defined in most disease states and/or insults, a lack of understanding remains in the regulatory networks that exist between the nucleus and mitochondrion as well as the import/export dynamics of RNA and other biomolecules within the mitochondrion itself. Nuclear influence on the type, quality, and concentration of proteins shuttled to the mitochondrion (anterograde signaling) and mitochondrial regulation of metabolites produced for nuclear DNA transcription and maintenance (retrograde signaling) are poorly understood processes. Likewise, the translocation of non-coding RNAs into mitochondria and their functional relevance is lacking. The underlying regulatory network of epigenomic modifications, competitive endogenous RNAs, and other transient modifications reveal a crucial, undiscovered nature surrounding mitochondrial biology. In examining these mechanisms, the heart, with a major reliance on mitochondrial ATP production for its contractile properties, is significantly impacted by changes at the mitochondrial level. Because of the mitochondrial dense nature and high energy demands of cardiac tissue, alterations in mitochondrial homeostasis have the capacity to significantly limit cardiac function. The purpose of this work is to understand how cardiac mitochondrion are altered in disease and pathological states, specifically in their adaptation to environmentally stimulated regulatory networks, such as epigenetic modifications and promotion/inhibition of non-coding RNAs.

Methods and Results: We examined both acute stress to mitochondrial regulation (inhalation toxicology) as well as chronic (type 2 diabetes mellitus). Inhalation of particulates at the nanoscale and its impact on molecular cardiovascular biology have been poorly evaluated. Through using a FVB transgenic microRNA-378a mouse knockout model, we examined the cardiovascular impact derived from altering the innate microRNA-378a response following acute nano- $\mathrm{TiO}_{2}$ inhalation exposure. Following inhalation exposure to nanomaterials, microRNA-378a was shown to be elevated. In complete knockout, and to a lesser extent partial knockout, of microRNA-378a, cardiac function was preserved. Through mitochondrial electron transport chain (ETC) complex activities and mitochondrial respiration, changes in substrate utilization were observed, specifically an increase in fatty acid metabolism in the knockout animals. MicroRNA-378a's regulation of mitofusin-1 (Mfn1), as well as other factors involved in mitochondrial metabolism, bioenergetics, and fission/fusion were shown to be altered in knockout and knockdown animals. 
The non-coding RNA network, which may serve as the primary response during acute exposure to inhaled toxicants, is dysregulated following inhalation exposure. While inhalation exposure serves as a means for inducing localized stress and observing the systemic alterations, diabetes is a chronic, systemic disease, affecting multiple organs and organ systems. In order to better diagnosis and treat type 2 diabetes, a better understanding is required of the cellular and molecular mechanisms during the onset and progression of the disease. In atrial tissue from 50 patients (30 non-diabetic and 20 type 2 diabetic) physiological, biochemical, genomic, and epigenomic factors were assessed in an attempt to better predict the pathogenesis of the disease in the heart. Using machine learning algorithms derived from Classification and Regression Trees (CART), we were able to determine that epigenetic factors were the most critical classifiers. Importantly, total, global 5-methylcytosine content of the genome, as well as methylation at the transcription factor A, mitochondrial (TFAM) promoter region, were most highly associated with diabetic status. Through using machine learning at the DNA level, this study begins to explore the potential of precision medicine-based approaches for disease classification and novel diagnostic measures. While machine learning can aid in the prediction of novel biomarkers, there remains much to be understood regarding the mitochondrion's role in diabetes, and the mechanisms of mitochondrial RNA import/export. Next-generation sequencing was performed on human patient and FVB mouse mitochondrial and cytoplasmic non-coding RNA populations. Assessment of non-coding RNA revealed a decreased RNA biodiversity in diabetic patients. Further, using a human, mouse, and cellular model, the binding of non-coding RNA to polynucleotide phosphorylase (PNPase) was confirmed, with a specific role of the PNPase KH and S1 binding domains crucial for long non-coding RNA binding. These findings suggest a role for PNPase in the import and/or export of long non-coding RNA during normal and pathogenic conditions.

Conclusions: Ultimately, the work summarized in the preceding experiments highlights how multiple pathological insults, whether chronic or acute, can influence the underlying molecular, regulatory networks in the heart. While overt cardiovascular and mitochondrial dysfunction follow insult, an emphasis on epigenetic control and non-coding RNA regulation may prove to be primary axes for therapeutic intervention. As we continue to pursue more informed and predictive assessments of cardiovascular dysfunction, the mitochondrion remains at the heart of the issue. 


\section{LIST OF ABBREVIATIONS AND DEFINITIONS}

$5 \mathrm{mC}$

$5 \mathrm{hmC}$

Anterograde Signaling

CART

ChIP

CLIP

$\mathrm{CpG}$

Cyto

$\mathrm{DbDb}$

ENM

H3K4me3

H3K27me3

IPA

$\mathrm{KH}$

LncRNA

MiRNA

Mito

ML

NcRNA

ND

$\mathrm{PM}_{0.1}, \mathrm{UFP}$

$\mathrm{PM}_{2.5}$

PNPase

PNPT1

Retrograde Signaling

S1 5-methylcytosine

5-hydroxymethylcytosine

Nuclear to Mitochondrial Signaling

Classification and Regression Trees

Chromatin Immunoprecipitation

Crosslinking-Immunoprecipitation

Cytosine followed Guanine (Nucleotides)

Cytoplasmic

Lepr $^{\mathrm{db}+/+}$ Mice

Engineered Nanomaterial

Histone 3 Lysine 4 trimethylation

Histone 3 Lysine 27 trimethylation

Ingenuity Pathway Analysis

KH RNA Binding Domain of PNPase

Long Non-Coding RNA

MicroRNA

Mitochondrial

Machine Learning

Non-Coding RNA

Non-Diabetic

Ultrafine Particulate Matter $<0.1 \mu \mathrm{m}$

Particulate Matter $<2.5 \mu \mathrm{m}$

Polynucleotide Phosphorylase

Polyribonucleotide Nucleotidyltransferase 1

Mitochondrion to Nucleus Signaling

S1 RNA Binding Domain of PNPase 
SHAP

SVM

T2DM

$\mathrm{TiO}_{2}$

$\mathrm{Tg}$

WT
SHapley Additive exPlanations

Support Vector Machines

Type 2 Diabetes Mellitus

Titanium Dioxide (Nano)

Transgenic

Wild type 


\section{TABLE OF CONTENTS}

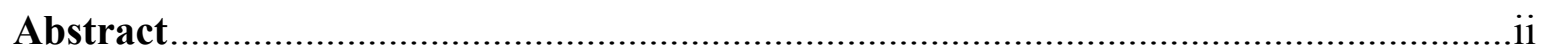

List of Abbreviations and Definitions ...................................................................

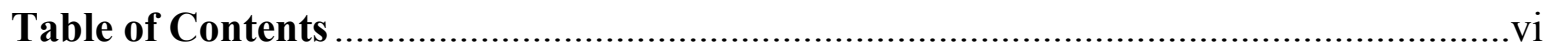

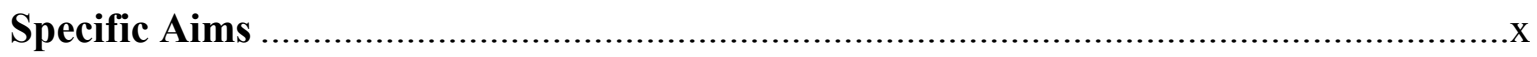

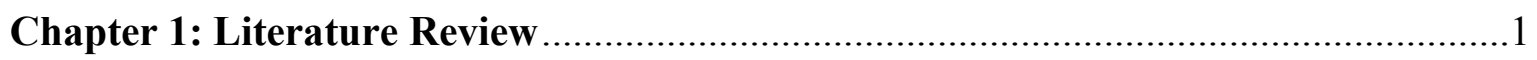

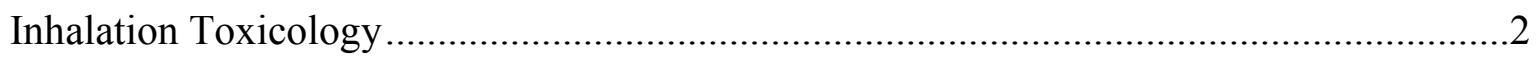

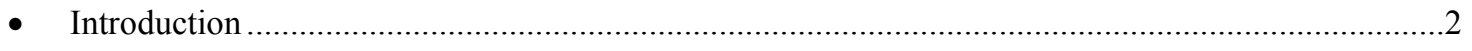

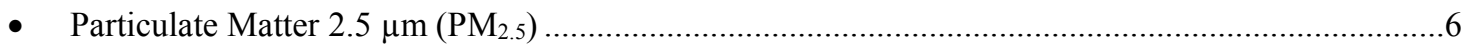

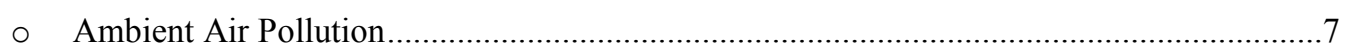

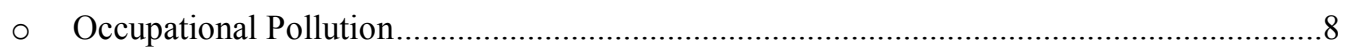

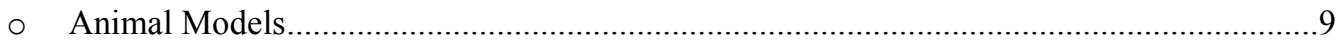

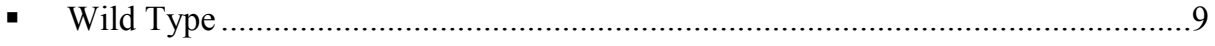

- Transgenic/Supplementation .........................................................................11

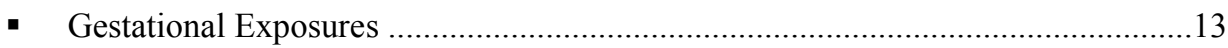

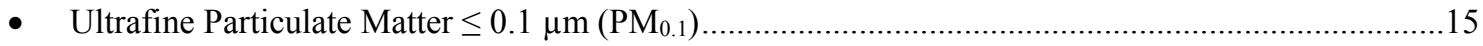

- Ambient Air and Occupational Pollution..........................................................................15

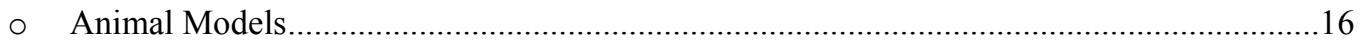

- Gestational Exposures ...........................................................................17

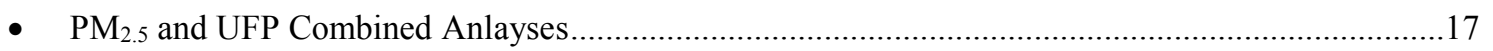

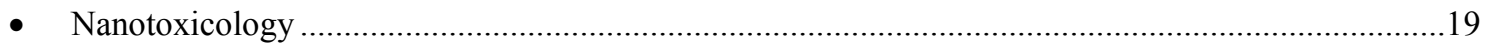

○ Animal Models...........................................................................................................

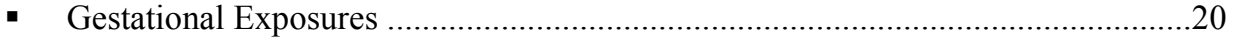

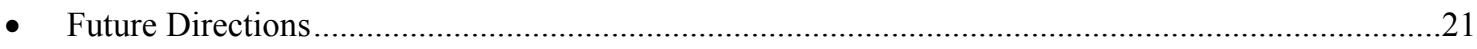

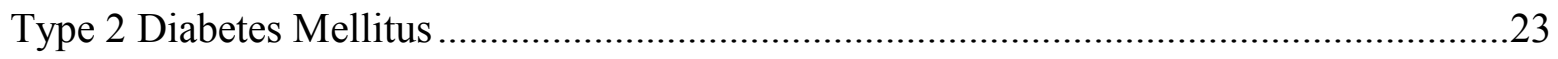

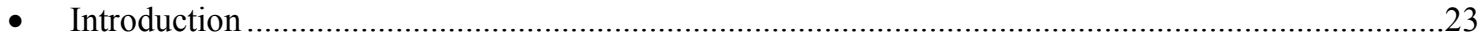

- MiRNA Regulation of Energy Substrate Metabolism in the Diabetic Heart........................................26

○ MiRNA Regulation of Insulin Signaling and Glycolysis....................................................26

- MiRNA Regulation of Fatty Acid Transport, Fatty Acid Oxidation, Citric Acid Cycle, Electron Transport Chain, and ATP Production .........................................................................2

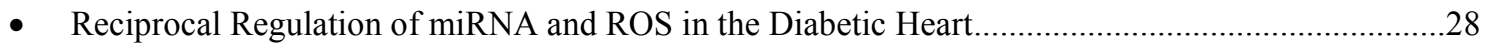

- MiRNA Regulation of Apoptosis in the Diabetic Heart......................................................................29 


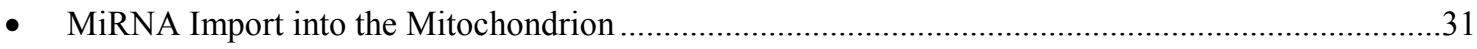

- Regulation of miRNAs Affecting the Diabetic and Non-Diabetic Cardiovascular System ...............32

- Regulation of miRNAs Altering the Epigenome of the Cardiovascular System .................................34

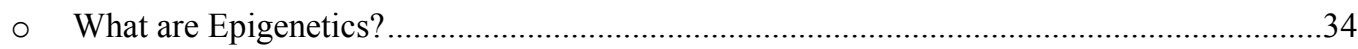

○ The Role of Epigenetics in Altering miRNA Expression ..............................................35

○ The Impact of Epigenetics and miRNA During Diabetes Mellitus ...................................37

- Exosomal Release, Transport, and Uptake of miRNA in the Cardiovascular System ......................39

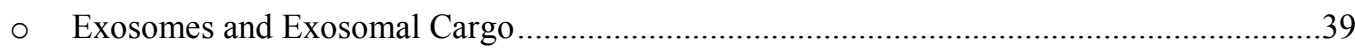

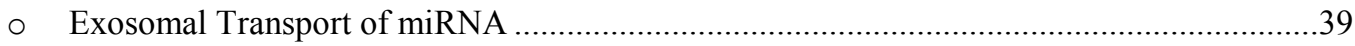

○ Exosomal Transport of miRNA in Diabetes Mellitus ....................................................41

- $\quad$ MiRNA Processing, LncRNA, and Other Regulators in the Cardiovascular System ......................42

○ Mechanisms of miRNA Processing and Post-transcriptional Control ...............................42

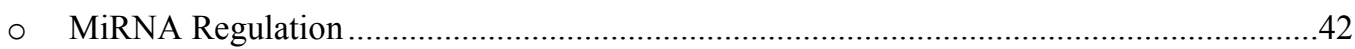

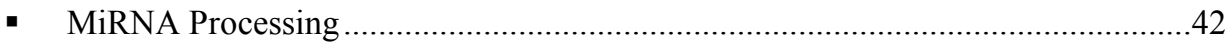

- MiRNA Post-Transcriptional Regulation ....................................................43

○ MiRNA Processing and LncRNA Regulation in the Diabetic Heart ...............................44

- $\quad$ Therapeutic Applications of miRNA in Diabetes Mellitus .........................................................45

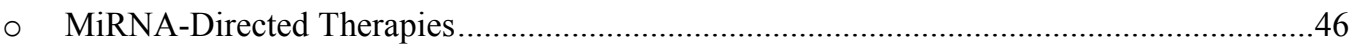

○ Epigenetics in miRNA Regulation during Therapeutic Intervention ................................47

- Exosomes in miRNA Regulation during Therapeutic Intervention .................................47

○ MiRNA Processing, Other Mechanism of Regulation, Therapeutic Intervention..................47

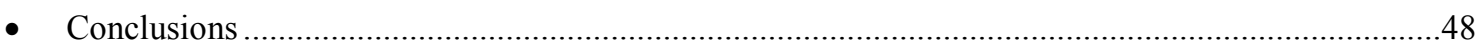

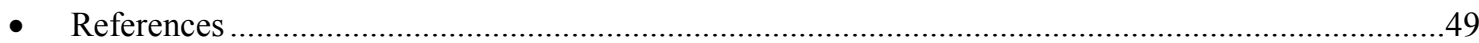

Chapter 2: Specific Aim 1 - MiRNA-378a as a Key Regulator of Cardiovascular Health

Following Engineered Nanomaterial Inhalation Exposure....................................69

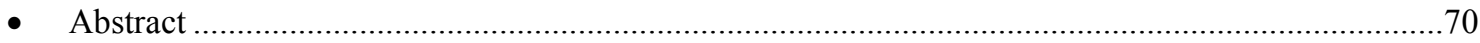

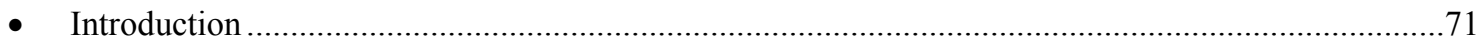

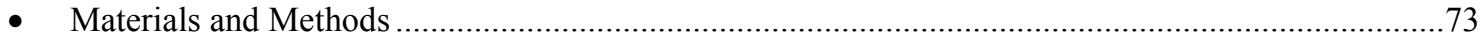

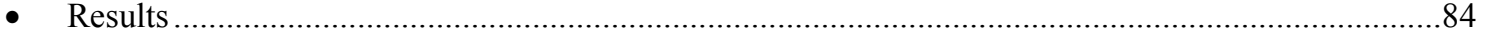

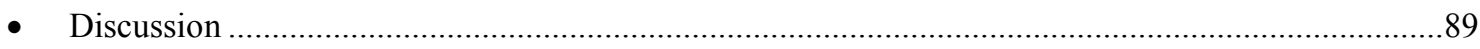

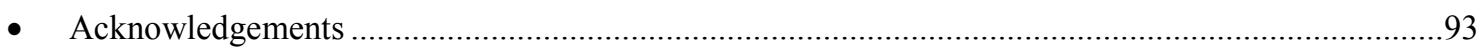

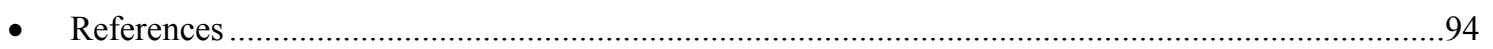

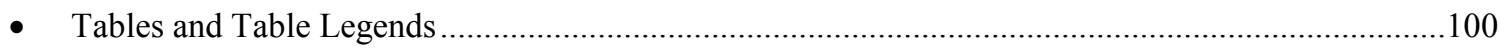

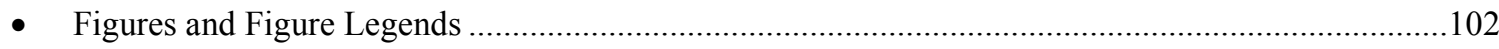

- Supplemental Tables and Table Legends ....................................................................... 120

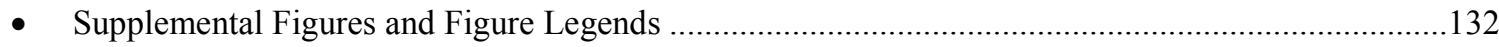


Chapter 3: Specific Aim 2 - Machine-Learning to Stratify Diabetic Patients using Novel

Cardiac Biomarkers and Integrative Genomics ............................................... 149

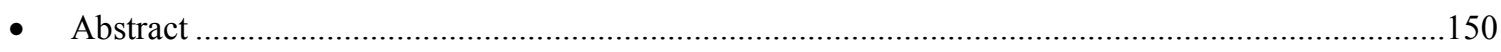

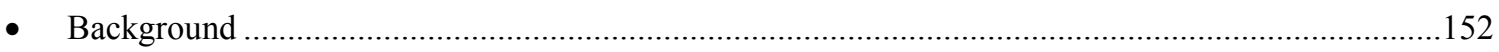

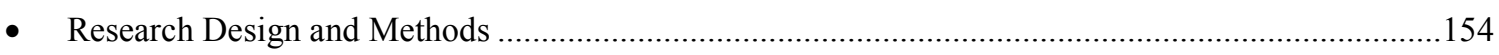

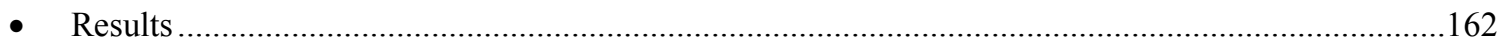

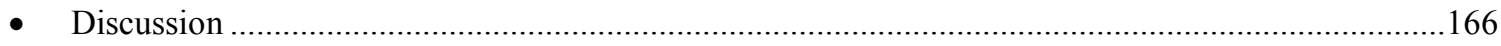

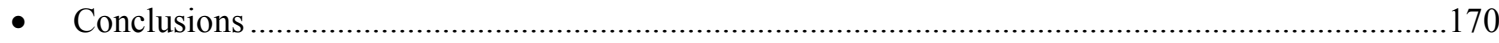

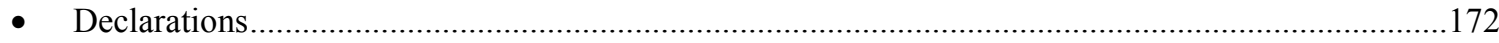

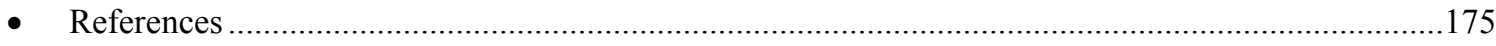

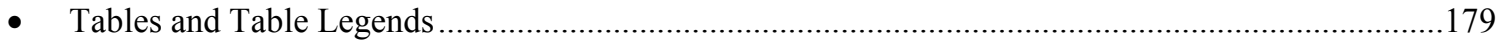

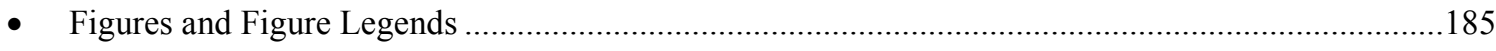

- Supplemental Tables and Table Legends ..............................................................................................204

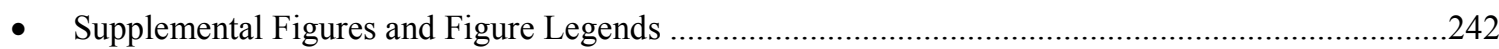

Chapter 4: Specific Aim 3 - The Mitochondrial Regulatory Network: Subcellular

Localization of Non-Coding RNA in the Heart .........................................................252

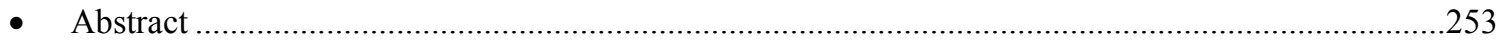

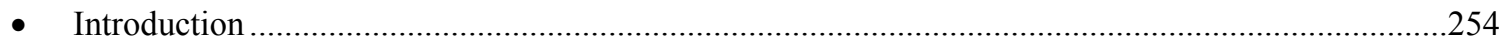

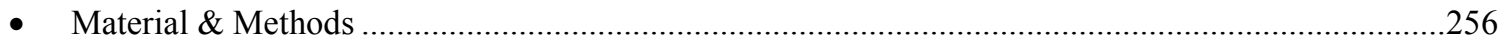

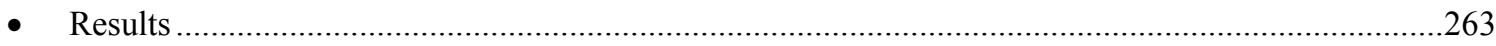

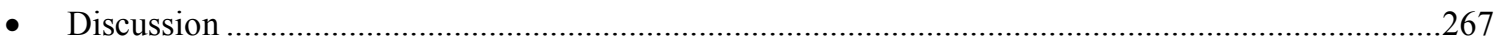

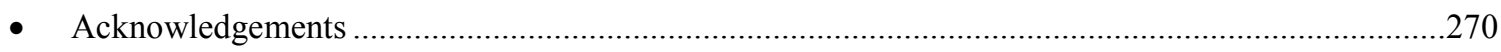

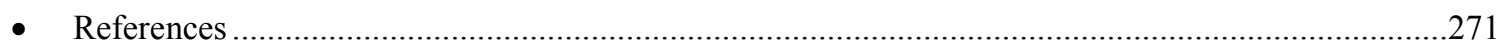

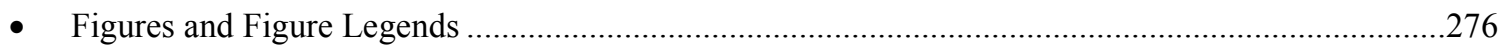

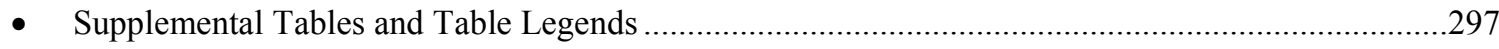

- Supplemental Figures and Figure Legends ……………….................................................................301

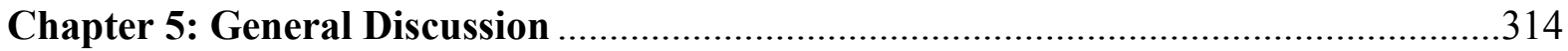

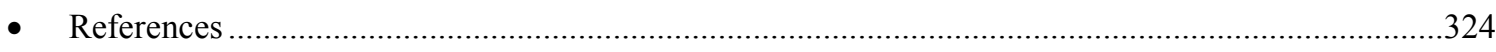

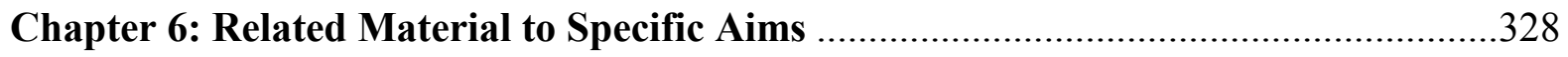

Section 1.1: Maternal Engineered Nanomaterial Exposure Disrupts Progeny Cardiac Function

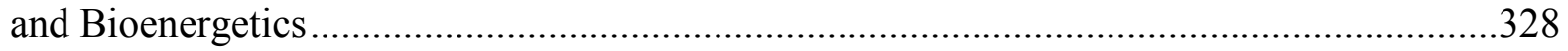

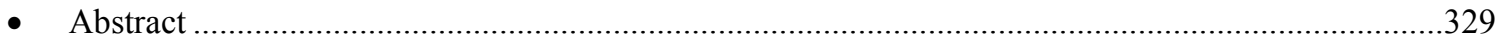




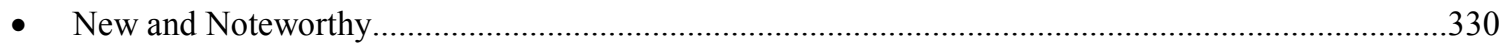

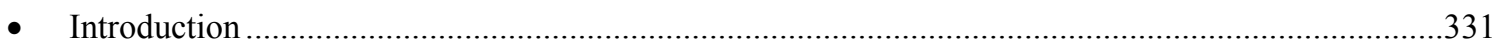

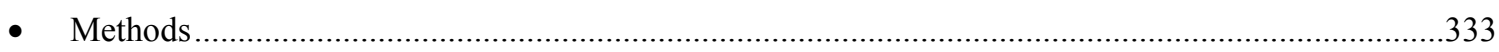

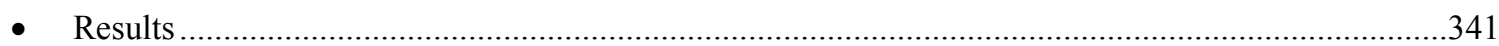

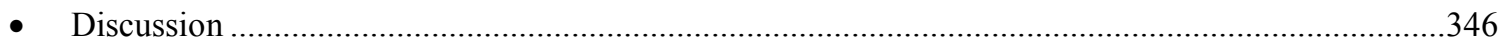

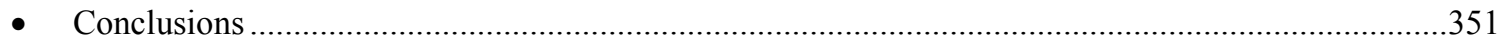

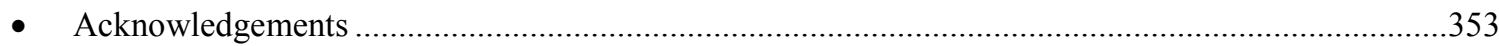

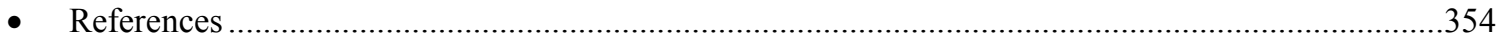

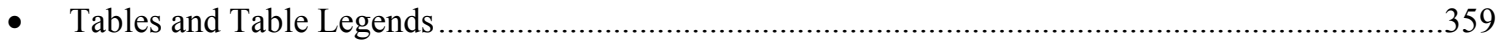

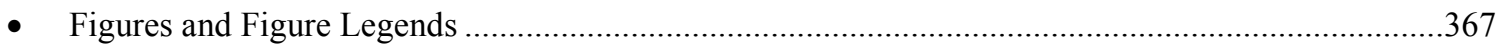

Section 1.2: Maternal Engineered Nanomaterial Inhalation During Gestation Alters the Fetal

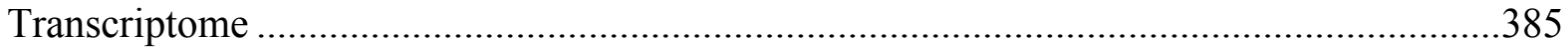

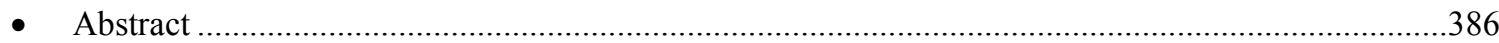

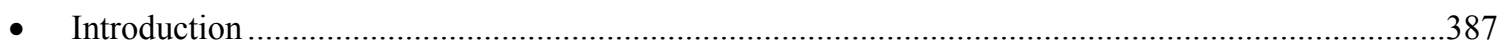

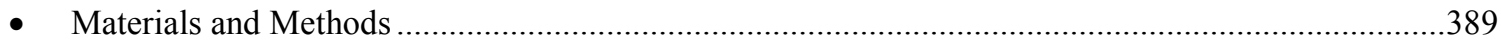

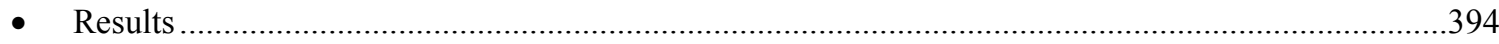

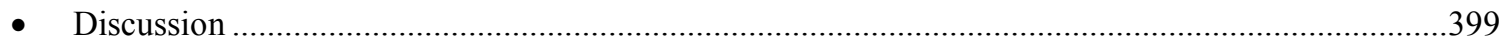

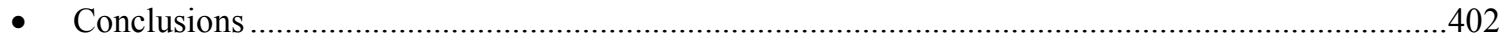

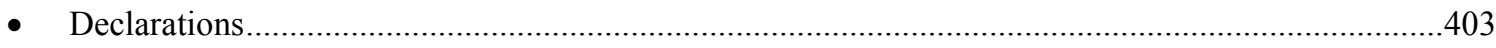

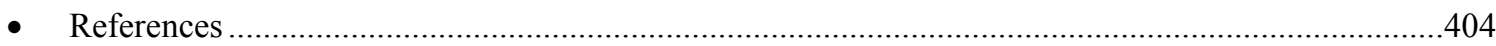

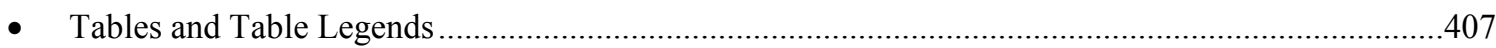

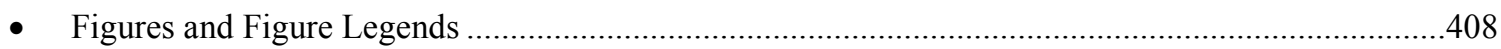

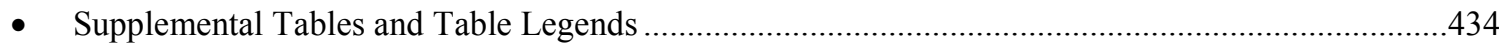

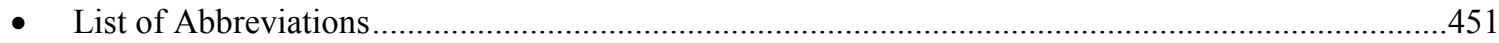

Section 2: Repository for Cardiovascular Studies ........................................................453

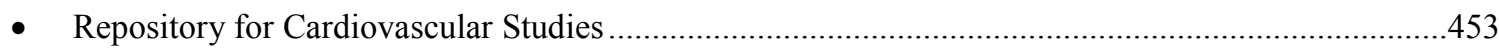

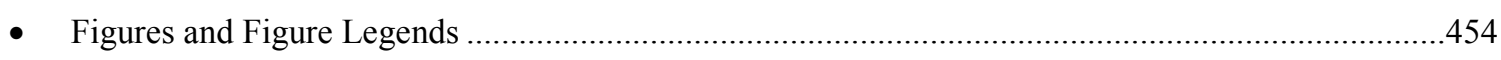

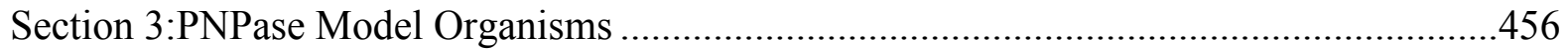

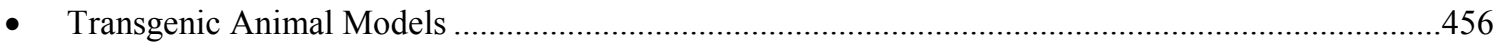

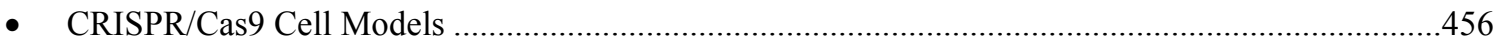

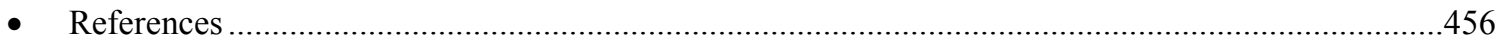

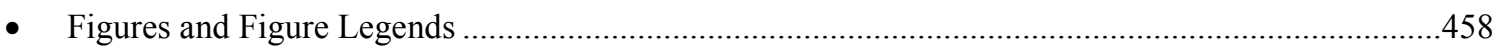

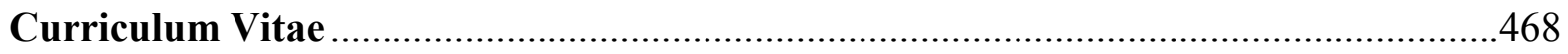




\section{Specific Aims:}

Maintenance of the cardiovascular system is vital in sustaining systemic homeostasis from both disease-related and xenobiotic-induced pathology. In the heart, diabetes mellitus $(5,8,9)$ and engineered nanomaterial (ENM) $(15,24,30)$ exposure affect cellular adaption through alterations in mitochondrial function. Variations to substrate utilization, increased reactive oxygen species, and eventual changes in mitochondrial bioenergetic capacity contribute to cellular dysfunction and ultimately cardiovascular complications through alterations to pump function (19). What remains unclear is how these environmental changes impact the regulatory networks of the heart, primarily the involvement of non-coding RNA (ncRNA) and epigenetics in altering mitochondrial health. The objective of this application is to understand how environmental factors, in the context of systemic disease and xenobiotic insult, alter cardiac function through ncRNAs and epigenetics. The long-term goal is to detect targets in these regulatory systems and therapeutically alter their expression to positively impact mitochondrial, cellular, and heart health.

My central hypothesis is that changes in the epigenome through DNA methylation and histone modifications alter the ncRNA transcriptome subsequently changing the import dynamics of microRNA (miRNA), long non-coding RNA (lncRNA), and other ncRNA into the mitochondrion and affecting mitochondrial function through RNA-RNA and RNA-protein interactions; ultimately, producing a feedback loop where mitochondrial substrate availability shapes nuclear epigenetic machinery. Following both diabetes mellitus $(3,7,13,32)$ and ENM exposure $(20,21,30)$ paradigms, epigenetic control of nuclear encoded-mitochondrial genes as well as transcription are altered and a shift in the ncRNA transcriptome in both the cytoplasm and mitochondrion is precipitated. This proposal seeks to expand the current knowledge of ncRNA and epigenetics as it relates to cardiovascular health following diabetic and ENM insult. There is a gap in knowledge regarding the mechanisms promoting and sustaining mitochondrial dysfunction following cardiac insult; justifying a need for research to expand our current understanding of the ncRNA/epigenetic interplay shaping environmental stimulation. This gap in knowledge will be addressed by experimentation directly assessing ncRNA altered in disease states and how these ncRNA can be targeted for therapeutic interventions, restoring mitochondrial function and ultimately cardiovascular function. 
Specific Aim 1: Determine the role of ncRNA in regulating cardiac and mitochondrial function following exposure to ENMs. Our working hypothesis is that ENM inhalation exposure alters ncRNA transcription and subsequent downstream regulation, affecting miRNA regulation of cardiac function through inhibiting/promoting cellular pathways. MiRNA-378a knockout and wild type mice will be exposed to nano titatinum dioxide $\left(\mathrm{Nano}-\mathrm{TiO}_{2}\right)$ and cardiac and mitochondrial function determined. Regulation of the miRNA-378a host gene and miRNA-378a targeted repression will be evaluated.

1.1 Determine the cardiovascular function of wild type, knockdown, and knockout expression for miRNA-378a using echocardiography and speckle-tracking stress strain.

1.2 Examine metabolic and ultrastructure effects of inhalation nano- $\mathrm{TiO}_{2}$ on mitochondria, and if miRNA-378a depletion can restore function.

1.3 Find specific targets of miRNA-378a that regulate metabolism, bioenergetics, and/or mitochondrial fission/fusion.

The expected outcomes of Specific Aim 1 are that miRNA-378a is upregulated following nano$\mathrm{TiO}_{2}$ exposure due to increased transcription of its host gene, repressing fatty acid metabolism, and that knockout of miRNA-378a can rescue mitochondrial function and ultrastructure through decreased regulation of constituents related to metabolism.

Specific Aim 2: Understand the cross talk between mitochondrial and nuclear regulator networks in diabetes mellitus using machine learning predictive algorithms. Our working hypothesis is that alterations to the genomic/epigenomic landscape in the nucleus can be correlated to mitochondrial function in patients with diabetes mellitus. Human right atrial tissue from type 2 diabetic and nondiabetic patients will be used to assess physiological, biochemical, genetic, and epigenetic markers and evaluated through predictive machine learning algorithms.

2.1 Tissue from 50 patients ( 30 non-diabetic and 20 type 2 diabetic) will be collected from the Ruby Memorial Hospital, Heart and Vascular Institute.

2.2 Biomarkers and risk factors measured through a variety of indices will be placed into a machine learning algorithm (SHapley Additive exPlanations) based around Classification and Regression Tress (CART). 
2.3 The most predictive biomarkers for diabetic status will be chosen and compared with the efficiency of common diagnostics, such as glycosylated hemoglobin (HbA1c).

The expected outcomes for Specific Aim 2 are that changes in substrate availability in the mitochondrion, e.g. S-adenosyl-L-methionine, likely regulate DNA methylation in the nuclear genome and that predictive machine learning algorithms can be used to measure the diagnostic and therapeutic potential of genetic/epigenetic modifications in diabetic onset and progression.

Specific Aim 3: Evaluate the mechanisms of non-coding RNA import/export involving the mitochondrion in diabetes mellitus and the role of Polynucleotide Phosphorylase (PNPase) in noncoding RNA binding. Our working hypothesis is that mitochondrial import of ncRNAs are significantly diminished in diabetic mitochondria and that PNPase can regulate the long noncoding import/export mechanisms of the mitochondrial regulatory network through its $\mathrm{KH}$ and S1 RNA binding domains. Using human patient, mouse, and cell models, the mitochondrial ncRNA network will be evaluated and how proteins, such as PNPase, may contribute to changes in ncRNA flux.

3.1 RNA from isolated mitochondria and cytoplasmic compartments from human right atrial tissue and FVB mouse whole heart will be assessed for lncRNA presence and potential interaction capacity with miRNA, mRNA, and other RNA species.

3.2 Cross-linking immunoprecipitation (CLIP) of PNPase in mitochondria to determine ncRNA interaction sites and species involved.

3.3 Using HL-1 cells, determine how the KH and S1 RNA binding domains of PNPase alter binding capacity with ncRNA.

The expected outcomes of Specific Aim 3 are that diabetes induces a reduction in total non-coding RNA biodiversity, altering the ncRNA regulatory network in the mitochondrion. PNPase, through the $\mathrm{KH}$ and S1 RNA binding domains directly interacts with lncRNA, potentially exporting/degrading specific lncRNA involved with the mitochondrion. 
The outcomes of these studies will significantly contribute toward the understanding of cardiovascular physiology, identification of therapeutic targets, and assist the development of treatment modalities for diabetic and ENM cardiovascular dysfunction. 


\section{$\underline{\text { Research Strategy }}$}

Significance: Cardiovascular diseases remain the top cause of death worldwide, with over 800,000 deaths in the United States alone, equating to 1 death every 40 seconds (2). Preservation and therapeutic treatment of the cardiovascular system is vital for mitigating co-morbidities and even death. Understanding how insults to the cardiovascular system can alter normal, homeostatic function can provide a more dynamic picture of the adaptive, molecular systems that exist; specifically, both through a systemic, chronic insult (diabetes mellitus) and through acute, direct action (engineered nanomaterial (ENM) exposure). Diabetes mellitus is a systemic pathology, resulting in cellular and tissue dysfunction across multiple organ systems $(1,4,23,27)$. Diabetic cardiomyopathy and other cardiovascular complications resulting from the disease comprise a major co-morbidity as well as the primary cause of mortality in patients with diabetes mellitus (12, 18). The cardiovascular system is also detrimentally affected through engineered nanomaterial exposure (ENM) $(17,22,24)$. Inhalation exposure to ENMs produce acute cardiac abnormalities and could contribute to sustained cardiovascular and systemic dysfunction over time $(10,25)$. Nano- $\mathrm{TiO}_{2}$ is one of the most prominently produced ENM $(11,16)$ and could pose a significant health risk occupationally, to consumers, or even environmentally.

The scientific premise for this dissertation work considers both cardiovascular physiology and molecular mechanisms related to mitochondrial structure and function. Currently, characterization of diabetes mellitus in the cardiovascular system has shown global epigenetic changes through DNA methylation and histone methylation $(28,31)$. With these changes, epigenetics have also been shown to manipulate ncRNA expression $(6,26)$. Though ncRNA such as miRNA and lncRNA have been shown to change following diabetic insult, their contribution to altering cardiac function has been limitedly explored, specifically in the context of mitochondrial health. Epigenetic and ncRNA alterations are less exaggerated following ENM inhalation exposure $(14,29)$ but the degree in alterations of the internal regulatory network provide a spectrum of ncRNA expression and epigenetic changes following cardiovascular insult. We have begun to assess the outcomes of epigenetics and ncRNA expression following both diabetic and ENM inhalation exposure. We have shown that following diabetes mellitus, the ncRNA in the mitochondrion are altered, and that specific proteins associated with the mitochondrion, Argonaut 2 (AGO2) and PNPase, are shown to be immunoprecipitated together and could provide a means 
for mitochondrial ncRNA import. Additionally, the expression of miRNAs involved with metabolism are altered following inhalation exposure to nano- $\mathrm{TiO}_{2}$, suggesting a role in regulating mitochondrial function.

Innovation: At current, no study has fully detailed the global ncRNA mitochondrial import/export pathway, the purpose of the ncRNA mitochondrial transcriptome, or how epigenetics influences the role of ncRNA and the mitochondrion. The proposed studies provide innovation conceptually, methodologically and mechanistically.

1. Conceptually Innovative: The central hypothesis proposes a regulatory network of how mitochondrial bioenergetics affect nuclear transcription, and vice versa. Through epigenetic control in the nucleus and transcription of ncRNAs which translocate to the mitochondrion and control bioenergetic regulation, the hypothesis provides a novel conceptual idea of the specific constituents involved in cellular homeostasis under pathological conditions.

2. Methodologically Innovative: While machine learning algorithms have been applied to biomarkers in predicting diabetes and other diseases, no study has used machine learning to assess patient-matched genetic/epigenetic features at base-pair resolution in diabetes. For the first time, we assess how machine learning can be used as a personalized medicine approach for identifying epigenetic changes in the diabetic heart.

3. Mechanistically Innovative: The primary ncRNA import pathway has yet to be elucidated. We propose PNPase as major constituents in the mitochondrial ncRNA import pathway. We further suggest that PNPase/lncRNA interactions through the KH and S1 RNA binding domains could explain alterations to mitochondrial ncRNA diversity.

The epigenetic/ncRNA model proposed provides not only innovative approaches, but also the propensity for therapeutic applications. AntagomiR therapy and lncRNA sponging provide simple and adaptive therapeutic intervention measures for regulating the expression of ncRNA in the mitochondrion. 


\section{References}

1. Alberti KG and Zimmet PZ. Definition, diagnosis and classification of diabetes mellitus and its complications. Part 1: diagnosis and classification of diabetes mellitus provisional report of a WHO consultation. Diabet Med 15: 539-553, 1998.

2. Benjamin EJ, Blaha MJ, Chiuve SE, Cushman M, Das SR, Deo R, de Ferranti SD, Floyd J, Fornage M, Gillespie C, Isasi CR, Jimenez MC, Jordan LC, Judd SE, Lackland D, Lichtman JH, Lisabeth L, Liu S, Longenecker CT, Mackey RH, Matsushita K, Mozaffarian D, Mussolino ME, Nasir K, Neumar RW, Palaniappan L, Pandey DK, Thiagarajan RR, Reeves MJ, Ritchey M, Rodriguez CJ, Roth GA, Rosamond WD, Sasson C, Towfighi A, Tsao CW, Turner MB, Virani SS, Voeks JH, Willey JZ, Wilkins JT, Wu JH, Alger HM, Wong SS, Muntner P, American Heart Association Statistics C, and Stroke Statistics S. Heart Disease and Stroke Statistics-2017 Update: A Report From the American Heart Association. Circulation 135: e146e603, 2017.

3. Bian Z, Li LM, Tang R, Hou DX, Chen X, Zhang CY, and Zen K. Identification of mouse liver mitochondria-associated miRNAs and their potential biological functions. Cell Res 20: 1076-1078, 2010.

4. Brownlee M. Biochemistry and molecular cell biology of diabetic complications. Nature 414: 813820, 2001.

5. Bugger $\mathbf{H}$ and Abel ED. Mitochondria in the diabetic heart. Cardiovasc Res 88: 229-240, 2010.

6. Chang CP and Han P. Epigenetic and IncRNA regulation of cardiac pathophysiology. Biochim Biophys Acta 1863: 1767-1771, 2016.

7. Cheng $\mathbf{Z}$ and Almeida FA. Mitochondrial alteration in type 2 diabetes and obesity: an epigenetic link. Cell Cycle 13: 890-897, 2014.

8. Dabkowski ER, Williamson CL, Bukowski VC, Chapman RS, Leonard SS, Peer CJ, Callery PS, and Hollander JM. Diabetic cardiomyopathy-associated dysfunction in spatially distinct mitochondrial subpopulations. Am J Physiol Heart Circ Physiol 296: H359-369, 2009.

9. Duncan JG. Mitochondrial dysfunction in diabetic cardiomyopathy. Biochim Biophys Acta 1813: 1351-1359, 2011.

10. Eisen EA, Costello S, Chevrier J, and Picciotto S. Epidemiologic challenges for studies of occupational exposure to engineered nanoparticles; a commentary. J Occup Environ Med 53: S57-61, 2011.

11. Gottschalk F, Lassen C, Kjoelholt J, Christensen F, and Nowack B. Modeling flows and concentrations of nine engineered nanomaterials in the Danish environment. Int J Environ Res Public Health 12: 5581-5602, 2015.

12. Grundy SM, Benjamin IJ, Burke GL, Chait A, Eckel RH, Howard BV, Mitch W, Smith SC, Jr., and Sowers JR. Diabetes and cardiovascular disease: a statement for healthcare professionals from the American Heart Association. Circulation 100: 1134-1146, 1999.

13. Guay C, Roggli E, Nesca V, Jacovetti C, and Regazzi R. Diabetes mellitus, a microRNA-related disease? Transl Res 157: 253-264, 2011.

14. Halappanavar S, Jackson P, Williams A, Jensen KA, Hougaard KS, Vogel U, Yauk CL, and Wallin H. Pulmonary response to surface-coated nanotitanium dioxide particles includes induction of acute phase response genes, inflammatory cascades, and changes in microRNAs: a toxicogenomic study. Environ Mol Mutagen 52: 425-439, 2011.

15. Hathaway QA, Nichols CE, Shepherd DL, Stapleton PA, McLaughlin SL, Stricker JC, Rellick SL, Pinti MV, Abukabda AB, McBride CR, Yi J, Stine SM, Nurkiewicz TR, and Hollander JM. Maternal-engineered nanomaterial exposure disrupts progeny cardiac function and bioenergetics. Am J Physiol Heart Circ Physiol 312: H446-H458, 2017.

16. Hendren CO, Mesnard X, Droge J, and Wiesner MR. Estimating production data for five engineered nanomaterials as a basis for exposure assessment. Environ Sci Technol 45: 2562-2569, 2011. 
17. Holland NA, Thompson LC, Vidanapathirana AK, Urankar RN, Lust RM, Fennell TR, and Wingard CJ. Impact of pulmonary exposure to gold core silver nanoparticles of different size and capping agents on cardiovascular injury. Part Fibre Toxicol 13: 48, 2016.

18. Kannel WB and McGee DL. Diabetes and cardiovascular disease. The Framingham study. JAMA 241: 2035-2038, 1979.

19. Lesnefsky EJ, Moghaddas S, Tandler B, Kerner J, and Hoppel CL. Mitochondrial dysfunction in cardiac disease: ischemia--reperfusion, aging, and heart failure. J Mol Cell Cardiol 33: 1065-1089, 2001.

20. Li S, Wang H, Qi Y, Tu J, Bai Y, Tian T, Huang N, Wang Y, Xiong F, Lu Z, and Xiao Z. Assessment of nanomaterial cytotoxicity with SOLiD sequencing-based microRNA expression profiling. Biomaterials 32 : 9021-9030, 2011.

21. Lu X, Miousse IR, Pirela SV, Melnyk S, Koturbash I, and Demokritou P. Short-term exposure to engineered nanomaterials affects cellular epigenome. Nanotoxicology 10: 140-150, 2016.

22. Miller MR, Raftis JB, Langrish JP, McLean SG, Samutrtai P, Connell SP, Wilson S, Vesey AT, Fokkens PHB, Boere AJF, Krystek P, Campbell CJ, Hadoke PWF, Donaldson K, Cassee FR, Newby DE, Duffin R, and Mills NL. Inhaled Nanoparticles Accumulate at Sites of Vascular Disease. ACS Nano 11: 45424552, 2017.

23. Nathan DM. Long-term complications of diabetes mellitus. N Engl J Med 328: 1676-1685, 1993.

24. Nichols CE, Shepherd DL, Hathaway QA, Durr AJ, Thapa D, Abukabda A, Yi J, Nurkiewicz TR, and Hollander JM. Reactive oxygen species damage drives cardiac and mitochondrial dysfunction following acute nano-titanium dioxide inhalation exposure. Nanotoxicology 12: 32-48, 2018.

25. Pirela SV, Martin J, Bello D, and Demokritou P. Nanoparticle exposures from nano-enabled tonerbased printing equipment and human health: state of science and future research needs. Crit Rev Toxicol 47: 678-704, 2017.

26. Sato F, Tsuchiya S, Meltzer SJ, and Shimizu K. MicroRNAs and epigenetics. FEBS J 278: 1598-1609, 2011.

27. Schalkwijk CG and Stehouwer CD. Vascular complications in diabetes mellitus: the role of endothelial dysfunction. Clin Sci (Lond) 109: 143-159, 2005.

28. Schones DE, Leung A, and Natarajan R. Chromatin Modifications Associated With Diabetes and Obesity. Arterioscler Thromb Vasc Biol 35: 1557-1561, 2015.

29. Snyder-Talkington BN, Dong C, Sargent LM, Porter DW, Staska LM, Hubbs AF, Raese R, McKinney W, Chen BT, Battelli L, Lowry DT, Reynolds SH, Castranova V, Qian Y, and Guo NL. mRNAs and miRNAs in whole blood associated with lung hyperplasia, fibrosis, and bronchiolo-alveolar adenoma and adenocarcinoma after multi-walled carbon nanotube inhalation exposure in mice. J Appl Toxicol 36: 161174, 2016.

30. Stapleton PA, Hathaway QA, Nichols CE, Abukabda AB, Pinti MV, Shepherd DL, McBride CR, Yi J, Castranova VC, Hollander JM, and Nurkiewicz TR. Maternal engineered nanomaterial inhalation during gestation alters the fetal transcriptome. Part Fibre Toxicol 15: 3, 2018.

31. Wahl S, Drong A, Lehne B, Loh M, Scott WR, Kunze S, Tsai PC, Ried JS, Zhang W, Yang Y, Tan S, Fiorito G, Franke L, Guarrera S, Kasela S, Kriebel J, Richmond RC, Adamo M, Afzal U, Ala-Korpela M, Albetti B, Ammerpohl O, Apperley JF, Beekman M, Bertazzi PA, Black SL, Blancher C, Bonder MJ, Brosch M, Carstensen-Kirberg M, de Craen AJ, de Lusignan S, Dehghan A, Elkalaawy M, Fischer K, Franco OH, Gaunt TR, Hampe J, Hashemi M, Isaacs A, Jenkinson A, Jha S, Kato N, Krogh V, Laffan M, Meisinger C, Meitinger T, Mok ZY, Motta V, Ng HK, Nikolakopoulou Z, Nteliopoulos G, Panico S, Pervjakova N, Prokisch H, Rathmann W, Roden M, Rota F, Rozario MA, Sandling JK, Schafmayer C, Schramm K, Siebert R, Slagboom PE, Soininen P, Stolk L, Strauch K, Tai ES, Tarantini L, Thorand B, Tigchelaar EF, Tumino R, Uitterlinden AG, van Duijn C, van Meurs JB, Vineis $P$, Wickremasinghe AR, Wijmenga $C$, Yang TP, Yuan W, Zhernakova A, Batterham RL, Smith GD, Deloukas P, Heijmans BT, Herder C, Hofman A, Lindgren CM, Milani L, van der Harst P, Peters A, Illig T, Relton CL, Waldenberger M, Jarvelin MR, Bollati V, Soong R, 
Spector TD, Scott J, McCarthy MI, et al. Epigenome-wide association study of body mass index, and the adverse outcomes of adiposity. Nature 541: 81-86, 2017.

32. Zhong $Q$ and Kowluru RA. Epigenetic changes in mitochondrial superoxide dismutase in the retina and the development of diabetic retinopathy. Diabetes 60: 1304-1313, 2011. 


\section{Chapter 1:}

\section{Literature Review}




\section{Inhalation Toxicology}

\section{Introduction}

Irrespective of diet, physical fitness, or other controllable risk factors, the quality, and content of the air we breathe is often unavoidable. Specifically, it has been shown that combustion products, particulate matter $<2.5 \mu \mathrm{m}$, and emissions of engineered materials at the nanoscale, all contribute to physiological disturbances $(59,116,117)$. While the pulmonary system is considered the first site of interaction with these particles, the heart and circulatory system may be subject to the most detrimental, long-term effects. Chronic and acute exposures to particulate matter $<2.5$ $\mu \mathrm{m}\left(\mathrm{PM}_{2.5}\right)$ and ultrafine particulate matter $<0.1 \mu \mathrm{m}\left(\mathrm{PM}_{0.1}\right.$, UFP) have been associated with an increased risk of cardiovascular disease, including a predisposition to cardiovascular morbidity and mortality $(21,205)$.

The overt, epidemiological risk of cardiovascular injury following inhalation exposure to toxicants has been well documented $(21,83)$ and includes increased propensity for ischemic heart disease, heart failure, arrhythmias, atherosclerosis, and other cardiovascular complications. While a large body of evidence suggests cardiovascular maladaptation following pulmonary exposure, within the last ten years studies have begun to examine the cellular and molecular mechanisms behind the induced cardiovascular decline. In this review, we will summarize those studies examining the genetic, epigenetic, proteomic, and metabolic adaptions to inhaled toxicants through both primary (the individual) and secondary (gestational, maternal and progeny) exposure. We will specifically examine the cardiovascular effects of ambient and occupational inhalation exposure from $\mathrm{PM}_{2.5}$, UFP, and engineered nanomaterials (ENMs) (Table 1.1). The purpose of this review is to provide a comparative narrative to the field of molecular cardiology on the mechanisms involved in various inhalation toxicology exposure paradigms, with the hope of inciting future investigations into areas germane to understanding the effects of cardiovascular inhalation toxicology.

Table 1.1: Summarized Recent Findings

\begin{tabular}{|c|c|c|c|}
\hline Study & Findings & Model/Exposure & Administered Concentration \\
\hline \multicolumn{4}{|c|}{$\mathbf{P M}_{2.5}$} \\
\hline \multicolumn{4}{|c|}{ Ambient Air Pollution } \\
\hline (143) & $\begin{array}{l}\text { Decreased methylation of Alu (carbon black) and } \\
\text { LINE-1 }\left(\mathrm{SO}_{4}\right)\end{array}$ & $\begin{array}{l}\text { Normative Aging Study, } 706 \\
\text { individuals }\end{array}$ & $\begin{array}{l}\mathrm{PM}_{2.5} \text { data collected from Harvard School of Public Health } \\
\text { during January } 1995 \text { to November } 2007\end{array}$ \\
\hline
\end{tabular}




\begin{tabular}{|c|c|c|c|}
\hline (272) & $\begin{array}{l}\text { Higher } \mathrm{PM}_{2.5} \text { correlated with increased blood } \\
\text { TLR2 methylation, flavonoid intake decreased } \\
\text { TLR2 methylation }\end{array}$ & $\begin{array}{l}\text { Normative Aging Study, } 573 \\
\text { participants }\end{array}$ & $\mathrm{PM}_{2.5}$ data collected from Harvard School of Public Health \\
\hline (47) & $\begin{array}{l}\mathrm{Fe}, \mathrm{Ni}, \mathrm{V} \text { were all correlated with SLFN11 } \\
\text { cg10911913 methylation }\end{array}$ & $\begin{array}{l}\text { Normative Aging Study, } 646 \\
\text { participants }\end{array}$ & $\begin{array}{l}\mathrm{PM}_{2.5}, 1 \text { year moving average, between } 1999 \text { and 2013, } \\
\text { Harvard University Countway Library }\end{array}$ \\
\hline (18) & $\begin{array}{l}\text { Fine and coarse } \mathrm{PM}_{2.5} \text { decreased Alu and TLR } 4 \\
\text { methylation }\end{array}$ & 15 local participants & $\begin{array}{l}\mathrm{PM}_{2.5} \text { collected in downtown Toronto. Administered } 250 \\
\text { and } 200 \mu \mathrm{g} / \mathrm{m}^{3} \text { per session }\end{array}$ \\
\hline (119) & $\begin{array}{l}54 \text { circulating miRNAs were correlated with dose } \\
\text { of exposure and pollution type }\end{array}$ & 24 non-smoking adults & $\begin{array}{l}\mathrm{PM}_{2.5} \text { collected on Oxford Street in London, 2-hour } \\
\text { exposure }\end{array}$ \\
\hline (131) & $\begin{array}{l}\text { Acute exposure resulted in elevated CRP, IL-6, and } \\
\text { TNFR2 }\end{array}$ & $\begin{array}{l}\text { Framingham Offspring cohort } \\
\text { cycle } 7 \text { and } 8\end{array}$ & $\begin{array}{l}\mathrm{PM}_{2.5} \text { collected the Boston Harvard Supersite and assessed } \\
\text { as 7-day moving average }\end{array}$ \\
\hline \multicolumn{4}{|c|}{ Occupational Exposure } \\
\hline (69) & $\begin{array}{l}\text { Increased LINE-1 methylation with increasing } \\
\text { dose of exposure }\end{array}$ & 66 male participants & $\begin{array}{l}\mathrm{PM}_{2.5} \text { collected January } 2010 \text { to June } 2012 \text { in Boilermaker } \\
\text { Union Local 29, Quincy, MA }\end{array}$ \\
\hline (23) & $\begin{array}{l}\text { Peripheral blood leukocytes mtDNA D-Loop } \\
\text { methylation was associated with exposure }\end{array}$ & 48 male participants & $\begin{array}{l}\mathrm{PM}_{2.5} \text { collected January } 2007 \text { to June } 2012 \text { in Boilermaker } \\
\text { Union Local 29, Quincy, MA }\end{array}$ \\
\hline (40) & $\begin{array}{l}\text { Elemental carbon ( } 48 \text { genes), } \mathrm{PM}_{2.5}(49 \text { genes }) \text { and } \\
\text { organic carbon ( } 260 \text { genes) differentially effected } \\
\text { the transcriptome }\end{array}$ & $\begin{array}{l}63 \text { pick-up and delivery drivers } \\
\text { and dock workers }\end{array}$ & $\begin{array}{l}\mathrm{PM}_{2.5} \text { collected February } 2009 \text { and October } 2010 \text { from } 10 \\
\text { trucking terminals in the northeastern US (CT, MA, MD, } \\
\text { NJ, NY, and PA). }\end{array}$ \\
\hline (81) & $\begin{array}{l}\text { ET-1 was reduced following exposure but was not } \\
\text { modified by exercise intensity }\end{array}$ & 18 male participants & $\begin{array}{l}\mathrm{PM}_{2.5} \text { collected a } 5.5-\mathrm{kW} \text { diesel engine under a constant } 2.5 \\
\mathrm{~kW} \text { load. Exposure of } 300 \mu \mathrm{g} / \mathrm{m}^{3} \text { over } 6 / 30 \text {-minute low and } \\
\text { high intensity cycling periods }\end{array}$ \\
\hline (155) & $\begin{array}{l}\text { Nootkatone pretreatment reduced thrombosis and } \\
\text { oxidative damage (NFE2L2) and heme turnover } \\
\text { (HO-1) }\end{array}$ & $\mathrm{BALB} / \mathrm{C}$ mice at 8 wks & $\begin{array}{l}\text { Diesel exhaust particles from the National Institute of } \\
\text { Standards and Technology, USA. 15 } \mu \mathrm{g} / \text { mouse } \\
\text { intratracheal instilled }\end{array}$ \\
\hline (82) & $\begin{array}{l}\text { Promoter methylation of microRNA-133a-2 in } \\
\text { progeny and cardiovascular stress decreased } \\
\text { microRNA 133a-2 expression }\end{array}$ & $\begin{array}{l}\text { Female C57BL/6 12-14 wks - } \\
12 \text { wk progeny }\end{array}$ & $\begin{array}{l}\mathrm{PM}_{2.5} \text { collected a single cylinder Yanmar diesel engine, } \\
\text { operating at } 82 \% \text { load } \sim 300 \mu \mathrm{g} / \mathrm{m}^{3} \text { for } 6 \mathrm{hrs} / \text { day for a total } \\
\text { of } 5 \text { days during pregnancy }\end{array}$ \\
\hline \multicolumn{4}{|c|}{ Animal Models } \\
\hline \multicolumn{4}{|c|}{ Wild Type } \\
\hline (166) & $\begin{array}{l}\text { Increased ketocholesterol (7-KCh) is poorly } \\
\text { cleared by CD36 macrophages }\end{array}$ & $\begin{array}{l}\text { ApoE } E^{-/-} \mathrm{Tg} \text { and } \mathrm{LDLR}^{-/-} \mathrm{Tg} \\
\text { mice } 8 \mathrm{wks}\end{array}$ & $\begin{array}{l}\mathrm{PM}_{2.5} \text { collected Columbus, } \mathrm{OH} \text { exposed for } 6 \text { months to } \\
9.1 \pm 7.3 \mu \mathrm{g} / \mathrm{m}^{3}\end{array}$ \\
\hline (177) & $\begin{array}{l}\text { ET-1, HSP70, CYP1A1, CYP1B1, laminin, } \\
\text { collagen, and calcium signaling proteins increased }\end{array}$ & Male BALB/c mice 7 to 8 wks & $\begin{array}{l}\mathrm{PM}_{2.5} \text { collected winter } 2008 \text { in an urban site in Milano, } \\
\text { Italy. Instilling intratracheally, on days } 0,3 \text { and } 6 \text {, at } 0.3 \\
\mathrm{mg} / \text { mouse }\end{array}$ \\
\hline (111) & 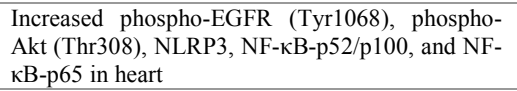 & BALB/c mice 6 to 8 wks & $\begin{array}{l}\mathrm{PM}_{2.5} \text { collected November } 2014 \text { in a major city of central } \\
\text { China. Intratracheal instillation of } 4.0 \mathrm{mg} / \mathrm{kg} \text { b.w. for } 5 \\
\text { consecutive days. }\end{array}$ \\
\hline (133) & In the heart, increased Caspase-3, Bax, and NF-kB & Male C57/BL6 mice 8 wks & $\begin{array}{l}\text { cted in, Nanjing, China. } 10 \mu \mathrm{g} \text { PM2.5 }(10 \mu \mathrm{l}) \\
\text { tranasal instillation. }\end{array}$ \\
\hline (244) & $\begin{array}{l}\text { Increased TNF- } \alpha \text { and IL- } 1 \beta \text {, along with NOX } 4 \text { and } \\
\text { NOX subunits as p6 } 67^{\text {phox }}, p 47^{\text {phox }} \text { and } 222^{\text {phox }} \text {, in the } \\
\text { heart }\end{array}$ & C57BL/6 mice 6 to 8 wks & $\begin{array}{l}\mathrm{PM}_{2.5} \text { collected September } 2013 \text { in Zhengzhou, Taiwan. } \\
\text { Instilled with } 1.5,3.0,6.0 \mathrm{mg} / \mathrm{kg} \text { BW } 5 \text { days } / \mathrm{wk} \text { for } 2 \text { wks }\end{array}$ \\
\hline (163) & 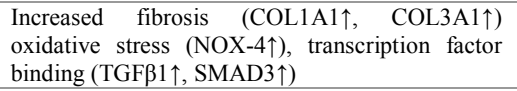 & $\begin{array}{l}\text { Female C57BL/6 } 10 \text { month and } \\
4 \text { wk old mice }\end{array}$ & $\begin{array}{l}\mathrm{PM}_{2.5} \text { collected } 2012 \text { and } 2013 \text { from Taiyuan, Northern } \\
\text { China. } 3 \mathrm{mg} / \mathrm{kg} \text {. b.w. } \mathrm{PM}_{2.5} \text { oropharyngeal aspiration every } \\
\text { other day for } 4 \text { wks }\end{array}$ \\
\hline (45) & $\begin{array}{l}\text { p-STAT3 } 1 \text { promoted microRNA- } 21 \text { expression, } \\
\text { advocating decreased TIMP3, increased MMP9 }\end{array}$ & $\begin{array}{l}\text { Male Sprague Dawley rats } 6 \\
\text { wks }\end{array}$ & $\begin{array}{l}\mathrm{PM}_{2.5} \text { collected in Beijing, China. Trachea drip, } 4 \mathrm{mg} / \mathrm{kg} \\
\text { b.w. every } 3 \text { days for } 36 \text { days }\end{array}$ \\
\hline (240) & $\begin{array}{l}\text { Hyperlipidemic heart increased JNK and P53 } \\
\text { phosphorylation and increased Bax and Caspase-3 } \\
\text { expression }\end{array}$ & $\begin{array}{l}\text { Male Wistar rats } 8 \text { wks on high } \\
\text { fat diet }\end{array}$ & $\begin{array}{l}\mathrm{PM}_{2.5} \text { collected October-December } 2012 \text { in Beijing, China. } \\
\text { Endotracheal instilling of suspension at } 0,4,40 \mathrm{mg} / \mathrm{kg}\end{array}$ \\
\hline (145) & $\begin{array}{l}\text { High intensity exercise stimulates reduced } \\
\text { inflammation (eHSP70 } \downarrow \text { ) }\end{array}$ & $\begin{array}{l}\text { Male B6.129SF2/J mice } 30 \\
\text { days old }\end{array}$ & $\begin{array}{l}\mathrm{PM}_{2.5} \text { collected in São Paulo, Brazil, } 5 \mu \mathrm{g} \text { nasotropic } \\
\text { instillation daily before exercise } 12 \mathrm{wks}\end{array}$ \\
\hline (266) & 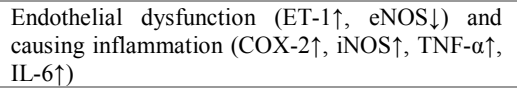 & Male C57BL/ 6 mice 6 to 8 wks & $\begin{array}{l}\mathrm{PM}_{2.5} \text {, collected Taiyuan, China. Intranasal instillation } \\
\text { every other day for } 6 \mathrm{hrs} / 28 \text { days. } 0.5 / 3.5 \mathrm{mg} / \mathrm{m}^{3} \mathrm{SO}_{2}, 0.2 / 2 \\
\mathrm{mg} / \mathrm{m}^{3} \mathrm{NO}_{2}, 1 / 10 \mathrm{mg} / \mathrm{kg} \mathrm{PM} 2.5\end{array}$ \\
\hline (269) & 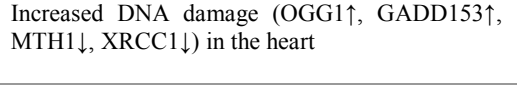 & Male Wistar rats & $\begin{array}{l}\mathrm{PM}_{2.5} \text { collected Taiyuan, China. Intratracheal instillation of } \\
1.5 \mathrm{mg} / \mathrm{kg} \text { b.w. PM } 2.5,1.6 \times 10^{-4} \mathrm{mg} / \mathrm{kg} \text { b.w. 1-NP, } \\
1.2 \times 10^{-4} \mathrm{mg} / \mathrm{kg} \text { b.w. 9-NA }\end{array}$ \\
\hline (249) & $\begin{array}{l}\text { Cardiac injury through changes in serum } \\
\text { biomarker activity (SERCA2 } \uparrow, \mathrm{cTnT} \uparrow, \mathrm{ANP} \uparrow \text {, and } \\
\mathrm{BNP} \uparrow \text { ) }\end{array}$ & Sprague Dawley rats 6 wks & $\begin{array}{l}\text { Silica nanoparticles (SiNPs) and methylmercury }(\mathrm{MeHg}) \\
\text { intratracheal instillation } 10 \text { times over } 30 \text { days, } 0.25 \mathrm{mg} / \mathrm{kg} \\
\text { for MeHg and } 2 \mathrm{mg} / \mathrm{kg} \text { SiNPs. }\end{array}$ \\
\hline (73) & $\begin{array}{l}\text { Heart failure markers (ANP } \uparrow, \quad \text { BNP } \uparrow) \text {, } \\
\text { inflammation (CRP } \uparrow, \quad I L-6 \uparrow, \quad \text { TNF- } \alpha \uparrow) \text { Also } \\
\text { ANGII } \uparrow \text { and ET- }-1 \uparrow \text { in serum }\end{array}$ & Sprague Dawley rats 6 wks & $\begin{array}{l}\text { Stöber technique was used to synthesize the SiNPs. Intra- } \\
\text { tracheal instillation of } 2 \mathrm{mg} / \mathrm{kg} \text { body SiNPs and/ or } 0.25 \\
\mathrm{mg} / \mathrm{kg} \text { of Pb }\end{array}$ \\
\hline \multicolumn{4}{|c|}{ Transgenic/Supplementation } \\
\hline (158) & $\begin{array}{l}\text { Atherosclerosis model increased NOX-4 subunits } \\
\mathrm{p} 22^{\text {phox }}, \mathrm{p} 47^{\text {phox }} \text { in cardiac tissue }\end{array}$ & Male $\mathrm{ApoE}^{-/ /} \mathrm{Tg} \mathrm{mi}$ & $\begin{array}{l}\mathrm{PM}_{2.5} \text { collected June-October } 2013 \text { in Shanghai, China. } 0 \text { - } \\
30 \mathrm{mg} / \mathrm{kg} \text { b.w installation }\end{array}$ \\
\hline (235) & $\begin{array}{l}\text { Fibrosis } \uparrow \text { and collagen } \uparrow \text { genes, lower antioxidant } \\
\text { genes (PRDX5 } 5 \text { ) }\end{array}$ & $\begin{array}{l}\text { Male AMPK } \alpha 2^{--\gamma} \operatorname{Tg} \text { mice } 6 \text { to } \\
8 \text { wks }\end{array}$ & $\begin{array}{l}\mathrm{PM}_{2.5} \text { collected April-October } 2016 \text { in Beijing, China. } \\
10 \mathrm{mg} / \mathrm{kg} \text { intratracheal instillation } / 6 \text { months }\end{array}$ \\
\hline (214) & $\begin{array}{l}\text { TRPA1 activation increases } \\
\text { cardiovascular } \\
\text { dyssynchrony. }\end{array}$ & $\begin{array}{l}\text { Trpa1 } 1^{\text {tmlKykw } / J} \quad\left(\operatorname{Trpa}^{-/}\right) \quad \mathrm{Tg} \\
\text { female mice }\end{array}$ & $\begin{array}{l}\mathrm{PM}_{2.5} \text { collected November-December of } 2014 \text { in Triangle } \\
\text { Park, NC. } 3 \text { hrs/day, } 2 \text { days/week, } 8 \text { total exposures through } \\
\text { a whole-body exposure system }\end{array}$ \\
\hline (268) & $\begin{array}{l}\text { Diabetic model induced NF-кB, COX-2, MAPK in } \\
\text { heart, IKB inhibitor restored function through NF- } \\
\text { KB reduction }\end{array}$ & KKay Tg mice 7 wks & $\begin{array}{l}\mathrm{PM}_{2.5} \text { collected Polaris in Columbus, Ohio. Intratracheally } \\
\text { administered for } 6 \mathrm{hr} / \mathrm{day}, 5 \text { day/week, for } 8 \text { weeks. } \mathrm{PM}_{2.5} \\
\text { dose of } 1.6 \mathrm{mg} / \mathrm{kg}\end{array}$ \\
\hline
\end{tabular}




\begin{tabular}{|c|c|c|c|}
\hline (90) & $\begin{array}{l}\text { Akt/eNOS phosphorylation } \downarrow \text { and } \\
\text { antioxidant treatment eased vascular } \\
\text { resistance/inflammation }\end{array}$ & $\begin{array}{l}\text { Male ecSOD Tg mice } 12 \text { wks } \\
\text { on high fat diet }\end{array}$ & $\begin{array}{l}\mathrm{PM}_{2.5} \text { collected June } 2009 \text { and December } 2010 \text { in } \\
\text { Louisville, KY. } 6 \mathrm{hr} / \text { day for } 9 \text { or } 30 \text { days, } 30-120 \mu \mathrm{g} / \mathrm{m}^{3} \\
\text { through a whole-body exposure system }\end{array}$ \\
\hline$(61)$ & $\begin{array}{l}\text { Vitamin E and omega-3 polyunsaturated fatty acid } \\
\text { administration decreased inflammation (TNF- } \alpha \downarrow \text {, } \\
\text { IL-1 } \beta \downarrow, \text { IL- } 6 \downarrow \text { ) }\end{array}$ & Sprague Dawley rats 6-8 wks & $\begin{array}{l}\mathrm{PM}_{2.5} \text { collected May-September } 2015 \text { in Shanghai, China. } \\
\text { Intratracheal instilled every other day for } 6 \text { days at } 10 \\
\mathrm{mg} / \mathrm{kg} \text { b.w., } 1.5 \mathrm{~mL} / \mathrm{kg} \text { b.w. }\end{array}$ \\
\hline (74) & 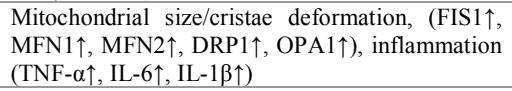 & Sprague Dawley rats & $\begin{array}{l}\mathrm{PM}_{2.5} \text { collected January } 2013 \text { in Taiyuan, China. } \\
\text { Intratracheal instillation of } 0.375,1.5,6.0 \text {, and } 24.0 \mathrm{mg} / \mathrm{kg} \\
\text { b.w. performed } 5 \text { times }\end{array}$ \\
\hline (261) & $\begin{array}{l}\text { SeY treatment reduced inflammation markers } \\
\text { (TNF- } \alpha \text {, IL-1 } \beta \text {, sICM- } 1 \text { ) }\end{array}$ & $\begin{array}{l}\text { Male Sprague Dawley rats } 7 \\
\text { wks }\end{array}$ & $\begin{array}{l}\mathrm{PM}_{2.5} \text { collected September-November } 2015 \text { in Shanghai, } \\
\text { China. Intratracheal instilled } / 40 \mathrm{mg} / \mathrm{kg}\end{array}$ \\
\hline$(60)$ & $\begin{array}{l}\text { N-Acetyl-L-cysteine reduced intracellular } \mathrm{Ca}^{2+} \\
\text { (RYR2 } \downarrow \text {, SERCA2a } \uparrow \text { ) }\end{array}$ & Male Balb/c mice 6 to 8 wks & $\begin{array}{l}\mathrm{PM}_{2.5} \text { collected Hebei, China. Intratracheal instilled } 0.5 \mathrm{mg} \\
\text { at Day } 0 \text { and Day } 2\end{array}$ \\
\hline (130) & $\begin{array}{l}\text { SOS1, CREB, GSK3b, and GRB2 expression } \\
\text { reduced, fucoidan treatment rescued all but CREB } \\
\text { levels. }\end{array}$ & C57BL/6J mice 8 wks & $\begin{array}{l}\mathrm{PM}_{2.5} \text { collected November-December } 2016 \text { in Taipei, } \\
\text { Taiwan. } 100 \mu \mathrm{g} / \mathrm{m}^{3}, 20 \text { days, } 6 \mathrm{hrs} / \text { day }\end{array}$ \\
\hline \multicolumn{4}{|c|}{ Gestational Exposure } \\
\hline (234) & $\begin{array}{l}\text { Dose-dependent increase of OPA1, MFN1, DRP1, } \\
\text { and FIS1 in progeny. }\end{array}$ & $\begin{array}{l}\text { Female Wistar rats } 10 \text { to } 12 \mathrm{wks} \\
-1 \text { day progeny }\end{array}$ & $\begin{array}{l}\mathrm{PM}_{2.5} \text { collected September } 2014 \text { to March } 2016 \text { in Harbin, } \\
\mathrm{China} \text {. Administered dropwise, } 3 \text { doses }(0.375 \mathrm{mg} / \mathrm{kg}, 1.5 \\
\mathrm{mg} / \mathrm{kg} \text {, and } 6.0 \mathrm{mg} / \mathrm{kg})\end{array}$ \\
\hline (211) & 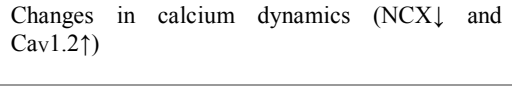 & $\begin{array}{l}\text { Female FVB mice } 12 \text { wks - } 14- \\
\text { day progeny }\end{array}$ & $\begin{array}{l}\mathrm{PM}_{2.5} \text { collected Columbus, Ohio. Exposed } 6 \mathrm{hr} / \mathrm{day}, 5 \\
\text { days/week, average } 91.78 \mu \mathrm{g} / \mathrm{m}^{3} \text { through a whole-body } \\
\text { inhalation system }\end{array}$ \\
\hline (212) & $\begin{array}{l}\text { Acetylation } \quad(\text { SIRT1 } \downarrow, \quad \text { SIRT2 } \downarrow) \text {, methylation } \\
(\text { DNMT1 } \uparrow, \quad \text { DNMT3a } \uparrow, \quad \text { DNMT3b } \uparrow), \quad \text { and } \mathrm{Ca}^{2+} \\
\text { markers altered (NCX } \downarrow, \text { p-PLN } \downarrow)\end{array}$ & $\begin{array}{l}\text { Female FVB mice } 12 \text { wks - } 12 \\
\text { wk progeny }\end{array}$ & $\begin{array}{l}\mathrm{PM}_{2.5} \text { collected Columbus, OH. } 6 \mathrm{hr} / \mathrm{day}, 7 \text { days } / \text { week } \\
\text { throughout pregnancy, average } 73.61 \mu \mathrm{gg} / \mathrm{m}^{3} \text { through a } \\
\text { whole-body inhalation system }\end{array}$ \\
\hline (210) & 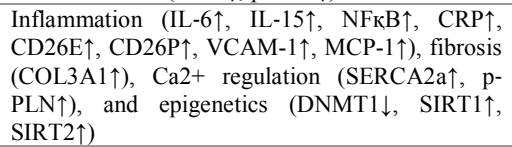 & $\begin{array}{l}\text { Paternal and maternal FVB } \\
\text { mice exposure - male } 12 \mathrm{wk} \\
\text { old progeny }\end{array}$ & $\begin{array}{l}\mathrm{PM}_{2.5} \text { collected Columbus, OH. Average } 38.58 \mu \mathrm{g} / \mathrm{m}^{3} \text { for } 6 \\
\text { hrs/day, } 5 \text { days/week for } 3 \text { months through a whole-body } \\
\text { inhalation system }\end{array}$ \\
\hline (36) & $\begin{array}{l}\text { Increased GATA4 and NKX2-5 and increased } \\
\text { inflammation (TNF- } \alpha \uparrow, \text { IL-1 } 1 \beta \uparrow \text { ), homocysteine } \\
\text { exacerbates effects }\end{array}$ & $\begin{array}{l}\text { Female Sprague Dawley rats } 10 \\
\text { wks - neonatal progeny }\end{array}$ & $\begin{array}{l}\mathrm{PM}_{2.5} \text { collected Fujian, China. Exposed during gestation } \\
\text { and lactation ( } \sim 42 \text { days) to } 36.5 \mu \mathrm{g} / \mathrm{m}^{3} \text { through a whole- } \\
\text { body inhalation system. }\end{array}$ \\
\hline (245) & $\begin{array}{l}\text { Increased histone acetyltransferase, H3K9ac, } \\
\text { GATA4, and MEF2c. Histone acetylation of } \\
\text { GATA4/MEF2c promoters }\end{array}$ & $\begin{array}{l}\text { Female C57BL/6 mice } 12 \text { wks } \\
-1 \text { day and } 16 \text { wk-old progeny }\end{array}$ & $\begin{array}{l}\mathrm{PM}_{2.5} \text { collected Chongqing, China. Gestational exposure } \\
\text { by ultrasonic nebulization, } 2 \text { hours a day during pregnancy, } \\
\text { at } \sim 300 \mu \mathrm{g} / \mathrm{m}^{3}\end{array}$ \\
\hline \multicolumn{4}{|c|}{ UFP } \\
\hline \multicolumn{4}{|c|}{ Ambient Air and Occupational Pollution } \\
\hline (56) & $\begin{array}{l}\text { Decreased blood plasminogen and } \\
\text { thrombomodulin, increased CRP and SR-A. } \\
\text { GSTM1 null participants had worse outcomes }\end{array}$ & $\begin{array}{l}34 \text { participants with metabolic } \\
\text { syndrome, } \sim 48 \text { years old }\end{array}$ & $\begin{array}{l}\text { UFP collected US EPA facility Chapel Hill NC. Whole } \\
\text { body exposure, average particle concentration } 189,000 \\
\text { particles } / \mathrm{cm}^{3} .2 \text { hour exposure }\end{array}$ \\
\hline (200) & $\begin{array}{l}\text { Platelet CD40L } \uparrow \text { and circulating CD } 40 \mathrm{~L} \downarrow \text {, with } \\
\text { increased plasma von Willebrand factor }\end{array}$ & $\begin{array}{l}19 \text { type } 2 \text { diabetics, } 30-60 \text { years } \\
\text { of age, never smoked }\end{array}$ & $\begin{array}{l}\text { UFP particles were generated. Elemental carbon UFP } \\
\text { through mouth piece for } 2 \mathrm{hr} \text { at } 50 \mu \mathrm{g} / \mathrm{m}^{3}\end{array}$ \\
\hline (126) & $\begin{array}{l}\text { CRP } \uparrow \text { and TNFRII } \uparrow \text { significantly associated with } \\
\text { white non-Hispanic }\end{array}$ & $\begin{array}{l}408 \text { individuals aged } 40- \\
91 \text { years }\end{array}$ & Ambient air pollution in Dorchester, South Boston \\
\hline (226) & 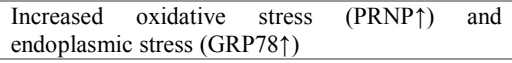 & $\begin{array}{l}30 \text { children and young adults } \\
\sim 20 \text { years of age }\end{array}$ & Ambient air pollution in Mexico City, Mexico \\
\hline (123) & 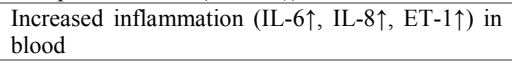 & $\begin{array}{l}\text { Men and women }(\mathrm{n}=52) 18 \text { to } \\
34 \text { years old }\end{array}$ & $\begin{array}{l}\text { UFP collected Sault Ste. Marie, Ontario, Canada, in the } \\
\text { summer of } 2010 \text {. Occupational air pollution }\end{array}$ \\
\hline \multicolumn{4}{|c|}{ Animal Models } \\
\hline (10) & $\begin{array}{l}\text { Increased } \mathrm{AT}_{1} \mathrm{R} \text { protein along with Acta } 1 \text { and } \\
\text { Col3a1 in the heart, with decreased HO-1 } \\
\text { expression }\end{array}$ & Male Sprague-Dawley rats & $\begin{array}{l}\text { UFP collected north of Mexico City, May to July, } 2009 \text {. } \\
\text { acute }(3 \text { days, } 5 \mathrm{hr} / \text { day) and subchronic ( } 8 \text { weeks, } 4 \\
\text { days/week, } 5 \mathrm{hr} / \text { day) to varying concentrations. }\end{array}$ \\
\hline (99) & $\begin{array}{l}\text { Mitochondrial mPTP deficits, with attenuated } \\
\text { beneficial impact of Cyclosporin A }\end{array}$ & Male Sprague-Dawley rats & $\begin{array}{l}\text { UFP collected US EPA facility Chapel Hill, NC. } 100 \mu \mathrm{g} \\
\text { UFP laryngopharynx instillation }\end{array}$ \\
\hline (220) & $\begin{array}{l}\text { CRP and fibrinogen increased in systemic } \\
\text { circulation }\end{array}$ & $\begin{array}{l}\text { Male } \\
\text { hypertensive rats (SHR), } 12-13 \\
\text { months old }\end{array}$ & $\begin{array}{l}\text { UFP generation. } 31 \mu \mathrm{g} \text { or } 0.024 \mathrm{~m}^{2} \mathrm{UFP} \text { deposition over } 24 \\
\text { hour time frame }\end{array}$ \\
\hline (263) & $\begin{array}{l}\text { Increasing UFP concentrations attenuated the } \\
\text { beneficial effects of captopril in reducing AngII. }\end{array}$ & $\begin{array}{l}\text { Spontaneously } \text { hypertensive } \\
\text { rats (SHR) }\end{array}$ & $\begin{array}{l}\text { UFP collected Beijing, China. UFP }(0.15 \mathrm{mg} / \mathrm{kg} \text {, } \\
0.45 \mathrm{mg} / \mathrm{kg} \text { and } 1.35 \mathrm{mg} / \mathrm{kg}) \text { and captopril administration } \\
\text { intratracheal instillation. }\end{array}$ \\
\hline \multicolumn{4}{|c|}{ Gestational Exposure } \\
\hline (151) & $\begin{array}{l}\text { Placental HSD11B2 DNA methylation, elevated } \\
\text { IL-1 } \beta \text {, IL-6 and MCP-1 levels }\end{array}$ & $\begin{array}{l}\text { Female C57BL/6J } p^{\text {un }} / p^{\text {un }} \text { mice, } \\
6 \text { wks old. GD } 17.5 \text { progeny }\end{array}$ & $\begin{array}{l}\text { UFP collected northern Mexico City collected from April } \\
\text { to June of } 2016.12 \mu \mathrm{g} \text { or } 400 \mu \mathrm{g} / \mathrm{kg} \text { intratracheal } \\
\text { instillation }\end{array}$ \\
\hline (173) & $\begin{array}{l}\text { Decreased IL-13 and IL-17 in lungs, with higher } \\
\text { serum levels of IL-10 }\end{array}$ & $\begin{array}{l}\text { Female } \mathrm{C} 57 \mathrm{BL} / 6 \text { and } \mathrm{BALB} / \mathrm{C} \\
\text { mice. Progeny } 0 \text { to } 4 \text { wks }\end{array}$ & $\begin{array}{l}\text { UFP generated. average mass concentration } 101.94 \mu \mathrm{g} / \mathrm{m}^{3} \text {, } \\
\text { 24-h daily mean dose of } 25 \mu \mathrm{g} / \mathrm{m}^{3}\end{array}$ \\
\hline \multicolumn{4}{|c|}{ PM$_{2.5}$ and UFP Combined Analyses } \\
\hline (43) & $\begin{array}{l}\text { Increased CRP and fibrinogen in blood to UFP, but } \\
\text { not } \mathrm{PM}_{2.5}\end{array}$ & $\begin{array}{l}135 \text { patients undergoing cardiac } \\
\text { catheterization }\end{array}$ & $\begin{array}{l}\text { Ambient air pollution. New York State Department of } \\
\text { Environmental Conservation (DEC) site in Rochester, NY }\end{array}$ \\
\hline (224) & $\begin{array}{l}\text { Elevated CRP and platelet count in blood for } \mathrm{PM}_{2.5} \text {, } \\
\text { but not UFP }\end{array}$ & 3275 participants & $\begin{array}{l}\text { Ambient air pollution. German Heinz Nixdorf Recall } \\
\text { Study, three adjacent cities (Essen, Mülheim, Bochum) }\end{array}$ \\
\hline (243) & $\begin{array}{l}\text { Mitochondrial haplotypes differentially effected } \\
\text { by exposure and IL- } 6 \text { and TNF- } \alpha \text { elevated. } \\
\text { Significant to quasi-ultrafine particles }<0.25 \mu \mathrm{m}\end{array}$ & 36 participants, $\sim 84$ years old & $\begin{array}{l}\text { Particulate matter collected from four retirement } \\
\text { communities in the Los Angeles air basin. }\end{array}$ \\
\hline
\end{tabular}




\begin{tabular}{|c|c|c|c|}
\hline (216) & $\begin{array}{l}\text { Dysregulated parasympathetic response, increased } \\
\text { IFN- } \gamma \text { methylation in blood to } \mathrm{PM}_{2.5} \text { and quasi- } \\
\text { ultrafine particles }<0.50 \mu \mathrm{m}\end{array}$ & 12 healthy participants & $\begin{array}{l}\text { Particulate matter collected Milan, Italy. Inhalation to } \\
\text { mixture }\left(\mathrm{PM}_{10}, \mathrm{PM}_{2.5}, \mathrm{PM}_{1.0} \text { and } \mathrm{PM}_{0.5} \mu \mathrm{m}\right) \text { over } 2 \text { sessions }\end{array}$ \\
\hline (68) & $\begin{array}{l}\text { Oxford cohort (hsa-miR-197-3p } \uparrow \text { and hsa-miR- } \\
99 \mathrm{a}-5 \mathrm{p} \uparrow \text { ) changes to } \mathrm{PM}_{2.5} \text {, but not UFP }\end{array}$ & $\begin{array}{l}59 \text { volunteers }(20 \text { healthy, } 20 \\
\text { COPD, } 19 \text { ischemic heart } \\
\text { disease) }\end{array}$ & $\begin{array}{l}\text { Ambient air pollution. Oxford Street or Hyde Park, } \\
\text { London, England. } 2 \text { hour walk }\end{array}$ \\
\hline (153) & $\begin{array}{l}69 \text { differentially methylated regions, } 13 \text { CpG sites, } \\
\text { including methylation of KNDC } 1 \text { and FAM50B }\end{array}$ & 157 non-smoking adults & $\begin{array}{l}\mathrm{PM}_{2.5} \text { collected indoor air pollution, measured in } 24 \text {-hour } \\
\text { intervals }\end{array}$ \\
\hline (137) & $\begin{array}{l}\text { VEGF, UCHL1, and cortisol positively correlated } \\
\text { with nickel, barium, and silver }\end{array}$ & $\begin{array}{l}53 \text { healthy participants, } 18- \\
60 \text { years old }\end{array}$ & $\begin{array}{l}\text { Particulate matter collected in downtown Toronto, Canada. } \\
130 \text {-min exposure }\end{array}$ \\
\hline \multicolumn{4}{|c|}{ Nanotoxicology } \\
\hline (105) & $\begin{array}{l}\text { Blood specific activation of } \mathrm{C} 3 \text {, with heart } \\
\text { activation of the complement cascade. }\end{array}$ & Female C57BL/6 mice 8 wks & $\begin{array}{l}\text { Nano- } \mathrm{TiO}_{2} \text {, UV-Titan } \mathrm{L} 181.6 .0 \mathrm{mg} \mathrm{Ti} / \mathrm{m}^{3}-10 \mathrm{mg} \mathrm{TiO} / \mathrm{m}^{3} \\
\text { intratracheal instillation }\end{array}$ \\
\hline (100) & 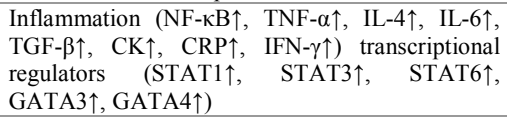 & CD-1 (ICR) male mice at 4 wks & $\begin{array}{l}\mathrm{Nano}^{-\mathrm{TiO}_{2}} \text { prepared through hydrolysis of } \mathrm{Ti}(\mathrm{OBu})_{4} \text {, nasal } \\
\text { instillation of mice every other day for } 6 \text { months. Treatment } \\
\text { of } 1.25-5 \mathrm{mg} / \mathrm{kg} \mathrm{TiO} \mathrm{TPs}_{2} \mathrm{NPs}\end{array}$ \\
\hline (78) & $\begin{array}{l}\text { Inhalation, compared to intra-arterial injection, } \\
\text { was more detrimental to cardiac tissues. } \\
\text { Inflammation and endothelial/epithelial pathways } \\
\text { perturbed }\end{array}$ & $\begin{array}{l}\text { Female BALB/cJ mice, } 10-12 \\
\text { wks old }\end{array}$ & $\begin{array}{l}\text { Carbon particles with primary particle diameter of } 10 \pm 2 \\
\mathrm{~nm} \text {. Intra-arterial infusion }\left(30 \mathrm{~mm}^{2} \text { per animal and }\right. \\
\left.\text { inhalation (lung deposited dose } 10,000 \mathrm{~mm}^{2}\right) . \text { Whole body } \\
\text { exposurr for } 4 \text { or } 24 \text { hours. }\end{array}$ \\
\hline (198) & $\begin{array}{l}\text { mPHGPx Tg mice reduced ROS and preserved } \\
\text { electron transport chain complex constituents in } \\
\text { the heart }\end{array}$ & $\begin{array}{l}\text { Male FVB and male mPHGPx } \\
\text { Tg mice } 10-12 \text { wks }\end{array}$ & $\begin{array}{l}\text { Nano- } \mathrm{TiO}_{2} \text {, particle size }(21 \mathrm{~nm}) / \mathrm{surface} \text { area }\left(48.08 \mathrm{~m}^{2} / \mathrm{g}\right) \text {. } \\
11.58 \pm 0.27 \mu \mathrm{g} \text { lung deposition, } 6 \mathrm{hr} \text { exposure through a } \\
\text { whole-body inhalation system }\end{array}$ \\
\hline (94) & $\begin{array}{l}\text { microRNA-378a } \mathrm{Tg} \text { mice preserved fusion } \\
(\uparrow \mathrm{MFN} 1) \text {, metabolism, and ultrastructure in the } \\
\text { heart }\end{array}$ & $\begin{array}{l}\text { FVB and miRNA-378a } \\
\text { knockout Tg mice aged 14-18 } \\
\text { weeks of age }\end{array}$ & $\begin{array}{l}\text { Nano- } \mathrm{TiO}_{2} \text {, particle size }(21 \mathrm{~nm}) / \mathrm{surface} \text { area }\left(48.08 \mathrm{~m}^{2} / \mathrm{g}\right) \text {. } \\
11.09 \mathrm{mg} / \mathrm{m}^{3} \text { concentration for } 4 \mathrm{hr} \text { exposure through a } \\
\text { whole-body inhalation system }\end{array}$ \\
\hline \multicolumn{4}{|c|}{ Gestational Exposure } \\
\hline (95) & $\begin{array}{l}\text { Increased cardiac mitochondrial proton leak, } \\
\text { ( } \uparrow \mathrm{UCP} 2) \text {, and fatty acid metabolism ( } \uparrow \mathrm{CPT} 1 \mathrm{~A} \text { and } \\
\uparrow \mathrm{PDH} \text { phosphorylation) }\end{array}$ & $\begin{array}{l}\text { Female Sprague Dawley rats } 10 \\
\text { to } 12 \mathrm{wks}-12 \mathrm{wks} \text { progeny }\end{array}$ & $\begin{array}{l}\text { Nano- } \mathrm{TiO}_{2} \text {, particle size }(21 \mathrm{~nm}) / \text { surface area }\left(48.08 \mathrm{~m}^{2} / \mathrm{g}\right) \\
7.79 \pm 0.26 \text { days, } 5 \mathrm{hr} / \text { day, } 10.35 \pm 0.13 \mathrm{mg} / \mathrm{m}^{3} \text { through a } \\
\text { whole-body inhalation system }\end{array}$ \\
\hline (199) & $\begin{array}{l}\text { Decreased State } 3 \text { and State } 4 \text { mitochondrial } \\
\text { respiration }\end{array}$ & $\begin{array}{l}\text { Female Sprague Dawley rats - } \\
\text { female } 11 \text { to } 16 \text { wks progeny }\end{array}$ & $\begin{array}{l}\text { Nano- } \mathrm{TiO}_{2} \text {, particle size }(21 \mathrm{~nm}) / \text { surface area }\left(48.08 \mathrm{~m}^{2} / \mathrm{g}\right) \\
6.8 \pm 0.5 \text { days } / 10.6 \pm 0.3 \mathrm{mg} / \mathrm{m}^{3} \text { through a whole-body } \\
\text { inhalation. }\end{array}$ \\
\hline (198) & $\begin{array}{l}\text { Cardiac mitochondrial epigenomic remodeling, } \\
\text { decreased transcriptomic immune response. }\end{array}$ & $\begin{array}{l}\text { Female Sprague Dawley rats } 10 \\
\text { to } 12 \text { wks - GD } 20 \text { fetal } \\
\text { progeny }\end{array}$ & $\begin{array}{l}\text { Nano- } \mathrm{TiO}_{2} \text {, particle size }(21 \mathrm{~nm}) / \text { surface area }\left(48.08 \mathrm{~m}^{2} / \mathrm{g}\right) \\
7.79 \pm 0.26 \text { days, } 5 \mathrm{hr} / \text { day, } 10.35 \pm 0.13 \mathrm{mg} / \mathrm{m}^{3} \text { through a } \\
\text { whole-body inhalation system }\end{array}$ \\
\hline
\end{tabular}

Table 1.1: Summarized recent findings of molecular mechanisms driving inhalation exposure in the cardiovascular system. LINE = long interspersed nuclear element, TLR $=$ Toll-like receptor, SLFN $=$ Schlafen Family Member, KNDC1 $=$ Kinase Non-Catalytic C-Lobe Domain Containing, FAM50B = family with sequence similarity 50 member $\mathrm{B}, \mathrm{CRP}=\mathrm{C}$-reactive protein, $\mathrm{IL}=$ interleukin, tumor necrosis factor receptor, $7-\mathrm{KCh}=7$-ketocholesterol, $\mathrm{CD}=$ cluster of differentiation, $\mathrm{ET}=$ endothelin, $\mathrm{HSP}-70=70-\mathrm{kDa}$ heat shock protein, $\mathrm{CYP}=$ cytochrome $\mathrm{P} 450, \mathrm{EGFR}=$ epidermal growth factor receptor, NLRP $=$ NOD-like receptor protein, NF-KB $=$ nuclear factor-kappa beta, $\mathrm{TNF}=$ tumor necrosis factor, NOX $=$ NADPH oxidase, COL3A1 = collagen type III alpha 1, COL1A1 = collagen type I alpha 1 , TGF $\beta=$ transforming growth factor beta, SMAD $=\mathrm{SMAD}$ transcription factor, STAT $=$ signal transducer and activator of transcription, TIMP3 = metalloproteinases 3 , MMP9 = matrix metalloproteinases 9 , JNK $=$ c-Jun NH2-terminal kinase, eNOS $=$ endothelial nitric oxide synthase, $\mathrm{COX}=$ cyclooxygenase, $\mathrm{iNOS}=$ inducible nitric oxide synthase, $\mathrm{OGG}=8$-oxoguanine DNA glycosylase, GADD $=$ growth arrest and DNA damage, $\mathrm{MTH}=$ MutT homolog, XRCC1 $=\mathrm{X}$-ray repair cross- complementing group $1, \mathrm{cTnT}=$ cardiac troponin $\mathrm{T}, \mathrm{ANP}=$ atrial natriuretic peptide, $\mathrm{BNP}=$ brain natriuretic peptide, $\mathrm{ANGII}=$ angiotensin $\mathrm{II}, \mathrm{PRDX}=$ peroxiredoxin, $\mathrm{TRPA} 1=$ transient receptor potential cation channel $\mathrm{A} 1, \mathrm{MAPK}=$ mitogen activated protein kinase, $\mathrm{I} \kappa \mathrm{B} \alpha=$ inhibitor of the transcription factor NF- $\mathrm{KB}$, FIS1 = mitochondrial fission 1, MFN = mitofusion, DRP $1=$ dynamin-related protein, OPA1 = mitochondrial dynamin like GTPase, $\mathrm{SeY}=$ selenium-enriched yeast, $\mathrm{sICAM}=$ soluble intercellular adhesion molecule, RYR2 = ryanodine receptor 2, SERCA2a $=$ sarcoplasmic/endoplasmic reticulum calcium ATPase 2a, SOS1 $=$ SOS Ras/Rac Guanine Nucleotide Exchange Factor 1, CREB = cAMP response element binding protein, GSK $=$ glycogen synthase kinase, $\mathrm{GRB}=$ Growth factor receptor-bound protein, $\mathrm{NCX}=\mathrm{Na}^{+} / \mathrm{Ca}^{2+}$ exchanger, $\mathrm{CaV} 1.2=$ calcium channel, voltagedependent, L type, alpha 1C subunit, SIRT $=$ sirtuin, DNMT $=$ DNA methyltransferase, $p$-PLN $=$ phosphorylated phospholamban, $\mathrm{VCAM}=$ vascular cell adhesion protein, $\mathrm{MCP}=$ monocyte chemoattractant protein, GATA = GATA binding protein, NKX2-5 = homeobox protein Nkx, H3K9ac = histone 3 lysine 9 acetylation, MEF = myocyte enhancer factor, NFE2L2 = nuclear factor erythroid-derived 2-like-2, HO-1 = heme oxygenase 1, C3 = complement 
factor 3, CK = creatine kinase, IFN = interferon, $\mathrm{mPHGPx}=$ phospholipid hydroperoxide glutathione peroxidase, $\mathrm{UCP} 2=$ uncoupling protein $2, \mathrm{CPT} 1 \mathrm{~A}=$ carnitine palmitoyltransferase $1 \mathrm{~A}, \mathrm{PDH}=$ pyruvate dehydrogenase.

\section{Particulate Matter $2.5 \mu \mathrm{m}\left(\mathbf{P M}_{2.5)}\right.$}

Particulate matter is the sum of all organic and inorganic compounds dispersed in the solid and liquid phases that can be carried by air (116). Decreasing the size of particulates, from 2.5-10 $\mu \mathrm{m}\left(\mathrm{PM}_{10}\right)$ to $<2.5 \mu \mathrm{m}\left(\mathrm{PM}_{2.5}\right)$, increases the capacity for the material to travel farther into the bronchoalveolar airways and allows for a broader interaction area $(75,247)$. With the heterogeneous nature of $\mathrm{PM}_{2.5}$, understanding the risks posed by inhalation exposure is difficult. At a physiological level, $\mathrm{PM}_{2.5}$ inhalation exposure has been correlated with vascular changes (206, 207) and alteration in heart structure (7). While the cardiovascular outcomes resulting from $\mathrm{PM}_{2.5}$ have been thoroughly examined, the interconnectedness between $\mathrm{PM}_{2.5}$ exposure and the cellular and molecular mechanisms involved lacks clear integration in the literature. We provide an illustration to help summarize the DNA, protein, and cellular changes observed following toxicant inhalation exposure, including $\mathrm{PM}_{2.5}$ (Figure 1.1).

\section{Figure 1.1: Cellular and Molecular Impacts on the Cardiovascular System}

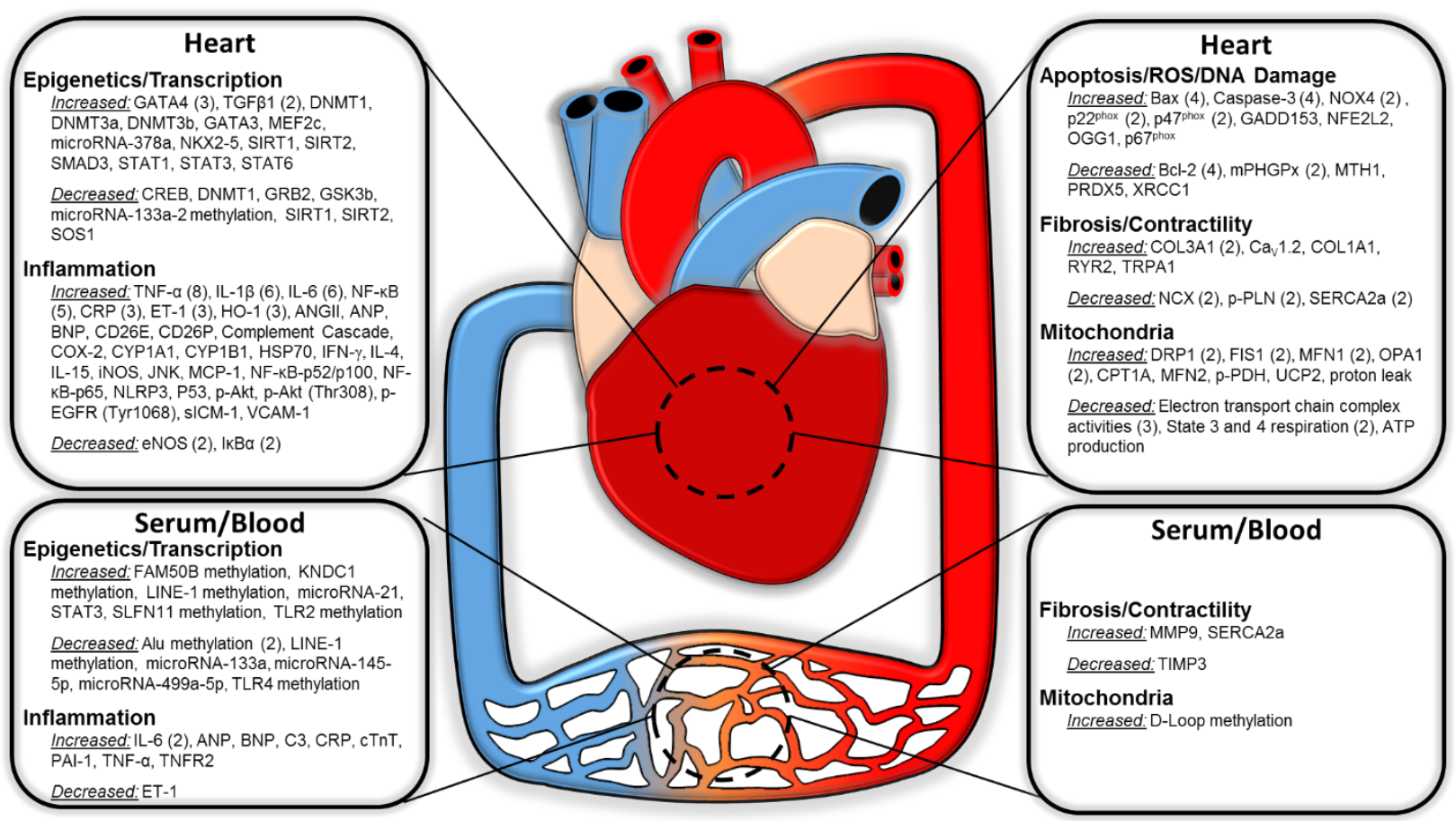


Figure 1.1: Summary of cardiovascular alterations observed following toxicant inhalation exposures. Changes in DNA, protein, and cellular expression levels observed in the heart, serum, and blood are displayed, followed by the number of references (if more than one) that have reported each respective change. GATA = GATA binding protein, TGF = transforming growth factor beta, DNMT = DNA methyltransferase, MEF = myocyte enhancer factor, NKX2$5=$ homeobox protein Nkx, SIRT $=$ sirtuin, SMAD $=$ SMAD transcription factor, STAT $=$ signal transducer and activator of transcription, $\mathrm{CREB}=\mathrm{cAMP}$ response element binding protein, GRB = Growth factor receptor-bound protein, GSK = glycogen synthase kinase, $\mathrm{SOS}=\mathrm{SOS}$ Ras/Rac Guanine Nucleotide Exchange Factor, $\mathrm{TNF}=$ tumor necrosis factor, $\mathrm{IL}=$ interleukin, $\mathrm{NF}-\mathrm{kB}=$ nuclear factor-kappa beta, $\mathrm{CRP}=\mathrm{C}$-reactive protein, $\mathrm{ET}=\mathrm{endothelin}, \mathrm{HO}$ $=$ heme oxygenase, $\mathrm{ANGII}=$ angiotensin II, ANP = atrial natriuretic peptide, $\mathrm{BNP}=$ brain natriuretic peptide, $\mathrm{CD} 26$ $=$ cluster of differentiation $26, \mathrm{COX}=$ cyclooxygenase, $\mathrm{CYP}=$ cytochrome P450, HSP-70 = 70-kDa heat shock protein, IFN = interferon, iNOS $=$ inducible nitric oxide synthase, JNK = c-Jun NH2-terminal kinase, $\mathrm{MCP}=$ monocyte chemoattractant protein, NLRP $=$ NOD-like receptor protein, $\mathrm{p}$-Akt $=$ phospho-Akt, EGFR $=$ epidermal growth factor receptor, sICAM = soluble intercellular adhesion molecule, VCAM = vascular cell adhesion protein, eNOS $=$ endothelial nitric oxide synthase, I $\kappa \mathrm{B} \alpha=$ inhibitor of the transcription factor NF- $\kappa \mathrm{B}, \mathrm{FAM} 50 \mathrm{~B}=$ family with sequence similarity 50 member $\mathrm{B}, \mathrm{KNDC}=$ Kinase Non-Catalytic C-Lobe Domain Containing, LINE = long interspersed nuclear element, SLFN = Schlafen Family Member, TLR = Toll-like receptor, C3 = complement factor $3, \mathrm{cTnT}=$ cardiac troponin $\mathrm{T}, \mathrm{PAI}=$ plasminogen activator inhibitor, $\mathrm{TNFR}=$ tumor necrosis factor receptor, $\mathrm{NOX}=$ NADPH oxidase, GADD = growth arrest and DNA damage, NFE2L2 = nuclear factor erythroid-derived 2-like-2, $\mathrm{OGG}=8$-oxoguanine DNA glycosylase, $\mathrm{mPHGPx}=$ phospholipid hydroperoxide glutathione peroxidase, $\mathrm{MTH}=$ MutT homolog, PRDX = peroxiredoxin, XRCC1 = X-ray repair cross- complementing group 1, COL3A1 = collagen type III alpha 1, CaV1.2 = calcium channel, voltage-dependent, L type, alpha 1C subunit, COL1A1= collagen type I alpha $1, \mathrm{RYR} 2$ = ryanodine receptor 2, TRPA1 $=$ transient receptor potential cation channel $\mathrm{A} 1, \mathrm{NCX}=\mathrm{Na}^{+} / \mathrm{Ca}^{2+}$ exchanger, p-PLN = phosphorylated phospholamban, SERCA2a $=$ sarcoplasmic/endoplasmic reticulum calcium ATPase 2a, DRP 1 = dynamin-related protein, FIS1 = mitochondrial fission $1, \mathrm{MFN}=$ mitofusion, OPA1 = mitochondrial dynamin like GTPase, CPT1A = carnitine palmitoyltransferase 1A, p-PDH = phosphorylated pyruvate dehydrogenase, UCP2 = uncoupling protein 2, MMP9 = matrix metalloproteinases 9, TIMP3= metalloproteinases 3 .

\section{Ambient Air Pollution}

In industrial cities and large metropolitan areas, the most pervasive medium for exposing individuals to aerosolized toxicants is through the ambient air. According to the World Health Organization (WHO), over three million people die every year from outdoor air pollution and nine out of ten people are exposed to poor air quality beyond the WHO limits (157). Half of all of the deaths resulting from ambient air pollution are primarily linked to cardiovascular disease (157).

At the human exposure level, studies have begun to examine the molecular adaptions in the heart, specifically through the Normative Aging Study, established by the United States Department of Veterans Affairs. In the Normative Aging Study, hypomethylation was shown in two repetitive elements (143). Over a 90-day period, increased carbon black levels were correlated with decreased methylation of $\mathrm{Alu}$, while $\mathrm{SiO}_{2}$ was associated with decreased methylation of long interspersed nuclear emlement-1 (LINE-1). Toll-like receptor 2 (TLR2) methylation was 
demonstrated to increase with the concentration of $\mathrm{PM}_{2.5}$ exposure, evidenced in a patient subset of the Normative Aging Study. Through the intake of flavonoids, TLR2 methylation was shown to decrease and ameliorate the effects on low frequency heart rate variability (272). Another study evaluating the Normative Aging Study analyzed gene pathways from blood collected from 646 participants, revealing that pathways such as cancer, signal transduction, and cell growth/death are affected by $\mathrm{PM}_{2.5}$ inhalation exposure (47). $\mathrm{CpG}$ methylation of Schlafen Family Member 11 (SLFN11), which is involved in regulating the innate response to fight cancer cells, was the most associated gene in their analysis. Other meta population studies have also investigated the cellular and molecular mechanisms of $\mathrm{PM}_{2.5}$ air pollution on the cardiovascular system $(18,119,131,153)$.

When measuring blood methylation levels in 15 healthy participants, the Alu repetitive transposable element was shown to be hypomethylated following concentrated ambient particles (CAPs) exposure. Decreased Alu methylation was also shown to be associated with higher diastolic blood pressure post-exposure (18). In a study examining the pollution of Oxford Street in London, England, 24 participants were recruited to give plasma samples after being exposed for 2 hours to ambient air conditions (119). The plasma evaluated contained circulating microRNAs that are involved in regulating cardiovascular function, with microRNA-133a, 145-5p, and 499a-5p differentially impacted. In a 3820 non-current smoking patient population that was part of the Framingham Heart Study, acute $\mathrm{PM}_{2.5}$ exposure was shown to be inversely correlated with Pselectin expression, suggesting decreased endothelial-surface cell adhesion (131). Generally, human studies evaluating exposure to $\mathrm{PM}_{2.5}$ have suggested global cellular alterations, including epigenome modifications, which may be indicated through transient inflammatory markers or repressive microRNA profiles. Nevertheless, the extent, and duration of epigenetic remodeling remains largely unknown in human populations.

\section{Occupational Pollution}

Most interactions with inhaled toxicants are from indirect or diluted sources, which may only interact acutely with a given individual. Alternatively, the occupational setting is one in which more concentrated, chronic exposures can result, leading to more severe cardiopulmonary outcomes. Understanding the occupational niche is important in predicating the longevity of complications arising from any given exposure. 
In studies examining occupational exposure of boilermakers, decreases in heart rate variability and epigenetic alterations following exposure in 66 male participants were observed (69). Methylation of LINE-1 was significantly increased in peripheral blood leukocytes of participants when exposed to $\mathrm{PM}_{2.5}$, but was not directly associated with declining heart rate variability. A following study examined blood from 48 male participants for methylation of mitochondrial DNA (23). While methylation of specific mitochondrial transcribed genes, mitochondrially encoded tRNA phenylalanine (MT-TF) and mitochondrial 12s RNA (MT-RNR1), were not associated with $\mathrm{PM}_{2.5}$ exposure, methylation of the mitochondrial promoter D-Loop was associated with both $\mathrm{PM}_{2.5}$ exposure and measures of heart rate variability (23). In blood from 63 trucking industry participants, 48 (elemental carbon), 260 (organic carbon), and $49\left(\mathrm{PM}_{2.5}\right)$ differentially regulated genes were associated with each exposure; these expression profiles indicating genes regulating apoptosis, DNA and metal binding, and chronic heart and lung disease pathways (40). Plasma examined from 18 males after cycling, revealed a significant increase in nitric oxide variants $\left(\mathrm{NO}_{\mathrm{x}}\right)$ with a significant decrease in eET-1, though, the results suggest that acute exposure to diesel exhaust did not alter flow-mediated dilation and may not alter overall performance (81).

Diesel exhaust exposures in animals have also helped to develop a better understanding of occupational exposures through combustion particulates. Nootkatone, a sesquiterpenoid in grapefruit, was used as a pretreatment strategy for diesel exhaust exposure, reducing blood clotting (plasminogen activator inhibitor-1 (PAI-1) and fibrinogen), inflammation (IL-6), and lipid peroxidation (155). The authors suggest that the prothrombotic, oxidative environment was induced through nootkatoone activation of nuclear factor erythroid-derived 2-like 2 (NFE2L2) and HO-1. Neonatal cardiomyocytes from those exposed to diesel exhaust in utero exhibited differential methylation of microRNA133a-2 at the promoter region. As the progeny aged, introducing stress, such as transverse aortic constriction, revealed increased microRNA-133a-2 and decreased protein tyrosine phosphatase, receptor type F (PTPRF) and peptidase domain containing associated with muscle regeneration 1 (PAMR1) expression. (82).

\section{Animal Models}

Wild Type 
Animal models have been pivotal in determining mechanisms of cardiovascular dysfunction following exposure to $\mathrm{PM}_{2.5}$. Examining the effects of $\mathrm{PM}_{2.5}$ exposure in the development of atherosclerosis, Rao, et al. discovered the increased abundance of an oxidized variant of cholesterol, 7-ketocholesterol $(7-\mathrm{KCh})$. By reducing the number of CD36-positive macrophages, 7-KCh, which is inefficiently disposed of by macrophages, is more easily cleared and accumulates less within the vasculature (166). Gene expression of the 70-kDa heat shock protein (HSP-70), heme oxygenase-1 (HO-1), and myeloperoxidase (MPO) increased in the heart of mice following $\mathrm{PM}_{2.5}$ exposure, with a total of 181 up-regulated and 178 down-regulated genes. The heart was shown to have greater than six times more differentially affected genes compared to the lungs, with collagen, laminin, and calcium handling related genes significantly increased (177). Cardiac inflammation and fibrosis were induced in the heart of mice exposed to $\mathrm{PM}_{2.5}$ with elevated expression of C-X-C motif chemokine ligand 1 (CXCL1), interleukin (IL) -6, and IL-18. Nuclear factor-kappa beta (NF-KB) and epidermal growth factor receptor (EGFR)/Akt signaling may be markers involved in the initial response to inhalation exposure (111). Li et al. further highlighted this with an inhalation exposure paradigm in mice that diminished cardiac function with increased apoptosis through Caspase-3, Bax and Bcl-2 in male mice. Specifically, the connection between NF-kB activation and subsequent activation of IL-1 $\beta$ and IL-6 in neonatal ventricular cardiomyocytes was elucidated mechanistically (133).

The role of ROS, and particularly NADPH oxidase 4 (Nox4), in mice was examined during the development of cardiovascular disease following $\mathrm{PM}_{2.5}$ exposure. Measures after exposure indicated increased inflammation markers (tumor necrosis factor- $\alpha$ (TNF- $\alpha$ ) and IL-1 $\beta$ ), myocardial apoptosis, and NOX4 and NOX4 associated subunits, suggesting that the progression of cardiovascular disease, may be dictated by ROS generation and the modification of antioxidant proteins, which are initially regulated by $\mathrm{PM}_{2.5}$ exposure. (244). In another investigation, genes linked to fibrosis, (collagen type I alpha 1 (Col1a1) and collagen type III alpha 1 (Col3a1)) and ROS production (NOX-4 and transforming growth factor beta 1 (TGF $\beta 1)$ ) were shown to be increased, matching the cardiac dysfunction and prooxidative environment found in the heart (163). Vascular endothelial cell permeability pathways were assessed in rats through signal transducer and activator of transcription 3 (STAT3) phosphorylation (45). Induction of STAT3 regulated microRNA-21 expression, which in turn, caused a decrease in metalloproteinases 3 (TIMP3) and stimulated matrix metalloproteinases 9 (MMP9) expression. Exploring the effects of 
hyperlipidemia during exposure, rats eating a high fat diet were predisposed to higher blood pressure and lower heart rate variability, which were accompanied by decreased expression of antioxidant proteins, including superoxide dismutase (SOD). Additionally, the authors found that apoptosis in hyperlipidemic rats was regulated through c-Jun NH2-terminal kinase (JNK) and P53 phosphorylation and downstream activation of Bax and Caspase-3 (240). The intracellular and extracellular levels of HSP-70 were used to assess inflammation through a variety of exercise modalities (145). Mice were exposed to $\mathrm{PM}_{2.5}$ during moderate and high intensity exercises, which revealed higher heart lipid peroxidation levels. However, only the high intensity exercise group showed an anti-inflammatory effect, reducing the extracellular to intracellular HSP70 level, or Hindex.

The use of multiple models of particulate toxicity can provide a means for detecting individual toxicants responsible for the resulting physiological insult. In a co-exposure model of $\mathrm{SO}_{2}, \mathrm{NO}_{2}$, and $\mathrm{PM}_{2.5}$, increased endothelin-1 (ET-1) and endothelial nitric oxide synthase (eNOS) expression suggested endothelial dysfunction, while upregulation of cyclooxygenase-2 (COX-2), inducible nitric oxide synthase (iNOS), TNF- $\alpha$, and IL-6 suggested inflammatory signaling. The co-exposure contributed to decreased blood pressure and increased cardiovascular complications when compared to $\mathrm{PM}_{2.5}$ alone (266). In rats, $\mathrm{PM}_{2.5}$, consisting of polycyclic aromatic hydrocarbons (PAHs) 1-nitropyrene (1-NP) and 9-nitroanthracene (9-NA), increased DNA damage and DNA damage response genes (8-oxoguanine DNA glycosylase (OGG1) and growth arrest and DNA damage 153 (GADD153)), as well as genes involved in ROS regulation (glutathione S-transferase (GST)), and coagulation (HO-1) (269).

\section{$\underline{\text { Transgenic/Supplementation }}$}

Animal models of $\mathrm{PM}_{2.5}$ have not only allowed for the investigation of cellular adaptations in the heart following exposure, they have also provided information regarding manipulation of the biological system. In an atherosclerotic transgenic mouse model ( $\mathrm{ApoE}^{-/-}$), cardiac autonomic nervous system dysfunction and oxidative stress were induced following exposure to $\mathrm{PM}_{2.5}$, though, the high-fat diet fed, atherosclerotic mice displayed a greater proclivity for oxidative damage (158). To examine the metabolic component of inhalation exposure, AMP-activated protein kinase $\alpha 2$ knockout (AMPK $\alpha 2^{-/}$) transgenic mice, which lack this crucial gene for fatty acid oxidation, were assessed for cardiovascular function (235). AMPK $\alpha 2^{-/-}$mice exhibited left 
ventricular dysfunction and an increased oxidative and inflammatory environment through decreased expression of peroxiredoxin 5 (PRDX5) and increased expression of NF-KB and TNF$\alpha$. Thompson et al. showed that a transgenic mouse model for transient receptor potential cation channel A1 (TRPA1) knockout acts protectively in reducing myocardial dyssynchrony in mice when exposed to $\mathrm{PM}_{2.5}$ CAPs and Acrolein co-exposure (214). The authors concluded that TRAPA1 acts to increase cardiac risk, specifically in exposures with heterogeneous compositions. Supplementation of vitamins, antioxidants, and other natural and artificial compounds can provide valuable information regarding the mechanisms involved in $\mathrm{PM}_{2.5}$ exposure.

Through intracerebroventricular injection of an IкB kinase-2 (IKK) analog (IMD-0354) into diabetic mice, inflammation induced by $\mathrm{PM}_{2.5}$ exposure through increased COX-2 and mitogen activated protein kinase (MAPK) was remediated. Increasing IKK, an inhibitor of NF$\mathrm{KB}$, blocked the inflammatory response and reduced cardiovascular injury (268). In aortic tissue, 9 days of CAPs exposure resulted in decreased phosphorylation of Akt and eNOS, subsequently suppressing inhibitor of the transcription factor NF- $\mathrm{KB}(\mathrm{I} \kappa \mathrm{B} \alpha)$ and producing an inflammatory response. By treating with an antioxidant, 4-hydroxy-2,2,6,6-tetramethylpiperidine-1-oxyl (TEMPOL), inflammation was alleviated, as well as insulin resistance in high fat diet fed mice (90). Supplementation of vitamin $\mathrm{E}$ and omega-3 polyunsaturated fatty acids prior to $\mathrm{PM}_{2.5}$ exposure in rats, attenuated cardiac injury and reduced inflammation through decreased expression of TNF- $\alpha$, IL-1 $\beta$, and IL-6 (61). At high doses of PM 2.5 , cardiac mitochondrial ultrastructure (size and cristae formation) changes were observed, potentially through changes in fission/fusion dynamics, with increases in mitochondrial fission 1 (FIS1), mitofusion (MFN) 1, MFN2, dynaminrelated protein 1 (DRP1), and mitochondrial dynamin like GTPase (OPA1). Furthermore,these authors went on to show increased inflammation (TNF- $\alpha$, IL-6, and IL-1 $\beta$ ) (74). Investigating the benefits of selenium yeast ( $\mathrm{SeY}$ ) supplementation, Sprague Dawley rats were pretreated with $\mathrm{SeY}$ for 28 days and subsequently exposed to $\mathrm{PM}_{2.5}$ (261). SeY pretreatment caused a significant increase in the total antioxidant capacity, total SOD expression, and a decrease in proinflammatory markers, TNF- $\alpha$ and IL- $1 \beta$ as compared to the $\mathrm{PM}_{2.5}$ exposed group.

A prooxidative environment in mice following $\mathrm{PM}_{2.5}$ exposure was characterized by an increase in intracellular free calcium, and a potential decrease in cardiac contractile function due to increased ryanodine receptor 2 (RYR2) and decreased sarcoplasmic/endoplasmic reticulum 
calcium ATPase 2a (SERCA2a). With inclusion of an antioxidant therapeutic approach from compound essential oils (CEOs), the heart reduced total ROS, returned the calcium levels back to control, and reduced the thrombosis factor HO-1 (60). Hypertrophy was investigated in a cohort of mice following exposure (130). Cardiac remodeling through hypertrophy and fibrosis were correlated with reduced SOS Ras/Rac Guanine Nucleotide Exchange Factor 1 (SOS1), cAMP response element binding protein (CREB), and glycogen synthase kinase 3 beta (GSK3b) expression, which was remediated through fucoidan administered therapeutically.

\section{Gestational Exposures}

Understanding the environmental impact on developing progeny is a growing field and germane to future evaluations of $\mathrm{PM}_{2.5}$ cardiotoxicity (Figure 1.2). One day old neonatal offspring from Wistar rats revealed higher myocardium apoptosis in a dose-dependent manner, potentially due to alterations in mitochondrial fission/fusion through OPA1, MFN1, DRP1, and FIS1 (234). In one day old neonate mice, an inflammatory and extracellular matrix remodeling phenotype (increased IL-6, IL-1ß, collagen-1, matrix metalloproteinase (MMP) 9, and MMP13 expression) was shown, revealing early fibrosis of the heart (211). Calcium regulated contraction has been shown to be affected throughout gestationally-exposed progeny development. Assesment of fourteen-day old progeny, revealed decreased cardiomyocyte contractility through calcium dynamics, with changes to proteins (SERCA-2A, phosphorylated phospholamban (p-PLN), $\mathrm{Na}^{+} / \mathrm{Ca}^{2+}$ exchanger (NCX), and calcium channel, voltage-dependent, L type, alpha 1C subunit (CaV1.2)) involved in calcium flux in the heart (211). At 12 weeks of age, progeny revealed cardiac dysfunction and increased collagen deposition in cardiac tissue, potentially through changes in epigenetic regulation (decreased Sirtuin (SIRT) 1 and SIRT2 and increased DNA methyltransferase (DNMT) 1, DNMT3a, and DNMT3b expression) and calcium handling proteins (decreased $\mathrm{Ca}^{2+}$-ATPase, p-PLN, and NCX) (212). 
Figure 1.2: Gestational Inhalation Exposure Paradigm

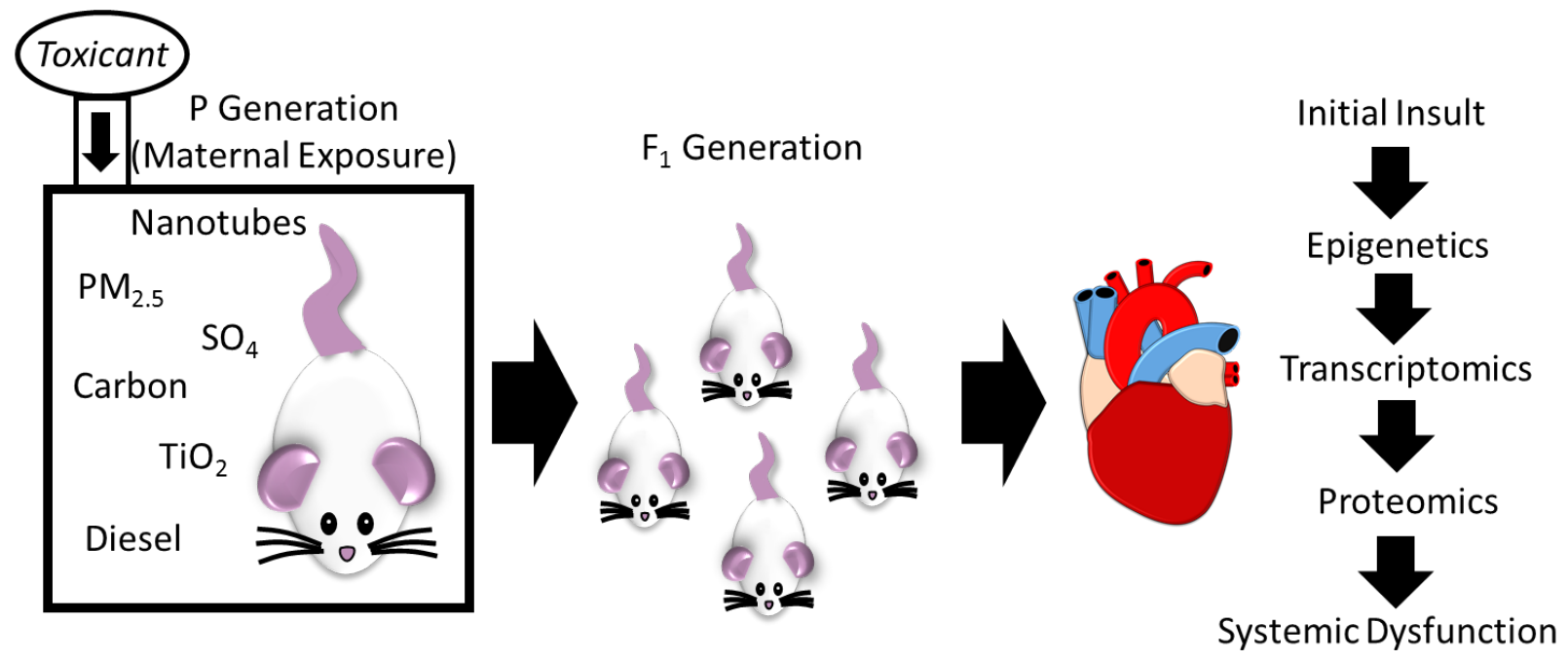

Figure 1.2: Molecular adaptations of the heart following gestational inhalation exposure. Exposure to particulates during pregnancy can result in maladaptive alterations in progeny cardiovascular function. Epigenetic alterations in the heart have the capacity to initiate persistent functional deficits that may continue into adulthood.

In a gestational exposure setting, wherein both parents were exposed to $\mathrm{PM}_{2.5}$, the resulting progeny were shown to have increased inflammation, fibrosis, and oxidative stress with an increase in calcium handling proteins (210). Interestingly, epigenetic changes suggest a repressive phenotype, with both the maintenance methyltransferase (DNMT1) and histone deacetylases (SIRT 1/2) increased in expression. Inflammation markers (TNF- $\alpha$ and IL-1 $\beta$ ) were significantly increased, along with a reduction in genes associated with cardiovascular development (GATA Binding Protein 4 (GATA4) and homeobox protein Nkx (NKX2-5)) in neonatal and weanling Sprague Dawley rats following exposure during gestation and lactation. The authors also detail the similarity in response of progeny exposed in utero to $\mathrm{PM}_{2.5}$ and those exposed through maternal intraperitoneally delivered homocysteine (36). In young adult mice following gestational exposure, cardiac hypertrophy was exhibited, with an increase in both total histone acetylation and promoter acetylation of hypertrophy genes (GATA4 and myocyte enhancer factor 2C (Mef2c)), indicating potential global epigenetic remodeling through transcriptional activation of acetylated regions (245). Interestingly, a cardiac hypertrophic/fibrotic phenotype and epigenetic remodeling have been reported in early development $(210,212,245)$. 


\section{Ultrafine Particulate Matter $\leq 0.1 \mu \mathrm{m}\left(\mathbf{P M}_{0.1}\right)$}

Similar to $\mathrm{PM}_{2.5}$, ultrafine particulate matter (UFP) has the capacity to penetrate deeply into the alveoli of the tracheobronchial airways (201), but, UFP exhibit an increased capacity for interstitialization and transport systemically throughout the body due to their high surface-tovolume ratios $(27,62)$. While natural sources of UFP exist, UFPs can be produced in high concentrations through combustion reactions in urban settings (113) which can be especially detrimental to human health (190). Specifically, the impact on vulnerable populations $(190,228)$ as well as the increased susceptibility of women to inhaled UFPs (191) make the study of particulate matter at the nanoscale so important. While investigations have begun to examine the cardiovascular deficits associated with UFP inhalation toxicology (205), the molecular and cellular mechanisms driving the associated dysfunction remain unlinked between exposure paradigms. We provide an illustration to help summarize the DNA, protein, and cellular changes observed following toxicant inhalation exposure, including nanomaterial/nanoparticle (Figure 1.1).

\section{Ambient Air and Occupational Pollution}

In individuals with metabolic disorder, UFP inhalation exposure was specifically detrimental to cardiac function in those without the glutathione s-transferase mu 1 (GTSM1) protein (56). In the complete cohort exposed to UFP, C-reactive protein (CRP), serum amyloid A (SAA) were increased with a subsequent decrease in plasminogen and thrombomodulin within blood, indicating an upregulation of inflammatory pathways. In a type 2 diabetic cohort, the inflammatory response is also modified following UFP inhalation exposure, increasing CD40 ligand (CD40L) expression in platelets with a resulting decrease in soluble CD40 in blood (200). Platelet CD40L expression and blood coagulation von Willebrand factor were both elevated following acute UFP exposure, indicating the adverse predisposition of type 2 diabetics to additional insults. To understand the relationship between ambient exposure and the time/concentration of the exposure, Lane et al. examined biomarkers in patients with varying levels of microenvironment exposures (time-activity adjusted) as well as the concentration of the UFPs (annual average particle number concentration) (126). Positive associations between time/concentration of exposure and blood CRP, IL-6, and tumor necrosis factor receptor 2 
(TNFRII) were found, with significant positive correlations of CRP and TNFRII with white nonHispanic participants, but not those of East Asian descent.

While most population-derived analyses use systemic blood to assess cardiovascular impact, post-mortem ventricular autopsies were performed on 30 children and young adults to assess the impact of urban UFP pollution (226). Compared to clean air controls, proteins involved in oxidative (prion protein (PRNP)), and endoplasmic reticulum (glucose regulated protein 78 (GRP78)) stress were significantly upregulated, and even disproportionately upregulated in the left, compared to right, ventricle of the UFP exposed cohort. These data suggest a compensation by the heart to attenuate inflammatory effects of air pollution, maybe specifically for UFP (226). In an occupational study of healthy individuals near a steel mill, increases in plasma levels of IL6, IL-8, and ET-1 were significantly associated with rises in $\mathrm{CO}$, UFP and $\mathrm{SO}_{2}$ concentrations in ambient air, with a positively correlation between BET-1 and CRP with cardiac dysfunction indexes (123). Proton ontology revealed a significant elevation of inflammation specific pathways.

\section{Animal Models}

In Sprague-Dawley rats, an acute and sub-chronic inhalation exposure to both $\mathrm{PM}_{2.5}$ and $\mathrm{PM}_{0.1}$ revealed significant upregulation of angiotensin II type 1 receptor $\left(\mathrm{AT}_{1} \mathrm{R}\right) \mathrm{mRNA}$ in the heart, with the sub-chronic exposure to both PMs causing an increased protein level (10). In the sub-chronic group for both PM concentrations, this also lead to decreased expression of HO-1 and increased expression of actin alpha 1 (Acta1), Col3a1, and IL-6. The authors suggest that cardiovascular complications, marked by coronary artery wall thickness, are linked to alterations within the endocrine system, specifically pathways related to angiotensin (10). UFP exposure has also been linked to mitochondrial dysfunction. Implementing a Langendorff isolated heart model in rats, ischemia/reperfusion was exacerbated in those rats exposed to UFP (99). Treatment with Cyclosporin A (CsA) in isolated hearts as well as decreased $\mathrm{Ca}^{2+}$ buffering in isolated mitochondria suggest that the mitochondrial permeability transition pore (mPTP) may be impacted. To understand the impacts of aging phenotypes to UFP inhalation exposure, a 12-13 month spontaneously hypertensive rats (SHRs) were exposed to ultrafine carbon particles (UFCP) (220). Increased serum CRP, plasma fibrinogen, and levels of ET-1 in the heart were all associated with UFCP inhalation exposure and increased blood pressure and heart rate. Additionally in the 
SHR model, angiotensin II (AngII) was shown to be elevated in the blood following exposure to UFCP through instillation, with increasing doses of UFCP attenuating the effectiveness of captopril, an angiotensin converting enzyme inhibitor (263).

\section{Gestational Exposures}

The gestational impact of UFP on growing progeny also provides valuable mechanistic knowledge regarding the modes of action of inhaled UFP on cardiovascular function. UFP exposure to pregnant $\mathrm{C} 57 \mathrm{BL} / 6 \mathrm{~J}$ dams lead to fetal resorption and increased blood pressure in adult mice progeny (151). Increases in maternal serum inflammation during gestation (i.e. elevated IL$1 \beta$, IL-6 and MCP-1 levels) and $\mathrm{AT}_{1} \mathrm{R}$ and angiotensin I-converting enzyme (ACE) activation in the lungs were suggested to contribute to progeny cardiovascular dysfunctions. Gestational exposure to UFP in mice was also performed to understand adaptability to immunological responses and innate immunity in progeny (173). Challenging progeny immune systems with house dust mite (HDM) allergen revealed a decreased immune response through lower IL-13 and IL-17, with higher serum levels of IL-10. This has also been demonstrated with UFP containing persistent free radicals (MCP230), which suppressed the $\mathrm{T}$ helper and $\mathrm{T}$ regulatory cells found within the lungs of offspring (239).

\section{PM2.5 and UFP Combined Analyses}

While many studies have examined the effects of either $\mathrm{PM}_{2.5}$ or UFP, some analyses allow for the direct comparison of both sizes of particulates within a single investigation. Levels of Delta$\mathrm{C}$, which is a compound known to exist in wood smoke, was found to be positively correlated with fibrinogen and myeloperoxidase (MPO), but not with $\mathrm{PM}_{2.5}$ or UFP (43). Additionally, the study identified $\mathrm{PM}_{2.5}$ or UFP as retaining a positive correlation with CRP and fibrinogen, sharing a similarity in the effects on blood coagulation with effects of Delta-C. In a meta-population study, including 3275 participants, CRP and platelet count was significantly increased in blood of participants matching long-term (2000-2008) rises in $\mathrm{PM}_{2.5}$, with no direct associations found for $\mathrm{PM}_{0.1 .}$.(224). The integrity of the mitochondrial DNA defines the respiratory potential of the mitochondrion, allowing for separation of individuals in to a variety of haplogroups (171). Ambient air pollution differentially effected individuals with diverse haplotype backgrounds, with 
the haplogroup $\mathrm{H}$ (more uncoupled) compared to haplogroup $\mathrm{U}$ (less uncoupled) being more detrimentally effected and elevated blood IL- 6 and TNF- $\alpha$ in both groups associated with quasiultrafine particles $<0.25 \mu \mathrm{m}(243)$.

To determine the epigenetic regulation of inflammatory markers, healthy subjects inhaled an array of particulate matter compositions $\left(\mathrm{PM}_{10}, \mathrm{PM}_{2.5}, \mathrm{PM}_{1.0}\right.$ and $\left.\mathrm{PM}_{0.5}\right)$ over two sessions (216). Blood samples following the trials revealed that increased interferon $\gamma$ (IFN- $\gamma$ ) methylation was significantly associated with $\mathrm{PM}_{2.5}$ as well as quasi-ultrafine particles $<0.50 \mu \mathrm{m}$, correlating with alterations in the parasympathetic nervous system. Examining the impact of urban air pollution in healthy, COPD, and ischemic heart disease participants, microRNA profiles in blood were assessed to identify associations between isolated pollutants. Nine differentially regulated microRNAs were significantly associated with $\mathrm{PM}_{2.5}$ in blood, while no associations were identified for UFP (68). The microRNA alterations for $\mathrm{PM}_{2.5}$ included an upregulation of hsa-miR197-3p and hsa-miR-99a-5p, playing potential roles in cancer $(68,256)$. Acute, 24 hour exposure to $\mathrm{PM}_{2.5}$ personal air pollution resulted in significant alterations to the DNA methylation profile in 157 healthy non-smoking adults (153).

Blood collected from those subjects revealed up to 69 differentially methylated regions with altered methylation status, linking to genes such as Kinase Non-Catalytic C-Lobe Domain Containing 1 (KNDC1) and family with sequence similarity 50 member B (FAM50B), which remain relatively obscure as to their functional role in the cardiovascular system. Additionally, ambient and personal exposure to UFP resulted in 31 differentially methylated regions in DNA (153). As a means of isolating out the specific toxicants responsible for cellular and molecular dysfunction following inhalation exposure, particulate matter at the coarse $\left(\mathrm{PM}_{10}\right)$, fine $\left(\mathrm{PM}_{2.5}\right)$, and ultrafine $\left(\mathrm{PM}_{0.1}\right)$ range were exposed to healthy volunteers and specific metals were correlated to physiological effects (137). As a result, increased serum concentrations of VEGF, traumatic brain injury marker ubiquitin C-terminal hydrolase L1 (UCHL1), and cortisol were significantly correlated with nickel, barium, and silver metal concentrations, respectively. While reports have generally shown the increased toxicity of smaller particulate matter, Liu et al. indicated that coarse $\left(\mathrm{PM}_{10}\right)$ exposures to CAPs caused significant changes in blood cortisol levels, where UFPs did not reveal any changes in biomarkers surveyed (138). 


\section{Nanotoxicology}

Studies investigating ultrafine particulate matter (UFP) allow for the examination of physiological perturbations in a fashion that is comparable to nanomaterials and nanoparticles. While inhalation studies examining ENMs and UFP typically differ in the number and standardization of compounds found within the mixtures, both share commonality in the nanometer scale that permits similar dispersion within the lungs during inhalation. Nanomaterials represent an ever-increasing vehicle for inhalation toxicology (121). Whether during the manufacturing of nanomaterials (121) or in their consumer use (154), production and rate of application continue to rise. Nanomaterials and nanoparticles, similar to $\mathrm{PM}_{0.1}$, exist exclusively at a biological scale that alters the dynamics of interactions with organic matter, potentially altering toxicities compared to their micrometer-sized equivalents. We provide an illustration to help summarize the DNA, protein, and cellular changes observed following toxicant inhalation exposure, including nanomaterial/nanoparticle (Figure 1.1).

\section{Animal Models}

Savi et al. showed that a direct translocation of nanomaterials to the heart, through inhalation exposure, could be linked to subsequent cardiovascular dysfunction (179), and others have since examined the molecular consequences of this interaction (105). In the heart, following inhalation exposure, translocation of nanomaterials was suggested to initiate the complement cascade, setting off a local immune response, as well as global complement factor 3 (C3) upregulation in the blood. Chronic exposure to nano- $\mathrm{TiO}_{2}$ in outbred mice led to a significant change in inflammation markers, including changes in transcription factors (STAT 1/3/6 and GATA 3/4) in the heart (100). Examining co-exposure models, silica nanoparticles (SiNP) and methylmercury ( $\mathrm{MeHg}$ ) induced an increase in activity of cardiac troponin $\mathrm{T}$ (cTnT), atrial natriuretic peptide (ANP) and brain natriuretic peptide (BNP), along with increased SERCA2 levels in rat serum (249).

In another model by the same group, Feng et al. applied a SiNPs and Pb co-exposure model and found similar effects in the heart, namely increased expression of inflammation markers (Creactive protein (CRP), IL-6, and TNF- $\alpha$ ) and upregulation of ANP and BNP following exposure 
in the serum (73). To evaluate the mechanistic effects of inhalation compared to intra-arterial infusion on extra-pulmonary organ susceptibility, Ganguly et al. employed carbon nanoparticles in the evaluation of cardiovascular function (78). While finding that inhalation exposure induced an inflammatory response within the lungs and intra-arterial infusion produced forms of systemic inflammation, cardiovascular transcripts and proteins related to inflammation and endothelial/epithelial activation in the aorta and heart were more significantly impacted through inhalation exposure than injected carbon nanoparticles. These results provide strong evidences for indirect (neuronal or inflammatory) in contrast to direct (nanoparticle contact) mechanisms of cardiovascular dysfunctions associated with xenobiotic exposures (78).

Only recently have transgenic mouse models been applied to examining nanomaterial exposure. In a single dose inhalation exposure, a transgenic mouse model for phospholipid hydroperoxide glutathione peroxidase (mPHGPx) was used to restore mitochondrial respiratory function and ROS levels following nano- $\mathrm{TiO}_{2}$ exposure (198). By increasing antioxidant defense, proteomics revealed that changes in mitochondrial composition, including individual electron transport chain complex constituents, were protected from the detrimental effects of exposure. In another study, a microRNA knockout transgenic mouse model (microRNA-378a) showed increased Mfn1 expression and altered mitochondrial ultrastructure through transmission electron microscopy, potentially through mitochondrial fusion dynamics (94).

\section{Gestational Exposures}

A limited number of studies have begun to examine the cardiovascular complications arising in progeny that were gestationally-exposed to nanomaterials/particles. The impact of gestational nanoparticle exposure on cardiac function and mitochondrial health, as well as transcriptomic and epigenomic landscape have been explored. Diastolic cardiac function and mitochondrial respiration were shown to be diminished in young adult progeny following nano$\mathrm{TiO}_{2}$ gestational exposure (95), with increases in mitochondrial proton leak, uncoupling protein 2 (UCP2), and changes in mitochondrial metabolism proteins (carnitine palmitoyltransferase 1A (CPT1A) and pyruvate dehydrogenase (PDH) phosphorylation). Previously, state 3 respiration was also shown to be decreased in the left ventricle following gestational exposure in young adult progeny (199). Ultimately, changes in global histone methylation, and the resulting epigenome, were considered for future evaluation. Using chromatin immunoprecipitation sequencing for 
histone 3 lysine 4/27 trimethyaltion (H3K4me3 and H3K27me3) coupled with the transcriptomic profiling of fetal cardiac tissue from gestationally-exposed dams (198), major pathways including immune adaption and organismal growth, were significantly altered. In the fetal progeny, cardiovascular outcomes at the young adult stage may actually be linked to liver and fetal development, evidenced through IPA protein ontology.

\section{Future Directions}

From a morning commute, to an occupational setting, to our very homes, exposure to particulates remains a central issue for debate and therapeutic/lifestyle interventions. Industrialization and increased urbanization will continue to exacerbate issues concerning air quality and human health. Although we provide a summarization of the molecular mechanisms driving cardiovascular decline following exposure, areas of research that are crucial in driving the field of molecular inhalation cardiovascular toxicology forward, still remain.

A need exists for more encompassing "omics" based approaches. While investigators have begun to examine molecular influencers of inhalation exposure in the heart, including protein and gene markers for inflammation, ROS generation, antioxidant defense, general and specific transcription factors, non-coding RNAs, cancer predisposition, and others, few studies have examined the complete cellular protein, gene, or metabolic regulatory networks. The integration of transcriptomics with epigenomics, proteomics, etc., provides a complete snapshot of the altered molecular state. In the heart, elucidating the primary causes of insult will require multi-omics integration through the identification of pathways altered, (i.e. metal-binding, DNA damage response, epigenetic factors, mitochondrial, or other protein pathways). True mechanistic understanding requires a more complete picture to elucidate causal and compensatory actions of the cell.

A necessity also exists for cellular models of exposure interactions. While it remains outside the scope of this review, in vitro models can provide a very granular, and controlled approach to mechanistic evaluations. Of note, authors have recently investigated the effects of $\mathrm{PM}_{2.5}$ exposure $(24,46,71,101,250,251,266)$, as well as the nanotoxicology $(72,103,104,249)$ in cells. Ultimately, work at the population, animal, and cellular level can all lead to better 
evaluation of particulate exposure to cardiovascular outcomes. Due to limitations in the standardization of particulate matter exposures, more research establishing the relationship between individual toxicants and specific molecular outcomes is needed in order to draw conclusive statements about the exposure and/or co-exposure etiology. Moving forward, research in molecular inhalation cardiovascular toxicology needs to continue to examine what the relevant pathways are to the heart and circulatory system, including mitochondrial function, calcium regulation, and paracrine/exosome release from the heart and systemic implications. As we continue to shift the paradigm of cardiovascular research in inhalation toxicology, much of the cellular and molecular mechanisms are yet to be discerned. How these mechanisms differ for each toxicant, longevity of exposure, and concentration of dose must be considered when characterizing pathways altered in the heart (Figure 1.3).

Figure 1.3: Cellular and Molecular Impacts on the Cardiovascular System

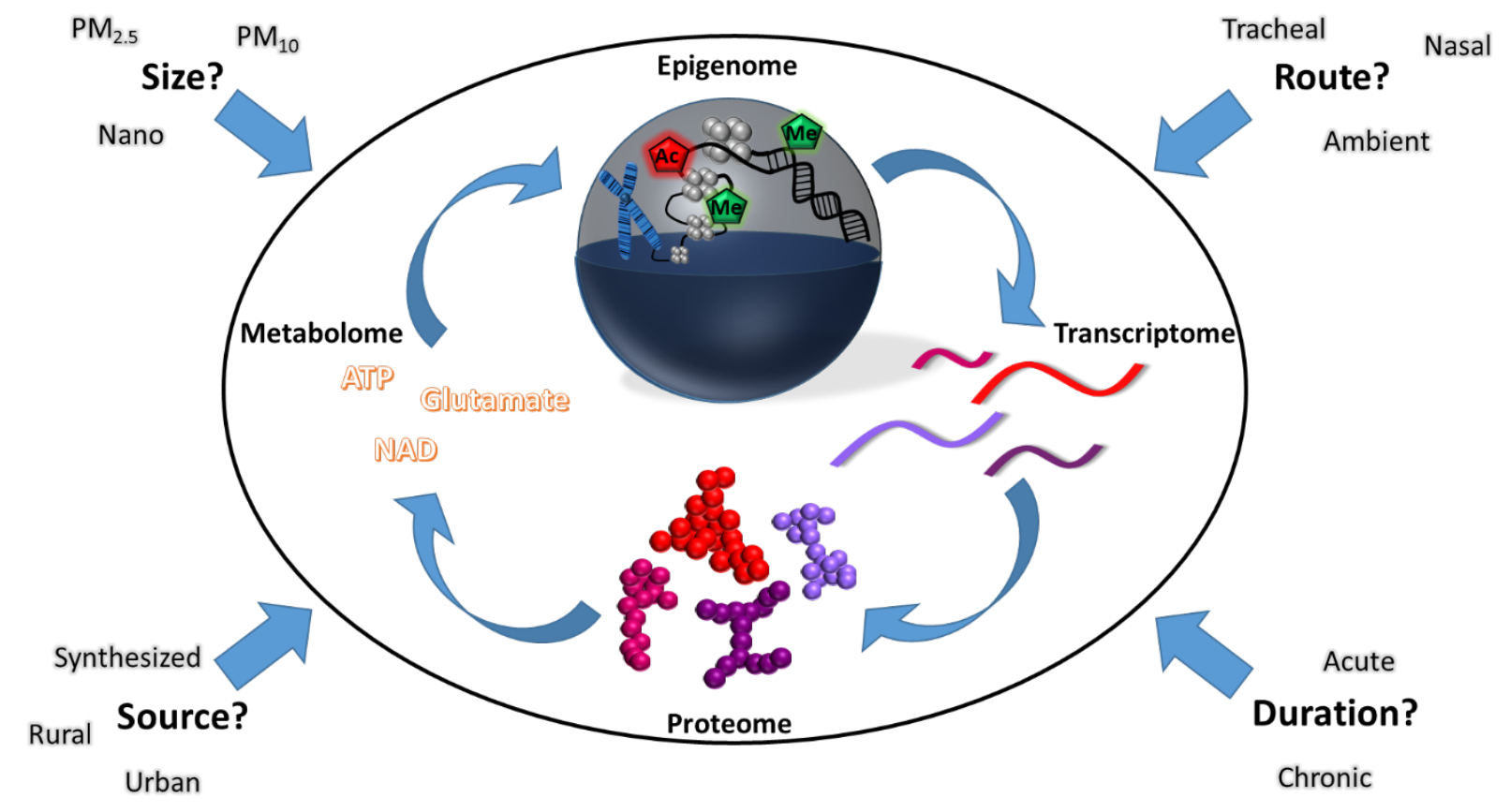

Figure 1.3: Overview of the variables that dictate the effects induced by toxicant exposures on the downstream cellular and molecular mechanisms in the heart: source of the toxicant, particle size and dose, method of exposure, and the duration of the exposure. Changes in any one of the four levels indicated above could have serious implications on the interpretation of broad-scale cellular alterations and how we should consider the basic mechanisms involved in inhalation exposure paradigms. 


\section{Type 2 Diabetes Mellitus}

\section{Introduction}

Diabetes mellitus affects 29.1 million Americans and 25.9\% of Americans age 65 and older. Adults with diabetes mellitus have two to four times the rate of mortality from heart disease as compared to those without diabetes mellitus, and 68 percent of diabetic patients age 65 and older die of heart disease $(1,2)$. The diabetic heart is metabolically characterized by insulin resistance, reduced cellular glucose import and oxidation, and increased mitochondrial fatty acid import and oxidation (197). This chronic alteration in energy substrate utilization and loss of the dynamic ability of the myocardium to metabolically adapt to its environment, such as upregulating anaerobic glycolysis during cardiac ischemia, initiates a pathological state termed diabetic cardiomyopathy $(5,142)$. Increased cardiac hypertrophy and fibrosis are structural changes observed in diabetic cardiomyopathy that are accompanied molecularly by increased oxidative stress, mitochondrial dysfunction, and cardiomyocyte apoptosis (35, 64, 106, 172, 273). Diabetic heart failure is the culmination of these pathologic insults that is characterized by severe contractile dysfunction of the myocardium (57).

The proteomic alterations of the heart in the diabetic state have been well characterized. Metabolically, the decrease in glucose import and oxidation in the diabetic cardiomyocyte is associated with decreased expression of insulin-stimulated glucose transporter type 4 (GLUT4) and decreased activity of pyruvate dehydrogenase (PDH) (197). The increase in mitochondrial fatty acid import and oxidation are found in conjunction with decreased acetyl-CoA carboxylase activity, decreased malonyl-CoA concentration, increased carnitine palmitoyl transferase I activity, and increased beta-hydroxyacyl-CoA dehydrogenase activity (197). Increased reactive oxygen species (ROS) generation in diabetic cardiomyopathy results from contributors such as excess fatty acid oxidation, increased NADPH oxidase activity, and uncoupled nitric oxide synthases (NOS) (106). Increased cardiomyocyte apoptosis in the diabetic setting is contributed to by the decreased expression of the anti-apoptotic protein B-cell lymphoma 2 (Bcl-2), and increased expression of the pro-apoptotic protein p53 $(168,270)$. At the heart of energy substrate metabolism, ROS generation, and apoptosis lies the mitochondrion.

While transcription factor regulation of gene expression, post-translational modification, and substrate inhibition via negative feedback have shown to be foundational in regulating protein expression and activity, microRNA (miRNA) and epigenetic mechanisms of regulation have more 
recently emerged (6). MiRNA are single-stranded noncoding RNA molecules, 22 nucleotides long, that associate into a multi-protein RNA-induced silencing complex (RISC) which inhibits its target mRNA species from being translated into a functional protein (15). Differential expression of miRNAs have been characterized in a variety of cardiovascular conditions including atherosclerosis, myocardial infarction, and heart failure to modulate processes such as energy substrate metabolism, fibrosis, and cardiac remodeling (162, 192, 264). Nuclear- and, more recently, mitochondrial-genome-encoded proteins which function in the mitochondria, have been shown to be regulated by miRNAs $(14,17,49,109)$, and may alter dynamics between the mitochondrion and nucleus (Figure 1.4). Since cardiomyocyte mitochondria are central to the pathogenesis of diabetic heart disease, the first section of this review will focus on miRNA regulation of key pathologic pathways intersecting at the mitochondrion. The following section will focus on the role of regulation on the miRNA regulator, including epigenetic, exosomal, processing, and post-transcriptional control (Figure 1.5). Finally, treatment strategies for cardiomyocyte energy substrate metabolism, pharmacologic manipulation and miRNA-based therapies for the treatment of diabetic heart disease will be discussed.

\section{Figure 1.4: Nuclear and Mitochondrial Signaling}

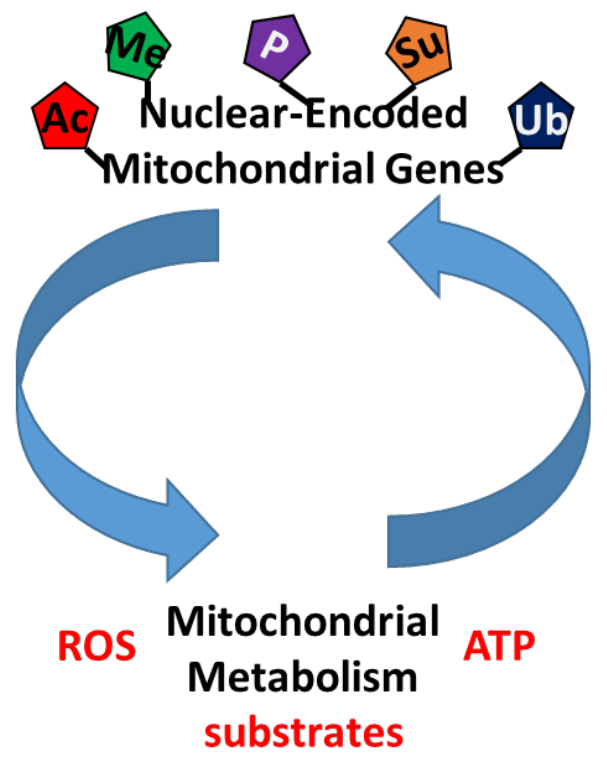

Figure 1.4: Feedback loops existing between the mitochondrion and nucleus. By epigenetically altering the transcription efficiency of nuclear-encoded mitochondrial proteins through changes in DNA or histone acetylation (Ac), methylation (Me), phosphorylation (P), sumoylation $(\mathrm{Su})$, and/or ubiquitination (Ub), the mitochondrion is affected (anterograde signaling). Alternatively, mitochondrial metabolism affects substrate availability for proteins necessary in causing epigenetic regulation (retrograde signaling).

\section{MiRNA Regulation of the Mitochondrion in the}

Diabetic Heart

MiRNAs can be transcribed through two primary pathways, either a canonical or non-canonical pathway (89, $149,242)$. In the canonical pathway, the pri-miRNA is transcribed from its existing exon and intron, containing one to several hairpin loop structures. After transcription, DiGeorge Syndrome critical region 8 (DGCR8) and Drosha, known as the microprocessing complex, will cut the pri-miRNA in the nucleus, resulting in a shorter ( $\sim 65 \mathrm{nt})$ pre-miRNA $(85,92)$. The pre-miRNA is then exported through Exportin-5 and RanGTP (254). 
Finally, in the cytoplasm, Dicer will edit the pre-miRNA resulting in the mature miRNA (19-22 nt) which can then associate with the RNA-induced silencing complex (RISC). In the noncanonical pathway, hairpin structures within introns can be spliced out, resulting in pre-miRNA that are referred to as mirtrons (242). After splicing, the mirtrons proceed through the same pathway as canonical miRNA in order to be exported from the nucleus.

\section{Figure 1.5: Processes of miRNA Regulation}

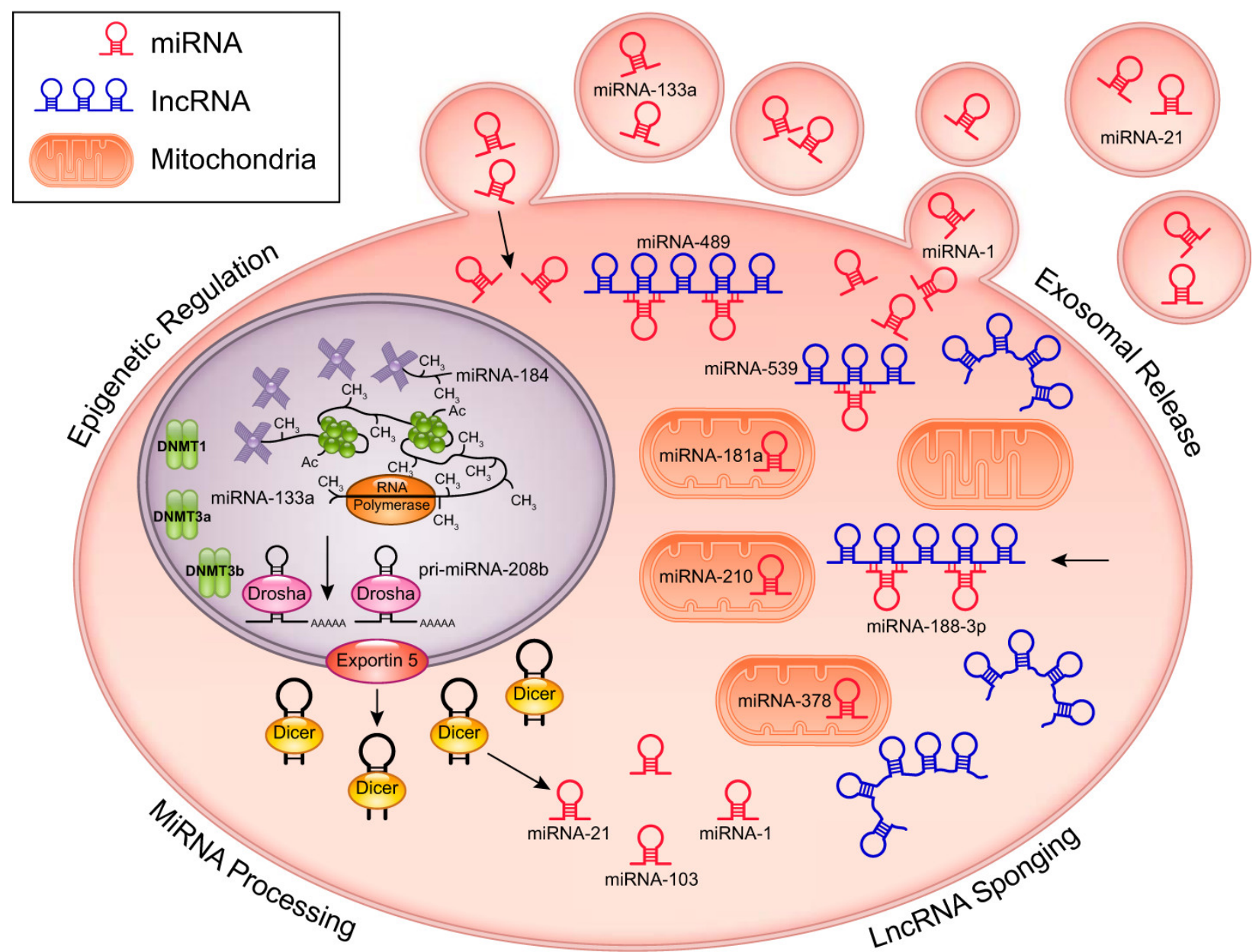

Figure 1.5: Regulation of miRNA regulators. The expression of miRNAs are controlled through multiple factors. Epigenetic regulation includes hypermethylation of the miRNA-184 CpG loci, pri-miRNA-208b tethering to increase EZH2 binding, and miRNA interaction with DNMT1, DNMT3a, and DNMT3b. Regulation of miRNA expression through miRNA processing is dependent on Dicer expression (miRNA-103) and protein-protein interactions with Dicer (miRNA-21). Other proteins, such as G3bp1, can affect processing (miRNA-1). LncRNA can regulate miRNA expression by acting as a "sponge," sequestering native and exogenous miRNAs, such as miRNA-188-3p, -539, and -489. Also, exosomal release/transport of miRNA-1, -21, and -133a can alter miRNA expression. EZH2 = Enhancer 
of zeste homolog 2; DNMT = DNA methyl transferase; G3bp1 = GTPase-activating protein-binding protein 1; $\operatorname{lncRNA}=$ long non-coding RNA.

Following export from the nucleus, miRNAs are then implicated in regulating the expression of proteins essential to mitochondrial health. Recently, miRNAs have been found to affect mitochondrial function through targeting pathways including energy substrate metabolism, ROS generation, and apoptosis. This section will focus on miRNA regulation of these processes within the cardiomyocyte in the context of the diabetic heart (Table 1.2).

\section{Table 1.2: MiRNA Regulation of Mitochondrial Pathways in the Diabetic Heart}

\begin{tabular}{|c|c|c|c|c|c|c|}
\hline miRNA & $\begin{array}{c}\text { Environmental } \\
\text { Regulation }\end{array}$ & $\begin{array}{c}\text { Expression in } \\
\text { DB Heart }\end{array}$ & $\begin{array}{c}\text { miRNA } \\
\text { Target }\end{array}$ & $\begin{array}{l}\text { miRNA Target } \\
\text { Function }\end{array}$ & DB Model & Source \\
\hline let-7 & Pro & Upregulated & IR, IRS-2 & Insulin Signaling & STZ, ischemia reperfusion & $(129,275)$ \\
\hline miRNA-195 & & Upregulated & SIRT1 & Insulin Signaling & $\mathrm{STZ} ; \mathrm{db} / \mathrm{db}$ & $(54,271)$ \\
\hline miRNA-216a & & Upregulated & CAV2 & Insulin Signaling & $\begin{array}{c}\text { Human diabetic; Ischemic } \\
\text { heart failure }\end{array}$ & $(84,125)$ \\
\hline $\begin{array}{l}\text { miRNA- } \\
199 a-3 p\end{array}$ & & Upregulated & CAV2 & Insulin Signaling & STZ & $(58,185)$ \\
\hline miRNA-133a & Epi, Exo & Downregulated & TR4 & $\begin{array}{l}\text { Fatty Acid } \\
\text { Transport }\end{array}$ & STZ & $(38,160)$ \\
\hline miRNA-210 & & Upregulated & ISCU1/2 & ETC Complex I & $\begin{array}{l}\text { Human diabetic; Ischemic } \\
\text { heart failure }\end{array}$ & $(30,84)$ \\
\hline miRNA-141 & & Upregulated & Slc25a3 & $\begin{array}{c}\text { Mitochondrial } \\
\text { Phosphate Carrier }\end{array}$ & STZ & (17) \\
\hline miRNA-378 & & Upregulated & ATP6 & ATP Production & STZ & $(109)$ \\
\hline miRNA-29a & & Downregulated & PGC-1a & $\begin{array}{l}\text { Fatty Acid } \\
\text { Oxidation }\end{array}$ & STZ & $(58,136)$ \\
\hline miRNA-30c & & Downregulated & p53 & Apoptosis & $\begin{array}{l}\text { Human diabetic; Rat diabetic } \\
\text { cardiomyopathy; High } \\
\text { glucose-treated H9C2 }\end{array}$ & (168) \\
\hline miRNA-181a & & Downregulated & $\mathrm{p} 53$ & Apoptosis & $\begin{array}{l}\text { Human diabetic; Rat diabetic } \\
\text { cardiomyopathy; High } \\
\text { glucose-treated H9C2 }\end{array}$ & (176) \\
\hline miRNA-30d & & Upregulated & FOXO3a & Survival & $\begin{array}{c}\text { STZ; } \\
\text { High glucose-treated H9C2 }\end{array}$ & $(132,257)$ \\
\hline miRNA-34a & & Upregulated & BCL-2 & Survival & High glucose-treated H9C2 & $(267)$ \\
\hline miRNA-1 & Exo, Pro & Upregulated & BCL-2 & Survival & $\begin{array}{c}\text { STZ; } \\
\text { High glucose-treated H9C2 }\end{array}$ & $(255,258)$ \\
\hline
\end{tabular}

Table 1.2: MiRNA regulation of mitochondrial pathways in the diabetic heart. The differential expression of each miRNA species in diabetes is indicated along with its protein target. The process or pathway the protein participates is given. The model(s) in which the reported observations have been made are noted. Environmental regulation refers to the participation of the miRNA in either epigenetic (Epi), exosomal transport (Exo), or miRNA processing (Pro) pathways known to influence the diabetic cardiovascular system.

\section{MiRNA Regulation of Energy Substrate Metabolism in the Diabetic Heart}

MiRNA Regulation of Insulin Signaling and Glycolysis 
The downregulation of insulin signaling components in the diabetic heart is influenced by miRNA regulation. Li et al. showed that let-7 miRNA was overexpressed in the myocardium of streptozotocin (STZ)-induced diabetic rats, while the protein expression of insulin-like growth factor 1 receptor (IGF-1R), insulin receptor (IR), and glucose transporter 4 (GLUT4) were significantly lower (129). Interestingly, they found that inhibition of let-7 via the administration of let-7 antimiR conferred cardioprotection against ischemia-reperfusion injury through increased expression of p-Akt and p-mTOR (129). IR and IRS2 are validated as direct targets of let-7, and the observation of a normalization of IGF-1R, IR, and GLUT4 expression upon administration of let-7 antimiR supports the assertion that let-7 targets insulin signaling and the glucose transport pathway $(129,275)$. Further, Greco et al. found that miRNA-216a was overexpressed in failing human hearts of patients with and without diabetes mellitus, and its expression was negatively correlated with left ventricular ejection fraction (84). MiRNA-216a targets and represses caveolin 2, a scaffolding protein and substrate of the IR that helps to recruit IRS-1 to the IR and propagate insulin signaling $(84,125)$. The role of miRNA-216a overexpression in regulating intermediary metabolism in HF patients with and without diabetes is unanswered and of much interest to the field. MiRNA-199a-3p has been observed to be overexpressed in STZ-induced diabetic mice, and it also has a validated target of caveolin $2(58,185)$. Zheng et al. showed that miRNA-195 is overexpressed in STZ-induced and $d b / d b$ mouse hearts as well as in cardiomyocytes isolated from $d b / d b$ mice, while sirtuin 1 (Sirt1) protein levels were significantly decreased (271). Inhibiting miRNA-195 increased Sirt1 expression in diabetic mice (271). Sirt1 is a downstream effector of the insulin signaling pathway indicating that miRNA not only serve to fine tune IR and IRS2, but also regulate the expression of proteins further along the insulin signaling cascade (54). Altogether, miRNA targeting of insulin signaling components of the diabetic heart may help explain the proteomic alterations of this pathway observed in the diabetic condition.

MiRNA Regulation of Fatty Acid Transport, Fatty Acid Oxidation, Citric Acid Cycle, Electron Transport Chain, and ATP Production

The upregulation of fatty acid import and oxidation in the diabetic heart is impacted by miRNA modulation. Chen et al. demonstrated that miRNA-133a was significantly decreased in the hearts of STZ-induced diabetic mice and miRNA-133a is a direct regulator of TR4 which induces the expression of lipid importer CD36 and promoter of lipid accumulation through solute 
carrier family 27 member 1 (Slc27a1/FATP1) $(38,160)$. This could help to explain the increase in CD36 expression in cardiac tissue of STZ-induced diabetic rats that has been reported (159). Peroxisome proliferator-activated receptor alpha (PPAR- $\alpha$ ) is the primary transcription factor responsible for the fatty acid oxidation gene expression program (181). Peroxisome proliferatoractivated receptor gamma coactivator 1-alpha (PGC-1 $\alpha$ ), an essential coactivator of PPAR- $\alpha$ and inducer of medium-chain acyl-CoA dehydrogenase, has been found to be directly regulated by the miRNA-29 family (miRNA-29a-c) $(134,136)$. Interestingly, miRNA-29a has been found to be decreased in STZ-induced diabetic hearts which may help explain the induction of PPAR- $\alpha$ and increased fatty acid oxidation (58). Greco et al. observed that miRNA-210 is upregulated in failing diabetic human hearts compared to failing non-diabetic human hearts (84). MiRNA-210 is a known negative regulator of iron-sulfur cluster assembly scaffold protein IscU 1 and 2 (ISCU1/2),

which play essential roles in the function of aconitase and Complex I of the electron transport chain (30). Baseler et al. observed a significant upregulation of miRNA-141 in STZ-induced diabetic mice and found that miRNA-141 is a direct negative regulator of solute carrier family 25 member 3 (Slc25a3) which is essential for inorganic phosphate import into the mitochondrial matrix, and thus ATP synthesis (17). It has also been shown that miRNA-378 was overexpressed in interfibrillar mitochondria (IFM), mitochondria located between the myofibrils, of STZ-induced diabetic mice, and that miRNA-378 is a direct negative regulator of ATP synthase F0 component ATP6 (109). These observations provide compelling evidence that miRNAs play a critical role in the regulation of energy metabolism in the diabetic heart.

\section{Reciprocal Regulation of miRNA and ROS in the Diabetic Heart}

Oxidative stress has been shown to be a result of and cause of miRNA dysregulation. Saito et al. demonstrated that glucose fluctuations in STZ-induced diabetic rats increased cardiomyocyte miRNA-200c and miRNA-141 levels, with an accompanying increase in ROS generation, upregulation of NADPH oxidase and thioredoxin-interacting protein, while decreasing catalase and superoxide dismutase activities (175). As glycemic control has been shown to attenuate some of the peripheral diabetic symptoms, diabetic cardiomyopathy progresses even after blood glucose normalization. One possible explanation for this finding comes from a study showing that even after glycemic control in STZ-induced diabetic mice, miRNA dysregulation of many myocardial damage pathways including oxidative stress (dysregulated miRNA-221, miRNA-146a, miRNA- 
34a, miRNA-210, miRNA-19b, miRNA-27a, miRNA-155) persisted (42). Due to its upregulation in cardiac fibrosis, hypertrophy, and oxidative stress in both diabetic and insulin treated groups compared to control, miRNA-125b seems to have broad-ranging effects in mediating diabetic cardiac dysfunction (42). Excess ROS production in STZ-induced diabetic cardiomyopathy has been linked to decreased expression of miRNA-499, miRNA-1, miRNA-133a, and miRNA-133b, as treatment with the antioxidant $\mathrm{N}$-acetylcysteine restored the levels of these miRNA species to normal (255). The authors further demonstrated that Junctin, a key component of cardiomyocyte calcium handling, is a direct target of miRNA-1 and is consequently upregulated in the diabetic heart, which has previously been shown to impair cardiac relaxation and induce cardiac hypertrophy and arrhythmia (255). The mechanisms by which miRNAs regulate ROS production and by which oxidative stress influences differential expression of miRNAs to regulate a variety of pathophysiological pathways in the diabetic heart remains a relatively unexplored area of research.

\section{MiRNA Regulation of Apoptosis in the Diabetic Heart}

The diabetic heart, in the setting of diabetic cardiomyopathy and diabetic heart failure, experiences an increased rate of cardiomyocyte apoptosis. This increase in apoptosis is determined by a variety of factors including lipotoxicity, glucotoxicity, and increased oxidative stress. MiRNA-34b is upregulated in diabetic heart failure, and it has been shown to promote apoptosis by acting as an important downstream effector of p53 $(19,84,97)$. MiRNA-30c and miRNA-181a have been shown to be downregulated in diabetic patients, a diabetic cardiomyopathy rat model, and high-glucose treated cardiomyocytes (168). P53 is a validated target of miRNA-30c and miRNA-181a, and the decreased levels of these miRNA species is correlated with increased p53pathway activation of hypertrophy and apoptosis (168). Upregulation of miRNA-30d in highglucose-treated cardiomyocytes and diabetic rats has also been shown to play an important role in cardiac mitochondrial-implicated pyroptosis where it has been verified to directly target and inhibit forkhead box O3 (FOXO3a), with downstream effects including decreased expression of apoptosis repressor with caspase recruitment domain (ARC) and upregulation of caspase-1 (132, 257). Zhao et al. showed that high-glucose-treated rat cardiomyocyte $\mathrm{H} 9 \mathrm{C} 2$ cells have enhanced expression of miRNA-34a, decreased expression of the miRNA-34a target anti-apoptotic protein Bcl-2, and increased apoptosis (267). Bcl-2 has been found to be targeted and downregulated by 
overexpression of miRNA-195 in a mouse model of STZ-induced type 1 diabetes, and inhibition of miRNA-195 reduced ROS production and inhibited apoptosis (271). Further, Yu et al. showed that rat cardiomyocyte H9C2 cells treated with high glucose had increased miRNA-1 expression, downregulation of the miRNA-1 target IGF-1, increased cytochrome-c release, and increased apoptosis (258). To add validity to the importance of miRNA-1 in high-glucose-mediated cardiomyocyte apoptosis, it has been found that high glucose in vitro and in vivo increases miRNA1/miRNA-206 expression precipitating post-translational modification of heat shock protein-60, a protein involved in protection against diabetic myocardial injury (183). Furthermore, Katare et al. found upregulation of miRNA-1, downregulation of the miRNA-1 targets proto-oncogene serine/threonine-protein kinase (Pim-1) and Bcl2 (anti-apoptotic proteins), and increased proapoptotic caspase-3 activity in the hearts of STZ-induced type 1 diabetic mice (115).

MiRNA impact on energy substrate metabolism, ROS interactions within the cell, and apoptotic pathways all show the significance of the interaction between miRNAs, mitochondria, and the diabetic heart (Figure 1.6). With the significant impact to cellular and physiological health from miRNAs in the diabetic heart, a more fundamental question arises to address the driving mechanisms behind changing miRNA expression. 
Figure 1.6: Signaling Pathways of the Diabetic Heart

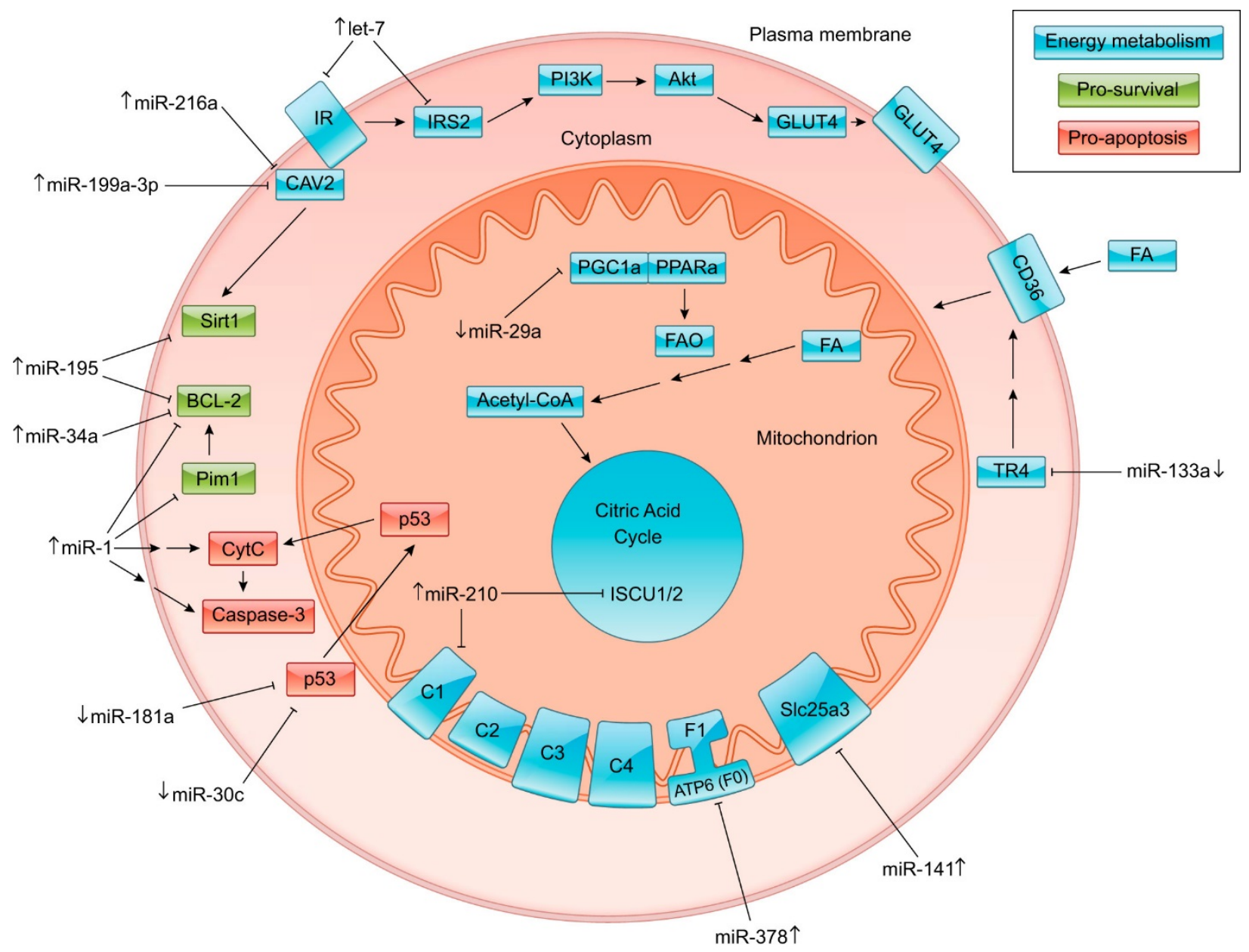

Figure 1.6: MiRNA influence on signaling pathways in the diabetic heart. MiRNA regulation of cardiomyocyte energy metabolism (blue), survival (green), and apoptosis (red) in the diabetic heart. The up or down arrow beside each miRNA species indicates its up- or down-regulation in the diabetic heart (Table 1.2). The marked line connecting each miRNA with its target demonstrates that the miRNA inhibits its target. Subcellular components have been labeled: plasma membrane (yellow), cytoplasm (green), and mitochondrion (blue). CAV2 = caveolin 2; IR = insulin receptor; IRS2 $=$ insulin receptor substrate 2 ; PI3K $=$ phosphatidylinositol-4,5-bisphosphate 3-kinase; GLUT4 = glucose transporter type 4; FA = fatty acid; CD36 = cluster of differentiation 36; TR4 = testicular receptor 4; FAO = fatty acid oxidation; PPARa $=$ peroxisome proliferator-activated receptor alpha; $\mathrm{PGCla}=$ peroxisome proliferatoractivated receptor gamma coactivator 1-alpha; Acetyl-CoA = acetyl coenzyme A; ISCU1/2 = iron-sulfur cluster assembly proteins; $\mathrm{C} 1-\mathrm{C} 4=$ electron transport chain complex 1-4; F0 = ATP synthase subunit F0; F1 = ATP synthase subunit $\mathrm{F} 1 ; \mathrm{Slc} 25 \mathrm{a} 3$ = mitochondrial phosphate carrier protein; $\mathrm{CytC}=$ cytochrome C; BCL-2 = B-cell lymphoma 2; Sirt1 $=$ sirtuin 1 .

\section{MiRNA Import into the Mitochondrion}


What still remains a highly relevant, and hotly debated, topic is the process involved in miRNA import into the mitochondrion. While it has been hypothesized that some miRNAs could be transcribed through the mitochondrial genome (196), predominately, miRNA import is favored as the mechanism for miRNA accumulation in the mitochondrion. Research has demonstrated the presence of miRNA within the mitochondrion $(11,50,120,188,265)$, and even how fluctuating concentrations within the organelle contributes toward the development of pathologies $(48,49,63$, $186,195)$. Two currently proposed mechanisms of miRNA import into the mitochondria include the direct movement across the mitochondrial membrane through a chaperone, Argonaut 2 (AGO2) (49, 265), and diffusion through small RNA import machinery, Polynucleotide Phosphorylase (PNPase) (186).

Both Das et al. and Zhang et al. have shown that $\mathrm{AGO} 2$ is present in the mitochondria, and that crosslinking immunoprecipitation reveals associations with miRNAs. $(49,265)$. While it has been suggested that $\mathrm{AGO} 2$ could have a localization sequence on its $\mathrm{N}$-terminus for mitochondrial targeting (12), no definitive research has validated the precise mechanisms involved in AGO2/miRNA mitochondrial import. A new mechanism proposed for mitochondrial miRNA import includes the mitochondrial inner membrane protein PNPase, and potential associations with AGO2. Wang et al., has demonstrated the importance of PNPase in facilitating the import of RNase P, 5S rRNA, and MRP RNAs $(232,233)$, suggested through stem-loop recognition. Recently, our group has shown that the regulation of PNPase can alter miRNA-378 levels within the mitochondrion (186), indicating that the function of PNPase may extend to other small RNA species. The expression of PNPase is also decreased during diabetes mellitus. Though this novel mechanism of miRNA import has not been fully characterized mechanistically, it provides a basis for future research.

\section{Regulation of miRNAs Affecting the Diabetic and Non-Diabetic Cardiovascular System}

As described above, many cardiovascular diseases including diabetic cardiomyopathy and diabetic heart failure have unique miRNA expression signatures. These regulatory molecules have an impact in modulating cellular pathways in health and disease. Although miRNAs are highly active in post-transcriptional repression and degradation with complex downstream effects, the miRNA expression landscape is just as complexly regulated by environmental stimuli; including 
mechanisms of epigenetic modulation, exosomal transport between cells and tissues, processing of pri- and pre-miRNA pathways, long non-coding RNA (lncRNA) "sponging," and many others

(Table 1.3).

Table 1.3: Regulation of miRNA in the Heart and Circulatory System

\begin{tabular}{|c|c|c|c|c|c|}
\hline miRNA & $\begin{array}{c}\text { Type of } \\
\text { Regulation }\end{array}$ & Pathway & $\begin{array}{c}\text { Expression in } \\
\text { Heart or } \\
\text { Circulatory } \\
\text { System } \\
\end{array}$ & Disease Model & Source \\
\hline $\begin{array}{c}\text { miRNA- } \\
133 a\end{array}$ & Epigenetic & $\begin{array}{c}\text { Class I and IIb HDACs decrease the } \\
\text { expression of miRNA-133a }\end{array}$ & Downregulated & $\begin{array}{l}\text { Mouse heart - transverse aortic } \\
\text { constriction model }\end{array}$ & (169) \\
\hline miRNA-184 & Epigenetic & $\begin{array}{l}\text { DNA hypermethylation through } \\
\text { DNMT1 }\end{array}$ & Downregulated & $\begin{array}{l}\text { HL-1 cardiomyocytes - } \\
\text { Arrhythmogenic } \\
\text { cardiomyopathy model }\end{array}$ & $(88)$ \\
\hline $\begin{array}{l}\text { pri-miR- } \\
208 \mathrm{~b}\end{array}$ & Epigenetic & Pri-miRNA tether to EZH2 & Upregulated & $\begin{array}{l}\text { Mouse heart - transverse aortic } \\
\text { constriction model }\end{array}$ & $(150)$ \\
\hline $\begin{array}{l}\text { *miRNA- } \\
375\end{array}$ & Epigenetic & CpG Island methylation & Upregulated & Human plasma & $\begin{array}{ll}(31, \\
203)\end{array}$ \\
\hline $\begin{array}{l}\text { *miRNA- } \\
145\end{array}$ & Epigenetic & Promoter hypomethylation & Upregulated & $\begin{array}{l}\text { Human saphenous vein smooth } \\
\text { muscle }\end{array}$ & (170) \\
\hline $\begin{array}{l}* \text { miRNA- } \\
125 \mathrm{~b}\end{array}$ & Epigenetic & $\begin{array}{c}\text { Suv39h1 3' UTR binding, decreasing } \\
\text { H3K9me3 expression }\end{array}$ & Upregulated & $\begin{array}{l}\text { Mouse microvascular smooth } \\
\text { muscle cells }-\mathrm{db} / \mathrm{db} \text { model }\end{array}$ & (227) \\
\hline $\begin{array}{l}* \text { miRNA- } \\
101\end{array}$ & Epigenetic & $\begin{array}{l}\text { Binding to EZH2, decreasing } \\
\text { H3K27me3 expression }\end{array}$ & Upregulated & $\begin{array}{l}\text { Human fetal embryonic stem } \\
\text { cells of the umbilical cord vein }\end{array}$ & (74) \\
\hline $\begin{array}{c}\text { *miRNA- } \\
133 \mathrm{a}\end{array}$ & Epigenetic & $\begin{array}{c}\text { Binding to DNMT1, DNMT3A, and } \\
\text { DNMT3B }\end{array}$ & Downregulated & $\begin{array}{l}\text { Ins2(+/-) Akita diabetic mice } \\
\text { and HL-1 cardiomyocytes }\end{array}$ & (34) \\
\hline $\begin{array}{c}\text { miRNA-1 } \\
\text { and miRNA- } \\
133 \mathrm{a}\end{array}$ & $\begin{array}{l}\text { Exosomal } \\
\text { Transport }\end{array}$ & $\begin{array}{l}\text { Potential biomarker for cardiomyocyte } \\
\text { death, released from injured } \\
\text { myocardium }\end{array}$ & $\begin{array}{l}\text { Upregulated } \\
\text { release into } \\
\text { serum }\end{array}$ & $\begin{array}{l}\text { Human serum from acute } \\
\text { myocardial infarction patients }\end{array}$ & (124) \\
\hline $\begin{array}{l}\text { miRNA-142- } \\
\qquad 3 \mathrm{p}\end{array}$ & $\begin{array}{l}\text { Exosomal } \\
\text { Transport }\end{array}$ & $\begin{array}{l}\text { Endothelial barrier integrity breakdown } \\
\text { through RAB11FIP2 interaction }\end{array}$ & $\begin{array}{l}\text { Upregulated } \\
\text { release into } \\
\text { serum }\end{array}$ & $\begin{array}{l}\text { Human serum from heart } \\
\text { transplant surgery patients }\end{array}$ & (202) \\
\hline miRNA-21 & $\begin{array}{l}\text { Exosomal } \\
\text { Transport }\end{array}$ & $\begin{array}{l}\text { Binds PDCD4, offering } \\
\text { cardioprotection }\end{array}$ & $\begin{array}{l}\text { Upregulated } \\
\text { release into } \\
\text { media }\end{array}$ & $\begin{array}{l}\text { Mouse cardiac progenitor cells } \\
\text { under oxidative stress }\end{array}$ & (246) \\
\hline miRNA-30a & $\begin{array}{l}\text { Exosomal } \\
\text { Transport }\end{array}$ & $\begin{array}{l}\text { HIF-1 } \alpha \text { upregulates miRNA-30a, } \\
\text { potentially offering cardioprotection } \\
\text { from autophagy }\end{array}$ & $\begin{array}{l}\text { Upregulated } \\
\text { release into } \\
\text { serum }\end{array}$ & $\begin{array}{l}\text { Human serum from acute } \\
\text { myocardial infarction patients }\end{array}$ & $(252)$ \\
\hline miRNA-143 & $\begin{array}{l}\text { Exosomal } \\
\text { Transport }\end{array}$ & $\begin{array}{l}\text { Upregulation of miRNA-143-3p, } \\
\text { compared to miRNA-143-5p. }\end{array}$ & $\begin{array}{l}\text { Upregulated } \\
\text { release into } \\
\text { media }\end{array}$ & $\begin{array}{c}\text { Human pulmonary artery } \\
\text { smooth muscle cells from } \\
\text { pulmonary arterial } \\
\text { hypertension patients }\end{array}$ & $(55)$ \\
\hline miRNA-194 & $\begin{array}{l}\text { Exosomal } \\
\text { Transport }\end{array}$ & $\begin{array}{c}\text { Contribution to the p53-response and } \\
\text { potential biomarker for ischemic heart } \\
\text { failure }\end{array}$ & $\begin{array}{l}\text { Upregulated } \\
\text { release into } \\
\text { serum }\end{array}$ & $\begin{array}{l}\text { Human serum from acute } \\
\text { myocardial infarction patients }\end{array}$ & (148) \\
\hline $\begin{array}{c}{ }^{*} \text { miRNA-1 } \\
\text { and miRNA- } \\
133 \mathrm{a} \\
\end{array}$ & $\begin{array}{l}\text { Exosomal } \\
\text { Transport }\end{array}$ & $\begin{array}{l}\text { Levels positively correlated with } \\
\text { predicting myocardial steatosis in type } \\
2 \text { diabetic patients }\end{array}$ & $\begin{array}{l}\text { Upregulated } \\
\text { release into } \\
\text { media }\end{array}$ & $\begin{array}{l}\text { HL-1 cardiomyocyte - lipid- } \\
\text { loaded preconditioning }\end{array}$ & (53) \\
\hline *miRNA-7 & $\begin{array}{l}\text { Exosomal } \\
\text { Transport }\end{array}$ & $\begin{array}{l}\text { Changes in serum levels were not } \\
\text { significantly contributed by exosomes }\end{array}$ & $\begin{array}{l}\text { Upregulated in } \\
\text { serum, not } \\
\text { changing in } \\
\text { exosomes }\end{array}$ & $\begin{array}{l}\text { Human serum from patients } \\
\text { with microvascular } \\
\text { complications }\end{array}$ & $(230)$ \\
\hline $\begin{array}{c}\text { miRNA- } \\
455, \\
\text { miRNA-29b, } \\
\text { miRNA-323- } \\
\text { 5p and } \\
\text { miRNA466 } \\
\end{array}$ & $\begin{array}{l}\text { Exosomal } \\
\text { Transport }\end{array}$ & Downregulation of MMP9 & $\begin{array}{l}\text { Upregulated } \\
\text { release into } \\
\text { serum }\end{array}$ & $\begin{array}{c}\text { Mouse }-\mathrm{db} / \mathrm{db} \text { model with } \\
\text { exercise }\end{array}$ & $(32)$ \\
\hline $\begin{array}{l}\text { *miRNA- } \\
320\end{array}$ & $\begin{array}{l}\text { Exosomal } \\
\text { Transport }\end{array}$ & $\begin{array}{c}\text { Targets IGF-1, Hsp20 and Ets2, } \\
\text { producing an anti-angiogenic effect in } \\
\text { cardiac endothelial cells }\end{array}$ & $\begin{array}{l}\text { Upregulated } \\
\text { release from } \\
\text { cardiomyocytes }\end{array}$ & $\begin{array}{l}\text { Rat cardiac endothelial cell and } \\
\text { cardiomyocyte co-culture - } \\
\text { Goto-Kakizaki (GK) model } \\
\end{array}$ & (241) \\
\hline
\end{tabular}




\begin{tabular}{|c|c|c|c|c|c|}
\hline miRNA-21 & $\begin{array}{l}\text { MiRNA } \\
\text { Processing }\end{array}$ & $\begin{array}{c}\text { DICER1 and phospho-SMAD-2 } \\
\text { interactions increase mature miRNA-21 } \\
\text { expression }\end{array}$ & Upregulated & $\begin{array}{l}\text { Mouse - transverse aortic } \\
\text { constriction model }\end{array}$ & $(79)$ \\
\hline miRNA-103 & $\begin{array}{l}\text { MiRNA } \\
\text { Processing }\end{array}$ & $\begin{array}{c}\text { Downregulation of Dicer leads to } \\
\text { decreased NF-KB activation through } \\
\text { decreased miRNA-103/KLF4 binding }\end{array}$ & Downregulated & $\begin{array}{l}\text { Mouse }- \text { high fat diet } \\
\text { apolipoprotein E knockout } \\
\text { model }\end{array}$ & (93) \\
\hline miRNA-1 & $\begin{array}{c}\text { MiRNA } \\
\text { Processing }\end{array}$ & $\begin{array}{c}\text { G3bp1 binds the stem loop of miRNA- } \\
1 \text {, decreasing its expression }\end{array}$ & Downregulated & $\begin{array}{l}\text { Rat cardiomyocyte - transverse } \\
\text { aortic constriction model }\end{array}$ & $(98)$ \\
\hline $\begin{array}{c}\text { *let-7a, } \\
\text { miRNA-130, } \\
\text { miRNA-142- } \\
\text { 3p, miRNA- } \\
\text { 148, } \\
\text { miRNA-338, } \\
\text { miRNA-345- } \\
\text { 3p, miRNA- } \\
\text { 384-3p, } \\
\text { miRNA-433, } \\
\text { miRNA-450, } \\
\text { miRNA-451, } \\
\text { miRNA-455, } \\
\text { miRNA-494, } \\
\text { miRNA-499, } \\
\text { miRNA-500, } \\
\text { miRNA-542- } \\
\text { 3p, miRNA- } \\
\text { 744, and } \\
\text { miRNA-872 }\end{array}$ & $\begin{array}{l}\text { MiRNA } \\
\text { Processing }\end{array}$ & $\begin{array}{l}\text { Dicer was shown to be downregulated, } \\
\text { though some miRNAs showed } \\
\text { increased expression }\end{array}$ & Upregulated & Ins2(+/-) Akita diabetic mice & (33) \\
\hline $\begin{array}{l}\text { miRNA-188- } \\
\qquad 3 \mathrm{p}\end{array}$ & $\begin{array}{l}\text { LncRNA } \\
\text { Sponging }\end{array}$ & $\begin{array}{c}\text { Increased expression of APF lncRNA } \\
\text { decreases miRNA-188-3p levels while } \\
\text { increasing ATG7 }\end{array}$ & Downregulated & $\begin{array}{c}\text { Mouse cardiomyocytes - } \\
\text { anoxia/re-oxygenation model }\end{array}$ & $(236)$ \\
\hline miRNA-539 & $\begin{array}{l}\text { LncRNA } \\
\text { Sponging }\end{array}$ & $\begin{array}{c}\text { Increased expression of CARL lncRNA } \\
\text { decreases miRNA-539 levels while } \\
\text { increasing PHB2 }\end{array}$ & Downregulated & $\begin{array}{c}\text { Mouse cardiomyocytes - } \\
\text { anoxia/re-oxygenation model }\end{array}$ & $(238)$ \\
\hline miRNA-489 & $\begin{array}{l}\text { LncRNA } \\
\text { Sponging }\end{array}$ & $\begin{array}{c}\text { Increased expression of CHRFL } \\
\text { lncRNA decreases miRNA-489 levels } \\
\text { while increasing Myd88 }\end{array}$ & Downregulated & $\begin{array}{l}\text { Mouse }- \text { angiotension II } \\
\text { treatment }\end{array}$ & (237) \\
\hline
\end{tabular}

Table 1.3: Regulation of miRNA in the heart and circulatory system. Each miRNA which is regulated, or involved in mechanisms altering miRNA expression, is listed for the heart and/or circulatory system. The type of regulation, the specific pathway involved, and the expression change of the miRNA are included. The disease model is stated, with an asterisk (*) next to the miRNA name, denoting the use of a diabetic model.

\section{Regulation of miRNAs Altering the Epigenome of the Cardiovascular System}

\section{What are Epigenetics?}

The most described forms of epigenetic regulation consist primarily of two transient alterations: DNA methylation and histone modification (28). DNA methylation proceeds through the addition of a 5' methyl group which occurs on cytosine nucleotides, where it is added by DNA methyltransferases (DNMT1, DNMT3a, and DNMT3b) (20). Most often, this methyl group addition will occur at $\mathrm{CpG}$ islands (paired cytosine and guanine nucleotides within a DNA sequence). The addition of methyl groups to a gene sequence attenuates transcription by decreasing 
the propensity of transcription factors and other transcription initiation proteins to bind to the sequence (112).

Histone modifications, such as acetylation, methylation, phosphorylation, etc., change the confirmation of chromatin by either relaxing or condensing the DNA/protein structure (16). The processes involved in histone modifications include histone methyltransferases (HMTs), which generally condense chromatin, and histone acetyl transferases (HATs), that primarily relax chromatin (274). The interplay of DNA methylation and histone modifications can significantly alter gene expression, specifically in diseases such as type 2 diabetes mellitus, such that the epigenome can be completely reshaped (229).

\section{The Role of Epigenetics in Altering miRNA Expression}

Of the variety of mechanisms known to regulate miRNA expression, the beginning of the regulatory chain starts at transcription. MiRNAs can either be intergenic, found within the introns of genes, or intragenic, found within the coding region of a gene, and can assume multi-directional or overlapping features with the gene $(41,146)$. At each gene locus containing a miRNA, the expression of the miRNA can be governed both by the DNA methylation of a promoter/intergenic/intragenic region or through histone confirmations around the reading frame (178). Researchers have begun to investigate how miRNAs can be controlled epigenetically in a variety of pathological conditions. Conversely, miRNAs can be implemented as a direct mechanism for altering cellular epigenetics. The epigenetic machinery of the cell contains multiple forms of epigenetic regulation: polycomb repressor complex 1 (PRC1), PRC2, histone deacetylases (HDACs), HATs, HMTs, and DNMTs (107, 178).

MiRNAs have been found to directly affect major constituents of the epigenetic machinery and many miRNAs have been identified with changed expression levels through epigenetic regulation. Ingenuity Pathway Analysis (IPA) can be implemented as a protein/miRNA ontology system through the use of known molecular association data, combined with predictive software analyzing sequence homology. Figure1.7 depicts how mature miRNAs are known to interact with the DNMT machinery, and also reveals miRNAs associated with the mitochondrion of the diabetic heart that theoretically could interact with DNMTs through their seed sequence regions. 


\section{Figure 1.7: DNA Methylation and MiRNA Involvement}

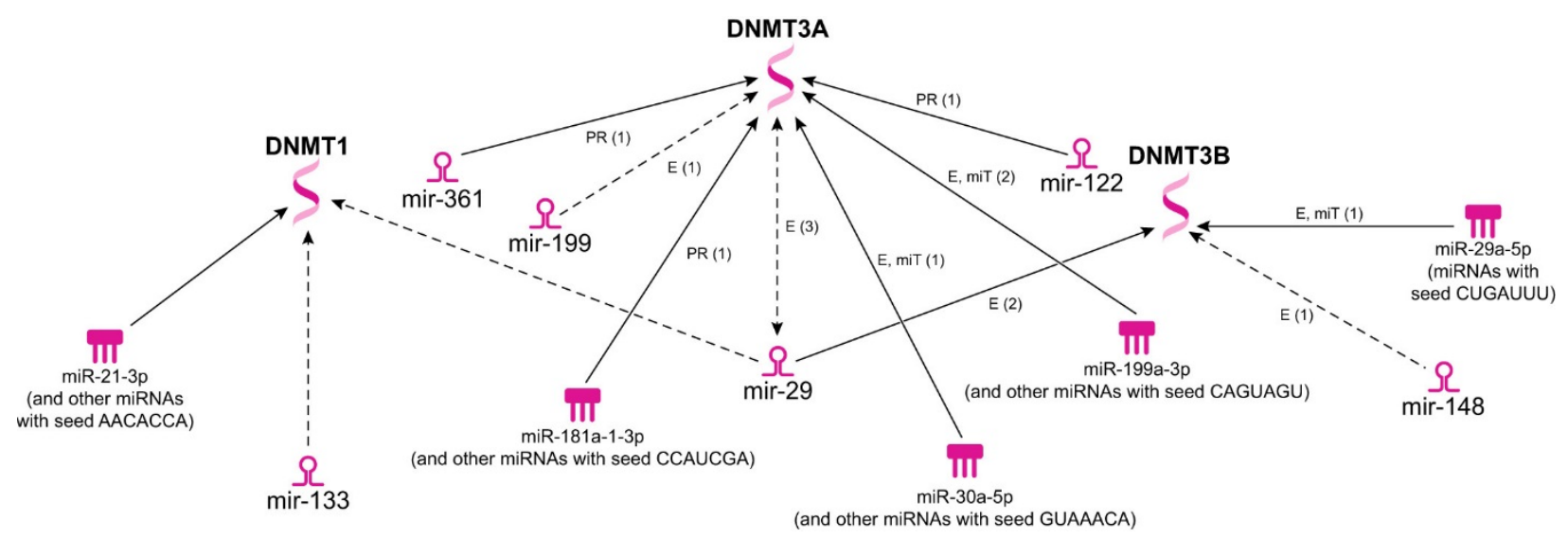

Figure 1.7: Predicted interactions of miRNA with DNMT1. Ingenuity Pathway Analysis (IPA) provides potential areas for future epigenetic/miRNA analyses. The predicted connections include mature miRNAs and miRNA seed sequences, which are suggested to interact with DNMT1, DNMT3a, or DNMT3b. MiRNAs with listed seed sequences can account for one or many miRNAs which contain similar regions suggested to promote protein/miRNA or mRNA/miRNA interactions with the DNMTs. DNMT1 $=$ DNA methyltransferase 1 , DNMT3a $=$ DNA methyltransferase 3a, DNMT3b = DNA methyltransferase 3b.

In human femoral artery atherosclerotic plaques, Aavik et al. measured global DNA methylation levels and found hypomethylation of the genome and two-thirds of the genes exhibiting a significant, differential methylation pattern, compared to normal mammary artery (3). One of the major findings was the 14q32 locus being significantly hypomethylated, which contains miRNAs $-127,-136,-410,-431,-432$, and -433 (3). The authors suggest that this epigenetic paradigm in atherosclerotic plaques is a potential mechanism for initiating the progression of the lesions. In the heart, during overload-induced fibrosis through transverse aortic constriction, class I and IIb HDACs were shown to negatively impact the heart through the regulation of miRNA133a (169). HDACs are known to increase in a pressure overload-induced model and by introducing HDAC inhibitors to overload-induced mice, miRNA-133a expression was restored and functional deficits were ameliorated.

Arrhythmogenic cardiomyopathy has been linked to mutations in specific genes, including plakophilin 2 (PKP2) $(8,223)$. A PKP2 knockdown model in HL-1 cardiomyocyte cells was used to evaluate miRNA expression, revealing 59 differentially regulated miRNAs (88). Of these differentially regulated miRNAs, miRNA-184 showed the most significant downregulation. The authors suggest that miRNA-184 expression is controlled through DNA hypermethylation of its 
gene loci through DNMT1. A newly proposed mechanism of miRNA/epigenetic interaction suggests that pri-miRNA can be used as tethers to recruit histone modification proteins to specific DNA sites (147). Enhancer of zester homolog-2 (EZH2) was shown to bind to the bi-directional promoter at the myosin heavy (MHC) chain genes in the heart and promote a switch from $\alpha-\mathrm{MHC}$ to the antisense $\beta$-MHC. Through RNA-chromatin immunoprecipitation experiments, the authors demonstrated the capacity for pri-miR-208b to tether specific genomic sites and recruit chromatin modification machinery.

\section{The Impact of Epigenetics and miRNA During Diabetes Mellitus}

With the progression toward type 2 diabetes mellitus (i.e. insulin resistance, increased adiposity, increased body-mass-index (BMI)), changes in the epigenome can be observed. A patient cohort $(n=10,261)$ revealed that genome-wide changes in DNA methylation in blood were linked to an increased BMI (229). The resulting differential methylation was found to encompass genes involved in lipid metabolism, inflammatory signaling, and substrate transport. The group also found that changes in genome-wide DNA methylation can be used as a predictive measure for the development of type 2 diabetes mellitus. Methylation of genes involved in the progression of type 2 diabetes mellitus also varies between ethnicities (76, 122, 152), with Indian Asians showing increased levels of DNA methylation at ABCG1, PHOSPHO1, SOCS3, SREBF1, and TXNIP. The increased methylation of these genetic loci in the Indian Asian population correlates to the increased risk of developing type 2 diabetes mellitus (29).

Pheiffer et al., found that miRNA expression in type 2 diabetes mellitus can be controlled through DNA methylation (161). Specifically, this group established the importance of intergenic regions in controlling miRNA expression and showed that most differentially regulated peaks between diabetic and non-diabetic groups were found in the intergenic regions, as opposed to the promoter or intragenic regions. These findings raise a number of new questions concerning miRNAs and epigenetics, such as how does epigenetic regulation occur in a specific pathology, such as diabetes mellitus, or tissue, such as the heart, and how can therapeutics take advantage of these processes? In plasma from type 2 diabetic patients, miRNA-375 was increased (203). Further, miRNA-375 has 17 different CpG islands within or surrounding the DNA loci, in which some of these regions were differentially regulated between diabetic and control populations. Other studies focusing on two different populations in China, Han and Kazak, revealed differences 
in the methylation pattern of miRNA-375 (31). The authors postulate that this may be a potential mechanism for the increased prevalence of type 2 diabetes mellitus in the Han population.

The role that miRNAs and epigenetics play in defining pathological states, such as diabetes mellitus, and how this interaction occurs in a variety of tissue types has been well-studied. What remains to be fully understood is the impact of the epigenetic/miRNA interplay within the diabetic heart and cardiovascular system. In the saphenous vein of human diabetic and non-diabetic patients, smooth muscle cells were cultured and miRNA-145 promoter methylation was measured (170). Expression of miRNA-145 was increased in the diabetic phenotype, but highly variable CpG island methylation between groups did not allow for any overall significant changes between groups. Though, specific CpG loci were shown to exhibit hypomethylation, irrespective of group. Microvascular smooth muscle cells (MVSMCs) from $d b / d b$ and control mice were used to assess the effects of miRNA-125b, which is overexpressed in the diabetic phenotype (227). MiRNA-125b was validated to bind to the suppressor of variegation 3-9 homolog 1 (Suv39h1) 3' UTR controlling the expression of histone 3 lysine 9 tri-methylation (H3K9me3). Increased miRNA$125 \mathrm{~b}$ expression resulted in less restrictive $\mathrm{H} 3 \mathrm{~K} 9 \mathrm{me} 3$ marks, ultimately increasing the expression of inflammatory genes.

Gestational diabetes mellitus can affect the health and future function of the growing progeny. In human fetal embryonic stem cells of the umbilical cord vein (HUVECs), EZH2 was decreased in mothers with gestational diabetes mellitus while miRNA-101 was increased (74). EZH2 is responsible for the tri-methylation of histone 3 lysine 27 (H3K27me3). EZH2 is also directly targeted by miRNA-101, resulting in decreased H3K27me3 in HUVECs from gestational diabetic mothers and subsequent altered gene transcriptional patterns. Through the use of Ins2(+/) Akita diabetic mice and HL-1 cardiomyocytes, Chavali et al. defined the role of miRNA-133a in regulating DNMTs (34). The group found that DNMT1, DNMT3a, and DNMT3b were inversely correlated to miRNA-133a (i.e. silencing of miRNA-133a induced overexpression of the DNMTs while expression of miRNA-133a inhibited DNMT expression). In the Akita model, miRNA-133a is decreased, with DNMT1 and DNMT3b being induced. Through involvement in both the processes of DNA methylation and histone modifications, the unique miRNA/epigenetic signature involved in the progression of a pathology, such as diabetes mellitus, could be an invaluable predictor of cardiac function and cardiovascular health. 


\section{Exosomal Release, Transport, and Uptake of miRNA in the Cardiovascular System}

Exosomes and Exosomal Cargo

Exosomes are cell derived vesicular carriers utilized for cell signaling and communication and have been implicated in numerous disease states, including diabetic cardiomyopathy (174) and various cancers (9). Generally, exosomes range in size from 50 to $100 \mathrm{~nm}(167,193,213)$, but small discrepancies in size are common in the literature. The central role of exosomes is the delivery of various cargo types including lipids, nucleic acids, proteins, RNAs, and miRNAs to different cells $(22,213,218,221)$. Exosomes transport cargo, transmitting a unique biochemical signal from the host cell to a specific target cell or tissue or, more systemically, by the blood stream. Exosomal cargo is of importance due to the specificity of the cargo loaded into each vesicle by the host cell. This cargo is then delivered to targeted acceptor cells and the exosomes release their protein and lipid cargo to activate downstream signaling events, or simply deliver genetic material including DNA and various RNAs (194). Secretion of exosomes occurs via multiple mechanisms, such as release upon exocytic fusion of multivesicular bodies with the plasma membrane and miRNA release through a ceramide-dependent mechanism (218). The different pathways available for exosome secretion depend on the cell types (213) and, currently, a lack of classification of the mechanisms of exosome biogenesis exists.

\section{Exosomal Transport of miRNA}

The transport of miRNAs via exosomes can directly impact cellular dynamics. Exosomal transport in the immune system through macrophages has also proven to be an important mechanism for changing cellular dynamics $(4,51,108,209)$, specifically through the regulation of inflammation during cardiac injury (231). This section will focus explicitly on the regulation of exosomal transport of miRNA within the heart and circulatory system.

MiRNA-1 and miRNA-133a are increased in the serum of patients who have suffered from an acute myocardial infarction (124). In a mouse model of myocardial infarction, the infarcted and peri-infarcted tissue display a significant reduction of miRNA-1 and miRNA-133a. Through measuring the exosomal release of miRNA-133a in dead, H9c2 cardiomyoblasts, the authors suggest that injured myocardium releases miRNA-133a and that this marker may be used in the serum to account for cardiomyocyte death. Sukma Dewi et al., measured the miRNAs of exosomes 
taken from the serum of patients who underwent heart transplant surgery, both with and without experiencing acute cardiac allograft rejection (202). These authors found miRNA-142-3p to be significantly increased in the exosomes. When the exosomes from the serum were used to treat endothelial cells, miRNA-142-3p also increased in the endothelial cells. After acute cardiac allograft rejection, the authors concluded that exosomes in the serum can affect endothelial barrier integrity through the interaction of miRNA-142-3p with RAB11 family interacting protein 2 (RAB11FIP2). The capacity for exosomes released from the heart to affect the systemic circulation and peripheral tissues has been explored (66). Serum was taken from patients before and after undergoing coronary artery bypass grafting, the authors found an increase in the total plasma concentration of exosomes, as well as increased miRNA cargo. They observed that high sensitivity cardiac troponin I was positively correlated with the plasma expression of total exosomes.

Cardiac progenitor cells (CPCs) were used to understand how exosomal release from cells can contribute toward alleviation of oxidative stress (246). During oxidative stress, it has been shown that CPCs released more miRNA-21, which targets the protein programmed cell death 4 (PDCD4), ultimately reducing the cleaved version of caspase-3. These authors suggest that CPCs may offer cardioprotection during oxidative stress through release of exosomal miRNA-21 to surrounding tissue. After acute myocardial infarction, exosomes isolated from the serum of patients contained increased levels of miRNA-30a (252). MiRNA-30a is also released in exosomes from cardiomyocytes after hypoxic conditioning. Hypoxia inducible factor (HIF)-1 $\alpha$ regulates the expression of miRNA-30a in exosomes released by the cell, which directly target autophagy machinery. In this way, cardiomyocytes may protect surrounding tissue in a hypoxic environment by releasing miRNA-30a and decreasing autophagy. During pulmonary arterial hypertension, the selective upregulation of miRNA-143-3p compared to miRNA-143-5p was shown in pulmonary artery smooth muscle cells (PASMCs) (55). PASMCs also excreted more miRNA-143-3p through exosomes to influence vascular endothelial cells. Blocking the actions of miRNA-143-3p resulted in decreased pulmonary hypertension in mouse models.

Following acute myocardial infarction, Matsumoto et al., examined the role of circulating miRNAs as a contributing factor to the incidence of heart failure (148). Eighteen days postinfarction, serum was collected and miRNA-192 was shown to be significantly increased, which plays a role in the p53 response. In the p53 pathway, miRNA-194 was shown to be significantly 
elevated in exosomes and the authors correlated serum levels of miRNAs-192, -194, and -34a in patients who experience acute myocardial infarction with the development of heart failure.

\section{Exosomal Transport of miRNA in Diabetes Mellitus}

Through the progression of diabetes mellitus, it is well documented that the miRNA profile changes throughout the body $(13,67,87,118,182,260)$. What is not entirely known is how the miRNA profile changes within exosomes. Questions concerning the transport of miRNAs in diabetes mellitus, the protective or detrimental effects of exosomes during the pathology from a temporal standpoint, and how exosomal transport of miRNAs may differ mechanistically between tissues, have begun to be explored.

In uncomplicated type 2 diabetes mellitus, the role of serum miRNAs in predicting myocardial steatosis was evaluated (53). These authors found that miRNA-1 and miRNA-133a were positively correlated with myocardial steatosis, and that miRNA-133a was elevated in type 2 diabetic patients. Using HL-1 cardiomyocytes, miRNA-1 and miRNA-133a were found to be higher in exosomes released from lipid-loaded preconditioning. Patients with type 2 diabetes mellitus may exhibit microvascular complications (230). MiRNA-7 was shown to be overexpressed in the serum of patients with type 2 diabetes mellitus or type 2 diabetes mellitus with microvascular complications compared to non-diabetics. These authors found that miRNA-7 was not primarily packaged in exosomes, but free floating in the serum. The authors concluded that miRNA-7 serum levels may have the potential to predict microvascular complications in type 2 diabetes mellitus. Examining the generational effects of diabetes mellitus and exosome delivery, Shi et al., found that mice made type 1 diabetic through STZ injections before mating had serum containing increased levels of exosomes with miRNAs involved in cardiac developmental regulation (187). They also showed that these exosomes can be directly transferred to the fetal pups, which may account for the observed increased in congenital heart defects. By injecting the exosomes of the diabetic mothers into the control pups, they also observed an increase in congenital heart defects in the $F_{1}$ generation.

The effects of exercise on the diabetic condition and how complications of the disease can be mitigated were explored through matrix metalloprotease 9 (MMP9) regulation (32). MMP9 was shown to be significantly decreased in a $d b / d b$ model after exercise. The authors demonstrated that exosomal transport of miRNAs decreasing the expression of MMP9 (i.e. miRNA-455, miRNA- 
29b, miRNA-323-5p and miRNA466) were increased after exercise. In the Goto-Kakizaki (GK) rat, a model of type 2 diabetes mellitus, cardiac endothelial cells (MCECs) co-cultured with cardiomyocytes from diabetic rats resulted in a decrease in proliferation and migration as compared to control, Wistar rats (241). Inhibition of exosome formation from diabetic mice restored function of MCECs, which was correlated with a significant decrease in miRNA-320 within MCECs. The importance of miRNA-320 is that it binds to targets which decrease angiogenesis. The authors concluded that in diabetes mellitus, cardiomyocytes may be involved in producing an anti-angiogenic effect on the surrounding environment.

MiRNA Processing, LncRNA, and Other Regulators of miRNA in the Cardiovascular System

Mechanisms of miRNA Processing and Post-transcriptional Control

Indirect mechanisms of miRNA regulation, such as epigenomic changes and alterations in exosomal transport, are not the only ways that miRNA levels can be affected in the diabetic heart. More direct means of miRNA alteration within the cell can include miRNA processing as well as regulation by small biomolecules or other RNA species. The milieu of the pathology, such as changes in glucose and other metabolite concentrations within the cell, can directly alter the efficiency of miRNA processing and modify the dynamics of cellular processes.

After transcription of miRNA, post-transcriptional regulation through long-noncoding RNAs (lncRNAs) can occur. It has been shown that lncRNAs have binding sites for miRNAs (110). One mechanism, referred to as "sponging," involves the expression of lncRNA in order to sequester miRNAs (96). This lncRNA/miRNA is considered part of a larger network of competing endogenous RNA (ceRNA), in which the cell uses both coding and non-coding RNA to expand the functional genetic information that a cell can produce (176). At current, this field is underexplored, with few lncRNAs and even fewer lncRNA/miRNA interactions characterized. Ultimately, the potential for information regarding the control, sequestering, and further posttranscriptional regulation of miRNAs is immense.

MiRNA Regulation

MiRNA Processing 
The most well-known and studied role of Drosha and Dicer are to regulate the transcription of miRNAs in developed organisms. Studies have shown that a complete depletion of Dicer from an embryonic time point can significantly alter the development of cardiac tissue $(180,189)$, likely through a depletion of most, if not all, miRNAs. Additionally, miRNA processing through Dicer has been shown to have epigenetic control (222). During hypoxic conditions, the demethylases KDM6A/B have decreased expression, resulting in increased H3K27me3. Other epigenetic mechanisms, such as DNA methylation or further histone modifications, could also likely control the expression and processing of Dicer or Drosha.

García et al. demonstrated that myocardial remodeling by miRNA-21 may be mechanistically controlled by the presence of SMAD2/3 and DICER1 (79). In mice undergoing pressure overload, SMAD2/3 and DICER1 expression was increased in the left ventricle, which was also upregulated in patients with aortic stenosis. MiRNA-21 was isolated bound to DICER1, while DICER1 and phosphorylated SMAD2/3 were shown to have protein-protein interactions. The authors concluded that this TGF $\beta$-dependent mechanism of phosphorylated SMAD2/3 and DICER1 regulating the increased, mature miRNA-21 processing could be important in myocardial remodeling. In some tissues, the downregulation of Dicer may be beneficial. It was shown that in endothelial cells where Dicer is downregulated, it decreased lesional macrophage content and monocyte adhesion in high fat diet apolipoprotein E knockout mice (93). Mechanistically, downregulation of Dicer also decreases miRNAs, such as miRNA-103, which binds Krüppel-like factor 4 (KLF4). KLF4 through NF-KB leads to an exacerbated atherosclerotic pathology, suggesting that increased Dicer expression is a maladaptation in endothelial cells.

Other than the canonical Drosha and Dicer miRNA editing pathway, other mechanisms have been proposed for editing miRNA. GTPase-activating protein (SH3 domain) binding protein 1 (G3bp1) is upregulated in cardiac hypertrophy and binds to the stem loop of miRNA-1 (98). MiRNA-1 is known to regulate important transcription and translational machinery in the cell. Overexpression of G3bp1 can lead to decreased miRNA-1 levels and therefore increased transcriptional and translational capacity of the cell. Though G3bp1 does not directly induce cardiac hypertrophy, this novel post-transcriptional regulation of miRNA by an endoribonuclease may be mimicked by other proteins as well.

\section{MiRNA Post-Transcriptional Regulation}


Gene regulation through lncRNA is becoming recognized as a mechanism for the control of pathological development. LncRNA named autophagy promoting factor (APF lncRNA) promotes autophagy in the heart through targeting miRNA-188-3p (236). MiRNA-188-3p targets autophagy-related protein 7 (ATG7) suppressing autophagy. Thus, regulation of APF lncRNA in the heart could be beneficial in reducing autophagy-related heart conditions, such as myocardial infarction and heart failure. Wang et al. also investigated the role of IncRNA named cardiac apoptosis-related lncRNA (CARL lncRNA) in regulating mitochondrial fission and apoptosis (238). CARL lncRNA targets miRNA-539, acting as a sponge, to increase the expression of the miRNA-539 target prohibitin-2 (PHB2). PHB2 is suggested to play a role in suppressing apoptosis and increasing mitochondrial fission. In mice, after angiotension II treatment, miRNA-489 expression was found to be reduced (237). By overexpressing miRNA-489 in mouse cardiomyocytes, the hypertrophic response was decreased and miRNA-489 was shown to target myeloid differentiation primary response gene 88 (Myd88) as the mechanism. MiRNA-489 expression was further found to be regulated by a lncRNA named cardiac hypertrophy related factor (CHRF lncRNA) which can act as an endogenous sponge and increase the hypertrophic response.

Acute myocardial infarction was experimentally induced in swine (114). Using global runon sequencing, RNA was taken from both the infarcted area and the peripheral, healthy cardiac tissue. In the infarcted tissue, 450 lncRNAs were differentially regulated. Some of the differentially regulated lncRNAs were novel, conserved sequences found within myocardial transcription factors in an antisense orientation. The sequencing experiment demonstrates the breadth of IncRNAs that could potentially impact miRNA and mitochondrial function. The regulation of miRNAs and the dynamic of cellular homeostasis in the heart and circulatory system surpass the scope of this review paper, with research teams reporting miRNA regulation through transcription factors $(26,102,164)$, gene expression $(37,88)$, other non-coding RNAs $(80,253)$, and even the microbiome (225).

\section{MiRNA Processing and LncRNA Regulation in the Diabetic Heart}

Limited research has been conducting in exploring the diabetic heart and the mechanisms of miRNA processing and post-transcriptional regulation. During gestational diabetes mellitus and pregnancy, it was demonstrated that the content of DGCR8, Drosha, and Dicer were increased 
compared to non-pregnant controls (165). Further, DGCR8, Drosha, and Dicer were more highly expressed in gestational diabetes mellitus than in those with normal pregnancies, suggesting a role for increased miRNA processing in diabetes mellitus. An increase in Dicer expression is also observed in Insulin2 mutant (Ins2+/-) Akita mice (33). In Akita hearts, even though Dicer expression is increased, let-7a, miRNA-130, miRNA-142-3p, miRNA-148, miRNA-338, miRNA345-3p, miRNA-384-3p, miRNA-433, miRNA-450, miRNA-451, miRNA-455, miRNA-494, miRNA-499, miRNA-500, miRNA-542-3p, miRNA-744, and miRNA-872 expression are decreased.

LncRNAs have been connected to the diabetic heart, being independent predictors of grade I diastolic dysfunction and left ventricular mass to left ventricular end-diastolic volume ratio in human blood (52). Also, blocking the function of lncRNA NONRATT021972 could serve as a therapeutic for diabetic cardiac autonomic neuropathy, decreasing tumor necrosis factor alpha (TNF- $\alpha$ ) and increasing insulin receptor substrate 1 (IRS1) expression (248). Though no studies at current have implicated lncRNAs in controlling miRNA expression in the diabetic heart, the impact of lncRNAs on the pathogenesis of the diabetic heart is noted, and miRNA targets are likely present.

\section{Therapeutic Applications of miRNA in Diabetes Mellitus}

Due to the significant cardiovascular complications that arise from diabetes mellitus, investigating the therapeutic applications that are available, or could be feasible options in the future, are important. The purpose of this section is to evaluate both current and potential future therapeutic applications in the diabetic heart. We will evaluate how direct targeting of miRNAs, exosomal transport of miRNAs, epigenetic manipulation of/by miRNAs, and other therapeutics involving miRNAs could impact patient health.

Targeting dysregulated energy substrate metabolism in the viable, but diseased myocardium, has been extensively investigated as a potential therapy for heart disease patients. Diabetic cardiomyopathy and diabetic heart failure are characterized by decreased glycolysis, decreased glucose oxidation, and increased fatty acid metabolism. Previous strategies to address these metabolic alterations have been to pharmacologically improve myocardial glucose import, increase pyruvate dehydrogenase activity to allow for greater glucose oxidation, and decrease fatty acid oxidation (208). These drugs include glucose transport activators (GLP-1 mimetics/agonists), 
pyruvate dehydrogenase activators (dichloroacetate, L-carnitine), carnitine palmitoyltransferase I inhibitors (perhexiline, etomoxir), and fatty acid oxidation inhibitors (ranolazine, trimetazine) (91, $140,141,144)$. Due to extensive regulation of fatty acid metabolism by the peroxisome proliferator-activated receptor (PPAR) family of transcription factors, PPAR agonists are being considered for improving mitochondrial function in diabetic cardiomyopathy (127). Interestingly, metoprolol, a beta-blocker, has been shown to inhibit fatty acid oxidation and improve cardiac function in STZ-induced diabetic rats (184). Recent studies of antianginal drugs have shown effects in improving myocardial oxygen utilization and efficiency of energy substrate metabolism (39).

\section{MiRNA-Directed Therapies}

As differential miRNA expression in cardiovascular disease have proven to modulate the expression of components of energy substrate metabolism, one may ask if normalizing the expression of miRNAs that target the most important metabolic players through the administration of antimiRs/antagomiRs or miRNA mimics would recalibrate energy substrate metabolism back to healthy flux. This strategy of pharmacologically regulating the regulators (miRNAs) has been successfully deployed preclinically to improve pathological cardiac abnormalities found in diabetic cardiomyopathy or diabetic heart failure. As an example, Thum et al. showed that administering an antagomiR specific for miRNA-21 reduced cardiac fibrosis in a mouse pressureoverload-induced model (215). Others have reported that antagomiR-mediated inhibition of miRNA-199b inhibited and reversed cardiac hypertrophy and fibrosis in mouse models of heart failure (44). Therapeutic inhibition of miRNA-208a via antimiR action has shown much promise in preventing pathological cardiac remodeling in hypertension-induced heart failure in Dahl hypertensive rats, and in improving systemic insulin sensitivity and glucose tolerance in a mouse model of high fat diet-induced obesity $(86,150)$. To our knowledge, there have been no preclinical miRNA-based studies to investigate the potential of normalizing the differential expression of miRNAs targeting components of energy substrate metabolism in the diseased diabetic heart. Because metabolic dysregulation is foundational to the etiology of diabetic heart disease and miRNA-based therapeutics may offer precise regulatory potential, we believe the field of miRNAbased therapies targeting energy substrate metabolism in diabetic cardiomyopathy and diabetic heart failure has a very bright future. 
Epigenetics in miRNA Regulation during Therapeutic Intervention

In $o b / o b$ mice, unacylated ghrelin was used as a therapeutic to increase resistance against oxidative stress (217). Unacylated ghrelin increased the expression of miRNA-126, leading to indirect increases in SIRT1 and decreased histone 3 lysate 56 deacetylation. The results of the study showed that unacylated ghrelin can reduce cell senesce by protecting endothelial cells from ROS damage. Curcumin (diferuloylmethane) has been implicated as a potential drug for modifying both epigenetic and miRNA expression. Curcumin supplementation has been suggested to inhibit DNA methylation through inhibition of the catalytic thiolate of DNMT1 (139). Restriction of DNA methylation may be a contributing factor toward modified miRNA expression after curcumin supplementation $(204,262)$. EGCG, which is an ester of epigallocatechin and gallic acid, has shown very similar results as curcumin. EGCG is a direct inhibitor of DNMT1 and also has been shown to alter as many as 61 miRNAs and their expression $(70,128,219)$.

\section{Exosomes in miRNA Regulation during Therapeutic Intervention}

Exosome based therapeutics currently revolve around the idea of collecting exosomes from healthy cell populations to directly transfer to those in a pathological setting. Gallet et al. showed that in pigs, cardiosphere-derived cell (CDC) exosomes, which were delivered through open-chest intramyocardial injection, improved the condition of acute and convalescent myocardial infarction subjects (77). Alleviation of conditions included decreased scarring and improved left ventricle ejection fraction. The content of CDCs has also been profiled, revealing that, aside from traditional miRNAs, exosomes derived from CDCs also contain non-coding RNA species, such as Y RNA, which are understudied (25). Future applications for the heart could even include the use of other modified exosomes. One such study examined the transport of a cargo protein through injected macrophage exosomes across the blood brain barrier (259). With the capacity to alter exosomal cargo, such as introducing specific miRNA or miRNA sponges, the potential therapeutic applications in the heart are numerous.

\section{MiRNA Processing and Other Mechanism of Regulation during Therapeutic Intervention}

In both diabetic human patients and mice, DICER1 protein levels were found to be increased in peripheral blood mononuclear cells (PBMCs) after treatment with Metformin (156). 
Metformin was shown to post-transcriptionally regulate the expression of RNA binding protein AU-binding factor 1 (AUF1), disrupting binding with DICER1 mRNA and leading to increased expression of DICER1 protein. Metformin also resulted in an increase in miRNA-20a, miRNA34a, miRNA-130a, miRNA-106b, miRNA-125, and let-7c, as well as a decrease in genes associated with senescence. Elgheznawy et al. demonstrated that platelets from diabetic human patients and mice had a lower expression of miRNA-142, miRNA-143, miRNA-155, and miRNA223 (65). In diabetic platelets, DICER1 expression was decreased, which the authors hypothesized was due to calpain cleavage of the enzyme. When diabetic mice were treated with a calpain inhibitor, miRNA-223 expression was restored.

Though lncRNAs have not yet been implemented in diabetic cardiovascular studies, their application could be effective. For cancer therapy, an interfering lncRNA (lncRNAi) was designed to contain specific complementary miRNA sequences which impacted the disease progression (135). The results included decreased abilities for proliferation, migration, and invasion of hepatocellular carcinoma cells.

\section{Conclusions}

MiRNAs are key players in the regulation, maintenance, and function of the cardiovascular system. The impact of miRNA on mitochondrial function in the diabetic heart is evidence that managing the expression of these small endogenous RNAs is important, and that the onset of pathologies may largely be controlled, and sustained, by miRNA. This review has examined how the miRNA profile is altered in the diabetic heart, and how the changes in miRNA expression specifically target mitochondrial function, through energy substrate utilization, apoptosis, and ROS production. Further, we examined how miRNA may be controlled in the cardiovascular system and diabetes mellitus through epigenetics, exosomes, processing, and post-transcriptional sequestration. While research concerning miRNA expression is maturing, studies focusing on the regulation of the regulating miRNAs is in its infancy. Much information can be gained mechanistically of disease and pathologies, specifically in the heart and diabetes mellitus, through

additional research in this area. Therapeutically, regulating the regulators provides a method with the potential for improved treatment strategies and health effects with greater longevity. 


\section{References}

1. Cardiovascular Disease \& Diabetes. In: American Heart Association. http://www.heart.org/HEARTORG/Conditions/More/Diabetes/WhyDiabetesMatters/Cardiovasc ular-Disease-Diabetes UCM 313865 Article.jsp/\#.WXtO7ITyupp, 2015.

2. Statistics About Diabetes. In: American Diabetes Association. http://www.diabetes.org/diabetes-basics/statistics/, 2017.

3. Aavik E, Lumivuori H, Leppanen O, Wirth T, Hakkinen SK, Brasen JH, Beschorner U, Zeller T, Braspenning M, van Criekinge W, Makinen K, and Yla-Herttuala S. Global DNA methylation analysis of human atherosclerotic plaques reveals extensive genomic hypomethylation and reactivation at imprinted locus 14q32 involving induction of a miRNA cluster. Eur Heart J 36: 993-1000, 2015.

4. Alipoor SD, Mortaz E, Tabarsi P, Farnia P, Mirsaeidi M, Garssen J, Movassaghi M, and Adcock IM. Bovis Bacillus Calmette-Guerin (BCG) infection induces exosomal miRNA release by human macrophages. J Transl Med 15: 105, 2017.

5. An D and Rodrigues B. Role of changes in cardiac metabolism in development of diabetic cardiomyopathy. Am J Physiol Heart Circ Physiol 291: H1489-1506, 2006.

6. Asrih $\mathbf{M}$ and Steffens S. Emerging role of epigenetics and miRNA in diabetic cardiomyopathy. Cardiovasc Pathol 22: 117-125, 2013.

7. Aung N, Sanghvi MM, Zemrak F, Lee AM, Cooper JA, Paiva JM, Thomson RJ, Fung K, Khanji MY, Lukaschuk E, Carapella V, Kim YJ, Munroe PB, Piechnik SK, Neubauer S, and Petersen SE. Association Between Ambient Air Pollution and Cardiac Morpho-Functional Phenotypes: Insights From the UK Biobank Population Imaging Study. Circulation 138: 21752186, 2018.

8. Awad MM, Calkins H, and Judge DP. Mechanisms of disease: molecular genetics of arrhythmogenic right ventricular dysplasia/cardiomyopathy. Nat Clin Pract Cardiovasc Med 5: 258-267, 2008.

9. Azmi AS, Bao B, and Sarkar FH. Exosomes in cancer development, metastasis, and drug resistance: a comprehensive review. Cancer Metastasis Rev 32: 623-642, 2013.

10. Aztatzi-Aguilar OG, Uribe-Ramirez M, Arias-Montano JA, Barbier O, and De Vizcaya-Ruiz A. Acute and subchronic exposure to air particulate matter induces expression of angiotensin and bradykinin-related genes in the lungs and heart: Angiotensin-II type-I receptor as a molecular target of particulate matter exposure. Part Fibre Toxicol 12: 17, 2015.

11. Bandiera $\mathbf{S}$, Mategot $\mathbf{R}$, Girard $\mathbf{M}$, Demongeot $\mathbf{J}$, and Henrion-Caude $\mathbf{A}$. MitomiRs delineating the intracellular localization of microRNAs at mitochondria. Free Radic Biol Med 64: 12-19, 2013.

12. Bandiera S, Ruberg S, Girard M, Cagnard N, Hanein S, Chretien D, Munnich A, Lyonnet S, and Henrion-Caude A. Nuclear outsourcing of RNA interference components to human mitochondria. PLoS One 6: e20746, 2011.

13. Banerjee J, Nema V, Dhas Y, and Mishra N. Role of MicroRNAs in Type 2 Diabetes and Associated Vascular Complications. Biochimie 139: 9-19, 2017.

14. Baradan R, Hollander JM, and Das S. Mitochondrial miRNAs in diabetes: just the tip of the iceberg. Can J Physiol Pharmacol: 1-7, 2017.

15. Bartel DP. MicroRNAs: target recognition and regulatory functions. Cell 136: 215-233, 2009.

16. Bartova E, Krejci J, Harnicarova A, Galiova G, and Kozubek S. Histone modifications and nuclear architecture: a review. J Histochem Cytochem 56: 711-721, 2008. 
17. Baseler WA, Thapa D, Jagannathan R, Dabkowski ER, Croston TL, and Hollander JM. miR-141 as a regulator of the mitochondrial phosphate carrier (Slc25a3) in the type 1 diabetic heart. Am J Physiol Cell Physiol 303: C1244-1251, 2012.

18. Bellavia A, Urch B, Speck M, Brook RD, Scott JA, Albetti B, Behbod B, North M, Valeri L, Bertazzi PA, Silverman F, Gold D, and Baccarelli AA. DNA hypomethylation, ambient particulate matter, and increased blood pressure: findings from controlled human exposure experiments. J Am Heart Assoc 2: e000212, 2013.

19. Benchimol S. p53-dependent pathways of apoptosis. Cell Death Differ 8: 1049-1051, 2001.

20. Bird A. DNA methylation patterns and epigenetic memory. Genes Dev 16: 6-21, 2002.

21. Brook RD, Rajagopalan S, Pope CA, 3rd, Brook JR, Bhatnagar A, Diez-Roux AV, Holguin F, Hong Y, Luepker RV, Mittleman MA, Peters A, Siscovick D, Smith SC, Jr., Whitsel L, Kaufman JD, American Heart Association Council on E, Prevention CotKiCD, Council on Nutrition PA, and Metabolism. Particulate matter air pollution and cardiovascular disease: An update to the scientific statement from the American Heart Association. Circulation 121: 2331-2378, 2010.

22. Buschow SI, Liefhebber JM, Wubbolts R, and Stoorvogel W. Exosomes contain ubiquitinated proteins. Blood Cells Mol Dis 35: 398-403, 2005.

23. Byun HM, Colicino E, Trevisi L, Fan T, Christiani DC, and Baccarelli AA. Effects of Air Pollution and Blood Mitochondrial DNA Methylation on Markers of Heart Rate Variability. $J$ Am Heart Assoc 5, 2016.

24. Cai C, Huang J, Lin Y, Miao W, Chen P, Chen X, Wang J, and Chen M. Particulate matter 2.5 induced arrhythmogenesis mediated by TRPC 3 in human induced pluripotent stem cellderived cardiomyocytes. Arch Toxicol, 2019.

25. Cambier L, de Couto G, Ibrahim A, Echavez AK, Valle J, Liu W, Kreke M, Smith RR, Marban L, and Marban E. Y RNA fragment in extracellular vesicles confers cardioprotection via modulation of IL-10 expression and secretion. EMBO Mol Med 9: 337-352, 2017.

26. Caporali A, Meloni M, Nailor A, Mitic T, Shantikumar S, Riu F, Sala-Newby GB, Rose L, Besnier M, Katare R, Voellenkle C, Verkade P, Martelli F, Madeddu P, and Emanueli C. p75(NTR)-dependent activation of NF-kappaB regulates microRNA-503 transcription and pericyte-endothelial crosstalk in diabetes after limb ischaemia. Nat Commun 6: 8024, 2015.

27. Cassee FR, Heroux ME, Gerlofs-Nijland ME, and Kelly FJ. Particulate matter beyond mass: recent health evidence on the role of fractions, chemical constituents and sources of emission. Inhal Toxicol 25: 802-812, 2013.

28. Cedar $\mathbf{H}$ and Bergman Y. Linking DNA methylation and histone modification: patterns and paradigms. Nat Rev Genet 10: 295-304, 2009.

29. Chambers JC, Loh M, Lehne B, Drong A, Kriebel J, Motta V, Wahl S, Elliott HR, Rota F, Scott WR, Zhang W, Tan ST, Campanella G, Chadeau-Hyam M, Yengo L, Richmond RC, Adamowicz-Brice M, Afzal U, Bozaoglu K, Mok ZY, Ng HK, Pattou F, Prokisch H, Rozario MA, Tarantini L, Abbott J, Ala-Korpela M, Albetti B, Ammerpohl O, Bertazzi PA, Blancher C, Caiazzo R, Danesh J, Gaunt TR, de Lusignan S, Gieger C, Illig T, Jha S, Jones S, Jowett J, Kangas AJ, Kasturiratne A, Kato N, Kotea N, Kowlessur S, Pitkaniemi J, Punjabi P, Saleheen D, Schafmayer C, Soininen P, Tai ES, Thorand B, Tuomilehto J, Wickremasinghe AR, Kyrtopoulos SA, Aitman TJ, Herder C, Hampe J, 
Cauchi S, Relton CL, Froguel P, Soong R, Vineis P, Jarvelin MR, Scott J, Grallert H, Bollati V, Elliott P, McCarthy MI, and Kooner JS. Epigenome-wide association of DNA methylation markers in peripheral blood from Indian Asians and Europeans with incident type 2 diabetes: a nested case-control study. Lancet Diabetes Endocrinol 3: 526-534, 2015.

30. Chan SY, Zhang YY, Hemann C, Mahoney CE, Zweier JL, and Loscalzo J. MicroRNA-210 controls mitochondrial metabolism during hypoxia by repressing the iron-sulfur cluster assembly proteins ISCU1/2. Cell Metab 10: 273-284, 2009.

31. Chang X, Li S, Li J, Yin L, Zhou T, Zhang C, Chen X, and Sun K. Ethnic differences in microRNA-375 expression level and DNA methylation status in type 2 diabetes of Han and Kazak populations. J Diabetes Res 2014: 761938, 2014.

32. Chaturvedi P, Kalani A, Medina I, Familtseva A, and Tyagi SC. Cardiosome mediated regulation of MMP9 in diabetic heart: role of mir29b and mir455 in exercise. J Cell Mol Med 19: 2153-2161, 2015.

33. Chavali V, Tyagi SC, and Mishra PK. Differential expression of dicer, miRNAs, and inflammatory markers in diabetic Ins2+/- Akita hearts. Cell Biochem Biophys 68: 25-35, 2014.

34. Chavali V, Tyagi SC, and Mishra PK. MicroRNA-133a regulates DNA methylation in diabetic cardiomyocytes. Biochem Biophys Res Commun 425: 668-672, 2012.

35. Chavali V, Tyagi SC, and Mishra PK. Predictors and prevention of diabetic cardiomyopathy. Diabetes Metab Syndr Obes 6: 151-160, 2013.

36. Chen H, Chen X, Hong X, Liu C, Huang H, Wang Q, Chen S, Chen H, Yang K, and Sun Q. Maternal exposure to ambient PM2.5 exaggerates fetal cardiovascular maldevelopment induced by homocysteine in rats. Environ Toxicol 32: 877-889, 2017.

37. Chen J, Young ME, Chatham JC, Crossman DK, Dell'Italia LJ, and Shalev A. TXNIP regulates myocardial fatty acid oxidation via miR-33a signaling. Am J Physiol Heart Circ Physiol 311: H64-75, 2016.

38. Chen S, Puthanveetil P, Feng B, Matkovich SJ, Dorn GW, 2nd, and Chakrabarti S. Cardiac miR-133a overexpression prevents early cardiac fibrosis in diabetes. J Cell Mol Med 18: 415-421, 2014.

39. Chong CR, Sallustio B, and Horowitz JD. Drugs that Affect Cardiac Metabolism: Focus on Perhexiline. Cardiovasc Drugs Ther 30: 399-405, 2016.

40. Chu JH, Hart JE, Chhabra D, Garshick E, Raby BA, and Laden F. Gene expression network analyses in response to air pollution exposures in the trucking industry. Environ Health 15: $101,2016$.

41. Corcoran DL, Pandit KV, Gordon B, Bhattacharjee A, Kaminski N, and Benos PV. Features of mammalian microRNA promoters emerge from polymerase II chromatin immunoprecipitation data. PLoS One 4: e5279, 2009.

42. Costantino S, Paneni F, Luscher TF, and Cosentino F. MicroRNA profiling unveils hyperglycaemic memory in the diabetic heart. Eur Heart J 37: 572-576, 2016.

43. Croft DP, Cameron SJ, Morrell CN, Lowenstein CJ, Ling F, Zareba W, Hopke PK, Utell MJ, Thurston SW, Thevenet-Morrison K, Evans KA, Chalupa D, and Rich DQ. Associations between ambient wood smoke and other particulate pollutants and biomarkers of systemic inflammation, coagulation and thrombosis in cardiac patients. Environ Res 154: 352-361, 2017.

44. da Costa Martins PA, Salic K, Gladka MM, Armand AS, Leptidis S, el Azzouzi H, Hansen A, Coenen-de Roo CJ, Bierhuizen MF, van der Nagel R, van Kuik J, de Weger R, de Bruin A, Condorelli G, Arbones ML, Eschenhagen T, and De Windt LJ. MicroRNA-199b 
targets the nuclear kinase Dyrk1a in an auto-amplification loop promoting calcineurin/NFAT signalling. Nat Cell Biol 12: 1220-1227, 2010.

45. Dai J, Chen W, Lin Y, Wang S, Guo X, and Zhang QQ. Exposure to Concentrated Ambient Fine Particulate Matter Induces Vascular Endothelial Dysfunction via miR-21. Int J Biol Sci 13: 868-877, 2017.

46. Dai J, Sun C, Yao Z, Chen W, Yu L, and Long M. Exposure to concentrated ambient fine particulate matter disrupts vascular endothelial cell barrier function via the IL-6/HIF-1alpha signaling pathway. FEBS Open Bio 6: 720-728, 2016.

47. Dai L, Mehta A, Mordukhovich I, Just AC, Shen J, Hou L, Koutrakis P, Sparrow D, Vokonas PS, Baccarelli AA, and Schwartz JD. Differential DNA methylation and PM2.5 species in a 450K epigenome-wide association study. Epigenetics 12: 139-148, 2017.

48. Das S, Bedja D, Campbell N, Dunkerly B, Chenna V, Maitra A, and Steenbergen C. miR-181c regulates the mitochondrial genome, bioenergetics, and propensity for heart failure in vivo. PLoS One 9: e96820, 2014.

49. Das S, Ferlito M, Kent OA, Fox-Talbot K, Wang R, Liu D, Raghavachari N, Yang Y, Wheelan SJ, Murphy E, and Steenbergen C. Nuclear miRNA regulates the mitochondrial genome in the heart. Circ Res 110: 1596-1603, 2012.

50. Dasgupta N, Peng Y, Tan Z, Ciraolo G, Wang D, and Li R. miRNAs in mtDNA-less cell mitochondria. Cell Death Discov 1: 15004, 2015.

51. de Couto G, Gallet R, Cambier L, Jaghatspanyan E, Makkar N, Dawkins JF, Berman BP, and Marban E. Exosomal MicroRNA Transfer Into Macrophages Mediates Cellular Postconditioning. Circulation 136: 200-214, 2017.

52. de Gonzalo-Calvo D, Kenneweg F, Bang C, Toro R, van der Meer RW, Rijzewijk LJ, Smit JW, Lamb HJ, Llorente-Cortes V, and Thum T. Circulating long-non coding RNAs as biomarkers of left ventricular diastolic function and remodelling in patients with well-controlled type 2 diabetes. Sci Rep 6: 37354, 2016.

53. de Gonzalo-Calvo D, van der Meer RW, Rijzewijk LJ, Smit JW, Revuelta-Lopez E, Nasarre L, Escola-Gil JC, Lamb HJ, and Llorente-Cortes V. Serum microRNA-1 and microRNA-133a levels reflect myocardial steatosis in uncomplicated type 2 diabetes. Sci Rep 7: 47, 2017.

54. de Kreutzenberg SV, Ceolotto G, Papparella I, Bortoluzzi A, Semplicini A, Dalla Man C, Cobelli C, Fadini GP, and Avogaro A. Downregulation of the longevity-associated protein sirtuin 1 in insulin resistance and metabolic syndrome: potential biochemical mechanisms. Diabetes 59: 1006-1015, 2010.

55. Deng L, Blanco FJ, Stevens H, Lu R, Caudrillier A, McBride M, McClure JD, Grant J, Thomas M, Frid M, Stenmark K, White K, Seto AG, Morrell NW, Bradshaw AC, MacLean MR, and Baker AH. MicroRNA-143 Activation Regulates Smooth Muscle and Endothelial Cell Crosstalk in Pulmonary Arterial Hypertension. Circ Res 117: 870-883, 2015.

56. Devlin RB, Smith CB, Schmitt MT, Rappold AG, Hinderliter A, Graff D, and Carraway MS. Controlled exposure of humans with metabolic syndrome to concentrated ultrafine ambient particulate matter causes cardiovascular effects. Toxicol Sci 140: 61-72, 2014.

57. Dhingra R and Vasan RS. Diabetes and the risk of heart failure. Heart Fail Clin 8: 125133, 2012.

58. Diao X, Shen E, Wang X, and Hu B. Differentially expressed microRNAs and their target genes in the hearts of streptozotocin-induced diabetic mice. Mol Med Rep 4: 633-640, 2011. 
59. Donaldson K, Tran L, Jimenez LA, Duffin R, Newby DE, Mills N, MacNee W, and Stone V. Combustion-derived nanoparticles: a review of their toxicology following inhalation exposure. Part Fibre Toxicol 2: 10, 2005.

60. Dong L, Sun W, Li F, Shi M, Meng X, Wang C, Meng M, Tang W, Liu H, Wang L, and Song $\mathbf{L}$. The harmful effects of acute PM2.5 exposure to the heart and a novel preventive and therapeutic function of CEOs. Sci Rep 9: 3495, 2019.

61. Du X, Jiang S, Bo L, Liu J, Zeng X, Xie Y, He Q, Ye X, Song W, and Zhao J. Combined effects of vitamin E and omega-3 fatty acids on protecting ambient PM2.5-induced cardiovascular injury in rats. Chemosphere 173: 14-21, 2017.

62. Du Y, Xu X, Chu M, Guo Y, and Wang J. Air particulate matter and cardiovascular disease: the epidemiological, biomedical and clinical evidence. J Thorac Dis 8: E8-E19, 2016.

63. Duarte FV, Palmeira CM, and Rolo AP. The Emerging Role of MitomiRs in the Pathophysiology of Human Disease. Adv Exp Med Biol 888: 123-154, 2015.

64. Duncan JG. Mitochondrial dysfunction in diabetic cardiomyopathy. Biochim Biophys Acta 1813: 1351-1359, 2011.

65. Elgheznawy A, Shi L, Hu J, Wittig I, Laban H, Pircher J, Mann A, Provost P, Randriamboavonjy V, and Fleming I. Dicer cleavage by calpain determines platelet microRNA levels and function in diabetes. Circ Res 117: 157-165, 2015.

66. Emanueli C, Shearn AI, Laftah A, Fiorentino F, Reeves BC, Beltrami C, Mumford A, Clayton A, Gurney M, Shantikumar S, and Angelini GD. Coronary Artery-Bypass-Graft Surgery Increases the Plasma Concentration of Exosomes Carrying a Cargo of Cardiac MicroRNAs: An Example of Exosome Trafficking Out of the Human Heart with Potential for Cardiac Biomarker Discovery. PLoS One 11: e0154274, 2016.

67. Erener S, Marwaha A, Tan R, Panagiotopoulos C, and Kieffer TJ. Profiling of circulating microRNAs in children with recent onset of type 1 diabetes. JCI Insight 2: e89656, 2017.

68. Espin-Perez A, Krauskopf J, Chadeau-Hyam M, van Veldhoven K, Chung F, Cullinan P, Piepers J, van Herwijnen M, Kubesch N, Carrasco-Turigas G, Nieuwenhuijsen M, Vineis P, Kleinjans JCS, and de Kok T. Short-term transcriptome and microRNAs responses to exposure to different air pollutants in two population studies. Environ Pollut 242: 182-190, 2018.

69. Fan T, Fang SC, Cavallari JM, Barnett IJ, Wang Z, Su L, Byun HM, Lin X, Baccarelli AA, and Christiani DC. Heart rate variability and DNA methylation levels are altered after shortterm metal fume exposure among occupational welders: a repeated-measures panel study. $B M C$ Public Health 14: 1279, 2014.

70. Fang MZ, Wang Y, Ai N, Hou Z, Sun Y, Lu H, Welsh W, and Yang CS. Tea polyphenol (-)-epigallocatechin-3-gallate inhibits DNA methyltransferase and reactivates methylationsilenced genes in cancer cell lines. Cancer Res 63: 7563-7570, 2003.

71. Feng L, Yang X, Asweto CO, Wu J, Zhang Y, Hu H, Shi Y, Duan J, and Sun Z. Genome-wide transcriptional analysis of cardiovascular-related genes and pathways induced by PM2.5 in human myocardial cells. Environ Sci Pollut Res Int 24: 11683-11693, 2017.

72. Feng L, Yang X, Asweto CO, Wu J, Zhang Y, Hu H, Shi Y, Duan J, and Sun Z. Lowdose combined exposure of nanoparticles and heavy metal compared with PM2.5 in human myocardial AC16 cells. Environ Sci Pollut Res Int 24: 27767-27777, 2017. 
73. Feng L, Yang X, Shi Y, Liang S, Zhao T, Duan J, and Sun Z. Co-exposure subacute toxicity of silica nanoparticles and lead acetate on cardiovascular system. Int J Nanomedicine 13: 7819-7834, 2018.

74. Floris I, Descamps B, Vardeu A, Mitic T, Posadino AM, Shantikumar S, Sala-Newby G, Capobianco G, Mangialardi G, Howard L, Dessole S, Urrutia R, Pintus G, and Emanueli C. Gestational diabetes mellitus impairs fetal endothelial cell functions through a mechanism involving microRNA-101 and histone methyltransferase enhancer of zester homolog-2. Arterioscler Thromb Vasc Biol 35: 664-674, 2015.

75. Franck U, Odeh S, Wiedensohler A, Wehner B, and Herbarth O. The effect of particle size on cardiovascular disorders--the smaller the worse. Sci Total Environ 409: 4217-4221, 2011.

76. Galanter JM, Gignoux CR, Oh SS, Torgerson D, Pino-Yanes M, Thakur N, Eng C, Hu D, Huntsman S, Farber HJ, Avila PC, Brigino-Buenaventura E, LeNoir MA, Meade K, Serebrisky D, Rodriguez-Cintron W, Kumar R, Rodriguez-Santana JR, Seibold MA, Borrell LN, Burchard EG, and Zaitlen N. Differential methylation between ethnic sub-groups reflects the effect of genetic ancestry and environmental exposures. Elife 6, 2017.

77. Gallet R, Dawkins J, Valle J, Simsolo E, de Couto G, Middleton R, Tseliou E, Luthringer D, Kreke M, Smith RR, Marban L, Ghaleh B, and Marban E. Exosomes secreted by cardiosphere-derived cells reduce scarring, attenuate adverse remodelling, and improve function in acute and chronic porcine myocardial infarction. Eur Heart J 38: 201-211, 2017.

78. Ganguly K, Ettehadieh D, Upadhyay S, Takenaka S, Adler T, Karg E, Krombach F, Kreyling WG, Schulz H, Schmid O, and Stoeger T. Early pulmonary response is critical for extra-pulmonary carbon nanoparticle mediated effects: comparison of inhalation versus intraarterial infusion exposures in mice. Part Fibre Toxicol 14: 19, 2017.

79. Garcia R, Nistal JF, Merino D, Price NL, Fernandez-Hernando C, Beaumont J, Gonzalez A, Hurle MA, and Villar AV. p-SMAD2/3 and DICER promote pre-miR-21 processing during pressure overload-associated myocardial remodeling. Biochim Biophys Acta 1852: 1520-1530, 2015.

80. Geng HH, Li R, Su YM, Xiao J, Pan M, Cai XX, and Ji XP. The Circular RNA Cdr1as Promotes Myocardial Infarction by Mediating the Regulation of miR-7a on Its Target Genes Expression. PLoS One 11: e0151753, 2016.

81. Giles LV, Tebbutt SJ, Carlsten C, and Koehle MS. The effect of low and high-intensity cycling in diesel exhaust on flow-mediated dilation, circulating NOx, endothelin-1 and blood pressure. PLoS One 13: e192419, 2018.

82. Goodson JM, Weldy CS, MacDonald JW, Liu Y, Bammler TK, Chien WM, and Chin MT. In utero exposure to diesel exhaust particulates is associated with an altered cardiac transcriptional response to transverse aortic constriction and altered DNA methylation. FASEB $J$ 31: 4935-4945, 2017.

83. Gordon $\mathbf{T}$ and Reibman J. Cardiovascular toxicity of inhaled ambient particulate matter. Toxicol Sci 56: 2-4, 2000.

84. Greco S, Fasanaro P, Castelvecchio S, D'Alessandra Y, Arcelli D, Di Donato M, Malavazos A, Capogrossi MC, Menicanti L, and Martelli F. MicroRNA dysregulation in diabetic ischemic heart failure patients. Diabetes 61: 1633-1641, 2012.

85. Gregory RI, Yan KP, Amuthan G, Chendrimada T, Doratotaj B, Cooch N, and Shiekhattar R. The Microprocessor complex mediates the genesis of microRNAs. Nature 432: 235-240, 2004. 
86. Grueter CE, van Rooij E, Johnson BA, DeLeon SM, Sutherland LB, Qi X, Gautron L, Elmquist JK, Bassel-Duby R, and Olson EN. A cardiac microRNA governs systemic energy homeostasis by regulation of MED13. Cell 149: 671-683, 2012.

87. Guay C and Regazzi R. Circulating microRNAs as novel biomarkers for diabetes mellitus. Nat Rev Endocrinol 9: 513-521, 2013.

88. Gurha P, Chen X, Lombardi R, Willerson JT, and Marian AJ. Knockdown of Plakophilin 2 Downregulates miR-184 Through CpG Hypermethylation and Suppression of the E2F1 Pathway and Leads to Enhanced Adipogenesis In Vitro. Circ Res 119: 731-750, 2016.

89. Ha M and Kim VN. Regulation of microRNA biogenesis. Nat Rev Mol Cell Biol 15: 509$524,2014$.

90. Haberzettl P, O'Toole TE, Bhatnagar A, and Conklin DJ. Exposure to Fine Particulate Air Pollution Causes Vascular Insulin Resistance by Inducing Pulmonary Oxidative Stress. Environ Health Perspect 124: 1830-1839, 2016.

91. Hamilton DJ. Metabolic Recovery of the Failing Heart: Emerging Therapeutic Options. Methodist Debakey Cardiovasc J 13: 25-28, 2017.

92. Han J, Lee Y, Yeom KH, Kim YK, Jin H, and Kim VN. The Drosha-DGCR8 complex in primary microRNA processing. Genes Dev 18: 3016-3027, 2004.

93. Hartmann P, Zhou Z, Natarelli L, Wei Y, Nazari-Jahantigh M, Zhu M, Grommes J, Steffens S, Weber C, and Schober A. Endothelial Dicer promotes atherosclerosis and vascular inflammation by miRNA-103-mediated suppression of KLF4. Nat Commun 7: 10521, 2016.

94. Hathaway QA, Durr AJ, Shepherd DL, Pinti MV, Brandebura AN, Nichols CE, Kunovac A, Goldsmith WT, Friend SA, Abukabda AB, Fink GK, Nurkiewicz TR, and Hollander JM. miRNA-378a as a key regulator of cardiovascular health following engineered nanomaterial inhalation exposure. Nanotoxicology: 1-20, 2019.

95. Hathaway QA, Nichols CE, Shepherd DL, Stapleton PA, McLaughlin SL, Stricker JC, Rellick SL, Pinti MV, Abukabda AB, McBride CR, Yi J, Stine SM, Nurkiewicz TR, and Hollander JM. Maternal-engineered nanomaterial exposure disrupts progeny cardiac function and bioenergetics. Am J Physiol Heart Circ Physiol 312: H446-H458, 2017.

96. Hausser $\mathbf{J}$ and Zavolan $\mathbf{M}$. Identification and consequences of miRNA-target interactions--beyond repression of gene expression. Nat Rev Genet 15: 599-612, 2014.

97. He L, He X, Lowe SW, and Hannon GJ. microRNAs join the p53 network--another piece in the tumour-suppression puzzle. Nat Rev Cancer 7: 819-822, 2007.

98. He M, Yang Z, Abdellatif M, and Sayed D. GTPase Activating Protein (Sh3 Domain) Binding Protein 1 Regulates the Processing of MicroRNA-1 during Cardiac Hypertrophy. PLoS One 10: e0145112, 2015.

99. Holland NA, Fraiser CR, Sloan RC, 3rd, Devlin RB, Brown DA, and Wingard CJ. Ultrafine Particulate Matter Increases Cardiac Ischemia/Reperfusion Injury via Mitochondrial Permeability Transition Pore. Cardiovasc Toxicol 17: 441-450, 2017.

100. Hong F, Wang L, Yu X, Zhou Y, Hong J, and Sheng L. Toxicological effect of TiO2 nanoparticle-induced myocarditis in mice. Nanoscale Res Lett 10: 1029, 2015.

101. Hu H, Wu J, Li Q, Asweto C, Feng L, Yang X, Duan F, Duan J, and Sun Z. Fine particulate matter induces vascular endothelial activation via IL-6 dependent JAK1/STAT3 signaling pathway. Toxicol Res (Camb) 5: 946-953, 2016.

102. Huang Z, Chen XJ, Qian C, Dong Q, Ding D, Wu QF, Li J, Wang HF, Li WH, Xie Q, Cheng X, Zhao N, Du YM, and Liao YH. Signal Transducer and Activator of Transcription 
3/MicroRNA-21 Feedback Loop Contributes to Atrial Fibrillation by Promoting Atrial Fibrosis in a Rat Sterile Pericarditis Model. Circ Arrhythm Electrophysiol 9, 2016.

103. Huerta-Garcia E, Ramos-Godinez MDP, Lopez-Saavedra A, Alfaro-Moreno E, Gomez-Crisostomo NP, Colin-Val Z, Sanchez-Barrera H, and Lopez-Marure R. Internalization of Titanium Dioxide Nanoparticles Is Mediated by Actin-Dependent Reorganization and Clathrin- and Dynamin-Mediated Endocytosis in H9c2 Rat Cardiomyoblasts. Chem Res Toxicol, 2019.

104. Huerta-Garcia E, Zepeda-Quiroz I, Sanchez-Barrera H, Colin-Val Z, Alfaro-Moreno E, Ramos-Godinez MDP, and Lopez-Marure R. Internalization of Titanium Dioxide Nanoparticles Is Cytotoxic for H9c2 Rat Cardiomyoblasts. Molecules 23, 2018.

105. Husain M, Wu D, Saber AT, Decan N, Jacobsen NR, Williams A, Yauk CL, Wallin $\mathbf{H}$, Vogel $\mathbf{U}$, and Halappanavar S. Intratracheally instilled titanium dioxide nanoparticles translocate to heart and liver and activate complement cascade in the heart of C57BL/6 mice. Nanotoxicology 9: 1013-1022, 2015.

106. Huynh K, Bernardo BC, McMullen JR, and Ritchie RH. Diabetic cardiomyopathy: mechanisms and new treatment strategies targeting antioxidant signaling pathways. Pharmacol Ther 142: 375-415, 2014.

107. Iorio MV, Piovan C, and Croce CM. Interplay between microRNAs and the epigenetic machinery: an intricate network. Biochim Biophys Acta 1799: 694-701, 2010.

108. Ismail N, Wang Y, Dakhlallah D, Moldovan L, Agarwal K, Batte K, Shah P, Wisler J, Eubank TD, Tridandapani S, Paulaitis ME, Piper MG, and Marsh CB. Macrophage microvesicles induce macrophage differentiation and miR-223 transfer. Blood 121: 984-995, 2013. 109. Jagannathan R, Thapa D, Nichols CE, Shepherd DL, Stricker JC, Croston TL, Baseler WA, Lewis SE, Martinez I, and Hollander JM. Translational Regulation of the Mitochondrial Genome Following Redistribution of Mitochondrial MicroRNA in the Diabetic Heart. Circ Cardiovasc Genet 8: 785-802, 2015.

110. Jalali S, Bhartiya D, Lalwani MK, Sivasubbu S, and Scaria V. Systematic transcriptome wide analysis of lncRNA-miRNA interactions. PLoS One 8: e53823, 2013.

111. Jin Y, Wu Z, Wang N, Duan S, Wu Y, Wang J, Wu W, and Feng F. Association of EGF Receptor and NLRs signaling with Cardiac Inflammation and Fibrosis in Mice Exposed to Fine Particulate Matter. J Biochem Mol Toxicol 30: 429-437, 2016.

112. Jones PA. Functions of DNA methylation: islands, start sites, gene bodies and beyond. Nat Rev Genet 13: 484-492, 2012.

113. Jorgensen RB, Buhagen M, and Foreland S. Personal exposure to ultrafine particles from PVC welding and concrete work during tunnel rehabilitation. Occup Environ Med 73: 467473, 2016.

114. Kaikkonen MU, Halonen P, Liu OH, Turunen TA, Pajula J, Moreau P, Selvarajan I, Tuomainen T, Aavik E, Tavi P, and Yla-Herttuala S. Genome-Wide Dynamics of Nascent Noncoding RNA Transcription in Porcine Heart After Myocardial Infarction. Circ Cardiovasc Genet 10, 2017.

115. Katare R, Caporali A, Zentilin L, Avolio E, Sala-Newby G, Oikawa A, Cesselli D, Beltrami AP, Giacca M, Emanueli C, and Madeddu P. Intravenous gene therapy with PIM-1 via a cardiotropic viral vector halts the progression of diabetic cardiomyopathy through promotion of prosurvival signaling. Circ Res 108: 1238-1251, 2011.

116. Katsouyanni K. Ambient air pollution and health. Br Med Bull 68: 143-156, 2003. 
117. Kessler R. Engineered nanoparticles in consumer products: understanding a new ingredient. Environ Health Perspect 119: a120-125, 2011.

118. Kong L, Zhu J, Han W, Jiang X, Xu M, Zhao Y, Dong Q, Pang Z, Guan Q, Gao L, Zhao J, and Zhao L. Significance of serum microRNAs in pre-diabetes and newly diagnosed type 2 diabetes: a clinical study. Acta Diabetol 48: 61-69, 2011.

119. Krauskopf J, Caiment F, van Veldhoven K, Chadeau-Hyam M, Sinharay R, Chung KF, Cullinan P, Collins P, Barratt B, Kelly FJ, Vermeulen R, Vineis P, de Kok TM, and Kleinjans JC. The human circulating miRNome reflects multiple organ disease risks in association with short-term exposure to traffic-related air pollution. Environ Int 113: 26-34, 2018. 120. Kren BT, Wong PY, Sarver A, Zhang X, Zeng Y, and Steer CJ. MicroRNAs identified in highly purified liver-derived mitochondria may play a role in apoptosis. RNA Biol 6: 65-72, 2009.

121. Kuhlbusch TAJ, Wijnhoven SWP, and Haase A. Nanomaterial exposures for worker, consumer and the general public. Nanoimpact 10: 11-25, 2018.

122. Kulkarni H, Kos MZ, Neary J, Dyer TD, Kent JW, Jr., Goring HH, Cole SA, Comuzzie AG, Almasy L, Mahaney MC, Curran JE, Blangero J, and Carless MA. Novel epigenetic determinants of type 2 diabetes in Mexican-American families. Hum Mol Genet 24: 5330-5344, 2015.

123. Kumarathasan P, Vincent R, Blais E, Bielecki A, Guenette J, Filiatreault A, Brion O, Cakmak S, Thomson EM, Shutt R, Kauri LM, Mahmud M, Liu L, and Dales R. Cardiovascular and inflammatory mechanisms in healthy humans exposed to air pollution in the vicinity of a steel mill. Part Fibre Toxicol 15: 34, 2018.

124. Kuwabara Y, Ono K, Horie T, Nishi H, Nagao K, Kinoshita M, Watanabe S, Baba O, Kojima Y, Shizuta S, Imai M, Tamura T, Kita T, and Kimura T. Increased microRNA-1 and microRNA-133a levels in serum of patients with cardiovascular disease indicate myocardial damage. Circ Cardiovasc Genet 4: 446-454, 2011.

125. Kwon H, Lee J, Jeong K, Jang D, and Pak Y. Fatty acylated caveolin-2 is a substrate of insulin receptor tyrosine kinase for insulin receptor substrate-1-directed signaling activation. Biochim Biophys Acta 1853: 1022-1034, 2015.

126. Lane KJ, Levy JI, Scammell MK, Peters JL, Patton AP, Reisner E, Lowe L, Zamore W, Durant JL, and Brugge D. Association of modeled long-term personal exposure to ultrafine particles with inflammatory and coagulation biomarkers. Environ Int 92-93: 173-182, 2016.

127. Lee TW, Bai KJ, Lee TI, Chao TF, Kao YH, and Chen YJ. PPARs modulate cardiac metabolism and mitochondrial function in diabetes. J Biomed Sci 24: 5, 2017.

128. Lee WJ, Shim JY, and Zhu BT. Mechanisms for the inhibition of DNA methyltransferases by tea catechins and bioflavonoids. Mol Pharmacol 68: 1018-1030, 2005.

129. Li J, Ren Y, Shi E, Tan Z, Xiong J, Yan L, and Jiang X. Inhibition of the Let-7 Family MicroRNAs Induces Cardioprotection Against Ischemia-Reperfusion Injury in Diabetic Rats. Ann Thorac Surg 102: 829-835, 2016.

130. Li KL and Lin YC. PM2.5 induced cardiac hypertrophy via CREB/GSK3b/SOS1 pathway and metabolomics alterations. Oncotarget 9: 30748-30760, 2018.

131. Li W, Dorans KS, Wilker EH, Rice MB, Ljungman PL, Schwartz JD, Coull BA, Koutrakis P, Gold DR, Keaney JF, Jr., Vasan RS, Benjamin EJ, and Mittleman MA. Shortterm exposure to ambient air pollution and circulating biomarkers of endothelial cell activation: The Framingham Heart Study. Environ Res 171: 36-43, 2019. 
132. Li X, Du N, Zhang Q, Li J, Chen X, Liu X, Hu Y, Qin W, Shen N, Xu C, Fang Z, Wei Y, Wang R, Du Z, Zhang Y, and Lu Y. MicroRNA-30d regulates cardiomyocyte pyroptosis by directly targeting foxo3a in diabetic cardiomyopathy. Cell Death Dis 5: e1479, 2014.

133. Li X, Geng J, Chen Y, Chen F, Liu C, Xu Q, Zhao J, Hu J, Xie J, and Xu B. Exposure to particulate matter induces cardiomyocytes apoptosis after myocardial infarction through NFkappaB activation. Biochem Biophys Res Commun 488: 224-231, 2017.

134. Li X, Monks B, Ge Q, and Birnbaum MJ. Akt/PKB regulates hepatic metabolism by directly inhibiting PGC-1alpha transcription coactivator. Nature 447: 1012-1016, 2007.

135. Li X, Su Y, Sun B, Ji W, Peng Z, Xu Y, Wu M, and Su C. An Artificially Designed Interfering lncRNA Expressed by Oncolytic Adenovirus Competitively Consumes OncomiRs to Exert Antitumor Efficacy in Hepatocellular Carcinoma. Mol Cancer Ther 15: 1436-1451, 2016.

136. Liang J, Liu C, Qiao A, Cui Y, Zhang H, Cui A, Zhang S, Yang Y, Xiao X, Chen Y, Fang F, and Chang Y. MicroRNA-29a-c decrease fasting blood glucose levels by negatively regulating hepatic gluconeogenesis. J Hepatol 58: 535-542, 2013.

137. Liu L, Urch B, Szyszkowicz M, Evans G, Speck M, Van Huang A, Leingartner K, Shutt RH, Pelletier G, Gold DR, Brook JR, Godri Pollitt K, and Silverman FS. Metals and oxidative potential in urban particulate matter influence systemic inflammatory and neural biomarkers: A controlled exposure study. Environ Int 121: 1331-1340, 2018.

138. Liu L, Urch B, Szyszkowicz M, Speck M, Leingartner K, Shutt R, Pelletier G, Gold DR, Scott JA, Brook JR, Thorne PS, and Silverman FS. Influence of exposure to coarse, fine and ultrafine urban particulate matter and their biological constituents on neural biomarkers in a randomized controlled crossover study. Environ Int 101: 89-95, 2017.

139. Liu Z, Xie Z, Jones W, Pavlovicz RE, Liu S, Yu J, Li PK, Lin J, Fuchs JR, Marcucci G, Li C, and Chan KK. Curcumin is a potent DNA hypomethylation agent. Bioorg Med Chem Lett 19: 706-709, 2009.

140. Lopaschuk GD. Treating ischemic heart disease by pharmacologically improving cardiac energy metabolism. Am J Cardiol 82: 14K-17K, 1998.

141. Lopatin YM, Rosano GM, Fragasso G, Lopaschuk GD, Seferovic PM, Gowdak LH, Vinereanu D, Hamid MA, Jourdain P, and Ponikowski P. Rationale and benefits of trimetazidine by acting on cardiac metabolism in heart failure. Int J Cardiol 203: 909-915, 2016.

142. Lorenzo O, Ramirez E, Picatoste B, Egido J, and Tunon J. Alteration of energy substrates and ROS production in diabetic cardiomyopathy. Mediators Inflamm 2013: 461967, 2013.

143. Madrigano J, Baccarelli A, Mittleman MA, Wright RO, Sparrow D, Vokonas PS, Tarantini L, and Schwartz J. Prolonged exposure to particulate pollution, genes associated with glutathione pathways, and DNA methylation in a cohort of older men. Environ Health Perspect 119: 977-982, 2011.

144. Magoon R, Choudhury A, Malik V, Sharma R, and Kapoor PM. Pharmacological update: New drugs in cardiac practice: A critical appraisal. Ann Card Anaesth 20: S49-S56, 2017. 145. Mai AS, Dos Santos AB, Beber LCC, Basso RDB, Sulzbacher LM, Goettems-Fiorin PB, Frizzo MN, Rhoden CR, Ludwig MS, and Heck TG. Exercise Training under Exposure to Low Levels of Fine Particulate Matter: Effects on Heart Oxidative Stress and Extra-to-Intracellular HSP70 Ratio. Oxid Med Cell Longev 2017: 9067875, 2017.

146. Marsico A, Huska MR, Lasserre J, Hu H, Vucicevic D, Musahl A, Orom U, and Vingron M. PROmiRNA: a new miRNA promoter recognition method uncovers the complex regulation of intronic miRNAs. Genome Biol 14: R84, 2013. 
147. Mathiyalagan P, Okabe J, Chang L, Su Y, Du XJ, and El-Osta A. The primary microRNA-208b interacts with Polycomb-group protein, Ezh2, to regulate gene expression in the heart. Nucleic Acids Res 42: 790-803, 2014.

148. Matsumoto S, Sakata Y, Suna S, Nakatani D, Usami M, Hara M, Kitamura T, Hamasaki T, Nanto S, Kawahara Y, and Komuro I. Circulating p53-responsive microRNAs are predictive indicators of heart failure after acute myocardial infarction. Circ Res 113: 322-326, 2013.

149. Miyoshi K, Miyoshi T, and Siomi H. Many ways to generate microRNA-like small RNAs: non-canonical pathways for microRNA production. Mol Genet Genomics 284: 95-103, 2010 .

150. Montgomery RL, Hullinger TG, Semus HM, Dickinson BA, Seto AG, Lynch JM, Stack C, Latimer PA, Olson EN, and van Rooij E. Therapeutic inhibition of miR-208a improves cardiac function and survival during heart failure. Circulation 124: 1537-1547, 2011.

151. Morales-Rubio RA, Alvarado-Cruz I, Manzano-Leon N, Andrade-Oliva MD, UribeRamirez M, Quintanilla-Vega B, Osornio-Vargas A, and De Vizcaya-Ruiz A. In utero exposure to ultrafine particles promotes placental stress-induced programming of reninangiotensin system-related elements in the offspring results in altered blood pressure in adult mice. Part Fibre Toxicol 16: 7, 2019.

152. Mosher MJ, Melton PE, Stapleton P, Schanfield MS, and Crawford MH. Patterns of DNA Methylation Across the Leptin Core Promoter in Four Diverse Asian and North American Populations. Hum Biol 88: 121-135, 2016.

153. Mostafavi N, Vermeulen R, Ghantous A, Hoek G, Probst-Hensch N, Herceg Z, Tarallo S, Naccarati A, Kleinjans JCS, Imboden M, Jeong A, Morley D, Amaral AFS, van Nunen E, Gulliver J, Chadeau-Hyam M, Vineis $\mathbf{P}$, and Vlaanderen J. Acute changes in DNA methylation in relation to $24 \mathrm{~h}$ personal air pollution exposure measurements: A panel study in four European countries. Environ Int 120: 11-21, 2018.

154. Nazarenko Y, Zhen H, Han T, Lioy PJ, and Mainelis G. Nanomaterial inhalation exposure from nanotechnology-based cosmetic powders: a quantitative assessment. J Nanopart Res 14, 2012.

155. Nemmar A, Al-Salam S, Beegam S, Yuvaraju P, and Ali BH. Thrombosis and systemic and cardiac oxidative stress and DNA damage induced by pulmonary exposure to diesel exhaust particles and the effect of nootkatone thereon. Am J Physiol Heart Circ Physiol 314: H917-H927, 2018.

156. Noren Hooten N, Martin-Montalvo A, Dluzen DF, Zhang Y, Bernier M, Zonderman AB, Becker KG, Gorospe M, de Cabo R, and Evans MK. Metformin-mediated increase in DICER1 regulates microRNA expression and cellular senescence. Aging Cell 15: 572-581, 2016. 157. Organization WH. Ambient air pollution: A global assessment of exposure and burden of disease: WHO Press, 2016.

158. Pei Y, Jiang R, Zou Y, Wang Y, Zhang S, Wang G, Zhao J, and Song W. Effects of Fine Particulate Matter (PM2.5) on Systemic Oxidative Stress and Cardiac Function in ApoE(-/-) Mice. Int J Environ Res Public Health 13, 2016.

159. Pelsers MM, Lutgerink JT, Nieuwenhoven FA, Tandon NN, van der Vusse GJ, Arends JW, Hoogenboom HR, and Glatz JF. A sensitive immunoassay for rat fatty acid translocase (CD36) using phage antibodies selected on cell transfectants: abundant presence of fatty acid translocase/CD36 in cardiac and red skeletal muscle and up-regulation in diabetes. Biochem J 337 ( Pt 3): 407-414, 1999. 
160. Peng XP, Huang L, and Liu ZH. miRNA-133a attenuates lipid accumulation via TR4CD36 pathway in macrophages. Biochimie 127: 79-85, 2016.

161. Pheiffer C, Erasmus RT, Kengne AP, and Matsha TE. Differential DNA methylation of microRNAs within promoters, intergenic and intragenic regions of type 2 diabetic, pre-diabetic and non-diabetic individuals. Clin Biochem 49: 433-438, 2016.

162. Pinti MV, Hathaway QA, and Hollander JM. Role of microRNA in metabolic shift during heart failure. Am J Physiol Heart Circ Physiol 312: H33-H45, 2017.

163. Qin G, Xia J, Zhang Y, Guo L, Chen R, and Sang N. Ambient fine particulate matter exposure induces reversible cardiac dysfunction and fibrosis in juvenile and older female mice. Part Fibre Toxicol 15: 27, 2018.

164. Qiu Y, Yang J, Bian S, Chen G, and Yu J. PPARgamma suppresses the proliferation of cardiac myxoma cells through downregulation of MEF2D in a miR-122-dependent manner. Biochem Biophys Res Commun 474: 560-565, 2016.

165. Rahimi G, Jafari N, Khodabakhsh M, Shirzad Z, and Dogaheh HP. Upregulation of microRNA processing enzymes Drosha and Dicer in gestational diabetes mellitus. Gynecol Endocrinol 31: 156-159, 2015.

166. Rao X, Zhong J, Maiseyeu A, Gopalakrishnan B, Villamena FA, Chen LC, Harkema JR, Sun Q, and Rajagopalan S. CD36-dependent 7-ketocholesterol accumulation in macrophages mediates progression of atherosclerosis in response to chronic air pollution exposure. Circ Res 115: 770-780, 2014.

167. Raposo G and Stoorvogel W. Extracellular vesicles: exosomes, microvesicles, and friends. J Cell Biol 200: 373-383, 2013.

168. Raut SK, Singh GB, Rastogi B, Saikia UN, Mittal A, Dogra N, Singh S, Prasad R, and Khullar M. miR-30c and miR-181a synergistically modulate p53-p21 pathway in diabetes induced cardiac hypertrophy. Mol Cell Biochem 417: 191-203, 2016.

169. Renaud L, Harris LG, Mani SK, Kasiganesan H, Chou JC, Baicu CF, Van Laer A, Akerman AW, Stroud RE, Jones JA, Zile MR, and Menick DR. HDACs Regulate miR-133a Expression in Pressure Overload-Induced Cardiac Fibrosis. Circ Heart Fail 8: 1094-1104, 2015.

170. Riches K, Huntriss J, Keeble C, Wood IC, O'Regan DJ, Turner NA, and Porter KE. Mapping the methylation status of the miR-145 promoter in saphenous vein smooth muscle cells from individuals with type 2 diabetes. Diab Vasc Dis Res 14: 122-129, 2017.

171. Ruiz-Pesini E, Mishmar D, Brandon M, Procaccio V, and Wallace DC. Effects of purifying and adaptive selection on regional variation in human mtDNA. Science 303: 223-226, 2004.

172. Russo I and Frangogiannis NG. Diabetes-associated cardiac fibrosis: Cellular effectors, molecular mechanisms and therapeutic opportunities. J Mol Cell Cardiol 90: 84-93, 2016.

173. Rychlik KA, Secrest JR, Lau C, Pulczinski J, Zamora ML, Leal J, Langley R, Myatt LG, Raju M, Chang RC, Li Y, Golding MC, Rodrigues-Hoffmann A, Molina MJ, Zhang R, and Johnson NM. In utero ultrafine particulate matter exposure causes offspring pulmonary immunosuppression. Proc Natl Acad Sci U S A 116: 3443-3448, 2019.

174. Sahoo S and Emanueli C. Exosomes in Diabetic Cardiomyopathy: The Next-Generation Therapeutic Targets? Diabetes 65: 2829-2831, 2016.

175. Saito S, Thuc LC, Teshima Y, Nakada C, Nishio S, Kondo H, Fukui A, Abe I, Ebata Y, Saikawa T, Moriyama M, and Takahashi N. Glucose Fluctuations Aggravate Cardiac Susceptibility to Ischemia/Reperfusion Injury by Modulating MicroRNAs Expression. Circ J 80: 186-195, 2016. 
176. Salmena L, Poliseno L, Tay Y, Kats L, and Pandolfi PP. A ceRNA hypothesis: the Rosetta Stone of a hidden RNA language? Cell 146: 353-358, 2011.

177. Sancini G, Farina F, Battaglia C, Cifola I, Mangano E, Mantecca P, Camatini M, and Palestini P. Health risk assessment for air pollutants: alterations in lung and cardiac gene expression in mice exposed to Milano winter fine particulate matter (PM2.5). PLoS One 9: e109685, 2014.

178. Sato F, Tsuchiya S, Meltzer SJ, and Shimizu K. MicroRNAs and epigenetics. FEBS J 278: 1598-1609, 2011.

179. Savi M, Rossi S, Bocchi L, Gennaccaro L, Cacciani F, Perotti A, Amidani D, Alinovi R, Goldoni M, Aliatis I, Lottici PP, Bersani D, Campanini M, Pinelli S, Petyx M, Frati C, Gervasi A, Urbanek K, Quaini F, Buschini A, Stilli D, Rivetti C, Macchi E, Mutti A, Miragoli M, and Zaniboni M. Titanium dioxide nanoparticles promote arrhythmias via a direct interaction with rat cardiac tissue. Part Fibre Toxicol 11: 63, 2014.

180. Saxena A and Tabin CJ. miRNA-processing enzyme Dicer is necessary for cardiac outflow tract alignment and chamber septation. Proc Natl Acad Sci U S A 107: 87-91, 2010.

181. Schilling JD. The mitochondria in diabetic heart failure: from pathogenesis to therapeutic promise. Antioxid Redox Signal 22: 1515-1526, 2015.

182. Sebastiani G, Nigi L, Grieco GE, Mancarella F, Ventriglia G, and Dotta F. Circulating microRNAs and diabetes mellitus: a novel tool for disease prediction, diagnosis, and staging? $J$ Endocrinol Invest 40: 591-610, 2017.

183. Shan ZX, Lin QX, Deng CY, Zhu JN, Mai LP, Liu JL, Fu YH, Liu XY, Li YX, Zhang YY, Lin SG, and Yu XY. miR-1/miR-206 regulate Hsp60 expression contributing to glucosemediated apoptosis in cardiomyocytes. FEBS Lett 584: 3592-3600, 2010.

184. Sharma V, Dhillon P, Wambolt R, Parsons H, Brownsey R, Allard MF, and McNeill JH. Metoprolol improves cardiac function and modulates cardiac metabolism in the streptozotocin-diabetic rat. Am J Physiol Heart Circ Physiol 294: H1609-1620, 2008.

185. Shatseva T, Lee DY, Deng Z, and Yang BB. MicroRNA miR-199a-3p regulates cell proliferation and survival by targeting caveolin-2. J Cell Sci 124: 2826-2836, 2011.

186. Shepherd DL, Hathaway QA, Pinti MV, Nichols CE, Durr AJ, Sreekumar S, Hughes KM, Stine SM, Martinez I, and Hollander JM. Exploring the mitochondrial microRNA import pathway through Polynucleotide Phosphorylase (PNPase). J Mol Cell Cardiol 110: 15-25, 2017.

187. Shi R, Zhao L, Cai W, Wei M, Zhou X, Yang G, and Yuan L. Maternal exosomes in diabetes contribute to the cardiac development deficiency. Biochem Biophys Res Commun 483: 602-608, 2017.

188. Shinde S and Bhadra U. A complex genome-microRNA interplay in human mitochondria. Biomed Res Int 2015: 206382, 2015.

189. Singh MK, Lu MM, Massera D, and Epstein JA. MicroRNA-processing enzyme Dicer is required in epicardium for coronary vasculature development. J Biol Chem 286: 41036-41045, 2011.

190. Sinharay R, Gong J, Barratt B, Ohman-Strickland P, Ernst S, Kelly FJ, Zhang JJ, Collins P, Cullinan P, and Chung KF. Respiratory and cardiovascular responses to walking down a traffic-polluted road compared with walking in a traffic-free area in participants aged 60 years and older with chronic lung or heart disease and age-matched healthy controls: a randomised, crossover study. Lancet 391: 339-349, 2018. 
191. Slezakova K, Peixoto C, Oliveira M, Delerue-Matos C, Pereira MDC, and Morais S. Indoor particulate pollution in fitness centres with emphasis on ultrafine particles. Environ Pollut 233: 180-193, 2018.

192. Small EM and Olson EN. Pervasive roles of microRNAs in cardiovascular biology. Nature 469: 336-342, 2011.

193. Sokolova V, Ludwig AK, Hornung S, Rotan O, Horn PA, Epple M, and Giebel B. Characterisation of exosomes derived from human cells by nanoparticle tracking analysis and scanning electron microscopy. Colloids Surf B Biointerfaces 87: 146-150, 2011.

194. Squadrito ML, Baer C, Burdet F, Maderna C, Gilfillan GD, Lyle R, Ibberson M, and De Palma M. Endogenous RNAs modulate microRNA sorting to exosomes and transfer to acceptor cells. Cell Rep 8: 1432-1446, 2014.

195. Sripada L, Singh K, Lipatova AV, Singh A, Prajapati P, Tomar D, Bhatelia K, Roy M, Singh R, Godbole MM, Chumakov PM, and Singh R. hsa-miR-4485 regulates mitochondrial functions and inhibits the tumorigenicity of breast cancer cells. J Mol Med (Berl) 95: 641-651, 2017.

196. Sripada L, Tomar D, and Singh R. Mitochondria: one of the destinations of miRNAs. Mitochondrion 12: 593-599, 2012.

197. Stanley WC, Lopaschuk GD, and McCormack JG. Regulation of energy substrate metabolism in the diabetic heart. Cardiovasc Res 34: 25-33, 1997.

198. Stapleton PA, Hathaway QA, Nichols CE, Abukabda AB, Pinti MV, Shepherd DL, McBride CR, Yi J, Castranova VC, Hollander JM, and Nurkiewicz TR. Maternal engineered nanomaterial inhalation during gestation alters the fetal transcriptome. Part Fibre Toxicol 15: 3, 2018.

199. Stapleton PA, Nichols CE, Yi J, McBride CR, Minarchick VC, Shepherd DL, Hollander JM, and Nurkiewicz TR. Microvascular and mitochondrial dysfunction in the female F1 generation after gestational TiO2 nanoparticle exposure. Nanotoxicology 9: 941-951, 2015.

200. Stewart JC, Chalupa DC, Devlin RB, Frasier LM, Huang LS, Little EL, Lee SM, Phipps RP, Pietropaoli AP, Taubman MB, Utell MJ, and Frampton MW. Vascular effects of ultrafine particles in persons with type 2 diabetes. Environ Health Perspect 118: 1692-1698, 2010. 201. Sturm R. Local lung deposition of ultrafine particles in healthy adults: experimental results and theoretical predictions. Ann Transl Med 4: 420, 2016.

202. Sukma Dewi I, Celik S, Karlsson A, Hollander Z, Lam K, McManus JW, Tebbutt S, Ng R, Keown P, McMaster R, McManus B, Ohman J, and Gidlof O. Exosomal miR-142-3p is increased during cardiac allograft rejection and augments vascular permeability through downregulation of endothelial RAB11FIP2 expression. Cardiovasc Res 113: 440-452, 2017.

203. Sun K, Chang X, Yin L, Li J, Zhou T, Zhang C, and Chen X. Expression and DNA methylation status of microRNA-375 in patients with type 2 diabetes mellitus. Mol Med Rep 9: 967-972, 2014.

204. Sun M, Estrov Z, Ji Y, Coombes KR, Harris DH, and Kurzrock R. Curcumin (diferuloylmethane) alters the expression profiles of microRNAs in human pancreatic cancer cells. Mol Cancer Ther 7: 464-473, 2008.

205. Sun Q, Hong X, and Wold LE. Cardiovascular effects of ambient particulate air pollution exposure. Circulation 121: 2755-2765, 2010.

206. Sun Q, Wang A, Jin X, Natanzon A, Duquaine D, Brook RD, Aguinaldo JG, Fayad ZA, Fuster V, Lippmann M, Chen LC, and Rajagopalan S. Long-term air pollution exposure 
and acceleration of atherosclerosis and vascular inflammation in an animal model. JAMA 294: 3003-3010, 2005.

207. Sun Q, Yue P, Kirk RI, Wang A, Moatti D, Jin X, Lu B, Schecter AD, Lippmann M, Gordon T, Chen LC, and Rajagopalan S. Ambient air particulate matter exposure and tissue factor expression in atherosclerosis. Inhal Toxicol 20: 127-137, 2008.

208. Taegtmeyer H. Cardiac metabolism as a target for the treatment of heart failure. Circulation 110: 894-896, 2004.

209. Tang N, Sun B, Gupta A, Rempel H, and Pulliam L. Monocyte exosomes induce adhesion molecules and cytokines via activation of NF-kappaB in endothelial cells. FASEB J 30: 3097-3106, 2016.

210. Tanwar V, Adelstein JM, Grimmer JA, Youtz DJ, Katapadi A, Sugar BP, Falvo MJ, Baer LA, Stanford KI, and Wold LE. Preconception Exposure to Fine Particulate Matter Leads to Cardiac Dysfunction in Adult Male Offspring. J Am Heart Assoc 7: e010797, 2018.

211. Tanwar V, Adelstein JM, Grimmer JA, Youtz DJ, Sugar BP, and Wold LE. PM2.5 exposure in utero contributes to neonatal cardiac dysfunction in mice. Environ Pollut 230: 116124, 2017.

212. Tanwar V, Gorr MW, Velten M, Eichenseer CM, Long VP, 3rd, Bonilla IM, Shettigar V, Ziolo MT, Davis JP, Baine SH, Carnes CA, and Wold LE. In Utero Particulate Matter Exposure Produces Heart Failure, Electrical Remodeling, and Epigenetic Changes at Adulthood. J Am Heart Assoc 6, 2017.

213. Thery C, Zitvogel L, and Amigorena S. Exosomes: composition, biogenesis and function. Nat Rev Immunol 2: 569-579, 2002.

214. Thompson LC, Walsh L, Martin BL, McGee J, Wood C, Kovalcik K, Pancras JP, Haykal-Coates N, Ledbetter AD, Davies D, Cascio WE, Higuchi M, Hazari MS, and Farraj AK. Ambient Particulate Matter and Acrolein Co-Exposure Increases Myocardial Dyssynchrony in Mice via TRPA1. Toxicol Sci 167: 559-572, 2019.

215. Thum T, Gross C, Fiedler J, Fischer T, Kissler S, Bussen M, Galuppo P, Just S, Rottbauer W, Frantz S, Castoldi M, Soutschek J, Koteliansky V, Rosenwald A, Basson MA, Licht JD, Pena JT, Rouhanifard SH, Muckenthaler MU, Tuschl T, Martin GR, Bauersachs J, and Engelhardt S. MicroRNA-21 contributes to myocardial disease by stimulating MAP kinase signalling in fibroblasts. Nature 456: 980-984, 2008.

216. Tobaldini E, Bollati V, Prado M, Fiorelli EM, Pecis M, Bissolotti G, Albetti B, Cantone L, Favero C, Cogliati C, Carrer P, Baccarelli A, Bertazzi PA, and Montano N. Acute particulate matter affects cardiovascular autonomic modulation and IFN-gamma methylation in healthy volunteers. Environ Res 161: 97-103, 2018.

217. Togliatto G, Trombetta A, Dentelli P, Gallo S, Rosso A, Cotogni P, Granata R, Falcioni R, Delale T, Ghigo E, and Brizzi MF. Unacylated ghrelin induces oxidative stress resistance in a glucose intolerance and peripheral artery disease mouse model by restoring endothelial cell miR-126 expression. Diabetes 64: 1370-1382, 2015.

218. Trajkovic K, Hsu C, Chiantia S, Rajendran L, Wenzel D, Wieland F, Schwille P, Brugger B, and Simons M. Ceramide triggers budding of exosome vesicles into multivesicular endosomes. Science 319: 1244-1247, 2008.

219. Tsang WP and Kwok TT. Epigallocatechin gallate up-regulation of miR-16 and induction of apoptosis in human cancer cells. J Nutr Biochem 21: 140-146, 2010. 
220. Upadhyay S, Stoeger T, George L, Schladweiler MC, Kodavanti U, Ganguly K, and Schulz H. Ultrafine carbon particle mediated cardiovascular impairment of aged spontaneously hypertensive rats. Part Fibre Toxicol 11: 36, 2014.

221. Valadi H, Ekstrom K, Bossios A, Sjostrand M, Lee JJ, and Lotvall JO. Exosomemediated transfer of mRNAs and microRNAs is a novel mechanism of genetic exchange between cells. Nat Cell Biol 9: 654-659, 2007.

222. van den Beucken T, Koch E, Chu K, Rupaimoole R, Prickaerts P, Adriaens M, Voncken JW, Harris AL, Buffa FM, Haider S, Starmans MHW, Yao CQ, Ivan M, Ivan C, Pecot CV, Boutros PC, Sood AK, Koritzinsky M, and Wouters BG. Hypoxia promotes stem cell phenotypes and poor prognosis through epigenetic regulation of DICER. Nat Commun 5: 5203, 2014.

223. van der Zwaag PA, Jongbloed JD, van den Berg MP, van der Smagt JJ, Jongbloed R, Bikker H, Hofstra RM, and van Tintelen JP. A genetic variants database for arrhythmogenic right ventricular dysplasia/cardiomyopathy. Hum Mutat 30: 1278-1283, 2009.

224. Viehmann A, Hertel S, Fuks K, Eisele L, Moebus S, Mohlenkamp S, Nonnemacher M, Jakobs H, Erbel R, Jockel KH, Hoffmann B, and Heinz Nixdorf Recall Investigator G. Long-term residential exposure to urban air pollution, and repeated measures of systemic blood markers of inflammation and coagulation. Occup Environ Med 72: 656-663, 2015.

225. Vikram A, Kim YR, Kumar S, Li Q, Kassan M, Jacobs JS, and Irani K. Vascular microRNA-204 is remotely governed by the microbiome and impairs endothelium-dependent vasorelaxation by downregulating Sirtuin1. Nat Commun 7: 12565, 2016.

226. Villarreal-Calderon R, Franco-Lira M, Gonzalez-Maciel A, Reynoso-Robles R, Harritt L, Perez-Guille B, Ferreira-Azevedo L, Drecktrah D, Zhu H, Sun Q, Torres-Jardon R, Aragon-Flores M, Calderon-Garciduenas A, Diaz P, and Calderon-Garciduenas L. Upregulation of mRNA ventricular PRNP prion protein gene expression in air pollution highly exposed young urbanites: endoplasmic reticulum stress, glucose regulated protein 78, and nanosized particles. Int J Mol Sci 14: 23471-23491, 2013.

227. Villeneuve LM, Kato M, Reddy MA, Wang M, Lanting L, and Natarajan R. Enhanced levels of microRNA-125b in vascular smooth muscle cells of diabetic $\mathrm{db} / \mathrm{db}$ mice lead to increased inflammatory gene expression by targeting the histone methyltransferase Suv39h1. Diabetes 59: 2904-2915, 2010.

228. Vora R, Zareba W, Utell MJ, Pietropaoli AP, Chalupa D, Little EL, Oakes D, Bausch J, Wiltshire J, and Frampton MW. Inhalation of ultrafine carbon particles alters heart rate and heart rate variability in people with type 2 diabetes. Part Fibre Toxicol 11: 31, 2014.

229. Wahl S, Drong A, Lehne B, Loh M, Scott WR, Kunze S, Tsai PC, Ried JS, Zhang W, Yang Y, Tan S, Fiorito G, Franke L, Guarrera S, Kasela S, Kriebel J, Richmond RC, Adamo M, Afzal U, Ala-Korpela M, Albetti B, Ammerpohl O, Apperley JF, Beekman M, Bertazzi PA, Black SL, Blancher C, Bonder MJ, Brosch M, Carstensen-Kirberg M, de Craen AJ, de Lusignan S, Dehghan A, Elkalaawy M, Fischer K, Franco OH, Gaunt TR, Hampe J, Hashemi M, Isaacs A, Jenkinson A, Jha S, Kato N, Krogh V, Laffan M, Meisinger C, Meitinger T, Mok ZY, Motta V, Ng HK, Nikolakopoulou Z, Nteliopoulos G, Panico S, Pervjakova N, Prokisch H, Rathmann W, Roden M, Rota F, Rozario MA, Sandling JK, Schafmayer C, Schramm K, Siebert R, Slagboom PE, Soininen P, Stolk L, Strauch K, Tai ES, Tarantini L, Thorand B, Tigchelaar EF, Tumino R, Uitterlinden AG, van Duijn C, van Meurs JB, Vineis P, Wickremasinghe AR, Wijmenga C, Yang TP, Yuan W, Zhernakova A, Batterham RL, Smith GD, Deloukas P, Heijmans BT, Herder C, Hofman A, Lindgren CM, Milani L, van 
der Harst P, Peters A, Illig T, Relton CL, Waldenberger M, Jarvelin MR, Bollati V, Soong R, Spector TD, Scott J, McCarthy MI, et al. Epigenome-wide association study of body mass index, and the adverse outcomes of adiposity. Nature 541: 81-86, 2017.

230. Wan S, Wang J, Wang J, Wu J, Song J, Zhang CY, Zhang C, Wang C, and Wang JJ. Increased serum miR-7 is a promising biomarker for type 2 diabetes mellitus and its microvascular complications. Diabetes Res Clin Pract 130: 171-179, 2017.

231. Wang C, Zhang C, Liu L, A X, Chen B, Li Y, and Du J. Macrophage-Derived mir-155Containing Exosomes Suppress Fibroblast Proliferation and Promote Fibroblast Inflammation during Cardiac Injury. Mol Ther 25: 192-204, 2017.

232. Wang G, Chen HW, Oktay Y, Zhang J, Allen EL, Smith GM, Fan KC, Hong JS, French SW, McCaffery JM, Lightowlers RN, Morse HC, 3rd, Koehler CM, and Teitell MA. PNPASE regulates RNA import into mitochondria. Cell 142: 456-467, 2010.

233. Wang G, Shimada E, Koehler CM, and Teitell MA. PNPASE and RNA trafficking into mitochondria. Biochim Biophys Acta 1819: 998-1007, 2012.

234. Wang H, Peng X, Cao F, Wang Y, Shi H, Lin S, Zhong W, and Sun J. Cardiotoxicity and Mechanism of Particulate Matter 2.5 (PM2.5) Exposure in Offspring Rats During Pregnancy. Med Sci Monit 23: 3890-3896, 2017.

235. Wang H, Shen X, Tian G, Shi X, Huang W, Wu Y, Sun L, Peng C, Liu S, Huang Y, Chen X, Zhang F, Chen Y, Ding W, and Lu Z. AMPKalpha2 deficiency exacerbates long-term PM2.5 exposure-induced lung injury and cardiac dysfunction. Free Radic Biol Med 121: 202-214, 2018.

236. Wang K, Liu CY, Zhou LY, Wang JX, Wang M, Zhao B, Zhao WK, Xu SJ, Fan LH, Zhang XJ, Feng C, Wang CQ, Zhao YF, and Li PF. APF lncRNA regulates autophagy and myocardial infarction by targeting miR-188-3p. Nat Commun 6: 6779, 2015.

237. Wang K, Liu F, Zhou LY, Long B, Yuan SM, Wang Y, Liu CY, Sun T, Zhang XJ, and Li PF. The long noncoding RNA CHRF regulates cardiac hypertrophy by targeting miR-489. Circ Res 114: 1377-1388, 2014.

238. Wang K, Long B, Zhou LY, Liu F, Zhou QY, Liu CY, Fan YY, and Li PF. CARL lncRNA inhibits anoxia-induced mitochondrial fission and apoptosis in cardiomyocytes by impairing miR-539-dependent PHB2 downregulation. Nat Commun 5: 3596, 2014.

239. Wang P, You D, Saravia J, Shen H, and Cormier SA. Maternal exposure to combustion generated PM inhibits pulmonary Th1 maturation and concomitantly enhances postnatal asthma development in offspring. Part Fibre Toxicol 10: 29, 2013.

240. Wang Q, Gan X, Li F, Chen Y, Fu W, Zhu X, Xu D, Long M, and Xu D. PM2.5 Exposure Induces More Serious Apoptosis of Cardiomyocytes Mediated by Caspase3 through JNK/ P53 Pathway in Hyperlipidemic Rats. Int J Biol Sci 15: 24-33, 2019.

241. Wang X, Huang W, Liu G, Cai W, Millard RW, Wang Y, Chang J, Peng T, and Fan GC. Cardiomyocytes mediate anti-angiogenesis in type 2 diabetic rats through the exosomal transfer of miR-320 into endothelial cells. J Mol Cell Cardiol 74: 139-150, 2014.

242. Winter J, Jung S, Keller S, Gregory RI, and Diederichs S. Many roads to maturity: microRNA biogenesis pathways and their regulation. Nat Cell Biol 11: 228-234, 2009.

243. Wittkopp S, Staimer N, Tjoa T, Gillen D, Daher N, Shafer M, Schauer JJ, Sioutas C, and Delfino RJ. Mitochondrial genetic background modifies the relationship between trafficrelated air pollution exposure and systemic biomarkers of inflammation. PLoS One 8: e64444, 2013. 
244. Wu F and Zhang J. The involvement of Nox4 in fine particulate matter exposure-induced cardiac injury in mice. J Toxicol Sci 43: 171-181, 2018.

245. Wu X, Pan B, Liu L, Zhao W, Zhu J, Huang X, and Tian J. In utero exposure to PM2.5 during gestation caused adult cardiac hypertrophy through histone acetylation modification. $J$ Cell Biochem 120: 4375-4384, 2019.

246. Xiao J, Pan Y, Li XH, Yang XY, Feng YL, Tan HH, Jiang L, Feng J, and Yu XY. Cardiac progenitor cell-derived exosomes prevent cardiomyocytes apoptosis through exosomal miR-21 by targeting PDCD4. Cell Death Dis 7: e2277, 2016.

247. Xing YF, Xu YH, Shi MH, and Lian YX. The impact of PM2.5 on the human respiratory system. J Thorac Dis 8: E69-74, 2016.

248. Xu H, Liu C, Rao S, He L, Zhang T, Sun S, Wu B, Zou L, Wang S, Xue Y, Jia T, Zhao S, Li G, Liu S, Li G, and Liang S. LncRNA NONRATT021972 siRNA rescued decreased heart rate variability in diabetic rats in superior cervical ganglia. Auton Neurosci 201: 1-7, 2016.

249. Yang X, Feng L, Zhang Y, Hu H, Shi Y, Liang S, Zhao T, Cao L, Duan J, and Sun Z. Co-exposure of silica nanoparticles and methylmercury induced cardiac toxicity in vitro and in vivo. Sci Total Environ 631-632: 811-821, 2018.

250. Yang X, Feng L, Zhang Y, Hu H, Shi Y, Liang S, Zhao T, Fu Y, Duan J, and Sun Z. Cytotoxicity induced by fine particulate matter (PM2.5) via mitochondria-mediated apoptosis pathway in human cardiomyocytes. Ecotoxicol Environ Saf 161: 198-207, 2018.

251. Yang X, Feng L, Zhang Y, Shi Y, Liang S, Zhao T, Sun B, Duan J, and Sun Z. Integrative analysis of methylome and transcriptome variation of identified cardiac diseasespecific genes in human cardiomyocytes after PM2.5 exposure. Chemosphere 212: 915-926, 2018. 252. Yang Y, Li Y, Chen X, Cheng X, Liao Y, and Yu X. Exosomal transfer of miR-30a between cardiomyocytes regulates autophagy after hypoxia. J Mol Med (Berl) 94: 711-724, 2016. 253. Yang ZG, Awan FM, Du WW, Zeng Y, Lyu J, Wu, Gupta S, Yang W, and Yang BB. The Circular RNA Interacts with STAT3, Increasing Its Nuclear Translocation and Wound Repair by Modulating Dnmt3a and miR-17 Function. Mol Ther, 2017.

254. Yi R, Qin Y, Macara IG, and Cullen BR. Exportin-5 mediates the nuclear export of premicroRNAs and short hairpin RNAs. Genes Dev 17: 3011-3016, 2003.

255. Yildirim SS, Akman D, Catalucci D, and Turan B. Relationship between downregulation of miRNAs and increase of oxidative stress in the development of diabetic cardiac dysfunction: junctin as a target protein of miR-1. Cell Biochem Biophys 67: 1397-1408, 2013.

256. Yoshimura A, Sawada K, Nakamura K, Kinose Y, Nakatsuka E, Kobayashi M, Miyamoto M, Ishida K, Matsumoto Y, Kodama M, Hashimoto K, Mabuchi S, and Kimura T. Exosomal miR-99a-5p is elevated in sera of ovarian cancer patients and promotes cancer cell invasion by increasing fibronectin and vitronectin expression in neighboring peritoneal mesothelial cells. BMC Cancer 18: 1065, 2018.

257. Yu J, Nagasu H, Murakami T, Hoang H, Broderick L, Hoffman HM, and Horng T. Inflammasome activation leads to Caspase-1-dependent mitochondrial damage and block of mitophagy. Proc Natl Acad Sci US A 111: 15514-15519, 2014.

258. Yu XY, Song YH, Geng YJ, Lin QX, Shan ZX, Lin SG, and Li Y. Glucose induces apoptosis of cardiomyocytes via microRNA-1 and IGF-1. Biochem Biophys Res Commun 376: 548-552, 2008.

259. Yuan D, Zhao Y, Banks WA, Bullock KM, Haney M, Batrakova E, and Kabanov AV. Macrophage exosomes as natural nanocarriers for protein delivery to inflamed brain. Biomaterials 142: 1-12, 2017. 
260. Zampetaki A, Kiechl S, Drozdov I, Willeit P, Mayr U, Prokopi M, Mayr A, Weger S, Oberhollenzer F, Bonora E, Shah A, Willeit J, and Mayr M. Plasma microRNA profiling reveals loss of endothelial miR-126 and other microRNAs in type 2 diabetes. Circ Res 107: 810$817,2010$.

261. Zeng X, Liu J, Du X, Zhang J, Pan K, Shan W, Xie Y, Song W, and Zhao J. The protective effects of selenium supplementation on ambient PM2.5-induced cardiovascular injury in rats. Environ Sci Pollut Res Int 25: 22153-22162, 2018.

262. Zhang J, Zhang T, Ti X, Shi J, Wu C, Ren X, and Yin H. Curcumin promotes apoptosis in A549/DDP multidrug-resistant human lung adenocarcinoma cells through an miRNA signaling pathway. Biochem Biophys Res Commun 399: 1-6, 2010.

263. Zhang X, Chen Y, Wei H, Qin Y, Hao Y, Zhu Y, Deng F, and Guo X. Ultrafine carbon black attenuates the antihypertensive effect of captopril in spontaneously hypertensive rats. Inhal Toxicol 26: 853-860, 2014.

264. Zhang $\mathbf{X}$ and Schulze PC. MicroRNAs in heart failure: Non-coding regulators of metabolic function. Biochim Biophys Acta 1862: 2276-2287, 2016.

265. Zhang X, Zuo X, Yang B, Li Z, Xue Y, Zhou Y, Huang J, Zhao X, Zhou J, Yan Y, Zhang H, Guo P, Sun H, Guo L, Zhang Y, and Fu XD. MicroRNA directly enhances mitochondrial translation during muscle differentiation. Cell 158: 607-619, 2014.

266. Zhang Y, Ji X, Ku T, and Sang N. Inflammatory response and endothelial dysfunction in the hearts of mice co-exposed to SO2, NO2, and PM2.5. Environ Toxicol 31: 1996-2005, 2016.

267. Zhao F, Li B, Wei YZ, Zhou B, Wang H, Chen M, Gan XD, Wang ZH, and Xiong SX. MicroRNA-34a regulates high glucose-induced apoptosis in H9c2 cardiomyocytes. J Huazhong Univ Sci Technolog Med Sci 33: 834-839, 2013.

268. Zhao J, Liu C, Bai Y, Wang TY, Kan H, and Sun Q. IKK inhibition prevents PM2.5exacerbated cardiac injury in mice with type 2 diabetes. J Environ Sci (China) 31: 98-103, 2015.

269. Zhao L, Zhang L, Chen M, Dong C, Li R, and Cai Z. Effects of Ambient Atmospheric PM2.5, 1-Nitropyrene and 9-Nitroanthracene on DNA Damage and Oxidative Stress in Hearts of Rats. Cardiovasc Toxicol, 2018.

270. Zhao Y, Ponnusamy M, Dong Y, Zhang L, Wang K, and Li P. Effects of miRNAs on myocardial apoptosis by modulating mitochondria related proteins. Clin Exp Pharmacol Physiol 44: 431-440, 2017.

271. Zheng D, Ma J, Yu Y, Li M, Ni R, Wang G, Chen R, Li J, Fan GC, Lacefield JC, and Peng T. Silencing of miR-195 reduces diabetic cardiomyopathy in C57BL/6 mice. Diabetologia 58: 1949-1958, 2015.

272. Zhong J, Colicino E, Lin X, Mehta A, Kloog I, Zanobetti A, Byun HM, Bind MA, Cantone L, Prada D, Tarantini L, Trevisi L, Sparrow D, Vokonas P, Schwartz J, and Baccarelli AA. Cardiac autonomic dysfunction: particulate air pollution effects are modulated by epigenetic immunoregulation of Toll-like receptor 2 and dietary flavonoid intake. $J$ Am Heart Assoc 4: e001423, 2015.

273. Zhou Q, Lv D, Chen P, Xu T, Fu S, Li J, and Bei Y. MicroRNAs in diabetic cardiomyopathy and clinical perspectives. Front Genet 5: 185, 2014.

274. Zhou VW, Goren A, and Bernstein BE. Charting histone modifications and the functional organization of mammalian genomes. Nat Rev Genet 12: 7-18, 2011.

275. Zhu H, Shyh-Chang N, Segre AV, Shinoda G, Shah SP, Einhorn WS, Takeuchi A, Engreitz JM, Hagan JP, Kharas MG, Urbach A, Thornton JE, Triboulet R, Gregory RI, 
Consortium D, Investigators M, Altshuler D, and Daley GQ. The Lin28/let-7 axis regulates glucose metabolism. Cell 147: 81-94, 2011. 


\section{Chapter 2: Specific Aim 1}

\section{MiRNA-378a as a Key Regulator of Cardiovascular Health Following Engineered Nanomaterial Inhalation Exposure}

Quincy A. Hathaway ${ }^{1,2,3}$, Andrya J. Durr ${ }^{1,2}$, Danielle L. Shepherd ${ }^{1,2}$, Mark V. Pinti ${ }^{1,2}$, Ashley N. Brandebura, ${ }^{4,5}$ Cody E. Nichols ${ }^{6}$, Amina Kunovac ${ }^{1,2}$, William T. Goldsmith ${ }^{3,7}$, Sherri A. Friend ${ }^{8}$, Alaeddin B. Abukabda ${ }^{3,7}$, Garrett K. Fink ${ }^{1}$, Timothy R. Nurkiewicz ${ }^{3,7}$, and John M. Hollander ${ }^{1,2}$

As published in Nanotoxicology. 2019 Feb 1:1-20. doi: 10.1080/17435390.2019.1570372. [Epub ahead of print]

${ }^{1}$ Division of Exercise Physiology, West Virginia University School of Medicine, Morgantown, WV, USA. ${ }^{2}$ Mitochondria, Metabolism \& Bioenergetics Working Group, West Virginia University School of Medicine, Morgantown, WV, USA. ${ }^{3}$ Toxicology Working Group, West Virginia University School of Medicine, Morgantown, WV, USA. ${ }^{4}$ Rockefeller Neuroscience Institute, West Virginia University School of Medicine, Morgantown, WV, USA. ${ }^{5}$ Department of Biochemistry, West Virginia University School of Medicine, Morgantown, WV, USA. ${ }^{6}$ Immunity, Inflammation, and Disease Laboratory, National Institute of Environmental Health Sciences, Research Triangle Park, NC, USA. ${ }^{7}$ Department of Physiology, Pharmacology \& Neuroscience, Morgantown, WV, USA, ${ }^{8}$ National Institute for Occupational Safety and Health, Morgantown, WV, USA

Running Title: MiRNA-378a and ENM exposure

Keywords: Cardiovascular, Mitochondria, Inhalation Exposure 


\begin{abstract}
Nano-titanium dioxide (nano- $\mathrm{TiO}_{2}$ ), though one of the most utilized and produced engineered nanomaterials (ENMs), diminishes cardiovascular function through dysregulation of metabolism and mitochondrial bioenergetics following inhalation exposure. The molecular mechanisms governing this cardiac dysfunction remain largely unknown. The purpose of this study was to elucidate molecular mediators that connect nano- $\mathrm{TiO}_{2}$ exposure with impaired cardiac function. Specifically, we were interested in the role of microRNA (miRNA) expression in the resulting dysfunction. Not only are miRNA global regulators of gene expression, but miRNA-based therapeutics provide a realistic treatment modality. Wild type and MiRNA-378a knockout mice were exposed to nano- $\mathrm{TiO}_{2}$ with an aerodynamic diameter of $182 \pm 1.70 \mathrm{~nm}$ and a mass concentration of $11.09 \mathrm{mg} / \mathrm{m}^{3}$ for four hours. Cardiac function, utilizing the Vevo 2100 Imaging System, electron transport chain complex activities, and mitochondrial respiration assessed cardiac and mitochondrial function. Immunoblotting and qPCR examined molecular targets of miRNA378a. MiRNA-378a-3p expression was increased 48 hours post inhalation exposure to nano- $\mathrm{TiO}_{2}$. Knockout of miRNA-378a preserved cardiac function following exposure as revealed by preserved E/A ratio and E/SR ratio. In knockout animals, complex I, III, and IV activities ( 2-6 fold) and fatty acid respiration ( $\sim 5$ fold) were significantly increased. MiRNA-378a regulated proteins involved in mitochondrial fusion, transcription, and fatty acid metabolism. MiRNA-378a-3p acts as a negative regulator of mitochondrial metabolic and biogenesis pathways. MiRNA-378a knockout animals provide a protective effect against nano- $\mathrm{TiO}_{2}$ inhalation exposure by altering mitochondrial structure and function. This is the first study to manipulate a miRNA to attenuate the effects of ENM exposure.
\end{abstract}




\section{Introduction}

The advent and advancement of clinical applications continues to drive utilization of engineered nanomaterials $(\mathrm{ENMs})$ in consumer goods $(5,19)$. As such, titanium dioxide $\left(\mathrm{TiO}_{2}\right)$ has become one of the most prolifically implemented ENMs $(25,32)$. While the beneficial manufacturing properties of ENMs are clear, the rate of incorporation in new products and devices outpaces our understanding of the toxicology of these materials. Though progress has been made outlining the consequences on organ systems and the physiological impact of ENM exposure, the molecular mechanisms governing their toxicity are poorly defined. Understanding how to manage the untoward consequences of ENM inhalation exposure therapeutically involves the exploitation of molecular pathways central to the etiology.

Systemic health effects following ENM exposure are linked to mitochondrial dysfunction, not only in the lungs (58) but also in the cardiovascular system $(29,52,69)$. The heart has been established as a significant contributor to the overall pathophysiological outcomes elicited by ENM exposure $(33,38,45,68)$. Cardiac mitochondrial function is crucial in maintaining/restoring cardiac homeostasis after ENM insult, as it is a central determinant of metabolic and bioenergetic capacity. Recently, particulate matter $\left(\mathrm{PM}_{2.5}\right)$ air pollution has been shown to alter microRNA (miRNA) expression in the serum of a human cohort in Shanghai, China (13). The correlation between serum miRNA expression and inflammatory cytokines involved in cardiovascular health reveals the clinical relevance of miRNAs in shaping the effects of inhalation exposure. Because miRNAs can influence numerous biological target axes, therapeutic interventions targeting their regulation offer distinct advantages to approaches designed to restore a single molecular target as they provide the potential for a coordinated response to pathological insults at numerous loci.

MiRNAs are transcribed as pri-miRNA species within the nucleus (processed through Drosha) and travel to the cytoplasm as pre-miRNA (processed through Dicer) where they eventually assume their mature miRNA conformation (64). The mature, miRNA species ( $22 \mathrm{nt})$ that originate in both intragenic and intergenic regions of the genome are known to individually function in regulating multiple genetic and epigenetic pathways (60). The manipulation of a miRNA that controls metabolic and mitochondrial function provides a potentially novel therapeutic strategy for controlling cellular insults precipitated by ENM exposure. In the heart, and other mitochondrially dense tissue, peroxisome proliferator-activated receptor gamma coactivator 
1-beta (Ppargc1b or PGC-1 $\beta$ ) is highly expressed (66), and is central in the control of metabolism (11). MiRNA-378a, which encodes both the $3 p$ and $5 p$ mature variants, resides within the first intron of the PGC- $1 \beta$ gene.

MiRNA-378a is involved in regulating apoptosis (39), insulin sensitivity (40), angiogenesis (34), metabolism (11), and other cellular processes $(23,73)$ implicating it in the coordination of cellular responses. Though multiple miRNAs regulate heart function $(18,22)$, the involvement of miRNA-378a with shifting substrate metabolism and regulation of mitochondrial bioenergetics (35) provides an intimate, and novel, connection between its expression and mitochondrial health in the heart. Carrer et al. reported on the propensity of miRNA-378a knockout mice to protect against metabolic insult (11). These researchers found that a high-fat diet induced obesity in wild type mice, but not miRNA-378a knockout mice, suggesting miRNA-378a may repress metabolic genes in response to environmental stimuli. The role of miRNA-378a in regulating metabolism and its high abundance in cardiac tissue (42) indicates that this small RNA may represent a therapeutic target for attenuation of metabolic disturbances and mitochondrial dysfunction resulting from pathological insult.

The objective of the current study was to determine whether manipulation of miRNA-378a expression could alter adverse cardiovascular and metabolic outcomes associated with ENM inhalation exposure. Our findings indicate that knockout, and knockdown to a lesser extent, of miRNA-378a improves cardiac and mitochondrial function by altering global bioenergetic and respiration capacity following nano- $\mathrm{TiO}_{2}$ inhalation exposure. This is the first study to determine the impact of altered miRNA expression on the response to ENM exposure in vivo. 


\section{Materials and Methods}

\section{MiRNA-378a Knockout Mouse Model}

The West Virginia University Animal Care and Use Committee approved all animal studies, which conformed to the most current National Institutes of Health (NIH) Guidelines for the Care and Use of Laboratory Animals manual. Specifics about the animal model have been previously published (11). Briefly, miRNA-378a is located within the first intron of the PGC-1 $\beta$ gene (Figure S2.1). Removal of the miRNA-378a loci was achieved by replacing the region with a neomycin cassette and LoxP sites. The recombination removed the miRNA-378a transcribed region but retained PGC-1 $\beta$ gene function. MiRNA-378a animals have been shown to be more metabolically active, specifically through mitochondrial fatty acid oxidation (11). Sperm, which was cryopreserved from the miRNA-378a knockout 129SvEv/C57BL/6 mouse line, was used for in vitro fertilization of FVB mice. The progeny heterozygous miRNA-378a FVB mice were bred for greater than 10 generations, establishing the congenic line.

\section{Experimental Model}

Mice were housed in the West Virginia University Health Sciences Center Animal Facility and given access to a rodent diet and water ad libitum. To verify presence of the knockout allele, traditional PCR was performed amplifying the first intronic region of the PGC- $1 \beta$ gene (Table $\mathrm{S} 2.1$ ). The WT allele amplifies a $400 \mathrm{bp}$ product while the transgenic allele amplifies a $470 \mathrm{bp}$ product. Between 14-18 weeks of age, mice were subjected to a single exposure of either control, filtered air (Sham exposure) or nano- $\mathrm{TiO}_{2}$ aerosol (Ex exposure). Animals used in the analyses, unless stated, included Con Sham $(n=6)$, Con Ex $(n=6)$, Het Sham $(n=7)$, Het Ex $(n=8)$, KO Sham $(n=4)$, and KO Ex $(n=4)$. Both male and female mice were used in the analyses.

\section{Engineered Nanomaterial}

Nano-TiO 2 P25 powder, purchased from Evonik (Aeroxide TiO2, Parsippany, NJ), was prepared through drying, sieving, and storing, as previously described $(41,54)$, and was composed of anatase $(80 \%)$ and rutile $(20 \%) \mathrm{TiO}_{2}$ with a primary particle size of $21 \mathrm{~nm}$. This particle has 
been previously characterized: Zeta potential $=-56.6 \mathrm{mV}(52)$ and specific surface area $=48.08$ $\mathrm{m}^{2} / \mathrm{g}(54,59)$ determined.

\section{Inhalation Exposure System}

Nano- $\mathrm{TiO}_{2}$ aerosols were created with a customized high-pressure acoustical generator (HPAG). $\mathrm{TiO}_{2}$ bulk nano-powders were aerosolized with the HPAG and fed into the whole-body exposure chamber. Figure 2.1A shows a representative transmission electron micrograph of nano$\mathrm{TiO}_{2}$ aerosols sampled from the chamber, imaged with the JEOL 1400 transmission electron microscope (Tokyo, Japan). A personal DataRAM (pDR-1500; Thermo Environmental Instruments Inc., Franklin, MA) sampled the exposure chamber air and utilized light scattering techniques to estimate the mass concentration in real-time. Feedback loops within the software automatically adjusted the acoustic energy to maintain a stable mass concentration during the exposure. A target DataRAM concentration of $10 \mathrm{mg} / \mathrm{m}^{3}$ for a period of 240 minutes was chosen in order to achieve a similar lung burden (7-10 $\mu \mathrm{g}$ /exposure) as that in previous studies $(52,67)$. The DataRAM signal during the exposure is shown in Figure 2.1B. A concurrent gravimetric measurement indicated an average mass concentration of $11.09 \mathrm{mg} / \mathrm{m}^{3}$ during the 240 minute period. Aerosol size distributions were examined from the exposure chamber with: 1) a highresolution electrical low-pressure impactor (ELPI+; Dekati, Tampere, Finland), (2) a scanning particle mobility sizer (SMPS 3938; TSI Inc., St. Paul, MN), and (3) an aerodynamic particle sizer (APS 3321; TSI Inc., St. Paul, MN). The ELPI+ data (Figure 2.1C) and the combined SMPS-APS data (Figure 2.1D) both indicated log-normal aerosol size distributions with Count Median Diameters of $182 \mathrm{~nm}$ and $190 \mathrm{~nm}$, respectively. Bedding material soaked with water was used in the exposure chamber to maintain a comfortable humidity during the exposure. Control animals were exposed to HEPA filtered air with similar chamber conditions in terms of temperature and humidity.

\section{Echocardiography-M-mode, B-Mode and PW Doppler Imaging}

Both one week prior to exposure and within 24 hours following exposure to control filtered air or nano- $\mathrm{TiO}_{2}$, ultrasound imaging was performed. Animals were initially anesthetized using 
$2.5 \%$ isoflurane, with a sustained rate of $1 \%$ isoflurane during imaging, in order to more appropriately replicate physiological conditions (36). To monitor the condition of the mice, a rectal temperature probe was used. Motion-mode (M-mode), Brightness-mode (B-mode), and Pulse Wave (PW) Doppler imaging acquisition and analysis have been previously described by our laboratory $(29,52,53,63,70)$. Briefly, images were procured with a linear array transducer at 32 $40 \mathrm{MHz}$, with a frame rate of 233-401 frames/second. Ultrasound images were taken using the Vevo 2100 Imaging System (Visual Sonics, Toronto, Canada). Briefly, imaging of the left ventricle in long-axis B-mode was achieved through placing the transducer left of the sternum. Rotation $90^{\circ}$, perpendicular to the animal, allowed for short-axis B-Mode images. Gating midway between the short-axis B-Mode images allowed for acquisition of M-mode parameters. Pulse Wave Doppler echocardiography measured mitral valve function, determining early (E-wave) and late (A-wave) diastolic filling of the left ventricle. Ejection Fraction is considered the percent of blood leaving the heart following each contraction and fractional shortening the change in left ventricular diameter. Total cardiac output was measured as the heart rate times the stroke volume per beat (volume of blood leaving the heart). All analyses were performed by one analyst. Three replicate analyses were performed for each echocardiographic measure per animal.

\section{Stress Strain-Speckle Tracking}

In both the short and long axis, left ventricular visualization B-Mode images were used for speckle-tracking-based strain analyses. The procedure for speckle-tracking-based strain analysis have been previously described by our laboratory $(29,52,63)$. As a summary, strain was measured as a function of total deformation length divided by the original length of a segment, computed for each cardiac cycle $(7,57)$. Peak strain and strain rates were measured across radial, circumferential, and longitudinal dimensions. Displacement length, velocity, strain, and strain rate were measured in the endocardium in the short (radial and circumferential dimensions) and long (radial and longitudinal) axes over a minimum of three cycles. Short-axis images of the left ventricle were split into anterior free, lateral, posterior, inferior free, posterior septum, and anterior septum while long-axis images were split into anterior base, anterior mid, anterior apex, posterior apex, posterior mid, and posterior base. The average of segmental values was used for analyses in this study. Assessment of Global Longitudinal Strain (GLS) in the left ventricle provides a speckle tracking- 
based valuation of myocardium contraction which correlates with left ventricular function $(6,21)$, and can be combined with PW Doppler E-wave (E/SR) to make clinical prognostics of left ventricular health (72).

\section{Mitochondrial Isolations}

48 hours post-exposure to control filtered air or nano- $\mathrm{TiO}_{2}$, mice were euthanized, the heart, lungs, and liver were excised, and cardiac and lung mitochondrial subpopulations were isolated for analyses as previously described (56), with modifications by our laboratory $(3,4,16)$. Samples were prepared through differential centrifugation, allowing for the compartmentalization of nuclear, cytoplasmic, and mitochondrial fractions. Mitochondrial subpopulations, subsarcolemmal (SSM) and interfibrillar (IFM) were combined to form a total mitochondrial population.

\section{Mitochondrial Respiration}

State 3 and state 4 respiration rates were analyzed in isolated mitochondria as previously described $(15,70)$ with adaptations. The Bradford method (9), with standardization to bovine serum albumin, provided normalization of protein concentrations for cardiac mitochondria loading. To measure oxygen consumption of mitochondria, a multi-unit ( 8 channel) Oxytherm Peltier Electrode apparatus (Hansatech Instruments, Norfolk, England) was utilized. Calibration of the machine was performed through maximal oxygen concentration in respiration buffer $(80 \mathrm{mM}$ $\mathrm{KCl}$, 50mM MOPS, $1 \mathrm{mmol} / 1 \mathrm{EGTA}, 5 \mathrm{mmol} / 1 \mathrm{KH}_{2} \mathrm{PO}_{4}$ and $1 \mathrm{mg} / \mathrm{ml} \mathrm{BSA}$ ) and zero oxygen concentration through $\mathrm{NaHSO}_{3}$. Oxygen concentration measurements were assessed using the $\mathrm{S} 1$ Clark type polarographic oxygen electrode disc, with a central platinum cathode and a concentric silver anode. Mitochondria were loaded into $500 \mathrm{~mL}$ of respiration buffer in the Peltier Chamber. Changes in oxygen levels were measured in real-time, providing both $\mathrm{nmol} \mathrm{O}_{2} / \mathrm{mL}$ consumption and rates per 10s, 30s, and $1 \mathrm{~min}$. Maximal complex I-mediated respiration was initiated by the addition of glutamate $(5 \mathrm{mM})$ and malate $(5 \mathrm{mM})$ for glucose mediated pathways or palmitoylcarnitine $(50 \mu \mathrm{M})$ and malate $(5 \mathrm{mM})$ for fatty-acid mediated pathways. Data for state $3(250 \mathrm{mM}$ 
ADP) and state 4 (ADP-limited) respiration were expressed as nmol of oxygen consumed $/ \mathrm{mL} / \mathrm{min}$, standardized to protein concentration.

\section{Electron Transport Chain Complex Activities}

Maximal activities of electron transport chain (ETC) complexes I, III, IV and V were measured as previously described $(29,62)$. The ETC is comprised of five protein complexes which transport electrons, in a redox fashion, to create a proton $\left(\mathrm{H}^{+}\right)$gradient which contributes toward the production of ATP. Complexes I (ubiquinone oxidoreductase), III (succinate dehydrogenase), and IV (cytochrome c reductase) can complex together to form a respirasome. Complex V (ATP Synthase) produces ATP from ADP using the proton gradient. The Bradford method (9), with standardization to bovine serum albumin, provided normalization of protein concentrations for cardiac and lung mitochondria loading. Activity was measured in complex I (reduction of decyclubiquinone), complex III (reduction of cytochrome c), complex IV (oxidation of reduced cytochrome c), and complex V (oligomycin-sensitive ATPase activity through pyruvate kinase and phosphoenolpyruvate.).

\section{Quantitative PCR}

RNA was isolated from $20 \mathrm{mg}$ of cardiac, lung, and liver tissue or from isolated cardiac mitochondria, using the miRNeasy Mini Kit (product no.: 217004, Qiagen, Hilden, Germany ) per manufacturer's instructions, with minor modifications. For tissue, $700 \mathrm{~mL}$ QIAzol was used for homogenization with a Polytron PowerGen 500 S1 tissue homogenizer (Fisher Scientific, Hampton, NH). Total RNA was isolated from both the whole tissue and mitochondrial samples and used in subsequent analyses. RNA was converted to cDNA with the First-strand cDNA Synthesis kit for miRNA (product no.: HP100042, Origene, Rockville, MD), per manufacturer's instructions. Differential expression of miRNA-378a-3p, miRNA-378a-5p, PGC-1 $\beta$, mitofusin 1 (Mfn1), and mediator complex subunit 19 (Med19) were measured. Primer design for qPCR is provided (Table S2.1). Briefly, primers for mRNA transcripts were derived using Primer-BLAST through NCBI, while miRNA forward primers were designed to span the 5' region of the sequence with a standard reverse to flank the poly-A tail following cDNA synthesis. Expression was 
normalized to GAPDH in whole tissue and U6 in isolated mitochondria. Experiments were performed on the Applied Biosystems 7900HT Fast Real-Time PCR system (Applied Biosystems, Foster City, CA), using 2X SYBR Green Master Mix. Quantification was achieved using the $2^{-}$ $\Delta \Delta \mathrm{C}_{\mathrm{T}}$ method (46).

\section{Western Blot Analyses}

Immunoblotting was performed on $4-12 \%$ gradient gels through MES SDS-PAGE, as previously described $(3,4,16,53,70)$. The Bradford method (9), with standardization to bovine serum albumin, provided normalization of protein concentrations for the cytoplasmic portion of cardiac tissue for loading. Primary antibodies used in the study included: anti-GAPDH (product no.: ab8245, Abcam, Cambridge, MA), anti-optic atrophy 1 (OPA1, product no.: ab42364, Abcam, Cambridge, MA), and anti-peroxisome proliferator-activated receptor alpha (PPAR $\alpha$, product no.: ab2779, Abcam, Cambridge, MA). All primary antibodies were used at a 1:1000 concentration. Secondary antibodies used included the following: goat anti-mouse IgG (H\&L) horseradish peroxidase (HRP) conjugate (product no. 31430, Thermo Fisher, Waltham, MA) and goat antirabbit IgG (H\&L) HRP conjugate (product no. ab6721, Abcam, Cambridge, MA). Standardization was determined through GAPDH expression. Pierce ${ }^{\circledR}$ ECL Western Blotting Substrate (product no.: 32106, Thermo Fisher) was used per manufacturer's instructions to detect tagged proteins through a G:Box Bioimaging system (Syngene, Frederick, MD). Data were captured using GeneSnap/GeneTools software (Syngene). Densitometry was analyzed using Image J Software $(\mathrm{NIH}$, Bethesda, MD). All values were expressed as optical density with arbitrary units.

\section{Mitochondrial Size, Internal Complexity, and Membrane Potential}

Flow cytometry was used to assess structural and functional parameters of mitochondria as previously described (15-17, 75), with modifications. Briefly, the LSRFortessa (BD Biosciences, Franklin Lakes, NJ) was used to perform flow cytometric analyses. To stain viable mitochondria, MitoTrackerTM Deep Red FM/633 (Thermo Fisher) was used to produce a threshold for gating. Measurements of size were assessed through Sphero AccuCount Blank Particles, $2.0 \mu \mathrm{m}$ (Spherotech Inc., Lake Forest, IL) sizing beads. Forward Scatter (FSC PMT-H) measured the 
absolute size of the mitochondria while the Side Scatter (SSC-H) measured the granularity of the mitochondria. The ratiometric mitochondrial dye JC-1 (5,5',6,6'-tetrachloro-1,1',3,3'tetraethylbenzimidazol carbocyanine iodide, Molecular Probes, Carlsbad, CA) was used to measure mitochondrial membrane potential. Changes in membrane potential were assessed as the ratio of orange to green fluorescence of JC-1. All flow cytometric measures were performed at the West Virginia University Flow Cytometry \& Single Cell Core Facility. FSC files were processed using the FCS Express Flow Research Edition (De Novo Software, Glendale, CA).

\section{Transmission Electron Microscopy of Mitochondria}

After excision of cardiac tissue, $\sim 1-2 \mathrm{~mm}^{3}$ pieces were cut and sent to the WVU Electron Microscopy Shared Facilities where they were processed and further imaged at the WVU Electron Microscopy Histopathology and Tissue Bank. Briefly, cardiac tissue was fixed in 2.5\% glutaraldehyde in 0.1 M PBS (pH 7.4) for 60 minutes. After washing in PBS, samples were incubated in $1 \%$ osmium tetroxide containing $1 \%$ potassium ferricyanide for 60 minutes. Following additional PBS washes, tissue was dehydrated using a graded series of alcohol dilutions and propylene oxide. Samples were then incubated overnight in a 1:1 mix of propylene oxide and EPON. Samples were incubated in pure EPON for 4 washes, each for 60 minutes. Samples were cured in pure EPON inside the BEEM ${ }^{\circledR}$ Embedding Capsules Size 3 (Electron Microscopy Sciences, Hatfield, PA) starting at $37^{\circ} \mathrm{C}$ for 24 hours and then $60^{\circ} \mathrm{C}$ for 48 hours. Tissue was sectioned using an Ultramicrotome Leica EM UC7 (Leica Microsystems, Wetzler, Germany) and stained with uranyl acetate and lead citrate. Sections were imaged using the JEOL JEM-2100 Transmission Electron Microscope (JOEL, Akishima, Tokyo, Japan). Semi-quantitative analyses of mitochondrial size was processed through Fiji (NIH, Bethesda, MD) and the Trainable Weka Segmentation (TWS) plugin by providing a probability map by which particle size could be determined. Selection of 5 mitochondria per group were used to perform analyses.

\section{In Situ Hybridization (ISH) for miRNA-378a}

After excision of cardiac tissue, the apex of the heart was removed $\left(\sim 5 \mathrm{~mm}^{3}\right)$ and prepared per manufacturer's instructions (Qiagen) $(31,61)$, with slight modifications. Tissue was treated 
with freshly prepared $4 \%$ paraformaldehyde in PBS and was incubated at $4^{\circ} \mathrm{C}$ overnight. Tissue was then directly transferred to $0.5 \mathrm{M}$ Sucrose/PBS and was incubated at $4^{\circ} \mathrm{C}$ overnight. Following incubation, tissue was flash frozen and samples taken to the WVU Pathology Laboratory of Translational Medicine to embed and section tissue. Tissue was cut in 12-14 $\mu \mathrm{M}$ sections and mounted on Superfrost/Plus slides. Sections were further fixed in $4 \%$ paraformaldehyde in PBS and was incubated at $4^{\circ} \mathrm{C}$ for 10 minutes and then washed with PBS 3 times for 5 minutes each. For epitope retrieval, slides were immersed in a citric acid bath $(10 \mathrm{mM}$ citric acid, $\mathrm{pH} 6.0)$ at $95^{\circ} \mathrm{C}$ for 15 minutes and then washed in PBS for 5 minutes at room temperature. Sections were acetylated $(2.33 \mathrm{~mL}$ of triethanolamine and $500 \mu \mathrm{L}$ acetic anhydride in $197 \mathrm{~mL}$ water) for 10 minutes. Slides were washed 3 times with PBS for 5 minutes each. Slides were hybridized using a hybridization buffer $(50 \%$ deionized formamide, $0.3 \mathrm{M} \mathrm{NaCl}, 20 \mathrm{mM}$ Tris $\mathrm{HCl}, \mathrm{pH} 8.0,5 \mathrm{mM}$ EDTA, $10 \mathrm{mM} \mathrm{Na}_{3} \mathrm{PO}_{4}$, pH 8.0, 10\% Dextran Sulfate, 1X Denhardt's solution, and $0.5 \mathrm{mg} / \mathrm{mL}$ of yeast RNA) (74) and $40 \mathrm{nM}$ of miRNA-378a-3p 3'-DIG labeled probe (Qiagen).

Total hybridization mixture was heated to $65^{\circ} \mathrm{C}$ to linearize the probe and $100 \mu \mathrm{L}$ of solution was added to slides, cover slipped, and hybridized at $56^{\circ} \mathrm{C}$ for 18 hours in a $\mathrm{HybEZ}{ }^{\mathrm{TM}}$ Hybridization System (Advanced Cell Diagnostics, Newark, CA). Coverslips were removed in 5X SSC buffer at room temperature for 20 minutes. Slides were then washed twice in $50 \%$ formamide, $0.1 \%$ Tween-20, and $1 \mathrm{X} \mathrm{SSC}$ at $56^{\circ} \mathrm{C}$. Following two washes in PBS for 15 minutes each, blocking solution (3\% Donkey serum, $0.1 \%$ Titron-100X, in PBS) was applied for 1 hour at room temperature. AP conjugated ant-DIG Fab fragments (1:1000) (Sigma-Aldrich, St. Louis, MO) were then applied to the blocking solution for 2 hours at room temperature. Following two washes in $0.1 \%$ Tween/PBS for 30 minutes each and $1 \mathrm{X}$ PBS for 10 minutes each, BM Purple AP Substrate (Sigma-Aldrich) and 2mM Levamisole were applied to slides for 6 days. Staining was quantified through light microscopy using the EVOS ${ }^{\mathrm{TM}}$ FL Auto Imaging System (Thermo Fisher). Briefly, using images derived with the same light intensity, Fiji (NIH, Bethesda, MD) was applied to measure intensity of BM Purple staining by using the $\log$ (max intensity/mean intensity) of Purple (R: 0.4643, G: 0.8303, B: 0.3083).

Citrate Synthase Activity 
Citrate synthase is key to metabolism through the production of citrate from acetyl-CoA and oxaloacetate, initiating the tricarboxylic acid (TCA) cycle. To determine the number of mitochondria in heart tissue, a colorimetric Citrate Synthase Assay (Sciencell, San Diego, CA) was employed per manufacturer's instructions (35). The Bradford method (9), with standardization to bovine serum albumin, provided normalization of protein concentrations for cardiac mitochondria. Briefly, $1 \mu \mathrm{g}$ of mitochondria was used to measure the colorimetric change produced from 5', 5'-Dithiobis 2-nitrobenzoic acid (DTNB) and CoA-SH forming of TNB at an absorbance maximum of $412 \mathrm{~nm}$.

\section{Hydrogen Peroxide $\left(\mathrm{H}_{2} \mathrm{O}_{2}\right)$ Assay}

To measure the concentration of $\mathrm{H}_{2} \mathrm{O}_{2}$ in the heart we used an Amplex ${ }^{\circledR}$ Red Hydrogen Peroxide/Peroxidase Assay Kit (Thermo Fisher, Waltham, MA) per manufacturer's instructions and as previously described (52), with modifications. Heart tissue was homogenized in NP-40 buffer (150 mM NaCl, $50 \mathrm{mM}$ Tris-HCl, 1\% Triton-100X, and $\mathrm{pH}$ adjusted to 7.4) and $50 \mu \mathrm{g}$ of protein, determined through the Bradford method (9), was used in the assay. The reaction of the Amplex ${ }^{\circledR}$ Red reagent (10-acetyl-3,7-dihydroxyphenoxazine) with $\mathrm{H}_{2} \mathrm{O}_{2}$ occurs at a stoichiometric ratio of $1: 1$. Sample $\mathrm{H}_{2} \mathrm{O}_{2}$ concentrations were determined through running a standard curve ( 0 to $20 \mu \mathrm{M} \mathrm{H}_{2} \mathrm{O}_{2}$ ), with absorbance measured at $560 \mathrm{~nm}$. Data was normalized to the number of mitochondria for each sample, using citrate synthase activity (described above).

\section{TargetScan}

The miRNA-mRNA binding prediction software TargetScan 7.1 Server was implemented in order to determine seed-sequence binding affinities $(1,44)$. Briefly, binding affinities were examined in the 3' untranslated region (UTR) of Mfn1 and Med19, where context++ scores (CSs) predicted the propensity for binding. CSs are derived from a variety of variables including 3' UTR length, supplementary pairing, local AU content, and conservation of target sequence. Seedsequence regions begin with the second base pair on the miRNA, starting from the 5' end, and can identically bind to eight (8mer), seven (7mer), or six (6mer) consecutive base pairs on the mRNA strand. http://www.targetscan.org/vert 71/ 
$\operatorname{IntaRNA}$

RNA-RNA interaction software IntaRNA 2.0 was used to assess miRNA binding in the 3' UTR of Mfn1 (mouse), MFN1 (human), Med19 (mouse), and MED19 (human) (10, 47, 76). Unlike TargetScan 7.1, IntaRNA considers free energy binding as well as intramolecular forces between base pairs. Ensembl was used to obtain the 3' UTR sequence for Mfn1 and Med19. The 3' UTR sequences were then run against the miRNA-378a-3p sequence (5' ACUGGACUUGGAGUCAGAAGG - 3') to determine complementarity. The interaction energy is a measure of binding potential.

Mfold

The UNAFold Web Server was used to evaluate miRNA folding, specifically through using the mfold software (78). The pre-miRNA-378a (5' AGGGCUCCUGACUCCAGGUCCUGUGUGUUACCUCGAAAUAGCACUGGACUUGGAG UCAGAAGGCCU - 3'), mature miRNA-378a-3p (5' - ACUGGACUUGGAGUCAGAAGG 3'), and mature miRNA-378a-5p (5' - CUCCUGACUCCAGGUCCUGUGU - 3') sequences from miRBase release 21 were used.

Ingenuity Pathway Analysis (IPA)

QIAGEN's IPA Server (December 2017 update) provides protein ontology and the capacity to correlate gene expression data through molecular pathways experimentally verified. The "PPAR $\alpha / \operatorname{RXR} \alpha$ Activation" pathway and graphical illustrations were used in the analysis www.qiagen.com/ingenuity.

\section{Statistics}

Significance was determined using either a two-tailed Student's t-test or one-way analysis of variance (ANOVA), where appropriate. Tukey's multiple comparisons test was implemented 
following the ANOVA to derive significance between multiple groups. Differences between groups were considered statistically different $(P \leq 0.05$, denoted by $*)$. All data are presented as the mean \pm standard error of the mean (SEM). 


\section{Results}

MiRNA-378a Expression following Nano-TiO $\mathrm{N}_{2}$ Exposure

Because the pre-miRNA-378a gene actually contains both a 3' (miRNA-378a-3p) and a 5' (miRNA-378a-5p) arm mature miRNA (42), expression of both miRNAs needed to be assessed in total heart homogenates and within isolated mitochondria. This tissue and organellar-specific approach allowed for the determination of miRNA-378a's involvement in regulating a variety of pathways (whole tissue) or those specifically related to mitochondrial bioenergetics and mitochondrial transcription (mitochondria). In whole heart tissue (Figure 2.2A), the expression of miRNA-378a-3p was significantly increased following exposure, while the $5 p$ strand was unchanged. Using in situ hybridization, miRNA-378a-3p was also shown to be significantly elevated in cardiac tissue, displaying universal, not localized, expression of the miRNA throughout the cell (Figure 2.2B). The gene that encodes these two mature miRNAs, PGC-1 $\beta$, was also shown to have increased expression following exposure (Figure S2.2A). The heart, in comparison to the lung and liver, was the only tissue where miRNA-378a-3p was increased, with a trend toward decreased expression in both the lung and liver following exposure (Figure S2.2B).

In mitochondria (Figure 2.2C), the 3p strand remained unchanged, while miRNA-378a-5p was increased after nano- $\mathrm{TiO}_{2}$ exposure. Though alterations in mitochondrial miRNA-378a-5p could potentially change dynamics surrounding mitochondrial metabolism (35), the relative abundance of the $5 \mathrm{p}$ strand to the $3 \mathrm{p}$ strand was significantly lower (Figure S2.2C) in both the whole heart tissue and the mitochondrion, likely contributing little to systemic cellular mechanisms. Genotyping was performed using PCR and gel agarose electrophoresis for the genic region surrounding miRNA-378a for wild type (WT), heterozygous knockout (Het) and total knockout (KO) animals; the size and characteristic folding of the pre-miRNA and mature strands are also represented (Figure 2.2D). The expression of miRNA-378a-3p and $5 p$ were then confirmed both in whole heart tissue and mitochondria through qPCR in transgenic animals (Figure S2.2D). The increased expression of miRNA-378a-3p in the whole heart homogenates following exposure provided a pathway for examining how miRNAs can alter cardiac function following exposure to nano- $\mathrm{TiO}_{2}$ through a transgenic model. 


\section{Cardiac Contractile Function and miRNA-378a}

A list of all M-mode parameters are provided in Table 2.1. Of note, a decrease in the ejection fraction (percentage of total blood leaving the heart) and fractional shortening (change in left ventricular diameter) between the WT Sham and WT Ex group was observed, though preserved in the Het and KO groups. The WT groups also had larger systolic volumes than both the Het and KO groups. M-mode characteristics are also provided for Het and KO mice preexposure, validating the sham exposure model (Table S2.2). PW Doppler was implemented to measure mitral valve function and filling parameters (Figure 2.3A). The E wave (early diastolic filling of the left ventricle) was shown to not be significantly altered between groups, with the Het group exhibiting a higher A wave (late diastolic filling of the left ventricle) following exposure (Figure 2.3B). As seen in other studies following nano- $\mathrm{TiO}_{2}$ exposure $(29,52)$, the WT group had an $\mathrm{E} / \mathrm{A}$ ratio of $\sim 2$. When the $\mathrm{E} / \mathrm{A}$ ratio increases, it indicates either more passive (early) filling or less active (late) filling of the left ventricle; this signifies a pathological shift in blood flow in the left ventricle and indicates diastolic dysfunction and increased left atrial pressure.

While the KO group preserved E/A ratio, the Het group had a significant decrease in E/A ratio. Short axis radial strain and strain rate were significantly decreased following exposure to nano- $\mathrm{TiO}_{2}$ in the WT and Het groups, respectively, while no changes were shown in the KO (Figure 2.3C). Also, long axis diastolic radial strain was increased in the WT group after exposure. Generally, decreases in systolic measures of strain infer impaired contractile properties of the left ventricle, while increases in diastolic measures of strain could suggest aberrant relaxation of the left ventricle. Strain measures represent the contraction and relaxation of the myocardium and subsequent cardiomyocyte contractility. The segmental changes in short axis systolic radial strain are illustrated as an example of the segmental analysis of speckle tracking stress-strain (Figure 2.3D).

In the long axis, global longitudinal strain (GLS) during end-diastole provides significant prognostic value in assessing cardiac health (21) (Figure 2.3E). By combining both the E wave and end-diastolic GLS (E/SR ratio), it provides both cardiac strain and mitral valve function in a prognostic assessment (72). An increased E/SR ratio in the WT and Het indicates increased susceptibility to adverse cardiac events, while the KO group remained unchanged (Figure 2.3E). Cardiac function following exposure is further captured in the left ventricle myocardial 
performance index ( $\left.\frac{\text { isovolumic relaxation time }(I V R T)+\text { isovolumic contraction time }(I V C T)}{\text { left ventricle ejection time }(\text { LVET) }}\right)$, revealing increased function in the KO group compared to the WT (Figure 2.3E). All other measures of diastolic (Table S2.3 and S2.4) and systolic (Table S2.5 and S2.6) stress-strain are included. Decreases in miRNA-378a expression attenuates the effect of nano- $\mathrm{TiO}_{2}$ exposure to the heart, preserving cardiac function following acute nano- $\mathrm{TiO}_{2}$ exposure.

\section{Mitochondrial Bioenergetics and miRNA-378a}

In the WT, changes to ETC activities and mitochondrial respiratory capacity were observed following exposure. Complex III activity and glucose-mediated state 3 respiration were decreased following nano- $\mathrm{TiO}_{2}$ exposure (Figure $\mathrm{S} 2.3 \mathrm{~A}$ and $\mathrm{S} 2.3 \mathrm{~B}$ ). When examining bioenergetic capacity of all the groups, a distinctive metabolic profile was observed. Irrespective of exposure, complex I, III, and IV activities were shown to be significantly increased in the Het and KO groups (Figure 2.4A), potentially contributing to the cardioprotection observed after exposure. Unlike in the WT mice, glucose-mediated metabolism was not the most significantly impacted metabolic pathway in the Het or $\mathrm{KO}$ animals (Figure 2.4B); the main pathway affected was fatty-acid metabolism (Figure $2.4 \mathrm{C}$ ). Both the Het and $\mathrm{KO}$ groups displayed a more robust fatty acid metabolic profile, which is illustrated in Figure 2.4D.

While exposure primarily affected glucose substrate utilization pathways in WT animals (Figure S2.3B), the Het and $\mathrm{KO}$ animals displayed decrements in fatty acid substrate utilization in state 3 and state 4 respiration, respectively. The increased utilization of fatty acid metabolites has been previously shown in the liver of miRNA-378a knockout animals (11), supporting the parallels described in the heart and suggesting a metabolic contribution toward attenuating the pathology. Lung tissue of the Het and KO animals showed conserved ETC complex activity following exposure (Figure S2.4A and S2.4B), indicating preservation of cardiac function following inhalation exposure may be a function of protection to multiple organ systems in the miRNA-378a knockout/knockdown animals. The increase in complex activities and fatty acid-driven state 3 and state 4 respiration in Het and $\mathrm{KO}$ animals revealed a bioenergetic role for cardioprotection following nano- $\mathrm{TiO}_{2}$ exposure. 


\section{Mitochondrial Ultrastructure}

Mitochondrial size, through flow cytometry (Figure S2.5A), was shown to increase following exposure in the WT group (Figure 2.5A), as well as in both the Het and KO groups, irrespective of exposure. The shape and size of mitochondria were also examined through transmission electron microscopy (TEM), confirming the increased size of mitochondria following ENM inhalation exposure in the WT group using a semi-quantitative approach (Figure 2.5B). Citrate synthase activity, used as a common marker of mitochondrial number/content and shown to be highly correlative (43), revealed decreased mitochondrial number in the WT group after exposure, while the Het and $\mathrm{KO}$ were protected from the effect (Figure S2.5B). In the WT group following exposure, though the size of the mitochondria increased, decreased mitochondrial number suggests a role in contributing to the dysfunctional respiratory capacity of the mitochondria (37). Alternatively, in the Het and KO groups, mitochondrial size was shown increased while mitochondrial size and number were unaffected by the exposure paradigm. The increased size and unchanging number of mitochondria in the Het and $\mathrm{KO}$ groups could relate to the increased bioenergetic capacity and potential protective mechanism observed following nano$\mathrm{TiO}_{2}$ inhalation exposure. Other measures of mitochondrial ultrastructure, such as internal complexity (Figure S2.5C) and membrane potential (Figure S2.5D) were measured and found to be unchanged. Measurements of $\mathrm{H}_{2} \mathrm{O}_{2}$ were performed to further understand mitochondrial stress/dysfunction. In the WT group following exposure, the concentration of $\mathrm{H}_{2} \mathrm{O}_{2}$ in cardiac tissue was significantly increased (Figure $\mathrm{S} 2.5 \mathrm{E}$ ). Following inhalation exposure to nano- $\mathrm{TiO}_{2}$, the density, size, and impact of ROS is altered, but knockout, or even knockdown, of miRNA-378a produces a protective phenotype.

\section{Targets of miRNA-378a and Pathways Affected}

Gene transcripts, which were related to mitochondrial function and revealed highest 3' UTR binding scores by miRNA-378a, were examined for further in-silico binding and expression. Mfn1 and Med19 were identified to be directly targeted by miRNA-378a-3p using bioinformatics software IntaRNA and TargetScan. MiRNA-378a-3p binding to the 3' untranslated region (UTR) was evaluated through both free energy binding affinity (IntaRNA) (Figure 2.5D) and seed sequence complementarity (TargetScan) (Figure S2.6A). The mRNA of Mfn1 and Med19 were 
found to be significantly increased following exposure in the Het and $\mathrm{KO}$ groups (Figure 2.5D), suggesting that the negative regulation of miRNA-378a-3p was degrading transcripts and limiting translation of these proteins. While Med19 is involved in general transcriptional activation of RNA Polymerase, Mfn1 is involved prolifically in mitochondrial fusion dynamics and the health of mitochondria.

Opa1, a direct mediator with Mfn1 of inducing mitochondrial fusion, was shown to be significantly increased in the Het and $\mathrm{KO}$ groups following inhalation exposure; likewise, expression of the fatty acid metabolism protein Ppara was also increased (Figure S2.6B). The knockdown/knockout of miRNA-378a-5p, which acts as a negative regulator of estrogen-related receptor gamma (EER $\gamma$ ) and GA-binding protein alpha chain (GABPA), could promote the expression of PGC-1 $\beta$ and subsequently interact with Ppar $\alpha$ (20). In the KO animal, PGC-1 $\beta$ is found to be significantly increased compared to the WT group, following inhalation exposure (Figure S2.6C). Following maternal nano- $\mathrm{TiO}_{2}$ exposure, $\mathrm{PGC}-1 \beta$ displayed increased histone 3 lysine 4 tri-methylation ( $\mathrm{H} 3 \mathrm{~K} 4 \mathrm{me} 3)$ association at its promoter region, suggesting an increased expression of both the gene and miRNA-378a through epigenetic mechanisms (Figure S2.6D) (67). Suppressing miRNA-378a during inhalation exposure could diminish cardiac dysfunction by offering cardioprotection through pathways including transcription, bioenergetics, fatty acid metabolism, and mitochondrial fusion (Figure 2.6A and 2.6B). 


\section{Discussion}

The scope and impact of research examining the potential human consequences of ENM exposure continues to grow, with considerations into the dose, physiochemical characteristics, route, and time course of exposure and the impact on human health and safety adding multiple layers of complexity. Nano- $\mathrm{TiO}_{2}$ remains a significant contributor to total ENM production, affecting both those involved in manufacturing and utilization of the material. The life cycle of ENMs, including byproducts of manufacturing, disposal through waste and recycling, use by consumers, and other mediums, eventually carry ENMs into the environment (26). The environmental sequestration of ENMs and their byproducts could then have secondary or tertiary impacts on human health (8). We provide evidence to support the therapeutic benefit of miRNA378a downregulation as a means of mediating the ENM toxicological response through metabolism, mitochondrial function, and other cellular processes during ENM inhalation exposure. For the first time, this study illustrates the impact of the manipulation of a miRNA in vivo on controlling toxicity to ENMs.

Under non-pathological states, altered miRNA content may have no overt consequences to basal physiological processes. As a stressor is introduced, such as disease, xenobiotic exposure, or caloric changes, specific miRNAs are needed to mediate the response and attempt to return the cell to homeostasis $(11,49,55)$. In the transgenic miRNA-378a knockout and knockdown animals, this has been highlighted in both caloric changes (11) and currently with ENM inhalation exposure. Adaptation to a stress has significantly diverse outcomes in transgenic versus wild type animals, though little or no genetic or phenotypic changes exist at a basal condition. Because miRNAs exist in a complicated web of regulatory non-coding RNA, compensatory mechanisms are present which can attenuate loss of function from a gene or genes $(27,71)$. In the transgenic animals used in this study, it is likely that the loss of miRNA-378a is compensated through increased expression of endogenous miRNAs that regulate similar functional pathways, such as the insulin response and fatty acid metabolism pathways. Additionally, epigenetic remodeling could occur, repressing the expression of previously targeted miRNA-378a transcripts.

Cellular and systemic dysfunction has been attributed to ENM-induced changes in miRNA expression $(2,14,28,50,51,65)$. While the contribution of miRNA expression in advancing ENM related insult is beginning to be understood, what lacks clarity are the explicit mechanisms 
governing these changes in expression. The interplay between the epigenome and miRNA expression provides a new area of research and hierarchy of control, which could potentially explain the regulation of multiple pathways. MiRNAs are known to directly regulate, and to be regulated by, epigenetic function $(30,60)$.

In one of the first studies to examine the effects of inhalation of ENM and miRNA expression, Halappanavar et al. showed that following inhalation exposure, miRNA-1 and -449 were elevated (28). MiRNA-1 is known to target histone deacetylase 4 (HDAC4) while miRNA449 has been shown to target HDAC1, resulting in global changes to the epigenetic histone profile in the cell (12). Down regulation of $\mathrm{HDACs}$ following nano- $\mathrm{TiO}_{2}$ exposure would suggest the activation of genes through open histone conformations, which we have reported in progeny following maternal exposure (67). Alternatively, the miRNA expression can be altered by epigenetic machinery. The expression of miRNA-378a is regulated through methylation in TGF$\beta 1$-treated LX-2 cells (77). In the current study, both the expression of the host gene PGC-1 $\beta$ and miRNA-378a-3p were shown to be increased following inhalation exposure to nano- $\mathrm{TiO}_{2}$, with epigenetic mechanisms known to alter the expression of the PGC-1 $1 \beta$ gene. The epigenetic landscape of miRNA-378a, and other altered miRNAs, could provide valuable information regarding transient and, potentially, sustained effects of ENM exposure on the heart and other organ systems.

The animal models employed in the study examine miRNA-378a across a stratum of normal (WT), knockdown (Het), and knockout (KO) expression levels. Intuitively, one could hypothesize that the health outcomes going from poorest to best prognosis would trend in a linear fashion from WT to Het to KO. While we expect the KO model to have increased mitochondrial fatty acid metabolism (24) and overall better health (11) following ENM exposure, the knockdown expression of miRNA-378a may still be prevalent enough to regulate maladaptive metabolic pathways, meaning that the linearity of functional measures in the three models may not exist in all cases. MiRNA's are multifaceted transcriptional and translational repressors that target multiple genic groups, making it difficult to speculate the intermediary effects that knockdown expression may have on any one cardiac, mitochondrial, or molecular measure (48). That being said, in general, we expected to see overall better function in the Het model compared to the WT control. 
The current study proposes the idea that targeting miRNAs can alter cardiac and mitochondrial function and adaptation to ENM inhalation exposure. A noted limitation to the study is the examination of systemic miRNA-378a knockout and knockdown when examining cardiovascular health following ENM inhalation exposure. The current work focuses on cardiac adaptation to ENM inhalation exposure, but does so through systemic knockdown of miRNA378a; a cardiac-specific knockout model could provide further indication into the absence of localized miRNA-378a expression following insult. We point to miRNA-378a as a target for attenuation of nano- $\mathrm{TiO}_{2}$ inhalation exposure, though it is likely that multiple miRNAs, and other coding and non-coding RNAs, are involved in the response to the insult. Our current work may be limited through the exploration of a single miRNA, but the outlined methodology provides an architype for transgenic animal use in examining ENM toxicology and signifies the importance of non-coding RNA in regulating the cardiovascular response.

The goal of ENM inhalation toxicology is to decrease the severity of biologic effects and/or prevention of exposure, ultimately increasing the positive impact of ENM utilization in human endeavors. The ability to therapeutically target a miRNA, or even group of miRNAs, could beneficially protect those exposed to ENMs. We show through the heterozygous knockout of miRNA-378a that even partial downregulation of a miRNA can beneficially alter the response to ENM inhalation exposure, producing a therapeutic effect. Ultimately, further investigation into the role of miRNAs following ENM exposure is needed as well as understanding their interconnection with the epigenome. Future studies could examine the epigenetic regulation of genes encoding miRNAs, such as the promotor region of PGC-1 $\beta$, and determining if ENM exposure provides transient or sustained epigenetic control of crucial genes and non-coding RNA.

Understanding the role of miRNAs in regulating the response to ENM inhalation exposure is important in defining the molecular consequences involved in the response. In this study, we discovered that miRNA-378a, a small non-coding RNA, was directly involved in regulating cardiac function following nano- $\mathrm{TiO}_{2}$ inhalation exposure. The knockout, and to a lesser extent knockdown, of miRNA-378a expression was associated with preserved cardiac function following exposure and increased bioenergetic capacity when compared to wild type animals. The mechanisms governing this functional difference are likely through bolstered mitochondrial health, including increased mitochondrial fusion and fatty acid metabolism, as well as changes to 
cellular transcription. This study investigates for the first time, the in vivo role of a miRNA in attenuating cardiovascular consequences following ENM inhalation exposure, setting miRNA378 a as a potential target for future investigations. Manipulation of miRNA-378a, or other miRNAs found in the heart or systemically, have the potential to improve health outcomes following ENM inhalation exposure. 


\section{Acknowledgements}

We would like to thank Eric Olson and the Transgenic Technology Center at University of Texas Southwestern Medical Center for the miRNA-378a knockout mouse model.

\section{Declaration of Interest}

The authors declare that they have no competing interests, financial or otherwise. This work was supported by: R01 HL-128485 (JMH), R01-ES015022 (TRN), AHA-17PRE33660333 (QAH), AHA-13PRE16850066 (CEN), DGE-1144676 (QAH, ABA, TRN), WV-INBRE support by NIH Grant P20GM103434, WVU Flow Cytometry \& Single Cell Core supported by MBRCC CoBRE Grant GM103488 and Fortessa S10 Grant OD016165, and the Community Foundation for the Ohio Valley Whipkey Trust.

\section{Disclaimer}

The findings and conclusions in this report are those of the authors and do not necessarily represent the official position of the National Institute for Occupational Safety and Health, Centers for Disease Control and Prevention. 


\section{References:}

1. Agarwal V, Bell GW, Nam JW, and Bartel DP. Predicting effective microRNA target sites in mammalian mRNAs. Elife 4, 2015.

2. Balansky R, Longobardi M, Ganchev G, Iltcheva M, Nedyalkov N, Atanasov P, Toshkova R, De Flora S, and Izzotti A. Transplacental clastogenic and epigenetic effects of gold nanoparticles in mice. Mutat Res 751-752: 42-48, 2013.

3. Baseler WA, Dabkowski ER, Jagannathan R, Thapa D, Nichols CE, Shepherd DL, Croston TL, Powell M, Razunguzwa TT, Lewis SE, Schnell DM, and Hollander JM. Reversal of mitochondrial proteomic loss in Type 1 diabetic heart with overexpression of phospholipid hydroperoxide glutathione peroxidase. Am J Physiol Regul Integr Comp Physiol 304: R553-565, 2013.

4. Baseler WA, Dabkowski ER, Williamson CL, Croston TL, Thapa D, Powell MJ, Razunguzwa TT, and Hollander JM. Proteomic alterations of distinct mitochondrial subpopulations in the type 1 diabetic heart: contribution of protein import dysfunction. Am J Physiol Regul Integr Comp Physiol 300: R186-200, 2011.

5. Besinis A, De Peralta T, Tredwin CJ, and Handy RD. Review of nanomaterials in dentistry: interactions with the oral microenvironment, clinical applications, hazards, and benefits. ACS Nano 9: 2255-2289, 2015.

6. Biering-Sorensen T, Biering-Sorensen SR, Olsen FJ, Sengelov M, Jorgensen PG, Mogelvang R, Shah AM, and Jensen JS. Global Longitudinal Strain by Echocardiography Predicts Long-Term Risk of Cardiovascular Morbidity and Mortality in a Low-Risk General Population: The Copenhagen City Heart Study. Circ Cardiovasc Imaging 10, 2017.

7. Blessberger $\mathbf{H}$ and Binder $\mathbf{T}$. Two dimensional speckle tracking echocardiography: clinical applications. Heart 96: 2032-2040, 2010.

8. Boxall AB, Tiede K, and Chaudhry Q. Engineered nanomaterials in soils and water: how do they behave and could they pose a risk to human health? Nanomedicine (Lond) 2: 919-927, 2007.

9. Bradford MM. A rapid and sensitive method for the quantitation of microgram quantities of protein utilizing the principle of protein-dye binding. Anal Biochem 72: 248-254, 1976.

10. Busch A, Richter AS, and Backofen R. IntaRNA: efficient prediction of bacterial sRNA targets incorporating target site accessibility and seed regions. Bioinformatics 24: 2849-2856, 2008.

11. Carrer M, Liu N, Grueter CE, Williams AH, Frisard MI, Hulver MW, Bassel-Duby R, and Olson EN. Control of mitochondrial metabolism and systemic energy homeostasis by microRNAs 378 and 378*. Proc Natl Acad Sci U S A 109: 15330-15335, 2012.

12. Chen JF, Mandel EM, Thomson JM, Wu Q, Callis TE, Hammond SM, Conlon FL, and Wang DZ. The role of microRNA-1 and microRNA-133 in skeletal muscle proliferation and differentiation. Nat Genet 38: 228-233, 2006.

13. Chen R, Li H, Cai J, Wang C, Lin Z, Liu C, Niu Y, Zhao Z, Li W, and Kan H. Fine Particulate Air Pollution and the Expression of microRNAs and Circulating Cytokines Relevant to Inflammation, Coagulation, and Vasoconstriction. Environ Health Perspect 126: 017007, 2018.

14. Chew WS, Poh KW, Siddiqi NJ, Alhomida AS, Yu LE, and Ong WY. Short- and longterm changes in blood miRNA levels after nanogold injection in rats--potential biomarkers of nanoparticle exposure. Biomarkers 17: 750-757, 2012.

15. Croston TL, Thapa D, Holden AA, Tveter KJ, Lewis SE, Shepherd DL, Nichols CE, Long DM, Olfert IM, Jagannathan R, and Hollander JM. Functional deficiencies of 
subsarcolemmal mitochondria in the type 2 diabetic human heart. Am J Physiol Heart Circ Physiol 307: H54-65, 2014.

16. Dabkowski ER, Baseler WA, Williamson CL, Powell M, Razunguzwa TT, Frisbee JC, and Hollander JM. Mitochondrial dysfunction in the type 2 diabetic heart is associated with alterations in spatially distinct mitochondrial proteomes. Am J Physiol Heart Circ Physiol 299: H529-540, 2010.

17. Dabkowski ER, Williamson CL, Bukowski VC, Chapman RS, Leonard SS, Peer CJ, Callery PS, and Hollander JM. Diabetic cardiomyopathy-associated dysfunction in spatially distinct mitochondrial subpopulations. Am J Physiol Heart Circ Physiol 296: H359-369, 2009.

18. Divakaran V and Mann DL. The emerging role of microRNAs in cardiac remodeling and heart failure. Circ Res 103: 1072-1083, 2008.

19. Dong H, Dong C, Ren T, Li Y, and Shi D. Surface-engineered graphene-based nanomaterials for drug delivery. J Biomed Nanotechnol 10: 2086-2106, 2014.

20. Eichner LJ, Perry MC, Dufour CR, Bertos N, Park M, St-Pierre J, and Giguere V. miR-378(*) mediates metabolic shift in breast cancer cells via the PGC-1beta/ERRgamma transcriptional pathway. Cell Metab 12: 352-361, 2010.

21. Ersboll M, Valeur N, Mogensen UM, Andersen MJ, Moller JE, Velazquez EJ, Hassager C, Sogaard P, and Kober L. Prediction of all-cause mortality and heart failure admissions from global left ventricular longitudinal strain in patients with acute myocardial infarction and preserved left ventricular ejection fraction. J Am Coll Cardiol 61: 2365-2373, 2013.

22. Espinoza-Lewis RA and Wang DZ. MicroRNAs in heart development. Curr Top Dev Biol 100: 279-317, 2012.

23. Gagan J, Dey BK, Layer R, Yan Z, and Dutta A. MicroRNA-378 targets the myogenic repressor MyoR during myoblast differentiation. J Biol Chem 286: 19431-19438, 2011.

24. Gerin I, Bommer GT, McCoin CS, Sousa KM, Krishnan V, and MacDougald OA. Roles for miRNA-378/378* in adipocyte gene expression and lipogenesis. Am J Physiol Endocrinol Metab 299: E198-206, 2010.

25. Gottschalk F, Lassen C, Kjoelholt J, Christensen F, and Nowack B. Modeling flows and concentrations of nine engineered nanomaterials in the Danish environment. Int J Environ Res Public Health 12: 5581-5602, 2015.

26. Gottschalk F and Nowack B. The release of engineered nanomaterials to the environment. J Environ Monit 13: 1145-1155, 2011.

27. Gurtan AM and Sharp PA. The role of miRNAs in regulating gene expression networks. J Mol Biol 425: 3582-3600, 2013.

28. Halappanavar S, Jackson P, Williams A, Jensen KA, Hougaard KS, Vogel U, Yauk CL, and Wallin H. Pulmonary response to surface-coated nanotitanium dioxide particles includes induction of acute phase response genes, inflammatory cascades, and changes in microRNAs: a toxicogenomic study. Environ Mol Mutagen 52: 425-439, 2011.

29. Hathaway QA, Nichols CE, Shepherd DL, Stapleton PA, McLaughlin SL, Stricker JC, Rellick SL, Pinti MV, Abukabda AB, McBride CR, Yi J, Stine SM, Nurkiewicz TR, and Hollander JM. Maternal-engineered nanomaterial exposure disrupts progeny cardiac function and bioenergetics. Am J Physiol Heart Circ Physiol 312: H446-H458, 2017.

30. Hathaway QA, Pinti MV, Durr AJ, Waris S, Shepherd DL, and Hollander JM. Regulating MicroRNA Expression: At the Heart of Diabetes Mellitus and the Mitochondrion. Am J Physiol Heart Circ Physiol: ajpheart 00520 02017, 2017. 
31. Heller S, Sheane CA, Javed Z, and Hudspeth AJ. Molecular markers for cell types of the inner ear and candidate genes for hearing disorders. Proc Natl Acad Sci U S A 95: 11400$11405,1998$.

32. Hendren CO, Mesnard X, Droge J, and Wiesner MR. Estimating production data for five engineered nanomaterials as a basis for exposure assessment. Environ Sci Technol 45: 25622569, 2011.

33. Holland NA, Thompson LC, Vidanapathirana AK, Urankar RN, Lust RM, Fennell TR, and Wingard CJ. Impact of pulmonary exposure to gold core silver nanoparticles of different size and capping agents on cardiovascular injury. Part Fibre Toxicol 13: 48, 2016.

34. Hua Z, Lv Q, Ye W, Wong CK, Cai G, Gu D, Ji Y, Zhao C, Wang J, Yang BB, and Zhang Y. MiRNA-directed regulation of VEGF and other angiogenic factors under hypoxia. PLoS One 1: e116, 2006.

35. Jagannathan R, Thapa D, Nichols CE, Shepherd DL, Stricker JC, Croston TL, Baseler WA, Lewis SE, Martinez I, and Hollander JM. Translational Regulation of the Mitochondrial Genome Following Redistribution of Mitochondrial MicroRNA in the Diabetic Heart. Circ Cardiovasc Genet 8: 785-802, 2015.

36. James JF, Hewett TE, and Robbins J. Cardiac physiology in transgenic mice. Circ Res 82: 407-415, 1998.

37. Johnson ML, Robinson MM, and Nair KS. Skeletal muscle aging and the mitochondrion. Trends Endocrinol Metab 24: 247-256, 2013.

38. Kan H, Wu Z, Young SH, Chen TH, Cumpston JL, Chen F, Kashon ML, and Castranova V. Pulmonary exposure of rats to ultrafine titanium dioxide enhances cardiac protein phosphorylation and substance P synthesis in nodose ganglia. Nanotoxicology 6: 736-745, 2012.

39. Kim SW, Kim HW, Huang W, Okada M, Welge JA, Wang Y, and Ashraf M. Cardiac stem cells with electrical stimulation improve ischaemic heart function through regulation of connective tissue growth factor and miR-378. Cardiovasc Res 100: 241-251, 2013.

40. Knezevic I, Patel A, Sundaresan NR, Gupta MP, Solaro RJ, Nagalingam RS, and Gupta M. A novel cardiomyocyte-enriched microRNA, miR-378, targets insulin-like growth factor 1 receptor: implications in postnatal cardiac remodeling and cell survival. J Biol Chem 287: 12913-12926, 2012.

41. Knuckles TL, Yi J, Frazer DG, Leonard HD, Chen BT, Castranova V, and Nurkiewicz TR. Nanoparticle inhalation alters systemic arteriolar vasoreactivity through sympathetic and cyclooxygenase-mediated pathways. Nanotoxicology 6: 724-735, 2012.

42. Krist B, Florczyk U, Pietraszek-Gremplewicz K, Jozkowicz A, and Dulak J. The Role of miR-378a in Metabolism, Angiogenesis, and Muscle Biology. Int J Endocrinol 2015: 281756, 2015.

43. Larsen S, Nielsen J, Hansen CN, Nielsen LB, Wibrand F, Stride N, Schroder HD, Boushel R, Helge JW, Dela F, and Hey-Mogensen M. Biomarkers of mitochondrial content in skeletal muscle of healthy young human subjects. J Physiol 590: 3349-3360, 2012.

44. Lewis BP, Burge CB, and Bartel DP. Conserved seed pairing, often flanked by adenosines, indicates that thousands of human genes are microRNA targets. Cell 120: 15-20, 2005. 45. Li Z, Hulderman T, Salmen R, Chapman R, Leonard SS, Young SH, Shvedova A, Luster MI, and Simeonova PP. Cardiovascular effects of pulmonary exposure to single-wall carbon nanotubes. Environ Health Perspect 115: 377-382, 2007.

46. Livak KJ and Schmittgen TD. Analysis of relative gene expression data using real-time quantitative PCR and the 2(-Delta Delta C(T)) Method. Methods 25: 402-408, 2001. 
47. Mann M, Wright PR, and Backofen R. IntaRNA 2.0: enhanced and customizable prediction of RNA-RNA interactions. Nucleic Acids Res 45: W435-W439, 2017.

48. Melman YF, Shah R, and Das S. MicroRNAs in heart failure: is the picture becoming less miRky? Circ Heart Fail 7: 203-214, 2014.

49. Mendell JT and Olson EN. MicroRNAs in stress signaling and human disease. Cell 148: 1172-1187, 2012.

50. $\quad$ Nagano T, Higashisaka K, Kunieda A, Iwahara Y, Tanaka K, Nagano K, Abe Y, Kamada H, Tsunoda S, Nabeshi H, Yoshikawa T, Yoshioka Y, and Tsutsumi Y. Liver-specific microRNAs as biomarkers of nanomaterial-induced liver damage. Nanotechnology 24: 405102, 2013.

51. Ng CT, Dheen ST, Yip WC, Ong CN, Bay BH, and Lanry Yung LY. The induction of epigenetic regulation of PROS1 gene in lung fibroblasts by gold nanoparticles and implications for potential lung injury. Biomaterials 32: 7609-7615, 2011.

52. Nichols CE, Shepherd DL, Hathaway QA, Durr AJ, Thapa D, Abukabda A, Yi J, Nurkiewicz TR, and Hollander JM. Reactive oxygen species damage drives cardiac and mitochondrial dysfunction following acute nano-titanium dioxide inhalation exposure. Nanotoxicology 12: 32-48, 2018.

53. Nichols CE, Shepherd DL, Knuckles TL, Thapa D, Stricker JC, Stapleton PA, Minarchick VC, Erdely A, Zeidler-Erdely PC, Alway SE, Nurkiewicz TR, and Hollander JM. Cardiac and mitochondrial dysfunction following acute pulmonary exposure to mountaintop removal mining particulate matter. Am J Physiol Heart Circ Physiol 309: H2017-2030, 2015.

54. Nurkiewicz TR, Porter DW, Hubbs AF, Cumpston JL, Chen BT, Frazer DG, and Castranova V. Nanoparticle inhalation augments particle-dependent systemic microvascular dysfunction. Part Fibre Toxicol 5: 1, 2008.

55. Olejniczak M, Kotowska-Zimmer A, and Krzyzosiak W. Stress-induced changes in miRNA biogenesis and functioning. Cell Mol Life Sci 75: 177-191, 2018.

56. Palmer JW, Tandler B, and Hoppel CL. Biochemical properties of subsarcolemmal and interfibrillar mitochondria isolated from rat cardiac muscle. J Biol Chem 252: 8731-8739, 1977.

57. Pavlopoulos $\mathbf{H}$ and Nihoyannopoulos $\mathbf{P}$. Strain and strain rate deformation parameters: from tissue Doppler to 2D speckle tracking. Int J Cardiovasc Imaging 24: 479-491, 2008.

58. Ruenraroengsak $\mathbf{P}$ and Tetley TD. Differential bioreactivity of neutral, cationic and anionic polystyrene nanoparticles with cells from the human alveolar compartment: robust response of alveolar type 1 epithelial cells. Part Fibre Toxicol 12: 19, 2015.

59. Sager TM, Kommineni C, and Castranova V. Pulmonary response to intratracheal instillation of ultrafine versus fine titanium dioxide: role of particle surface area. Part Fibre Toxicol 5: 17, 2008.

60. Sato F, Tsuchiya S, Meltzer SJ, and Shimizu K. MicroRNAs and epigenetics. FEBS J 278: 1598-1609, 2011.

61. Schaeren-Wiemers $\mathbf{N}$ and Gerfin-Moser A. A single protocol to detect transcripts of various types and expression levels in neural tissue and cultured cells: in situ hybridization using digoxigenin-labelled cRNA probes. In: Histochemistry, 1993, p. 431-440.

62. Shepherd DL, Hathaway QA, Pinti MV, Nichols CE, Durr AJ, Sreekumar S, Hughes KM, Stine SM, Martinez I, and Hollander JM. Exploring the mitochondrial microRNA import pathway through Polynucleotide Phosphorylase (PNPase). J Mol Cell Cardiol 110: 15-25, 2017.

63. Shepherd DL, Nichols CE, Croston TL, McLaughlin SL, Petrone AB, Lewis SE, Thapa D, Long DM, Dick GM, and Hollander JM. Early detection of cardiac dysfunction in 
the type 1 diabetic heart using speckle-tracking based strain imaging. J Mol Cell Cardiol 90: 7483, 2016.

64. Shukla GC, Singh J, and Barik S. MicroRNAs: Processing, Maturation, Target Recognition and Regulatory Functions. Mol Cell Pharmacol 3: 83-92, 2011.

65. Snyder-Talkington BN, Dong C, Sargent LM, Porter DW, Staska LM, Hubbs AF, Raese R, McKinney W, Chen BT, Battelli L, Lowry DT, Reynolds SH, Castranova V, Qian Y, and Guo NL. mRNAs and miRNAs in whole blood associated with lung hyperplasia, fibrosis, and bronchiolo-alveolar adenoma and adenocarcinoma after multi-walled carbon nanotube inhalation exposure in mice. J Appl Toxicol 36: 161-174, 2016.

66. St-Pierre J, Lin J, Krauss S, Tarr PT, Yang R, Newgard CB, and Spiegelman BM. Bioenergetic analysis of peroxisome proliferator-activated receptor gamma coactivators 1alpha and 1beta (PGC-1alpha and PGC-1beta) in muscle cells. J Biol Chem 278: 26597-26603, 2003.

67. Stapleton PA, Hathaway QA, Nichols CE, Abukabda AB, Pinti MV, Shepherd DL, McBride CR, Yi J, Castranova VC, Hollander JM, and Nurkiewicz TR. Maternal engineered nanomaterial inhalation during gestation alters the fetal transcriptome. Part Fibre Toxicol 15: 3, 2018.

68. Stapleton PA, Minarchick VC, Cumpston AM, McKinney W, Chen BT, Sager TM, Frazer DG, Mercer RR, Scabilloni J, Andrew ME, Castranova V, and Nurkiewicz TR. Impairment of coronary arteriolar endothelium-dependent dilation after multi-walled carbon nanotube inhalation: a time-course study. Int J Mol Sci 13: 13781-13803, 2012.

69. Stapleton PA, Nichols CE, Yi J, McBride CR, Minarchick VC, Shepherd DL, Hollander JM, and Nurkiewicz TR. Microvascular and mitochondrial dysfunction in the female F1 generation after gestational TiO2 nanoparticle exposure. Nanotoxicology 9: 941-951, 2015.

70. Thapa D, Nichols CE, Lewis SE, Shepherd DL, Jagannathan R, Croston TL, Tveter KJ, Holden AA, Baseler WA, and Hollander JM. Transgenic overexpression of mitofilin attenuates diabetes mellitus-associated cardiac and mitochondria dysfunction. $J$ Mol Cell Cardiol 79: 212-223, 2015.

71. Thomson DW and Dinger ME. Endogenous microRNA sponges: evidence and controversy. Nat Rev Genet 17: 272-283, 2016.

72. Wang J, Khoury DS, Thohan V, Torre-Amione G, and Nagueh SF. Global diastolic strain rate for the assessment of left ventricular relaxation and filling pressures. Circulation 115: 1376-1383, 2007.

73. Wang P, Gu Y, Zhang Q, Han Y, Hou J, Lin L, Wu C, Bao Y, Su X, Jiang M, Wang $\mathbf{Q}, \mathbf{L i} \mathbf{N}$, and Cao X. Identification of resting and type I IFN-activated human NK cell miRNomes reveals microRNA-378 and microRNA-30e as negative regulators of NK cell cytotoxicity. $J$ Immunol 189: 211-221, 2012.

74. Wilkinson DG. In Situ Hybridization: A Practical Approach: Oxford University Press, 1999.

75. Williamson CL, Dabkowski ER, Baseler WA, Croston TL, Alway SE, and Hollander JM. Enhanced apoptotic propensity in diabetic cardiac mitochondria: influence of subcellular spatial location. Am J Physiol Heart Circ Physiol 298: H633-642, 2010.

76. Wright PR, Georg J, Mann M, Sorescu DA, Richter AS, Lott S, Kleinkauf R, Hess WR, and Backofen R. CopraRNA and IntaRNA: predicting small RNA targets, networks and interaction domains. Nucleic Acids Res 42: W119-123, 2014. 
77. Yu F, Yang J, Huang K, Pan X, Chen B, Dong P, and Zheng J. The EpigeneticallyRegulated microRNA-378a Targets TGF-beta2 in TGF-beta1-Treated Hepatic Stellate Cells. Cell Physiol Biochem 40: 183-194, 2016.

78. Zuker M. Mfold web server for nucleic acid folding and hybridization prediction. Nucleic Acids Res 31: 3406-3415, 2003. 
Table 2.1: M-Mode Cardiac Function

\begin{tabular}{|c|c|c|c|c|c|c|c|}
\hline Calculation & Units & WT Sham ${ }^{1}$ & $\mathbf{W T} \mathbf{E x ^ { 2 }}$ & Het Sham ${ }^{3}$ & Het $\mathbf{E x}^{4}$ & KO Sham 5 & KO Ex ${ }^{6}$ \\
\hline $\mathrm{CO}$ & $\mathrm{mL} / \mathrm{min}$ & $13.04 \pm 1.15$ & $11.00 \pm 1.41$ & $12.11 \pm 2.22$ & $12.79 \pm 1.97$ & $12.34 \pm 0.82$ & $14.35 \pm 0.62$ \\
\hline Diameter;d & $\mathrm{mm}$ & $2.33 \pm 0.06$ & $2.15 \pm 0.13$ & $2.52 \pm 0.15$ & $2.58 \pm 0.14$ & $2.62 \pm 0.07$ & $2.82 \pm 0.29$ \\
\hline Diameter;s & $\mathrm{mm}$ & $1.01 \pm 0.03$ & $1.03 \pm 0.08$ & $0.83 \pm 0.09$ & $0.85 \pm 0.08$ & $0.79 \pm 0.08$ & $0.99 \pm 0.21$ \\
\hline $\mathbf{E F}$ & $\%$ & $87.39 \pm 1.16$ & $\begin{array}{c}84.17 \pm 1.75 \\
6\end{array}$ & $89.07 \pm 1.54$ & $87.43 \pm 1.89$ & $90.05 \pm 0.79$ & $91.60 \pm 0.45$ \\
\hline FS & $\%$ & $56.44 \pm 1.62$ & $\begin{array}{c}52.14 \pm 2.11 \\
6\end{array}$ & $57.88 \pm 2.06$ & $55.86 \pm 2.50$ & $59.86 \pm 0.80$ & $62.96 \pm 2.74$ \\
\hline LV Mass & $\mathrm{mg}$ & $\begin{array}{c}108.88 \pm \\
10.24\end{array}$ & $\begin{array}{c}111.87 \pm \\
10.05\end{array}$ & $100.21 \pm 6.94$ & $132.61 \pm 8.47$ & $\begin{array}{c}122.29 \pm \\
10.26\end{array}$ & $108.32 \pm 9.97$ \\
\hline $\begin{array}{l}\text { LV Mass } \\
\text { Corr }\end{array}$ & $\mathrm{mg}$ & $87.10 \pm 8.20$ & $89.50 \pm 8.04$ & $80.17 \pm 5.55$ & $106.08 \pm 6.78$ & $97.84 \pm 8.21$ & $86.66 \pm 7.97$ \\
\hline SV & $\mathrm{uL}$ & $27.16 \pm 1.38$ & $22.06 \pm 2.62$ & $22.24 \pm 3.19$ & $23.62 \pm 3.26$ & $24.22 \pm 1.46$ & $31.49 \pm 4.82$ \\
\hline$V ; d$ & $\mathrm{uL}$ & $30.86 \pm 1.44$ & $29.65 \pm 1.92$ & $25.13 \pm 3.13$ & $25.18 \pm 2.13$ & $25.41 \pm 1.66$ & $29.66 \pm 2.82$ \\
\hline $\mathbf{V} ; \mathbf{s}$ & $\mathrm{uL}$ & $\begin{array}{c}2.63 \pm 0.36 \\
3,4,5,6\end{array}$ & $\begin{array}{c}2.93 \pm 0.29 \\
3,4,5,6\end{array}$ & $1.45 \pm 0.40$ & $1.55 \pm 0.28$ & $1.19 \pm 0.31$ & $1.68 \pm 0.14$ \\
\hline
\end{tabular}


Table 2.1: Ultrasound imaging following nano- $\mathrm{TiO}_{2}$ inhalation exposure. Measurements were taken for at least three consecutive systolic and diastolic peaks and troughs, respectively, for each animal. Significance $(P \leq 0.05)$ is denoted for a specific category through bold text and reference to the sample number that it is significantly different from. Sample numbers are listed in the column heading. $\mathrm{WT}=$ wild type, $\mathrm{Het}=$ heterozygous for the miRNA-378a allele, $\mathrm{KO}=$ knockout for the miRNA-378a allele, Sham $=$ control filtered air exposed, $\mathrm{Ex}=$ nano- $\mathrm{TiO}_{2}$ exposed, $\mathrm{CO}=$ cardiac output, Diameter; $\mathrm{d}=$ diastolic diameter, Diameter;s $=$ systolic diameter, $\mathrm{EF}=$ ejection fraction, FS = fractional shortening, LV Mass = left ventricular mass, LV Mass Corr $=$ left ventricular mass corrected, $\mathrm{SV}=$ stroke volume, $\mathrm{V} ; \mathrm{d}=$ volume during diastole, $\mathrm{V} ; \mathrm{s}=$ volume during systole. 
Figure 2.1
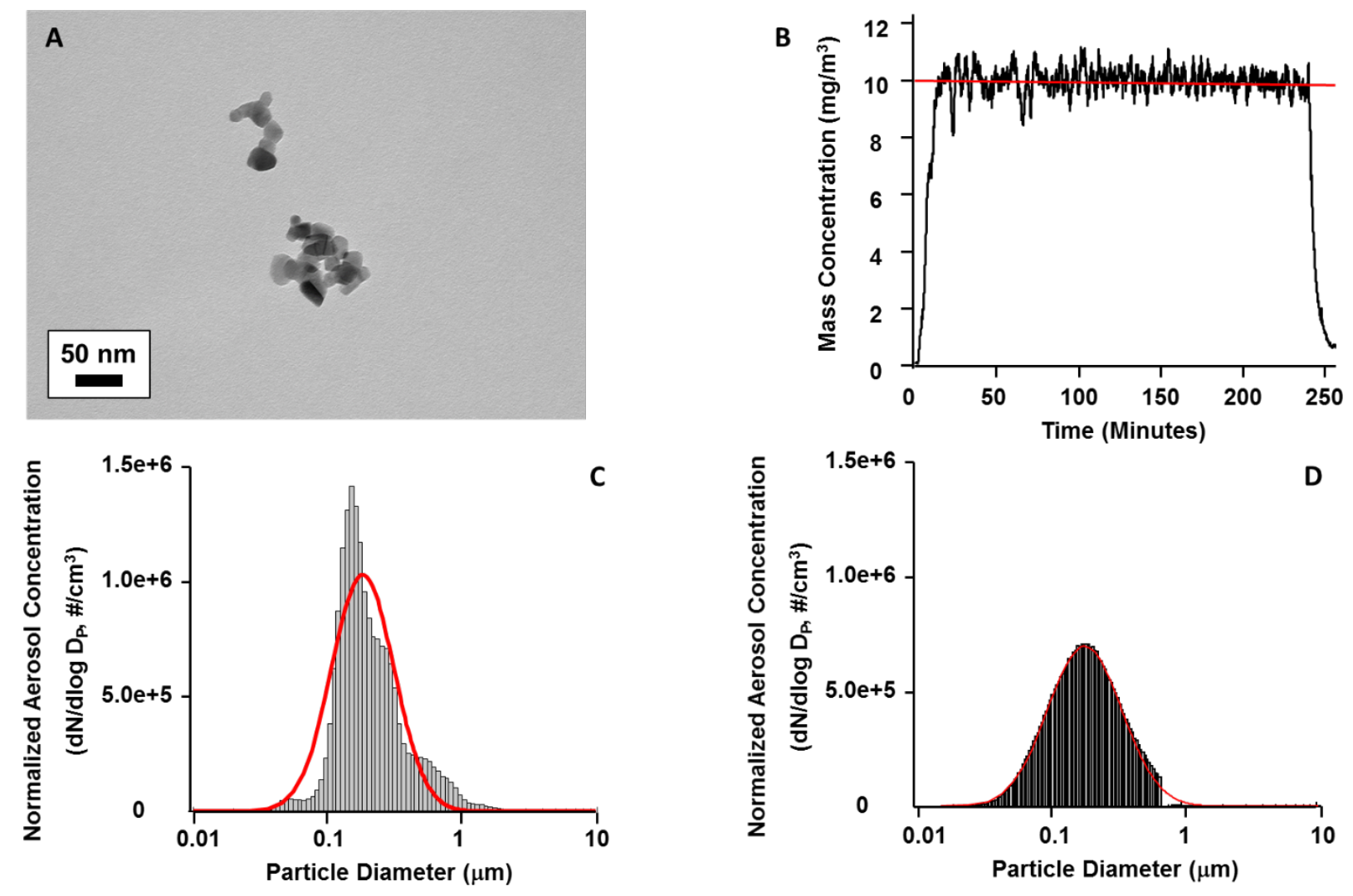
Figure 2.1: $\mathrm{Nano}_{-} \mathrm{TiO}_{2}$ aerosol characterizations. (A) Representative transmission electron micrograph of aerosolized agglomerates collected on a filter. Bar is $50 \mathrm{~nm}$. (B) Real-time estimation of the aerosol mass concentration measured with a light scattering device. The red line represents the target concentration of $10 \mathrm{mg} / \mathrm{m}^{3}$. The black line is the measured concentration during the exposure period. Note that computerized feedback system (see Methods) maintains a stable concentration. Mean DataRAM aerosol concentration during this exposure was $9.88 \pm 0.03$ $\mathrm{mg} / \mathrm{m}^{3}$. Data was sampled every 2 seconds. (C) High resolution ELPI aerodynamic diameter measurements. The red line designates the log normal distribution obtained with the log probability plot method. This produced a count median diameter of $182 \mathrm{~nm}$ with a geometric standard deviation of 1.70. (D) SMPS (light gray bars) and APS (dark gray bars) mobility diameter measurements. The red line designates the log normal distribution obtained with the log probability plot method. This produced a count median diameter of $190 \mathrm{~nm}$ with a geometric standard deviation of 1.88. Note: APS bar values are nominal and difficult to visualize. 
Figure 2.2

A miRnA-378a-3p - Tissue

miRNA-378a-5p - Tissue
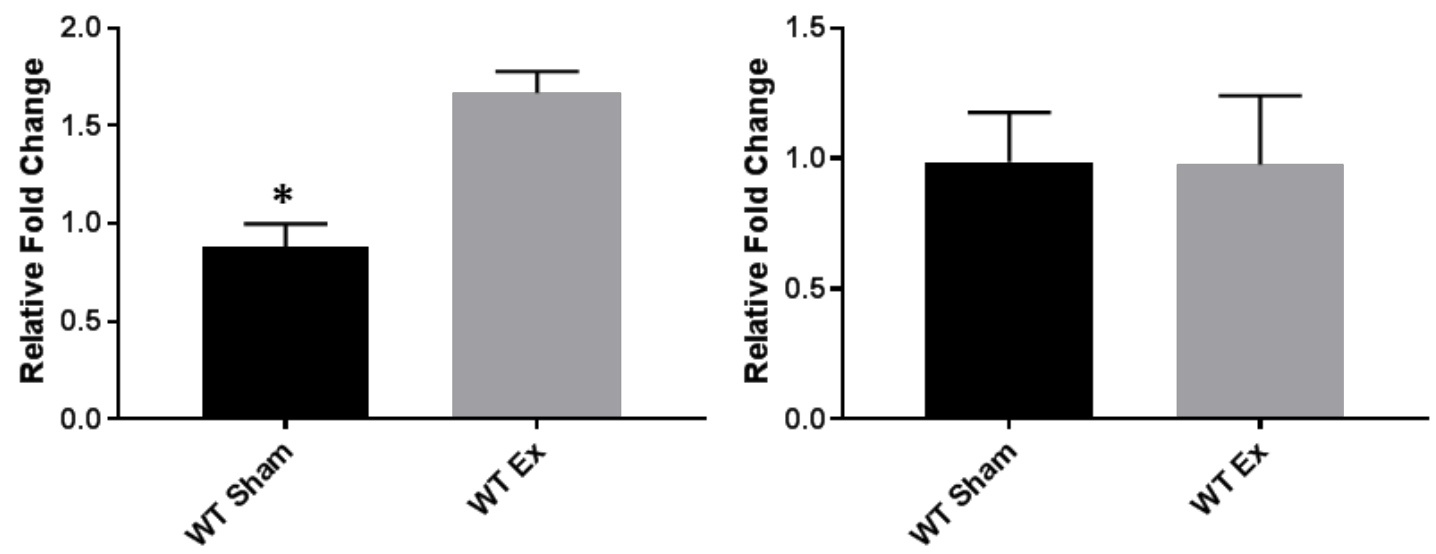

B

WT Sham - miRNA-378a-3p

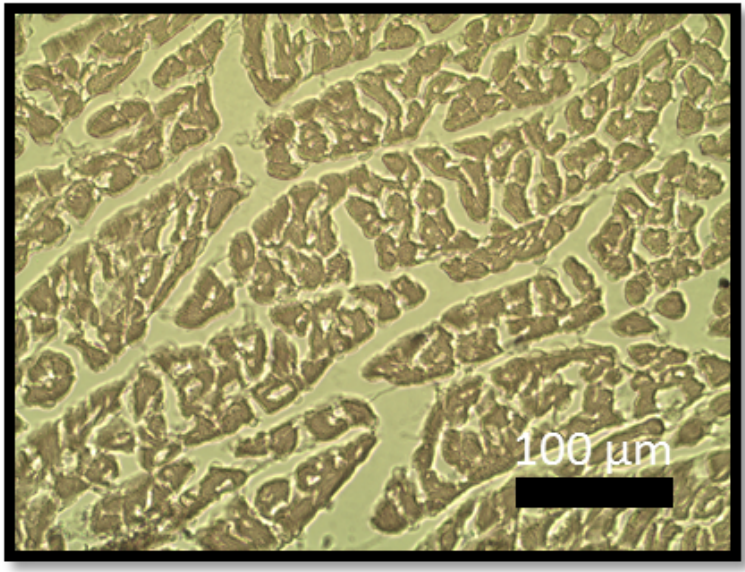

WT Ex - miRNA-378a-3p
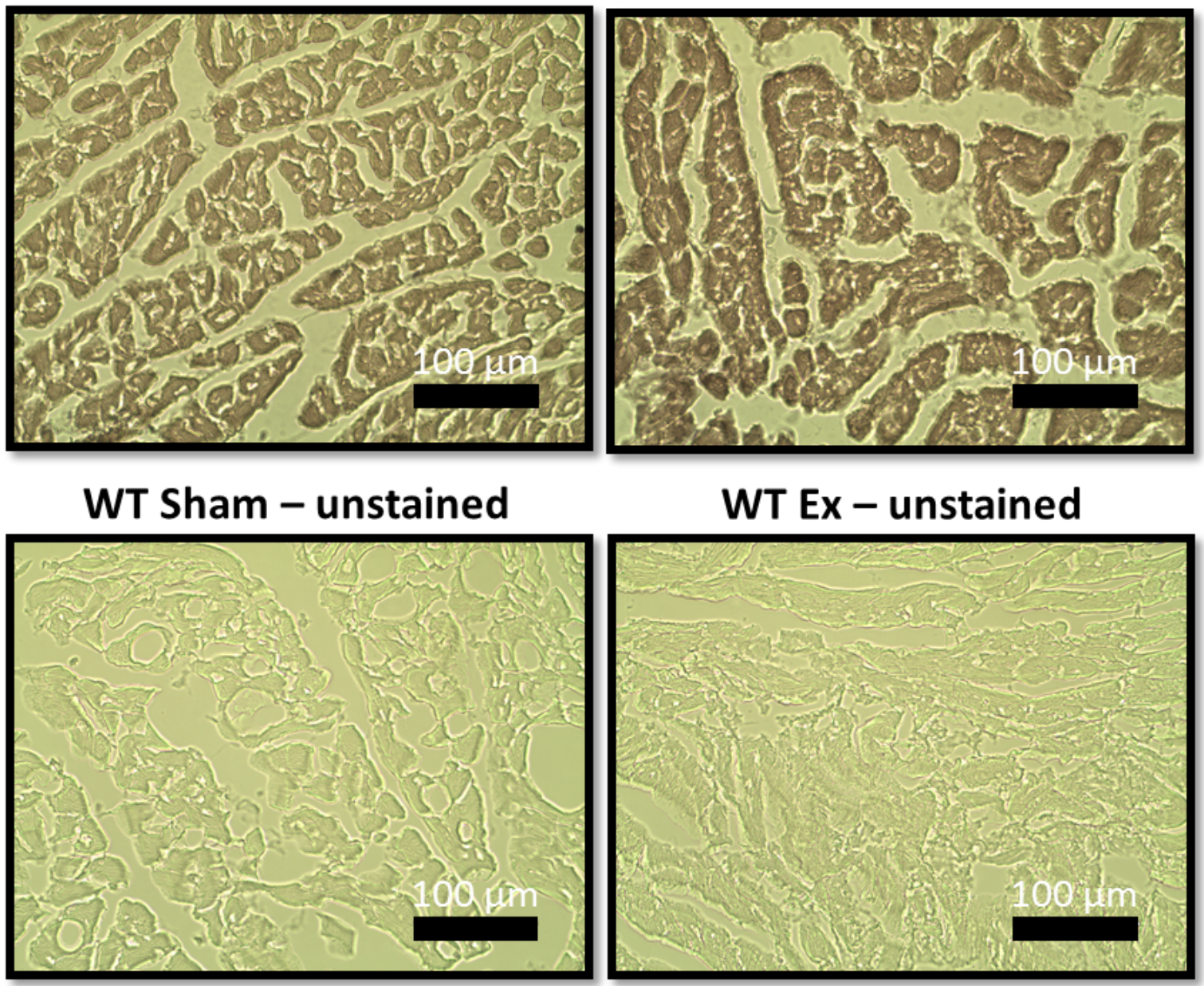

WT Ex - unstained

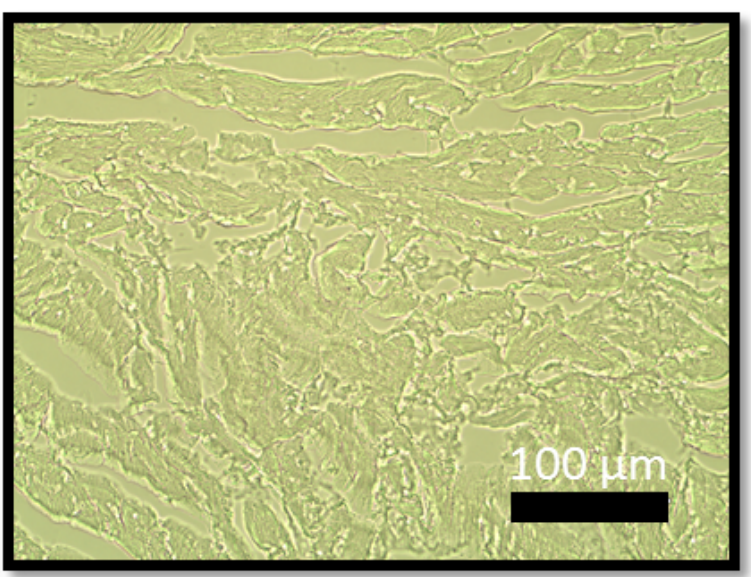


Figure 2.2

C

miRNA-378a-3p - Mitochondria

miRNA-378a-5p - Mitochondria
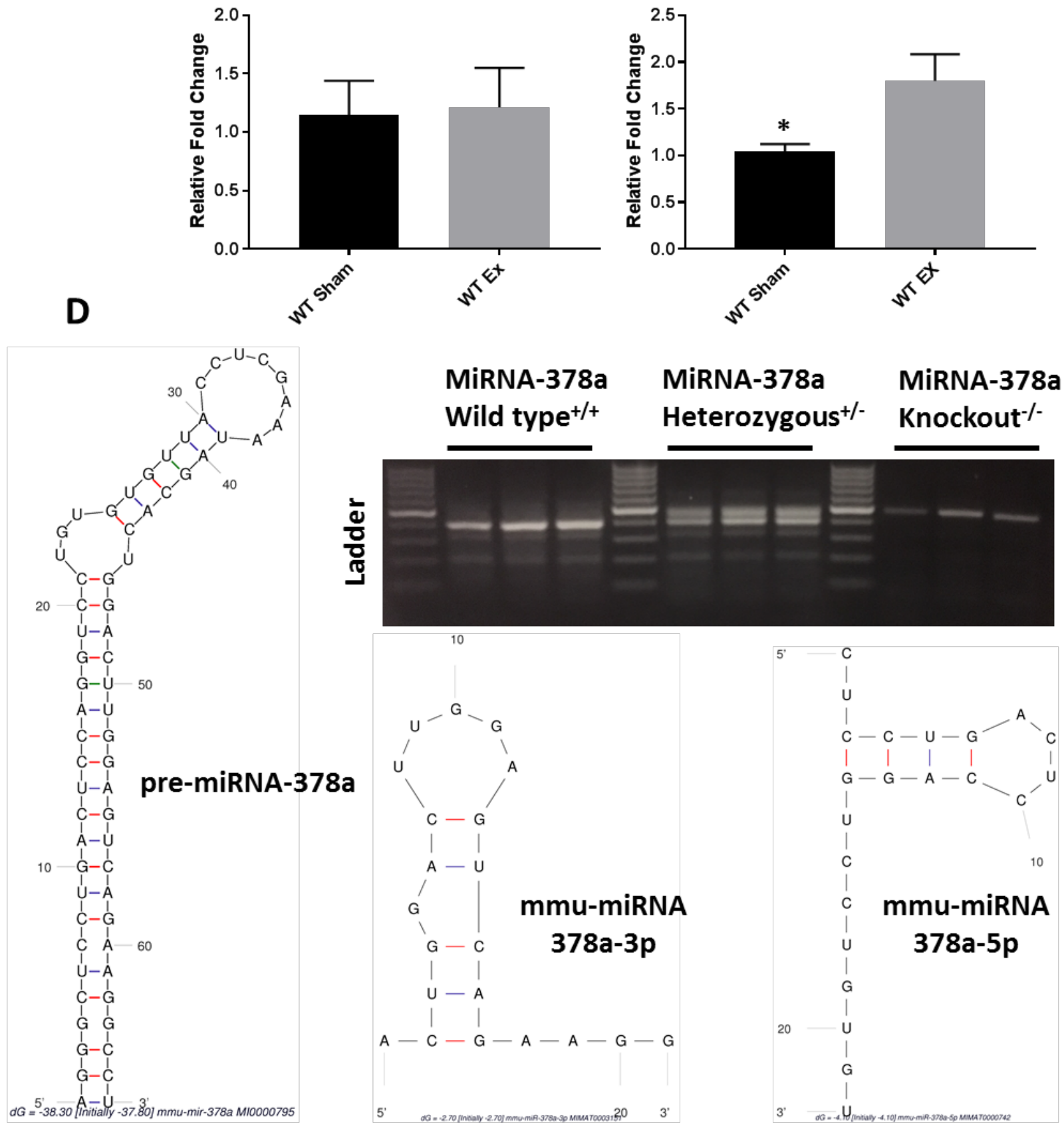
Figure 2.2: Expression of miRNA-378a following nano- $\mathrm{TiO}_{2}$ inhalation and miRNA-378a animal models. Following inhalation exposure to control filtered air or nano- $\mathrm{TiO}_{2} \mathrm{qPCR}$ was implemented to assess miRNA-378a-3p and miRNA-378a-5p expression in (A) whole heart tissue. (B) Representative images of in situ hybridization of miRNA-378a-3p in cardiac tissue performed under light microscopy ( $\mathrm{n}=4$ for both groups). (C) qPCR was also used to measure miRNA-378a$3 p$ and miRNA-378a-5p expression in isolated mitochondria (D) Genotyping through PCR amplification and agarose gel electrophoresis for the miRNA-378a loci in WT, Het, and KO animals. Additionally, RNA folding software Mfold evaluated the likely folding characteristics of the miRNA-378a species. Groups are considered significantly different if $P \leq 0.05=*$. All data are presented as the mean \pm standard error of the mean $(\mathrm{SEM}) . \mathrm{WT}=$ wild type, Sham $=$ control filtered air exposed, $\mathrm{Ex}=$ nano- $\mathrm{TiO}_{2}$ exposed. 
Figure 2.3

A
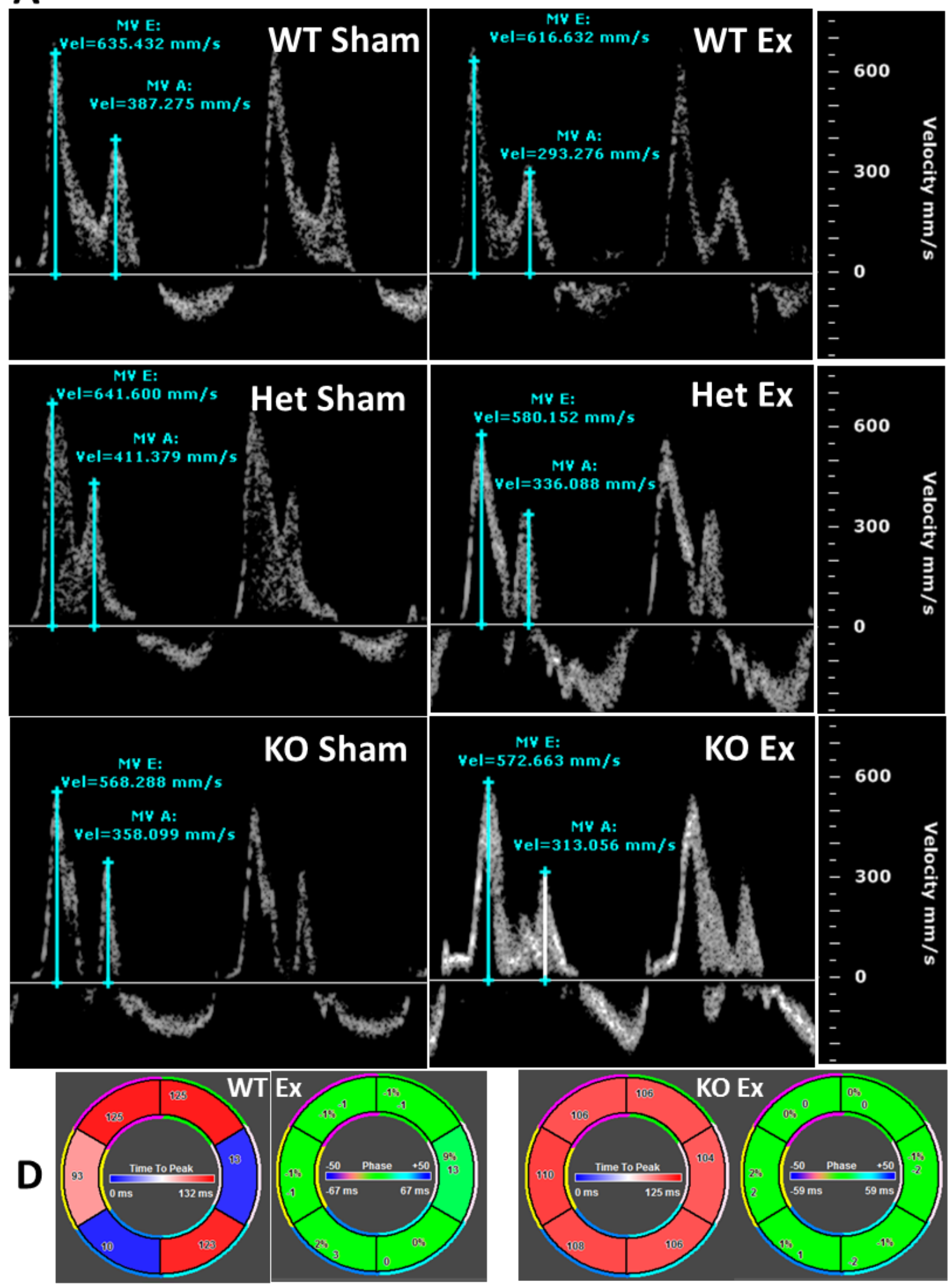
Figure 2.3

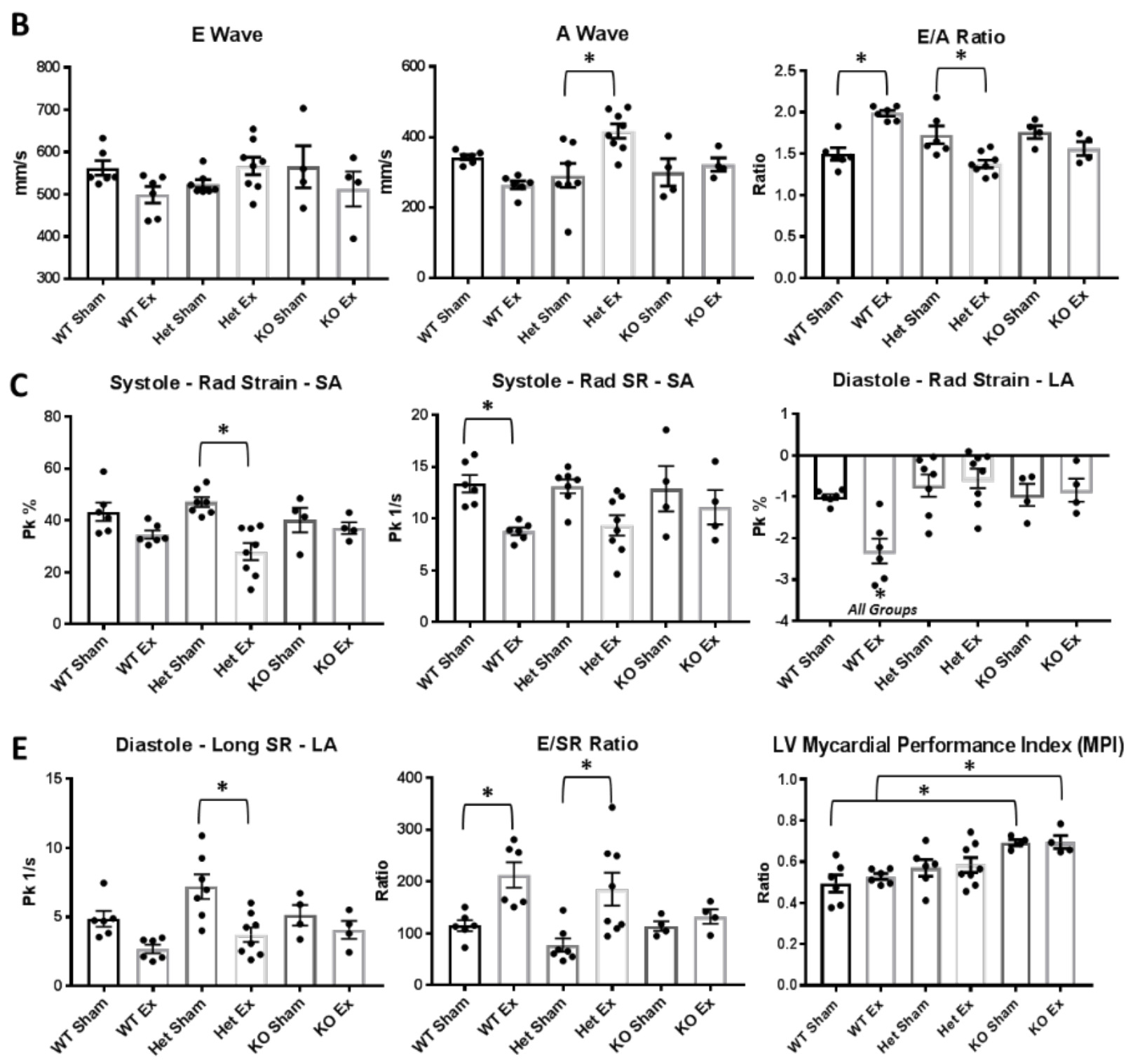


Figure 2.3: Cardiac function following nano- $\mathrm{TiO}_{2}$ inhalation exposure. (A) $\mathrm{PW}$ Doppler images illustrate changes in E and A wave velocities following exposure. (B) E and A wave velocities, as well as E/A wave ratios, are represented from PW Doppler imaging. Speckle-tracking analyses from B-Mode short and long axis images for (C) systolic and diastolic strain and strain rate. (D) An illustration of dyssynchrony of the endocardium during short axis systolic radial stain (indicated by color) in the WT nano- $\mathrm{TiO}_{2}$ exposed animals compared to conserved strain parameters in the $\mathrm{KO}$ exposed animals. (E) The diastolic longitudinal strain rate coupled with the E wave velocities provided a prognostic cardiac health index for the left ventricle (LV), while the LV myocardial performance index (MPI) further provided a prognostic index of the left ventricular ejection parameters. Groups are considered significantly different if $P \leq 0.05=*$. All data are presented as the mean \pm standard error of the mean $(\mathrm{SEM}) . \mathrm{WT}=$ wild type, Het $=$ heterozygous for the miRNA-378a allele, $\mathrm{KO}=$ knockout for the miRNA-378a allele, Sham $=$ control filtered air exposed, $\mathrm{Ex}=$ nano- $\mathrm{TiO}_{2}$ exposed, $\mathrm{SA}=$ left ventricular short axis, $\mathrm{LA}=$ left ventricular long axis, Long $=$ longitudinal, $\mathrm{Rad}=$ radial . 
Figure 2.4
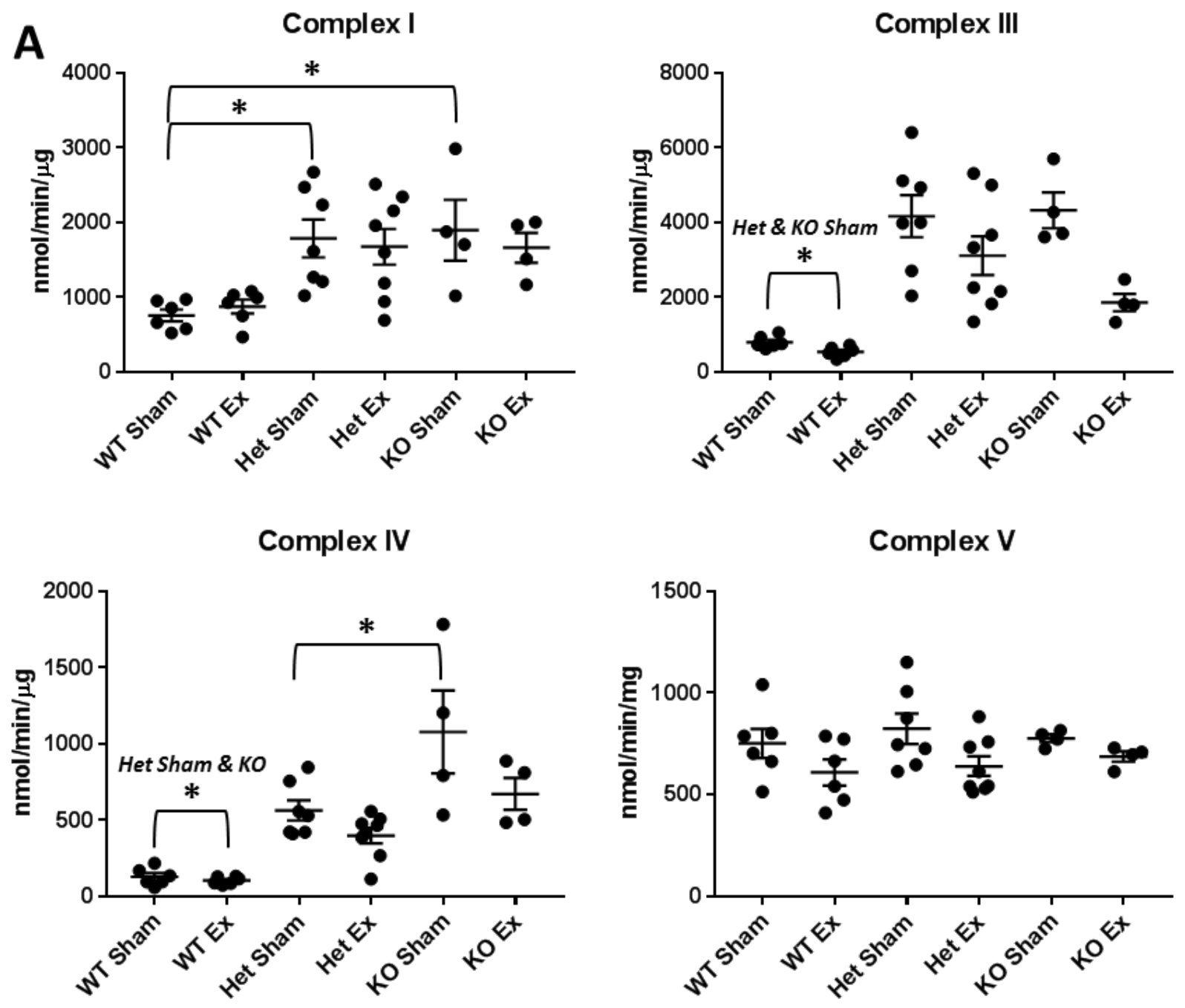
Figure 2.4

B

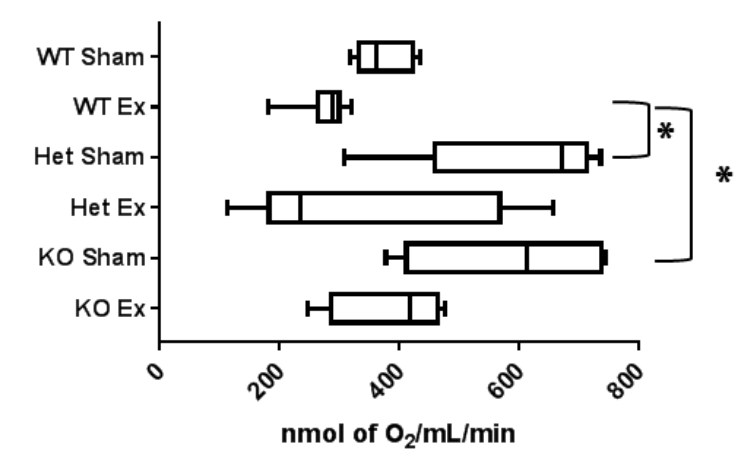

Glutamine - State 4

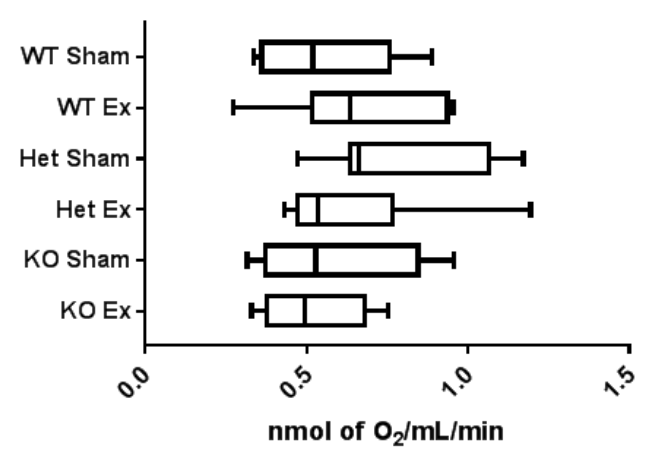

C P-Carnitine - State 3

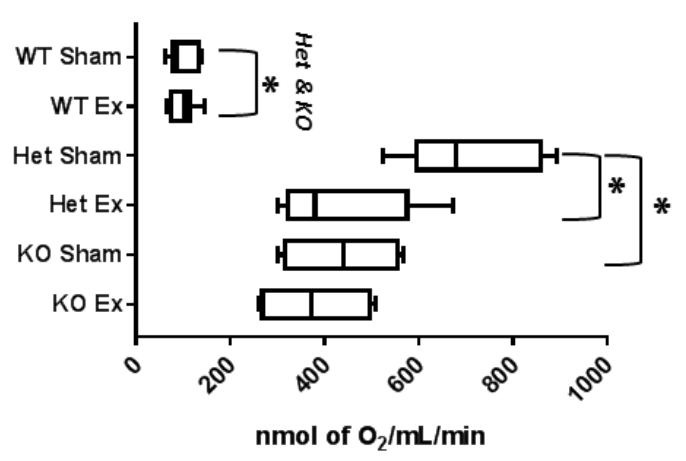

P-Carnitine - State 4

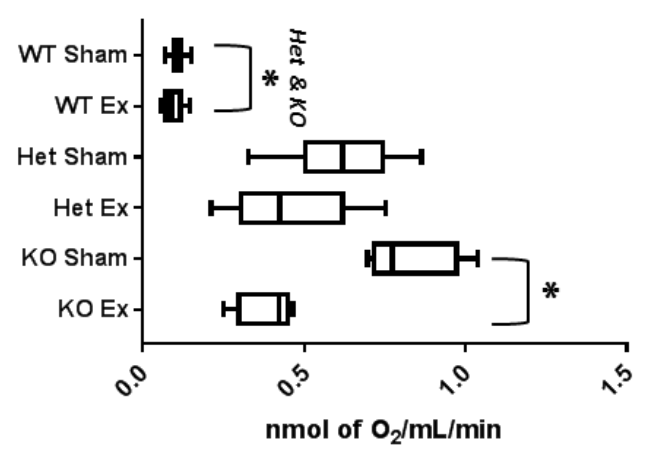


Figure 2.4
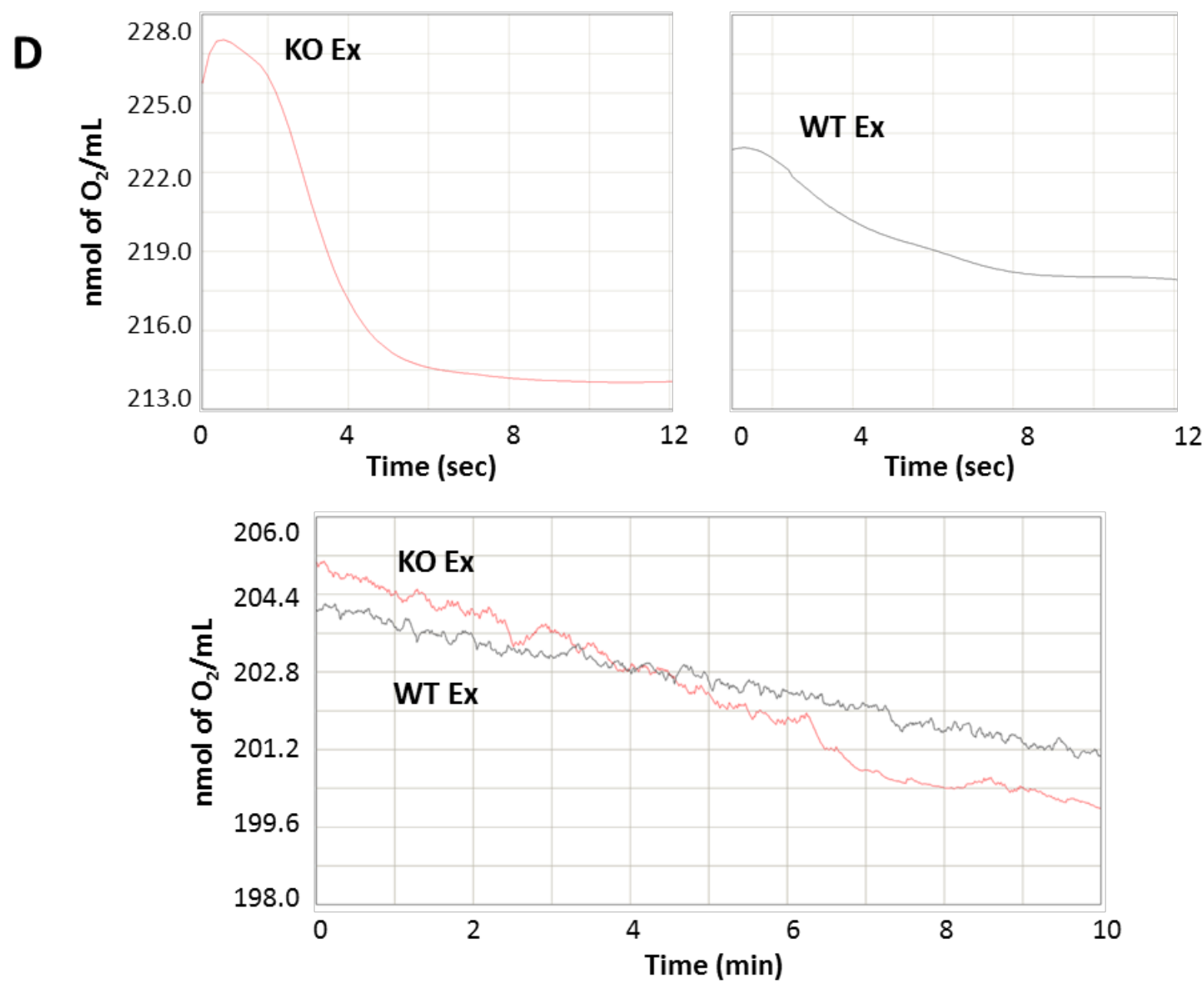
Figure 2.4: Bioenergetic function of cardiac mitochondria following nano- $\mathrm{TiO}_{2}$ inhalation exposure. (A) Electron transport chain complex activities were measured for complexes I, III, IV, and V. Mitochondrial State 3 and State 4 respiration were measured through (B) glucose mediated pathways and (C) fatty acid mediated pathways. (D) State 3 and State 4 respiration changes between a representative $\mathrm{WT}$ and $\mathrm{KO}$ animal illustrates changes in oxygen consumption when using p-carnitine for fatty acid mediated respiration. Groups are considered significantly different if $P \leq 0.05=*$. All data are presented as the mean \pm standard error of the mean $(\mathrm{SEM}) . \mathrm{WT}=$ wild type, $\mathrm{Het}=$ heterozygous for the miRNA-378a allele, $\mathrm{KO}=$ knockout for the miRNA-378a allele, Sham $=$ control filtered air exposed, $\mathrm{Ex}=$ nano- $\mathrm{TiO}_{2}$ exposed, $\mathrm{P}-$ Carnitine $=$ palmitoyl carnitine. 
Figure 2.5
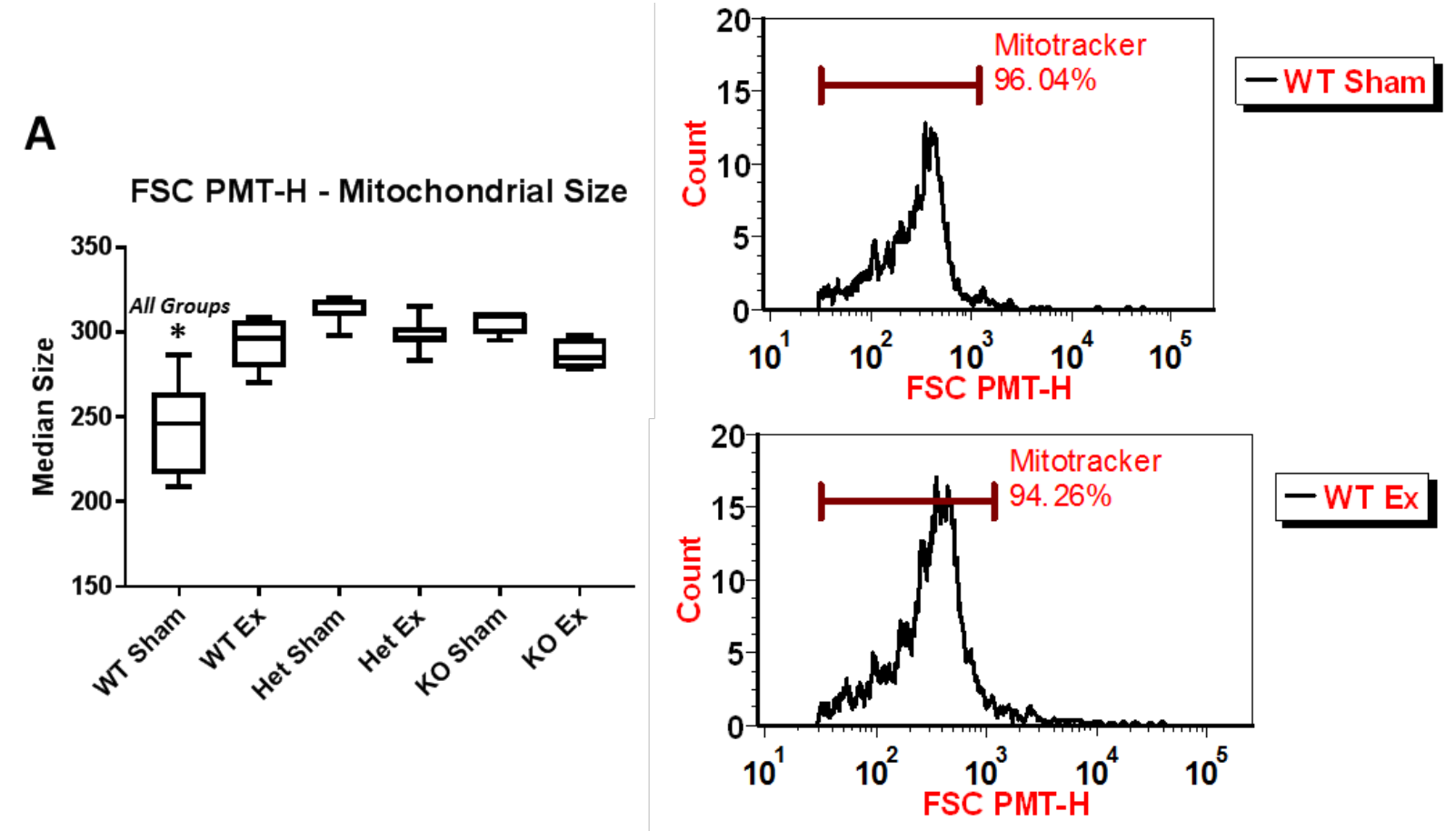
Figure 2.5
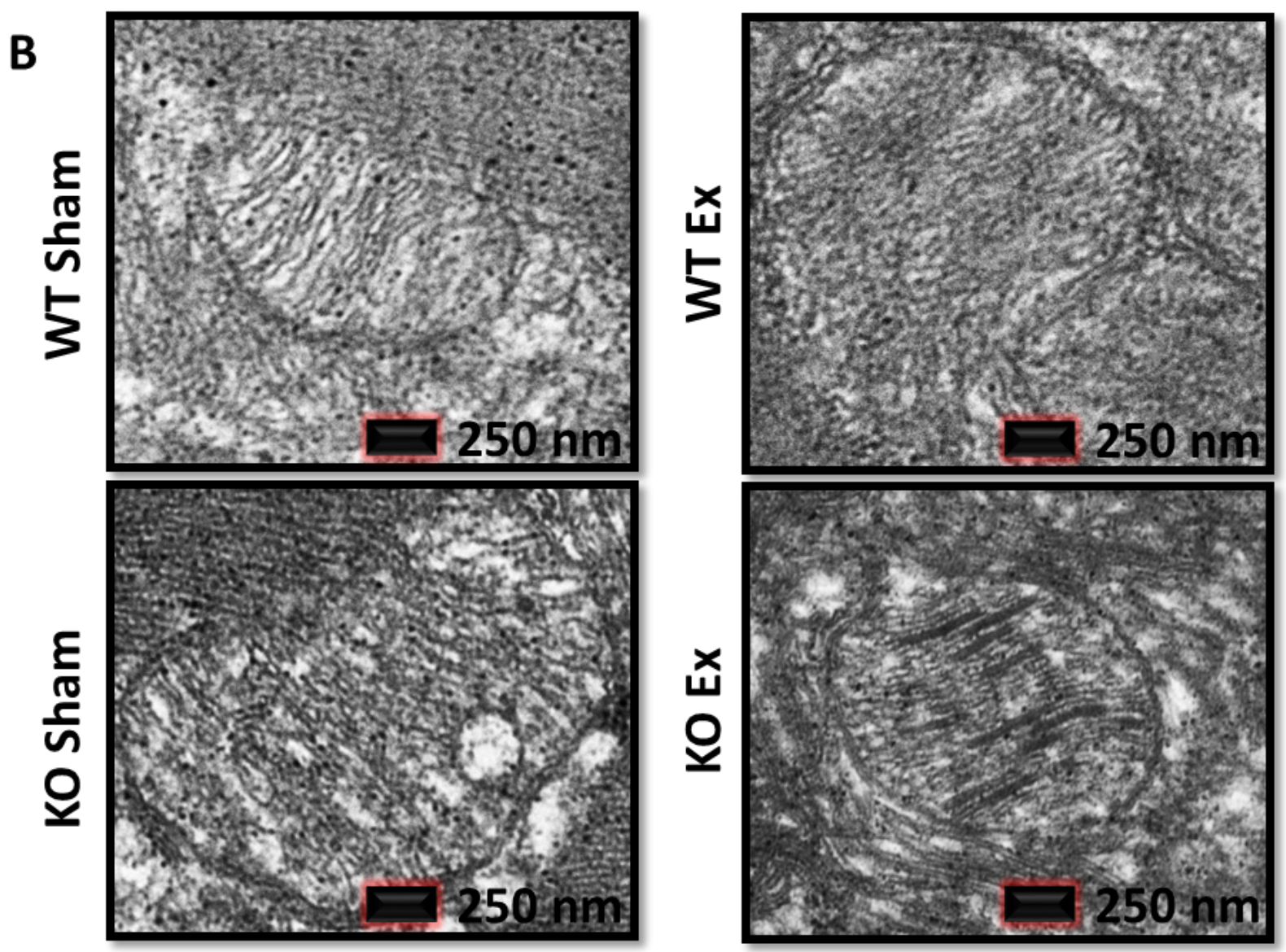
Figure 2.5

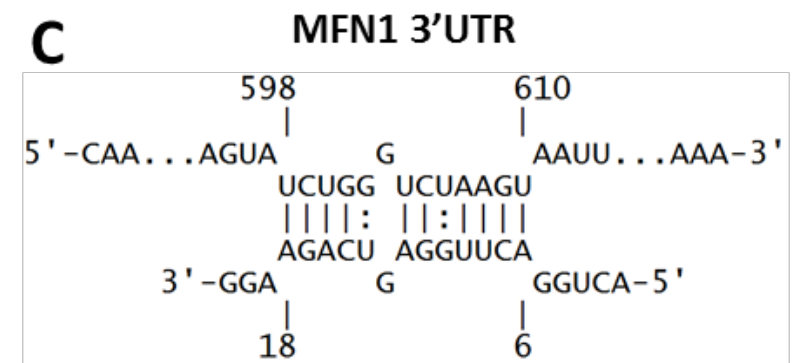

Interaction Energy $=-9.01139 \mathrm{kcal} / \mathrm{mol}$

Mfn1 3'UTR
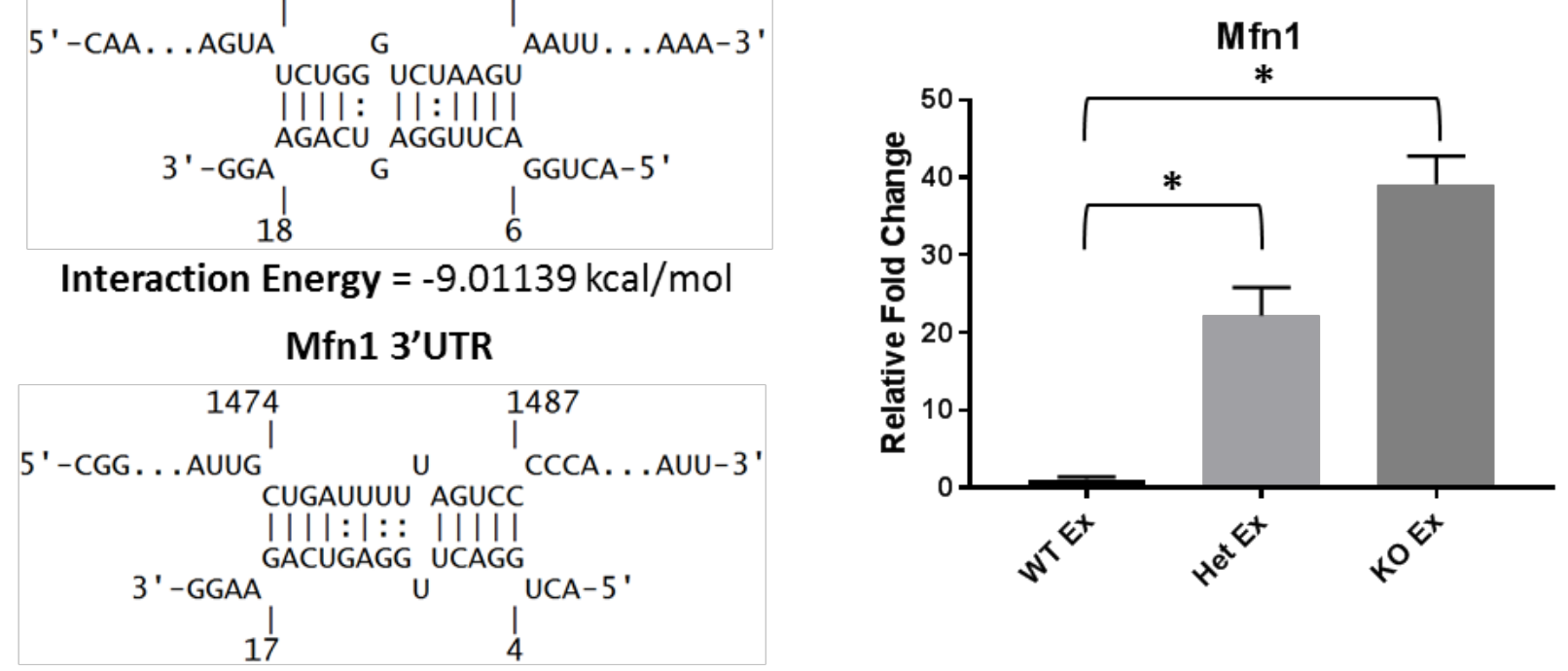

Interaction Energy $=-8.20488 \mathrm{kcal} / \mathrm{mol}$
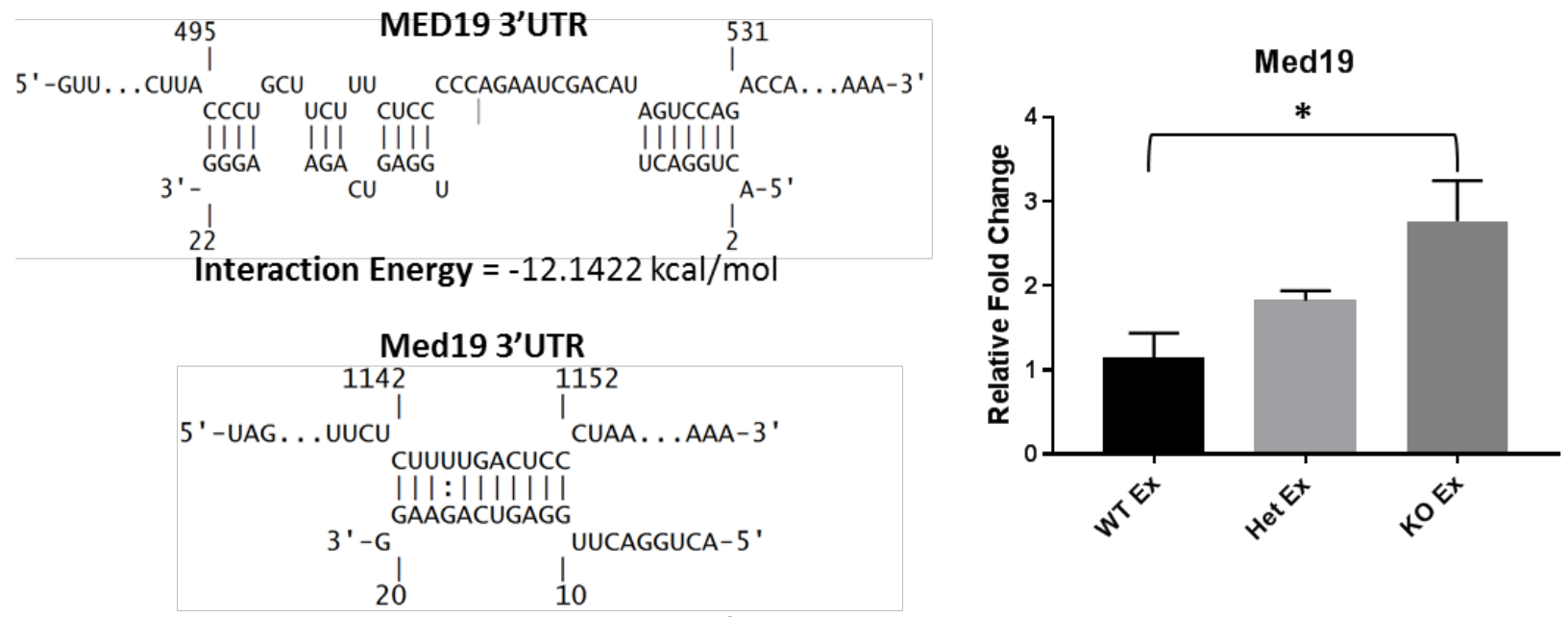

Interaction Energy $=-11.8936 \mathrm{kcal} / \mathrm{mol}$ 
Figure 2.5: Mechanisms governing mitochondrial function and molecular targets of miRNA-378a following inhalation exposure. (A) Mitochondrial size was determined through forward scatter, gating mitochondria positively stained with MitoTracker ${ }^{\mathrm{TM}}$ Deep Red FM/633; representative images of the WT sham and nano- $\mathrm{TiO}_{2}$ exposure are illustrated. (B) Representative Transmission Electron Microscopy (TEM) images depicting changes to mitochondrial size $(\mathrm{n}=1$ for each group). (C) Binding interactions and qPCR for both human (MFN1 and MED19) and mouse (Mfn1 and Med19) using IntaRNA 2.0. Interaction energies are calculated as binding propensity through free energy binding near the seed sequence region. Groups are considered significantly different if $P \leq 0.05=*$. All data are presented as the mean \pm standard error of the mean $(\mathrm{SEM}) . \mathrm{WT}=$ wild type, Het $=$ heterozygous for the miRNA-378a allele, $\mathrm{KO}=$ knockout for the miRNA-378a allele, Sham $=$ control filtered air exposed, $\mathrm{Ex}=$ nano- $\mathrm{TiO}_{2}$ exposed, FSC PMT-H $=$ forward scatter, Mfn1 = mitofusin 1, Med19 = mediator complex subunit 19, 3' UTR = 3' untranslated region. 
Figure 2.6

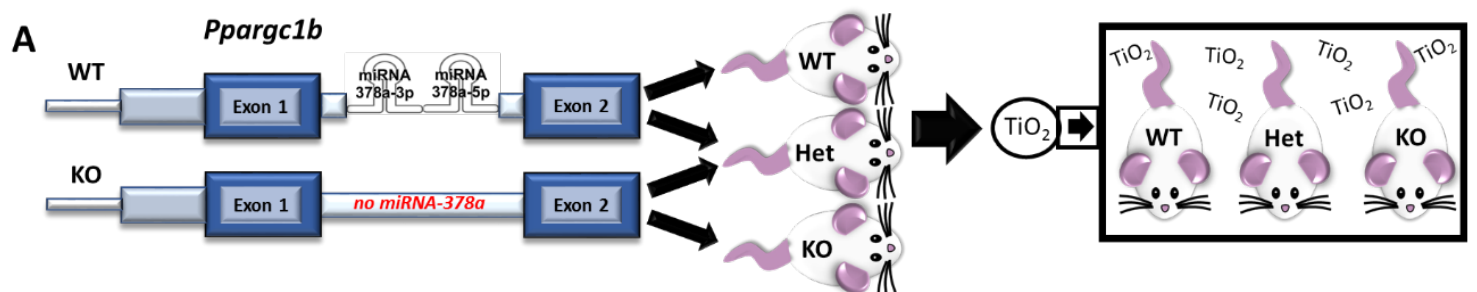

B

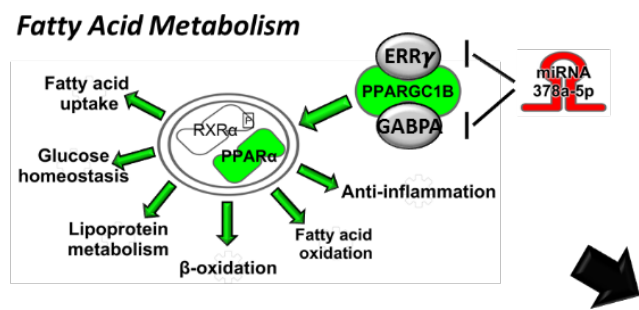

Bioenergetics
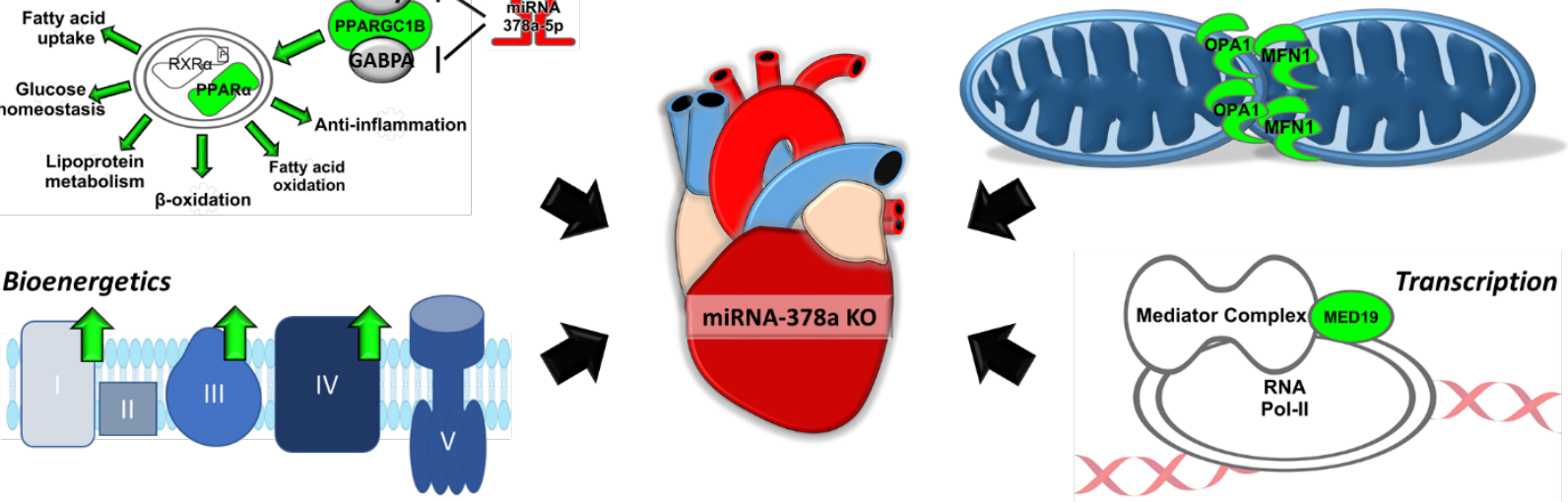
Figure 2.6: General schema of miRNA-378a interactions following inhalation exposure. (A) Transgenic mouse model, illustrating miRNA-378a wild type and knockout alleles. Mice were exposed to a total nano- $\mathrm{TiO}_{2}$ deposition of $9.88 \pm 0.03 \mathrm{mg} / \mathrm{m}^{3}$. Cardiac issue was excised for subsequent experimental procedures. (B) Molecular changes in the $\mathrm{KO}$ nano- $\mathrm{TiO}_{2}$ exposed heart compared to WT exposed. Med19 was shown to be significantly elevated, an important cofactor in regulating cellular transcription. Metabolically, fatty acid metabolism and electron transport chain complex activities were elevated, likely through mediators such as Ppar $\alpha$ and Ppargc1b and enhanced mitochondrial ultrastructure. Increased mitochondrial fusion (Mfn1 and Opa1) contributes to the integrity of mitochondria following exposure. These molecular pathways altered with the repression of miRNA-378a contribute to elevated cardiac function in KO compared to the WT mice. WT $=$ wild type, Het $=$ heterozygous for the miRNA-378a allele, $\mathrm{KO}=$ knockout for the miRNA-378a allele, Mfn1 = mitofusin 1, Med19 = mediator complex subunit 19, 3' UTR = 3' untranslated region, OPA1 = optic atrophy $1, \operatorname{PPAR} \alpha=$ peroxisome proliferator-activated receptor alpha, Ppargc1b or PGC-1 $\beta$ = peroxisome proliferator-activated receptor gamma coactivator 1beta. 
Table S2.1

\begin{tabular}{lll}
\hline Gene Name & Sequence & Orientation \\
Ppargc1b & CAGCCTCAGTTCCAGAAGTCAG & Forward \\
Ppargc1b & CACCGAAGTGAGGTGCTTATGC & Reverse \\
Mfn1 & CCAGGTACAGATGTCACCACAG & Forward \\
Mfn1 & TTGGAGAGCCGCTCATTCACCT & Reverse \\
Med19 & ATGCTGTCTGGTTTCCGCCTCC & Forward \\
Med19 & GCGGCTCTGTTTGTGCTTATGC & Reverse \\
Gapdh & CATCACTGCCACCCAGAAGACTG & Forward \\
Gapdh & ATGCCAGTGAGCTTCCCGTTCAG & Reverse \\
miRNA-378a-3p & GCAGACTGGACTTGGAGT & Forward \\
miRNA-378a-5p & TCCTGACTCCAGGTCCT & Forward \\
Standard Reverse & GAACATGTCTGCGTATCTC & Reverse \\
miRNA-378a & & Forward \\
Genotype & GGCCAACTTGGGAAATGTA & \\
miRNA-378a & & Reverse \\
Genotype & AGCTCACATGCAAACACAGG & \\
\hline
\end{tabular}


Table S2.1: Primer design for qPCR and genotyping. Primers were designed using Primer-BLAST through NCBI and Primer3. Sequence information was compiled from the University of Santa Cruz (UCSC) genome browser from the mouse GRCm38/mm10 assembly. MiRNA sequences were analyzed based on the miRBase 21 release. 
Table S2.2

\begin{tabular}{|c|c|c|c|c|c|c|c|c|c|}
\hline Calculation & Units & $\begin{array}{l}\text { Het Sham } \\
\text { Pre }\end{array}$ & $\begin{array}{c}\text { Het Sham } \\
\text { Post }\end{array}$ & $\begin{array}{c}\text { Het Ex } \\
\text { Pre }\end{array}$ & $\begin{array}{l}\text { Het Ex } \\
\text { Post }\end{array}$ & $\begin{array}{c}\text { KO Sham } \\
\text { Pre }\end{array}$ & $\begin{array}{c}\text { KO Sham } \\
\text { Post }\end{array}$ & $\begin{array}{c}\text { KO Ex } \\
\text { Pre }\end{array}$ & $\begin{array}{l}\text { KO Ex } \\
\text { Post }\end{array}$ \\
\hline $\begin{array}{c}\text { CO (LV } \\
\text { Trace) }\end{array}$ & $\mathrm{mL} / \mathrm{min}$ & $\begin{array}{c}13.12 \pm \\
1.43\end{array}$ & $\begin{array}{l}12.11 \pm \\
2.22\end{array}$ & $\begin{array}{c}13.66 \pm \\
1.32\end{array}$ & $\begin{array}{c}12.79 \pm \\
1.97\end{array}$ & $\begin{array}{l}10.96 \pm \\
2.03\end{array}$ & $\begin{array}{c}12.34 \pm \\
0.82\end{array}$ & $\begin{array}{c}12.48 \pm \\
2.62\end{array}$ & $\begin{array}{c}14.35 \pm \\
0.62\end{array}$ \\
\hline $\begin{array}{l}\text { Diameter;d } \\
\text { (LV Trace) }\end{array}$ & $\mathrm{mm}$ & $\begin{array}{c}2.54 \pm \\
0.16\end{array}$ & $\begin{array}{c}2.52 \pm \\
0.15\end{array}$ & $\begin{array}{c}2.84 \pm \\
0.18\end{array}$ & $\begin{array}{c}2.58 \pm \\
0.14\end{array}$ & $\begin{array}{c}2.54 \pm \\
0.21\end{array}$ & $\begin{array}{c}2.62 \pm \\
0.07\end{array}$ & $\begin{array}{c}2.52 \pm \\
0.20\end{array}$ & $\begin{array}{c}2.82 \pm \\
0.29\end{array}$ \\
\hline $\begin{array}{l}\text { Diameter;s } \\
\text { (LV Trace) }\end{array}$ & $\mathrm{mm}$ & $\begin{array}{c}0.68 \pm \\
0.06\end{array}$ & $\begin{array}{c}0.83 \pm \\
0.09\end{array}$ & $\begin{array}{c}1.12 \pm \\
0.19\end{array}$ & $\begin{array}{c}0.85 \pm \\
0.08\end{array}$ & $\begin{array}{c}0.81 \pm \\
0.13\end{array}$ & $\begin{array}{c}0.79 \pm \\
0.08\end{array}$ & $\begin{array}{c}0.71 \pm \\
0.11\end{array}$ & $\begin{array}{c}0.99 \pm \\
0.21\end{array}$ \\
\hline $\mathbf{E F}$ & $\%$ & $\begin{array}{c}87.17 \pm \\
0.51\end{array}$ & $\begin{array}{c}89.07 \pm \\
1.54\end{array}$ & $\begin{array}{c}86.62 \pm \\
1.44\end{array}$ & $\begin{array}{c}87.43 \pm \\
1.89\end{array}$ & $\begin{array}{c}89.47 \pm \\
0.69\end{array}$ & $\begin{array}{c}90.05 \pm \\
0.79\end{array}$ & $\begin{array}{c}88.63 \pm \\
0.90\end{array}$ & $\begin{array}{c}91.60 \pm \\
0.45\end{array}$ \\
\hline FS & $\%$ & $\begin{array}{c}55.33 \pm \\
0.88\end{array}$ & $\begin{array}{l}57.88 \pm \\
2.06\end{array}$ & $\begin{array}{c}54.30 \pm \\
1.54\end{array}$ & $\begin{array}{c}55.86 \pm \\
2.50\end{array}$ & $\begin{array}{c}56.80 \pm \\
0.98\end{array}$ & $\begin{array}{c}59.86 \pm \\
0.80\end{array}$ & $\begin{array}{c}57.22 \pm \\
1.35\end{array}$ & $\begin{array}{c}62.96 \pm \\
2.74\end{array}$ \\
\hline $\begin{array}{c}\text { LV Mass (LV } \\
\text { Trace) }\end{array}$ & $\mathrm{mg}$ & $\begin{array}{c}101.51 \pm \\
11.52\end{array}$ & $\begin{array}{l}100.21 \pm \\
6.94\end{array}$ & $\begin{array}{c}119.63 \pm \\
9.81\end{array}$ & $\begin{array}{c}132.61 \pm \\
8.47\end{array}$ & $\begin{array}{c}113.24 \pm \\
18.63\end{array}$ & $\begin{array}{c}122.29 \pm \\
10.26\end{array}$ & $\begin{array}{c}103.44 \pm \\
3.96\end{array}$ & $\begin{array}{c}108.32 \pm \\
9.97\end{array}$ \\
\hline $\begin{array}{l}\text { LV Mass Corr } \\
\text { (LV Trace) }\end{array}$ & mg & $\begin{array}{c}84.20 \pm \\
9.22\end{array}$ & $\begin{array}{l}80.17 \pm \\
5.55\end{array}$ & $\begin{array}{l}95.70 \pm \\
7.85\end{array}$ & $\begin{array}{c}106.08 \pm \\
6.78\end{array}$ & $\begin{array}{c}90.59 \pm \\
14.91\end{array}$ & $\begin{array}{c}97.84 \pm \\
8.21\end{array}$ & $\begin{array}{c}82.75 \pm \\
3.17\end{array}$ & $\begin{array}{c}86.66 \pm \\
7.97\end{array}$ \\
\hline SV (LV Trace) & $\mathrm{uL}$ & $\begin{array}{c}23.36 \pm \\
3.45\end{array}$ & $\begin{array}{c}22.24 \pm \\
3.19\end{array}$ & $\begin{array}{c}24.05 \pm \\
3.43\end{array}$ & $\begin{array}{c}23.62 \pm \\
3.26\end{array}$ & $\begin{array}{c}22.69 \pm \\
4.38\end{array}$ & $\begin{array}{c}24.22 \pm \\
1.46\end{array}$ & $\begin{array}{c}22.73 \pm \\
4.13\end{array}$ & $\begin{array}{c}31.49 \pm \\
4.82\end{array}$ \\
\hline $\begin{array}{l}\text { V;d (LV } \\
\text { Trace) }\end{array}$ & $\mathrm{uL}$ & $\begin{array}{c}24.16 \pm \\
3.48\end{array}$ & $\begin{array}{c}25.13 \pm \\
3.13\end{array}$ & $\begin{array}{c}25.74 \pm \\
2.43\end{array}$ & $\begin{array}{c}25.18 \pm \\
2.13\end{array}$ & $\begin{array}{c}24.09 \pm \\
4.82\end{array}$ & $\begin{array}{c}25.41 \pm \\
1.66\end{array}$ & $\begin{array}{c}23.72 \pm \\
4.36\end{array}$ & $\begin{array}{c}29.66 \pm \\
2.82\end{array}$ \\
\hline $\begin{array}{c}\text { V;s (LV } \\
\text { Trace) }\end{array}$ & $\mathrm{uL}$ & $\begin{array}{c}1.81 \pm \\
0.18\end{array}$ & $\begin{array}{c}1.45 \pm \\
0.40\end{array}$ & $\begin{array}{c}1.69 \pm \\
0.52\end{array}$ & $\begin{array}{c}1.55 \pm \\
0.28\end{array}$ & $\begin{array}{c}1.40 \pm \\
0.49\end{array}$ & $\begin{array}{c}1.19 \pm \\
0.31\end{array}$ & $\begin{array}{c}1.00 \pm \\
0.39\end{array}$ & $\begin{array}{c}1.68 \pm \\
0.14\end{array}$ \\
\hline
\end{tabular}


Table S2.2: Ultrasound imaging one week prior and following nano- $\mathrm{TiO}_{2}$ inhalation exposure in the Het and KO groups. Measurements were taken for at least three consecutive systolic and diastolic peaks and troughs, respectively, for each animal. Significance $(P \leq 0.05)$ is denoted for a specific category through bold text and reference to the sample number that it is significantly different from. Sample numbers are listed in the column heading. Het $=$ heterozygous for the miRNA-378a allele, $\mathrm{KO}=$ knockout for the miRNA-378a allele, Sham $=$ control filtered air exposed, $\mathrm{Ex}=$ nano- $\mathrm{TiO}_{2}$ exposed, Pre $=$ one week prior to inhalation exposure, Post $=24$ post inhalation exposure, $\mathrm{CO}=$ cardiac output, Diameter; $\mathrm{d}=$ diastolic diameter, Diameter; $=$ systolic diameter, $\mathrm{EF}=$ ejection fraction, $\mathrm{FS}=$ fractional shortening, $\mathrm{LV}$ Mass = left ventricular mass, $\mathrm{LV}$ Mass Corr $=$ left ventricular mass corrected, $\mathrm{SV}=$ stroke volume, $\mathrm{V} ; \mathrm{d}=$ volume during diastole, $\mathrm{V} ; \mathrm{s}=$ volume during systole. 
Table S2.3

\begin{tabular}{|c|c|c|c|c|c|c|c|c|c|c|c|}
\hline Measures & Units & $\begin{array}{c}\text { WT } \\
\text { Sham }\end{array}$ & WT Ex ${ }^{2}$ & $\begin{array}{c}\text { Het Sham } \\
\text { Pre }^{3}\end{array}$ & $\begin{array}{c}\text { Het Sham } \\
{ }_{4}^{4} \\
\text { Post }\end{array}$ & $\begin{array}{c}\text { Het Ex } \\
\text { Pre }\end{array}$ & $\begin{array}{c}\text { Het Ex } \\
\text { Post }^{6}\end{array}$ & $\begin{array}{c}\text { KO Sham } \\
\text { Pre }^{7}\end{array}$ & $\begin{array}{c}\text { KO Sham } \\
\text { Post }\end{array}$ & $\begin{array}{c}\text { KO Ex } \\
\text { Pre }\end{array}$ & $\begin{array}{c}\text { KO Ex } \\
\text { Post }\end{array}$ \\
\hline Heart Rate & beats/min & $\begin{array}{c}538.22 \pm \\
14.49\end{array}$ & $\begin{array}{c}545.81 \pm \\
9.99\end{array}$ & $\begin{array}{c}506.19 \pm \\
25.00\end{array}$ & $\begin{array}{c}519.79 \pm \\
21.03\end{array}$ & $\begin{array}{c}548.25 \pm \\
27.64\end{array}$ & $\begin{array}{c}544.15 \pm \\
24.07\end{array}$ & $\begin{array}{c}498.40 \pm \\
37.06\end{array}$ & $\begin{array}{c}530.93 \pm \\
10.26\end{array}$ & $\begin{array}{c}531.20 \pm \\
38.12\end{array}$ & $\begin{array}{c}529.08 \pm \\
11.34\end{array}$ \\
\hline $\begin{array}{l}\text { Radial Velocity } \\
\text { (Endo) }\end{array}$ & $\mathrm{Pk} \mathrm{cm} / \mathrm{s}$ & $\begin{array}{c}-2.05 \pm \\
0.14\end{array}$ & $\begin{array}{c}-2.21 \pm \\
0.16\end{array}$ & $\begin{array}{c}-2.01 \pm \\
0.26\end{array}$ & $\begin{array}{c}-2.16 \pm \\
0.33\end{array}$ & $\begin{array}{c}-2.37 \pm \\
0.27\end{array}$ & $\begin{array}{c}-2.13 \pm \\
0.35\end{array}$ & $\begin{array}{c}-1.61 \pm \\
0.14\end{array}$ & $\begin{array}{l}-2.20 \pm \\
0.05\end{array}$ & $\begin{array}{c}-2.24 \pm \\
0.35\end{array}$ & $\begin{array}{c}-2.05 \pm \\
0.27\end{array}$ \\
\hline $\begin{array}{c}\text { Radial } \\
\text { Displacement } \\
\text { (Endo) }\end{array}$ & $\mathrm{Pk} \mathrm{mm}$ & $0.00 \pm 0.00$ & $\begin{array}{c}-0.01 \pm \\
0.00\end{array}$ & $0.00 \pm 0.00$ & $0.00 \pm 0.00$ & $0.00 \pm 0.00$ & $\begin{array}{c}-0.01 \pm \\
0.00\end{array}$ & $0.00 \pm 0.00$ & $\begin{array}{c}-0.01 \pm \\
0.00\end{array}$ & $0.00 \pm 0.00$ & $\begin{array}{c}0.00 \pm \\
0.00\end{array}$ \\
\hline $\begin{array}{l}\text { Radial Strain } \\
\text { (Endo) }\end{array}$ & $\mathrm{Pk} \%$ & $\begin{array}{c}-\mathbf{- 0 . 5 8} \pm \\
\mathbf{0 . 2 7} \\
\mathbf{3 , 4 , 5 , 6 , 7 , 8 , 9 , 1 0}\end{array}$ & $\begin{array}{c}-\mathbf{0 . 4 8} \pm \\
0.08 \\
3,4,5,6,6,7,8,9,10\end{array}$ & $\begin{array}{l}-2.22 \pm \\
1.13\end{array}$ & $\begin{array}{c}-2.18 \pm \\
0.41\end{array}$ & $\begin{array}{c}-3.51 \pm \\
1.24\end{array}$ & $\begin{array}{c}-2.20 \pm \\
0.42\end{array}$ & $\begin{array}{l}-3.14 \pm \\
2.10\end{array}$ & $\begin{array}{c}-2.54 \pm \\
0.38\end{array}$ & $\begin{array}{c}-3.85 \pm \\
1.93\end{array}$ & $\begin{array}{c}-2.48 \pm \\
0.34\end{array}$ \\
\hline $\begin{array}{l}\text { Radial Strain } \\
\text { Rate (Endo) }\end{array}$ & $\mathrm{Pk} 1 / \mathrm{s}$ & $\begin{array}{c}-10.54 \pm \\
0.93\end{array}$ & $\begin{array}{c}-14.36 \pm \\
0.61\end{array}$ & $\begin{array}{c}-14.98 \pm \\
1.62\end{array}$ & $\begin{array}{c}-19.81 \pm \\
1.58 \\
1,5,6,7,8,9,10\end{array}$ & $\begin{array}{c}-13.51 \pm \\
1.53\end{array}$ & $\begin{array}{c}-12.60 \pm \\
1.69\end{array}$ & $\begin{array}{c}-7.79 \pm \\
0.94\end{array}$ & $\begin{array}{c}-12.82 \pm \\
1.21\end{array}$ & $\begin{array}{c}-11.82 \pm \\
1.76\end{array}$ & $\begin{array}{c}-10.88 \pm \\
1.02\end{array}$ \\
\hline $\begin{array}{l}\text { Circumferential } \\
\text { Velocity (Endo) }\end{array}$ & Pk deg/s & $\begin{array}{c}-289.64 \pm \\
37.03\end{array}$ & $\begin{array}{c}-397.82 \pm \\
59.93\end{array}$ & $\begin{array}{c}-327.13 \pm \\
78.10\end{array}$ & $\begin{array}{c}-393.17 \pm \\
47.03\end{array}$ & $\begin{array}{c}-416.00 \pm \\
63.33\end{array}$ & $\begin{array}{c}-319.16 \pm \\
46.13\end{array}$ & $\begin{array}{c}-399.62 \pm \\
101.16\end{array}$ & $\begin{array}{c}-484.66 \pm \\
103.14\end{array}$ & $\begin{array}{c}-415.47 \pm \\
83.05\end{array}$ & $\begin{array}{c}-384.98 \pm \\
57.72\end{array}$ \\
\hline $\begin{array}{l}\text { Circumferential } \\
\text { Displacement } \\
\text { (Endo) }\end{array}$ & Pk deg & $\begin{array}{c}-2.75 \pm \\
0.62\end{array}$ & $\begin{array}{c}-2.79 \pm \\
0.74\end{array}$ & $\begin{array}{c}-1.77 \pm \\
0.53\end{array}$ & $\begin{array}{c}-2.95 \pm \\
1.12\end{array}$ & $\begin{array}{c}-1.03 \pm \\
0.53\end{array}$ & $\begin{array}{c}-1.77 \pm \\
0.51\end{array}$ & $\begin{array}{c}-1.30 \pm \\
0.50\end{array}$ & $\begin{array}{l}-0.90 \pm \\
0.05\end{array}$ & $\begin{array}{c}-2.54 \pm \\
0.64\end{array}$ & $\begin{array}{l}-1.79 \pm \\
0.99\end{array}$ \\
\hline $\begin{array}{c}\text { Circumferential } \\
\text { Strain (Endo) }\end{array}$ & $\mathrm{Pk} \%$ & $0.27 \pm 0.060$ & $0.31 \pm 0.1$ & $50.65 \pm 0.43$ & $0.86 \pm 0.621$ & $1.02 \pm 1.42$ & $0.80 \pm 0.70$ & $1.86 \pm 1.41$ & $\underset{1,2,3,4,6}{2.11 \pm 0.78}$ & $\underset{1,2,3,4,6}{2.24}$ & $\begin{array}{c}1.50 \pm \\
0.49 \\
2\end{array}$ \\
\hline $\begin{array}{l}\text { Circumferential } \\
\text { Strain Rate } \\
\text { (Endo) }\end{array}$ & $\mathrm{Pk} 1 / \mathrm{s}$ & $\begin{array}{c}-19.16 \pm \\
2.11\end{array}$ & $\begin{array}{c}-14.76 \pm \\
0.76\end{array}$ & $\begin{array}{l}25.97 \pm \\
5.85\end{array}$ & $\begin{array}{c}21.67 \pm \\
4.04\end{array}$ & $\begin{array}{l}32.99 \pm \\
8.35\end{array}$ & $\begin{array}{c}23.38 \pm \\
8.35\end{array}$ & $\begin{array}{c}14.75 \pm \\
2.38\end{array}$ & $\begin{array}{c}14.75 \pm \\
2.38\end{array}$ & $\begin{array}{c}23.39 \pm \\
6.30\end{array}$ & $\begin{array}{c}23.39 \pm \\
6.30\end{array}$ \\
\hline
\end{tabular}


Table S2.3: B-Mode images were used in the short axis to examine circumferential and radial stress-strain parameters during diastole. Ultrasound imaging one week prior (Het and KO groups) and following nano- $\mathrm{TiO}_{2}$ inhalation exposure (WT, Het, and $\left.\mathrm{KO}\right)$. Significance $(P \leq 0.05)$ is denoted for a specific category through bold text and reference to the sample number that it is significantly different from. Sample numbers are listed in the column heading. WT $=$ Wild type, Het $=$ heterozygous for the miRNA-378a allele, $\mathrm{KO}=$ knockout for the miRNA-378a allele, Sham $=$ control filtered air exposed, $\mathrm{Ex}=$ nano- $\mathrm{TiO}_{2}$ exposed, Pre $=$ one week prior to inhalation exposure, Post $=24$ post inhalation exposure. 
Table S2.4

\begin{tabular}{|c|c|c|c|c|c|c|c|c|c|c|c|}
\hline Measures & Units & WT Sham ${ }^{1}$ & WT Ex ${ }^{2}$ & $\begin{array}{c}\text { Het Sham } \\
{ }^{3} \\
\text { Pre }\end{array}$ & $\begin{array}{c}\text { Het Sham } \\
\text { Post }\end{array}$ & $\begin{array}{c}\text { Het Ex } \\
\text { Pre }\end{array}$ & $\begin{array}{c}\text { Het Ex } \\
\text { Post }^{6}\end{array}$ & $\begin{array}{c}\text { KO Sham } \\
\text { Pre }^{7}\end{array}$ & $\begin{array}{c}\text { KO Sham } \\
\text { Post }\end{array}$ & $\begin{array}{c}\text { KO Ex } \\
\text { Pre }\end{array}$ & $\begin{array}{c}\text { KO Ex } \\
\text { Post }\end{array}$ \\
\hline Heart Rate & beats $/ \mathrm{min}$ & $\begin{array}{c}538.22 \pm \\
14.49\end{array}$ & $\begin{array}{c}545.81 \pm \\
9.99\end{array}$ & $\begin{array}{c}506.19 \pm \\
25.00\end{array}$ & $\begin{array}{c}519.79 \pm \\
21.03\end{array}$ & $\begin{array}{c}548.25 \pm \\
27.64\end{array}$ & $\begin{array}{c}544.15 \pm \\
24.07\end{array}$ & $\begin{array}{c}498.40 \pm \\
37.06\end{array}$ & $\begin{array}{c}530.93 \pm \\
10.26\end{array}$ & $\begin{array}{c}531.20 \pm \\
38.12\end{array}$ & $\begin{array}{c}529.08 \pm \\
11.34\end{array}$ \\
\hline $\begin{array}{c}\text { Radial } \\
\text { Velocity } \\
\text { (Endo) }\end{array}$ & $\mathrm{Pk} \mathrm{cm} / \mathrm{s}$ & $\begin{array}{c}-1.31 \pm \\
0.18\end{array}$ & $\begin{array}{c}-1.19 \pm \\
0.17\end{array}$ & $\begin{array}{c}-1.45 \pm \\
0.13\end{array}$ & $\begin{array}{c}-1.33 \pm \\
0.20\end{array}$ & $\begin{array}{c}-1.29 \pm \\
0.13\end{array}$ & $\begin{array}{c}-1.19 \pm \\
0.20\end{array}$ & $\begin{array}{c}-0.99 \pm \\
0.12\end{array}$ & $\begin{array}{c}-1.24 \pm \\
0.20\end{array}$ & $\begin{array}{c}-1.33 \pm \\
0.22\end{array}$ & $\begin{array}{c}-0.90 \pm \\
0.09\end{array}$ \\
\hline $\begin{array}{c}\text { Radial } \\
\text { Displacement } \\
\text { (Endo) }\end{array}$ & $\mathrm{Pk} \mathrm{mm}$ & $\begin{array}{c}-0.01 \pm \\
0.01\end{array}$ & $\begin{array}{c}-0.02 \pm \\
0.01\end{array}$ & $\begin{array}{c}-0.01 \pm \\
0.01\end{array}$ & $\begin{array}{c}-0.01 \pm \\
0.01\end{array}$ & $\begin{array}{c}-0.01 \pm \\
0.00\end{array}$ & $\begin{array}{c}-0.01 \pm \\
0.00\end{array}$ & $\begin{array}{c}-0.01 \pm \\
0.00\end{array}$ & $0.00 \pm 0.00$ & $\begin{array}{c}-0.01 \pm \\
0.01\end{array}$ & $\begin{array}{c}-0.01 \pm \\
0.00\end{array}$ \\
\hline $\begin{array}{l}\text { Radial Strain } \\
\text { (Endo) }\end{array}$ & $\mathrm{Pk} \%$ & $\begin{array}{l}-0.73 \pm \\
0.32\end{array}$ & $\begin{array}{c}-2.31 \pm \\
0.30 \\
1,3,4,5,6,7,8,9,10\end{array}$ & $\begin{array}{c}-0.55 \pm \\
0.22\end{array}$ & $\begin{array}{c}-0.73 \pm \\
0.27\end{array}$ & $\begin{array}{l}-0.36 \pm \\
0.22\end{array}$ & $\begin{array}{c}-0.53 \pm \\
0.24\end{array}$ & $\begin{array}{c}-0.79 \pm \\
0.34\end{array}$ & $\begin{array}{c}-0.95 \pm \\
0.27\end{array}$ & $\begin{array}{c}-0.38 \pm \\
0.26\end{array}$ & $\begin{array}{c}-0.84 \pm \\
0.28\end{array}$ \\
\hline $\begin{array}{l}\text { Radial Strain } \\
\text { Rate (Endo) }\end{array}$ & $\mathrm{Pk} 1 / \mathrm{s}$ & $\begin{array}{c}-6.52 \pm \\
1.08\end{array}$ & $\begin{array}{c}-6.30 \pm \\
0.54\end{array}$ & $\begin{array}{c}-7.34 \pm \\
0.37\end{array}$ & $\begin{array}{c}-8.85 \pm \\
1.44\end{array}$ & $\begin{array}{c}-6.56 \pm \\
0.94\end{array}$ & $\begin{array}{l}-6.76 \pm \\
1.05\end{array}$ & $\begin{array}{c}-5.03 \pm \\
0.76\end{array}$ & $\begin{array}{c}-7.15 \pm \\
1.69\end{array}$ & $\begin{array}{c}-6.68 \pm \\
1.57\end{array}$ & $\begin{array}{c}-5.90 \pm \\
1.88\end{array}$ \\
\hline $\begin{array}{l}\text { Longitudinal } \\
\text { Velocity } \\
\text { (Endo) }\end{array}$ & $\mathrm{Pk} \mathrm{deg} / \mathrm{s}$ & $\begin{array}{c}-0.81 \pm \\
0.13\end{array}$ & $\begin{array}{c}-0.77 \pm \\
0.15\end{array}$ & $\begin{array}{c}-0.71 \pm \\
0.14\end{array}$ & $\begin{array}{c}-0.75 \pm \\
0.14\end{array}$ & $\begin{array}{l}-0.55 \pm \\
0.05\end{array}$ & $\begin{array}{l}-0.66 \pm \\
0.05\end{array}$ & $\begin{array}{l}-0.92 \pm \\
0.22\end{array}$ & $\begin{array}{c}-0.68 \pm \\
0.10\end{array}$ & $\begin{array}{c}-0.77 \pm \\
0.19\end{array}$ & $\begin{array}{c}-0.64 \pm \\
0.04\end{array}$ \\
\hline $\begin{array}{l}\text { Longitudinal } \\
\text { Displacement } \\
\text { (Endo) }\end{array}$ & Pk deg & $\begin{array}{c}-0.01 \pm \\
0.00\end{array}$ & $\begin{array}{c}-0.02 \pm \\
0.01\end{array}$ & $\begin{array}{c}-0.02 \pm \\
0.01\end{array}$ & $\begin{array}{c}-0.02 \pm \\
0.01\end{array}$ & $\begin{array}{c}-0.03 \pm \\
0.01\end{array}$ & $\begin{array}{c}-0.05 \pm \\
0.02\end{array}$ & $\begin{array}{c}-0.01 \pm \\
0.00\end{array}$ & $\begin{array}{c}-0.02 \pm \\
0.02\end{array}$ & $\begin{array}{c}-0.02 \pm \\
0.01\end{array}$ & $\begin{array}{c}-0.05 \pm \\
0.04\end{array}$ \\
\hline $\begin{array}{l}\text { Longitudinal } \\
\text { Strain } \\
\text { (Endo) }\end{array}$ & $\mathrm{Pk} \%$ & $0.61 \pm 0.20$ & $1.10 \pm 0.38$ & $0.45 \pm 0.20$ & $1.08 \pm 0.36$ & $0.47 \pm 0.13$ & $0.59 \pm 0.30$ & $1.01 \pm 0.30$ & $1.04 \pm 0.27$ & $0.96 \pm 0.96$ & $0.59 \pm 0.26$ \\
\hline $\begin{array}{l}\text { Longitudinal } \\
\text { Strain Rate } \\
\text { (Endo) }\end{array}$ & $\mathrm{Pk} 1 / \mathrm{s}$ & $4.86 \pm 0.57$ & $\underset{3,4,5}{2.68} \pm 0.31$ & $6.47 \pm 0.74$ & $7.19 \pm 0.89$ & $5.93 \pm 0.38$ & $\underset{3,4}{3.72} \pm 0.53$ & $4.40 \pm 0.23$ & $5.13 \pm 0.74$ & $4.62 \pm 0.80$ & $4.07 \pm 0.65$ \\
\hline
\end{tabular}


Table S2.4: B-Mode images were used in the long axis to examine circumferential and longitudinal stress-strain parameters during diastole. Ultrasound imaging one week prior (Het and $\mathrm{KO}$ groups) and following nano- $\mathrm{TiO}_{2}$ inhalation exposure (WT, Het, and $\mathrm{KO}$ ). Significance $(P \leq 0.05)$ is denoted for a specific category through bold text and reference to the sample number that it is significantly different from. Sample numbers are listed in the column heading. $\mathrm{WT}=$ Wild type, Het $=$ heterozygous for the miRNA-378a allele, $\mathrm{KO}=$ knockout for the miRNA-378a allele, Sham $=$ control filtered air exposed, $\mathrm{Ex}=$ nano- $\mathrm{TiO}_{2}$ exposed, $\mathrm{Pre}=$ one week prior to inhalation exposure, Post $=24$ post inhalation exposure. 
Table S2.5

\begin{tabular}{|c|c|c|c|c|c|c|c|c|c|c|c|}
\hline Measures & Units & $\begin{array}{c}\text { WT } \\
\text { Sham }\end{array}$ & WT Ex ${ }^{2}$ & $\begin{array}{c}\text { Het Sham } \\
\text { Pre }^{3}\end{array}$ & $\begin{array}{c}\text { Het Sham } \\
{ }^{4} \\
\text { Post }\end{array}$ & $\begin{array}{c}\text { Het Ex } \\
\text { Pre }\end{array}$ & $\begin{array}{c}\text { Het Ex } \\
{ }^{6} \\
\text { Post }\end{array}$ & $\begin{array}{c}\text { KO Sham } \\
\text { Pre }^{7}\end{array}$ & $\begin{array}{c}\text { KO Sham } \\
\text { Post }\end{array}$ & $\begin{array}{c}\text { KO Ex } \\
\text { Pre }\end{array}$ & $\begin{array}{c}\text { KO Ex } \\
\text { Post }\end{array}$ \\
\hline Heart Rate & beats $/ \mathrm{min}$ & $\begin{array}{c}538.22 \pm \\
14.49\end{array}$ & $\begin{array}{c}545.81 \pm \\
9.99\end{array}$ & $\begin{array}{c}506.19 \pm \\
25.00\end{array}$ & $\begin{array}{c}519.79 \pm \\
21.03\end{array}$ & $\begin{array}{c}548.25 \pm \\
27.64\end{array}$ & $\begin{array}{c}544.15 \pm \\
24.07\end{array}$ & $\begin{array}{c}498.40 \pm \\
37.06\end{array}$ & $\begin{array}{c}530.93 \pm \\
10.26\end{array}$ & $\begin{array}{c}531.20 \pm \\
38.12\end{array}$ & $\begin{array}{c}529.08 \pm \\
11.34\end{array}$ \\
\hline $\begin{array}{l}\text { Radial Velocity } \\
\text { (Endo) }\end{array}$ & $\mathrm{Pk} \mathrm{cm} / \mathrm{s}$ & $2.38 \pm 0.21$ & $11.97 \pm 0.06$ & $1.94 \pm 0.20$ & $2.22 \pm 0.35$ & $2.46 \pm 0.21$ & $1.90 \pm 0.21$ & $1.78 \pm 0.36$ & $2.66 \pm 0.112$ & $2.26 \pm 0.43$ & $\begin{array}{c}2.22 \pm \\
0.19\end{array}$ \\
\hline $\begin{array}{c}\text { Radial } \\
\text { Displacement } \\
\text { (Endo) }\end{array}$ & $\mathrm{Pk} \mathrm{mm}$ & $0.77 \pm 0.07$ & $70.70 \pm 0.03$ & $0.58 \pm 0.03$ & $0.63 \pm 0.09$ & $0.63 \pm 0.03$ & $30.54 \pm 0.06$ & $0.50 \pm 0.05$ & $0.58 \pm 0.040$ & $0.51 \pm 0.06$ & $\begin{array}{c}0.57 \pm \\
0.03\end{array}$ \\
\hline $\begin{array}{l}\text { Radial Strain } \\
\text { (Endo) }\end{array}$ & $\mathrm{Pk} \%$ & $\begin{array}{c}42.39 \pm \\
4.11\end{array}$ & $\begin{array}{c}35.24 \pm \\
1.97\end{array}$ & $\begin{array}{l}41.95 \pm \\
2.73\end{array}$ & $\begin{array}{c}47.09 \pm \\
1.85 \\
1,2,6\end{array}$ & $\begin{array}{c}40.55 \pm \\
3.11\end{array}$ & $\begin{array}{c}27.97 \pm \\
3.31\end{array}$ & $\begin{array}{c}38.04 \pm \\
2.84\end{array}$ & $\begin{array}{c}40.12 \pm \\
4.68\end{array}$ & $\begin{array}{c}39.62 \pm \\
4.96\end{array}$ & $\begin{array}{c}37.04 \pm \\
2.28\end{array}$ \\
\hline $\begin{array}{c}\text { Radial Strain } \\
\text { Rate (Endo) }\end{array}$ & $\mathrm{Pk} 1 / \mathrm{s}$ & $\begin{array}{c}11.87 \pm \\
1.13\end{array}$ & $\underset{1,4,5}{8.92 \pm 0.44}$ & $\begin{array}{c}12.15 \pm \\
1.24\end{array}$ & $\begin{array}{c}12.82 \pm \\
1.08\end{array}$ & $\begin{array}{c}14.18 \pm \\
1.61\end{array}$ & $9.35 \pm 0.99$ & $\begin{array}{c}11.01 \pm \\
2.69\end{array}$ & $\begin{array}{c}12.88 \pm \\
2.18\end{array}$ & $\begin{array}{c}12.38 \pm \\
2.25\end{array}$ & $\begin{array}{c}11.09 \pm \\
1.67\end{array}$ \\
\hline $\begin{array}{l}\text { Circumferential } \\
\text { Velocity (Endo) }\end{array}$ & $\mathrm{Pk}$ deg $/ \mathrm{s}$ & $\begin{array}{c}387.12 \pm \\
94.80\end{array}$ & $\begin{array}{l}310.20 \pm \\
71.63\end{array}$ & $\begin{array}{l}321.54 \pm \\
55.53\end{array}$ & $\begin{array}{c}461.01 \pm \\
34.60\end{array}$ & $\begin{array}{c}635.88 \pm \\
159.07\end{array}$ & $\begin{array}{c}337.21 \pm \\
61.29\end{array}$ & $\begin{array}{c}452.42 \pm \\
103.81\end{array}$ & $\begin{array}{c}476.84 \pm \\
22.23\end{array}$ & $\begin{array}{c}452.37 \pm \\
105.52\end{array}$ & $\begin{array}{c}451.66 \pm \\
81.90\end{array}$ \\
\hline $\begin{array}{l}\text { Circumferential } \\
\text { Displacement } \\
\text { (Endo) }\end{array}$ & Pk deg & $4.78 \pm 1.92$ & $23.61 \pm 1.675$ & $5.94 \pm 0.70$ & $7.80 \pm 1.019$ & $9.38 \pm 3.77$ & $75.36 \pm 1.07$ & $6.27 \pm 1.61$ & $6.50 \pm 1.245$ & $5.81 \pm 1.60$ & $\begin{array}{c}6.58 \pm \\
2.08\end{array}$ \\
\hline $\begin{array}{c}\text { Circumferential } \\
\text { Strain (Endo) }\end{array}$ & $\mathrm{Pk} \%$ & $\begin{array}{c}-48.51 \pm \\
3.88\end{array}$ & $\begin{array}{c}-40.36 \pm \\
3.38\end{array}$ & $\begin{array}{c}-37.61 \pm \\
3.75\end{array}$ & $\begin{array}{c}-43.61 \pm \\
3.61\end{array}$ & $\begin{array}{c}-42.24 \pm \\
2.62\end{array}$ & $\begin{array}{c}-28.78 \\
2.63 \\
1,4,5\end{array}$ & $\begin{array}{c}-34.46 \pm \\
4.31\end{array}$ & $\begin{array}{c}-38.65 \pm \\
3.48\end{array}$ & $\begin{array}{c}-36.35 \pm \\
5.39\end{array}$ & $\begin{array}{c}-39.32 \pm \\
3.57\end{array}$ \\
\hline $\begin{array}{l}\text { Circumferential } \\
\text { Strain Rate } \\
\text { (Endo) }\end{array}$ & $\mathrm{Pk} \mathrm{1/s}$ & $\begin{array}{c}-29.02 \pm \\
3.20\end{array}$ & $\begin{array}{c}-22.37 \pm \\
1.15\end{array}$ & $\begin{array}{c}-22.73 \pm \\
4.42\end{array}$ & $\begin{array}{c}-27.44 \pm \\
6.30\end{array}$ & $\begin{array}{c}-27.83 \pm \\
5.95\end{array}$ & $\begin{array}{c}-23.14 \pm \\
4.99\end{array}$ & $\begin{array}{c}-19.65 \pm \\
6.55\end{array}$ & $\begin{array}{c}-33.70 \pm \\
3.90\end{array}$ & $\begin{array}{c}-28.86 \pm \\
11.48\end{array}$ & $\begin{array}{c}-28.29 \pm \\
4.83\end{array}$ \\
\hline
\end{tabular}


Table S2.5: B-Mode images were used in the short axis to examine circumferential and radial stress-strain parameters during systole. Ultrasound imaging one week prior (Het and KO groups) and following nano- $\mathrm{TiO}_{2}$ inhalation exposure (WT, Het, and $\left.\mathrm{KO}\right)$. Significance $(P \leq 0.05)$ is denoted for a specific category through bold text and reference to the sample number that it is significantly different from. Sample numbers are listed in the column heading. WT = Wild type, Het $=$ heterozygous for the miRNA-378a allele, $\mathrm{KO}=$ knockout for the miRNA-378a allele, Sham $=$ control filtered air exposed, $\mathrm{Ex}=$ nano- $\mathrm{TiO}_{2}$ exposed, Pre $=$ one week prior to inhalation exposure, Post $=24$ post inhalation exposure. 


\section{Table S2.6}

\begin{tabular}{|c|c|c|c|c|c|c|c|c|c|c|c|}
\hline Measures & Units & WT Sham ${ }^{1}$ & WT Ex ${ }^{2}$ & $\begin{array}{c}\text { Het Sham } \\
{ }^{3}{ }^{3}\end{array}$ & $\begin{array}{c}\text { Het Sham } \\
\text { Post }\end{array}$ & $\begin{array}{c}\text { Het Ex } \\
\text { Pre }\end{array}$ & $\begin{array}{c}\text { Het Ex } \\
{ }^{6} \\
\text { Post }\end{array}$ & $\begin{array}{c}\text { KO Sham } \\
\text { Pre }^{7}\end{array}$ & $\begin{array}{c}\text { KO Sham } \\
\text { Post }\end{array}$ & $\begin{array}{c}\text { KO Ex } \\
\text { Pre }^{9}\end{array}$ & $\begin{array}{c}\text { KO Ex } \\
\text { Post }\end{array}$ \\
\hline Heart Rate & beats/min & $\begin{array}{c}538.22 \pm \\
14.49\end{array}$ & $\begin{array}{c}545.81 \pm \\
9.99\end{array}$ & $\begin{array}{c}506.19 \pm \\
25.00\end{array}$ & $\begin{array}{c}526.40 \pm \\
24.25\end{array}$ & $\begin{array}{c}548.25 \pm \\
27.64\end{array}$ & $\begin{array}{c}544.15 \pm \\
24.24\end{array}$ & $\begin{array}{c}498.40 \pm \\
37.06\end{array}$ & $\begin{array}{c}530.93 \pm \\
10.26\end{array}$ & $\begin{array}{c}531.20 \pm \\
38.12\end{array}$ & $\begin{array}{c}529.08 \pm \\
11.34\end{array}$ \\
\hline $\begin{array}{l}\text { Radial } \\
\text { Velocity } \\
\text { (Endo) }\end{array}$ & $\mathrm{Pk} \mathrm{cm} / \mathrm{s}$ & $1.31 \pm 0.12$ & $1.25 \pm 0.11$ & $1.14 \pm 0.08$ & $1.09 \pm 0.14$ & $1.18 \pm 0.09$ & $1.14 \pm 0.13$ & $0.96 \pm 0.17$ & $1.03 \pm 0.11$ & $1.22 \pm 0.21$ & $0.91 \pm 0.08$ \\
\hline $\begin{array}{c}\text { Radial } \\
\text { Displacement } \\
\text { (Endo) }\end{array}$ & $\mathrm{Pk} \mathrm{mm}$ & $0.42 \pm 0.06$ & $0.37 \pm 0.04$ & $0.43 \pm 0.02$ & $0.35 \pm 0.04$ & $0.37 \pm 0.02$ & $0.34 \pm 0.04$ & $0.32 \pm 0.04$ & $0.35 \pm 0.04$ & $0.38 \pm 0.04$ & $0.28 \pm 0.02$ \\
\hline $\begin{array}{l}\text { Radial Strain } \\
\text { (Endo) }\end{array}$ & $\mathrm{Pk} \%$ & $\begin{array}{c}18.65 \pm \\
2.94\end{array}$ & $\begin{array}{c}14.92 \pm \\
3.37\end{array}$ & $\begin{array}{c}21.46 \pm \\
1.16\end{array}$ & $\begin{array}{c}16.89 \pm \\
1.25\end{array}$ & $\begin{array}{c}19.51 \pm \\
1.95\end{array}$ & $\begin{array}{c}18.46 \pm \\
0.86\end{array}$ & $\begin{array}{c}13.97 \pm \\
2.69\end{array}$ & $\begin{array}{c}14.56 \pm \\
0.77\end{array}$ & $\begin{array}{c}14.87 \pm \\
2.24\end{array}$ & $\begin{array}{c}13.14 \\
1.12 \\
3\end{array}=$ \\
\hline $\begin{array}{l}\text { Radial Strain } \\
\text { Rate (Endo) }\end{array}$ & $\mathrm{Pk} 1 / \mathrm{s}$ & $5.36 \pm 0.60$ & $3.79 \pm 0.24$ & $4.65 \pm 0.34$ & $6.82 \pm 1.46$ & $5.21 \pm 0.53$ & $5.27 \pm 0.54$ & $3.43 \pm 0.51$ & $4.37 \pm 0.26$ & $4.23 \pm 0.26$ & $3.54 \pm 0.35$ \\
\hline $\begin{array}{c}\text { Longitudinal } \\
\text { Velocity } \\
\text { (Endo) }\end{array}$ & $\mathrm{Pk} \mathrm{deg} / \mathrm{s}$ & $0.90 \pm 0.06$ & $0.53 \pm 0.08$ & $0.58 \pm 0.08$ & $0.63 \pm 0.15$ & $0.61 \pm 0.05$ & $0.73 \pm 0.19$ & $0.70 \pm 0.21$ & $0.67 \pm 0.09$ & $0.74 \pm 0.25$ & $0.60 \pm 0.13$ \\
\hline $\begin{array}{l}\text { Longitudinal } \\
\text { Displacement } \\
\text { (Endo) }\end{array}$ & Pk deg & $0.23 \pm 0.02$ & $0.17 \pm 0.04$ & $0.12 \pm 0.03$ & $0.11 \pm 0.04$ & $0.10 \pm 0.02$ & $0.07 \pm 0.01$ & $0.20 \pm 0.07$ & $0.10 \pm 0.04$ & $0.16 \pm 0.08$ & $0.10 \pm 0.03$ \\
\hline $\begin{array}{l}\text { Longitudinal } \\
\text { Strain } \\
\text { (Endo) }\end{array}$ & $\mathrm{Pk} \%$ & $\begin{array}{c}-10.58 \pm \\
1.74\end{array}$ & $\begin{array}{c}-7.10 \pm \\
0.74 \\
3\end{array}$ & $\begin{array}{c}-11.55 \pm \\
0.59\end{array}$ & $\begin{array}{c}-9.41 \pm \\
1.31\end{array}$ & $\begin{array}{c}-9.45 \pm \\
0.69\end{array}$ & $\begin{array}{c}-8.02 \pm \\
0.66\end{array}$ & $\begin{array}{c}-8.46 \pm \\
1.78\end{array}$ & $\begin{array}{c}-9.55 \pm \\
0.60\end{array}$ & $\begin{array}{c}-9.11 \pm \\
1.39\end{array}$ & $\begin{array}{c}-8.80 \pm \\
0.61\end{array}$ \\
\hline $\begin{array}{l}\text { Longitudinal } \\
\text { Strain Rate } \\
\text { (Endo) }\end{array}$ & $\mathrm{Pk} 1 / \mathrm{s}$ & $\begin{array}{c}-3.90 \pm \\
0.42\end{array}$ & $\begin{array}{c}-3.09 \pm \\
0.23\end{array}$ & $\begin{array}{c}-4.13 \pm \\
0.23\end{array}$ & $\begin{array}{c}-4.73 \pm \\
0.75\end{array}$ & $\begin{array}{c}-4.02 \pm \\
0.37\end{array}$ & $\begin{array}{c}-3.36 \pm \\
0.33\end{array}$ & $\begin{array}{c}-3.61 \pm \\
0.45\end{array}$ & $\begin{array}{c}-3.91 \pm \\
0.29\end{array}$ & $\begin{array}{c}-3.84 \pm \\
0.58\end{array}$ & $\begin{array}{c}-3.57 \pm \\
0.50\end{array}$ \\
\hline
\end{tabular}


Table S2.6: B-Mode images were used in the long axis to examine circumferential and longitudinal stress-strain parameters during systole. Ultrasound imaging one week prior (Het and KO groups) and following nano- $\mathrm{TiO}_{2}$ inhalation exposure (WT, Het, and KO). Significance $(P \leq$ 0.05 ) is denoted for a specific category through bold text and reference to the sample number that it is significantly different from. Sample numbers are listed in the column heading. WT $=$ Wild type, Het $=$ heterozygous for the miRNA-378a allele, $\mathrm{KO}=$ knockout for the miRNA-378a allele, Sham $=$ control filtered air exposed, $\mathrm{Ex}=$ nano- $\mathrm{TiO}_{2}$ exposed, $\mathrm{Pre}=$ one week prior to inhalation exposure, Post $=24$ post inhalation exposure. 


\section{Figure S2.1}

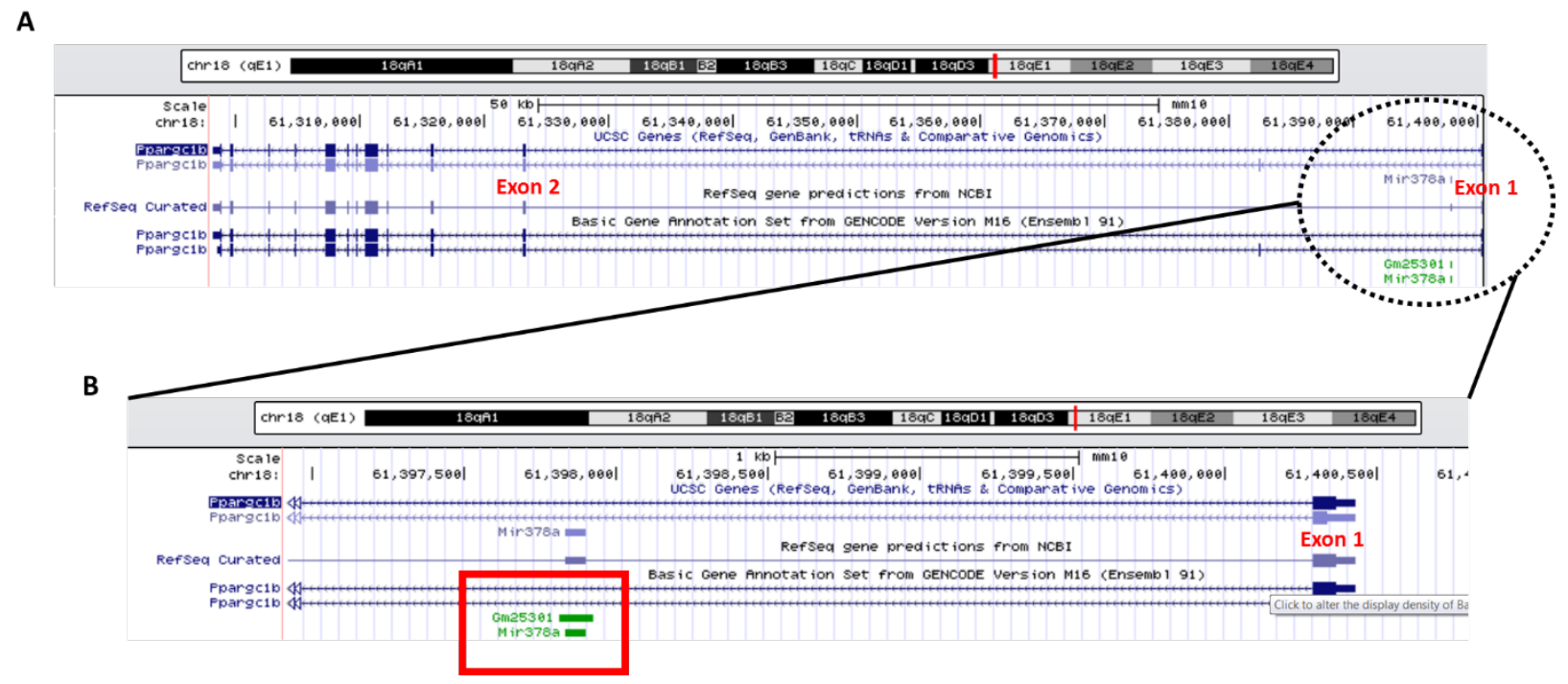


Figure S2.1: The genomic loci of Ppargc1b. (A) Total exonic and intronic region of Ppargclb, with miRNA-378a being transcribed in the first intron. (B) Magnified view of the first intron of Ppargc1b. Image was taken from the University of Santa Cruz (UCSC) genome browser from the mouse GRCm38/mm10 assembly. Ppargclb = Peroxisome proliferator-activated receptor gamma coactivator 1-beta. 
Figure S2.2
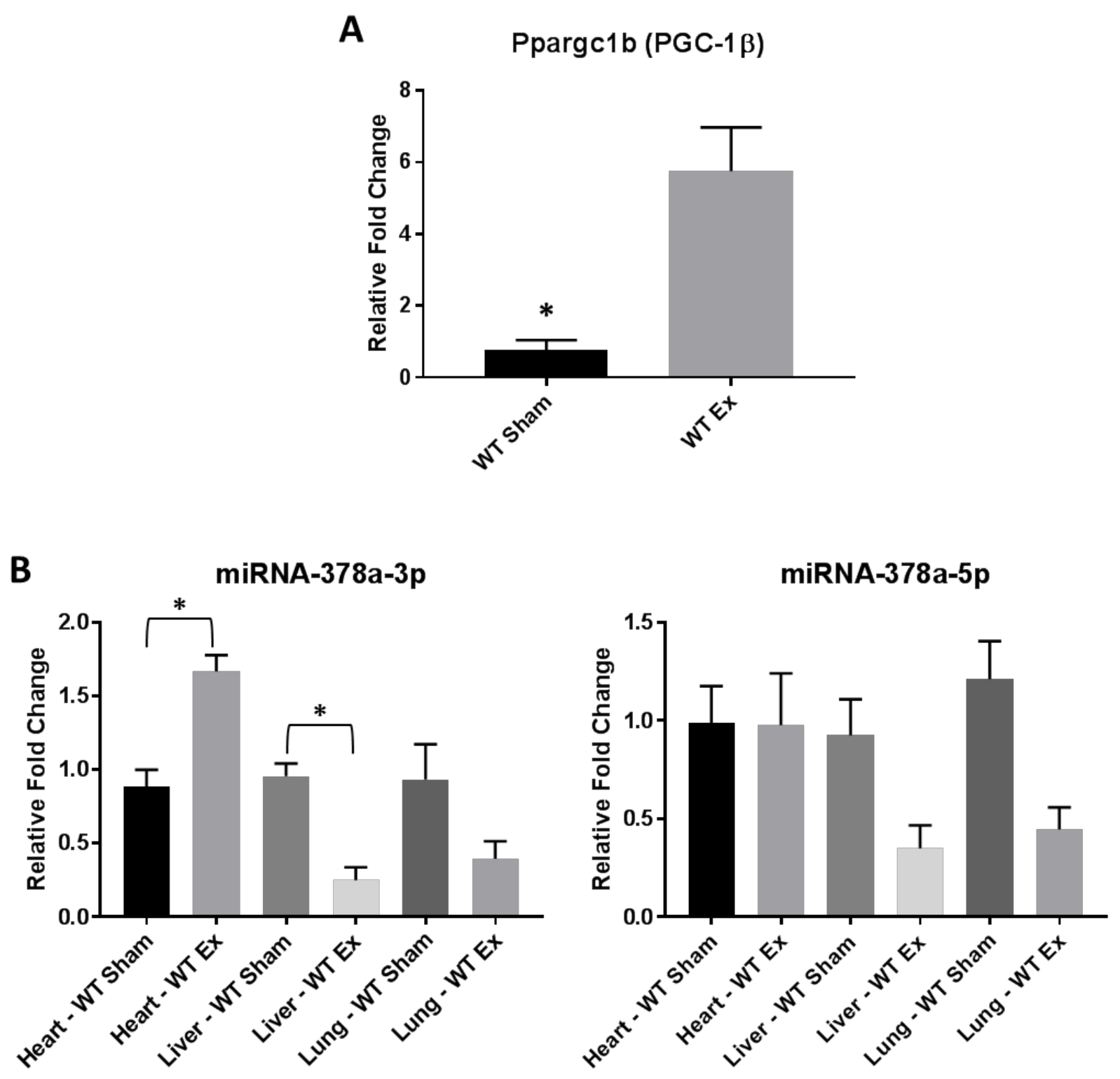
Figure S2.2

C

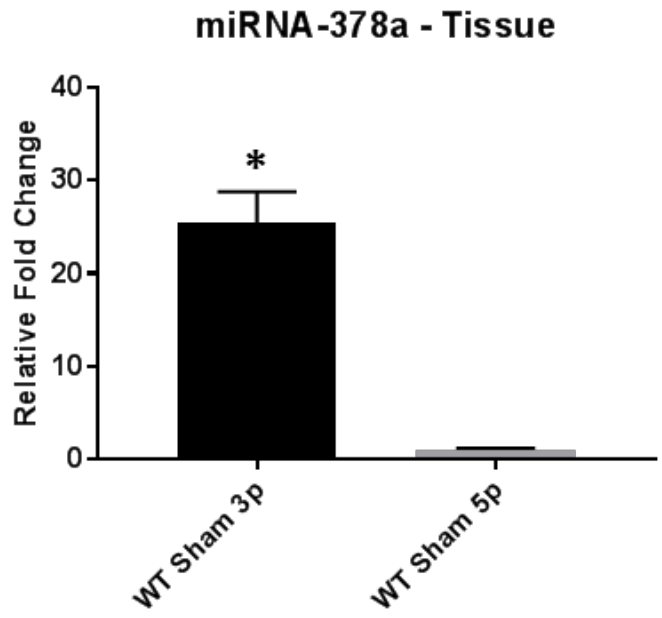

D miRnA-378a-3p - Tissue

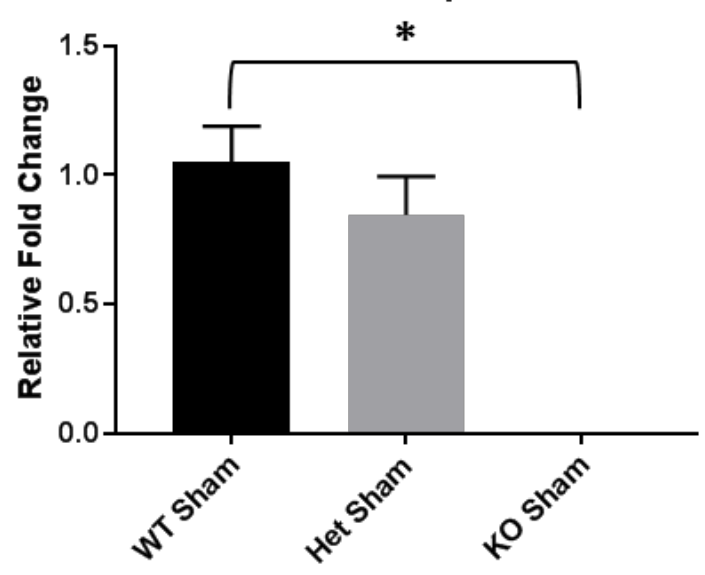

miRNA-378a-3p - Mitochondria

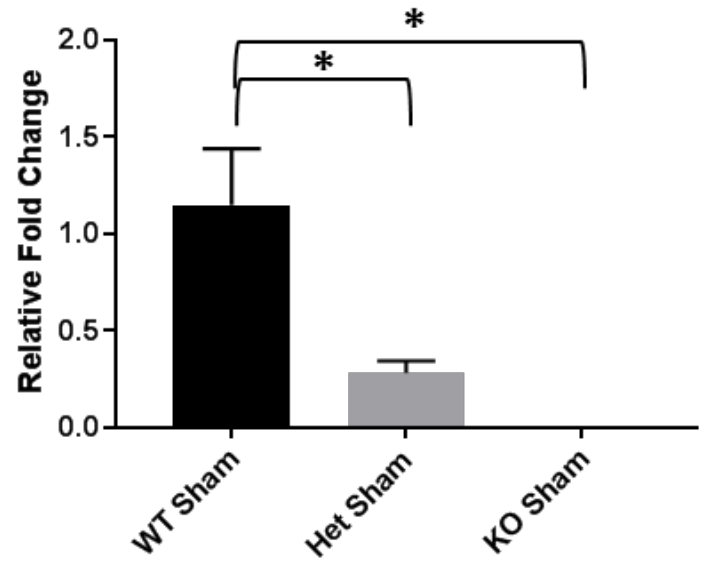

miRNA-378a - Mitochondria
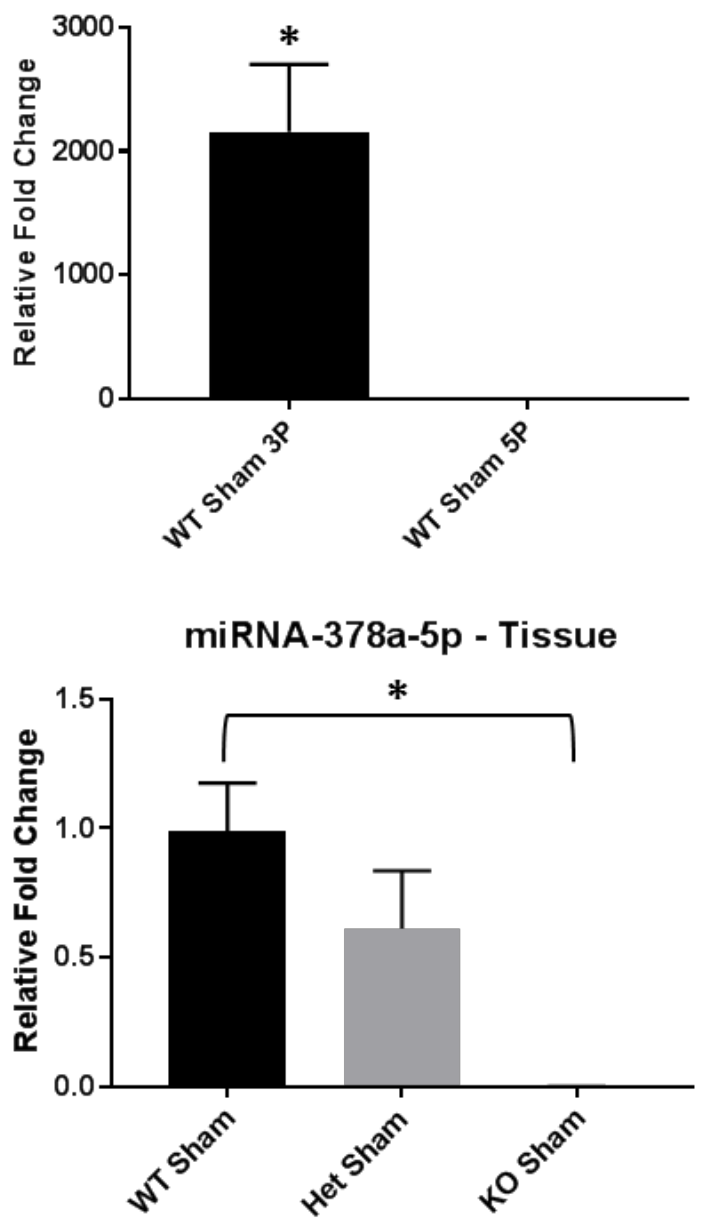

miRNA-378a-5p - Mitochondria

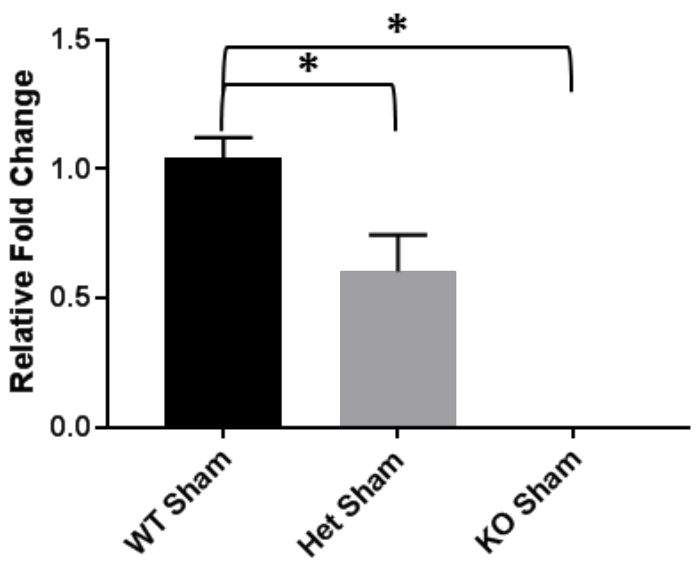


Figure S2.2: Expression of miRNA-378a in other tissues and transgenic animals. (A) qPCR of Ppargc1b (PGC-1 $\beta$ ), the miRNA-378a host gene, following nano- $\mathrm{TiO}_{2}$ exposure. (B) $\mathrm{qPCR}$ in the heart, lung, and liver for miRNA-378a-3p and miRNA-378a-5p. Using the transgenic animals (C) The relative abundance of miRNA-378a-3p versus miRNA-378a-5p was also verified. With the knockdown and knockout transgenic animals, (D) qPCR confirmed miRNA-378a-3p and miRNA378a-5p expression in the whole heart tissue and isolated mitochondria. Groups are considered significantly different if $P \leq 0.05=*$. All data are presented as the mean \pm standard error of the mean $(\mathrm{SEM}) . \mathrm{WT}=$ wild-type Het $=$ heterozygous for the miRNA-378a allele, $\mathrm{KO}=$ knockout for the miRNA-378a allele, Sham $=$ control filtered air exposed, $\mathrm{Ex}=$ nano- $\mathrm{TiO}_{2}$ exposed, Ppargc $1 \mathrm{~b}$ or PGC-1 $\beta$ = peroxisome proliferator-activated receptor gamma coactivator 1-beta. 
Figure S2.3

A
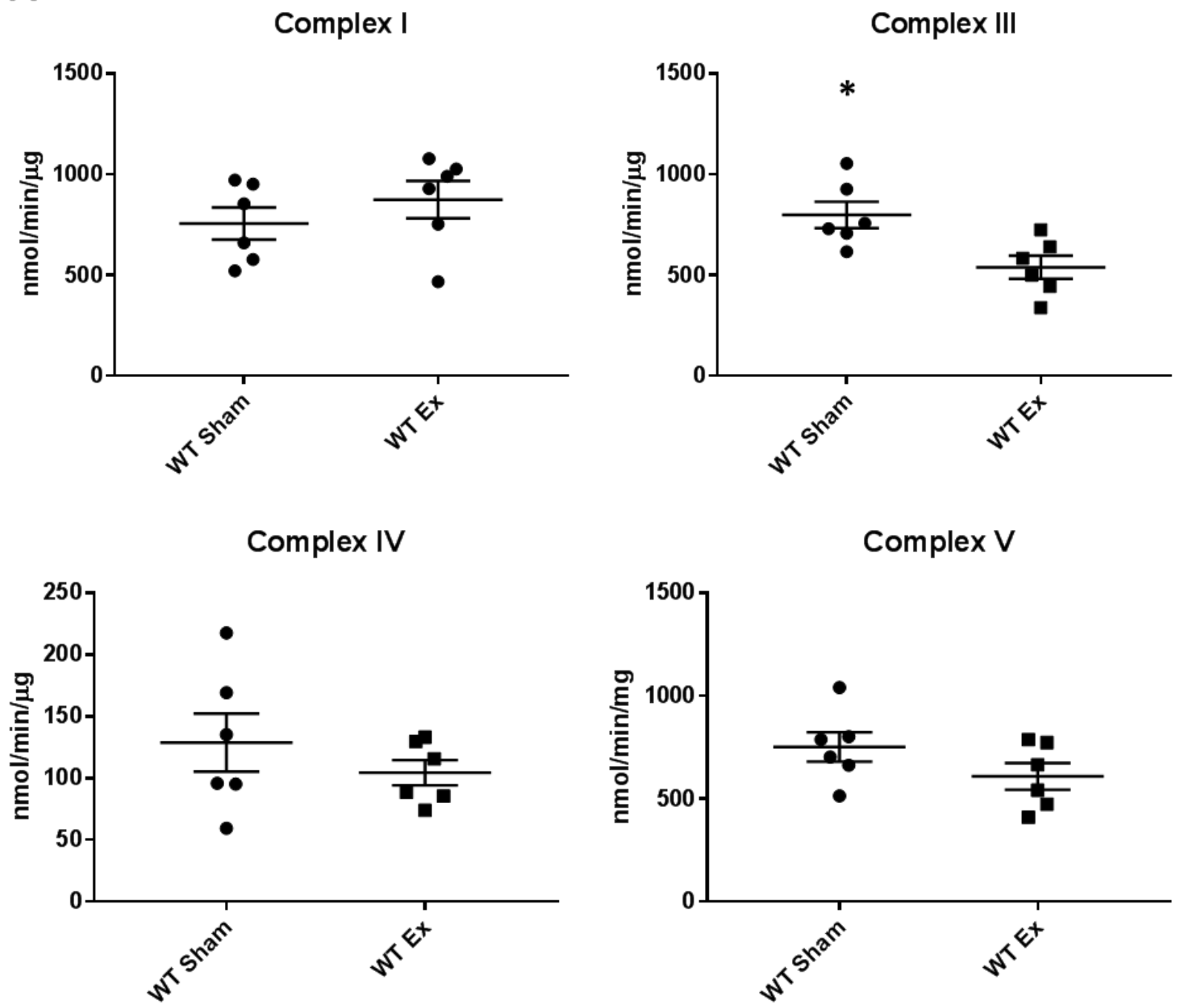
Figure S2.3

B
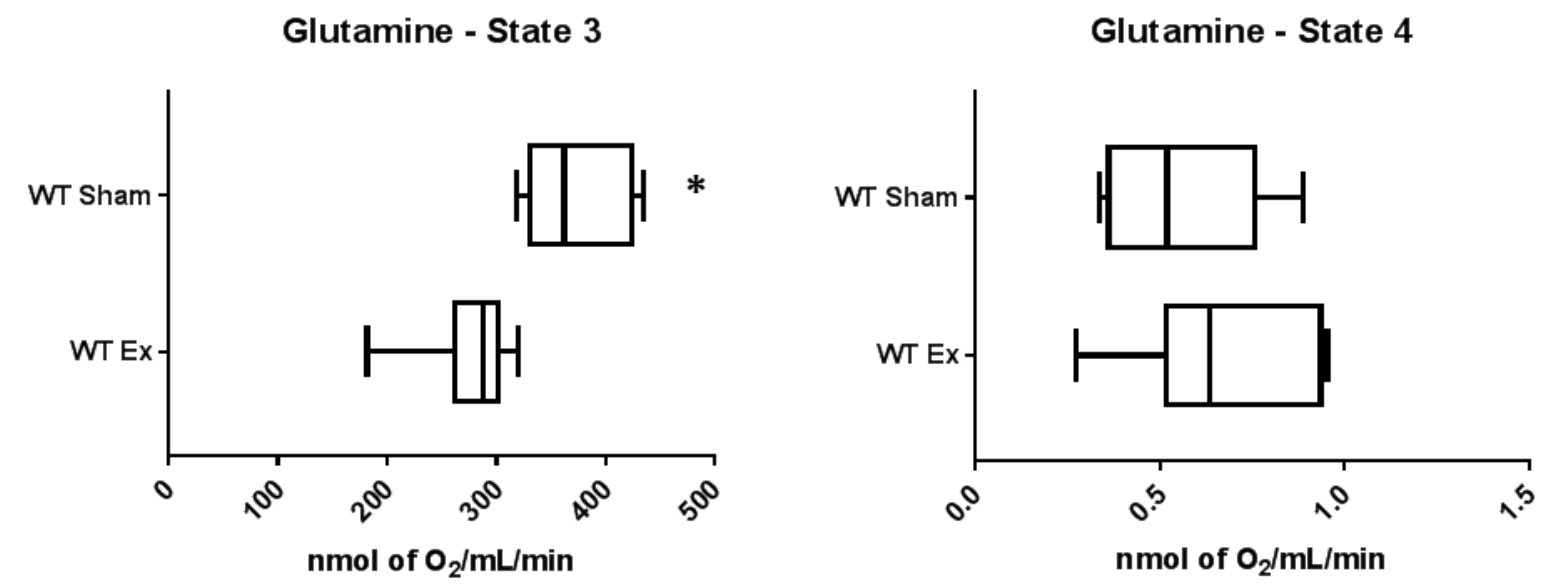

P-Carnitine - State 3

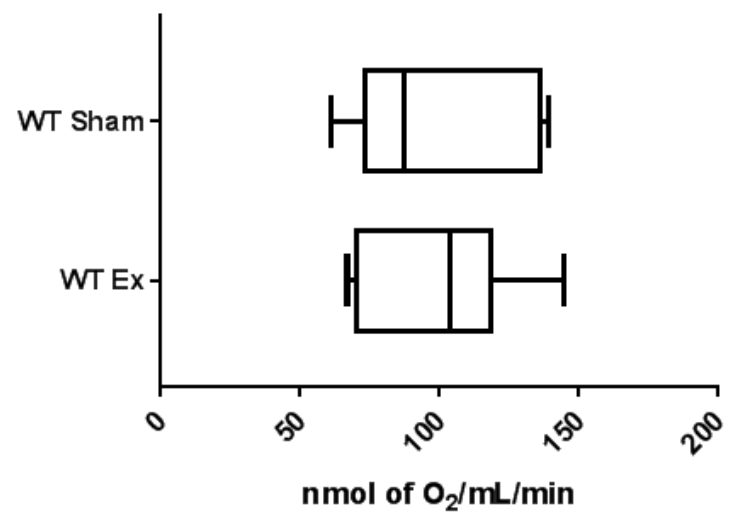

P-Carnitine - State 4

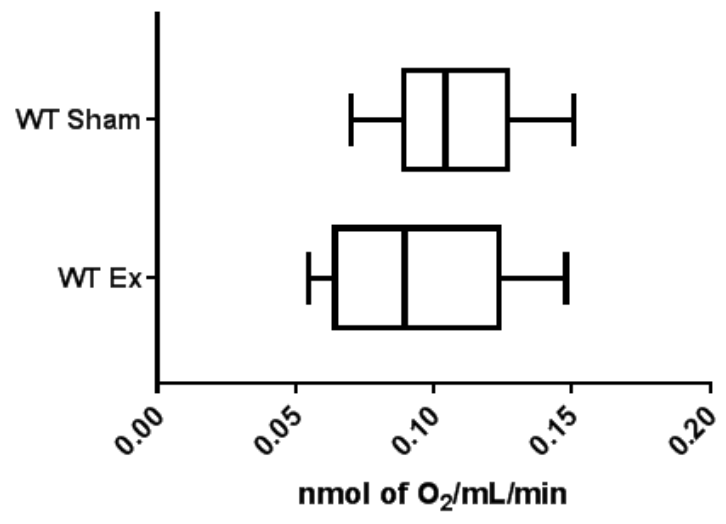


Figure S2.3: Bioenergetic function of cardiac mitochondria following nano- $\mathrm{TiO}_{2}$ inhalation exposure in the WT. (A) Electron transport chain complex activities were measured for complexes I, III, IV, and V in the WT. (B) Mitochondrial State 3 and State 4 respiration were measured through glucose mediated pathways and fatty acid mediated pathways in the WT. Groups are considered significantly different if $P \leq 0.05=*$. All data are presented as the mean \pm standard error of the mean $(\mathrm{SEM}) . \mathrm{WT}=$ Wild type, Sham $=$ control filtered air exposed, $\mathrm{Ex}=$ nano- $\mathrm{TiO}_{2}$ exposed, P-Carnitine = palmitoyl carnitine. 
Figure S2.4
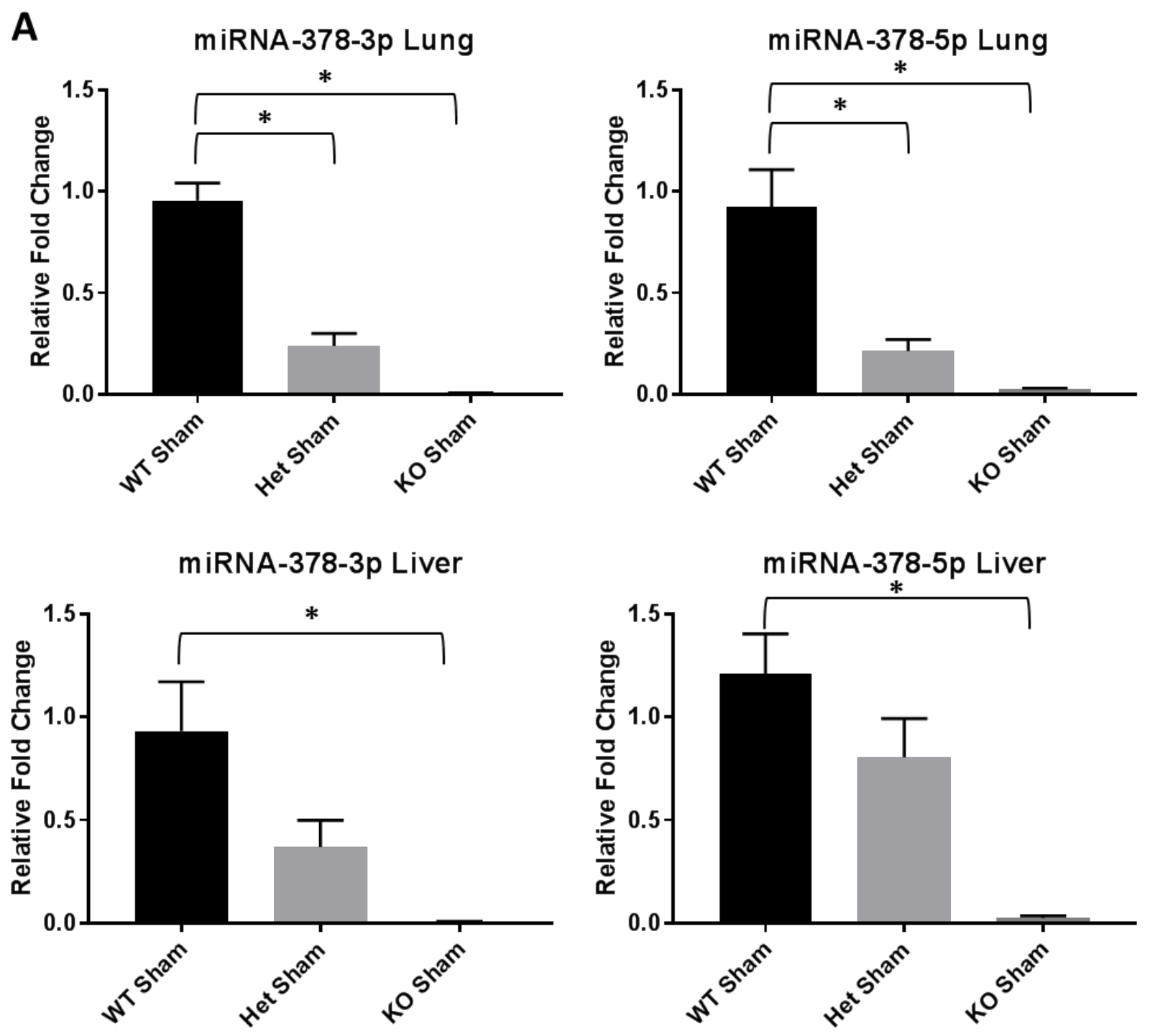
Figure S2.4

B

Complex I

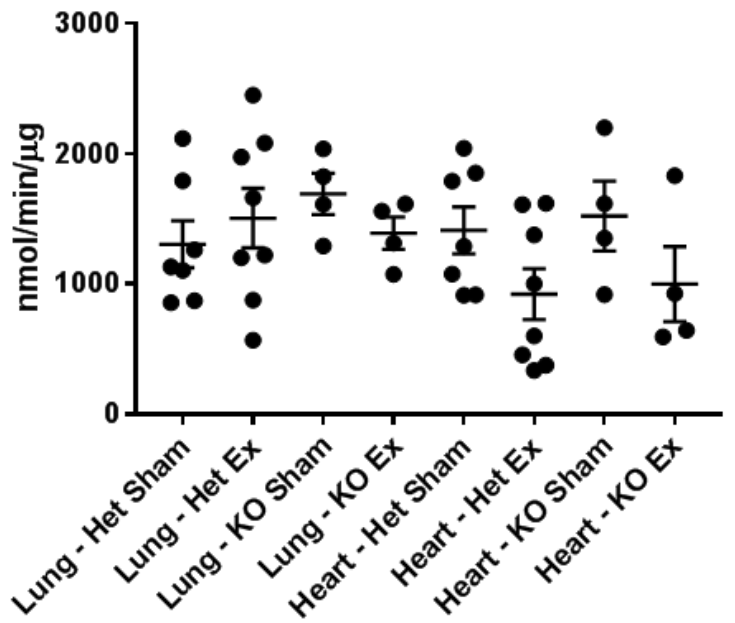

Complex IV

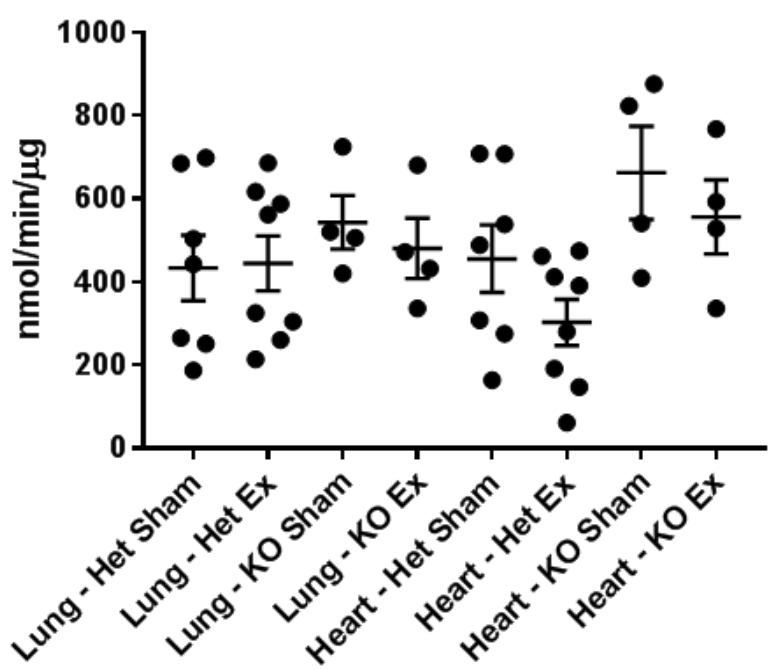

Complex III

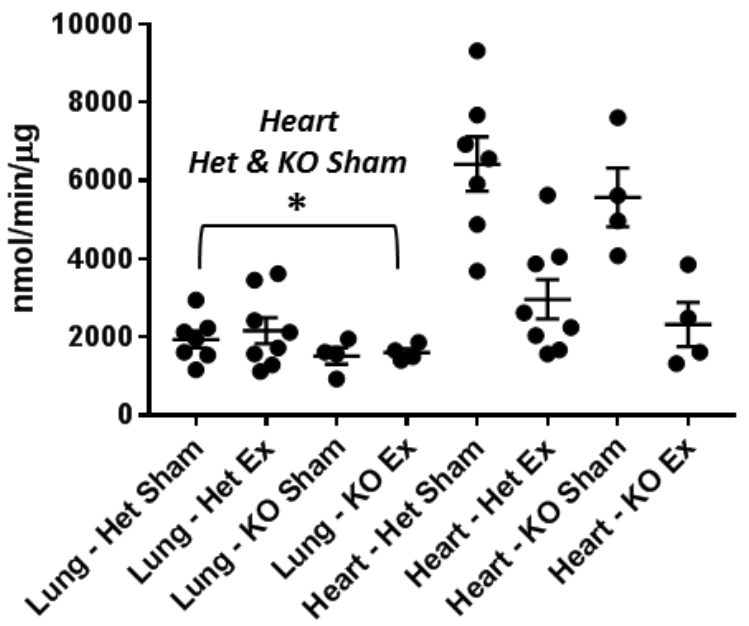

Complex V

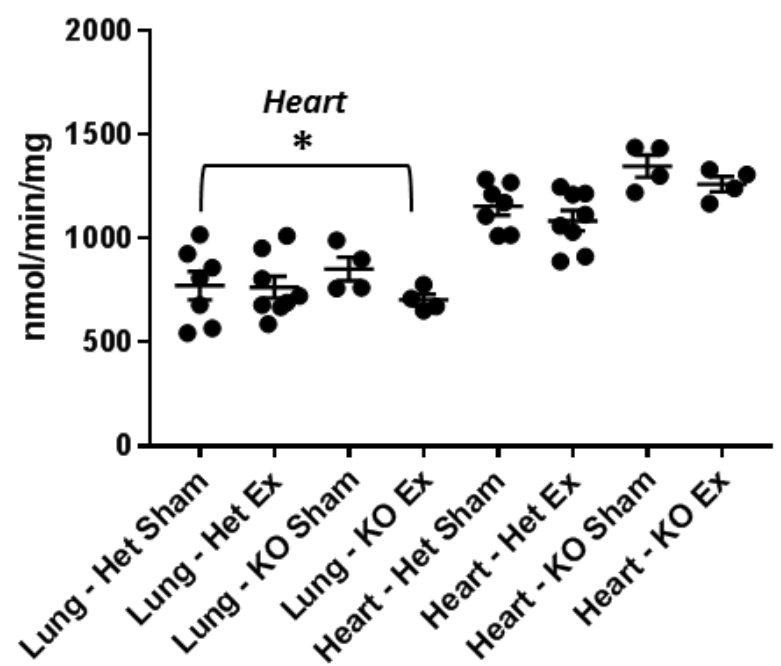


Figure S2.4: Expression of miRNA-378a and its bioenergetic effects on other tissues. (A) qPCR was implemented to assess expression of miRNA-378a-3p and miRNA-378a-5p in the lung and liver. (B) Electron transport chain complex activities were measured for complexes I, III, IV, and $\mathrm{V}$ in the lung and liver for the Het and KO groups. Groups are considered significantly different if $P \leq 0.05=*$. All data are presented as the mean \pm standard error of the mean $(\mathrm{SEM})$. WT $=$ Wild type, $\mathrm{Het}=$ heterozygous for the miRNA-378a allele, $\mathrm{KO}=$ knockout for the miRNA-378a allele, Sham $=$ control filtered air exposed, $\mathrm{Ex}=$ nano- $\mathrm{TiO}_{2}$ exposed. 
Figure S2.5

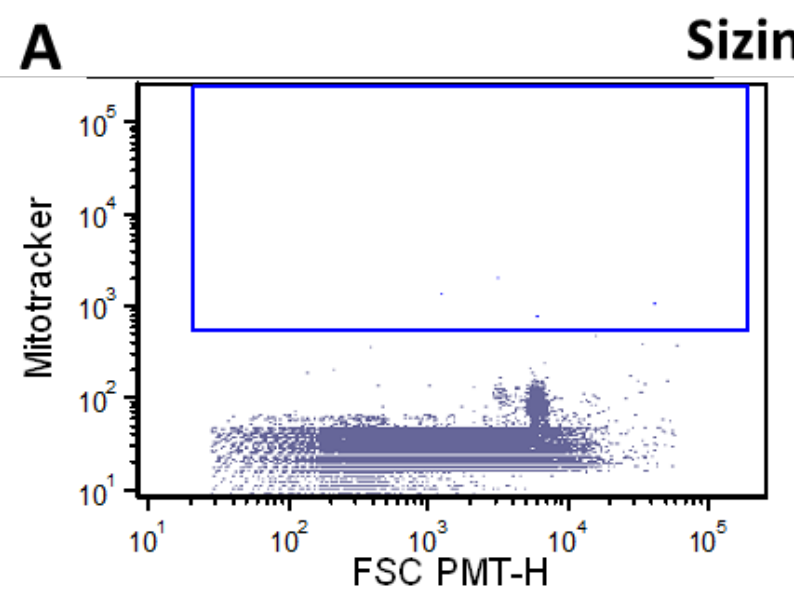

Sizing Beads

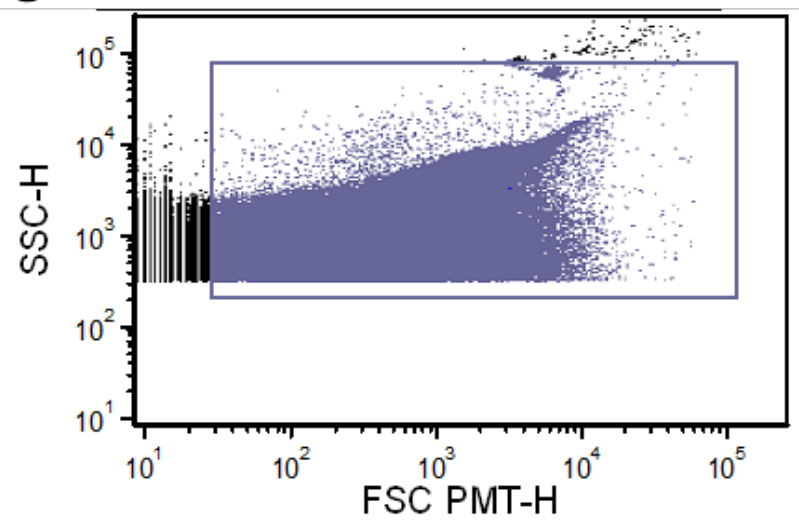

Isolated Mitochondria
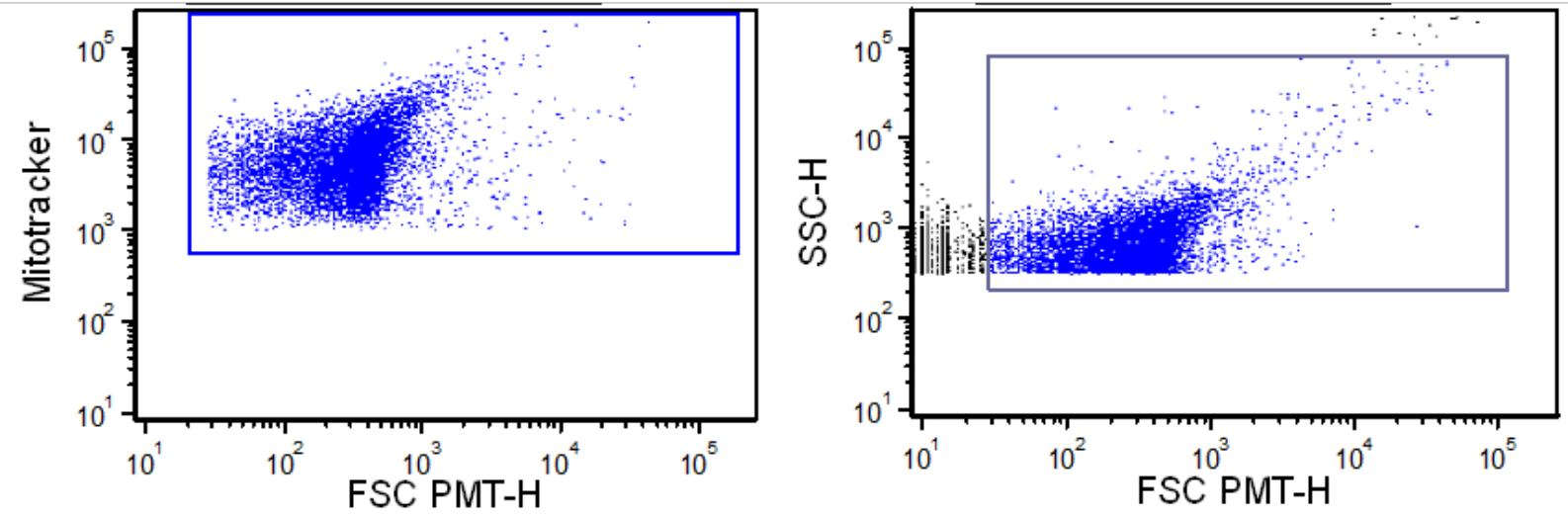
Figure S2.5
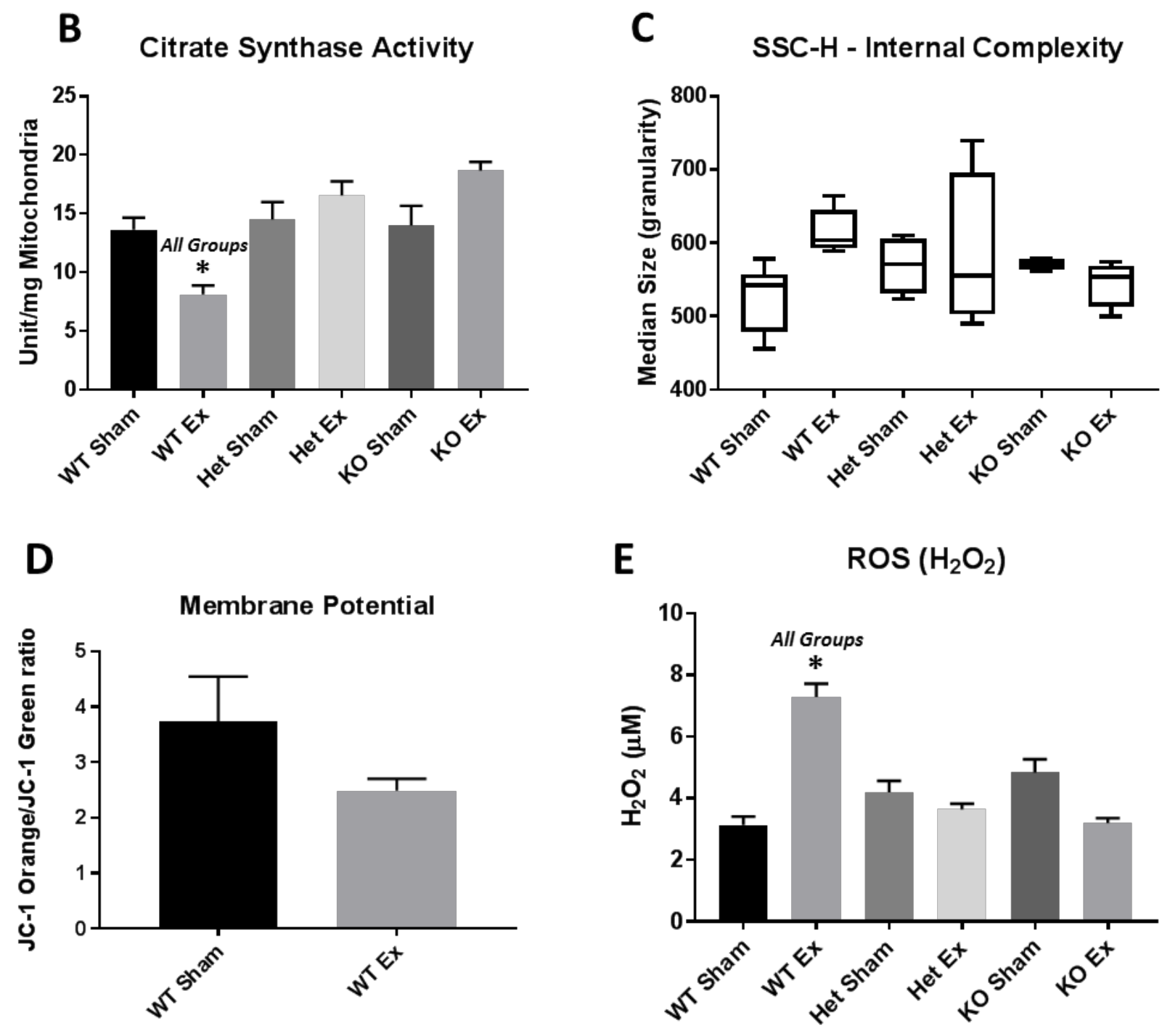
Figure S2.5: Mitochondrial structure and function and the regulation of the Ppargclb host gene. (A) Flow cytometry Sphero AccuCount Blank Particles, $2.0 \mu \mathrm{m}$ sizing beads and MitoTracker ${ }^{\mathrm{TM}}$ Deep Red FM/633 staining allowed for measurements of mitochondrial size and structure. (B) Citrate synthase activity was used to measure mitochondrial number/content on isolated mitochondria. Flow cytometry assessed mitochondria through (C) internal complexity and (D) membrane potential implementing MitoTrackerTM Deep Red FM/633 and JC-1 staining, respectively. (E) Cardiac tissue was assessed for $\mathrm{H}_{2} \mathrm{O}_{2}$ concentration, normalized to mitochondrial number. Groups are considered significantly different if $P \leq 0.05=*$. All data are presented as the mean \pm standard error of the mean $(\mathrm{SEM}) . \mathrm{FSC}$ PMT-H $=$ forward scatter, $\mathrm{SSC}-\mathrm{H}=$ side scatter, $\mathrm{WT}=\mathrm{Wild}$ type, $\mathrm{Het}=$ heterozygous for the miRNA-378a allele, $\mathrm{KO}=$ knockout for the miRNA378a allele, Sham $=$ control filtered air exposed, $\mathrm{Ex}=$ nano- $\mathrm{TiO}_{2}$ exposed. 
Figure S2.6

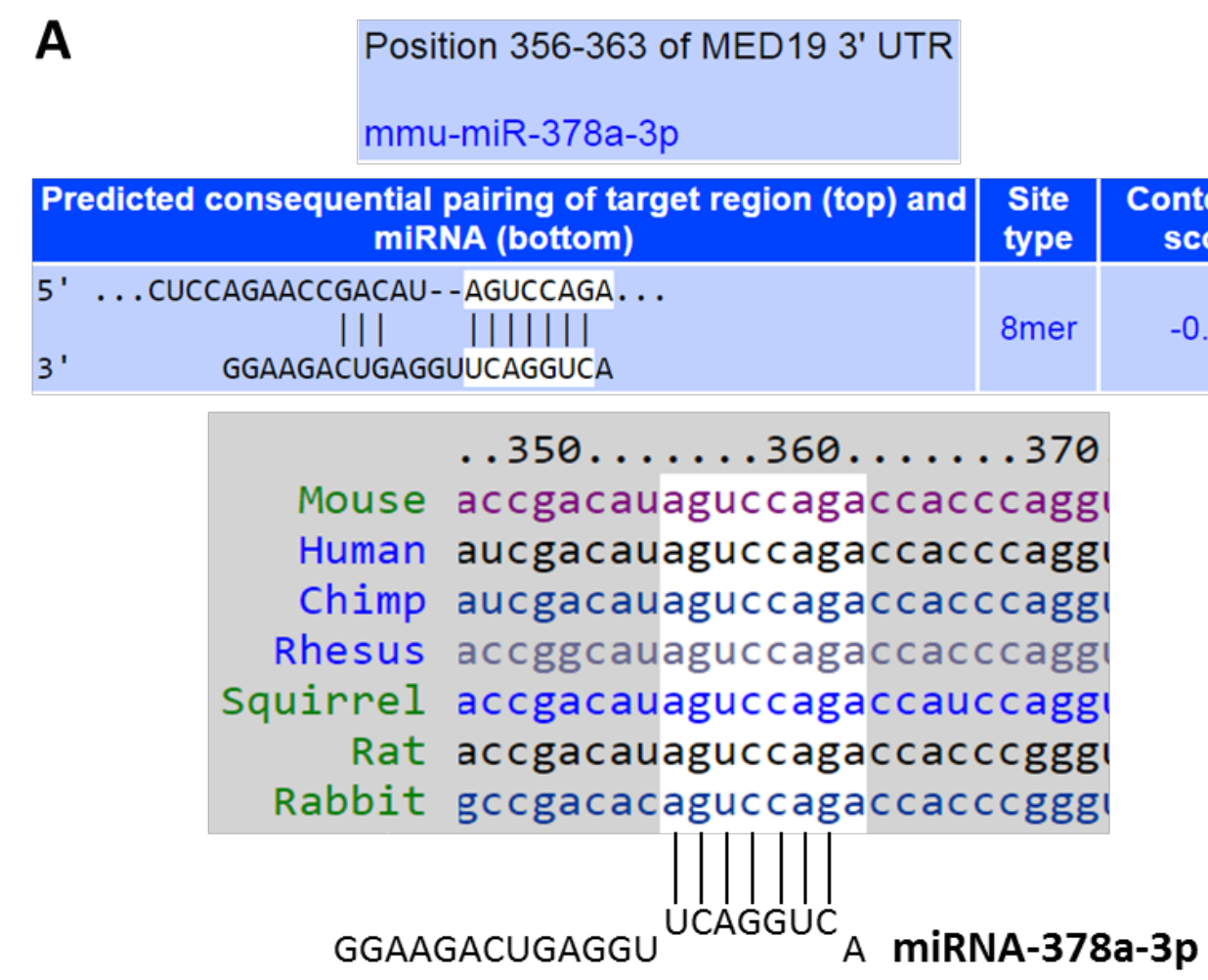

Position $1335-1342$ of MFN1 3' UTR

mmu-miR-378a-3p

\begin{tabular}{|c|c|c|}
\hline $\begin{array}{l}\text { Predicted consequential pairing of target region (top) and } \\
\text { miRNA (bottom) }\end{array}$ & $\begin{array}{l}\text { Site } \\
\text { type }\end{array}$ & $\begin{array}{l}\text { Context++ } \\
\text { score }\end{array}$ \\
\hline 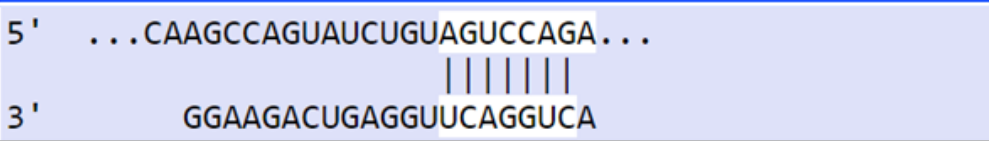 & $8 \mathrm{mer}$ & -0.29 \\
\hline
\end{tabular}

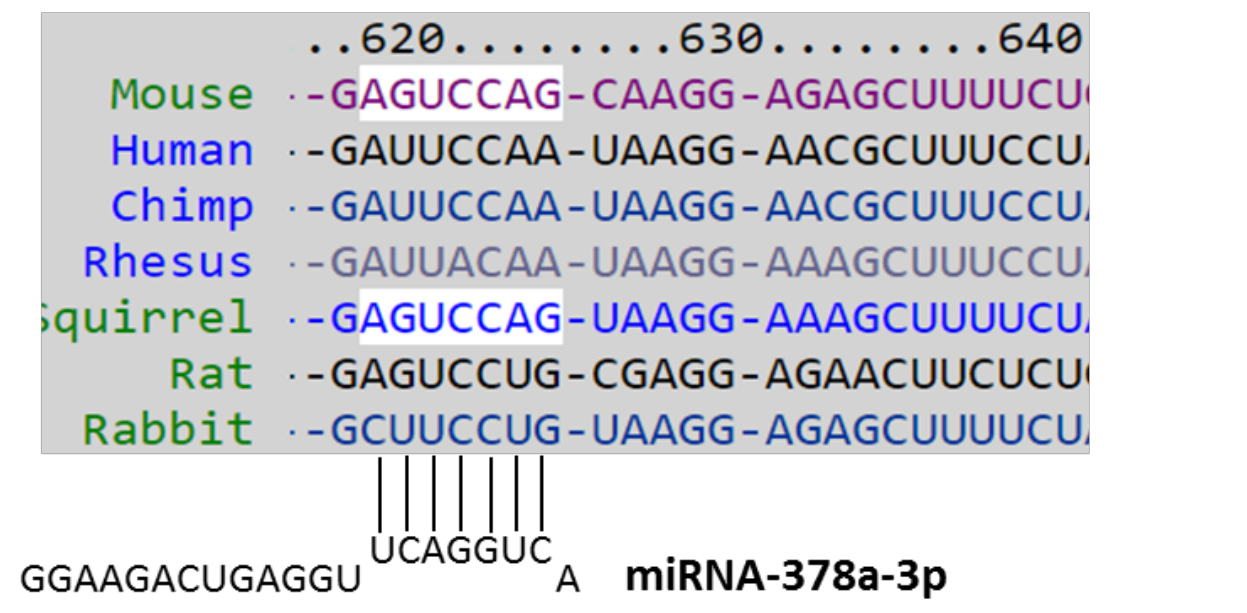


Figure S2.6

B
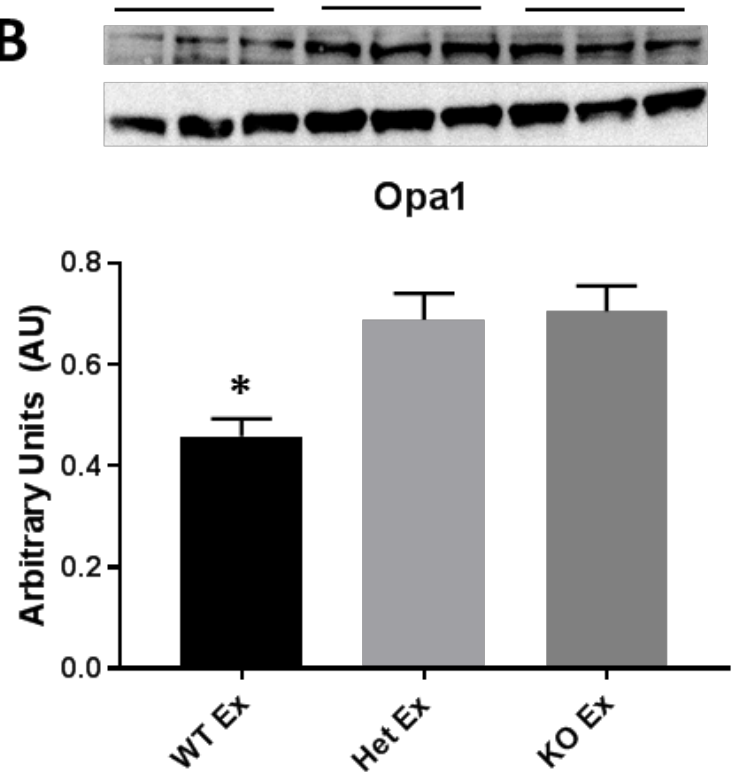

C
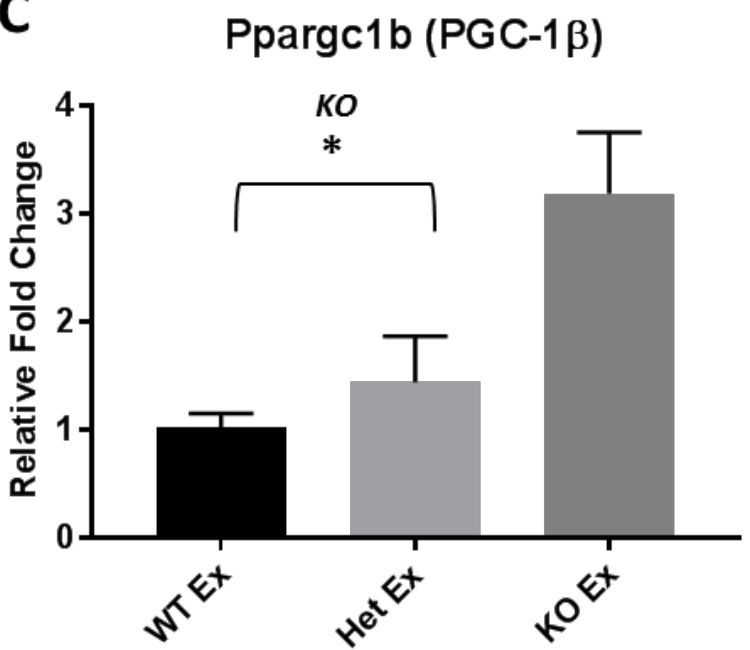

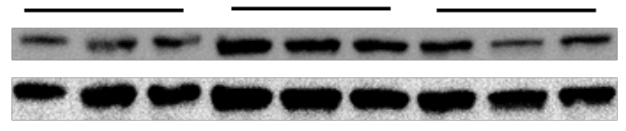

Ppar $\alpha$

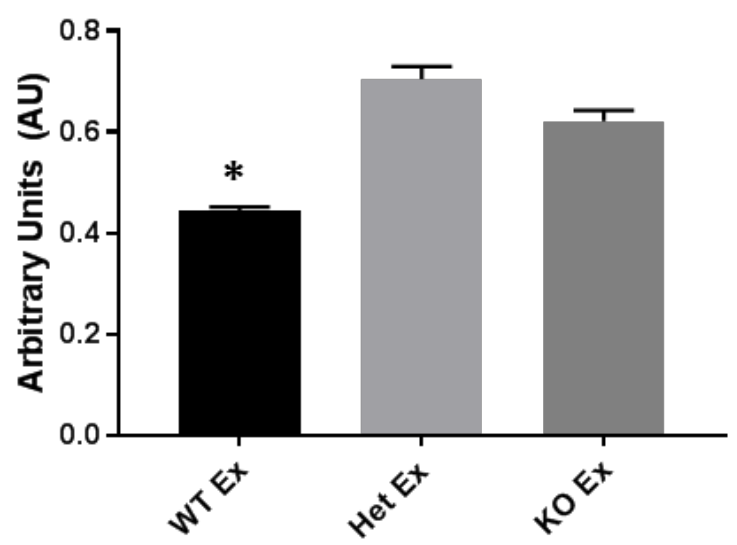

D Ppargc1b (PGC-1 $1 \beta)$

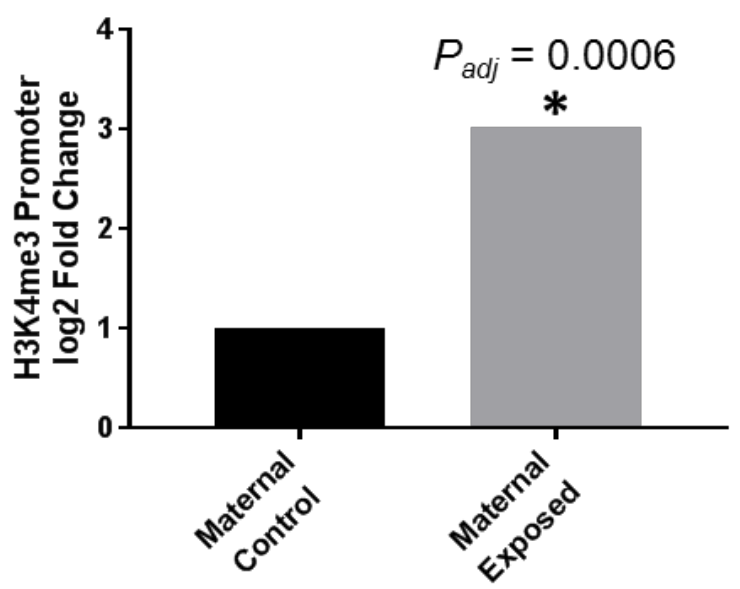


Figure S2.6: Seed sequence homology in the 3'UTR of Med19 and Mfn1. Through TargetScan, miRNA-378a-3p was shown to bind the 3' UTR of (A) Med19 and Mfn1 with seed sequence homology. "Position" refers to the location in the 3'UTR region that binding occurs. The "Site Type" refers to the specific binding of the miRNA to the mRNA strand by the number of bases that share continued homology. The "Context Score" (0 to -0.99) is an index of the confidence of binding, with a more negative score instilling higher confidence. (B) Immunoblotting of additional proteins involved in mitochondrial fusion (Opa1) and fatty acid metabolism (Ppara). (C) Increased transcription of the miRNA-378a host gene, Ppargc1b, occurs following inhalation exposure. (D) Chromatin Immunoprecipitation (ChIP) Sequencing of $\mathrm{H} 3 \mathrm{~K} 4 \mathrm{me} 3$ in gestational nano- $\mathrm{TiO}_{2}$ exposed progeny revealed increased $\mathrm{H} 3 \mathrm{~K} 4 \mathrm{me} 3$ association at the Ppargclb promoter loci, suggesting increased epigenetic regulation and subsequent expression of the gene. Groups are considered significantly different if $P \leq 0.05=*$. All data are presented as the mean \pm standard error of the mean $(\mathrm{SEM}) . P$-adjusted values $=P$ values derived from DESeq2 and calculated with the False Discovery Rate $(\mathrm{FDR})=0.05$. Groups are considered significantly different if $P_{a d j} \leq 0.05$ $={ }^{*}$. Mfn1 $=$ Mitofusin 1, Med19 $=$ mediator complex subunit 19, $3^{\prime}$ UTR $=3^{\prime}$ ' untranslated region, $\mathrm{WT}=$ Wild type, $\mathrm{Het}=$ heterozygous for the miRNA-378a allele, $\mathrm{KO}=$ knockout for the miRNA$378 \mathrm{a}$ allele, Sham $=$ control filtered air exposed, $\mathrm{Ex}=$ nano- $\mathrm{TiO}_{2}$ exposed, $\mathrm{Ppargc} 1 \mathrm{~b}=$ Peroxisome proliferator-activated receptor gamma coactivator 1-beta, H3K4me3 = Histone 3 lysine 3 trimethylation. 


\section{Chapter 3: Specific Aim 2 \\ Machine-Learning to Stratify Diabetic Patients using Novel Cardiac Biomarkers and Integrative Genomics}

Quincy A. Hathaway, ${ }^{1,2}$ Skyler M. Roth, ${ }^{3}$ Mark V. Pinti, ${ }^{4}$ Daniel C. Sprando, ${ }^{5}$ Amina Kunovac,, 2 Andrya J. Durr, ${ }^{1,2}$ Chris C. Cook, ${ }^{6}$ Garrett K. Fink, ${ }^{1}$ Tristen B. Cheuvront, ${ }^{3}$ Jasmine H. Grossman, ${ }^{3}$ Ghadah A. Aljahli, ${ }^{3}$ Andrew D. Taylor, ${ }^{1,2}$ Andrew P. Giromini, ${ }^{5}$ Jessica L. Allen, ${ }^{3}$ and *John M. Hollander ${ }^{1,2}$

As published in Cardiovasc Diabetol. 2019 Jun 11;18(1):78. doi: 10.1186/s12933-019-0879-0. [Epub ahead of print]

${ }^{1}$ Division of Exercise Physiology, West Virginia University School of Medicine, Morgantown 26505, WV, USA.

${ }^{2}$ Mitochondria, Metabolism \& Bioenergetics Working Group, West Virginia University School of Medicine, Morgantown 26505, WV, USA. ${ }^{3}$ Department of Chemical and Biomedical Engineering, West Virginia University, Morgantown 26505, WV, USA. ${ }^{4}$ West Virginia University School of Pharmacy, Morgantown 26505, WV, USA. ${ }^{5}$ West Virginia University School of Medicine, Morgantown 26505, WV, USA. ${ }^{6}$ Cardiovascular and Thoracic Surgery, West Virginia University School of Medicine, Morgantown 26505, WV, USA.

*Corresponding Author:

John M. Hollander, Ph.D., F.A.H.A.

Division of Exercise Physiology

West Virginia University School of Medicine

PO Box 9227

1 Medical Center Drive

Morgantown, WV 26506

Tel: 1-(304) 293-3683

Fax: 1-(304) 293-7105

Email: jhollander@hsc.wvu.edu 


\begin{abstract}
Background - Diabetes mellitus is a chronic disease that impacts an increasing percentage of people each year. Among its comorbidities, diabetics are two to four times more likely to develop cardiovascular diseases. While HbAlc remains the primary diagnostic for diabetics, its ability to predict long-term, health outcomes across diverse demographics, ethnic groups, and at a personalized level are limited. The purpose of this study was to provide a model for precision medicine through the implementation of machine learning algorithms using multiple cardiac biomarkers as a means for predicting diabetes mellitus development.
\end{abstract}

Methods - Right atrial appendages from 50 patients, 30 non-diabetic and 20 type 2 diabetic, were procured from the WVU Ruby Memorial Hospital. Machine-learning was applied to physiological, biochemical, and sequencing data for each patient. Supervised learning implementing SHapley Additive exPlanations (SHAP) allowed binary (no diabetes or type 2 diabetes) and multiple classification (no diabetes, prediabetes, and type 2 diabetes) of the patient cohort with and without the inclusion of HbA1c levels. Findings were validated through Logistic Regression (LR), Linear Discriminant Analysis (LDA), Gaussian Naïve Bayes (NB), Support Vector Machine (SVM), and Classification and Regression Tree (CART) models with 10-fold cross validation.

Results - Total nuclear methylation and hydroxymethylation were highly correlated to diabetic status, with nuclear methylation and mitochondrial electron transport chain (ETC) activities achieving superior testing accuracies in the predictive model ( 84\% testing, binary). Mitochondrial DNA SNPs found in the D-Loop region (SNP-73G, -16126C, and -16362C) were highly associated with diabetes mellitus. The $\mathrm{CpG}$ island of transcription factor A, mitochondrial (TFAM) revealed CpG24 (chr10:58385262, $P=0.003)$ and CpG29 (chr10:58385324, $P=0.001)$ as markers correlating with diabetic progression. When combining the most predictive factors from each set, total nuclear methylation and $\mathrm{CpG} 24$ methylation were the best diagnostic measures in both binary and multiple classification sets.

Conclusions - Using machine-learning, we were able to identify novel as well as the most relevant biomarkers associated with type 2 diabetes mellitus by integrating physiological, biochemical, and sequencing datasets. Ultimately, this approach may be used as a guideline for future investigations into disease pathogenesis and novel biomarker discovery. 
Keywords: Epigenetics, Mitochondria, Heart, Machine-Learning, CART, SHAP 


\section{Background}

A disconnect continues to persist in the diagnosis and pathogenesis of diabetes-induced cardiovascular dysfunction. While diabetics are at a two to four fold greater risk of developing cardiovascular diseases $(7,22)$, understanding how the numerous biochemical markers involved in the pathology integrate and influence disease progression has not been fully explicated. In a clinical setting, the ability to better calculate prognostics of a patient's health through the integration of biomarkers facilitates the potential for developing personalized and generalized Medicine, as well as treatment strategies (50). While glycated hemoglobin (HbAlc) remains a hallmark for disease diagnosis (8), other biomarkers may exist that more unequivocally define the severity of the pathology, characterize the mechanisms involved, and/or provide a better predictive tool of future cardiovascular events.

Diabetes mellitus is a multi-faceted disease, consisting of systemic comorbidities which necessitate a variety of treatment modalities and stratify those affected with the disease (12). Before the implementation of machine-learning algorithms in Medicine, linear statistical models have highlighted measures, such as $\mathrm{HbAlc}$, as diagnostic staples for the evaluation of diabetes mellitus onset and progression (40). By exploring these previously published metadata sets, machine-learning has been applied in refining the accuracy of biomarkers used to characterize the pathology as well as to highlight vulnerable populations in need of clinical intervention (27). Machine-learning has also revealed that coupling HbAlc with additional biomarkers, such as 8hydroxy-2-deoxyguanosine (8-OhdG) and other metabolites, can increase the accuracy of the predictive model and better characterize the severity of the disease (26).

In cardiology, machine-learning approaches have been applied primarily to imaging-based diagnostics, including echocardiography and computed tomography angiography to evaluate cardiovascular health and outcomes $(1,59)$. It is estimated that machine-learning applications in the field of cardiovascular research will continue to grow at an exponential rate (51). While imagederived deep learning models are increasing in popularity, little is known about the predicative power of machine-learning models on basic genomic, epigenomic, proteomic, and metabolomic profiles of the heart. While the beginning of the "big data" age was characterized by the accumulation and compartmentalization of datasets, the recent advent of combining metadata, deep sequencing, and “omics"-based approaches warrants the union between hierarchical 
predictive algorithms and biological processes. As more patients have access to their personal "omics" profiles, machine-learning will be instrumental in providing feedback for the individual patient and the general population of patients impacted by the disease, enhancing health practice by the caregiver.

While demographic information and physical examination data are more easily procured from patients, the genomic and transcriptomic characterization of a tissue or cell type provides a much finer granularity and uniqueness when predicting phenotypic outcomes in patients (36). HbA1c, fasting blood glucose, and BMI are examples of easily accessible, valuable measurements when evaluating diabetes progression and onset (40,43), but genetic components, including epigenetic, epitranscriptomic, single nucleotide polymorphisms (SNPs), and others, provide a wealth of undiscovered information for disease classification. This genetic component may be specifically important when understanding the pathogenesis of diabetes in ethnic groups, when BMI $(2,44)$ and HbAlc (14) show distinct differences between ethnicities. Though applying patient-matched, genomic information is currently unrealistic for disease diagnosis, it may hold the key for revealing commonalities across ethnic and demographic groups when classifying diabetic onset, progression, and severity.

In the current study, machine-learning was used as a predictive tool to integrate cardiac physiological, biochemical, genomic, and epigenomic biomarker data in a patient-matched fashion and enable determination of type 2 diabetic status. In 50 patients, machine-learning algorithms revealed the interconnectedness between diabetic classification, mitochondrial function, and methylation status. Our study highlights how novel biomarkers can be used to augment existing diagnostic standards as well as provide new, and more precise, methods for identifying the development and severity of type 2 diabetes mellitus in potentially at-risk populations, such as those with prediabetes. While we examine physiological, biochemical, and molecular datasets using machine-learning algorithms, our goal was to understand which features possessed the best predictive accuracies and if these specific features could be used alone, or in conjunction, with $\mathrm{HbAlc}$. The purpose for the inclusion of models that do not rise above $50 \%$ predictive accuracy was to contrast them against those models that do rise above $50 \%$ in the absence of $\mathrm{HbAlc}$, to determine which biomarkers are the best overall predictors. 


\section{Research Design and Methods}

Study Approval

All tissue and patient information was acquired in a double de-identified fashion, and was approved by the West Virginia University Institutional Review Board and Institutional Biosafety Committee (19). Patients were all consented by the Heart and Vascular Institute, J.W. Ruby Memorial Hospital at the West Virginia University School of Medicine. Right atrial appendages were removed during open-heart and/or valvular surgeries. Both groups of patients (non-diabetic and type 2 diabetic) who were receiving open-heart surgery could elect for their tissues to be used for research purposes, with no direct or indirect incentivisation. A total of fifty patients were selected for the study (between August 2016 and May 2018), thirty of which were non-diabetic (ND) and twenty that were type 2 diabetic (T2DM) and existed along a spectrum of measured HbA1c levels. Patient inclusion into the study was determined by (a) election for open heart surgery and release of tissue for research purposes, (b) was not undergoing surgery due to heart failure, and (c) demographic data was provided. Patient characteristics are provided in Table 3.1, listing patients classified as non-diabetic and those who have been clinically diagnosed as type 2 diabetic. Patient information is also provided for separation of the groups into those with no diabetes, prediabetes, and type 2 diabetes (Table S3.1). Table S3.1 contains the matching fifty patient cohort analyzed in Table 3.1 but with the creation of a new non-diabetic cohort $(\mathrm{n}=16)$, comprised of those individuals with an HbAlc $<5.7$, and prediabetic cohort $(n=14)$, comprised of clinically non-diabetic patients whose HbA1c is between 5.7 and 6.4.

\section{Mitochondrial Isolation}

Mitochondria were isolated from a portion of the right atrial appendage as previously described (45), with modifications by our laboratory $(4,5,20)$. Mitochondrial subpopulations of subsarcolemmal and interfibrillar mitochondria were extracted and combined to achieve a total mitochondrial population.

Electron Transport Chain (ETC) Complex Activities 
A portion of the tissue from all 50 patients was homogenized using a Polytron PowerGen $500 \mathrm{~S} 1$ tissue homogenizer (Fisher Scientific, Hampton, NH) in NP-40 buffer (150 mM NaCl, 50 $\mathrm{mM}, \mathrm{pH} 8.0$ Tris-Cl, and 1.0\% NP-40). Protein homogenates were used to measure electron transport chain complexes I, III, IV, and V (ATP synthase) spectrophotometrically, as previously described (3) and implemented by our laboratory (4, 23, 24, 52, 53). Protein concentrations were normalized using the Bradford method, with standardization to bovine serum albumin (9).

\section{Citrate Synthase Activity}

Isolated mitochondria from all 50 patients, was used to measure citrate synthase activity using a colorimetric Citrate Synthase Assay Kit (Sciencell, San Diego, CA), as previously described (25). Citrate synthase activity, normalized to protein content, was used to determine mitochondrial content.

\section{Methyltransferase}

Using a colorometric Methyltransferase Assay Kit (Caymen, Ann Arbor, Michigan), Sadenosylmethionine (SAM)-dependent methyltransferase activity was assessed, per manufacturer's instructions. Briefly, nuclear homogenates were used to assess total SAMdependent methyltransferase activity in all 50 patients.

\section{DNA $5 \mathrm{mC}$ and $5 \mathrm{hmC}$ Quantification}

Using a DNeasy Blood \& Tissue Kit (Qiagen, Hilden, Germany), DNA was isolated from both $10 \mathrm{mg}$ of atrial appendage tissue and mitochondria, per manufacturer's instructions. Levels of 5-methylcytosine $(5 \mathrm{mC})$ and 5-hydroxymethylcytosine $(5 \mathrm{hmC})$ were measured through a $5 \mathrm{mC}$ and 5hmC ELISA Kit (Zymo Research, Irvine, CA), per manufacturer's instructions. DNA was quantified using a NanoDrop ${ }^{\text {TM }} 1000$ Spectrophotometer (Thermo Fisher, Waltham, MA). 100 ng of nuclear (tissue extract) and mitochondrial DNA were used to assess total $5 \mathrm{mC}$ and $5 \mathrm{hmC}$ content spectrophotometrically for all 50 patients. 


\section{Western Blotting}

Using $4-12 \%$ gradient gels, immunoblotting was performed through MES SDS-PAGE, as previously described $(18,20,23,42,58)$. Protein was normalized using the Bradford method. Primary antibodies used in the study included: anti-TFAM, transcription factor A, mitochondrial, 1:500 (SCBT, Dallas, TX), anti-GAPDH 1:1,000 (Abcam, Cambridge, MA). The secondary antibody used in the study was a goat anti-mouse $\operatorname{IgG}(\mathrm{H} \& \mathrm{~L})$ horseradish peroxidase (HRP) conjugate 1:10,000 (Thermo Fisher). GAPDH expression was used to normalize protein content. Chemiluminescence was measured through Radiance Chemiluminescent Substrate (Azure Biosystems, Dublin, CA), per manufacturer's instructions and imaged using the G:Box Bioimaging system (Syngene, Frederick, MD). Images were captured through GeneSnap/GeneTools software (Syngene). Densitometry was analyzed using ImageJ and Fiji Software (NIH, Bethesda, MD). Data is represented as optical density with arbitrary units.

\section{Chromatin Immunoprecipitation (ChIP) - qPCR}

The SimpleChIP ${ }$ Plus Sonication Chromatin IP Kit (Cell Signaling Technology, Danvers, MA) was used, per manufacturer's instructions. Briefly, $100 \mathrm{mg}$ of atrial tissue was minced into $\sim 2 \mathrm{~mm}^{3}$ pieces and treated with freshly prepared $37 \%$ formaldehyde for 30 minutes. Sonicated DNA was assessed for sheering and further immunoprecipitated with anti-TFAM (SCBT) bound Protein $\mathrm{G}$ magnetic beads overnight at $4^{\circ} \mathrm{C}$. The beads were washed, DNA reverse cross-linked, and DNA eluted as previously described $(52,56)$. DNA bound to TFAM was further examined through qPCR. 2\% chromatin inputs for each sample were used for normalization of expression. An Applied Biosystems 7900HT Fast Real-Time PCR system (Applied Biosystems, Foster City, CA) was used to assess expression through SYBR Green. Quantification was achieved using the $2^{-\Delta \Delta \mathrm{CT}}$ method (49). All primer pairs to assess the mitochondrial D-Loop are provided (Table S3.2).

Overhang-Bisulfite Sequencing 
DNA was extracted from patient tissue as described above. DNA was bisulfite-treated using the EZ DNA Methylation-Lightning Kit (Zymo Research), per manufacturer's instructions. Primers were designed for the CpG island of TFAM; primer set 1 amplified the 3' end and primer set 2 amplified the 5' end of the $\mathrm{CpG}$ island (Table S3.2). Bisulfite DNA was prepared for sequencing using a 2-Step PCR amplification process (6). Step-1 PCR adapters included a base pairing region ( $\sim 23 \mathrm{bp}$ ) and an overhang Illumina adapter arm ( $\sim 33 \mathrm{bp})$. Bisulfite DNA was PCR amplified using Step-1 primers utilizing Platinum ${ }^{\mathrm{TM}}$ Taq DNA Polymerase (Thermo Fisher), run on 2\% agarose gels, extracted through a QIAquick Gel Extraction Kit (Qiagen), and DNA purified. DNA was then further amplified using Step-2 Illumina barcoded adapters and prepared using a 300-cycle MiSeq Reagent Micro Kit v2 (Illumina, San Diego, CA). PCR amplicons were sequenced on the MiSeq with paired-end (PE) 250 base pair reads. Files were aligned to the bisulfite converted reference genome GRCh38 release 94 implementing Bismark $(31,32)$. Alignment was obtained through Bismark using the Bowtie2 (33) engine using "non-directional" and "paired-end." Complete sequencing code is provided (https://github.com/qahathaway/WVU_Machine-Learning-50/tree/master).

\section{Mitochondrial SNP Sequencing}

Mitochondrial DNA was isolated as described above and further amplified using the REPLI-g Mitochondrial DNA Kit (Qiagen), per manufacturer's instructions. Libraries for amplified DNA were prepared using the MiSeq Reagent Kit v3 (Illumina). Mitochondrial DNA was sequenced on the MiSeq with paired-end (PE) 300 base pair reads. Files were aligned to the reference genome GRCh38 release 94 through Bowtie2 using "sensitive-local." BAM files were sorted, run through variant calling, and single-nucleotide polymorphisms (SNPs) were identified in the mitochondria through SAMtools $(21,34,35)$. Complete sequencing code is provided (https://github.com/qahathaway/WVU_Machine-Learning-50/tree/master).

\section{Machine-Learning Algorithms}

Decision tree classifier algorithms were created in python (v3.6.5) using scikit-learn (46) and pandas (41) libraries (Figure 3.1A). In binary classification, patient labels were determined 
through previous clinical diagnoses, where diabetic status was retained even if current HbAlc levels were below $6.5 \%$. In multiple classification, patients with an HbAlc value in the range of $5.7 \%$ to $6.4 \%$ were designated as having prediabetes. Due to this, the HbA1c feature was excluded from all tested trials, and the derived accuracies are in comparison to that of the "perfect" accuracy obtained from HbA1c classification. A decision tree classifier model was created using the functions of scikit-learn tree. The data file was split into $80 \%$ training and $20 \%$ testing partitions using a defined seed value. Different seeds were chosen for each set to maintain the training and testing set distributions. Selected seed values maintained a binary classification testing set of 5 diabetics and 5 non-diabetics. In the multi-classification testing set, seed values maintained a distribution of 3 diabetics, 3 non-diabetics, and 4 pre-diabetics. Seeds were only chosen such that the resulting training and testing accuracies were similar; ensuring that the created classification tree did not over fit to the small sample size and remained generalizable to future testing samples.

Within the model, 10-fold cross validation was implemented. CART analysis was then performed on each of the datasets using the scikit-learn model, and the features of importance extracted using the feature importance parameter. The physiological/biochemical, genomic, and epigenomic datasets were combined into a single file to serve as the "all features" dataset used to extract the best and most influential biomarkers. For each trial, selected combinations of biomarkers from each dataset were utilized, and within each trial CART analysis was performed five times. After each of the five iterations, average feature importance, average training, and average testing accuracies were obtained. Standard deviations were taken over each of the five iterations per trial. For each dataset, the first iteration of CART analysis included all biomarkers of each set. If the average feature importance was less than $1 \%$, the feature was no longer included in subsequent trials. After all iterations, if the average feature importance was less than or equal to $8 \%$ it was assigned to a category titled "other." These same trials were repeated with other default scikit-learn machine-learning frameworks (Logistic Regression, Linear Discriminant Analysis, KNearest Neighbors Classifier, Gaussian Naïve Bayes, and Support Vector Machine). 10-fold cross validation and the same seed parameters were used in analysis of these 5 models and the resulting training and testing accuracies are provided (Tables S3.3-S3.10). The 6 models tested include few modifications and utilize no additional regularization techniques aside from those default to the scikit-learn library models. Only the Support Vector Machine model received modifications, with the probability parameter set to "true" to provide probability estimates for each data point and a 
linear kernel used over the default Radial Basis Function 'rbf' kernel. Code for analyses is also provided (https://github.com/qahathaway/WVU_Machine-Learning-50/tree/master).

\section{Machine-Learning Feature Extraction and Accuracy}

To determine which model(s) would provide the most accurate predictions on the current data, we assessed the 345 total features across all 6 models in binary (Table 3.2) and multiple (Table 3.3) classification of diabetic status. Through assessment of individual datasets (i.e. physiological/biochemical, genetic, and epigenetic), a set of 18 features was further used to classify diabetic status in binary (Table S3.11) and multiple (Table S3.12) classification. Model analysis was enacted for each dataset, and the established 10-fold cross validation and seed parameters for binary and multi-classification were utilized. Each dataset was tested five times per model. Averages were obtained for training accuracy, training standard deviation, testing accuracy, testing standard deviation, model average F1 score, and area under the curve (AUC). AUC values were provided for each of the 6 tested algorithms for the testing data under binary classification using the roc_auc_score function of scikit-learn, but not for multiple classification. AUC was determined as 1.0 for LR and SVM models when evaluating the 345 total features due to the large sample size of biomarkers. From the available 345 features, 2 predictors were chosen that perfectly distinguished diabetic and nondiabetic status for this particular dataset. As such, these AUC values were removed from Table 3.2 for LR and SVM, as this was not an accurate indicator of the model's predictive ability. As the feature set was restricted to the 18 "best" features from each dataset, AUC values of 1.0 were no longer observed (Tables S3.11 and S3.12).

Extracted important features and corresponding feature bias within each model, with the exception of KNN, were determined and are provided for binary and multiple classification. CART feature importance was extracted from the trained model using the feature importance parameter. For the NB model, feature influence was determined using the predict_log_proba function to return the most important biomarker per class in both binary $(0,1)$ and multiple $(0,1,2)$ classification schemes. Feature importance was not determined for the KNN model due to the restrictions of the default model. LDA, LR, and SVM feature influence was determined by taking the magnitude of the model coefficients, coef_ parameter, times the standard deviation of that biomarker in the testing data. The resulting values are ranked based off magnitude and are reported 
with sign under the "Important Feature Bias" (Tables 3.2 and 3.3, Tables S3.11 and S3.12). A positive influence value indicates a biomarker favoring classification towards one label while a negative value favors the opposite classification label. The larger the magnitude, the more strongly that feature shifts classification.

\section{SHapley Additive exPlanations (SHAP)}

SHAP framework, from slundberg (https://github.com/slundberg/shap), was used to visually explain the classification trees developed for the 50 patient samples using an XGBoost model (Figure 3.1B) $(16,38,39)$. Figure 3.1B illustrates how SHAP plots are presented in three dimensions, with the " $\mathrm{X}$ " and "Y" dimensions plotted spatially while the " $Z$ " dimension is indicated only through color; allowing for the examination of how variables, " $\mathrm{X}$ " and " $\mathrm{Z}$ ", can influence the nature of the model and classification of patients, "Y", into non-diabetic and diabetic cohorts. Through Jupyter Notebook (v0.34.9) (30) with a Python 3 kernel and importing pandas, shap, and xgboost libraries, SHAP plots were derived. Delineation of binary and multiple classification systems are defined above. The entire 50 patient population was utilized during training of the XGBoost model and both the patient biomarker values and patient labels were provided during training. The XGBoost and SHAP tree explainer utilized were left unaltered. The number of influential features shown in the summary plot were selected using the max display parameter. Plot generation utilized force plot, dependency plot, and summary plot SHAP functions. Force plots depict the effect of biomarker values on the model's output. Dependency plots relate specific biomarker values to model prediction and show how the chosen biomarker depends on other tested biomarkers. Summary plots depict the top influential biomarkers and how they influence the model prediction. Code for analyses is also provided (https://github.com/qahathaway/WVU Machine-Learning-50/tree/master)

\section{Statistics}

Significance was determined using a two-tailed Student's t-test or one-way analysis of variance (ANOVA), where appropriate. Tukey's multiple comparisons test was implemented following the ANOVA to derive significance between multiple groups. Differences between 
groups were considered statistically different if $P \leq 0.05$, denoted by * if statistically different from non-diabetic or \# if statistically different from pre-diabetic. All data are presented as the mean \pm standard error of the mean (SEM).

\section{Data Availability}

Mitochondrial DNA-Seq: Sequence Read Archive PRJNA520920

https://dataview.ncbi.nlm.nih.gov/?search=SUB5124294

TFAM Promoter Methylation Amplicon-Seq: Sequence Read Archive PRJNA520920

https://dataview.ncbi.nlm.nih.gov/?search=SUB5125264

Bioinformatics and Machine-learning Scripts: Github

https://github.com/qahathaway/WVU_Machine-Learning-50/tree/master 


\section{Results}

Alterations to the interaction networks that exist between the nucleus and mitochondrion play a significant role in the development of diabetic cardiomyopathy $(11,17,47,60)$. As a result, we wanted to determine how observed changes in these parameters could predict diabetic status using machine-learning algorithms. All of the machine-learning algorithms in this study implemented to draw conclusions were constructed around tree ensembles, such as Classification and Regression Trees (CART). CART algorithms proved to have the overall highest testing and training accuracies when compared to other models (Tables S3.3-S3.10), while also performing superiorly in multiple classification of prediabetes (Tables 3.2-3.3). When examining the testing, training, and area under the curve (AUC) values that depict model performance, CART performed consistently at, or near, the top of the six models in both the binary (Table 3.2) and multiple (Table 3.3) classification sets when assessing all 345 features. SHapley Additive exPlanations (SHAP) which implement CART functions were used to provide binary (non-diabetic or type 2 diabetic) as well as multiple (non-diabetic, prediabetic, and type 2 diabetic) classification analyses. SHAP analysis maps a particular biomarker's numeric values to a computationally defined SHAP value that represents the degree to which specific biomarker values classify the patient to a particular label (non-diabetic or type 2 diabetic). We wanted to demonstrate how machine-learning algorithms, applied across a variety of health outcome datasets, could be implemented to identify novel biomarkers, with and without $\mathrm{HbA1c}$, to provide better assessment of type 2 diabetes mellitus. By presenting each dataset distinctly, we were able to assess which biomarkers provided the best overall predictive power.

\section{Physiological and Biochemical Analyses}

Those with type 2 diabetes mellitus had significantly lower electron transport chain (ETC) complex I and III activities, along with a decreased methyltransferase activity (Table S3.13). Using CART analysis and machine-learning, total nuclear methylation, total mitochondrial hydroxymethylation, and total nuclear hydroxymethylation were shown to be the most important factors influencing the model (Figure 3.2A). Total nuclear methylation was also shown to be significantly increased in type 2 diabetics (Figure 3.2B) with a corresponding decrease in total nuclear hydroxymethylation (Figure 3.2C). Nuclear methylation increased as HbA1c levels 
increased (Figure 3.2D) while the rate of hydroxymethylation, generally inversely correlated with methylation levels, decreased as HbA1c increased (Figure 3.2E). Methyltransferase activity, total mitochondrial hydroxymethylation, total nuclear methylation, and total nuclear hydroxymethylation were shown to be important features in predicting type 2 diabetes mellitus in the absence of HbAlc (Figure 3.2F). High s-adenosyl methionine (SAM) methyltransferase activity was also shown to be strongly associated with lower total nuclear methylation levels in the absence of HbA1c (Figure 3.2G).

A decrease in mitochondrial ETC complex III activity was associated with a higher BMI (Figure 3.2H). While those who were considered to be prediabetic (HbA1c 5.7-6.4) did not show significant differences between any of the biochemical measures except total TFAM CpG methylation (Table S3.14), total nuclear methylation was still shown to provide partial classification of patients into non-diabetic, prediabetic, and type 2 diabetic designations (Figure 3.2I-J). CART 10-fold cross validation confirmed findings for binary (testing (0.838), training (0.7448)) and multiple (testing (0.598), training (0.545)) classification (Figure S3.1 A-D).

\section{Genomic Analyses}

The complete mitochondrial genomes of all patients were sequenced, and a list of all single nucleotide polymorphisms (SNPs) was compiled. The binary nature of SNPs, i.e. either being converted or not, allowed the dynamic HbAlc levels to influence the machine-learning model much more efficiently (Figure 3.3A). When HbAlc was removed, classification of diabetic or nondiabetic through SNPs was much more apparent, revealing that the 16362 base pair was most significantly impacted (Figure 3.3B). When examining the distribution of SNPs across the mitochondrial genome, the most significant area for base pair alterations to occur was shown to be the D-Loop, or control region (Figure 3.3C).

The D-Loop (mtDNA 16,025bp - 576bp), as compared to all other regions in the mitochondrial genome, contained the highest frequency of SNPs used to predict type 2 diabetes mellitus (Figure 3.3A-B, Figure S3.2A-B). We further investigated how transcription factor binding could be altered at the D-Loop through chromatin immunoprecipitation (ChIP) of mitochondrial transcription factor A, mitochondrial (TFAM). Though protein levels of TFAM 
were unchanged (Figure 3.3A), ChIP-qPCR revealed decreased binding of TFAM to the proximal and distal end of the control region in type 2 diabetics (Figure S3.3B). SNPs near the replication of the $\mathrm{H}$ strand (Figure 3.3D) or at the end of the D-Loop region (Figure 3.3E) could impact TFAM binding and mitochondrial genome transcription. CART 10-fold cross validation confirmed findings for binary (testing (0.79), training (0.92)) and multiple (testing (0.576), training (0.808)) classification (Figure S3.2 A-D).

\section{Epigenomic Analyses}

The cytosine nucleotide followed by a guanine nucleotide $(\mathrm{CpG})$ island of TFAM was examined (Figure 3.4A), using overhang bisulfite PCR to amplify regions of the island for sequencing (Figure 3.4B). Though total methylation of the gene was low $(\sim 3 \%)$ and showed no significant differences between non-diabetic and type 2 diabetic patients (Table S3.11), sitespecific $\mathrm{CpG}$ island methylation changes revealed significant differences between groups (Figure 3.4A). Specifically, the $24^{\text {th }}(\mathrm{CpG} 24)$ and $29^{\text {th }}(\mathrm{CpG} 29) \mathrm{CpGs}$ in the amplified region revealed significant hypomethylation in type 2 diabetic patients (Figure 3.4C-D).

Without using the HbA1c parameter, methylation levels at $\mathrm{CpG} 24,1,29$, and 35 were shown to be significant contributors to the prediction of diabetic status (Figure 3.4E). When comparing the interactions of $\mathrm{CpG} 24$ and $\mathrm{CpG} 29$, methylation levels at $\mathrm{CpG} 24$ were shown to allow distinct separation of the non-diabetic and type 2 diabetic population (Figure 3.4F). CpG24 methylation remained a primary predictor, even in the presence of HbAlc for multiple classification (Figure 3.4G). Examining total methylation of the TFAM CpG island, prediabetics exhibited an overall increase in methylation, while non-diabetics and type 2 diabetics with similar HbA1c levels showed much lower expression (Figure 3.4H). CART 10-fold cross validation confirmed findings for binary (testing (0.79), training (0.925)) and multiple (testing (0.668), training (0.767)) classification (Figure S3.4 A-D).

Best/Combined Analyses 
Those physiological, biochemical, genomic, and/or epigenomic markers that provided the best association within their class for predicting type 2 diabetes mellitus status were used in the final analyses. With the combined list of features, CART algorithms continued to perform consistently at, or near, the top for testing and training accuracies in binary (Table S3.13) and multiple (Table S3.14) classification. Total nuclear hydroxymethylation and total nuclear methylation levels provided the most powerful predictors in delineating between binary (nondiabetic and type 2 diabetic) (Figure 3.5A) and multiple (non-diabetic, prediabetic, type 2 diabetic) (Figure 3.5B) classifications, distinguishing them as potentially suitable biomarkers to accompany diagnostic practices using $\mathrm{HbA} 1 \mathrm{c}$. When using machine-learning to predict diabetic status without HbA1c, CpG24 methylation status and total nuclear methylation proved to be the most powerful predictors in both the binary (Figure 3.5C) and multiple (Figure 3.5D) classification datasets. Ultimately, both in the prediction of type 2 diabetes mellitus (Figure 3.5E) and in assessing the onset (Figure 3.5F), CpG24 hypomethylation was strongly correlated with total nuclear hypermethylation. CART 10-fold cross validation confirmed findings for binary (testing (0.78), training (0.832)) and multiple (testing (0.67), training (0.542)) classification (Figure S3.5 A-D). Within our datasets, CpG24 methylation status and total nuclear methylation provided the best predictive measures for assessing type 2 diabetes mellitus. The incorporation of physiological, biochemical, genetic, and epigenetic features with machine-learning algorithms exemplifies the potential for more informative diagnostics in the future, as well as personalized approaches to generalized treatment modalities (Figure 3.6). 


\section{Discussion}

Machine-learning can be applied as a systems biology approach, integrating multiple classes of biometric data to assess the importance of specific factors, while also predicting future outcomes. Whereas conventional assessments of disease identification exist, more detailed genomic and epigenomic testing is likely to reveal a comprehensive, systemic valuation of an etiology. To-date, studies have applied machine-learning algorithms in examining the physiological, biochemical, and/or genetic components of disease onset or progression (15). The advantage of our current study is through the assimilation of patient-matched data across a variety of critically impacted systems, providing an archetype for developing novel, descriptive, diagnostic measures through machine-learning algorithms that are specific for each disease type. By individually representing our datasets in Figures 3.2-3.4, we were able to reach more conclusive data in Figure 3.5 by choosing the most predictive features for our final model. For the first time, a multi-omics, machine-learning approach was used to assess the progression and development of type 2 diabetes mellitus in a patient population, identifying potential biomarkers for cardiovascular risk and revealing the fundamental role of genetics in the pathology.

\section{Molecular Pathogenesis and Machine Learning}

While clinical practice has recently experienced a surge in deep learning applications used for non-invasive imaging (29), implementing machine-learning algorithms to the fundamental biochemistry and cellular and molecular processes of the body is now only blossoming. Onset and progression of type 2 diabetes has been traditionally measured through blood glucose levels, but, the multifaceted aspects of the disease could create variability in prognosis between vastly different demographic and ethnic groups. Owusu Adjah et al. recently identified BMI as a risk factor for determining ethnic group disposition to type 2 diabetes mellitus (44). Specifically, the relationship between BMI and increased incidence of diabetes mellitus is non-linear; some groups, such as South Asian populations, were more disposed to developing the disease even at lower BMIs. While the current manuscript examines cardiovascular tissue, other less invasive approaches have been used to apply machine-learning algorithms. By retrieving blood from the basilica vein, circulating biomarkers were examined for their role in predicting early recurrence of atrial fibrillation following cryoballoon ablation (10). Support vector machines confirmed that 
decreased levels of creatine-kinase (CK-MB) and Troponin $\mathrm{T}$ ( TnT) were associated with increased early recurrence of atrial fibrillation following cryoballoon ablation. Additionally, a unique, non-invasive approach for potentially diagnosing type 2 diabetes in patients was performed through the examination of toenails. Carter et al., through a variety of machine learning algorithms, focused on 22 elements, including aluminum, cesium, nickel, vanadium, and zinc, and was able to get an AUC of 0.90 when predicting diabetic status using a random forest model (13).

Similar to parts of the aims of this study, other groups have attempted to use machine learning to separate diabetic and non-diabetic patients without the inclusion of blood glucose or HbA1c (62). In a testing set of 13700 patients from the Luzhou, China region, random forest machine learning algorithms provided a 0.7225 accuracy when predicting diabetic status from physical examination data in the absence of blood glucose (62). Also using a random forest model, Tang et al. revealed how $\mathrm{CpG}$ island methylation data, combined with miRNA expression profiles, can be instrumental in cancer pathogenesis (57); implementing this two-feature selection process, they were able to identify the best tissue specific features, ultimately allowing for the identification of the originating tissue where tumor progression began. In a similar fashion, the machine learning algorithm HeteSim (54), which examines heterogeneous datasets and calculates their relatedness, was employed in ascribing how gene profiles can be related to phenotypic outcomes, specifically in the validation and prediction of genes classified within major diseases (61).

While understanding how to better form prognoses and treat cardiac dysfunction in patients with type 2 diabetes mellitus remains a critical mission, more than 80 million American adults, most of which are undiagnosed, are prediabetic (48). In the current work, we have implemented predictive algorithms to assess biomarkers likely involved in the onset, as well as prediabetic progression, of type 2 diabetes mellitus. Although multiple classification categories further reduce the predictive power of the model, separation into distinct groupings revealed a unique phenotype for prediabetics (Figure 3.4H). The effects of diabetes mellitus on the body is a high glucose stressed condition, altering substrate metabolism and causing systemic inflammation (55). Due to this environmental change, researchers have shown how epigenetic changes occur across most, if not all, tissues that are impacted by diabetes mellitus $(37,60)$.

In the cardiovascular system, the heart, circulatory system, and regulating immune system are all transcriptionally regulated through epigenetic alterations $(17,28)$, resulting in cellular 
adaptations to the environmental stress. Examining atrial appendages, the results obtained in this study are a direct reflection of changes within the heart. While blood is more easily acquired in type 2 diabetic patients, cardiac tissue, which is mitochondrially rich, provides a direct connection between physiological dysfunction observed in the heart and the impact of altered genomic profiles in the mitochondrion and nucleus. Machine-learning, which at current has been applied to very few genetic applications, may play a significant role in defining the epigenome of those with diabetes mellitus, likely unveiling genes and molecular pathways first impacted by the pathology.

\section{The Challenges of Machine Learning in the Clinical Setting}

Machine-learning algorithms produce generalizations as they are inherently predictive, which means a smaller sample size can occasionally result in increased emphasis on outliers within the patient dataset and determination of the outliers' biomarker features to be most influential in disease diagnosis. With a limited 50 patient dataset, there are concerns of overfitting the model, where the derived classification tree would have branches for each patient sample encountered during training. If this was to occur, the produced tree would fail future test cases while maintaining near perfect training accuracy, which was not observed. 10-fold cross validation ensured that no single developed tree was composed solely of outliers or a group of patient data of one label type, allowing patients of different labels to train the algorithm. Additionally, choosing seed values provided an even patient distribution during model training and testing. Both 10-fold cross validation and setting a seed allowed the derived models to not over fit the training data. With this being said, it should be noted that the small sample size limits the conclusions and predictions made by the machine-learning algorithms within the manuscript, and future investigations will need to validate specific features, including CpG24 of TFAM and global nuclear DNA methylation.

For developed frameworks and the implemented SHAP visualization, the results are inherently regulated by $\mathrm{HbAlc}$ since patient $\mathrm{HbAlc}$ values were used to assign the labels from which the machine-learning algorithm then proceeded to train. $\mathrm{HbA1c}$ is used as a guide in this study to help clarify how clinically assessed progression of diabetes (commonly through $\mathrm{HbAlc}$ levels) is related to the biochemical and genetic signatures found in the heart. Although no specific biomarker or biomarker combinations can replace HbAlc due to the apparent diagnostic bias in 
this study (essentially $\sim 100 \%$ accuracy when included), they can provide predictive accuracies near that of $\mathrm{HbA1c}$. While previous clinical diagnoses determined a patients' diabetic status in this study, some patients diagnosed as type 2 diabetics had HbAlc levels within normal ranges; begging the question of whether sustained, or attenuated, health effects can be accurately assessed through HbA1c levels alone when intervention (lifestyle or medicinally) occurs? Ultimately, this study provides a machine-learning algorithm utilizing the respective advantages of HbAlc in combination with other biomarkers to help circumvent the limitations of modern $\mathrm{HbA} 1 \mathrm{c}$ diagnosis, as well as introduces completely novel cardiac risk stratification paradigms for patients with type 2 diabetes mellitus.

The quantity and diversity of omics-based approaches continues to expand. Convenience and increasingly inexpensive options for biometric-based valuations incite a growing demand for the incorporation and meaningful explanation of large and diverse patient datasets. The methodology outlined in this manuscript can serve as an archetype for the development and implementation of machine-learning to other disciplines seeking to evaluate disease progression. By using various health outcomes datasets, we were able to identify, and combine, the most prominent biomarkers into an accurate predictive algorithm engineered around 50 patients. While we have identified specific genetic features that are highly predictive in 50 patients, as a much larger patient population is applied to this model, the prioritization of other features is likely to occur, enhancing the diagnostic potential for the individual diabetic or prediabetic patient. Indeed, this is the advantage of using machine-learning models, in that they continue to learn and develop more accurate predictions as the number of features and sampled population grows. 


\section{Conclusions}

Our work highlights the importance of identifying biomarkers in systems known to be disturbed during the disease (i.e. the mitochondrion and nucleus), and further applying these biological systems to personalized prognostics. By implementing classification tree, machinelearning algorithms to cardiac tissue from type 2 diabetic patients, we determined that hypermethylation of the nuclear genome was predictive of diabetic status and that it may provide added benefit to diagnostic applications in the future. Additionally, through our machine-learning model, as little as a $\sim 5 \%$ change in methylation status of a gene promoter could provide valuable predictive data when determining diabetic status. Defining new diagnostic parameters, better predicting future health outcomes, and specializing modalities of care begins with the integration of "big data" into machine-learning systems; this study reveals how integration of data assists in the determination of diabetic status in the heart. 


\section{Abbreviations}

5hmC: 5-hydroxymethylcytosine

5mC: 5-methylcytosine

AUC: Area Under the Curve

CART: Classification and Regression Trees

CpG: Cytosine Nucleotide followed by a Guanine Nucleotide

ETC: Electron Transport Chain

HbA1c: Glycated Hemoglobin

LR: Logistic Regression

LDA: Linear Discriminant Analysis

KNN: K-Nearest Neighbors

NB: Naive Bayes

SHAP: SHapley Additive exPlanations

SVM: Support Vector Machine

T2DM: Type 2 Diabetes Mellitus

TFAM: Transcritption Factor A, Mitochondrial 


\section{Declarations}

Ethics approval and consent to participate

All tissue and patient information was acquired in a double de-identified fashion, and was approved by the West Virginia University Institutional Review Board and Institutional Biosafety Committee. Patients were all consented by the Heart and Vascular Institute, J.W. Ruby Memorial Hospital at West Virginia University School of Medicine. Right atrial appendages were removed during open-heart and/or valvular surgeries (Refer to Methods, section Study Approval).

\section{Consent for publication}

N/A

Availability of data and material

The datasets generated and/or analysed during the current study, including sequencing files and computer code, are available (Refer to Methods, section Data Availability). Primary used and/or analysed during the current study are available from the corresponding author on reasonable request.

\section{Competing interests}

The authors declare that they have no competing interests.

\section{Funding}

This work was supported by: R01 HL-128485 (JMH), American Heart Association AHA17PRE33660333 (QAH), West Virginia IDeA Network of Biomedical Research WV-INBRE support by NIH Grant P20GM103434, and the Community Foundation for the Ohio Valley Whipkey Trust. Portions of this work have been accepted in abstract form to Experimental Biology, Orlando, FL 2019.

\section{Authors' Contributions}

Designing research studies (QAH, SMR, MVP, JMH), conducting experiments (QAH, MVP, AK, AJD, GKF, ADT, APG), acquiring data (QAH, MVP), surgical procedures (CCC), analyzing data (QAH, SMR, MVP, TBC, JHG, GAA, JMH), writing the manuscript (QAH, SMR, MVP, DCS, 
CCC, JLA, JMH). The author Quincy A. Hathaway had full access to all the data in the study and takes responsibility for the integrity of the data and the accuracy of the data analysis. All authors read and approved the final manuscript.

\section{Acknowledgements}

The authors have declared that no conflict of interest exists. We would like to acknowledge the WVU Genomics Core Facility, Morgantown WV for support provided to help make this publication possible. We would like to thank the West Virginia University Ruby Memorial Hospital Heart and Vascular Institute as well as surgeons Vinay Badwar, Harold Roberts, Muhammad Salman, and Lawrence Wei for promoting this collaborative work. 


\section{Additional Material}

File name: Additional File 1

File format: DOCX

Title of data: Supplemental Material

Description of data: Supplemental data to the primary manuscript including, patient characteristics, primer design, and 10-fold cross validation for machine learning algorithms. For each specific data set, we applied six different machine-learning models (CART, LR, LDA, KNN, NB, SVM) and determined which model would yield the best predictions on the data sets. CART yielded the best result, the other test/train accuracies are provided for comparison and support of our conclusions. 


\section{References}

1. Al'Aref SJ, Anchouche K, Singh G, Slomka PJ, Kolli KK, Kumar A, Pandey M, Maliakal G, van Rosendael AR, Beecy AN, Berman DS, Leipsic J, Nieman K, Andreini D, Pontone G, Schoepf UJ, Shaw LJ, Chang HJ, Narula J, Bax JJ, Guan Y, and Min JK. Clinical applications of machine learning in cardiovascular disease and its relevance to cardiac imaging. Eur Heart J, 2018.

2. Almajwal AM, Al-Baghli NA, Batterham MJ, Williams PG, Al-Turki KA, and AlGhamdi AJ. Performance of body mass index in predicting diabetes and hypertension in the Eastern Province of Saudi Arabia. Ann Saudi Med 29: 437-445, 2009.

3. Barrientos A, Fontanesi F, and Diaz F. Evaluation of the mitochondrial respiratory chain and oxidative phosphorylation system using polarography and spectrophotometric enzyme assays. Curr Protoc Hum Genet Chapter 19: Unit19 13, 2009.

4. Baseler WA, Dabkowski ER, Jagannathan R, Thapa D, Nichols CE, Shepherd DL, Croston TL, Powell M, Razunguzwa TT, Lewis SE, Schnell DM, and Hollander JM. Reversal of mitochondrial proteomic loss in Type 1 diabetic heart with overexpression of phospholipid hydroperoxide glutathione peroxidase. Am J Physiol Regul Integr Comp Physiol 304: R553-565, 2013.

5. Baseler WA, Dabkowski ER, Williamson CL, Croston TL, Thapa D, Powell MJ, Razunguzwa TT, and Hollander JM. Proteomic alterations of distinct mitochondrial subpopulations in the type 1 diabetic heart: contribution of protein import dysfunction. $\mathrm{Am} \mathrm{J}$ Physiol Regul Integr Comp Physiol 300: R186-200, 2011.

6. Bernstein DL, Kameswaran V, Le Lay JE, Sheaffer KL, and Kaestner KH. The BisPCR(2) method for targeted bisulfite sequencing. Epigenetics Chromatin 8: 27, 2015.

7. Bertoluci MC and Rocha VZ. Cardiovascular risk assessment in patients with diabetes. Diabetol Metab Syndr 9: 25, 2017.

8. Bonora $\mathbf{E}$ and Tuomilehto J. The pros and cons of diagnosing diabetes with A1C. Diabetes Care 34 Suppl 2: S184-190, 2011.

9. Bradford MM. A rapid and sensitive method for the quantitation of microgram quantities of protein utilizing the principle of protein-dye binding. Anal Biochem 72: 248-254, 1976.

10. Budzianowski J, Hiczkiewicz J, Burchardt P, Pieszko K, Rzezniczak J, Budzianowski P, and Korybalska K. Predictors of atrial fibrillation early recurrence following cryoballoon ablation of pulmonary veins using statistical assessment and machine learning algorithms. Heart Vessels 34: 352-359, 2019.

11. Bugger $\mathbf{H}$ and Abel ED. Mitochondria in the diabetic heart. Cardiovasc Res 88: 229-240, 2010 .

12. Capobianco E. Systems and precision medicine approaches to diabetes heterogeneity: a Big Data perspective. Clin Transl Med 6: 23, 2017.

13. Carter JA, Long CS, Smith BR, Smith TL, and Donati GL. Combining elemental analysis of toenails and machine learning techniques as a non-invasive diagnostic tool for the robust classification of type-2 diabetes. Expert Syst Appl 115: 245-255, 2019.

14. Cavagnolli G, Pimentel AL, Freitas PA, Gross JL, and Camargo JL. Effect of ethnicity on HbA1c levels in individuals without diabetes: Systematic review and meta-analysis. PLoS One 12: e0171315, 2017.

15. Chaudhary K, Poirion OB, Lu L, and Garmire LX. Deep Learning-Based Multi-Omics Integration Robustly Predicts Survival in Liver Cancer. Clin Cancer Res 24: 1248-1259, 2018. 
16. Chen T and C. G. XGBoost: A Scalable Tree Boosting System. In: Proceedings of the 22nd ACM SIGKDD International Conference on Knowledge Discovery and Data Mining. San Francisco, California, USA: ACM, 2016, p. 785-794.

17. Costantino S, Libby P, Kishore R, Tardif JC, El-Osta A, and Paneni F. Epigenetics and precision medicine in cardiovascular patients: from basic concepts to the clinical arena. Eur Heart J 39: 4150-4158, 2018.

18. Croston TL, Shepherd DL, Thapa D, Nichols CE, Lewis SE, Dabkowski ER, Jagannathan R, Baseler WA, and Hollander JM. Evaluation of the cardiolipin biosynthetic pathway and its interactions in the diabetic heart. Life Sci 93: 313-322, 2013.

19. Croston TL, Thapa D, Holden AA, Tveter KJ, Lewis SE, Shepherd DL, Nichols CE, Long DM, Olfert IM, Jagannathan R, and Hollander JM. Functional deficiencies of subsarcolemmal mitochondria in the type 2 diabetic human heart. Am J Physiol Heart Circ Physiol 307: H54-65, 2014.

20. Dabkowski ER, Baseler WA, Williamson CL, Powell M, Razunguzwa TT, Frisbee JC, and Hollander JM. Mitochondrial dysfunction in the type 2 diabetic heart is associated with alterations in spatially distinct mitochondrial proteomes. Am J Physiol Heart Circ Physiol 299: H529-540, 2010.

21. Danecek P, Auton A, Abecasis G, Albers CA, Banks E, DePristo MA, Handsaker RE, Lunter G, Marth GT, Sherry ST, McVean G, Durbin R, and Genomes Project Analysis G. The variant call format and VCFtools. Bioinformatics 27: 2156-2158, 2011.

22. Gregg EW, Cheng YJ, Srinivasan M, Lin J, Geiss LS, Albright AL, and Imperatore G. Trends in cause-specific mortality among adults with and without diagnosed diabetes in the USA: an epidemiological analysis of linked national survey and vital statistics data. Lancet 391: 2430-2440, 2018.

23. Hathaway QA, Durr AJ, Shepherd DL, Pinti MV, Brandebura AN, Nichols CE, Kunovac A, Goldsmith WT, Friend SA, Abukabda AB, Fink GK, Nurkiewicz TR, and Hollander JM. miRNA-378a as a key regulator of cardiovascular health following engineered nanomaterial inhalation exposure. Nanotoxicology: 1-20, 2019.

24. Hathaway QA, Nichols CE, Shepherd DL, Stapleton PA, McLaughlin SL, Stricker JC, Rellick SL, Pinti MV, Abukabda AB, McBride CR, Yi J, Stine SM, Nurkiewicz TR, and Hollander JM. Maternal-engineered nanomaterial exposure disrupts progeny cardiac function and bioenergetics. Am J Physiol Heart Circ Physiol 312: H446-H458, 2017.

25. Jagannathan R, Thapa D, Nichols CE, Shepherd DL, Stricker JC, Croston TL, Baseler WA, Lewis SE, Martinez I, and Hollander JM. Translational Regulation of the Mitochondrial Genome Following Redistribution of Mitochondrial MicroRNA in the Diabetic Heart. Circ Cardiovasc Genet 8: 785-802, 2015.

26. Jelinek HF, Stranieri A, Yatsko A, and Venkatraman S. Data analytics identify glycated haemoglobin co-markers for type 2 diabetes mellitus diagnosis. Comput Biol Med 75: 9097, 2016.

27. Kavakiotis I, Tsave O, Salifoglou A, Maglaveras N, Vlahavas I, and Chouvarda I. Machine Learning and Data Mining Methods in Diabetes Research. Comput Struct Biotechnol J 15: 104-116, 2017.

28. Keating ST, Plutzky J, and El-Osta A. Epigenetic Changes in Diabetes and Cardiovascular Risk. Circ Res 118: 1706-1722, 2016.

29. Klang E. Deep learning and medical imaging. J Thorac Dis 10: 1325-1328, 2018. 
30. Kluyver T, Ragan-Kelley B, Pérez F, Granger B, Bussonnier M, Frederic J, Kelley K, Hamrick J, Grout J, Corlay S, Ivanov P, Avila D, Abdalla S, Willing C, and team Jd. Jupyter Notebooks - a publishing format for reproducible computational workflows. In: 20th International Conference on Electronic Publishing, edited by Loizides F and Scmidt B. Göttingen, Germany, 2016, p. 87-90.

31. Krueger $\mathbf{F}$ and Andrews SR. Bismark: a flexible aligner and methylation caller for Bisulfite-Seq applications. Bioinformatics 27: 1571-1572, 2011.

32. Krueger F, Kreck B, Franke A, and Andrews SR. DNA methylome analysis using short bisulfite sequencing data. Nat Methods 9: 145-151, 2012.

33. Langmead B and Salzberg SL. Fast gapped-read alignment with Bowtie 2. Nat Methods 9: 357-359, 2012.

34. Li H. A statistical framework for SNP calling, mutation discovery, association mapping and population genetical parameter estimation from sequencing data. Bioinformatics 27: 29872993, 2011.

35. Li H, Handsaker B, Wysoker A, Fennell T, Ruan J, Homer N, Marth G, Abecasis G, Durbin R, and Genome Project Data Processing S. The Sequence Alignment/Map format and SAMtools. Bioinformatics 25: 2078-2079, 2009.

36. Libbrecht MW and Noble WS. Machine learning applications in genetics and genomics. Nat Rev Genet 16: 321-332, 2015.

37. Ling $\mathbf{C}$ and Groop L. Epigenetics: a molecular link between environmental factors and type 2 diabetes. Diabetes 58: 2718-2725, 2009.

38. Lundberg SM, Erion GG, and Lee S-I. Consistent Individualized Feature Attribution for Tree Ensembles. CoRR abs/1802.03888, 2018.

39. Lundberg SM and Lee SI. A Unified Approach to Interpreting Model Predictions. Adv Neur In 30, 2017.

40. Massi-Benedetti M. Changing targets in the treatment of type 2 diabetes. Curr Med Res Opin 22 Suppl 2: S5-13, 2006.

41. McKinney W. Data Structures for Statistical Computing in Python. Proc. of the 9th Python in Science Conf., SCIPY 2010, 2010.

42. Nichols CE, Shepherd DL, Knuckles TL, Thapa D, Stricker JC, Stapleton PA, Minarchick VC, Erdely A, Zeidler-Erdely PC, Alway SE, Nurkiewicz TR, and Hollander JM. Cardiac and mitochondrial dysfunction following acute pulmonary exposure to mountaintop removal mining particulate matter. Am J Physiol Heart Circ Physiol 309: H2017-2030, 2015.

43. Norberg M, Eriksson JW, Lindahl B, Andersson C, Rolandsson O, Stenlund H, and Weinehall L. A combination of HbAlc, fasting glucose and BMI is effective in screening for individuals at risk of future type 2 diabetes: OGTT is not needed. $J$ Intern Med 260: 263-271, 2006.

44. Owusu Adjah ES, Bellary S, Hanif W, Patel K, Khunti K, and Paul SK. Prevalence and incidence of complications at diagnosis of T2DM and during follow-up by BMI and ethnicity: a matched case-control analysis. Cardiovasc Diabetol 17: 70, 2018.

45. Palmer JW, Tandler B, and Hoppel CL. Biochemical properties of subsarcolemmal and interfibrillar mitochondria isolated from rat cardiac muscle. J Biol Chem 252: 8731-8739, 1977.

46. Pedregosa F, Varoquaux G, Gramfort A, Michel V, Thirion B, Grisel O, Blondel M, Prettenhofer P, Weiss R, Dubourg V, Vanderplas J, Passos A, Cournapeau D, Brucher M, Perrot M, and Duchesnay E. Scikit-learn: Machine Learning in Python. J Mach Learn Res 12: 2825-2830, 2011. 
47. Pinti MV, Fink GK, Hathaway QA, Durr AJ, Kunovac A, and Hollander JM. Mitochondrial Dysfunction in Type 2 Diabetes Mellitus: An Organ-Based Analysis. Am J Physiol Endocrinol Metab, 2019.

48. Prevention CfDCa. Prediabetes: Your Chance to Prevent Type 2 Diabetes. In: Diabetes, edited by National Center for Chronic Disease Prevention and Health Promotion DoDT, 2018.

49. Schmittgen TD and Livak KJ. Analyzing real-time PCR data by the comparative C(T) method. Nat Protoc 3: 1101-1108, 2008.

50. Scirica BM. Use of Biomarkers in Predicting the Onset, Monitoring the Progression, and Risk Stratification for Patients with Type 2 Diabetes Mellitus. Clin Chem 63: 186-195, 2017.

51. Shameer K, Johnson KW, Glicksberg BS, Dudley JT, and Sengupta PP. Machine learning in cardiovascular medicine: are we there yet? Heart 104: 1156-1164, 2018.

52. Shepherd DL, Hathaway QA, Nichols CE, Durr AJ, Pinti MV, Hughes KM, Kunovac A, Stine SM, and Hollander JM. Mitochondrial proteome disruption in the diabetic heart through targeted epigenetic regulation at the mitochondrial heat shock protein 70 (mtHsp70) nuclear locus. J Mol Cell Cardiol 119: 104-115, 2018.

53. Shepherd DL, Hathaway QA, Pinti MV, Nichols CE, Durr AJ, Sreekumar S, Hughes KM, Stine SM, Martinez I, and Hollander JM. Exploring the mitochondrial microRNA import pathway through Polynucleotide Phosphorylase (PNPase). J Mol Cell Cardiol 110: 15-25, 2017.

54. Shi C, Kong XN, Huang Y, Yu PS, and Wu B. HeteSim: A General Framework for Relevance Measure in Heterogeneous Networks. Ieee T Knowl Data En 26: 2479-2492, 2014.

55. Shoelson SE, Lee J, and Goldfine AB. Inflammation and insulin resistance. J Clin Invest 116: 1793-1801, 2006.

56. Stapleton PA, Hathaway QA, Nichols CE, Abukabda AB, Pinti MV, Shepherd DL, McBride CR, Yi J, Castranova VC, Hollander JM, and Nurkiewicz TR. Maternal engineered nanomaterial inhalation during gestation alters the fetal transcriptome. Part Fibre Toxicol 15: 3, 2018.

57. Tang W, Wan S, Yang Z, Teschendorff AE, and Zou Q. Tumor origin detection with tissue-specific miRNA and DNA methylation markers. Bioinformatics 34: 398-406, 2018.

58. Thapa D, Nichols CE, Lewis SE, Shepherd DL, Jagannathan R, Croston TL, Tveter KJ, Holden AA, Baseler WA, and Hollander JM. Transgenic overexpression of mitofilin attenuates diabetes mellitus-associated cardiac and mitochondria dysfunction. J Mol Cell Cardiol 79: 212-223, 2015.

59. Tsay D and Patterson C. From Machine Learning to Artificial Intelligence Applications in Cardiac Care. Circulation 138: 2569-2575, 2018.

60. Villeneuve LM and Natarajan R. The role of epigenetics in the pathology of diabetic complications. Am J Physiol Renal Physiol 299: F14-25, 2010.

61. Zeng XX, Liao YL, Liu YS, and Zou Q. Prediction and Validation of Disease Genes Using HeteSim Scores. Ieee Acm T Comput Bi 14: 687-695, 2017.

62. Zou Q, Qu K, Luo Y, Yin D, Ju Y, and Tang H. Predicting Diabetes Mellitus With Machine Learning Techniques. Front Genet 9: 515, 2018. 
Table 3.1

\begin{tabular}{lll}
\hline \multicolumn{1}{c}{ Parameter } & \multicolumn{1}{c}{ Non-Diabetic } & \multicolumn{1}{c}{ Type 2 Diabetic } \\
\hline Age & $61.97 \pm 2.449$ & $61.16 \pm 3.047$ \\
\hline Sex & Male $=26$, Female $=4$ & Male $=15$, Female $=5$ \\
\hline BMI $\left(\mathrm{kg} / \mathrm{m}^{2}\right)$ & $29.13 \pm 1.08$ & $29.14 \pm 1.448$ \\
Coronary Artery Disease & $73.33 \% \pm 8.212 \%$ & $\mathbf{1 0 0 \%} \pm \mathbf{0 . 0 \%} \%$ \\
Hypertension & $86.67 \% \pm 6.312 \%$ & $94.74 \% \pm 5.263 \%$ \\
Valvular Disease & $26.67 \% \pm 8.212 \%$ & $15.79 \% \pm 8.595 \%$ \\
\hline HbAlc & $5.567 \pm 0.07898$ & $\mathbf{8 . 0 1 6} \pm \mathbf{0 . 4 0 2 4} \%$ \\
\hline
\end{tabular}


Table 3.1: Patient characteristics and demographic information. Groups are considered significantly different if $P \leq 0.05=*$ compared to non-diabetic. All data are presented as the mean \pm standard error of the mean $(\mathrm{SEM}) . \mathrm{HbA} 1 \mathrm{c}=$ glycated hemoglobin. 
Table 3.2

\begin{tabular}{|c|c|c|c|c|c|c|c|c|}
\hline Model & Training & $\begin{array}{l}\text { Training } \\
\text { (StDev) }\end{array}$ & Testing & $\begin{array}{l}\text { Testing } \\
\text { (StDev) }\end{array}$ & $\begin{array}{c}\text { F1 } \\
\text { Score }\end{array}$ & Important Features & Important Feature Bias & AUC \\
\hline LR & 0.608 & 0.301 & 0.667 & 0.0 & 0.640 & $\begin{array}{c}\text { Complex III, Complex I, } \\
\text { CpG31, CpG28, CpG30, } \\
\text { Complex IV, CpG8, } \\
\text { CpG4, CpG12, Age }\end{array}$ & $\begin{array}{c}(-2.688),(-1.688),(1.648),(- \\
1.163),(-1.016),(0.982) \\
(0.945),(0.887),(0.882) \\
(0.848)\end{array}$ & NA \\
\hline LDA & 0.567 & 0.203 & 0.556 & 0.0 & 0.400 & $\begin{array}{l}\text { SNP16245, SNP16344, } \\
\text { SNP151, SNP5463, } \\
\text { SNP4295, SNP13722, } \\
\text { SNP94, SNP15884, } \\
\text { SNP9055, SNP477 }\end{array}$ & $\begin{array}{c}(-3.896 E+15),(-3.896 E+15) \\
(-3.896 E+15),(-3.896 E+15) \\
(-2.719 E+15),(-2.719 E+15) \\
(3.398 E+14),(3.398 E+14) \\
(3.398 E+14), 0.266\end{array}$ & 0.700 \\
\hline KNN & 0.642 & 0.239 & 0.444 & 0.0 & 0.430 & NA & NA & 0.600 \\
\hline NB & 0.725 & 0.227 & 0.778 & 0.0 & 0.780 & $\begin{array}{c}\text { Mito } 5 \mathrm{hmC} \\
\text { Methyltransferase }\end{array}$ & $(1.000),(0.000)$ & 0.775 \\
\hline SVM & 0.583 & 0.337 & 0.667 & 0.0 & 0.640 & $\begin{array}{c}\text { Complex III, CpG31, } \\
\text { Complex I, CpG28, } \\
\text { CpG8, CpG22, CpG12, } \\
\text { CpG29, CpG4, CpG35 }\end{array}$ & $\begin{array}{c}(-0.732),(0.488),(-0.443),(- \\
0.372),(0.350),(-0.349) \\
(0.322),(-0.260),(0.259) \\
(0.257)\end{array}$ & NA \\
\hline CART & 0.790 & 0.209 & 0.711 & 0.1 & 0.714 & $\begin{array}{l}\text { CpG 24, CpG 28, Nuc } \\
5 m C, C p G 11, C p G 23 \\
\text { CpG1, CpG4 }\end{array}$ & $\begin{array}{c}(0.587 \%),(0.213 \%) \\
(0.040 \%),(0.040 \%) \\
(0.040 \%),(0.040 \%),(0.040 \%)\end{array}$ & 0.715 \\
\hline
\end{tabular}




\section{Table 3.2: Overview of 6 machine-learning model analysis on all 345 features in binary}

classification. Model analysis was conducted five times and averages are reported for the resulting training accuracy, training standard deviation, testing accuracy, testing standard deviation, F1 score, and area under the curve (AUC). Important biomarker features associated with each trained model are provided along with the associated influence value for each feature. Important features are listed in order of influence within the model. LR, LDA, SVM feature bias exists as an influence parameter where magnitude dictates feature influence. A positive influence value indicates the biomarker favors classification towards one label while a negative value indicates favorable classification of the opposite label. The larger the magnitude, the more strongly that feature shifts classification. NB feature influence indicates the most important biomarker per class in binary $(0,1)$ classification schemes. CART feature bias percentages indicate feature influence on the created classification tree. Larger percentages indicate a feature that arises near the beginning of a tree before subsequent branching. Influence is not provided for KNN due to model restrictions. 
Table 3.3

\begin{tabular}{|c|c|c|c|c|c|c|c|}
\hline Model & Training & $\begin{array}{l}\text { Training } \\
\text { (StDev) }\end{array}$ & Testing & $\begin{array}{l}\text { Testing } \\
\text { (StDev) }\end{array}$ & $\begin{array}{c}\text { F1 } \\
\text { Score }\end{array}$ & Important Features & Important Feature Bias \\
\hline LR & 0.333 & 0.207 & 0.444 & 0.0 & 0.430 & $\begin{array}{c}\text { Complex V, CpG35, BMI, } \\
\text { CpG38, CpG18, CpG40, } \\
\text { CpG19, CpG23, Complex IV, } \\
\text { CpG25 }\end{array}$ & $\begin{array}{c}(-2.417),(-2.214),(1.942),(- \\
1.541),(-1.313),(-0.994),(- \\
0.881),(-0.824),(-0.812) \\
(0.8071)\end{array}$ \\
\hline LDA & 0.433 & 0.178 & 0.333 & 0.0 & 0.170 & $\begin{array}{l}\text { SNP11167, SNP10506, } \\
\text { SNP16309, SNP16343, } \\
\text { SNP2294, SNP14139, } \\
\text { SNP16162, SNP3672, } \\
\text { SNP8642, SNP143 }\end{array}$ & $\begin{array}{c}(-4.623 E+14),(-4.623 E+14),(- \\
4.623 E+14),(-4.623 E+14),(- \\
4.623 E+14),(-4.623 E+14),(- \\
4.623 E+14),(-4.623 E+14),(- \\
4.623 E+14),(5.779 E+13)\end{array}$ \\
\hline KNN & 0.358 & 0.239 & 0.444 & 0.0 & 0.450 & NA & NA \\
\hline NB & 0.425 & 0.243 & 0.778 & 0.0 & 0.780 & $\begin{array}{l}\text { Methyltransferase, Mito } \\
5 \mathrm{hmC} \text {, Nuc } 5 \mathrm{hmC}\end{array}$ & $(0.000),(1.000),(2.000)$ \\
\hline SVM & 0.442 & 0.163 & 0.556 & 0.0 & 0.520 & $\begin{array}{c}\text { Complex V, BMI, Complex } \\
\text { III, Complex I, Complex IV, } \\
\text { CpG31, Age, CpG19, CpG22, } \\
\text { CpG6 }\end{array}$ & $\begin{array}{c}(-0.943),(0.754),(0.561),(- \\
0.383),(-0.344),(0.307),(- \\
0.287),(-0.268),(-0.210), \\
(0.198)\end{array}$ \\
\hline CART & 0.660 & 0.257 & 0.556 & 0.0 & 0.558 & $\begin{array}{c}\text { CpG24, TFAM CpG, TFAM } \\
\text { Non-CpG, BMI, SNP94, } \\
\text { Complex IV, SNP8557, } \\
\text { CpG7, SNP242, SNP13722, } \\
\text { Complex III, Mito 5mC }\end{array}$ & $\begin{array}{l}(0.328 \%),(0.206 \%),(0.176 \%), \\
(0.137 \%),(0.016 \%),(0.045 \%), \\
(0.016 \%),(0.016 \%),(0.016 \%), \\
(0.016 \%),(0.016 \%),(0.016 \%)\end{array}$ \\
\hline
\end{tabular}


Table 3.3: Overview of 6 machine-learning model analysis on all 345 features in multiple

classification. Model analysis was conducted five times and averages are reported for the resulting training accuracy, training standard deviation, testing accuracy, testing standard deviation, and F1 score. Important biomarker features associated with each trained model are provided along with the associated influence value for each feature. Important features are listed in order of influence within the model. LR, LDA, SVM feature bias exists as an influence parameter where magnitude dictates feature influence. A positive influence value indicates the biomarker favors classification towards one label while a negative value indicates favorable classification of the opposite label. The larger the magnitude, the more strongly that feature shifts classification. NB feature influence indicates the most important biomarker per class in multiple $(0,1,2)$ classification schemes. CART feature bias percentages indicate feature influence on the created classification tree. Larger percentages indicate a feature that arises near the beginning of a tree before subsequent branching. Influence is not provided for $\mathrm{KNN}$ due to model restrictions. 
Figure 3.1

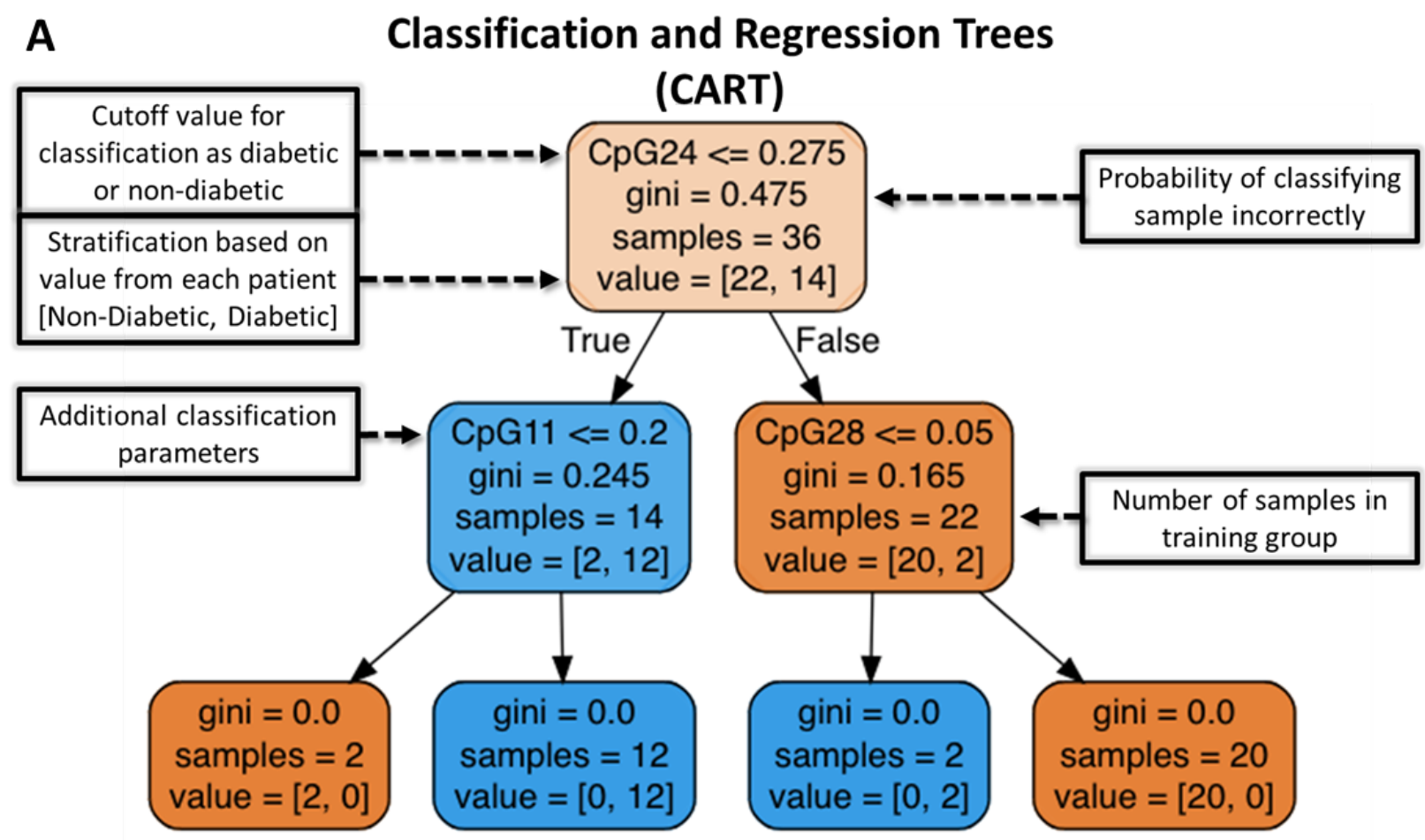


Figure 3.1

B
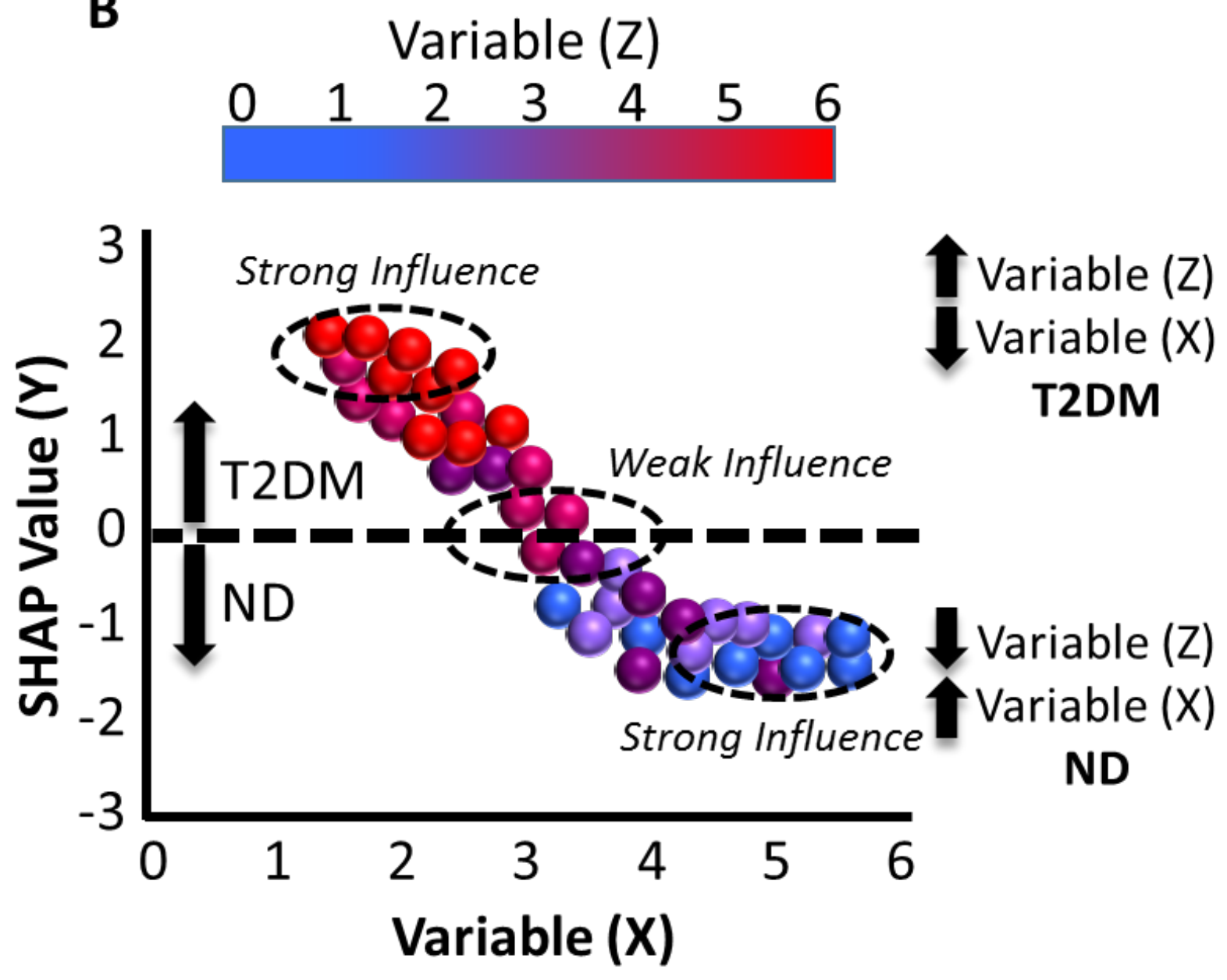
Figure 3.1: Overview of machine-learning using Classification and Regression Trees (CART) and SHapley Additive exPlanations (SHAP). (A) Classification trees begin with a specific parameter that most successfully partitions the samples, such as CpG24 methylation, and determine the probability of correctly delineating a population into classifications, such as nondiabetic and diabetic, through a discrete value of the parameter (e.g. 0.275). The delineation is then given a probability score (i.e. 0.475 , or a $47.5 \%$ chance of classifying the sample incorrectly), assigned a label, and further passed on to other parameters in the tree (e.g. CpG11 methylation and CpG28 methylation). As the samples progress through the tiers of the tree, the gini impurity gets smaller, more accurately delineating samples that make it to that particular "truth" statement. (B) An example of how SHAP illustrates sample distribution. The "SHAP Value" delineates between a condition being true (value $>0.0, \mathrm{~T} 2 \mathrm{DM}$ ) and it being false (value $<0.0$, ND). The more a specific value of a sample influences the composition of the model, the farther the point will migrate away from zero on the y-axis. If the value of a sample does not influence the model, it will reside near or at zero on the $y$-axis. In the example, a larger value of " $X$ " and lower value of " $Z$ " are highly predictive of the patient being ND, with these values strongly influencing the model "Y". CpG = cytosine nucleotide followed by a guanine nucleotide, ND = non-diabetic, T2DM = type 2 diabetic. 
Figure 3.2

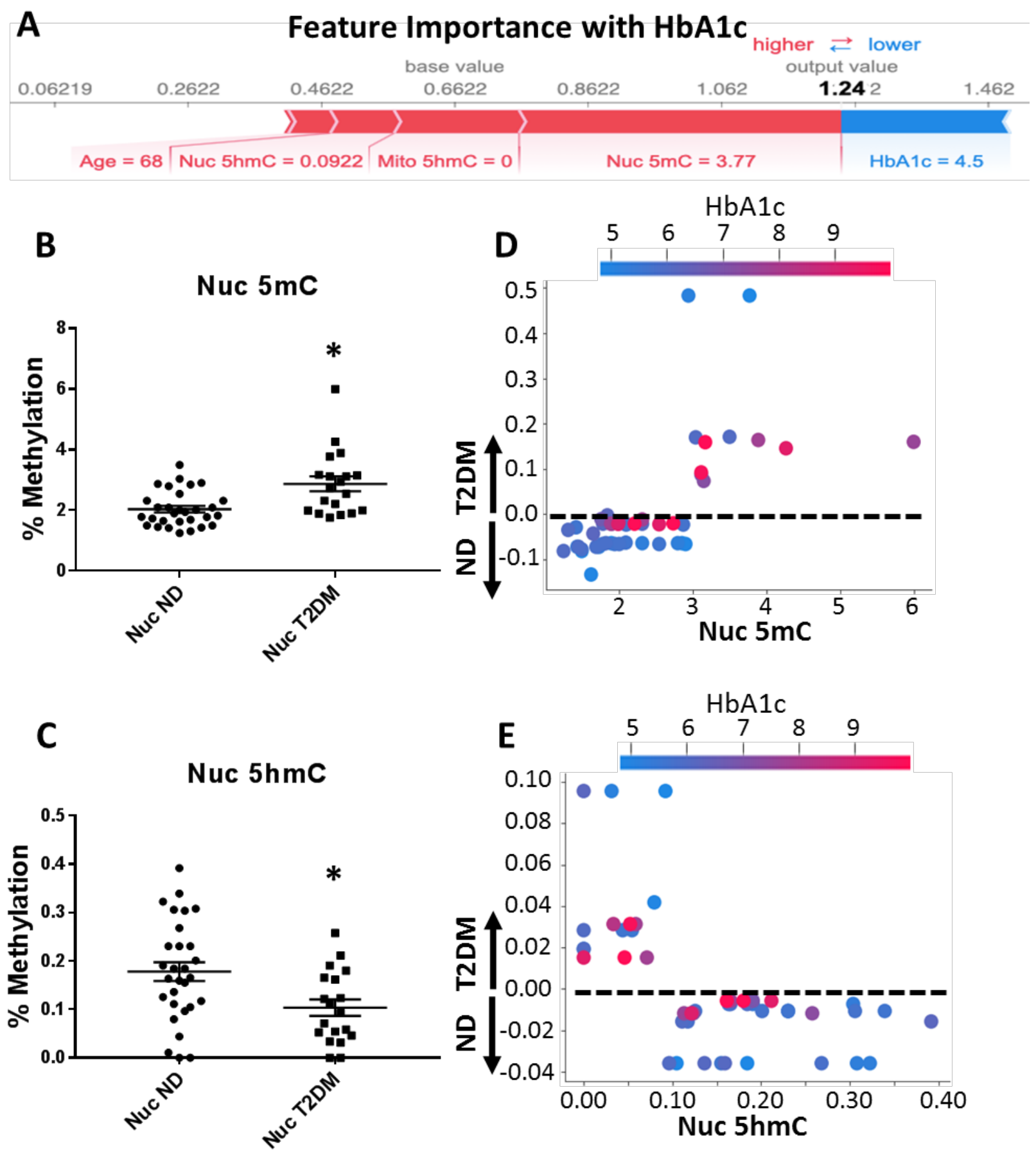


Figure 3.2
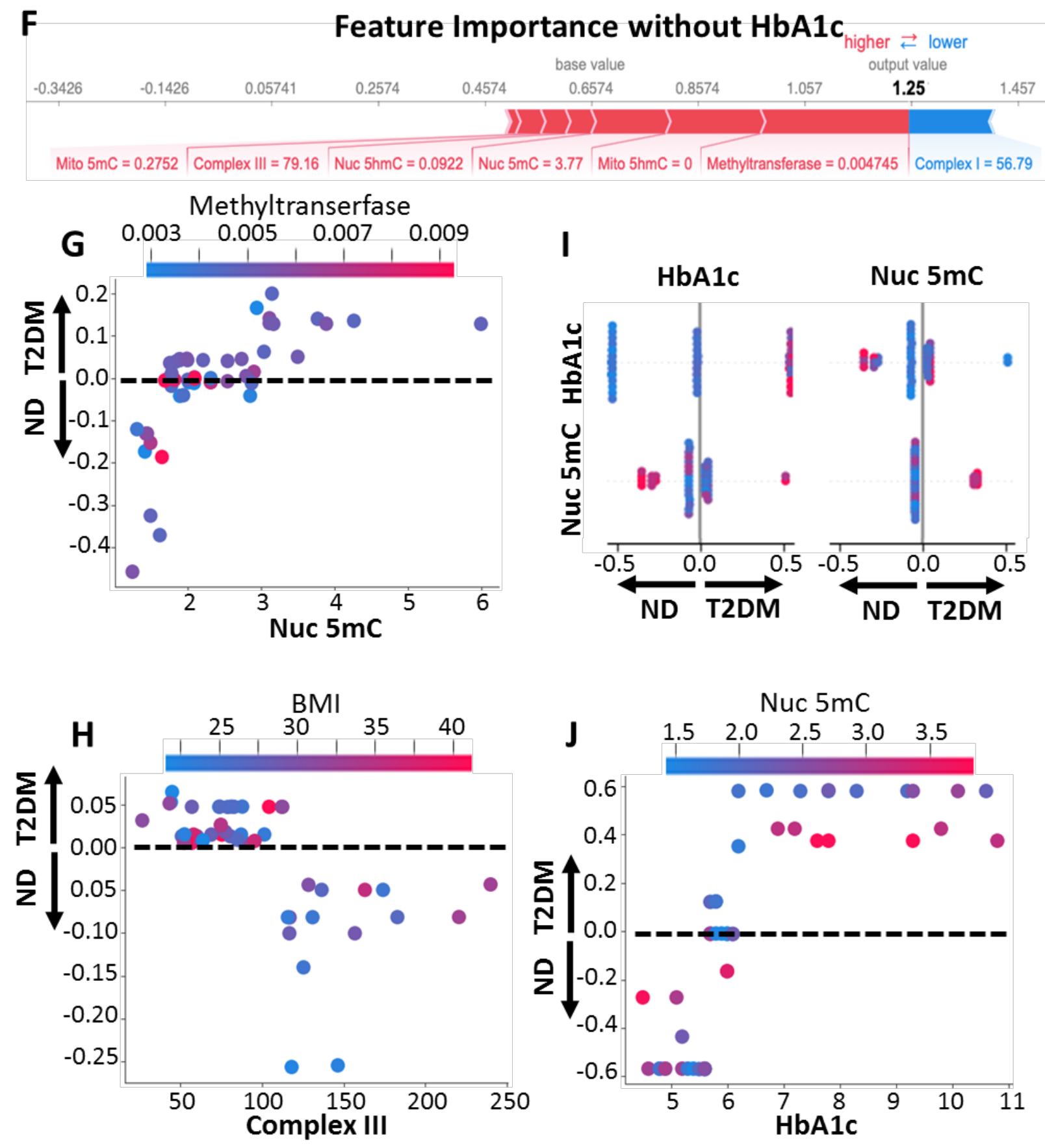


\section{Figure 3.2: Feature importance of physiological and biochemical characteristics from}

patients. (A) Using HbA1c for binary classification representing the factors positively (red) and negatively (blue) impacting the construction of the model, with size of the bars depicting importance. The (B) total nuclear methylation and (C) total nuclear hydroxymethylation of patients. SHAP binary depiction of the interaction between (D) total nuclear methylation and (E) total nuclear hydroxymethylation and HbA1c levels. (F) Not including HbA1c for binary classification representing the factors positively (red) and negatively (blue) impacting the construction of the model, with size of the bars depicting importance. SHAP binary depiction without $\mathrm{HbAlc}$ of the interaction between $(\mathrm{G})$ total nuclear methylation and methyltransferase activity and (H) electron transport chain complex III and BMI. Examining the multi classification effects of prediabetes, (I) A modified T-Plot where the main effects of biomarkers on the prediction output are shown along the diagonal axis whereas interaction effects are shown off the diagonal. SHAP depiction of patient separation with the individual and correlated effects of HbAlc and total nuclear methylation. SHAP multi depiction of the interaction between $(\mathrm{J})$ total nuclear methylation and HbA1c. SHAP values $>0.0$ are diabetic (T2DM), SHAP values $<0.0$ are non-diabetic (ND), SHAP values $=0$ are either ND or T2DM without influence on the model. Groups are considered significantly different if $P \leq 0.05=*$ compared to non-diabetic. All data are presented as the mean \pm standard error of the mean $(\mathrm{SEM}) . \mathrm{ND}=$ non-diabetic, $\mathrm{T} 2 \mathrm{DM}=$ type 2 diabetic, $\mathrm{Nuc}=$ nuclear, Mito $=$ mitochondrial, $5 \mathrm{mC}=5$-methylcytosine, 5hmC, 5- hydroxymethylcytosine, $\mathrm{HbA} 1 \mathrm{c}=$ glycated hemoglobin, binary $=$ no diabetes and diabetes, multi $=$ no diabetes, prediabetes, and type 2 diabetes. 
Figure 3.3
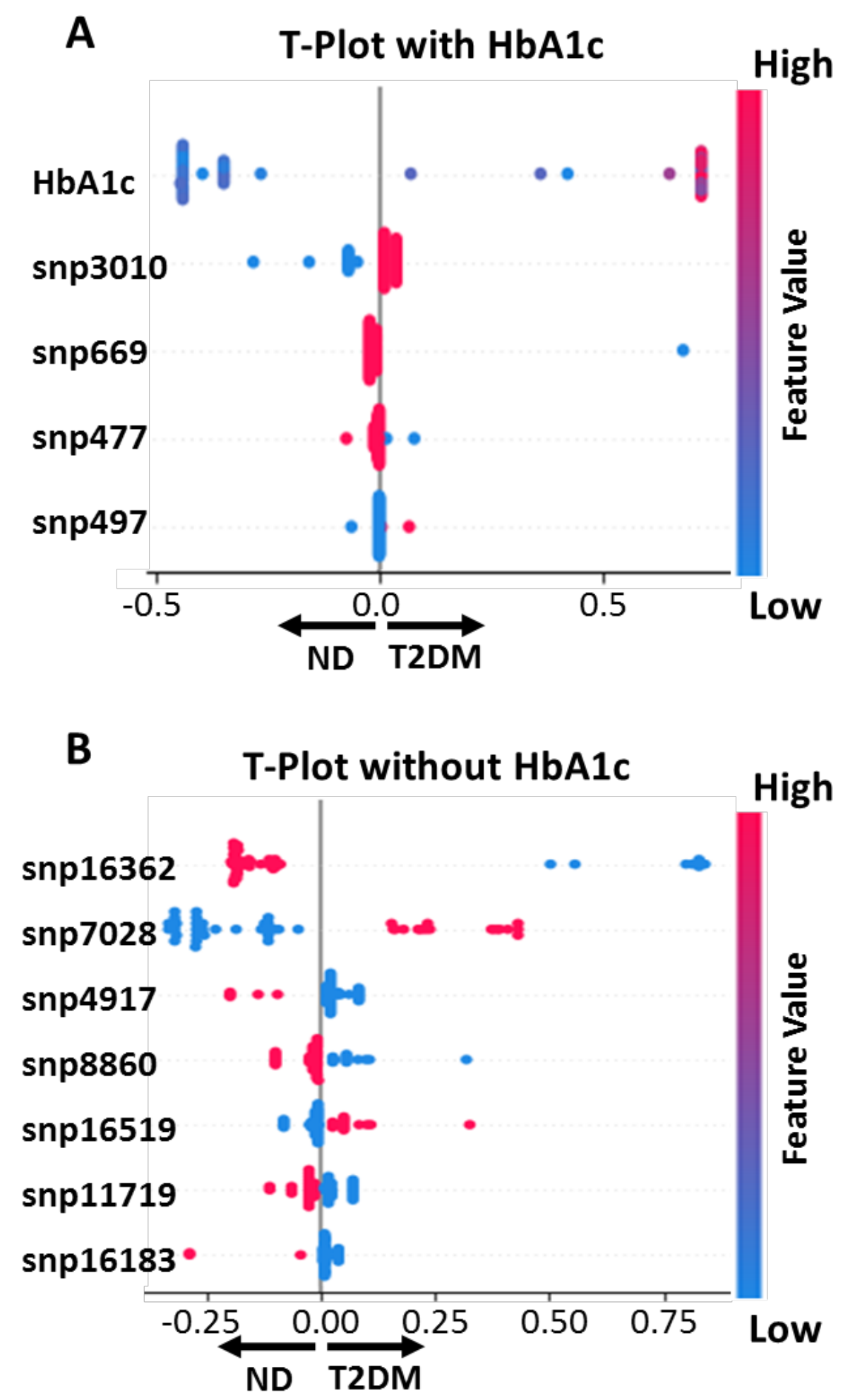
Figure 3.3
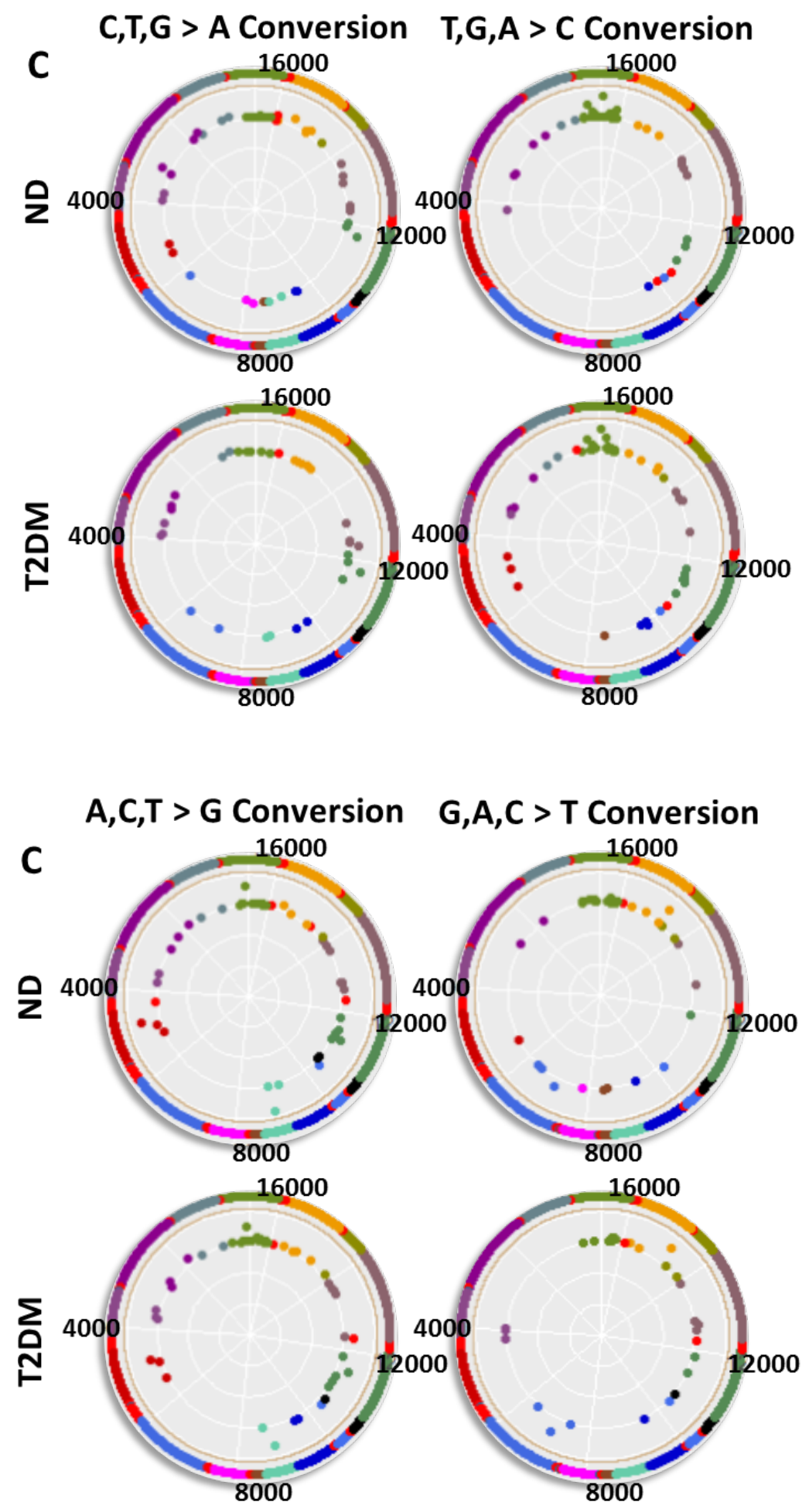
Figure 3.3
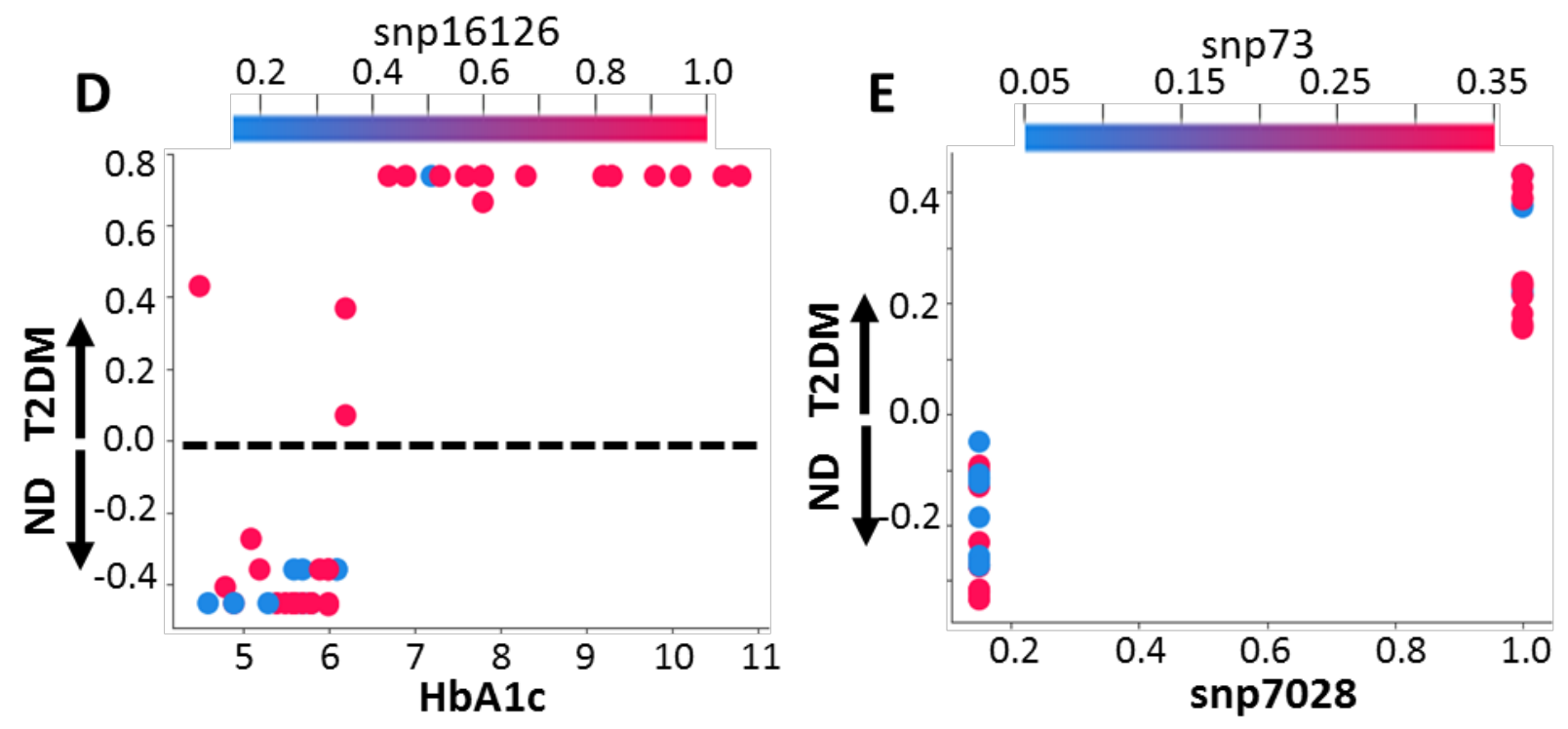
Figure 3.3: Feature importance of mitochondrial DNA SNPs from patients. (A) The most important predictive parameters using binary classification with $\mathrm{HbA1c}$, the absolute value of a feature being high (red) or low (blue) depicting diabetic (right-side) or non-diabetic (left-side) status. (B) The most important predictive parameters using binary classification without HbA1c, the absolute value of a feature being high (red) or low (blue) depicting diabetic (right-side) or nondiabetic (left-side) status. (C) Frequency of mitochondrial DNA SNPs by nucleotide converted in ND and T2DM patients; increasing frequency of SNPs occurring in the patient population are depicted by movement closer to the mitochondrial DNA strand. (D) SHAP binary depiction with HbA1c of the interaction between snp16126 and HbA1c. (E) SHAP binary depiction without HbA1c of the interaction between snp7028 and snp73. SHAP values $>0.0$ are diabetic (T2DM), SHAP values $<0.0$ are non-diabetic (ND), SHAP values $=0$ are either ND or T2DM without influence on the model. $\mathrm{ND}=$ non-diabetic, $\mathrm{T} 2 \mathrm{DM}=$ type 2 diabetic, HbA1c $=$ glycated hemoglobin, binary $=$ no diabetes and diabetes, multi $=$ no diabetes, prediabetes, and type 2 diabetes. 
Figure 3.4
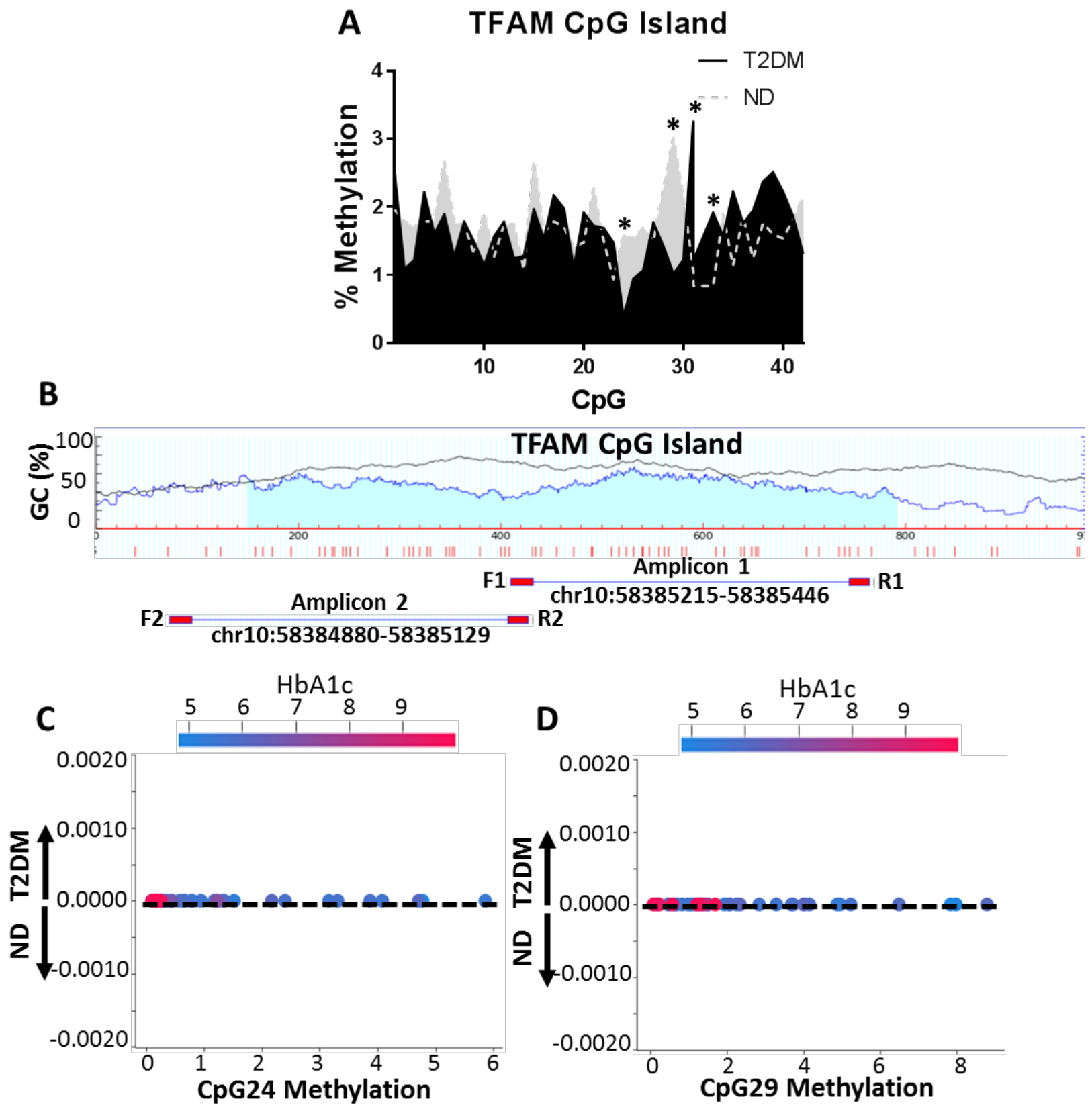
Figure 3.4

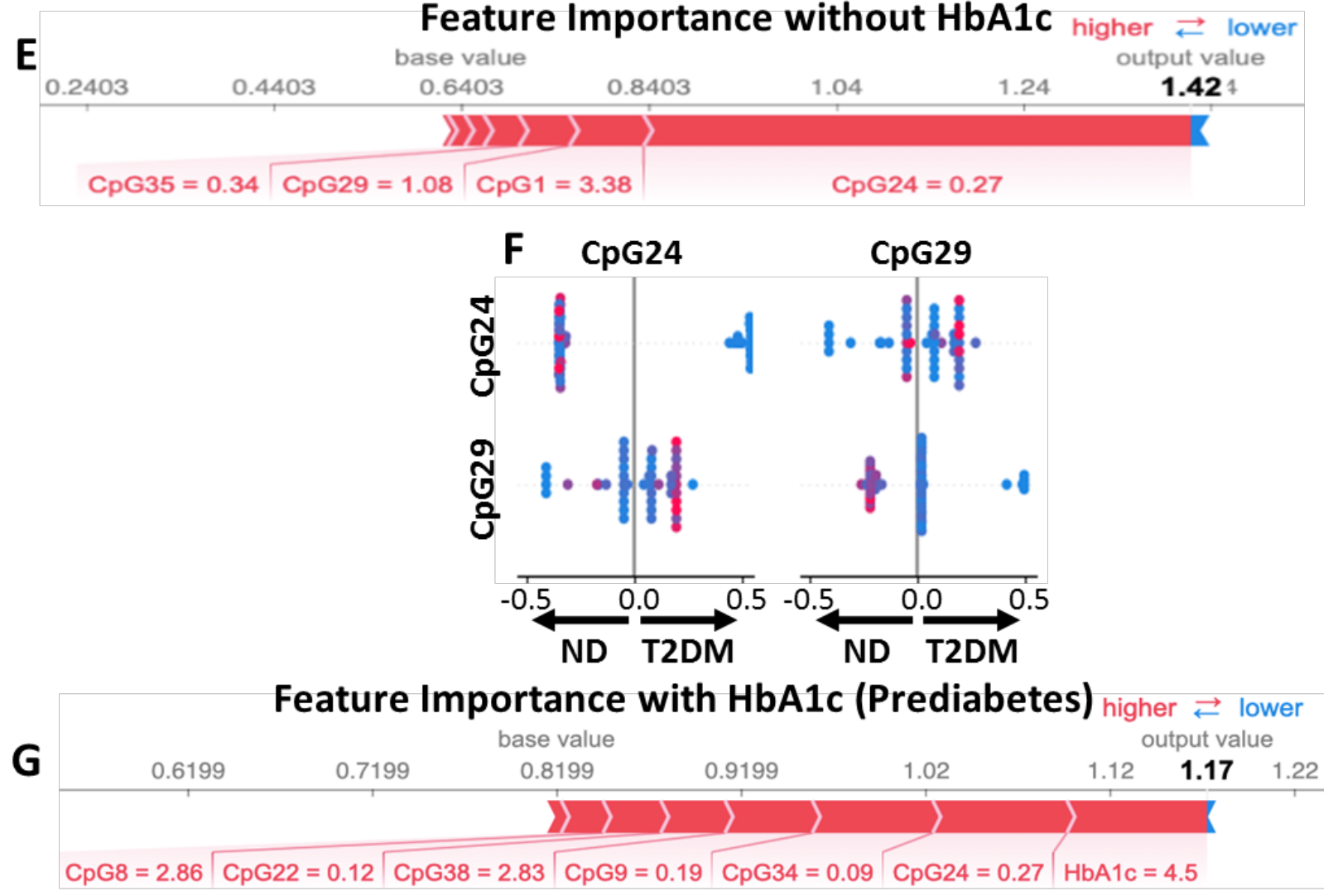

Feature Importance without HbA1c higher $\rightleftarrows$ lower base value output value

Feature Importance with HbA ๘ low

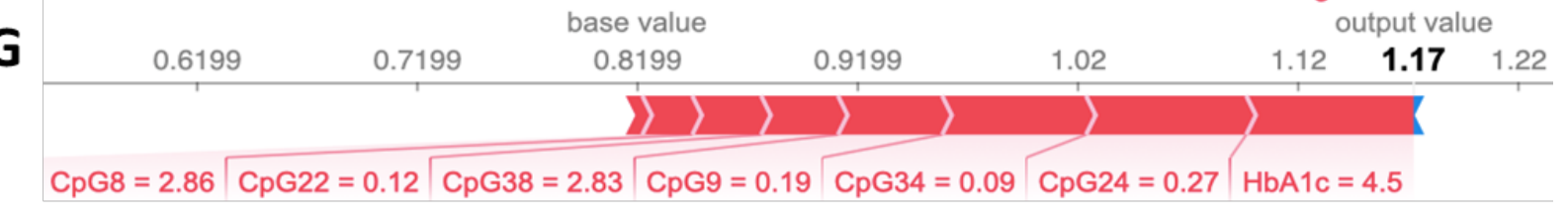

\section{H}

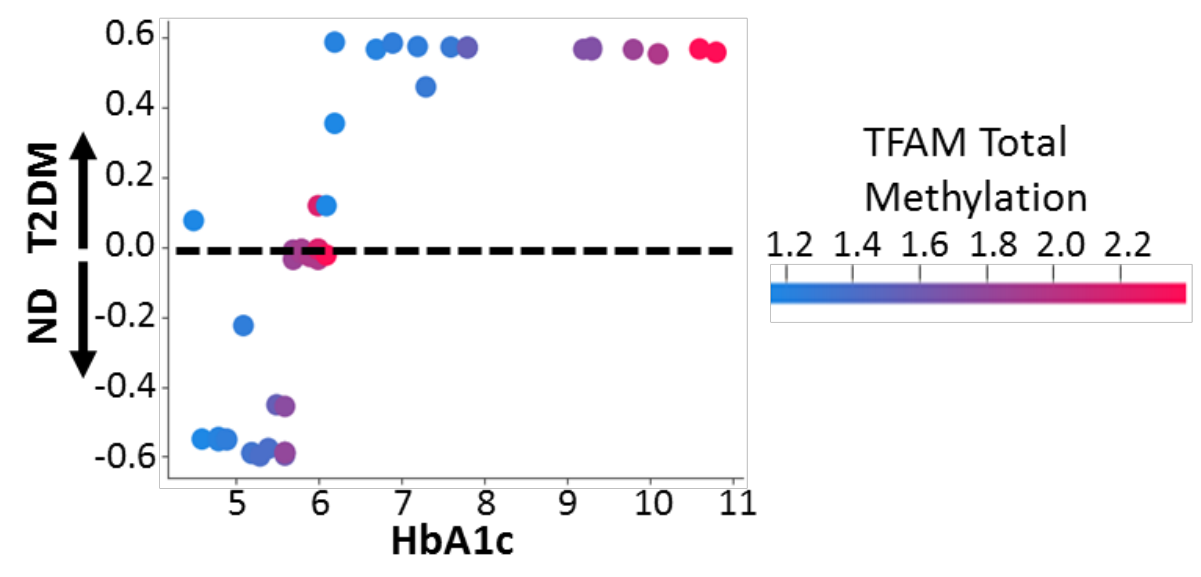


Figure 3.4: Feature importance of CpG island methylation of TFAM from patients. (A) Methylation across the promoter $\mathrm{CpG}$ region of the TFAM gene was determined using overhang bisulfite sequencing. (B) Experimental paradigm for amplification of the bisulfite-converted DNA for $23 \mathrm{CpG}$ sites proximal (Amplicon 1) and $19 \mathrm{CpG}$ sites distal (Amplicon 2) to the TFAM start site. SHAP binary depiction with HbA1c of the interaction between (C) CpG24 methylation and HbA1c and (D) CpG29 methylation and HbA1c. (E) Not including HbA1c for binary classification representing the factors positively (red) and negatively (blue) impacting the construction of the model, with size of the bars depicting importance. (F) A modified T-Plot where the main effects of biomarkers on the prediction output are shown along the diagonal axis whereas interaction effects are shown off the diagonal. SHAP binary depiction without HbA1c of patient separation with the individual and correlated effects of $\mathrm{CpG} 24$ methylation and $\mathrm{CpG} 29$ methylation. (G) Using $\mathrm{HbA} 1 \mathrm{c}$ for multi classification representing the factors positively (red) and negatively (blue) impacting the construction of the model, with size of the bars depicting importance. (H) SHAP multi depiction with $\mathrm{HbAlc}$ of the interaction between TFAM gene total methylation and HbA1c. SHAP values $>0.0$ are diabetic (T2DM), SHAP values $<0.0$ are non-diabetic (ND), SHAP values $=0$ are either ND or T2DM without influence on the model. Groups are considered significantly different if $P \leq 0.05=*$ compared to non-diabetic. All data are presented as the mean \pm standard error of the mean $(\mathrm{SEM}) . \mathrm{ND}=$ non-diabetic, $\mathrm{T} 2 \mathrm{DM}=$ type 2 diabetic, HbA1c $=$ glycated hemoglobin, $\mathrm{CpG}=$ cytosine nucleotide followed by a guanine nucleotide, $\mathrm{TFAM}=$ transcription factor A, mitochondrial, binary = no diabetes and diabetes, multi = no diabetes, prediabetes, and type 2 diabetes. 
Figure 3.5

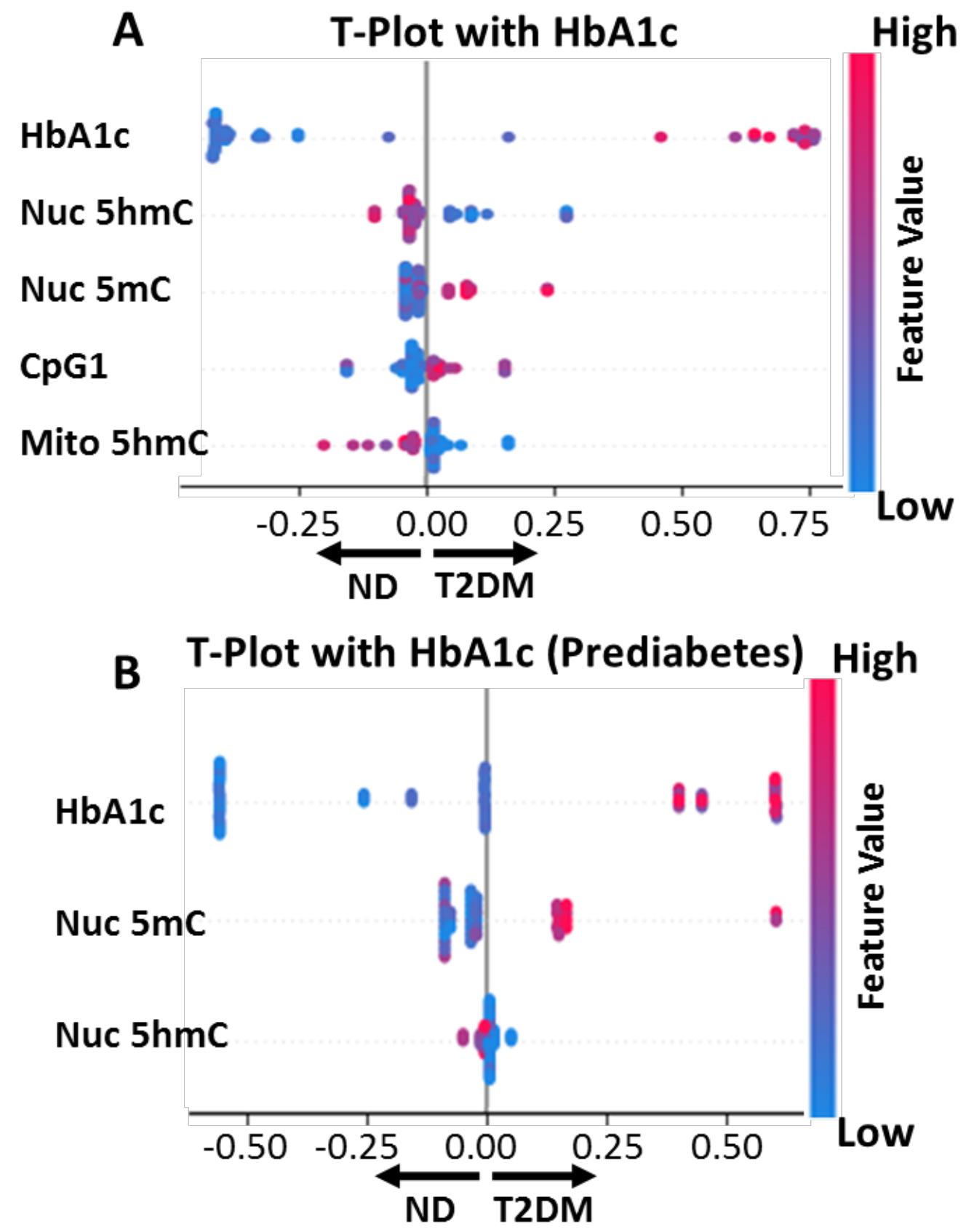


Figure 3.5
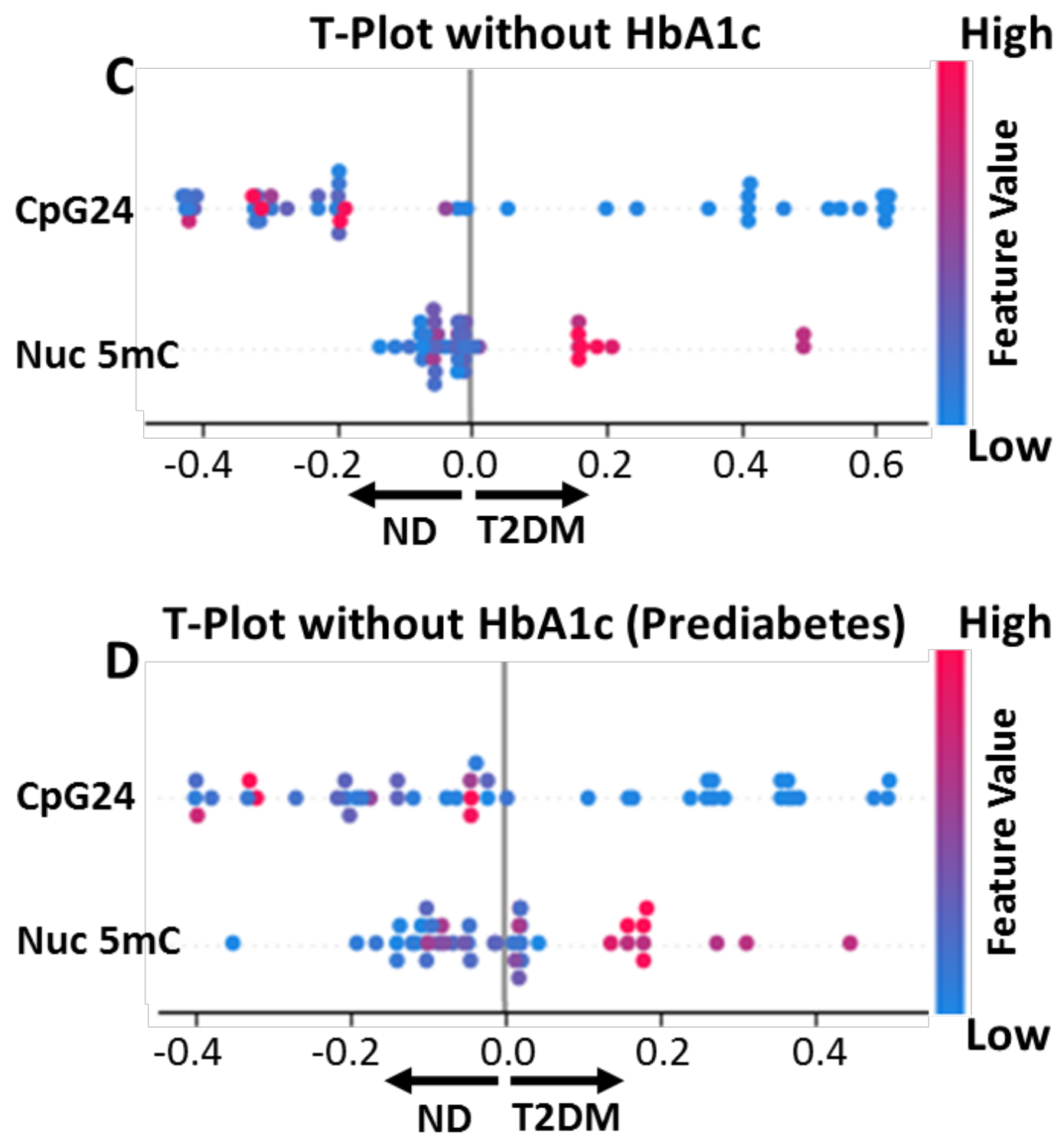
Figure 3.5
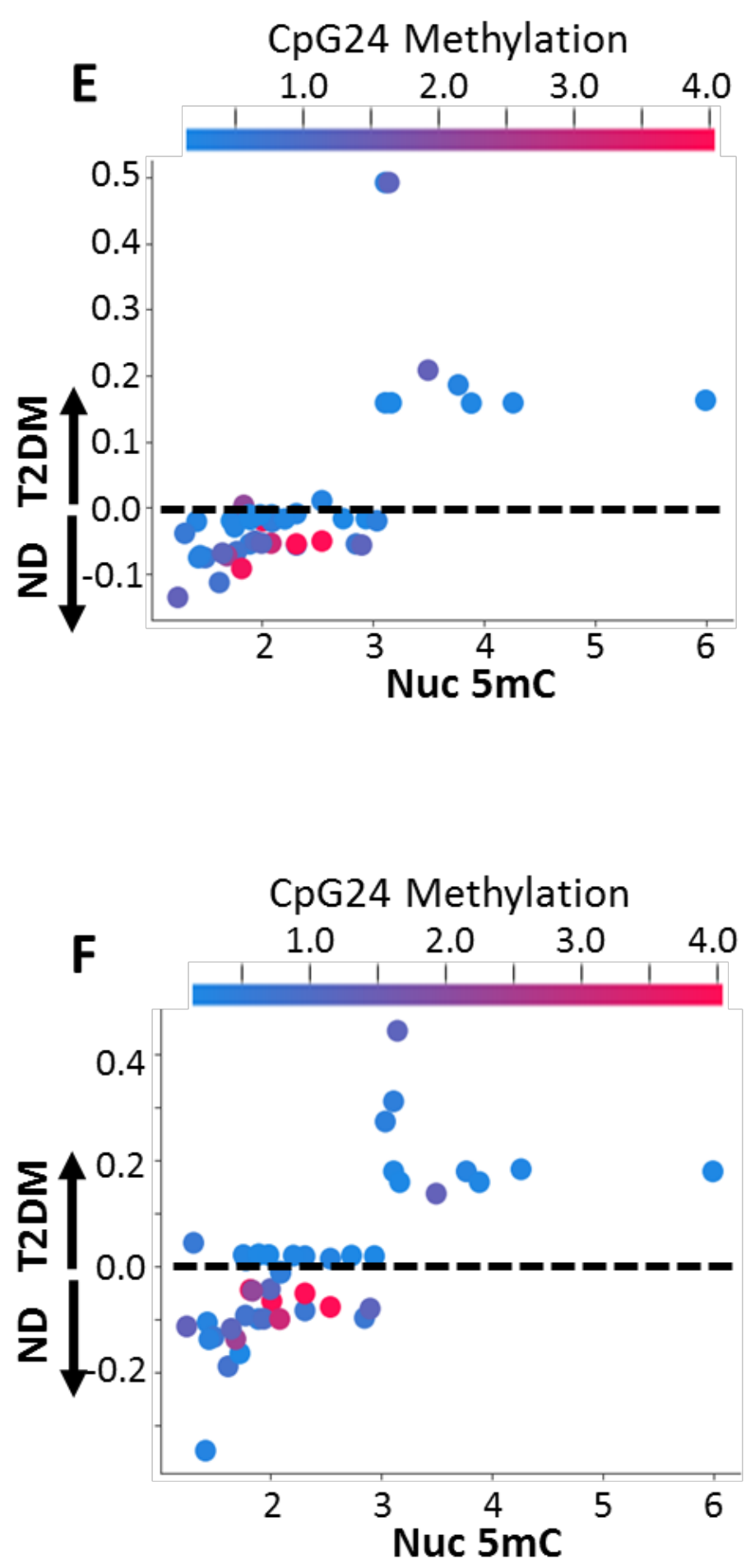
Figure 3.5: Feature importance of best factors combined from patients. The most important predictive parameters using (A) binary and (B) multi classification with $\mathrm{HbAlc}$, the absolute value of a feature being high (red) or low (blue) depicting diabetic (right-side) or non-diabetic (left-side) status. The most important predictive parameters using (C) binary and (D) multi classification without $\mathrm{HbA1c}$, the absolute value of a feature being high (red) or low (blue) depicting diabetic (right-side) or non-diabetic (left-side) status. SHAP (E) binary and (F) multi depiction without HbA1c of the interaction between total nuclear methylation and CpG24 methylation. SHAP values $>0.0$ are diabetic $($ T2DM), SHAP values $<0.0$ are non-diabetic (ND), SHAP values $=0$ are either $\mathrm{ND}$ or T2DM without influence on the model. $\mathrm{ND}=$ non-diabetic, T2DM = type 2 diabetic, HbA1c = glycated hemoglobin, $\mathrm{CpG}=$ cytosine nucleotide followed by a guanine nucleotide, Nuc $=$ nuclear, $5 \mathrm{mC}=5$-methylcytosine, binary $=$ no diabetes and diabetes, multi $=$ no diabetes, prediabetes, and type 2 diabetes. 


\section{Figure 3.6}

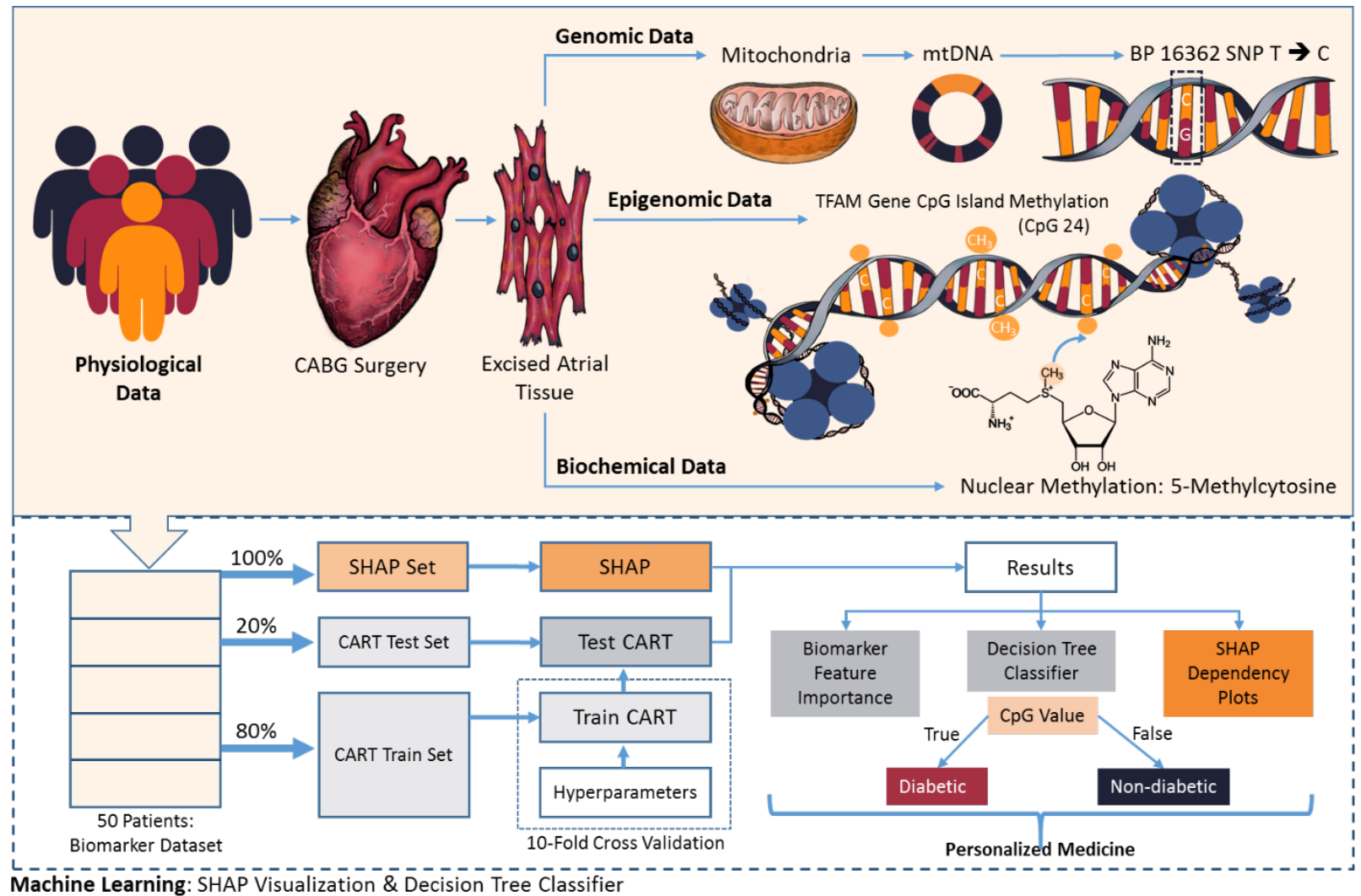


Figure 3.6: Overview of machine-learning pipeline implementing biological variables across a spectrum of gathered information. From the patient population undergoing coronary artery bypass graft surgery (CABG), physiological parameters (demographics, health reports, etc.) and atrial tissue were used for subsequent analyses. From cardiac tissue genomic (mitochondrial DNA), epigenomic (TFAM promoter $\mathrm{CpG}$ methylation), and biochemical (nuclear and mitochondrial function) were assessed. Cumulatively, the biological data was processed through tree ensembles in SHAP and validated through CART analysis with 10-Fold Cross Validation. Using these machine-learning algorithms, graphical depictions and biomarker feature importance are able to be derived, allowing for prediction of the onset and progression of diabetes. Ultimately, by using biological data at the genomic and epigenomic level, it allows for a precision medicine approaches and more personalized diagnostics and prognostics. TFAM = transcription factor A, mitochondrial, $\mathrm{mtDNA}=$ mitochondrial $\mathrm{DNA}, \mathrm{CpG}=$ cytosine nucleotide followed by a guanine nucleotide, $\mathrm{CART}=$ Classification and Regression Trees, $\mathrm{SHAP}=$ SHapley Additive exPlanations. 
Table S3.1

\begin{tabular}{llll}
\hline \multicolumn{1}{c}{ Parameter } & \multicolumn{1}{c}{ Non-Diabetic } & \multicolumn{1}{c}{ Pre-Diabetic } & \multicolumn{1}{c}{ Type 2 Diabetic } \\
\hline Age & $58.06 \pm 3.692$ & $66.43 \pm 2.799$ & $61.16 \pm 3.047$ \\
\hline Sex & Male $=15$, Female $=1$ & Male $=11$, Female $=3$ & Male $=14$, Female $=5$ \\
\hline BMI $\left(\mathrm{kg} / \mathrm{m}^{2}\right)$ & $30.11 \pm 1.405$ & $28.01 \pm 1.669$ & $29.14 \pm 1.448$ \\
Coronary Artery Disease & $68.75 \% \pm 11.97 \%$ & $78.57 \% \pm 11.38 \%$ & $100 \% \pm 0.0 \%$ \\
Hypertension & $81.25 \% \pm 10.08 \%$ & $92.86 \% \pm 7.143 \%$ & $94.74 \% \pm 5.263 \%$ \\
\hline Valvular Disease & $31.25 \% \pm 11.97 \%$ & $21.43 \% \pm 11.38 \%$ & $15.79 \% \pm 8.595 \%$ \\
\hline HbAlc & $5.263 \pm 0.08892$ & $5.914 \pm 0.0443$ & $\mathbf{8 . 0 1 6} \pm \mathbf{0 . 4 0 2 4} * \#$ \\
\hline
\end{tabular}


Table S3.1: Patient characteristics and demographic information for non-diabetic, pre-diabetic, and type 2 diabetic patients. Groups are considered significantly different if $P \leq 0.05=*$ compared to non-diabetic or \# compared to pre-diabetic. All data are presented as the mean \pm standard error of the mean $(\mathrm{SEM}) . \mathrm{HbAlc}=$ glycated hemoglobin. 
Table S3.2

\begin{tabular}{|c|c|c|c|c|}
\hline Software & Purpose & Primer Name & Sequence & Product Size \\
\hline \multirow{6}{*}{$\begin{array}{l}\text { MethPrim } \\
\text { er } 2.0\end{array}$} & \multirow{6}{*}{$\begin{array}{l}\text { Bisulfite } \\
\text { Overhang } \\
\text { PCR } \\
\text { Sequencin } \\
\text { g }\end{array}$} & TFAM 1 F1 & $\begin{array}{l}\text { TCGTCGGCAGCGTCAGATGTGTATAAGAGACAGGGGATAG } \\
\text { AGGTGGTTTAATAG }\end{array}$ & \multirow{6}{*}{399} \\
\hline & & TFAM 1 R1 & $\begin{array}{l}\text { GTCTCGTGGGCTCGGAGATGTGTATAAGAGACAGATACAC } \\
\text { AACTCTACTCCAAACCTTC }\end{array}$ & \\
\hline & & TFAM 1 F2 & $\begin{array}{l}\text { TCGTCGGCAGCGTCAGATGTGTATAAGAGACAGNGGGATA } \\
\text { GAGGTGGTTTAATAG }\end{array}$ & \\
\hline & & TFAM 1 R2 & $\begin{array}{l}\text { GTCTCGTGGGCTCGGAGATGTGTATAAGAGACAGNATACA } \\
\text { CAACTCTACTCCAAACCTTC }\end{array}$ & \\
\hline & & TFAM 1 F3 & $\begin{array}{l}\text { TCGTCGGCAGCGTCAGATGTGTATAAGAGACAGNNGGGAT } \\
\text { AGAGGTGGTTTAATAG }\end{array}$ & \\
\hline & & TFAM 1 R3 & $\begin{array}{l}\text { GTCTCGTGGGCTCGGAGATGTGTATAAGAGACAGNNATAC } \\
\text { ACAACTCTACTCCAAACCTTC }\end{array}$ & \\
\hline \multirow{6}{*}{$\begin{array}{l}\text { MethPrim } \\
\text { er } 2.0\end{array}$} & \multirow{6}{*}{$\begin{array}{l}\text { Bisulfite } \\
\text { Overhang } \\
\text { PCR } \\
\text { Sequencin } \\
\text { g }\end{array}$} & TFAM 2 F1 & $\begin{array}{l}\text { TCGTCGGCAGCGTCAGATGTGTATAAGAGACAGAAAATGA } \\
\text { AGATTAATGGGTTTTA }\end{array}$ & \multirow{6}{*}{356} \\
\hline & & TFAM 2 R1 & $\begin{array}{l}\text { GTCTCGTGGGCTCGGAGATGTGTATAAGAGACAGCTATTA } \\
\text { AACCACCTCTATCCC }\end{array}$ & \\
\hline & & TFAM 2 F2 & $\begin{array}{l}\text { TCGTCGGCAGCGTCAGATGTGTATAAGAGACAGNAAAATG } \\
\text { AAGATTAATGGGTTTTA }\end{array}$ & \\
\hline & & TFAM 2 R2 & $\begin{array}{l}\text { GTCTCGTGGGCTCGGAGATGTGTATAAGAGACAGNCTATT } \\
\text { AAACCACCTCTATCCC }\end{array}$ & \\
\hline & & TFAM 2 F3 & $\begin{array}{l}\text { TCGTCGGCAGCGTCAGATGTGTATAAGAGACAGNNAAAAT } \\
\text { GAAGATTAATGGGTTTTA }\end{array}$ & \\
\hline & & TFAM 2 R3 & $\begin{array}{l}\text { GTCTCGTGGGCTCGGAGATGTGTATAAGAGACAGNNCTAT } \\
\text { TAAACCACCTCTATCCC }\end{array}$ & \\
\hline \multirow{14}{*}{ Primer3 } & \multirow{14}{*}{$\begin{array}{c}\text { Chromatin } \\
\text { Immunopr } \\
\text { ecipitation } \\
\text { qPCR }\end{array}$} & D-Loop F1 & CATGGGGAAGCAGATTTGGG & \multirow{2}{*}{174} \\
\hline & & D-Loop R1 & TTGTAAGCATGGGGAGGGG & \\
\hline & & D-Loop F2 & TACATTACTGCCAGCCACCA & \multirow{2}{*}{113} \\
\hline & & D-Loop R2 & CATGGGGACGAGAAGGGATT & \\
\hline & & D-Loop F3 & CTTGACCACCATCCTCCGT & \multirow{2}{*}{137} \\
\hline & & D-Loop R3 & AGAGCTCCCGTGAGTGGTTA & \\
\hline & & D-Loop F4 & GAGCTCTCCATGCATTTGGT & \multirow{2}{*}{91} \\
\hline & & D-Loop R4 & TACTGCGACATAGGGTGCTC & \\
\hline & & D-Loop F5 & GAGCACCCTATGTCGCAGTA & \multirow{2}{*}{169} \\
\hline & & D-Loop R5 & CTGTGTGGAAAGTGGCTGTG & \\
\hline & & D-Loop F6 & GTCTGCACAGCCACTTTCC & \multirow{2}{*}{225} \\
\hline & & D-Loop R6 & TAGTATGGGAGTGGGAGGGG & \\
\hline & & D-Loop F7 & ССССТСССАСТСССАТАСТА & \multirow{2}{*}{183} \\
\hline & & D-Loop R7 & GGTGATGTGAGCCCGTCTAA & \\
\hline
\end{tabular}


Table S3.2: Primer design. Using MethPrimer 2.0 for bisulfite converted DNA and Primer3 for chromatin immunoprecipitation, primers were designed to measure TFAM CpG island methylation and TFAM binding to the D-Loop region of the mitochondrial DNA, respectively. TFAM $=$ transcription factor A, mitochondrial. 
Table S3.3

\begin{tabular}{|c|c|c|c|c|c|}
\hline \multicolumn{6}{|c|}{ SEED $=2$} \\
\hline & \multicolumn{5}{|c|}{ CART Binary } \\
\hline & $\begin{array}{c}\text { Features Implemented in Training and } \\
\text { Testing by Trial }\end{array}$ & $\begin{array}{c}\text { Training } \\
\text { (Average } \\
\text { Accuracy) }\end{array}$ & $\begin{array}{l}\text { Testing } \\
\text { (F1 Score) }\end{array}$ & $\begin{array}{l}\text { Train St.D } \\
\text { Average }\end{array}$ & Test St. D \\
\hline TRIAL 1 & Nuc 5mC, Complex I, III, IV & 0.73 & 0.836 & 0.1778 & 0.092 \\
\hline TRIAL 2 & Methyl, Complex III, IV, V & 0.6782 & 0.74 & 0.1902 & 0.055 \\
\hline TRIAL 3 & Methyl, Nuc 5hm, Complex I & 0.67 & 0.67 & 0.2072 & 0.000 \\
\hline TRIAL 4 & Complex I, III, IV & 0.573 & 0.482 & 0.2218 & 0.085 \\
\hline TRIAL 5 & $\begin{array}{c}\text { Methyl, Nuc 5mC, Complex I, Citrate } \\
\text { Synthase }\end{array}$ & 0.7448 & 0.604 & 0.2012 & 0.054 \\
\hline \multirow[t]{3}{*}{ TRIAL 6} & Methyl, Complex I, III & 0.652 & 0.7 & 0.2252 & 0.000 \\
\hline & \multicolumn{5}{|c|}{ LR Binary } \\
\hline & $\begin{array}{c}\text { Features Implemented in Training and } \\
\text { Testing by Trial }\end{array}$ & $\begin{array}{c}\text { Training } \\
\text { (Average } \\
\text { Accuracy) }\end{array}$ & $\begin{array}{l}\text { Testing } \\
\text { (F1 Score) }\end{array}$ & $\begin{array}{l}\text { Train St.D } \\
\text { Average }\end{array}$ & Test St. D \\
\hline TRIAL 1 & Nuc 5mC, Complex I, III, IV & 0.791667 & 0.7 & 0.107044 & $\mathbf{0}$ \\
\hline TRIAL 2 & Methyl, Complex III, IV, V & 0.583333 & 0.4 & 0.182574 & $\mathbf{0}$ \\
\hline TRIAL 3 & Methyl, Nuc 5hm, Complex I & 0.708333 & 0.52 & 0.201556 & $\mathbf{0}$ \\
\hline TRIAL 4 & Complex I, III, IV & 0.608333 & 0.6 & 0.186525 & $\mathbf{0}$ \\
\hline TRIAL 5 & $\begin{array}{c}\text { Methyl, Nuc 5mC, Complex I, Citrate } \\
\text { Synthase }\end{array}$ & 0.725 & 0.6 & 0.175 & 0 \\
\hline \multirow[t]{3}{*}{ TRIAL 6} & Methyl, Complex I, III & 0.633333 & 0.38 & 0.217945 & $\mathbf{0}$ \\
\hline & \multicolumn{5}{|c|}{ LDA Binary } \\
\hline & $\begin{array}{c}\text { Features Implemented in Training and } \\
\text { Testing by Trial }\end{array}$ & $\begin{array}{c}\text { Training } \\
\text { (Average } \\
\text { Accuracy) }\end{array}$ & $\begin{array}{c}\text { Testing } \\
\text { (F1 Score) }\end{array}$ & $\begin{array}{l}\text { Train St.D } \\
\text { Average }\end{array}$ & Test St. D \\
\hline TRIAL 1 & Nuc 5mC, Complex I, III, IV & 0.741667 & 0.7 & 0.114564 & $\mathbf{0}$ \\
\hline TRIAL 2 & Methyl, Complex III, IV, V & 0.558333 & 0.4 & 0.175 & $\mathbf{0}$ \\
\hline TRIAL 3 & Methyl, Nuc 5hm, Complex I & 0.683333 & 0.7 & 0.210159 & $\mathbf{0}$ \\
\hline TRIAL 4 & Complex I, III, IV & 0.608333 & 0.6 & 0.149304 & $\mathbf{0}$ \\
\hline TRIAL 5 & $\begin{array}{c}\text { Methyl, Nuc 5mC, Complex I, Citrate } \\
\text { Synthase }\end{array}$ & 0.675 & 0.8 & 0.195256 & $\mathbf{0}$ \\
\hline \multirow[t]{3}{*}{ TRIAL 6} & Methyl, Complex I, III & 0.633333 & 0.49 & 0.187083 & $\mathbf{0}$ \\
\hline & \multicolumn{5}{|c|}{ KNN Binary } \\
\hline & $\begin{array}{c}\text { Features Implemented in Training and } \\
\text { Testing by Trial }\end{array}$ & $\begin{array}{c}\text { Training } \\
\text { (Average } \\
\text { Accuracy) }\end{array}$ & $\begin{array}{c}\text { Testing } \\
\text { (F1 Score) }\end{array}$ & $\begin{array}{c}\text { Train St.D } \\
\text { Average }\end{array}$ & Test St. D \\
\hline TRIAL 1 & Nuc 5mC, Complex I, III, IV & 0.675 & 0.45 & 0.317214 & $\mathbf{0}$ \\
\hline TRIAL 2 & Methyl, Complex III, IV, V & 0.825 & 0.2 & 0.195256 & $\mathbf{0}$ \\
\hline
\end{tabular}




\begin{tabular}{|c|c|c|c|c|c|}
\hline TRIAL 3 & Methyl, Nuc 5hm, Complex I & 0.633333 & 0.67 & 0.15 & $\mathbf{0}$ \\
\hline TRIAL 4 & Complex I, III, IV & 0.675 & 0.45 & 0.317214 & $\mathbf{0}$ \\
\hline TRIAL 5 & $\begin{array}{c}\text { Methyl, Nuc 5mC, Complex I, Citrate } \\
\text { Synthase }\end{array}$ & 0.633333 & 0.67 & 0.15 & $\mathbf{0}$ \\
\hline \multirow[t]{3}{*}{ TRIAL 6} & Methyl, Complex I, III & 0.675 & 0.38 & 0.275 & $\mathbf{0}$ \\
\hline & \multicolumn{5}{|c|}{ NB Binary } \\
\hline & $\begin{array}{c}\text { Features Implemented in Training and } \\
\text { Testing by Trial }\end{array}$ & $\begin{array}{l}\text { Training } \\
\text { (Average } \\
\text { Accuracy) }\end{array}$ & $\begin{array}{c}\text { Testing } \\
\text { (F1 Score) }\end{array}$ & $\begin{array}{l}\text { Train St.D } \\
\text { Average }\end{array}$ & Test St. D \\
\hline TRIAL 1 & Nuc 5mC, Complex I, III, IV & 0.7 & 0.38 & 0.217945 & $\mathbf{0}$ \\
\hline TRIAL 2 & Methyl, Complex III, IV, V & 0.625 & 0.23 & 0.321131 & $\mathbf{0}$ \\
\hline TRIAL 3 & Methyl, Nuc 5hm, Complex I & 0.65 & 0.7 & 0.229129 & $\mathbf{0}$ \\
\hline TRIAL 4 & Complex I, III, IV & 0.6 & 0.29 & 0.229129 & $\mathbf{0}$ \\
\hline TRIAL 5 & $\begin{array}{c}\text { Methyl, Nuc 5mC, Complex I, Citrate } \\
\text { Synthase }\end{array}$ & 0.675 & 0.7 & 0.275 & $\mathbf{0}$ \\
\hline \multirow[t]{3}{*}{ TRIAL 6} & Methyl, Complex I, III & 0.675 & 0.49 & 0.296859 & $\mathbf{0}$ \\
\hline & \multicolumn{5}{|c|}{ SVM Binary } \\
\hline & $\begin{array}{c}\text { Features Implemented in Training and } \\
\text { Testing by Trial }\end{array}$ & $\begin{array}{c}\text { Training } \\
\text { (Average } \\
\text { Accuracy) } \\
\end{array}$ & $\begin{array}{l}\text { Testing } \\
\text { (F1 Score) }\end{array}$ & $\begin{array}{l}\text { Train St.D } \\
\text { Average }\end{array}$ & Test St. D \\
\hline TRIAL 1 & Nuc 5mC, Complex I, III, IV & $\mathbf{0 . 6 3 3 3 3 3}$ & $\mathbf{0 . 3 3}$ & 0.187083 & $\mathbf{0}$ \\
\hline TRIAL 2 & Methyl, Complex III, IV, V & 0.633333 & 0.52 & 0.187083 & $\mathbf{0}$ \\
\hline TRIAL 3 & Methyl, Nuc 5hm, Complex I & 0.608333 & 0.52 & 0.149304 & $\mathbf{0}$ \\
\hline TRIAL 4 & Complex I, III, IV & 0.633333 & $\mathbf{0 . 3 3}$ & 0.187083 & $\mathbf{0}$ \\
\hline TRIAL 5 & $\begin{array}{c}\text { Methyl, Nuc 5mC, Complex I, Citrate } \\
\text { Synthase }\end{array}$ & 0.658333 & 0.67 & 0.184278 & $\mathbf{0}$ \\
\hline TRIAL 6 & Methyl, Complex I, III & 0.608333 & 0.52 & 0.149304 & $\mathbf{0}$ \\
\hline
\end{tabular}


Table S3.3: Machine learning analyses for physiological/biochemical features using binary classification. CART $=$ Classification and Regression Trees, LR $=$ Logistic Regression, LDA = Linear Discriminant Analysis, KNN = K-Nearest Neighbors, NB = Naïve Bayes, SVC = Support Vector Machines. Nuc $=$ Nuclear, Mito $=$ Mitochondrial, $5 \mathrm{mC}=5$-methylcytosine, $5 \mathrm{hmC}=5$ hydroxymethylcytosine, Methyl = S-adenosyl methionine methyltransferase activity, St.D. = standard deviation, binary $=$ non-diabetic and type 2 diabetic, multi $=$ non-diabetic, prediabetic, and type 2 diabetic. 
Table S3.4

\begin{tabular}{|c|c|c|c|c|c|}
\hline \multicolumn{6}{|c|}{ SEED $=22$} \\
\hline & \multicolumn{5}{|c|}{ CART Multiple } \\
\hline & $\begin{array}{c}\text { Features Implemented in Training and } \\
\text { Testing by Trial }\end{array}$ & $\begin{array}{c}\text { Training } \\
\text { (Average } \\
\text { Accuracy) } \\
\end{array}$ & $\begin{array}{c}\text { Testing } \\
\text { (F1 Score) }\end{array}$ & $\begin{array}{l}\text { Train St.D } \\
\text { Average }\end{array}$ & Test St. D \\
\hline TRIAL 1 & Mito 5hmC, Nuc 5mC, Complex I, III, V & 0.49 & 0.35 & 0.2968 & 0.000 \\
\hline TRIAL 2 & $\begin{array}{c}\text { Nuc 5mC, Complex I, III, IV, V, Citrate } \\
\text { Synthase }\end{array}$ & 0.44 & 0.48 & 0.2828 & 0.079 \\
\hline TRIAL 3 & Methyl, Complex III, V & 0.545 & 0.598 & 0.26 & 0.045 \\
\hline TRIAL 4 & Nuc 5mC, Complex III, V & 0.52 & 0.412 & 0.2266 & 0.049 \\
\hline TRIAL 5 & Methyl, Nuc 5mC, Citrate Synthase & 0.4532 & 0.464 & 0.2362 & 0.048 \\
\hline \multirow[t]{3}{*}{ TRIAL 6} & $\begin{array}{c}\text { Methyl, Nuc 5mC, Complex III, Citrate } \\
\text { Synthase }\end{array}$ & 0.4748 & 0.36 & 0.1812 & 0.060 \\
\hline & \multicolumn{5}{|c|}{ LR Multiple } \\
\hline & $\begin{array}{c}\text { Features Implemented in Training and } \\
\text { Testing by Trial }\end{array}$ & $\begin{array}{c}\text { Training } \\
\text { (Average } \\
\text { Accuracy) } \\
\end{array}$ & $\begin{array}{l}\text { Testing } \\
\text { (F1 Score) }\end{array}$ & $\begin{array}{l}\text { Train St.D } \\
\text { Average }\end{array}$ & Test St. D \\
\hline TRIAL 1 & Mito 5hmC ,Nuc 5mC, Complex I, III, V & 0.5 & 0.28 & 0.33541 & $\mathbf{0}$ \\
\hline TRIAL 2 & $\begin{array}{c}\text { Nuc 5mC, Complex I, III, IV, V, Citrate } \\
\text { Synthase }\end{array}$ & 0.5 & 0.26 & 0.316228 & $\mathbf{0}$ \\
\hline TRIAL 3 & Methyl, Complex III, V & 0.525 & 0.1 & 0.283945 & $\mathbf{0}$ \\
\hline TRIAL 4 & Nuc 5mC, Complex III, V & 0.525 & 0.27 & 0.283945 & $\mathbf{0}$ \\
\hline TRIAL 5 & Methyl, Nuc 5mC, Citrate Synthase & 0.45 & 0.22 & 0.291548 & $\mathbf{0}$ \\
\hline \multirow[t]{3}{*}{ TRIAL 6} & $\begin{array}{c}\text { Methyl, Nuc 5mC, Complex III, Citrate } \\
\text { Synthase }\end{array}$ & 0.5 & 0.12 & 0.295804 & 0 \\
\hline & \multicolumn{5}{|c|}{ LDA Multiple } \\
\hline & $\begin{array}{c}\text { Features Implemented in Training and } \\
\text { Testing by Trial }\end{array}$ & $\begin{array}{c}\text { Training } \\
\text { (Average } \\
\text { Accuracy) }\end{array}$ & $\begin{array}{c}\text { Testing } \\
\text { (F1 Score) }\end{array}$ & $\begin{array}{l}\text { Train St.D } \\
\text { Average }\end{array}$ & Test St. D \\
\hline TRIAL 1 & Mito 5hmC ,Nuc 5mC, Complex I, III, V & 0.475 & 0.15 & 0.325 & $\mathbf{0}$ \\
\hline TRIAL 2 & $\begin{array}{l}\text { Nuc 5mC, Complex I, III, IV, V, Citrate } \\
\text { Synthase }\end{array}$ & 0.525 & 0.32 & 0.283945 & $\mathbf{0}$ \\
\hline TRIAL 3 & Methyl, Complex III, V & 0.6 & 0.11 & 0.339116 & $\mathbf{0}$ \\
\hline TRIAL 4 & Nuc 5mC, Complex III, V & 0.55 & 0.28 & 0.331662 & $\mathbf{0}$ \\
\hline TRIAL 5 & Methyl, Nuc 5mC, Citrate Synthase & 0.5 & 0.4 & 0.37081 & $\mathbf{0}$ \\
\hline \multirow[t]{3}{*}{ TRIAL 6} & $\begin{array}{c}\text { Methyl, Nuc 5mC, Complex III, Citrate } \\
\text { Synthase }\end{array}$ & 0.5 & 0.4 & 0.353553 & 0 \\
\hline & \multicolumn{5}{|c|}{ KNN Multiple } \\
\hline & $\begin{array}{c}\text { Features Implemented in Training and } \\
\text { Testing by Trial }\end{array}$ & $\begin{array}{c}\text { Training } \\
\text { (Average } \\
\text { Accuracy) }\end{array}$ & $\begin{array}{l}\text { Testing } \\
\text { (F1 Score) }\end{array}$ & $\begin{array}{l}\text { Train St.D } \\
\text { Average }\end{array}$ & Test St. D \\
\hline TRIAL 1 & Mito 5hmC ,Nuc 5mC, Complex I, III, V & 0.55 & 0.1 & 0.291548 & $\mathbf{0}$ \\
\hline
\end{tabular}




\begin{tabular}{|c|c|c|c|c|c|}
\hline TRIAL 2 & $\begin{array}{c}\text { Nuc 5mC, Complex I, III, IV, V, Citrate } \\
\text { Synthase }\end{array}$ & 0.575 & 0.15 & 0.296859 & $\mathbf{0}$ \\
\hline TRIAL 3 & Methyl, Complex III, V & 0.516667 & 0.1 & 0.229129 & $\mathbf{0}$ \\
\hline TRIAL 4 & Nuc 5mC, Complex III, V & 0.516667 & 0.1 & 0.229129 & $\mathbf{0}$ \\
\hline TRIAL 5 & Methyl, Nuc 5mC, Citrate Synthase & 0.425 & 0.23 & 0.336341 & $\mathbf{0}$ \\
\hline \multirow[t]{3}{*}{ TRIAL 6} & $\begin{array}{c}\text { Methyl, Nuc 5mC, Complex III, Citrate } \\
\text { Synthase }\end{array}$ & 0.491667 & 0.3 & 0.215542 & $\mathbf{0}$ \\
\hline & \multicolumn{5}{|c|}{ NB Multiple } \\
\hline & $\begin{array}{c}\text { Features Implemented in Training and } \\
\text { Testing by Trial }\end{array}$ & $\begin{array}{l}\text { Training } \\
\text { (Average } \\
\text { Accuracy) } \\
\end{array}$ & $\begin{array}{c}\text { Testing } \\
\text { (F1 Score) }\end{array}$ & $\begin{array}{l}\text { Train St.D } \\
\text { Average }\end{array}$ & Test St. D \\
\hline TRIAL 1 & Mito $5 \mathrm{hmC}$,Nuc $5 \mathrm{mC}$, Complex I, III, V & 0.4 & 0.15 & 0.3 & $\mathbf{0}$ \\
\hline TRIAL 2 & $\begin{array}{c}\text { Nuc 5mC, Complex I, III, IV, V, Citrate } \\
\text { Synthase }\end{array}$ & 0.45 & 0.22 & 0.269258 & $\mathbf{0}$ \\
\hline TRIAL 3 & Methyl, Complex III, V & 0.6 & 0.1 & 0.254951 & $\mathbf{0}$ \\
\hline TRIAL 4 & Nuc 5mC, Complex III, V & 0.475 & 0.15 & 0.283945 & $\mathbf{0}$ \\
\hline TRIAL 5 & Methyl, Nuc 5mC, Citrate Synthase & 0.491667 & 0.48 & 0.267317 & $\mathbf{0}$ \\
\hline \multirow[t]{3}{*}{ TRIAL 6} & $\begin{array}{c}\text { Methyl, Nuc 5mC, Complex III, Citrate } \\
\text { Synthase }\end{array}$ & 0.475 & 0.22 & 0.378319 & $\mathbf{0}$ \\
\hline & \multicolumn{5}{|c|}{ SVM Multiple } \\
\hline & $\begin{array}{c}\text { Features Implemented in Training and } \\
\text { Testing by Trial }\end{array}$ & $\begin{array}{l}\text { Training } \\
\text { (Average } \\
\text { Accuracy) }\end{array}$ & $\begin{array}{c}\text { Testing } \\
\text { (F1 Score) }\end{array}$ & $\begin{array}{l}\text { Train St.D } \\
\text { Average }\end{array}$ & Test St. D \\
\hline TRIAL 1 & Mito $5 \mathrm{hmC}$,Nuc $5 \mathrm{mC}$, Complex I, III, V & 0.366667 & 0.14 & 0.217945 & $\mathbf{0}$ \\
\hline TRIAL 2 & $\begin{array}{c}\text { Nuc 5mC, Complex I, III, IV, V, Citrate } \\
\text { Synthase }\end{array}$ & 0.416667 & 0.14 & 0.241523 & $\mathbf{0}$ \\
\hline TRIAL 3 & Methyl, Complex III, V & 0.366667 & 0.14 & 0.269258 & $\mathbf{0}$ \\
\hline TRIAL 4 & Nuc 5mC, Complex III, V & 0.366667 & 0.14 & 0.269258 & $\mathbf{0}$ \\
\hline TRIAL 5 & Methyl, Nuc 5mC, Citrate Synthase & 0.5 & 0.23 & 0.316228 & $\mathbf{0}$ \\
\hline TRIAL 6 & $\begin{array}{c}\text { Methyl, Nuc 5mC, Complex III, Citrate } \\
\text { Synthase }\end{array}$ & 0.466667 & 0.48 & 0.298608 & $\mathbf{0}$ \\
\hline
\end{tabular}


Table S3.4: Machine learning analyses for physiological/biochemical features using multi classification. CART $=$ Classification and Regression Trees, $\mathrm{LR}=$ Logistic Regression, $\mathrm{LDA}=$ Linear Discriminant Analysis, KNN = K-Nearest Neighbors, NB = Naïve Bayes, SVC $=$ Support Vector Machines. Nuc $=$ Nuclear, Mito $=$ Mitochondrial, $5 \mathrm{mC}=5$-methylcytosine, $5 \mathrm{hmC}=5$ hydroxymethylcytosine, Methyl $=$ S-adenosyl methionine methyltransferase activity, St.D. $=$ standard deviation, binary $=$ non-diabetic and type 2 diabetic, multi $=$ non-diabetic, prediabetic, and type 2 diabetic. 
Table S3.5

\begin{tabular}{|c|c|c|c|c|c|}
\hline \multicolumn{6}{|c|}{$\mathrm{SEED}=16$} \\
\hline & \multicolumn{5}{|c|}{ CART Binary } \\
\hline & $\begin{array}{c}\text { Features Implemented in Training and } \\
\text { Testing by Trial }\end{array}$ & $\begin{array}{r}\text { Training } \\
\text { (Average } \\
\text { Accuracy) }\end{array}$ & $\begin{array}{c}\text { Testing } \\
\text { (F1 Score) }\end{array}$ & $\begin{array}{l}\text { Train St.D } \\
\text { Average }\end{array}$ & Test St. D \\
\hline TRIAL 1 & $\begin{array}{c}72,310,3010,4769,8697,11419,11812 \\
14233,15904,16362\end{array}$ & 0.92 & 0.70 & 0.17 & $\mathbf{0}$ \\
\hline TRIAL 2 & $310,3010,4769,11419,16362$ & 0.92 & 0.58 & 0.17 & $\mathbf{0}$ \\
\hline TRIAL 3 & $310,4769,16362$ & 0.77 & 0.58 & 0.18 & $\mathbf{0}$ \\
\hline \multirow[t]{3}{*}{ TRIAL 4} & $72,310,16362$ & 0.72 & 0.79 & 0.31 & $\mathbf{0}$ \\
\hline & \multicolumn{5}{|c|}{ LR Binary } \\
\hline & $\begin{array}{c}\text { Features Implemented in Training and } \\
\text { Testing by Trial }\end{array}$ & $\begin{array}{l}\text { Training } \\
\text { (Average } \\
\text { Accuracy) }\end{array}$ & $\begin{array}{c}\text { Testing } \\
\text { (F1 Score) }\end{array}$ & $\begin{array}{l}\text { Train St.D } \\
\text { Average }\end{array}$ & Test St. D \\
\hline TRIAL 1 & $\begin{array}{c}72,310,3010,4769,8697,11419,11812 \\
14233,15904,16362 \\
\end{array}$ & 0.7833 & 0.67 & 0.2506 & 0 \\
\hline TRIAL 2 & $310,3010,4769,11419,16362$ & 0.7833 & 0.67 & 0.2506 & $\mathbf{0}$ \\
\hline TRIAL 3 & $310,4769,16362$ & 0.7833 & 0.67 & 0.2506 & $\mathbf{0}$ \\
\hline \multirow[t]{3}{*}{ TRIAL 4} & $72,310,16362$ & 0.7833 & 0.67 & 0.2506 & $\mathbf{0}$ \\
\hline & \multicolumn{5}{|c|}{ LDA Binary } \\
\hline & $\begin{array}{c}\text { Features Implemented in Training and } \\
\text { Testing by Trial }\end{array}$ & $\begin{array}{r}\text { Training } \\
\text { (Average } \\
\text { Accuracy) }\end{array}$ & $\begin{array}{c}\text { Testing } \\
\text { (F1 Score) }\end{array}$ & $\begin{array}{l}\text { Train St.D } \\
\text { Average }\end{array}$ & Test St. D \\
\hline TRIAL 1 & $\begin{array}{c}72,310,3010,4769,8697,11419,11812 \\
14233,15904,16362 \\
\end{array}$ & 0.8083 & 0.58 & 0.2983 & 0 \\
\hline TRIAL 2 & $310,3010,4769,11419,16362$ & $\mathbf{0 . 8 9 1 7}$ & 0.58 & 0.175 & $\mathbf{0}$ \\
\hline TRIAL 3 & $310,4769,16362$ & 0.7083 & 0.67 & 0.2083 & $\mathbf{0}$ \\
\hline \multirow[t]{3}{*}{ TRIAL 4} & $72,310,16362$ & 0.75 & 0.67 & 0.2838 & $\mathbf{0}$ \\
\hline & \multicolumn{5}{|c|}{ KNN Binary } \\
\hline & $\begin{array}{c}\text { Features Implemented in Training and } \\
\text { Testing by Trial }\end{array}$ & $\begin{array}{l}\text { Training } \\
\text { (Average } \\
\text { Accuracy) }\end{array}$ & $\begin{array}{c}\text { Testing } \\
\text { (F1 Score) }\end{array}$ & $\begin{array}{l}\text { Train St.D } \\
\text { Average }\end{array}$ & Test St. D \\
\hline TRIAL 1 & $\begin{array}{c}72,310,3010,4769,8697,11419,11812 \\
14233,15904,16362\end{array}$ & 0.8917 & 0.45 & 0.175 & $\mathbf{0}$ \\
\hline TRIAL 2 & $310,3010,4769,11419,16362$ & 0.8917 & 0.45 & 0.175 & $\mathbf{0}$ \\
\hline TRIAL 3 & $310,4769,16362$ & 0.7583 & 0.52 & 0.2399 & $\mathbf{0}$ \\
\hline \multirow[t]{2}{*}{ TRIAL 4} & $72,310,16362$ & 0.7333 & 0.33 & 0.2261 & $\mathbf{0}$ \\
\hline & \multicolumn{5}{|c|}{ NB Binary } \\
\hline
\end{tabular}




\begin{tabular}{|c|c|c|c|c|c|}
\hline & $\begin{array}{c}\text { Features Implemented in Training and } \\
\text { Testing by Trial }\end{array}$ & $\begin{array}{l}\text { Training } \\
\text { (Average } \\
\text { Accuracy) }\end{array}$ & $\begin{array}{c}\text { Testing } \\
\text { (F1 Score) }\end{array}$ & $\begin{array}{l}\text { Train St.D } \\
\text { Average }\end{array}$ & Test St. D \\
\hline TRIAL 1 & $\begin{array}{c}72,310,3010,4769,8697,11419,11812 \\
14233,15904,16362\end{array}$ & 0.775 & 0.67 & 0.2473 & $\mathbf{0}$ \\
\hline TRIAL 2 & $310,3010,4769,11419,16362$ & 0.7833 & 0.67 & 0.2506 & $\mathbf{0}$ \\
\hline TRIAL 3 & $310,4769,16362$ & 0.7833 & 0.67 & 0.2506 & $\mathbf{0}$ \\
\hline \multirow[t]{3}{*}{ TRIAL 4} & $72,310,16362$ & 0.7833 & 0.67 & 0.2506 & $\mathbf{0}$ \\
\hline & \multicolumn{5}{|c|}{ SVM Binary } \\
\hline & $\begin{array}{c}\text { Features Implemented in Training and } \\
\text { Testing by Trial }\end{array}$ & $\begin{array}{l}\text { Training } \\
\text { (Average } \\
\text { Accuracy) }\end{array}$ & $\begin{array}{c}\text { Testing } \\
\text { (F1 Score) }\end{array}$ & $\begin{array}{c}\text { Train St.D } \\
\text { Average }\end{array}$ & Test St. D \\
\hline TRIAL 1 & $\begin{array}{c}72,310,3010,4769,8697,11419,11812 \\
14233,15904,16362\end{array}$ & 0.6 & 0.33 & 0.2409 & $\mathbf{0}$ \\
\hline TRIAL 2 & $310,3010,4769,11419,16362$ & 0.7833 & 0.67 & 0.2506 & $\mathbf{0}$ \\
\hline TRIAL 3 & $310,4769,16362$ & 0.7833 & 0.67 & 0.2506 & $\mathbf{0}$ \\
\hline TRIAL 4 & $72,310,16362$ & 0.7833 & 0.67 & 0.2506 & $\mathbf{0}$ \\
\hline
\end{tabular}


Table S3.5: Machine learning analyses for mitochondrial SNP features using binary classification. CART $=$ Classification and Regression Trees, LR $=$ Logistic Regression, LDA $=$ Linear Discriminant Analysis, KNN = K-Nearest Neighbors, NB = Naïve Bayes, $\mathrm{SVC}=$ Support Vector Machines. St.D. $=$ standard deviation, binary $=$ non-diabetic and type 2 diabetic, multi $=$ nondiabetic, prediabetic, and type 2 diabetic. 
Table S3.6

\begin{tabular}{|c|c|c|c|c|c|}
\hline \multicolumn{6}{|c|}{$\mathrm{SEED}=87$} \\
\hline & \multicolumn{5}{|c|}{ CART Multiple } \\
\hline & $\begin{array}{c}\text { Features Implemented in Training and } \\
\text { Testing by Trial }\end{array}$ & $\begin{array}{l}\text { Training } \\
\text { (Average } \\
\text { Accuracy) }\end{array}$ & $\begin{array}{c}\text { Testing } \\
\text { (F1 Score) }\end{array}$ & $\begin{array}{c}\text { Train St.D } \\
\text { Average }\end{array}$ & Test St. D \\
\hline TRIAL 1 & $\begin{array}{c}114,143,151,195,310,3010,4295,4769, \\
7028,8860,9055,9840,10398,11812, \\
12246,13401,14766,15191,15223,16124, \\
16183,16189,16270,16336,16344,16362, \\
16390,16519\end{array}$ & 0.67 & 0.576 & 0.23 & 0.009 \\
\hline TRIAL 2 & $4295,7028,10398,16362,16390$ & 0.808 & 0.5 & 0.171 & $\mathbf{0}$ \\
\hline TRIAL 3 & $7028,10398,16362$ & 0.758 & 0.5 & 0.181 & $\mathbf{0}$ \\
\hline \multirow[t]{3}{*}{ TRIAL 4} & $\begin{array}{c}114,143,151,195,310,16124,16183 \\
16189,16270,16336,16344,16362,16390 \\
16519\end{array}$ & 0.33 & 0.12 & 0.309 & $\mathbf{0}$ \\
\hline & \multicolumn{5}{|c|}{ LR Multiple } \\
\hline & $\begin{array}{c}\text { Features Implemented in Training and } \\
\text { Testing by Trial }\end{array}$ & $\begin{array}{l}\text { Training } \\
\text { (Average } \\
\text { Accuracy) }\end{array}$ & $\begin{array}{c}\text { Testing } \\
\text { (F1 Score) }\end{array}$ & $\begin{array}{l}\text { Train St.D } \\
\text { Average }\end{array}$ & Test St. D \\
\hline TRIAL 1 & $\begin{array}{c}114,143,151,195,310,3010,4295,4769, \\
7028,8860,9055,9840,10398,11812, \\
12246,13401,14766,15191,15223,16124, \\
16183,16189,16270,16336,16344,16362, \\
16390,16519\end{array}$ & 0.6667 & 0.38 & 0.2789 & $\mathbf{0}$ \\
\hline TRIAL 2 & $4295,7028,10398,16362,16390$ & 0.6167 & 0.5 & 0.2333 & $\mathbf{0}$ \\
\hline TRIAL 3 & $7028,10398,16362$ & 0.6417 & 0.5 & 0.2329 & $\mathbf{0}$ \\
\hline \multirow[t]{3}{*}{ TRIAL 4} & $\begin{array}{c}114,143,151,195,310,16124,16183 \\
16189,16270,16336,16344,16362,16390 \\
16519\end{array}$ & 0.3917 & 0.15 & 0.2238 & $\mathbf{0}$ \\
\hline & \multicolumn{5}{|c|}{ LDA Multiple } \\
\hline & $\begin{array}{c}\text { Features Implemented in Training and } \\
\text { Testing by Trial }\end{array}$ & $\begin{array}{l}\text { Training } \\
\text { (Average } \\
\text { Accuracy) }\end{array}$ & $\begin{array}{c}\text { Testing } \\
\text { (F1 Score) }\end{array}$ & $\begin{array}{c}\text { Train St.D } \\
\text { Average }\end{array}$ & Test St. D \\
\hline TRIAL 1 & $\begin{array}{c}114,143,151,195,310,3010,4295,4769, \\
7028,8860,9055,9840,10398,11812, \\
12246,13401,14766,15191,15223,16124, \\
16183,16189,16270,16336,16344,16362, \\
16390,16519\end{array}$ & 0.575 & 0.58 & 0.2621 & $\mathbf{0}$ \\
\hline TRIAL 2 & $4295,7028,10398,16362,16390$ & 0.7833 & 0.5 & 0.159 & $\mathbf{0}$ \\
\hline TRIAL 3 & $7028,10398,16362$ & 0.7583 & 0.5 & 0.1805 & $\mathbf{0}$ \\
\hline TRIAL 4 & $\begin{array}{c}114,143,151,195,310,16124,16183 \\
16189,16270,16336,16344,16362,16390 \\
16519\end{array}$ & 0.3833 & 0.15 & 0.2533 & $\mathbf{0}$ \\
\hline & \multicolumn{5}{|c|}{ KNN Multiple } \\
\hline
\end{tabular}




\begin{tabular}{|c|c|c|c|c|c|}
\hline & $\begin{array}{c}\text { Features Implemented in Training and } \\
\text { Testing by Trial }\end{array}$ & $\begin{array}{l}\text { Training } \\
\text { (Average } \\
\text { Accuracy) }\end{array}$ & $\begin{array}{c}\text { Testing } \\
\text { (F1 Score) }\end{array}$ & $\begin{array}{l}\text { Train St.D } \\
\text { Average }\end{array}$ & Test St. D \\
\hline TRIAL 1 & $\begin{array}{c}114,143,151,195,310,3010,4295,4769, \\
7028,8860,9055,9840,10398,11812, \\
12246,13401,14766,15191,15223,16124, \\
16183,16189,16270,16336,16344,16362, \\
16390,16519\end{array}$ & 0.5417 & 0.25 & 0.2451 & $\mathbf{0}$ \\
\hline TRIAL 2 & $4295,7028,10398,16362,16390$ & 0.7583 & 0.5 & 0.1805 & $\mathbf{0}$ \\
\hline TRIAL 3 & $7028,10398,16362$ & 0.7583 & 0.5 & 0.1805 & $\mathbf{0}$ \\
\hline \multirow[t]{3}{*}{ TRIAL 4} & $\begin{array}{c}114,143,151,195,310,16124,16183 \\
16189,16270,16336,16344,16362,16390 \\
16519\end{array}$ & 0.35 & 0.14 & 0.2784 & $\mathbf{0}$ \\
\hline & \multicolumn{5}{|c|}{ NB Multiple } \\
\hline & $\begin{array}{c}\text { Features Implemented in Training and } \\
\text { Testing by Trial }\end{array}$ & $\begin{array}{l}\text { Training } \\
\text { (Average } \\
\text { Accuracy) }\end{array}$ & $\begin{array}{c}\text { Testing } \\
\text { (F1 Score) }\end{array}$ & $\begin{array}{c}\text { Train St.D } \\
\text { Average }\end{array}$ & Test St. D \\
\hline TRIAL 1 & $\begin{array}{c}114,143,151,195,310,3010,4295,4769, \\
7028,8860,9055,9840,10398,11812, \\
12246,13401,14766,15191,15223,16124, \\
16183,16189,16270,16336,16344,16362, \\
16390,16519\end{array}$ & 0.45 & 0.35 & 0.2303 & $\mathbf{0}$ \\
\hline TRIAL 2 & $4295,7028,10398,16362,16390$ & 0.6167 & 0.68 & 0.2048 & $\mathbf{0}$ \\
\hline TRIAL 3 & $7028,10398,16362$ & 0.5417 & 0.68 & 0.1873 & $\mathbf{0}$ \\
\hline \multirow[t]{3}{*}{ TRIAL 4} & $\begin{array}{c}114,143,151,195,310,16124,16183 \\
16189,16270,16336,16344,16362,16390 \\
16519\end{array}$ & 0.3917 & 0.46 & 0.3119 & $\mathbf{0}$ \\
\hline & \multicolumn{5}{|c|}{ SVM Multiple } \\
\hline & $\begin{array}{c}\text { Features Implemented in Training and } \\
\text { Testing by Trial }\end{array}$ & $\begin{array}{l}\text { Training } \\
\text { (Average } \\
\text { Accuracy) }\end{array}$ & $\begin{array}{c}\text { Testing } \\
\text { (F1 Score) }\end{array}$ & $\begin{array}{l}\text { Train St.D } \\
\text { Average }\end{array}$ & Test St. D \\
\hline TRIAL 1 & $\begin{array}{c}114,143,151,195,310,3010,4295,4769, \\
7028,8860,9055,9840,10398,11812, \\
12246,13401,14766,15191,15223,16124, \\
16183,16189,16270,16336,16344,16362, \\
16390,16519\end{array}$ & 0.45 & 0.14 & 0.2014 & $\mathbf{0}$ \\
\hline TRIAL 2 & $4295,7028,10398,16362,16390$ & 0.4917 & 0.14 & 0.1557 & $\mathbf{0}$ \\
\hline TRIAL 3 & $7028,10398,16362$ & 0.5417 & 0.3 & 0.1502 & $\mathbf{0}$ \\
\hline TRIAL 4 & $\begin{array}{c}114,143,151,195,310,16124,16183 \\
16189,16270,16336,16344,16362,16390 \\
16519\end{array}$ & 0.45 & 0.14 & 0.2014 & $\mathbf{0}$ \\
\hline
\end{tabular}


Table S3.6: Machine learning analyses for mitochondrial SNP features using multi classification. CART $=$ Classification and Regression Trees, $\mathrm{LR}=$ Logistic Regression, LDA $=$ Linear Discriminant Analysis, KNN = K-Nearest Neighbors, NB = Naïve Bayes, $\mathrm{SVC}=$ Support Vector Machines. St.D. $=$ standard deviation, binary $=$ non-diabetic and type 2 diabetic, multi $=$ nondiabetic, prediabetic, and type 2 diabetic. 
Table S3.7

\begin{tabular}{|c|c|c|c|c|c|}
\hline \multicolumn{6}{|c|}{$\mathrm{SEED}=8$} \\
\hline & \multicolumn{5}{|c|}{ CART Binary } \\
\hline & $\begin{array}{c}\text { Features Implemented in Training and } \\
\text { Testing by Trial }\end{array}$ & $\begin{array}{l}\text { Training } \\
\text { (Average } \\
\text { Accuracy) }\end{array}$ & $\begin{array}{c}\text { Testing } \\
\text { (F1 Score) }\end{array}$ & $\begin{array}{c}\text { Train St.D } \\
\text { Average }\end{array}$ & Test St. D \\
\hline TRIAL 1 & $\begin{array}{c}\text { CpG 24, CpG 26, CpG 28, CpG 29, CpG } \\
39\end{array}$ & 0.86336 & 0.742 & 0.17244 & 0.06572671 \\
\hline TRIAL 2 & CpG 24, CpG 28, CpG 29, CpG 39 & 0.88336 & 0.718 & 0.15724 & 0.06572671 \\
\hline TRIAL 3 & CpG 24, CpG 26, CpG 28, CpG 29 & 0.89002 & 0.79 & 0.12708 & $\mathbf{0}$ \\
\hline TRIAL 4 & CpG 24, CpG 28, CpG 29 & 0.925 & 0.79 & 0.1146 & $\mathbf{0}$ \\
\hline \multirow[t]{3}{*}{ TRIAL 5} & CpG 24, CpG 29 & 0.9 & 0.67 & 0.1225 & $\mathbf{0}$ \\
\hline & \multicolumn{5}{|c|}{ LR Binary } \\
\hline & $\begin{array}{c}\text { Features Implemented in Training and } \\
\text { Testing by Trial }\end{array}$ & $\begin{array}{l}\text { Training } \\
\text { (Average } \\
\text { Accuracy) }\end{array}$ & $\begin{array}{c}\text { Testing } \\
\text { (F1 Score) }\end{array}$ & $\begin{array}{c}\text { Train St.D } \\
\text { Average }\end{array}$ & Test St. D \\
\hline TRIAL 1 & $\begin{array}{c}\mathrm{CpG} 24, \mathrm{CpG} 26, \mathrm{CpG} 28, \mathrm{CpG} 29, \mathrm{CpG} \\
39\end{array}$ & 0.733333 & 0.49 & 0.189297 & $\mathbf{0}$ \\
\hline TRIAL 2 & CpG 24, CpG 28, CpG 29, CpG 39 & 0.791667 & 0.6 & 0.190941 & $\mathbf{0}$ \\
\hline TRIAL 3 & CpG 24, CpG 26, CpG 28, CpG 29 & 0.775 & 0.7 & 0.190212 & $\mathbf{0}$ \\
\hline TRIAL 4 & CpG 24, CpG 28, CpG 29 & 0.833333 & 0.58 & 0.139443 & $\mathbf{0}$ \\
\hline \multirow[t]{3}{*}{ TRIAL 5} & CpG 24, CpG 29 & 0.766667 & 0.8 & 0.238048 & $\mathbf{0}$ \\
\hline & \multicolumn{5}{|c|}{ LDA Binary } \\
\hline & $\begin{array}{c}\text { Features Implemented in Training and } \\
\text { Testing by Trial }\end{array}$ & $\begin{array}{l}\text { Training } \\
\text { (Average } \\
\text { Accuracy) }\end{array}$ & $\begin{array}{c}\text { Testing } \\
\text { (F1 Score) }\end{array}$ & $\begin{array}{c}\text { Train St.D } \\
\text { Average }\end{array}$ & Test St. D \\
\hline TRIAL 1 & $\begin{array}{c}\mathrm{CpG} 24, \mathrm{CpG} 26, \mathrm{CpG} 28, \mathrm{CpG} 29, \mathrm{CpG} \\
39\end{array}$ & 0.758333 & 0.58 & 0.141667 & $\mathbf{0}$ \\
\hline TRIAL 2 & CpG 24, CpG 28, CpG 29, CpG 39 & 0.808333 & 0.58 & 0.129368 & $\mathbf{0}$ \\
\hline TRIAL 3 & CpG 24, CpG 26, CpG 28, CpG 29 & 0.758333 & 0.58 & 0.087003 & $\mathbf{0}$ \\
\hline TRIAL 4 & CpG 24, CpG 28, CpG 29 & 0.808333 & 0.58 & 0.129368 & $\mathbf{0}$ \\
\hline \multirow[t]{3}{*}{ TRIAL 5} & CpG 24, CpG 29 & 0.733333 & 0.58 & 0.226078 & $\mathbf{0}$ \\
\hline & \multicolumn{5}{|c|}{ KNN Binary } \\
\hline & $\begin{array}{c}\text { Features Implemented in Training and } \\
\text { Testing by Trial }\end{array}$ & $\begin{array}{l}\text { Training } \\
\text { (Average } \\
\text { Accuracy) }\end{array}$ & $\begin{array}{l}\text { Testing } \\
\text { (F1 Score) }\end{array}$ & $\begin{array}{c}\text { Train St.D } \\
\text { Average }\end{array}$ & Test St. D \\
\hline TRIAL 1 & $\begin{array}{c}\mathrm{CpG} 24, \mathrm{CpG} 26, \mathrm{CpG} 28, \mathrm{CpG} 29, \mathrm{CpG} \\
39\end{array}$ & 0.625 & 0.45 & 0.214897 & $\mathbf{0}$ \\
\hline TRIAL 2 & CpG 24, CpG 28, CpG 29, CpG 39 & 0.65 & 0.49 & 0.181812 & $\mathbf{0}$ \\
\hline TRIAL 3 & CpG 24, CpG 26, CpG 28, CpG 29 & 0.683333 & 0.45 & 0.177951 & $\mathbf{0}$ \\
\hline TRIAL 4 & CpG 24, CpG 28, CpG 29 & 0.783333 & 0.7 & 0.113039 & $\mathbf{0}$ \\
\hline
\end{tabular}




\begin{tabular}{|c|c|c|c|c|c|}
\hline TRIAL 5 & CpG 24, CpG 29 & 0.858333 & 0.9 & 0.182764 & $\mathbf{0}$ \\
\hline & \multicolumn{5}{|c|}{ NB Binary } \\
\hline & $\begin{array}{c}\text { Features Implemented in Training and } \\
\text { Testing by Trial }\end{array}$ & $\begin{array}{l}\text { Training } \\
\text { (Average } \\
\text { Accuracy) }\end{array}$ & $\begin{array}{c}\text { Testing } \\
\text { (F1 Score) }\end{array}$ & $\begin{array}{l}\text { Train St.D } \\
\text { Average }\end{array}$ & Test St. D \\
\hline TRIAL 1 & $\begin{array}{c}\mathrm{CpG} 24, \mathrm{CpG} 26, \mathrm{CpG} 28, \mathrm{CpG} 29, \mathrm{CpG} \\
39\end{array}$ & 0.7 & 0.52 & 0.201384 & $\mathbf{0}$ \\
\hline TRIAL 2 & CpG 24, CpG 28, CpG 29, CpG 39 & 0.7 & 0.52 & 0.201384 & $\mathbf{0}$ \\
\hline TRIAL 3 & CpG 24, CpG 26, CpG 28, CpG 29 & $\mathbf{0 . 7 5 8 3 3 3}$ & 0.52 & 0.234076 & $\mathbf{0}$ \\
\hline TRIAL 4 & CpG 24, CpG 28, CpG 29 & $\mathbf{0 . 7 8 3 3 3 3}$ & 0.52 & 0.194365 & $\mathbf{0}$ \\
\hline \multirow[t]{3}{*}{ TRIAL 5} & CpG 24, CpG 29 & $\mathbf{0 . 7 8 3 3 3 3}$ & 0.52 & 0.224227 & $\mathbf{0}$ \\
\hline & \multicolumn{5}{|c|}{ SVM Binary } \\
\hline & $\begin{array}{c}\text { Features Implemented in Training and } \\
\text { Testing by Trial }\end{array}$ & $\begin{array}{l}\text { Training } \\
\text { (Average } \\
\text { Accuracy) }\end{array}$ & $\begin{array}{c}\text { Testing } \\
\text { (F1 Score) }\end{array}$ & $\begin{array}{l}\text { Train St.D } \\
\text { Average }\end{array}$ & Test St. D \\
\hline TRIAL 1 & $\begin{array}{c}\text { CpG 24, CpG 26, CpG 28, CpG 29, CpG } \\
39\end{array}$ & 0.625 & 0.7 & 0.289276 & $\mathbf{0}$ \\
\hline TRIAL 2 & CpG 24, CpG 28, CpG 29, CpG 39 & 0.716667 & 0.6 & 0.236291 & $\mathbf{0}$ \\
\hline TRIAL 3 & CpG 24, CpG 26, CpG 28, CpG 29 & $\mathbf{0 . 7 8 3 3 3 3}$ & $\mathbf{0 . 5 8}$ & 0.217945 & $\mathbf{0}$ \\
\hline TRIAL 4 & CpG 24, CpG 28, CpG 29 & $\mathbf{0 . 7 8 3 3 3 3}$ & $\mathbf{0 . 5 8}$ & 0.15899 & $\mathbf{0}$ \\
\hline TRIAL 5 & CpG 24, CpG 29 & 0.783333 & 0.67 & 0.194365 & $\mathbf{0}$ \\
\hline
\end{tabular}


Table S3.7: Machine learning analyses for Transcription Factor A, Mitochondrial (TFAM) CpG island methylation features using binary classification. CART $=$ Classification and Regression Trees, $\mathrm{LR}=$ Logistic Regression, LDA $=$ Linear Discriminant Analysis, $\mathrm{KNN}=\mathrm{K}-\mathrm{Nearest}$ Neighbors, NB $=$ Naïve Bayes, $\mathrm{SVC}=$ Support Vector Machines. $\mathrm{CpG}=$ cytosine nucleotide followed by a guanine nucleotide, St.D. $=$ standard deviation, binary $=$ non-diabetic and type 2 diabetic, multi $=$ non-diabetic, prediabetic, and type 2 diabetic. 
Table S3.8

\begin{tabular}{|c|c|c|c|c|c|}
\hline \multicolumn{6}{|c|}{$\mathrm{SEED}=22$} \\
\hline & \multicolumn{5}{|c|}{ CART Multiple } \\
\hline & $\begin{array}{c}\text { Features Implemented in Training and } \\
\text { Testing by Trial }\end{array}$ & $\begin{array}{l}\text { Training } \\
\text { (Average } \\
\text { Accuracy) }\end{array}$ & $\begin{array}{c}\text { Testing } \\
\text { (F1 Score) }\end{array}$ & $\begin{array}{l}\text { Train St.D } \\
\text { Average }\end{array}$ & Test St. D \\
\hline TRIAL 1 & $\begin{array}{c}\text { CpG 1, CpG 5, CpG 14, CpG 20, CpG 23, } \\
\text { CpG 24, CpG 33, TFAM CpG }\end{array}$ & 0.71836 & 0.668 & 0.19942 & 0.05761944 \\
\hline TRIAL 2 & $\begin{array}{c}\text { CpG 1, CpG 14, CpG 20, CpG 23, CpG } \\
24, \text { CpG 33, TFAM CpG }\end{array}$ & 0.69832 & 0.666 & 0.20908 & 0.06024948 \\
\hline TRIAL 3 & $\begin{array}{c}\text { CpG 1, CpG 20, } \\
\text { CpG 23, CpG 24, TFAM }\end{array}$ & 0.73168 & 0.6 & 0.19586 & $\mathbf{0}$ \\
\hline TRIAL 4 & $\begin{array}{c}\text { CpG 1, CpG 14, CpG 20, CpG 23, CpG } \\
24, \text { CpG 35, TFAM CpG }\end{array}$ & 0.76668 & 0.644 & 0.21556 & 0.06024948 \\
\hline \multirow[t]{3}{*}{ TRIAL 5} & $\begin{array}{c}\text { CpG 1, CpG 20, CpG 23, CpG 24, CpG } \\
\text { 35, TFAM CpG }\end{array}$ & 0.74834 & 0.644 & 0.19818 & 0.06024948 \\
\hline & \multicolumn{5}{|c|}{ LR Multiple } \\
\hline & $\begin{array}{c}\text { Features Implemented in Training and } \\
\text { Testing by Trial }\end{array}$ & $\begin{array}{l}\text { Training } \\
\text { (Average } \\
\text { Accuracy) }\end{array}$ & $\begin{array}{c}\text { Testing } \\
\text { (F1 Score) }\end{array}$ & $\begin{array}{l}\text { Train St.D } \\
\text { Average }\end{array}$ & Test St. D \\
\hline TRIAL 1 & $\begin{array}{c}\text { CpG 1, CpG 5, CpG 14, CpG 20, CpG 23, } \\
\text { CpG 24, CpG 33, TFAM CpG }\end{array}$ & 0.491667 & 0.15 & 0.242813 & $\mathbf{0}$ \\
\hline TRIAL 2 & $\begin{array}{c}\text { CpG 1, CpG 14, CpG 20, CpG 23, CpG } \\
24, \text { CpG 33, TFAM CpG }\end{array}$ & 0.491667 & 0.26 & 0.215542 & 0 \\
\hline TRIAL 3 & $\begin{array}{c}\text { CpG 1, CpG 20, CpG 23, CpG 24, TFAM } \\
\text { CpG }\end{array}$ & 0.475 & 0.28 & 0.190212 & $\mathbf{0}$ \\
\hline TRIAL 4 & $\begin{array}{c}\text { CpG 1, CpG 14, CpG 20, CpG 23, CpG } \\
\text { 24, CpG 35, TFAM CpG }\end{array}$ & 0.491667 & 0.26 & 0.242813 & $\mathbf{0}$ \\
\hline \multirow[t]{3}{*}{ TRIAL 5} & $\begin{array}{c}\text { CpG 1, CpG 20, CpG 23, CpG 24, CpG } \\
\text { 35, TFAM CpG }\end{array}$ & 0.466667 & 0.27 & 0.253311 & $\mathbf{0}$ \\
\hline & \multicolumn{5}{|c|}{ LDA Multiple } \\
\hline & $\begin{array}{c}\text { Features Implemented in Training and } \\
\text { Testing by Trial }\end{array}$ & $\begin{array}{l}\text { Training } \\
\text { (Average } \\
\text { Accuracy) }\end{array}$ & $\begin{array}{c}\text { Testing } \\
\text { (F1 Score) }\end{array}$ & $\begin{array}{c}\text { Train St.D } \\
\text { Average }\end{array}$ & Test St. D \\
\hline TRIAL 1 & $\begin{array}{c}\text { CpG 1, CpG 5, CpG 14, CpG 20, CpG 23, } \\
\text { CpG 24, CpG 33, TFAM CpG }\end{array}$ & 0.466667 & 0.22 & 0.227303 & $\mathbf{0}$ \\
\hline TRIAL 2 & $\begin{array}{c}\text { CpG 1, CpG 14, CpG 20, CpG 23, CpG } \\
24, \text { CpG 33, TFAM CpG }\end{array}$ & 0.466667 & 0.28 & 0.227303 & $\mathbf{0}$ \\
\hline TRIAL 3 & $\begin{array}{c}\text { CpG 1, CpG 20, CpG 23, CpG 24, TFAM } \\
\text { CpG }\end{array}$ & 0.475 & 0.28 & 0.190212 & $\mathbf{0}$ \\
\hline TRIAL 4 & $\begin{array}{c}\text { CpG 1, CpG 14, CpG 20, CpG 23, CpG } \\
\text { 24, CpG 35, TFAM CpG }\end{array}$ & 0.441667 & 0.28 & 0.283945 & $\mathbf{0}$ \\
\hline \multirow[t]{3}{*}{ TRIAL 5} & $\begin{array}{c}\text { CpG 1, CpG 20, CpG 23, CpG 24, CpG } \\
\text { 35, TFAM CpG }\end{array}$ & 0.441667 & 0.28 & 0.283945 & $\mathbf{0}$ \\
\hline & \multicolumn{5}{|c|}{ KNN Multiple } \\
\hline & $\begin{array}{c}\text { Features Implemented in Training and } \\
\text { Testing by Trial }\end{array}$ & $\begin{array}{l}\text { Training } \\
\text { (Average } \\
\text { Accuracy) }\end{array}$ & $\begin{array}{c}\text { Testing } \\
\text { (F1 Score) }\end{array}$ & $\begin{array}{l}\text { Train St.D } \\
\text { Average }\end{array}$ & Test St. D \\
\hline
\end{tabular}




\begin{tabular}{|c|c|c|c|c|c|}
\hline TRIAL 1 & $\begin{array}{c}\text { CpG 1, CpG 5, CpG 14, CpG 20, CpG 23, } \\
\text { CpG 24, CpG 33, TFAM CpG }\end{array}$ & 0.5 & 0.24 & 0.235702 & $\mathbf{0}$ \\
\hline TRIAL 2 & $\begin{array}{c}\text { CpG 1, CpG 14, CpG 20, CpG 23, CpG } \\
24, \text { CpG 33, TFAM CpG }\end{array}$ & 0.475 & 0.31 & 0.247347 & $\mathbf{0}$ \\
\hline TRIAL 3 & $\begin{array}{c}\text { CpG 1, CpG 20, CpG 23, CpG 24, TFAM } \\
\text { CpG }\end{array}$ & 0.45 & 0.38 & 0.167498 & $\mathbf{0}$ \\
\hline TRIAL 4 & $\begin{array}{c}\text { CpG 1, CpG 14, CpG 20, CpG 23, CpG } \\
24, \text { CpG 35, TFAM CpG }\end{array}$ & 0.308333 & 0.38 & 0.186525 & $\mathbf{0}$ \\
\hline \multirow[t]{3}{*}{ TRIAL 5} & $\begin{array}{c}\text { CpG 1, CpG 20, CpG 23, CpG 24, CpG } \\
\text { 35, TFAM CpG }\end{array}$ & 0.416667 & 0.38 & 0.247207 & $\mathbf{0}$ \\
\hline & \multicolumn{5}{|c|}{ NB Multiple } \\
\hline & $\begin{array}{c}\text { Features Implemented in Training and } \\
\text { Testing by Trial }\end{array}$ & $\begin{array}{l}\text { Training } \\
\text { (Average } \\
\text { Accuracy) }\end{array}$ & $\begin{array}{c}\text { Testing } \\
\text { (F1 Score) }\end{array}$ & $\begin{array}{l}\text { Train St.D } \\
\text { Average }\end{array}$ & Test St. D \\
\hline TRIAL 1 & $\begin{array}{c}\text { CpG 1, CpG 5, CpG 14, CpG 20, CpG 23, } \\
\text { CpG 24, CpG 33, TFAM CpG }\end{array}$ & 0.45 & 0.13 & 0.124722 & $\mathbf{0}$ \\
\hline TRIAL 2 & $\begin{array}{c}\text { CpG 1, CpG 14, CpG 20, CpG 23, CpG } \\
\text { 24, CpG 33, TFAM CpG }\end{array}$ & 0.475 & 0.26 & 0.153885 & $\mathbf{0}$ \\
\hline TRIAL 3 & $\begin{array}{c}\text { CpG 1, CpG 20, CpG 23, CpG 24, TFAM } \\
\text { CpG }\end{array}$ & 0.55 & 0.11 & 0.256038 & $\mathbf{0}$ \\
\hline TRIAL 4 & $\begin{array}{c}\text { CpG 1, CpG 14, CpG 20, CpG 23, CpG } \\
\text { 24, CpG 35, TFAM CpG }\end{array}$ & 0.45 & 0.28 & 0.201384 & $\mathbf{0}$ \\
\hline \multirow[t]{3}{*}{ TRIAL 5} & $\begin{array}{c}\text { CpG 1, CpG 20, CpG 23, CpG 24, CpG } \\
\text { 35, TFAM CpG }\end{array}$ & 0.5 & 0.28 & 0.207498 & $\mathbf{0}$ \\
\hline & \multicolumn{5}{|c|}{ SVM Multiple } \\
\hline & $\begin{array}{c}\text { Features Implemented in Training and } \\
\text { Testing by Trial }\end{array}$ & $\begin{array}{l}\text { Training } \\
\text { (Average } \\
\text { Accuracy) }\end{array}$ & $\begin{array}{c}\text { Testing } \\
\text { (F1 Score) }\end{array}$ & $\begin{array}{c}\text { Train St.D } \\
\text { Average }\end{array}$ & Test St. D \\
\hline TRIAL 1 & $\begin{array}{c}\text { CpG 1, CpG 5, CpG 14, CpG 20, CpG 23, } \\
\text { CpG 24, CpG 33, TFAM CpG }\end{array}$ & 0.5 & 0.07 & 0.260875 & $\mathbf{0}$ \\
\hline TRIAL 2 & $\begin{array}{c}\text { CpG 1, CpG 14, CpG 20, CpG 23, CpG } \\
\text { 24, CpG 33, TFAM CpG }\end{array}$ & 0.483333 & 0.16 & 0.235112 & $\mathbf{0}$ \\
\hline TRIAL 3 & $\begin{array}{c}\mathrm{CpG} 1, \mathrm{CpG} 20, \mathrm{CpG} 23, \mathrm{CpG} 24, \mathrm{TFAM} \\
\mathrm{CpG}\end{array}$ & 0.425 & 0.17 & 0.237024 & $\mathbf{0}$ \\
\hline TRIAL 4 & $\begin{array}{c}\text { CpG 1, CpG 14, CpG 20, CpG 23, CpG } \\
\text { 24, CpG 35, TFAM CpG }\end{array}$ & 0.508333 & 0.07 & 0.248468 & $\mathbf{0}$ \\
\hline TRIAL 5 & $\begin{array}{c}\text { CpG 1, CpG 20, CpG 23, CpG 24, CpG } \\
\text { 35, TFAM CpG }\end{array}$ & 0.508333 & 0.17 & 0.272463 & $\mathbf{0}$ \\
\hline
\end{tabular}


Table S3.8: Machine learning analyses for Transcription Factor A, Mitochondrial (TFAM) CpG island methylation features using multi classification. CART $=$ Classification and Regression Trees, $\mathrm{LR}=$ Logistic Regression, LDA $=$ Linear Discriminant Analysis, $\mathrm{KNN}=\mathrm{K}-\mathrm{Nearest}$ Neighbors, NB $=$ Naïve Bayes, $\mathrm{SVC}=$ Support Vector Machines. $\mathrm{CpG}=$ cytosine nucleotide followed by a guanine nucleotide, TFAM $\mathrm{CpG}=$ total $\mathrm{CpG}$ methylation of the TFAM promoter region, St.D. $=$ standard deviation, binary $=$ non-diabetic and type 2 diabetic, multi $=$ non-diabetic, prediabetic, and type 2 diabetic. 
Table S3.9

\begin{tabular}{|c|c|c|c|c|c|}
\hline \multicolumn{6}{|c|}{$\mathrm{SEED}=15$} \\
\hline & \multicolumn{5}{|c|}{ CART Binary } \\
\hline & $\begin{array}{c}\text { Features Implemented in Training and } \\
\text { Testing by Trial }\end{array}$ & $\begin{array}{l}\text { Training } \\
\text { (Average } \\
\text { Accuracy) }\end{array}$ & $\begin{array}{c}\text { Testing } \\
\text { (F1 Score) }\end{array}$ & $\begin{array}{l}\text { Train St.D } \\
\text { Average }\end{array}$ & Test St. D \\
\hline TRIAL 1 & $\begin{array}{c}\text { Methyl, Mito 5hmC, Nuc 5hmC, Mito } \\
\text { 5mC, Nuc 5mC, CpG 11, CpG 23, CpG 24, } \\
\text { CpG 28 }\end{array}$ & 0.786666 & 0.758 & 0.2043988 & 0.0491935 \\
\hline TRIAL 2 & $\begin{array}{c}\text { Methyl, Mito 5hmC, Nuc 5hmC, Mito } \\
\text { 5mC, Nuc 5mC, CpG } 1 \text { - 24, TFAM CpG, } \\
\text { TFAM Non-CpG }\end{array}$ & 0.8083318 & 0.714 & 0.2168344 & 0.06024948 \\
\hline TRIAL 3 & $\begin{array}{l}\text { Methyl, Mito 5hmC, Nuc 5hmC, Mito } \\
\text { 5mC, Nuc 5mC, CpG 24, TFAM CpG, } \\
\text { TFAM Non-CpG }\end{array}$ & 0.831667 & 0.67 & 0.2166188 & $\mathbf{0}$ \\
\hline TRIAL 4 & Methyl, Nuc 5mC, CpG 1 - 24 & 0.7899998 & 0.736 & 0.209388 & 0.06024948 \\
\hline \multirow[t]{3}{*}{ TRIAL 5} & $\begin{array}{c}\text { Nuc 5mC, CpG 1,CpG 7,CpG 8,CpG } \\
\text { 9,CpG 11,CpG 12,CpG 22,CpG 23,CpG } \\
\text { 24,CpG 28 }\end{array}$ & 0.743333 & 0.78 & 0.1964948 & $\mathbf{0}$ \\
\hline & \multicolumn{5}{|c|}{ LR Binary } \\
\hline & $\begin{array}{c}\text { Features Implemented in Training and } \\
\text { Testing by Trial }\end{array}$ & $\begin{array}{l}\text { Training } \\
\text { (Average } \\
\text { Accuracy) }\end{array}$ & $\begin{array}{c}\text { Testing } \\
\text { (F1 Score) }\end{array}$ & $\begin{array}{c}\text { Train St.D } \\
\text { Average }\end{array}$ & Test St. D \\
\hline TRIAL 1 & $\begin{array}{c}\text { Methyl, Mito 5hmC, Nuc 5hmC, Mito } \\
\text { 5mC, Nuc 5mC, CpG 11, CpG 23, CpG 24, } \\
\text { CpG 28 }\end{array}$ & 0.7 & 0.56 & 0.279384 & $\mathbf{0}$ \\
\hline TRIAL 2 & $\begin{array}{c}\text { Methyl, Mito 5hmC, Nuc 5hmC, Mito } \\
\text { 5mC, Nuc 5mC, CpG } 1 \text { - 24, TFAM CpG, } \\
\text { TFAM Non-CpG }\end{array}$ & 0.633333 & 0.67 & 0.208167 & $\mathbf{0}$ \\
\hline TRIAL 3 & $\begin{array}{l}\text { Methyl, Mito 5hmC, Nuc 5hmC, Mito } \\
\text { 5mC, Nuc 5mC, CpG 24, TFAM CpG, } \\
\text { TFAM Non-CpG }\end{array}$ & 0.758333 & 0.66 & 0.18047 & $\mathbf{0}$ \\
\hline TRIAL 4 & Methyl, Nuc 5mC, CpG 1 - 24 & 0.683333 & 0.66 & 0.2 & $\mathbf{0}$ \\
\hline \multirow[t]{3}{*}{ TRIAL 5} & $\begin{array}{c}\text { Nuc 5mC, CpG 1,CpG 7,CpG 8,CpG } \\
\text { 9,CpG 11,CpG 12,CpG 22,CpG 23,CpG } \\
\text { 24,CpG 28 }\end{array}$ & 0.658333 & 0.66 & 0.253996 & $\mathbf{0}$ \\
\hline & \multicolumn{5}{|c|}{ LDA Binary } \\
\hline & $\begin{array}{c}\text { Features Implemented in Training and } \\
\text { Testing by Trial }\end{array}$ & $\begin{array}{l}\text { Training } \\
\text { (Average } \\
\text { Accuracy) }\end{array}$ & $\begin{array}{c}\text { Testing } \\
\text { (F1 Score) }\end{array}$ & $\begin{array}{c}\text { Train St.D } \\
\text { Average }\end{array}$ & Test St. D \\
\hline TRIAL 1 & $\begin{array}{c}\text { Methyl, Mito 5hmC, Nuc 5hmC, Mito } \\
\text { 5mC, Nuc 5mC, CpG 11, CpG 23, CpG 24, } \\
\text { CpG 28 }\end{array}$ & 0.766667 & 0.22 & 0.238048 & $\mathbf{0}$ \\
\hline TRIAL 2 & $\begin{array}{c}\text { Methyl, Mito 5hmC, Nuc 5hmC, Mito } \\
\text { 5mC, Nuc 5mC, CpG } 1 \text { - 24, TFAM CpG, } \\
\text { TFAM Non-CpG }\end{array}$ & 0.541667 & 0.43 & 0.250693 & $\mathbf{0}$ \\
\hline TRIAL 3 & $\begin{array}{c}\text { Methyl, Mito 5hmC, Nuc 5hmC, Mito } \\
\text { 5mC, Nuc 5mC, CpG 24, TFAM CpG, } \\
\text { TFAM Non-CpG }\end{array}$ & 0.775 & 0.22 & 0.325 & 0 \\
\hline
\end{tabular}




\begin{tabular}{|c|c|c|c|c|c|}
\hline TRIAL 4 & Methyl, Nuc 5mC, CpG 1 - 24 & 0.525 & 0.44 & 0.276512 & $\mathbf{0}$ \\
\hline \multirow[t]{3}{*}{ TRIAL 5} & $\begin{array}{c}\text { Nuc 5mC, CpG 1,CpG 7,CpG 8,CpG } \\
\text { 9,CpG 11,CpG 12,CpG 22,CpG 23,CpG } \\
\text { 24,CpG 28 }\end{array}$ & 0.683333 & 0.78 & 0.306866 & $\mathbf{0}$ \\
\hline & \multicolumn{5}{|c|}{ KNN Binary } \\
\hline & $\begin{array}{c}\text { Features Implemented in Training and } \\
\text { Testing by Trial }\end{array}$ & $\begin{array}{l}\text { Training } \\
\text { (Average } \\
\text { Accuracy) }\end{array}$ & $\begin{array}{c}\text { Testing } \\
\text { (F1 Score) }\end{array}$ & $\begin{array}{c}\text { Train St.D } \\
\text { Average }\end{array}$ & Test St. D \\
\hline TRIAL 1 & $\begin{array}{c}\text { Methyl, Mito 5hmC, Nuc 5hmC, Mito } \\
\text { 5mC, Nuc 5mC, CpG 11, CpG 23, CpG 24, } \\
\text { CpG } 28\end{array}$ & 0.608333 & 0.66 & 0.295921 & $\mathbf{0}$ \\
\hline TRIAL 2 & $\begin{array}{c}\text { Methyl, Mito 5hmC, Nuc 5hmC, Mito } \\
\text { 5mC, Nuc 5mC, CpG } 1 \text { - 24, TFAM CpG, } \\
\text { TFAM Non-CpG }\end{array}$ & 0.608333 & 0.78 & 0.229885 & $\mathbf{0}$ \\
\hline TRIAL 3 & $\begin{array}{l}\text { Methyl, Mito 5hmC, Nuc 5hmC, Mito } \\
\text { 5mC, Nuc 5mC, CpG 24, TFAM CpG, } \\
\text { TFAM Non-CpG }\end{array}$ & 0.716667 & 0.76 & 0.236291 & $\mathbf{0}$ \\
\hline TRIAL 4 & Methyl, Nuc 5mC, CpG 1 - 24 & 0.633333 & 0.78 & 0.230338 & $\mathbf{0}$ \\
\hline \multirow[t]{3}{*}{ TRIAL 5} & $\begin{array}{c}\text { Nuc 5mC, CpG 1,CpG 7,CpG 8,CpG } \\
\text { 9,CpG 11,CpG 12,CpG 22,CpG 23,CpG } \\
\text { 24,CpG 28 }\end{array}$ & 0.641667 & 0.66 & 0.258333 & $\mathbf{0}$ \\
\hline & \multicolumn{5}{|c|}{ NB Binary } \\
\hline & $\begin{array}{c}\text { Features Implemented in Training and } \\
\text { Testing by Trial }\end{array}$ & $\begin{array}{l}\text { Training } \\
\text { (Average } \\
\text { Accuracy) }\end{array}$ & $\begin{array}{c}\text { Testing } \\
\text { (F1 Score) }\end{array}$ & $\begin{array}{l}\text { Train St.D } \\
\text { Average }\end{array}$ & Test St. D \\
\hline TRIAL 1 & $\begin{array}{c}\text { Methyl, Mito 5hmC, Nuc 5hmC, Mito } \\
\text { 5mC, Nuc 5mC, CpG 11, CpG 23, CpG 24, } \\
\text { CpG } 28\end{array}$ & 0.691667 & 0.33 & 0.300578 & $\mathbf{0}$ \\
\hline TRIAL 2 & $\begin{array}{c}\text { Methyl, Mito 5hmC, Nuc 5hmC, Mito } \\
\text { 5mC, Nuc 5mC, CpG } 1 \text { - 24, TFAM CpG, } \\
\text { TFAM Non-CpG }\end{array}$ & 0.566667 & 0.67 & 0.355121 & $\mathbf{0}$ \\
\hline TRIAL 3 & $\begin{array}{l}\text { Methyl, Mito 5hmC, Nuc 5hmC, Mito } \\
\text { 5mC, Nuc 5mC, CpG 24, TFAM CpG, } \\
\text { TFAM Non-CpG }\end{array}$ & 0.691667 & 0.54 & 0.255631 & $\mathbf{0}$ \\
\hline TRIAL 4 & Methyl, Nuc 5mC, CpG 1 - 24 & 0.508333 & 0.77 & 0.366003 & $\mathbf{0}$ \\
\hline \multirow[t]{3}{*}{ TRIAL 5} & $\begin{array}{c}\text { Nuc 5mC, CpG 1,CpG 7,CpG 8,CpG } \\
\text { 9,CpG 11,CpG 12,CpG 22,CpG 23,CpG } \\
\text { 24,CpG 28 }\end{array}$ & 0.6 & 0.56 & 0.357071 & $\mathbf{0}$ \\
\hline & \multicolumn{5}{|c|}{ SVM Binary } \\
\hline & $\begin{array}{c}\text { Features Implemented in Training and } \\
\text { Testing by Trial }\end{array}$ & $\begin{array}{l}\text { Training } \\
\text { (Average } \\
\text { Accuracy) }\end{array}$ & $\begin{array}{l}\text { Testing } \\
\text { (F1 Score) }\end{array}$ & $\begin{array}{l}\text { Train St.D } \\
\text { Average }\end{array}$ & Test St. D \\
\hline TRIAL 1 & $\begin{array}{c}\text { Methyl, Mito 5hmC, Nuc 5hmC, Mito } \\
\text { 5mC, Nuc 5mC, CpG 11, CpG 23, CpG 24, } \\
\text { CpG } 28\end{array}$ & 0.625 & 0.66 & 0.321131 & 0 \\
\hline TRIAL 2 & $\begin{array}{c}\text { Methyl, Mito 5hmC, Nuc 5hmC, Mito } \\
\text { 5mC, Nuc 5mC, CpG } 1 \text { - 24, TFAM CpG, } \\
\text { TFAM Non-CpG }\end{array}$ & 0.591667 & 0.61 & 0.208999 & 0 \\
\hline
\end{tabular}




\begin{tabular}{|c|c|c|c|c|c|}
\hline TRIAL 3 & $\begin{array}{c}\text { Methyl, Mito 5hmC, Nuc 5hmC, Mito } \\
\text { 5mC, Nuc 5mC, CpG 24, TFAM CpG, } \\
\text { TFAM Non-CpG }\end{array}$ & $\mathbf{0 . 7 6 6 6 6 7}$ & $\mathbf{0 . 6 1}$ & $\mathbf{0 . 2 3 8 0 4 8}$ & $\mathbf{0}$ \\
\hline TRIAL 4 & Methyl, Nuc 5mC, CpG 1 - 24 & $\mathbf{0 . 5 9 1 6 6 7}$ & $\mathbf{0 . 6 1}$ & $\mathbf{0 . 2 0 8 9 9 9}$ & $\mathbf{0}$ \\
\hline TRIAL 5 & $\begin{array}{c}\text { Nuc 5mC, CpG 1,CpG 7,CpG 8,CpG } \\
\text { 9, CpG 11, CpG 12,CpG 22,CpG 23,CpG } \\
24, \text { CpG 28 }\end{array}$ & $\mathbf{0 . 7 0 8 3 3 3}$ & $\mathbf{0 . 7 6}$ & $\mathbf{0 . 2 3 0 4 8 9}$ & $\mathbf{0}$ \\
\hline
\end{tabular}


Table S3.9: Machine learning analyses for all combined features using binary classification. CART $=$ Classification and Regression Trees, $\mathrm{LR}=$ Logistic Regression, $\mathrm{LDA}=$ Linear Discriminant Analysis, KNN = K-Nearest Neighbors, NB = Naïve Bayes, $\mathrm{SVC}=$ Support Vector Machines. Nuc $=$ Nuclear, Mito $=$ Mitochondrial, $5 \mathrm{mC}=5$-methylcytosine, $5 \mathrm{hmC}=5$ hydroxymethylcytosine, Methyl $=$ S-adenosyl methionine methyltransferase activity, $\mathrm{CpG}=$ cytosine nucleotide followed by a guanine nucleotide, TFAM CpG $=$ total $\mathrm{CpG}$ methylation of the TFAM promoter region, TFAM Non-CpG = total methylation of the TFAM promoter region not at $\mathrm{CpG}$ sites, St.D. $=$ standard deviation, binary $=$ non-diabetic and type 2 diabetic, multi $=$ nondiabetic, prediabetic, and type 2 diabetic. 
Table S3.10

\begin{tabular}{|c|c|c|c|c|c|}
\hline \multicolumn{6}{|c|}{$\mathrm{SEED}=86$} \\
\hline & \multicolumn{5}{|c|}{ CART Multiple } \\
\hline & $\begin{array}{c}\text { Features Implemented in Training and } \\
\text { Testing by Trial }\end{array}$ & $\begin{array}{l}\text { Training } \\
\text { (Average } \\
\text { Accuracy) }\end{array}$ & $\begin{array}{c}\text { Testing } \\
\text { (F1 Score) }\end{array}$ & $\begin{array}{l}\text { Train St.D } \\
\text { Avg. }\end{array}$ & Test St. D \\
\hline TRIAL 1 & $\begin{array}{c}\text { Mito 5mC, Nuc 5mC, Complex III, BMI, } \\
\text { CpG 7, CpG 24, CpG 28, TFAM CpG, } \\
\text { TFAM Non-CpG }\end{array}$ & 0.7916666 & 0.558 & 0.224768 & 0.00447214 \\
\hline TRIAL 2 & $\begin{array}{c}\text { Nuc 5mC, BMI, CpG 24, CpG 28, TFAM } \\
\text { CpG, TFAM Non-CpG }\end{array}$ & 0.8016666 & 0.56 & 0.2271884 & $\mathbf{0}$ \\
\hline TRIAL 3 & $\begin{array}{c}\text { BMI, CpG 24, TFAM CpG, TFAM Non- } \\
\text { CpG }\end{array}$ & 0.7316668 & 0.56 & 0.2568122 & $\mathbf{0}$ \\
\hline TRIAL 4 & $\begin{array}{l}\text { BMI, CpG 24, CpG 28, TFAM CpG, } \\
\text { TFAM Non-CpG }\end{array}$ & 0.8366664 & 0.56 & 0.234217 & $\mathbf{0}$ \\
\hline TRIAL 5 & BMI, CpG 24, TFAM CpG & 0.6933334 & 0.594 & 0.2764508 & 0.09476286 \\
\hline TRIAL 6 & BMI, CpG 24, TFAM Non-CpG & 0.63 & $\mathbf{0 . 3 3}$ & 0.20036 & $\mathbf{0}$ \\
\hline \multirow[t]{3}{*}{ TRIAL 7} & BMI, CpG 24 & 0.6266668 & 0.258 & 0.2756018 & 0.0752994 \\
\hline & \multicolumn{5}{|c|}{ LR Multiple } \\
\hline & $\begin{array}{c}\text { Features Implemented in Training and } \\
\text { Testing by Trial }\end{array}$ & $\begin{array}{l}\text { Training } \\
\text { (Average } \\
\text { Accuracy) } \\
\end{array}$ & $\begin{array}{c}\text { Testing } \\
\text { (F1 Score) }\end{array}$ & $\begin{array}{l}\text { Train St.D } \\
\quad \text { Avg. }\end{array}$ & Test St. D \\
\hline TRIAL 1 & $\begin{array}{c}\text { Mito 5mC, Nuc 5mC, Complex III, BMI, } \\
\text { CpG 7, CpG 24, CpG 28, TFAM CpG, } \\
\text { TFAM Non-CpG }\end{array}$ & 0.516667 & 0.55 & 0.213437 & $\mathbf{0}$ \\
\hline TRIAL 2 & $\begin{array}{c}\text { Nuc 5mC, BMI, CpG 24, CpG 28, TFAM } \\
\text { CpG, TFAM Non-CpG }\end{array}$ & 0.55 & 0.55 & 0.145297 & $\mathbf{0}$ \\
\hline TRIAL 3 & $\begin{array}{c}\text { BMI, CpG 24, TFAM CpG, TFAM Non- } \\
\text { CpG }\end{array}$ & 0.516667 & 0.18 & 0.189297 & $\mathbf{0}$ \\
\hline TRIAL 4 & $\begin{array}{l}\text { BMI, CpG 24, CpG 28,TFAM CpG, TFAM } \\
\text { Non-CpG }\end{array}$ & 0.516667 & 0.13 & 0.189297 & $\mathbf{0}$ \\
\hline TRIAL 5 & BMI, CpG 24, TFAM CpG & 0.575 & 0.18 & 0.191667 & $\mathbf{0}$ \\
\hline TRIAL 6 & BMI, CpG 24, TFAM Non-CpG & 0.558333 & 0.18 & 0.220637 & $\mathbf{0}$ \\
\hline \multirow[t]{3}{*}{ TRIAL 7} & BMI, CpG 24 & 0.533333 & 0.18 & 0.211476 & $\mathbf{0}$ \\
\hline & \multicolumn{5}{|c|}{ LDA Multiple } \\
\hline & $\begin{array}{c}\text { Features Implemented in Training and } \\
\text { Testing by Trial }\end{array}$ & $\begin{array}{l}\text { Training } \\
\text { (Average } \\
\text { Accuracy) }\end{array}$ & $\begin{array}{c}\text { Testing } \\
\text { (F1 Score) }\end{array}$ & $\begin{array}{c}\text { Train St.D } \\
\text { Avg. }\end{array}$ & Test St. D \\
\hline TRIAL 1 & $\begin{array}{c}\text { Mito 5mC, Nuc 5mC, Complex III, BMI, } \\
\text { CpG 7, CpG 24, CpG 28, TFAM CpG, } \\
\text { TFAM Non-CpG }\end{array}$ & 0.525 & 0.67 & 0.129368 & $\mathbf{0}$ \\
\hline TRIAL 2 & $\begin{array}{l}\text { Nuc 5mC, BMI, CpG 24, CpG 28, TFAM } \\
\text { CpG, TFAM Non-CpG }\end{array}$ & 0.533333 & 0.43 & 0.15 & $\mathbf{0}$ \\
\hline TRIAL 3 & $\begin{array}{c}\text { BMI, CpG 24, TFAM CpG, TFAM Non- } \\
\text { CpG }\end{array}$ & 0.475 & 0.44 & 0.232886 & $\mathbf{0}$ \\
\hline TRIAL 4 & $\begin{array}{l}\text { BMI, CpG 24, CpG 28,TFAM CpG, TFAM } \\
\text { Non-CpG }\end{array}$ & 0.541667 & 0.44 & 0.125 & $\mathbf{0}$ \\
\hline
\end{tabular}




\begin{tabular}{|c|c|c|c|c|c|}
\hline TRIAL 5 & BMI, CpG 24, TFAM CpG & 0.55 & 0.34 & 0.214735 & $\mathbf{0}$ \\
\hline TRIAL 6 & BMI, CpG 24, TFAM Non-CpG & 0.475 & 0.19 & 0.232886 & $\mathbf{0}$ \\
\hline \multirow[t]{3}{*}{ TRIAL 7} & BMI, CpG 24 & 0.525 & 0.1 & 0.232886 & $\mathbf{0}$ \\
\hline & \multicolumn{5}{|c|}{ KNN Multiple } \\
\hline & $\begin{array}{c}\text { Features Implemented in Training and } \\
\text { Testing by Trial }\end{array}$ & $\begin{array}{r}\text { Training } \\
\text { (Average } \\
\text { Accuracy) } \\
\end{array}$ & $\begin{array}{c}\text { Testing } \\
\text { (F1 Score) }\end{array}$ & $\begin{array}{l}\text { Train St.D } \\
\text { Avg. }\end{array}$ & Test St. D \\
\hline TRIAL 1 & $\begin{array}{c}\text { Mito 5mC, Nuc 5mC, Complex III, BMI, } \\
\text { CpG 7, CpG 24, CpG 28, TFAM CpG, } \\
\text { TFAM Non-CpG }\end{array}$ & 0.325 & 0.3 & 0.215542 & $\mathbf{0}$ \\
\hline TRIAL 2 & $\begin{array}{c}\text { Nuc 5mC, BMI, CpG 24, CpG 28, TFAM } \\
\text { CpG, TFAM Non-CpG }\end{array}$ & 0.366667 & 0.33 & 0.284312 & $\mathbf{0}$ \\
\hline TRIAL 3 & $\begin{array}{c}\text { BMI, CpG 24, TFAM CpG, TFAM Non- } \\
\text { CpG }\end{array}$ & 0.383333 & 0.31 & 0.26405 & $\mathbf{0}$ \\
\hline TRIAL 4 & $\begin{array}{c}\text { BMI, CpG 24, CpG 28,TFAM CpG, TFAM } \\
\text { Non-CpG }\end{array}$ & 0.375 & 0.34 & 0.294038 & $\mathbf{0}$ \\
\hline TRIAL 5 & BMI, CpG 24, TFAM CpG & 0.383333 & 0.31 & 0.286744 & $\mathbf{0}$ \\
\hline TRIAL 6 & BMI, CpG 24, TFAM Non-CpG & 0.408333 & 0.31 & 0.26207 & $\mathbf{0}$ \\
\hline \multirow[t]{3}{*}{ TRIAL 7} & BMI, CpG 24 & 0.433333 & 0.13 & 0.23214 & $\mathbf{0}$ \\
\hline & \multicolumn{5}{|c|}{ NB Multiple } \\
\hline & $\begin{array}{c}\text { Features Implemented in Training and } \\
\text { Testing by Trial }\end{array}$ & $\begin{array}{l}\text { Training } \\
\text { (Average } \\
\text { Accuracy) }\end{array}$ & $\begin{array}{c}\text { Testing } \\
\text { (F1 Score) }\end{array}$ & $\begin{array}{c}\text { Train St.D } \\
\text { Avg. }\end{array}$ & Test St. D \\
\hline TRIAL 1 & $\begin{array}{c}\text { Mito 5mC, Nuc 5mC, Complex III, BMI, } \\
\text { CpG 7, CpG 24, CpG 28, TFAM CpG, } \\
\text { TFAM Non-CpG }\end{array}$ & 0.558333 & 0.4 & 0.16266 & $\mathbf{0}$ \\
\hline TRIAL 2 & $\begin{array}{c}\text { Nuc 5mC, BMI, CpG 24, CpG 28, TFAM } \\
\text { CpG, TFAM Non-CpG }\end{array}$ & 0.608333 & 0.56 & 0.229885 & $\mathbf{0}$ \\
\hline TRIAL 3 & $\begin{array}{c}\text { BMI, CpG 24, TFAM CpG, TFAM Non- } \\
\text { CpG }\end{array}$ & 0.608333 & 0.47 & 0.273988 & $\mathbf{0}$ \\
\hline TRIAL 4 & $\begin{array}{l}\text { BMI, CpG 24, CpG 28,TFAM CpG, TFAM } \\
\text { Non-CpG }\end{array}$ & 0.591667 & 0.47 & 0.208999 & $\mathbf{0}$ \\
\hline TRIAL 5 & BMI, CpG 24, TFAM CpG & 0.55 & 0.18 & 0.183333 & $\mathbf{0}$ \\
\hline TRIAL 6 & BMI, CpG 24, TFAM Non-CpG & 0.566667 & 0.18 & 0.203443 & $\mathbf{0}$ \\
\hline \multirow[t]{3}{*}{ TRIAL 7} & BMI, CpG 24 & 0.55 & 0.18 & 0.214735 & $\mathbf{0}$ \\
\hline & \multicolumn{5}{|c|}{ SVM Multiple } \\
\hline & $\begin{array}{c}\text { Features Implemented in Training and } \\
\text { Testing by Trial }\end{array}$ & $\begin{array}{l}\text { Training } \\
\text { (Average } \\
\text { Accuracy) } \\
\end{array}$ & $\begin{array}{c}\text { Testing } \\
\text { (F1 Score) }\end{array}$ & $\begin{array}{l}\text { Train St.D } \\
\quad \text { Avg. }\end{array}$ & Test St. D \\
\hline TRIAL 1 & $\begin{array}{c}\text { Mito 5mC, Nuc 5mC, Complex III, BMI, } \\
\text { CpG 7, CpG 24, CpG 28, TFAM CpG, } \\
\text { TFAM Non-CpG }\end{array}$ & 0.35 & 0.17 & 0.152753 & $\mathbf{0}$ \\
\hline TRIAL 2 & $\begin{array}{c}\text { Nuc 5mC, BMI, CpG 24, CpG 28, TFAM } \\
\text { CpG, TFAM Non-CpG }\end{array}$ & 0.491667 & 0.31 & 0.29451 & $\mathbf{0}$ \\
\hline TRIAL 3 & $\begin{array}{c}\text { BMI, CpG 24, TFAM CpG, TFAM Non- } \\
\text { CpG }\end{array}$ & 0.408333 & 0.08 & 0.275 & $\mathbf{0}$ \\
\hline
\end{tabular}




\begin{tabular}{|c|c|c|c|c|c|}
\hline TRIAL 4 & BMI, CpG 24, CpG 28,TFAM CpG, TFAM & $\mathbf{0 . 3 4 1 6 6 7}$ & $\mathbf{0 . 1 3}$ & $\mathbf{0 . 2 9 4 5 1}$ & $\mathbf{0}$ \\
\hline TRIAL 5 & BMI, CpG 24, TFAM CpG & $\mathbf{0 . 3 8 3 3 3 3}$ & $\mathbf{0 . 1 8}$ & $\mathbf{0 . 2 9 8 6 0 8}$ & $\mathbf{0}$ \\
\hline TRIAL 6 & BMI, CpG 24, TFAM Non-CpG & $\mathbf{0 . 4 9 1 6 6 7}$ & $\mathbf{0 . 0 8}$ & $\mathbf{0 . 2 4 8 4 6 8}$ & $\mathbf{0}$ \\
\hline TRIAL 7 & BMI, CpG 24 & $\mathbf{0 . 4 6 6 6 6 7}$ & $\mathbf{0 . 1 8}$ & $\mathbf{0 . 2 1 1 4 7 6}$ & $\mathbf{0}$ \\
\hline
\end{tabular}


Table S3.10: Machine learning analyses for all combined features using multi classification. CART $=$ Classification and Regression Trees, LR $=$ Logistic Regression, LDA $=$ Linear Discriminant Analysis, KNN = K-Nearest Neighbors, NB = Naïve Bayes, SVC = Support Vector Machines. Nuc $=$ Nuclear, Mito $=$ Mitochondrial, $5 \mathrm{mC}=5$-methylcytosine, $5 \mathrm{hmC}=5$ hydroxymethylcytosine, $\mathrm{CpG}=$ cytosine nucleotide followed by a guanine nucleotide, TFAM CpG $=$ total $\mathrm{CpG}$ methylation of the TFAM promoter region, TFAM Non-CpG = total methylation of the TFAM promoter region not at $\mathrm{CpG}$ sites, St.D. = standard deviation, binary = non-diabetic and type 2 diabetic, multi $=$ non-diabetic, prediabetic, and type 2 diabetic. 
TableS3.11

\begin{tabular}{|c|c|c|c|c|c|c|c|c|}
\hline Model & Training & $\begin{array}{l}\text { Training } \\
\text { (StDev) }\end{array}$ & Testing & $\begin{array}{l}\text { Testing } \\
\text { (StDev) }\end{array}$ & F1 Score & Important Features & Important Feature Bias & $A U C$ \\
\hline LR & 0.808 & 0.171 & 0.667 & 0.000 & 0.670 & $\begin{array}{l}\text { CpG 35, CpG 29, } \\
\text { Mito 5mC, CpG 1, } \\
\text { Nuc 5mC, 16362, } \\
\text { SNP7028, CpG 24, } \\
\text { SNP16519, } \\
\text { SNP11719 }\end{array}$ & $\begin{array}{c}(2.656),(-1.496),(- \\
0.699),(0.583),(0.535), \\
(-0.359),(0.311),(- \\
0.304),(0.099),(-0.066)\end{array}$ & 0.700 \\
\hline LDA & 0.917 & 0.129 & 0.778 & 0.000 & 0.780 & $\begin{array}{c}\text { SNP4295, SNP4917, } \\
\text { SNP7028, } \\
\text { SNP16362, Nuc } \\
\text { 5hmC, SNP16519, } \\
72, \text { Nuc 5mC, } \\
\text { SNP8860, CpG } 29\end{array}$ & $\begin{array}{c}(-1.050 \mathrm{E}+16),(-8.170) \\
(7.367),(-6.313),(- \\
3.659),(3.472),(3.113) \\
(2.483),(-2.119),(- \\
1.359)\end{array}$ & 0.800 \\
\hline KNN & 0.750 & 0.236 & 0.556 & 0.000 & 0.560 & NA & N & 0.675 \\
\hline NB & 0.683 & 0.249 & 0.778 & 0.000 & 0.780 & $\begin{array}{c}\text { Mito } 5 \mathrm{hmC} \\
\text { Methyltransferase }\end{array}$ & (1.000), (0.000) & 0.725 \\
\hline SVM & 0.750 & 0.242 & 0.667 & 0.000 & 0.670 & $\begin{array}{c}\text { CpG 35, CpG 29, } \\
\text { SNP16362, Mito } \\
\text { 5mC, Nuc 5mC, } \\
\text { SNP7028, 72, CpG } \\
\text { 24, SNP16519, CpG } \\
1\end{array}$ & $\begin{array}{c}(2.240),(-0.892),(- \\
0.467),(-0.395),(0.313), \\
(0.225),(0.216),(- \\
0.210),(0.204),(0.190)\end{array}$ & 0.650 \\
\hline CART & 0.833 & 0.212 & 0.711 & 0.061 & 0.692 & $\begin{array}{c}\text { CpG 24, Nuc 5mC, } \\
\text { CpG } 1\end{array}$ & $\begin{array}{c}\text { (58.711\%), }(22.547 \%) \\
(18.742 \%)\end{array}$ & 0.740 \\
\hline
\end{tabular}


Table S3.11: Overview of 6 machine-learning model analysis on 18 selected features in binary classification. Model analysis was conducted five times and averages are reported for the resulting training accuracy, training standard deviation, testing accuracy, testing standard deviation, F1 score, and area under the curve (AUC). Important biomarker features associated with each trained model are provided along with the associated influence value for each feature. Important features are listed in order of influence within the model. LR, LDA, SVM feature bias exists as an influence parameter where magnitude dictates feature influence. A positive influence value indicates the biomarker favors classification towards one label while a negative value indicates favorable classification of the opposite label. The larger the magnitude, the more strongly that feature shifts classification. NB feature influence indicates the most important biomarker per class in binary $(0,1)$ classification schemes. CART feature bias percentages indicate feature influence on the created classification tree. Larger percentages indicate a feature that arises near the beginning of a tree before subsequent branching. Influence is not provided for KNN due to model restrictions. The 18 selected features include: Methyltransferase, Mito 5hmC, Nuc 5hmC, Mito 5mC, Nuc 5mC, CpG1, CpG24, CpG29, CpG35, SNP72, SNP4295, SNP4917, SNP7028, SNP8860, SNP11719, SNP16354, SNP16362, and SNP16519. 
Table S3.12

\begin{tabular}{|c|c|c|c|c|c|c|c|}
\hline Model & Training & $\begin{array}{l}\text { Training } \\
\text { (StDev) }\end{array}$ & Testing & $\begin{array}{l}\text { Testing } \\
\text { (StDev) }\end{array}$ & F1 Score & Important Features & Important Feature Bias \\
\hline LR & 0.408 & 0.209 & 0.444 & 0.000 & 0.380 & $\begin{array}{l}\text { Nuc 5mC, CpG1, CpG29, } \\
\text { SNP16362, SNP7028, } \\
\text { CpG35, SNP16519, } \\
\text { SNP11719, CpG24, Mito } \\
\text { 5mC }\end{array}$ & $\begin{array}{c}(-0.381),(-0.244),(0.200) \\
(0.191),(-0.168),(-0.122) \\
(-0.099),(0.081),(0.047) \\
(0.031)\end{array}$ \\
\hline LDA & 0.392 & 0.291 & 0.667 & 0.000 & 0.640 & $\begin{array}{c}\text { CpG35, SNP16362, } \\
\text { SNP7028, CpG29, } \\
\text { SNP4917, SNP11719, Nuc } \\
\text { 5mC, Nuc 5hmC, } \\
\text { Methyltransferase, Mito } \\
\text { 5mC }\end{array}$ & $\begin{array}{c}(-2.932),(1.759),(-1.431), \\
(1.323),(1.232),(1.151),(- \\
1.144),(0.899),(-0.767), \\
(0.640)\end{array}$ \\
\hline KNN & 0.400 & 0.288 & 0.222 & 0.000 & 0.180 & NA & NA \\
\hline NB & 0.367 & 0.312 & 0.444 & 0.000 & 0.350 & $\begin{array}{c}\text { Nuc } 5 \mathrm{hmC} \text {, Mito } 5 \mathrm{hmC}, \\
\text { Methyltransferase }\end{array}$ & (2.000), (1.000), (0.000) \\
\hline SVM & 0.467 & 0.264 & 0.333 & 0.000 & 0.360 & $\begin{array}{l}\text { CpG35, Nuc 5mC, Mito } \\
\text { 5mC, CpG24, SNP7028, } \\
\text { CpG29, SNP11719, CpG1, } \\
\text { SNP4917, Mito 5hmC }\end{array}$ & $\begin{array}{c}(0.770),(0.645),(-0.560) \\
(-0.348),(-0.195),(-0.165), \\
(0.137),(0.028),(0.026), \\
(0.011)\end{array}$ \\
\hline CART & 0.492 & 0.352 & 0.556 & 0.000 & 0.570 & $\begin{array}{c}\text { CpG 24, } \\
\text { Methyltransferase, CpG } \\
\text { 29, Nuc 5mC, CpG 1, Mito } \\
\text { 5hmC, Mito 5mC, Nuc } \\
\text { 5hmC, SNP11719 }\end{array}$ & $\begin{array}{c}(32.762 \%),(21.973 \%), \\
(19.800 \%),(8.501 \%), \\
(5.598 \%),(3.357 \%), \\
(2.975 \%),(1.678 \%), \\
(1.678 \%)\end{array}$ \\
\hline
\end{tabular}


Table S3.12: Overview of 6 machine-learning model analysis on 18 selected features in multiple classification. Model analysis was conducted five times and averages are reported for the resulting training accuracy, training standard deviation, testing accuracy, testing standard deviation, and F1 score. Important biomarker features associated with each trained model are provided along with the associated influence value for each feature. Important features are listed in order of influence within the model. LR, LDA, SVM feature bias exists as an influence parameter where magnitude dictates feature influence. A positive influence value indicates the biomarker favors classification towards one label while a negative value indicates favorable classification of the opposite label. The larger the magnitude, the more strongly that feature shifts classification. NB feature influence indicates the most important biomarker per class in multiple $(0,1,2)$ classification schemes. CART feature bias percentages indicate feature influence on the created classification tree. Larger percentages indicate a feature that arises near the beginning of a tree before subsequent branching. Influence is not provided for KNN due to model restrictions. The 18 selected features include: Methyltransferase, Mito 5hmC, Nuc 5hmC, Mito 5mC, Nuc 5mC, CpG1, CpG24, CpG29, CpG35, SNP72, SNP4295, SNP4917, SNP7028, SNP8860, SNP11719, SNP16354, SNP16362, and SNP16519. 
Table S3.13

\begin{tabular}{|c|c|c|}
\hline Parameter & Non-Diabetic & Type 2 Diabetic \\
\hline Nuclear 5mC (\% Methylation) & $2.039 \pm 0.1063$ & $2.873 \pm 0.2445 *$ \\
\hline $\begin{array}{l}\text { Mitochondrial } 5 \mathrm{mC}(\% \\
\text { Methylation) }\end{array}$ & $1.776 \pm 0.3107$ & $1.644 \pm 0.3658$ \\
\hline Nuclear 5hmC (\% Methylation) & $0.178 \pm 0.01935$ & $0.1035 \pm 0.0171 *$ \\
\hline $\begin{array}{l}\text { Mitochondrial } 5 \mathrm{hmC}(\% \\
\text { Methylation) }\end{array}$ & $0.08123 \pm 0.02082$ & $0.04837 \pm 0.01984$ \\
\hline Methyltransferase $(\mu \mathrm{mol} / \mathrm{min} / \mathrm{mL})$ & $0.005841 \pm 0.0004433$ & $0.004895 \pm 0.0001608 *$ \\
\hline Complex I (nmol/min/ $\mathrm{gg})$ & $45.24 \pm 3.302$ & $35.14 \pm 3.193 *$ \\
\hline Complex III (nmol/min/ $\mu \mathrm{g})$ & $106.8 \pm 9.509$ & $79.7 \pm 6.337 *$ \\
\hline Complex IV (nmol/min/ $/ \mathrm{g})$ & $15.28 \pm 1.7$ & $16.52 \pm 2.193$ \\
\hline Complex V (nmol/min/mg) & $134.7 \pm 14.34$ & $114.2 \pm 10.1$ \\
\hline $\begin{array}{l}\text { Citrate Synthase (Unit/mg } \\
\text { mitochondria) }\end{array}$ & $0.02384 \pm 0.00215$ & $0.02021 \pm 0.0009824$ \\
\hline TFAM CpG (\% Methylation) & $1.663 \pm 0.07412$ & $1.568 \pm 0.1121$ \\
\hline TFAM Non-CpG (\% Methylation) & $1.267 \pm 0.05839$ & $1.237 \pm 0.08055$ \\
\hline
\end{tabular}


Table S3.13: Biochemical features assessed in the non-diabetic and type 2 diabetic cohort. Groups are considered significantly different if $P \leq 0.05=*$ compared to non-diabetic. All data are presented as the mean \pm standard error of the mean (SEM). $5 \mathrm{mC}=5$-methylcytosine, $5 \mathrm{hmC}=5$ hydroxymethylcytosine, $\mathrm{CpG}=$ cytosine nucleotide followed by a guanine nucleotide, Complex $=$ electron transport chain complex activity, TFAM = transcription factor A, mitochondrial. 
Table S3.14

\begin{tabular}{|c|c|c|c|}
\hline Parameter & Non-Diabetic & Pre-Diabetic & Type 2 Diabetic \\
\hline $\begin{array}{l}\text { Nuclear } 5 \mathrm{mC}(\% \\
\text { Methylation })\end{array}$ & $2.057 \pm 0.1247$ & $2.019 \pm 0.1829$ & $2.873 \pm 0.2445 * \#$ \\
\hline $\begin{array}{l}\text { Mitochondrial } 5 \mathrm{mC}(\% \\
\text { Methylation) }\end{array}$ & $1.448 \pm 0.261$ & $1.432 \pm 0.2627$ & $1.166 \pm 0.191$ \\
\hline $\begin{array}{l}\text { Nuclear 5hmC (\% } \\
\text { Methylation) }\end{array}$ & $0.1839 \pm 0.02649$ & $0.1617 \pm 0.02873$ & $0.107 \pm 0.0169$ \\
\hline $\begin{array}{l}\text { Mitochondrial } 5 \mathrm{hmC}(\% \\
\text { Methylation })\end{array}$ & $0.07123 \pm 0.03244$ & $0.0964 \pm 0.02049$ & $0.05024 \pm 0.02082$ \\
\hline $\begin{array}{l}\text { Methyltransferase } \\
(\mu \mathrm{mol} / \mathrm{min} / \mathrm{mL})\end{array}$ & $0.006339 \pm 0.0006806$ & $0.004878 \pm 0.0004467$ & $0.005185 \pm 0.0002661$ \\
\hline Complex I (nmol/min/ $\mu \mathrm{g})$ & $42.69 \pm 4.352$ & $48.14 \pm 5.091$ & $35.14 \pm 3.193$ \\
\hline Complex III (nmol/min/ $\mu \mathrm{g})$ & $105.2 \pm 11.83$ & $108.7 \pm 15.72$ & $79.7 \pm 6.337$ \\
\hline Complex IV (nmol/min/ $\mu \mathrm{g})$ & $13.67 \pm 1.738$ & $17.12 \pm 3.057$ & $16.52 \pm 2.193$ \\
\hline Complex V (nmol/min/mg) & $119.81 \pm 3.07$ & $151.7 \pm 26.76$ & $114.2 \pm 10.1$ \\
\hline $\begin{array}{l}\text { Citrate Synthase (Unit/mg } \\
\text { mitochondria) }\end{array}$ & $0.01961 \pm 0.001418$ & $0.02868 \pm 0.004009$ & $0.02021 \pm 0.0009824$ \\
\hline $\begin{array}{l}\text { TFAM CpG ( } \% \\
\text { Methylation) }\end{array}$ & $1.45 \pm 0.05362$ & $1.907 \pm 0.1179 *$ & $1.568 \pm 0.1121$ \\
\hline $\begin{array}{l}\text { TFAM Non-CpG }(\% \\
\text { Methylation })\end{array}$ & $1.2 \pm 0.03096$ & $1.343 \pm 0.1192$ & $1.237 \pm 0.08055$ \\
\hline
\end{tabular}


Table S3.14: Biochemical features assessed in the non-diabetic and type 2 diabetic cohort. Groups are considered significantly different if $P \leq 0.05=*$ compared to non-diabetic or \# compared to pre-diabetic. All data are presented as the mean \pm standard error of the mean (SEM). $5 \mathrm{mC}=5$ mehtylcytosine, $5 \mathrm{hmC}=5$-hydroxymethylcytosine, $\mathrm{CpG}=$ cytosine nucleotide followed by a guanine nucleotide, Complex $=$ electron transport chain complex activity, TFAM $=$ transcription factor A, mitochondrial. 
Figure S3.1

A

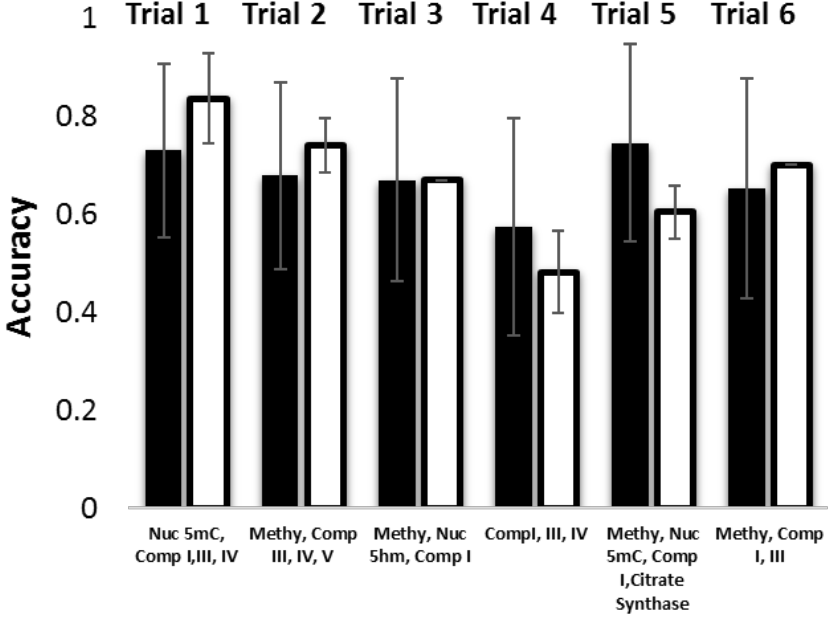

C

1

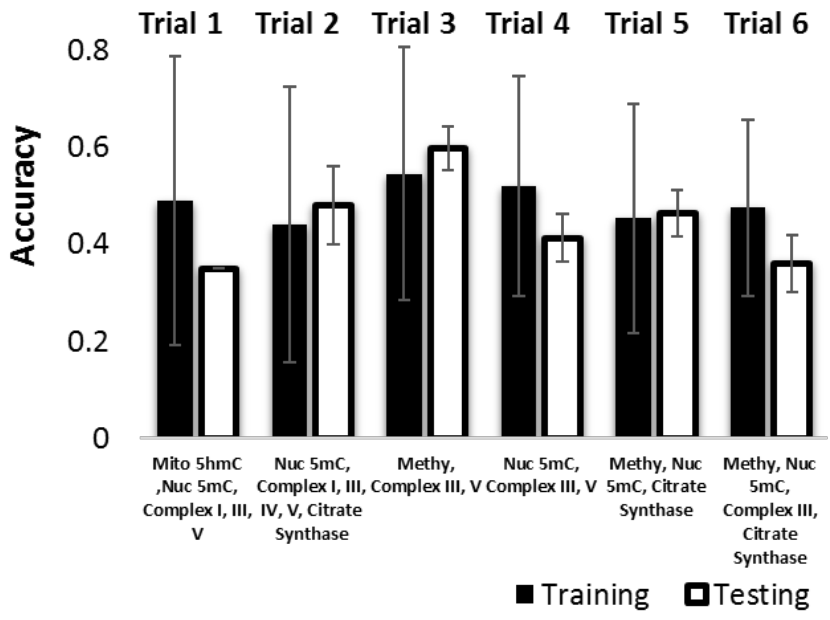

B

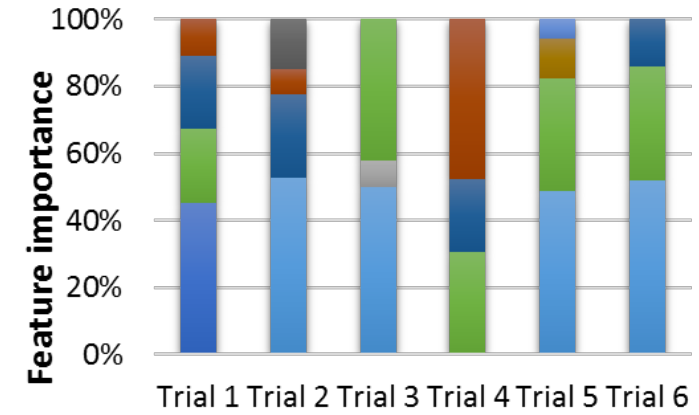

$\begin{array}{ll}\square \text { Methyltransferase } & \text { Nuc } 5 \mathrm{hmC} \\ \text { Nuc } 5 \mathrm{mC} & \text { Complex I } \\ \square \text { Complex III } & \text { Complex IV } \\ \text { Complex V } & \text { Citrate Synthase }\end{array}$

Other (<8\%)

D

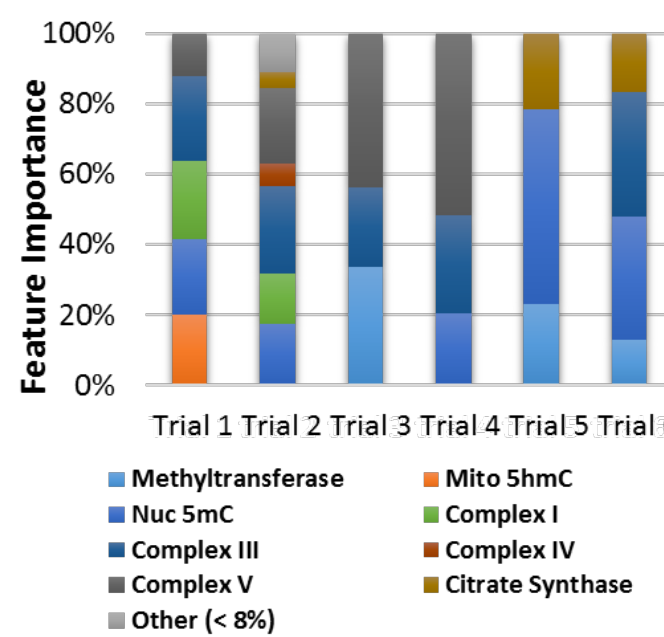


Figure S3.1: Feature importance using CART for physiological and biochemical characteristics from patients. (A) Training and testing accuracies for combinations of parameters that gave the most predictive outcomes in binary classification. (B) Percentage of individual feature importance by trial for binary classification. (C) Training and testing accuracies for combinations of parameters that gave the most predictive outcomes in multi classification. (D) Percentage of individual feature importance by trial for multi classification. Nuc $=$ nuclear, Mito $=$ mitochondrial, 5mC $=5$-methylcytosine, $5 \mathrm{hmC}, 5$-hydroxymethylcytosine, Methyl $=$ S-adenosyl methionine methyltransferase activity, binary $=$ non-diabetic and type 2 diabetic, multi $=$ non-diabetic, prediabetic, and type 2 diabetic. 
Figure S3.2
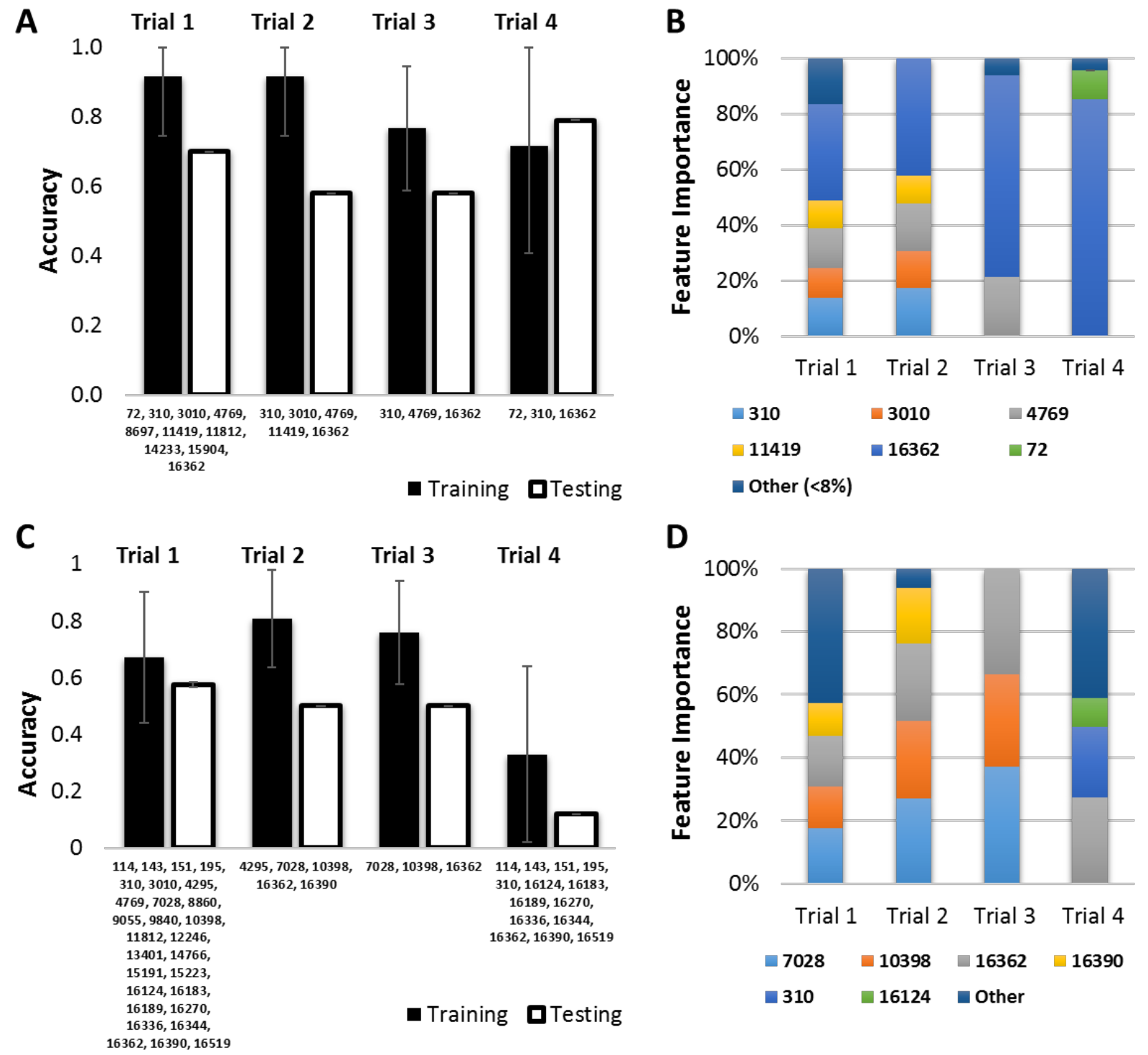
Figure S3.2: Feature importance using CART for mitochondrial DNA SNPs from patients. (A) Training and testing accuracies for combinations of parameters that gave the most predictive outcomes in binary classification. (B) Percentage of individual feature importance by trial for binary classification. (C) Training and testing accuracies for combinations of parameters that gave the most predictive outcomes in multi classification. (D) Percentage of individual feature importance by trial for multi classification. Numbers are indicative of the single nucleotide polymorphism (SNP) found in the mitochondrial DNA. Binary = non-diabetic and type 2 diabetic, multi $=$ non-diabetic, prediabetic, and type 2 diabetic. 
Figure S3.3

A

TFAM

VDAC

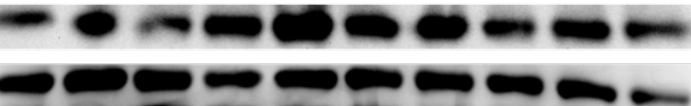

B
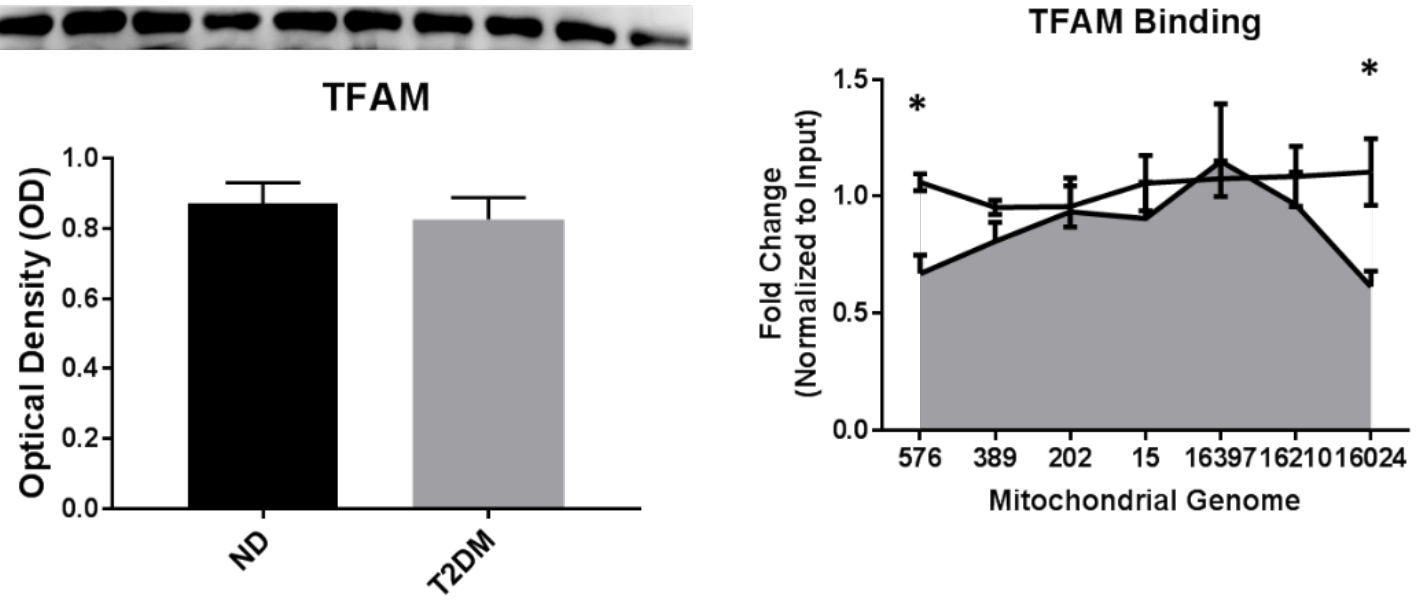
Figure S3.3: Expression and binding affinity of TFAM. (A) Expression of TFAM in isolated mitochondria from non-diabetic $(n=5$, left) and type 2 diabetic $(n=5$, right) patients. (B) Binding affinity of TFAM to the D-Loop region of mitochondrial DNA using chromatin immunoprecipitation. Groups are considered significantly different if $P \leq 0.05=*$ compared to non-diabetic. All data are presented as the mean \pm standard error of the mean (SEM). TFAM = transcription factor A, mitochondrial. 
Figure S3.4

A

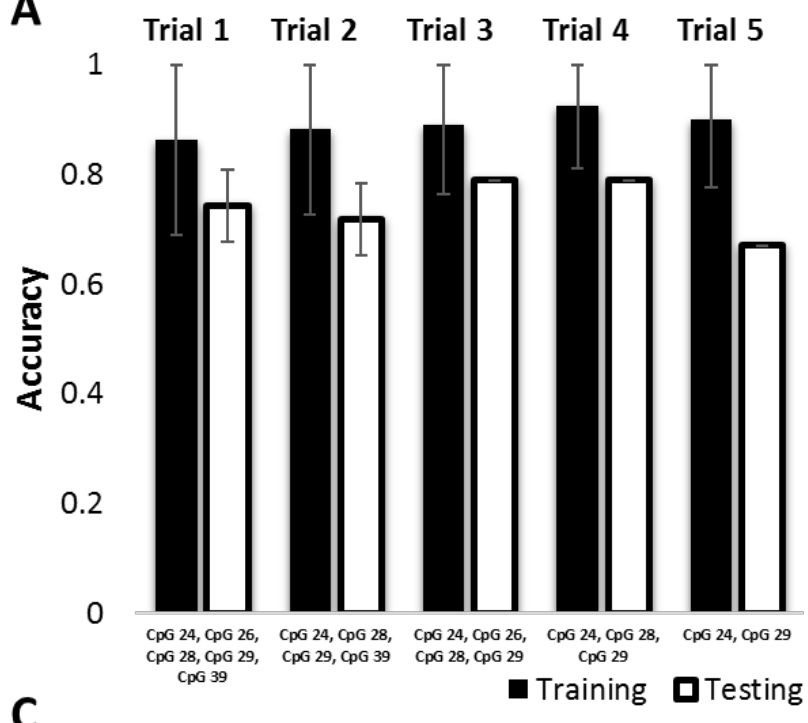

C

1 Trial $1 \quad$ Trial 2 Trial $3 \quad$ Trial $4 \quad$ Trial 5

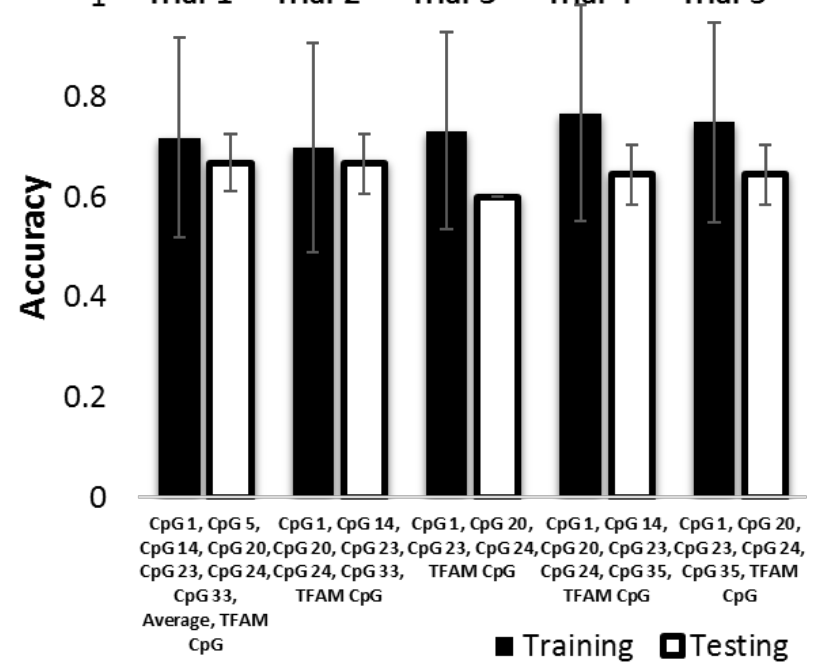

B

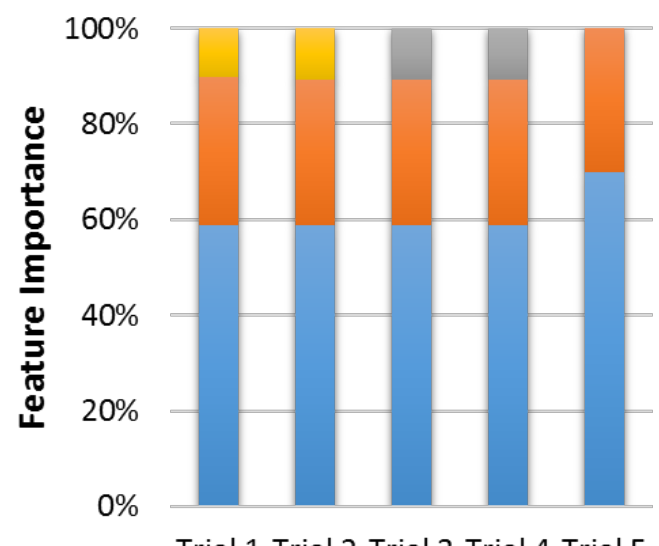

Trial 1 Trial 2 Trial 3 Trial 4 Trial 5

a CpG $24 \square$ CpG $29 \square$ CpG $28 \square$ Other $(<8 \%)$

D

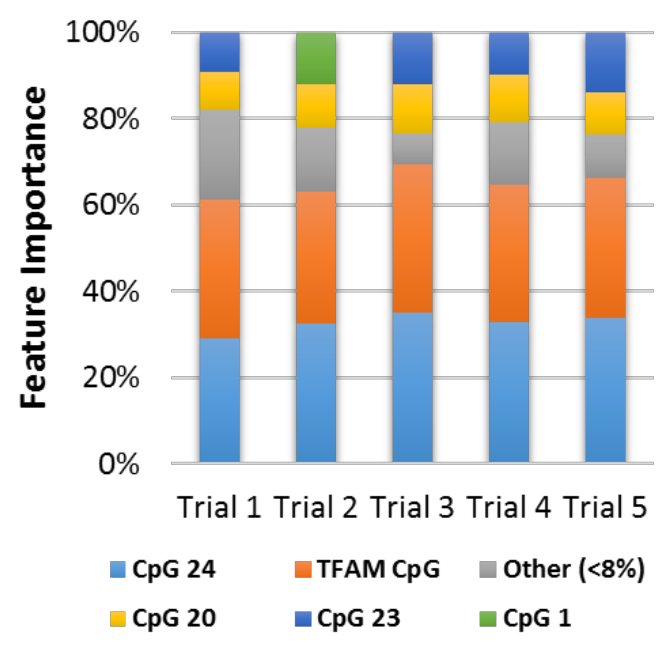


Figure S3.4: Feature importance using CART for $\mathrm{CpG}$ island methylation of TFAM from patients. (A) Training and testing accuracies for combinations of parameters that gave the most predictive outcomes in binary classification. (B) Percentage of individual feature importance by trial for binary classification. (C) Training and testing accuracies for combinations of parameters that gave the most predictive outcomes in multi classification. (D) Percentage of individual feature importance by trial for multi classification. $\mathrm{CpG}=$ cytosine nucleotide followed by a guanine nucleotide, TFAM $\mathrm{CpG}=$ total $\mathrm{CpG}$ methylation of the TFAM promoter region, $\mathrm{TFAM}=$ transcription factor A, mitochondrial, binary = non-diabetic and type 2 diabetic, multi = nondiabetic, prediabetic, and type 2 diabetic. 
Figure S3.5

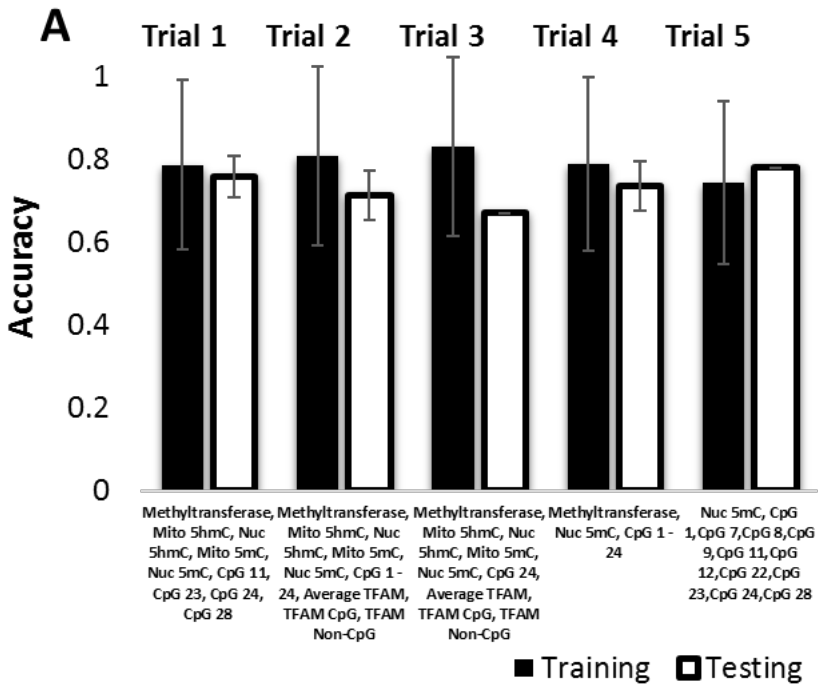

C Trial 1 Trial 2 Trial 3 Trial 4 Trial 5 Trial 6 Trial 7

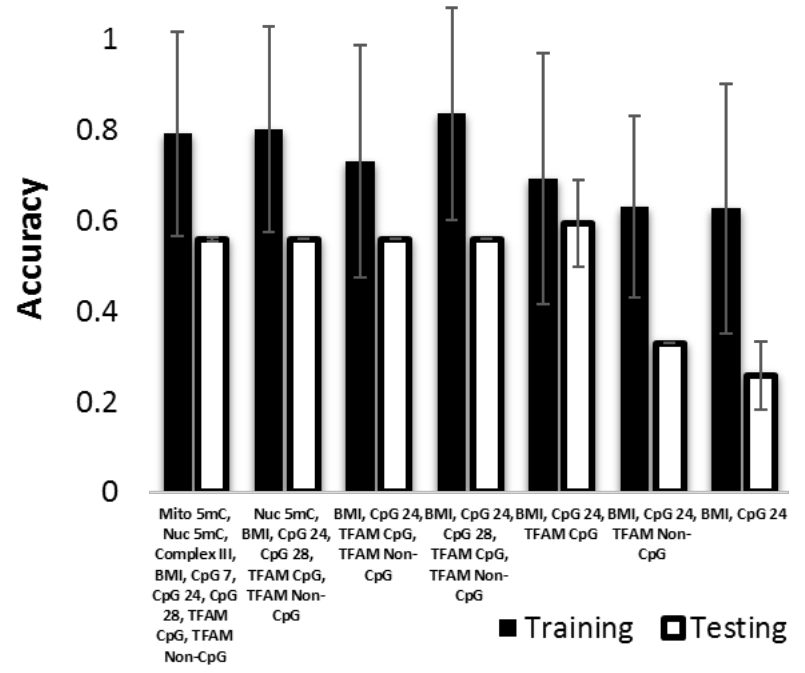

B

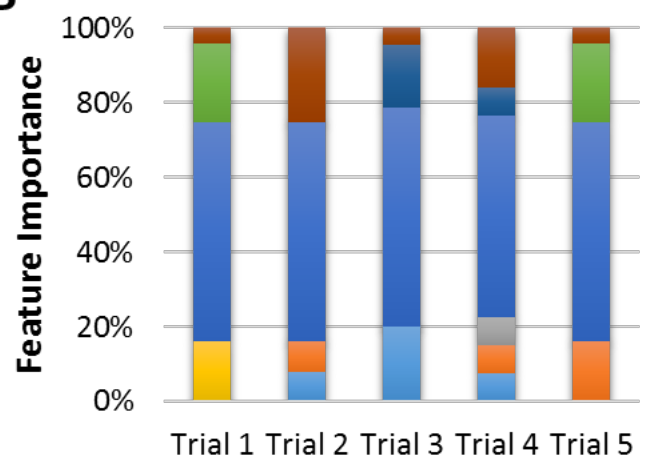

Nuclear $5 \mathrm{mC}$

CpG 8

CpG 24

CpG 28

口 Other $(<8 \%)$

D

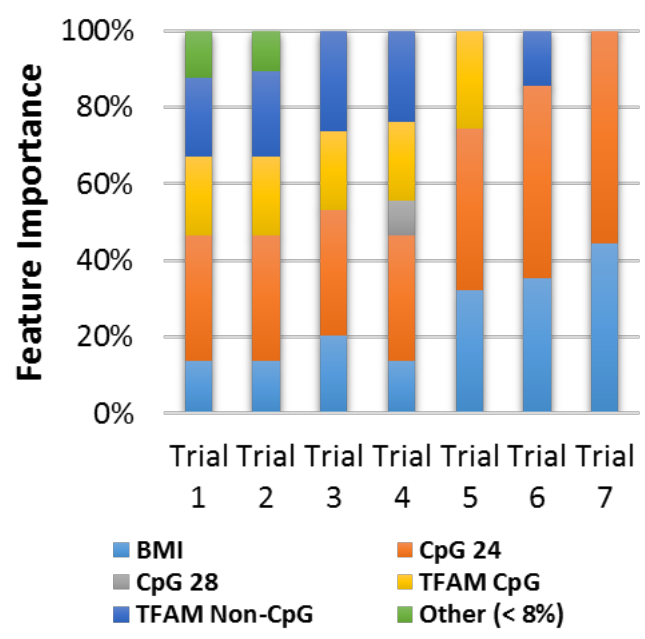


Figure S3.5: Feature importance using CART for all factors combined from patients. (A) Training and testing accuracies for combinations of parameters that gave the most predictive outcomes in binary classification. (B) Percentage of individual feature importance by trial for binary classification. (C) Training and testing accuracies for combinations of parameters that gave the most predictive outcomes in multi classification. (D) Percentage of individual feature importance by trial for multi classification. $\mathrm{Nuc}=$ nuclear, Mito $=$ mitochondrial, $5 \mathrm{mC}=5$-methylcytosine, 5hmC, 5-hydroxymethylcytosine, Methylytransferase $=$ S-adenosyl methionine methyltransferase activity, $\mathrm{CpG}=$ cytosine nucleotide followed by a guanine nucleotide, $\mathrm{TFAM} \mathrm{CpG}=$ total $\mathrm{CpG}$ methylation of the TFAM promoter region, TFAM Non- $\mathrm{CpG}=$ total methylation of the TFAM promoter region not at $\mathrm{CpG}$ sites, $\mathrm{TFAM}=$ transcription factor $\mathrm{A}$, mitochondrial, binary $=$ nondiabetic and type 2 diabetic, multi $=$ non-diabetic, prediabetic, and type 2 diabetic. 


\title{
Chapter 4: Specific Aim 3
}

\section{The Mitochondrial Regulatory Network: Subcellular Localization of Non-Coding RNA in the Heart}

\author{
Quincy A. Hathaway ${ }^{1,2}$, Andrew D. Taylor ${ }^{1,2}$, Amina Kunovac ${ }^{1,2}$, Mark V. Pinti ${ }^{2,3}$, Chris C. \\ Cook $^{4}$, Garrett K. Fink ${ }^{1}$, Andrya J. Durr ${ }^{1,2}$, Danielle L. Shepherd ${ }^{1,2}$ Aaron R. Robart ${ }^{5}$, and John \\ M. Hollander ${ }^{1,2}$ \\ Manuscript Prepared for Submission to Nucleic Acids Research \\ ${ }^{1}$ Division of Exercise Physiology, West Virginia University School of Medicine, Morgantown, WV, USA. \\ ${ }^{2}$ Mitochondria, Metabolism \& Bioenergetics Working Group, West Virginia University School of Medicine, \\ Morgantown, WV, USA. ${ }^{3}$ West Virginia University School of Pharmacy, Morgantown, WV, USA. ${ }^{4}$ Cardiovascular \\ and Thoracic Surgery, West Virginia University School of Medicine, Morgantown 26505, WV, USA, ${ }^{5}$ Department \\ of Biochemistry, West Virginia University School of Medicine, Morgantown, WV, USA.
}

Corresponding Author:

John M. Hollander, Ph.D., F.A.H.A.

Division of Exercise Physiology

West Virginia University School of Medicine

PO Box 9227

1 Medical Center Drive

Morgantown, WV 26506

Tel: 1-(304) 293-3683

Fax: 1-(304) 293-7105

Email: jhollander@hsc.wvu.edu

Keywords: Long Non-Coding RNA, MicroRNA, Machine Learning, PNPase, Type 2 Diabetes 


\begin{abstract}
Non-coding RNAs (ncRNAs) mediate processes such as transcription, translation, and other diverse biological functions within the cell. The purpose of the current study is to characterize the subcellular localization of ncRNA within the mitochondrion, and to understand how import/export mechanisms may dictate changes in RNA diversity during disease progression. Human patient and FVB/NJ mouse cardiac tissues were collected from diabetics $(\mathrm{n}=6)$ and nondiabetics $(\mathrm{n}=6)$. RNA from isolated subcellular compartments and crosslinking immunoprecipitation (CLIP) with Polynucleotide Phosphorylase (PNPase) were sequenced on the Illumina HiSeq and MiSeq. In HL-1 cells, qPCR of PNPase CLIP knockout mutants (KH and S1 knockout) was performed to evaluate mitochondrial/lncRNA interactions. LncRNA targeting sequences and secondary structures were validated through Classification and Regression Trees (CART) and Support Vector Machines (SVM). LncRNAs (MALAT1, NEAT1, and KCNQ1OT1), small nuclear RNA, small nucleolar RNA, and others were discovered in cardiac mitochondria, with diabetic insult significantly reducing expression of ncRNAs. PNPase was shown to bind lncRNA, with a reduced ( $\sim 80 \%)$ binding affinity in the absence of its RNA binding domains. We identified small, stem-loop secondary structures on lncRNA, which could promote mitochondrial/lncRNA interactions. PNPase may function as a lncRNA import/export protein, facilitating cellular regulation in disease states.
\end{abstract}




\section{Introduction}

Long non-coding RNAs (lncRNAs) have emerged as a class of regulatory RNAs that assume multiple responsibilities within the cell. LncRNAs are known to be involved in both nuclear and cytoplasmic cellular processes such as regulating chromatin structure, histone modifications, mRNA transcription, microRNA (miRNA) sponging, and regulation of protein translation and modifications $(64,67)$. With increasing study of the roles of lncRNA in the cytoplasm, the implications of lncRNAs in other subcellular regions remains largely unexplored. The mitochondrion represents an isolated spatially-dense region within the cell that could significantly change the stoichiometric interactions between lncRNAs and other RNA species. Recent investigations have assessed the involvement of lncRNA in altering mitochondrial function (23), but the direct interactions of IncRNA, and other ncRNA, within the mitochondrion remain largely unknown.

The mitochondrion, which encodes for less than $1 \%$ of its own protein components, relies heavily on nuclear transcription and translation of mitochondrial proteins. At the nuclear and cytoplasmic levels, ncRNA can regulate transcription/translation of nuclear-encoded mitochondrial proteins. A lncRNA antisense of PTEN induced putative kinase 1 (naPINK1) was shown to regulate mitochondrial dynamics by inhibiting expression of PTEN mRNA (46). Likewise, miRNAs, such as miRNA-663, have been shown to influence mitochondrial composition by regulating the expression of electron transport chain complex I, II, III, and IV assembly factors (9). While these provide examples of how ncRNAs can impact mitochondrial assembly, ultrastructure, and overall function from outside of the mitochondrion, the regulatory network of ncRNAs within the organelle can also influence function. The mitochondrial genome is comprised of 2 rRNAs, 22 tRNAs, and 13 coding mRNAs (56). Work from our group $(26,48)$, and others (36), has demonstrated miRNAs imported into the mitochondria regulate the mitochondrial genome, largely by inhibiting translation of mitochondrial-encoded genes. Additionally, miRNAs have been shown to activate mitochondrial DNA transcription (69) and the production of miRNAs $(2)$ and $\operatorname{lncRNAs}(18,43)$ from the mitochondrial genome itself.

With the focus typically centered on a very limited number of imported miRNAs and their effects on mitochondrial transcription and health, aspects of miRNA diversity are often lost entirely. Expression profiles within the mitochondrion reveal large quantities of miRNAs (1, 26, 
$28,53)$, even in the absence of mitochondrial DNA (16). While ongoing research is continuing to expand our understanding of ncRNA regulation of the mitochondrial genome, it begs the question: why would a large ncRNA regulatory network be required for maintaining 13 mitochondrial transcribed genes? Imported miRNAs have been identified to alter expression of the mitochondrial genome, but a large portion of miRNAs have shown little, or no, homology with mitochondrial derived sequences $(26,53)$. In understanding the abundance of ncRNAs within the mitochondrion, import/export pathways may provide details regarding the criteria necessary for ncRNA to become included in a mitochondrial regulatory network.

Polynucleotide phosphorylase (PNPase) is a ribonuclease conserved across bacterial, archaeal, and eukaryotic domains of life $(49,54)$. In bacteria, PNPase is a primary regulator of mRNA degradation (25), modifying the cellular response to temperature change (68) and biofilm formation (42), as well as functioning as a processing enzyme for long RNA (45). Progressing through the evolutionary timeline, eukaryotic PNPase is localized specifically to the intermembrane space of the mitochondrion, seemingly lacking its previously defined functional roles. In 2010, Wang et al. first established an RNA import function for PNPase in mammalian systems (60), revealing that tRNA and ribozyme machinery are transported by PNPase into the mitochondrion. Additionally, changes in PNPase expression have resulted in alterations in cellular function and miRNA import $(48,50)$. PNPase, through its KH and S1 RNA binding domains, may provide functional context for ncRNA regulation in the mitochondrion.

In the current study, we investigated the ncRNA regulatory network in the mitochondrion and its changes during disease progression. Specifically, tested if other nuclear-encoded ncRNAs, such as lncRNAs, are present within the mitochondrion. Through comparison of both cytoplasmic and isolated mitochondrial ncRNA populations, we identified hundreds of ncRNAs in the mitochondrion, including MALAT1, NEAT1, and KCNQ1OT1 lncRNAs, that were significantly decreased in diabetes mellitus. MALAT1, NEAT1, and KCNQ1OT1 were shown to bind to PNPase, which may act as a shuttle for import/export of lncRNA mediated by recognition of specific, RNA secondary structures. With the present data, we suggest that the mitochondrion has a dynamic ncRNA regulatory network that can be negatively impacted during disease progression. 


\section{Material \& Methods}

Study Approval and Patient Population

The West Virginia University Institutional Review Board and Institutional Biosafety Committee approved the studies and data generated from this work, including all tissue and patient information acquired (14). Patients were all consented by the Heart and Vascular Institute, J.W. Ruby Memorial Hospital at West Virginia University School of Medicine. Right atrial appendages were removed during open-heart and/or valvular surgeries and all tissue and data were stored in a double de-identified process. There was no incentive provided for patients. Surgeries were performed on both diabetic and non-diabetic patients, following consultation with the surgical team. Non-diabetic patients (HbA1c $<5.7$ with no previous diagnosis of diabetes mellitus) and diabetic patients (HbAlc $>5.7$ or with a previous diagnosis of diabetes mellitus) were designated in the study. Patients' tissue was used irrespective of sex, race, or ethnicity.

\section{Murine Model}

FVB/NJ male and female mice were housed in the West Virginia University Health Sciences Center Animal Facility and given access to a rodent diet and water ad libitum. FVB/NJ wild type mice were bred with FVB/NJ Lepr $^{d b+/}$ mice to obtain a homozygous diabetic model. Animals in the wild type and diabetic groups were aged to 25 weeks and were euthanized for molecular assays using sedation through isoflurane and cervical dislocation. We have previously reported on the cardiovascular deficits of this model $(15,47,48)$. Both sexes were evaluated indiscriminately.

\section{Mitochondrial Isolations}

Mitochondria were isolated from both human right atrial tissue and mouse whole heart. Mitochondrial subpopulations were isolated for analyses as previously described (39), with modifications by our laboratory $(3,4,15)$. Differential centrifugation allowed for the compartmentalization of nuclear, cytoplasmic, and mitochondrial fractions. Mitochondrial subpopulations, subsarcolemmal and interfibrillar, were combined to form a total mitochondrial 
population. Mitochondria were further purified through use of a sucrose gradient $(23 \%, 15 \%, 10 \%$ and $3 \%$ percoll solution), as previously described (26).

\section{PNPase Overexpression Constructs}

Express Cloning Vectors were synthesized through GenScript (Piscataway, NJ) using the pcDNA3.1+N-eGFP backbone. Sequences for the full length PNPase protein (FL), KH domain knockout of exon 23 of PNPase (KH), and the S1 domain knockout of the C-terminus of PNPase (S1) were cloned into vectors, complete sequence information is provided (Figure S4.1). Vectors were then transfected into a bacterial cell line (DH5 $)$, grown through antibiotic selection, and plasmid DNA was isolated for transfection.

\section{Cell culture}

Immortalized HL-1 murine cardiomyocytes were cultured at $37^{\circ} \mathrm{C}, 5 \% \mathrm{CO}_{2}$, as previously described $(12,26,48)$. Cells were supplemented with Claycomb media (Sigma Aldrich, St. Louis, MO) containing 10\% FBS, glutamine $(2 \mathrm{mM})$, penicillin/streptomycin, and norepinephrine $(0.1$ $\mathrm{mM}$ ) (12). Cells were seeded and transfected at 70-80\% confluence. Lipofectamine 3000 (Thermo Fisher, Waltham, MA) was used to transfect cells, per manufacturer's instructions. Briefly, cells were divided into five groups; no plasmid control (NP), pcDNA3.1+N-eGFP only (GFP), FL, KH, and S1 ( $\mathrm{n}=10$ for each group of independent transfections). In the GFP, FL, KH, and S1 sets, 10 $\mu \mathrm{g}$ of each respective plasmid was transfected. Media, with the Lipofectamine 3000, remained on the cells for 48 hours followed by cell imaging using the EVOSTM FL Auto Imaging System (Thermo Fisher) on the GFP fluorescent and phase contrast channels. Cells were washed with PBS, dissociated with $0.05 \%$ Trypsin, and preserved at $-80^{\circ} \mathrm{C}$.

\section{Mitochondrial Bioenergetics}

The GFP, FL, KH, and S1 groups were plated on a Seahorse XF96 V3 cell culture

microplate $(\mathrm{n}=8)$ at a density of 45,000 cells, determined by the Countess II FL Automated Cell Counter (Thermo Fisher). Cells were incubated overnight and the following day analyzed with a 
Seahorse XF Cell Mito Stress Test Kit (Agilent Technologies, Santa Clara, CA) on the Seahorse XF96 Analyzer (Agilent Technologies), per manufacturer's instructions. Information regarding performing the assay has been previously described (22).

\section{Crosslinking Immunoprecipitation (CLIP)}

Cells in the NP, GFP, FL, KH, and S1 groups ( $\mathrm{n}=5$ each), as well as diabetic and nondiabetic human and mouse homogenized heart tissue $(n=5$ each), were re-suspended in PBS and transferred to a 24-well plate on ice. Crosslinking was performed using the CX-2000 Crosslinker (Analytik Jena, Upland, CA) at $\left(400 \mathrm{~mJ} / \mathrm{cm}^{2}\right) 5$ times, as previously described (26) with the following modifications. After crosslinking, cells were centrifuged $(1,200 \mathrm{~g})$ for 7 minutes and subsequently frozen $\left(-80^{\circ} \mathrm{C}\right)$ for further use. $75 \mu \mathrm{L}$ per sample of Dynabeads ${ }^{\mathrm{TM}}$ Protein $\mathrm{G}$ (Thermo Fisher) were prepared through 3 washes with NP-40 buffer (20mM Tris (pH 8.0), $137 \mathrm{mM} \mathrm{NaCl}$, $10 \%$ Glycerol, 1\% Triton X100, 2mM EDTA, 0.1mM PMSF). After the third wash, the supernatant on the beads was discarded and $5 \mu \mathrm{g}$ of anti-GFP was added, along with NP-40 buffer, to reach a total volume of $100 \mu \mathrm{L}$. Beads were incubated overnight at $4^{\circ} \mathrm{C}$. The next day, each sample was resuspended in NP-40 Buffer to a total volume of $1 \mathrm{~mL}$, and protein concentrations determined through the Bradford Method (6). $100 \mu \mathrm{g}$ of protein from each sample was added to a new tube followed by RNAse I treatment (1:500 dilution, Ambion ${ }^{\mathrm{TM}}$ RNase I, Thermo Fisher) for 3 minutes at $37^{\circ} \mathrm{C}$, which allowed for partial digestion of RNA bound to PNPase. The reaction was stopped by incubating the samples on ice. The supernatant on the beads solution was then removed and the protein mixture was added and incubated at $4^{\circ} \mathrm{C}$ for 4 hours. Following the incubation, beads were washed 3 times in NP-40 buffer, final supernatant removed, and a mixture of water/NuPAGETM LDS Sample Buffer (4X) (Thermo Fisher) was added to the beads. The beads solution was heated at $70^{\circ} \mathrm{C}$ for 15 minutes and assessed through immunoblotting.

\section{Western Blotting}

Using $4-12 \%$ gradient gels, immunoblotting was performed through MOPS SDS-PAGE, as previously described $(13,21,22,38,57)$. Using the Bradford Method, protein concentrations were normalized. Primary antibodies implemented in the study: anti-GFP, 1:1000 ((B-2): sc-9996, 
SCBT, Dallas, TX), anti-PNPase, ((C-5): sc-271973, SCBT, Dallas, TX), anti-PNPase, ((D-1): sc271479, SCBT, Dallas, TX), and anti-GAPDH 1:1000 (Abcam, Cambridge, MA). Goat antimouse IgG (H\&L) horseradish peroxidase (HRP) conjugate 1:10,000 (Thermo Fisher) was used as the secondary antibody. Normalization of protein content was through GAPDH expression. Chemiluminescence quantified with Radiance Chemiluminescent Substrate (Azure Biosystems, Dublin, CA), per manufacturer's instructions and imaged using the G:Box Bioimaging system (Syngene, Frederick, MD). Images were taken with GeneSnap/GeneTools software (Syngene). Densitometry was analyzed using ImageJ and Fiji Software (NIH, Bethesda, MD). For CLIP samples, the area of nitrocellulose membrane containing the GFP (cells) or PNPase (human and mouse) fluorescent region was excised and saved for RNA analysis.

\section{RNA Isolation/Quantitative PCR}

Using the miRNeasy Mini Kit (product no.: 217004, Qiagen, Hilden, Germany), per manufacturer's instructions, RNA was isolated from $20 \mathrm{mg}$ of human right atrial tissue and mouse whole heart and from nitrocellulose membrane containing RNA derived from CLIP samples. Each sample was homogenized in QIAzol lysis reagent before proceeding. Total RNA was isolated for each group and RNA to be analyzed through qPCR was converted to cDNA through the HighCapacity RNA-to-cDNA ${ }^{\mathrm{TM}}$ Kit (Thermo Fisher), per manufacturer's instructions. Differential gene expression was assessed for MT-ND3 and MT-ATP6 in mouse cytoplasmic and mitochondrial fractions, as well as Malat1-201 and Neat1-201 in mitochondrial fractions. Malat1 expression was also measured in HL-1 cells following PNPase CLIP (as described above). All primer sequences are provided (Table S4.1). Primers were designed through NCBI Primer BLAST and Primer3 (59). Experiments were performed on the Applied Biosystems 7900HT Fast RealTime PCR system (Applied Biosystems, Foster City, CA), using 2X SYBR Green Master Mix. Quantification was achieved using the $2^{-\Delta \Delta C}$ T method (32), standardized to U6 and Gapdh expression where appropriate.

\section{Long non-coding RNA (LncRNA) Sequencing}


RNA, isolated from human atrial appendages and mouse whole heart (as described above), was sequenced through the West Virginia University Genomics Core Facility. Briefly, RNA was selected for long non-coding RNA analysis using a Ribo-Zero rRNA Removal Kit (Illumina, San Diego, CA). Samples were run on the HiSeq 2500 (Illumina) in 51bp paired end reads achieving $\sim 30$ million clustered reads per sample. Generated Fastq files were processed through HISAT2 $(27,41)$ under standard parameters, with the exception of quality scoring control specified under “--phred33." The reference genome used in generating BAM files included fasta formatted DNA from Ensemble release 95 for human (GRCh38) and mouse (GRCm38). Differential gene expression was performed in the R environment, through DESeq2 (35). Visualization through ggplot2 (63) and packages vidger, EnhancedVolcano, and others. The complete differential expression profiles of mitochondrial and cytoplasmic groups are included (Additional File 1).

\section{Small ncRNA Sequencing}

RNA isolated from human atrial appendages and mouse whole heart (as described above) was sequenced through the West Virginia University Genomics Core Facility. Small RNA was selected using the NEXTFLEX ${ }^{\circledR}$ Small RNA-Seq Kit v3 (Bioo Scientific, Austin, TX). Samples were run on the HiSeq 2500 in Rapid Run (Illumina) in 64bp single end reads on two lanes achieving 12 million clustered reads per sample. Adapter trimming through cutadapt (37) (5TGGAATTCTCGGGTGCCAAGG -3) allowed for isolation of genomic reads. Generated Fastq files were processed through Bowtie (29) using the parameters, “-a -m 6 --best --strata --chunkmbs 250." The reference genome used in generating BAM files included fasta formatted DNA from Ensemble release 94 for human (GRCh38). Differential gene expression was assessed in the R environment, as indicated above. MiRNA sequencing data was analyzed by the use of IPA (QIAGEN Inc., https://www.qiagenbioinformatics.com/products/ingenuitypathway-analysis). The complete differential expression profiles are included (Additional File 1).

\section{High-Throughput Sequencing Crosslinking Immunoprecipitation (HITS-CLIP)}

RNA isolated from human atrial appendages and mouse whole heart (as described above) was sequenced through the West Virginia University Genomics Core Facility. RNA was selected 
for using the NEXTFLEX ${ }^{\circledR}$ Small RNA-Seq Kit v3 (Bioo Scientific). Samples were run on the MiSeq in 36bp single end reads achieving $~ 1$ million clustered reads per sample. Adapter trimming through cutadapt and sequence alignment was performed as indicated above. Generated Fastq files were processed through Bowtie using the parameters, "-a -m 6 --best --strata --chunkmbs 250." The reference genome used in generating BAM files included fasta formatted DNA from Ensemble release 96 for human (GRCh38) and mouse (GRCm38). Differential gene expression was assessed in the $\mathrm{R}$ environment, as indicated above. The raw counts values for each sample are included (Additional File 1).

\section{Mitochondrial Targeting Sequences}

To determine if lncRNA that were found in the mitochondrion and associated with PNPase had sequence or secondary structure homology, RNAfold through the ViennaRNA Package was employed (34). RNAfold was used in conjunction with LncFinder (20) and seqinR (10) to evaluate folding parameters of both the full length lncRNA, as well as isolated regions (12-20 nucleotides) shown to bind to PNPase in CLIP analyses. Isolated regions were identified for 50 human and 50 mouse lncRNA labeled "positive" (found in CLIP Seq) and 100 lncRNA labeled "negative" (randomly generated sequences (55)). The isolated regions from genes identified within the PNPase CLIP were flanked by surrounding nucleotides within the genome to achieve 60 nucleotide sequences implemented in the subsequent RNAfold and machine learning applications. The randomly generated sequences also consisted of 60 nucleotides. The sequences directly identified in the CLIP Seq, as well as flanking regions, are provided for each positive control gene (Additional File 1).

\section{Machine Learning for RNA Sequences}

Using 10-fold cross validation, classification of "positive" and "negative" 60 nucleotide isolated regions was assessed through Classification and Regression Tress (CART) in randomForest (30) and rfUtilities (17). Machine learning algorithms were performed on 105 features, which included primary sequence and secondary structure information (Additional File 1). Briefly, rf.crossValidation was used with a randomForest object to provide 10 -fold cross 
validation. An unsupervised learning approach was applied to only the positive 60 nucleotide regions through $r$ f.unsupervised, with the identification of four specific clusters. As a confirmatory measure, Support Vector Machines (SVM), using the svm_cv function of LncFinder, was implemented to determine 10 -fold cross validation of full length and isolated regions.

\section{Statistics}

A two-sided Student's t-test or one-way analysis of variance (ANOVA) were used to determine statistical differences, where appropriate. Multiple groups were assessed through Tukey's multiple comparisons test following the ANOVA. Differences between groups were considered statistically different if $P \leq 0.05$, denoted by *. Data are presented as the mean \pm standard error of the mean (SEM), when appropriate. For all sequencing data, raw counts $>2$ were considered in statistical analyses. The false-discovery rate (FDR) was set to 0.05 and all significance was determined through a $P$ adjusted value $\left(P_{a d j}\right)<0.05$ using Wald testing. 


\section{Results}

Mitochondrial Isolation Purity

To evaluate the subcellular localization of non-coding RNA (ncRNA), assessments of fractionation purity are needed to confirm subsequent findings and accuracy. Tissue homogenization, differential centrifugation, and sucrose gradient separation allow for the enrichment of pure populations of mitochondria (Figure 4.1A). Mitochondrial contamination was determined through the presence of cytoplasmic ribosomal RNA (rRNA) within mitochondrial RNA fractions (Figure 4.1B). Typically considered a measure for degradation of RNA, proper isolation of mitochondrial RNA retains minimal traces of cytoplasmic rRNA $(1,26)$. Additionally, qPCR of mitochondrially derived mRNA revealed a significant difference ( $\sim 40-50$ fold) between mitochondrial and cytoplasmic compartments (Figure 4.1C). Sequencing data indicated a reduced expression of mitochondrial transcribed genes within the cytoplasm compared to mitochondria by 30 - 500 fold (Additional File 1). Mitochondrial populations revealed a clear separation from cytoplasmic portions of the cell.

\section{Mitochondrial MiRNAs}

To understand the cellular mechanisms governing mitochondrial import of nuclearencoded miRNAs, abundance, fold change, and ontology pathways were assessed. In human patient isolated mitochondria, the twenty most abundant miRNAs are provided (Figure 4.2A). In the presence of diabetic insult, 225 miRNAs were shown to be significantly downregulated within mitochondria, with one miRNA revealing a significant upregulation (Figure 4.2B). Of the miRNAs differentially impacted, Ingenuity Pathway Analysis (IPA) suggested that proteins outside of the mitochondrion were those most significantly impacted, with an emphasis on metabolic pathways (Figure 4.2C). When further examining the cellular pathways impacted by nuclear-encoded miRNAs within mitochondria, their key functions included cellular development, survival, and growth (Figure 4.2D). The complete miRNA expression profile is provided (Additional File 1). MiRNAs are both abundantly found within the mitochondrion and have altered expression profiles in disease states, suggesting significant fluctuations in import/export of miRNAs. 
Long non-coding RNA (IncRNA) Characterization of the Mitochondrion

While miRNAs have already been identified as ncRNA species in the mitochondrion, we wanted to assess if other ncRNAs interacted with the mitochondrion, and if more advanced mitochondrial regulatory networks exist and are altered during diabetic insult. Using a long noncoding RNA (lncRNA) sequencing approach on sucrose-purified mitochondria, we found that a variety of ncRNA were differentially expressed in both the diabetic human and mouse heart (Figure 4.3A). Of these, the top genes, as delineated by an adjusted $P$ value $\left(P_{a d j}\right)$, are illustrated for the human (MALAT1) and mouse (Mhrt) sequencing results (Figure 4.3B). PCA plots illustrated the dispersion between cytoplasmic and mitochondrial RNA isolates, with a greater diversity observed between human mitochondria in the disease state, but not the cytoplasm (Figure 4.3C). Differential expression profiles for all ncRNA genes in human and mouse (mitochondrial and cytoplasmic) cohorts are provided (Additional File 1). LncRNAs, as well as other ncRNAs, are present in the mitochondria with diabetic insult significantly altering expression profiles.

\section{Diabetic Mellitus ncRNA Loss of Diversity}

To understand how the ncRNA expression profiles are altered between the subcellular compartments and disease states, we assessed the number of differential genes per group (Figure 4.4A). Using these comparisons, we also wanted to understand the general $\log _{2}$ fold change between groups. In diabetic compared to non-diabetic mitochondria, the majority of differentially expressed genes were downregulated (Figure 4.4B). Of the two largest lncRNA populations, the top 5 differentially expressed transcripts are displayed for antisense and long-intergenic ncRNA (lincRNA), determined by $P_{a d j}<0.05$ and containing the highest read counts in each group (Figure 4.4C). Because MALAT1, NEAT1, KCNQ1OT1, and Mhrt (no human equivalent) revealed similar expression profiles between human and mouse groups (Figure 4.4C), we wanted to evaluate the expression profiles of these genes when compared between different disease states and subcellular compartments (Figure 4.4D). MALAT1, NEAT1, and KCNQ1OT1 in human and mouse models revealed a marked downregulation in diabetic mitochondria with unchanging or increased expression in diabetic cytoplasm (Additional File 1, Figure 4.4D). Malat1 and Neat1 expression were further confirmed through qPCR (Figure S4.2A). In a disease state, diminished 
localization of lncRNA and other ncRNAs in the mitochondrion (Figure S4.2B) mimic the miRNA response, suggesting a decrease in total ncRNA diversity.

\section{Polynucleotide Phosphorylase (PNPase) Interactions with ncRNA}

With data suggesting the immense diversity of the mitochondrial ncRNA regulatory network, and that in diabetes mellitus this network becomes impaired, we wanted to evaluate mitochondrial ncRNA import/export mechanisms. Cross-linking immunoprecipitation (CLIP) of PNPase in human and mouse cohorts revealed a propensity for longer ncRNAs to bind to the protein (Additional File 1). A gene comparison between human and mouse PNPase CLIP revealed 31 homologous ncRNA, including MALAT1, NEAT1, and KCNQ1OT1 (Figure 4.5A). The composition of ncRNA bound to PNPase is displayed (Figure 4.5B), with antisense and lincRNA transcripts being the most prevalent. We wanted to further investigate how PNPase may be involved in shuttling mechanics for ncRNA through its $\mathrm{KH}$ and S1 RNA binding domains. Using an HL-1 immortalized mouse cardiomyocyte cell line, we transfected cells with overexpression vectors (Figure 4.5C). Overexpression of constructs was demonstrated through immunoblotting, with a shift in size between the full length, KH domain knockout, and S1 domain knockout (Figure 4.5D). Using CLIP for PNPase in each of the cell variants, RNA was purified in each of the overexpression groups through SDS-PAGE gel electrophoresis, with a representative gel illustrated (Figure 4.5E). Examining the RNA bound to PNPase, MALAT1 expression was shown to be significantly downregulated when the $\mathrm{S} 1$ domain was removed from PNPase (Figure 4.5F), suggesting its role in RNA-protein interactions. Functionally, basal respiration and ATP production were significantly decreased in both the $\mathrm{KH}$ and $\mathrm{S} 1$ domain knockout groups, with maximal respiration and proton leak decreased in the S1 domain knockout group (Figure S4.2C). These results suggest that PNPase has binding affinity for lncRNA, most likely through the KH and/or S1 RNA binding domains.

\section{Mitochondrial RNA Targeting Sequence}

Examining the CLIP PNPase sequencing data, we wanted to determine if a mitochondrial RNA targeting sequence or secondary structure exists. A 60 nucleotide region, directly centered 
on reads acquired from sequencing, was implemented to determine RNA primary sequence and secondary structure homology through RNAfold (34). RNAfold secondary structures for MALAT1, NEAT1, and KCNQ1OT1 illustrate that RNA sequences bound to PNPase are found in stem-loop structures in both the full length (Figure S4.3) and 60 nucleotide isolated regions (Figure 4.6A). Implementing 10-fold cross validation for Support Vector Machines (SVM) and Classification and Regression Trees (CART), machine learning was applied to 50 human and 50 mouse lncRNA 60 nucleotide reads found to bind to PNPase (positives) and 100 randomly generated 60 nucleotide sequences (negatives) (55) (Table S4.2). Both SVM and CART attained close to $75 \%$ prediction accuracy on the entire dataset, for classification of either positive or negative 60 nucleotide sequences (Figure 4.6B) (Table S4.2). Of note, due to the random generation of the 60 base-pair regions, it is possible for some of the negative controls to share similar properties with the positive group, limiting the predictive power of the model.

Area under the curve (AUC) averaged $\sim 0.75$, with various iterations ranging from $0.65-$ 0.85 (Figure 4.6C). To determine the degree of heterogeneity between 60 nucleotide regions identified as positives, unsupervised learning through CART was applied to examine separation into four subtypes (Figure 4.6D). 10-fold cross validation was further applied to each of the subtypes compared to the 100 negative sequences, (Table S4.2), revealing the distinct primary and secondary structure differences between sequences identified to bind to PNPase (Figure 4.6E). Further, parameters of the LncFinder algorithms, including acguD (Figure S4.4A) and acguACGU (Figure S4.4B), suggest that RNA bound to PNPase has a greater propensity for unpaired loop structures, with differences also in the folding energy (Figure S4.4C) and sequence GC content (Figure S4.4D). Both supervised (Additional File 1) and unsupervised CART models suggest that secondary, not primary sequence, is important for RNA identification by the mitochondrion. Predominately, a stem-loop and internal loop structure may be necessary for recognition of lncRNA by PNPase (Figure 4.7). 


\section{Discussion}

Our knowledge of the mitochondrion has continued to broaden, with the discovery of new regulatory functions of the organelle and how these diverse functions impact tissue and cellular health. For the last ten years in the field of mitochondrial biology, a question has persisted regarding the purpose and function of nuclear-encoded miRNAs in the mitochondrion. In this study, for the first time, we suggest the existence of a novel mitochondrial regulatory network, which helps to clarify the presence, and purpose, of miRNAs in mitochondria. Our data indicate that the primary purpose of ncRNAs in mitochondria are not to regulate the mitochondrial genome, but rather to provide a repository of regulatory RNA, which can be altered during disease states. We suggest that PNPase, through interaction with long non-coding RNA (lncRNA), may play a significant role in regulating this response within the mitochondrion.

The interaction of lncRNA with mitochondrial function has been shown through a variety of alternative pathways. One of the most prominently studied pathways is the interaction of lncRNA with genes and genomic regions responsible for contributing to substrate utilization within the cell. Altering metabolic function has been shown through sponging of miRNAs that inhibit mRNA $(52,62)$, interactions with proteins directly (44), and through regulation of transcription in the nucleus (19). It has even been proposed, and further validated, that the mitochondrial genome itself produces lncRNAs $(18,43)$. In all of these investigations, few have assessed how nuclear produced lncRNAs can interact directly with the mitochondrion. The complete lncRNA interaction network that exists as part of the mitochondrial axis could play a pivotal role in both the maintenance of mitochondrial function and the sequestration of other ncRNAs.

Kren et al. was one of the first to postulate the idea of sequestration (28). In a microarray study discovering 15 miRNAs, the authors suggested that due to miRNAs inhibiting multiple mRNA targets, those mRNA may be shuttled into the mitochondria and regulated during specific cellular conditions. While the field has generally moved away from this idea of sequestration, there may be significant biological advantages to operating under those conditions. The data in this manuscript help support the concept of sequestration in four ways. 1) The number of miRNAs, lncRNAs, and other ncRNAs found within and/or directly interacting with the mitochondrion is likely greater than 1,000 different species. The mitochondrial genome, composed of 13 transcribed 
genes, and the mitochondrial proteome, composed of $\sim 1,200$, lack an inherent diversity for targeting by all of the ncRNAs (8). 2) The stoichiometric ratio of some RNAs, specifically lncRNA and miRNA, could be significantly elevated in a sub-compartment of the cell $(33,58)$. LncRNA, which may exist in the cytoplasm at a relatively low abundance, can be amplified through localization into the mitochondrion. 3) MALAT1, NEAT1, KCNQ1OT1, and many of the other lncRNAs found in the sequencing data have verified sponging regions for nuclear-encoded miRNAs in the mitochondria (40). 4) The mitochondrial matrix provides an environment promoting lncRNA folding/processing. High concentrations of magnesium and manganese ions could increase the stabilization of lncRNA secondary and tertiary structures $(24,51,66)$, along with further processing through small nuclear RNA (snoRNA) (65) (Additional File 1).

In the current study, we found that MALAT1, along with NEAT1, KCNQ1OT1, and others, may be important lncRNAs in mitochondria during diabetic progression. The increase of MALAT1 has been linked to a variety of pathogenic responses in diabetes mellitus $(5,11,31)$. Reduction, or complete knockout, of MALAT1 expression contributed to reduced ROS, inflammatory signaling, and negative regulatory effects to the genome. We showed that MALAT1, though not significantly elevated in the cytoplasm, was decreased in diabetic mitochondria. While we depict a movement toward decreased ncRNA diversity in diabetic mitochondria, it could be that under more overt stress, e.g. infection or injury, the mitochondrial regulatory network is less adaptable to attenuating the response. Additionally, reduction of lncRNA in the mitochondria may not necessarily mean that ncRNA is transferred to other locals within the cell; it could suggest that lncRNAs are degraded in the mitochondria, limiting the potential for sequestration of other ncRNAs, such as miRNA.

The bacterial version of PNPase exists as an enzyme with a primary purpose of modifying and degrading RNA species (7). The mammalian homolog, instead of remaining a free-floating enzyme, is localized to the mitochondrial intermembrane space. While not directly accessible to the cytoplasm or mitochondrial matrix, studies have reported the ability of PNPase to interact with and transport RNAs into the mitochondrion $(60,61)$. The KH and S1 RNA binding domains of PNPase are suggested as the mechanism for channeling ncRNA through the mitochondrial intermembrane space into the matrix (60). Importantly, Wang et al. suggested that a stem-loop sequence on the RNA was necessary for mitochondrial import (60). These findings support a 
mechanism whereby PNPase recognition requires RNA secondary structure as opposed to sequence specificity, which is consistent with our work, including the PNPase CLIP sequencing and machine learning approaches. The framework from LncFinder and the machine learning algorithms begins to provide an archetype for designing personalized lncRNA sequences to target to the mitochondrion; specifically, through incorporating stem-loop structures with more unpaired bases within the loop structures.

We have observed that ncRNAs, including lncRNA, are present in the mitochondrion and that overall diversity in the diabetic mitochondrion is lost. While not focused on in this manuscript, we observed the presence of other ncRNAs, such as snoRNA and small nuclear RNA (snRNA), which may prove to have their own unique biological role, import mechanism, and persistence pattern in the mitochondrion. To determine if ncRNA are degraded or further shuttled to other compartments of the cell, future investigations should evaluate the total cellular levels of lncRNA and miRNA that are downregulated in mitochondria following diabetic insult. The current study provides a more complete understanding of the ncRNA regulatory network of the mitochondrion, while revealing changes that occur during pathological insult. The shared features of lncRNA bound to PNPase highlight a novel mitochondrial RNA import mechanism, through the presence of stem-loop secondary structures, and suggest the presence of an interaction network with mitochondria. Within the mitochondrion, lncRNA would have the capacity to interact with other ncRNAs, such as miRNAs, possibly through sponging and regulation of cellular processes. The expanded mitochondrial regulatory network proposed in this study provides a new perspective on mitochondrial biology and continues to expand our conception of mitochondria as the "powerhouse of the cell." 


\section{Data Availability}

All source code and sequencing data files have been made freely available:

Source code at Github: https://github.com/qahathaway/Mitochondria PNPase LncRNA.

Sequencing data and sample identification at NCBI SRA under BioProject: PRJNA553501

Differential expression analyses (Additional File 1)

\section{Supplementary Data}

Supplementary data is included in the study, specifically supporting data (Tables S4.1-4.2, Figure S4.1-4.4) and differential expression analyses for next-generation sequencing experiments (Additional File 1)

\section{Funding}

This work was supported by: The National Heart, Lung, and Blood Institute [R01 HL128485] (JMH), American Heart Association [AHA-17PRE33660333] (QAH), West Virginia IDeA Network of Biomedical Research WV-INBRE support by National Institute of Health Grant [P20GM103434], and the Community Foundation for the Ohio Valley Whipkey Trust.

\section{Conflict of Interest}

The authors have declared that no conflict of interest exists.

\section{Acknowledgements}

We would like to acknowledge the WVU Genomics Core Facility, Morgantown WV as well as the Marshall University Genomics Core Facility, Huntington, WV for support provided to help make this publication possible. We would like to thank the West Virginia University Ruby Memorial Hospital Heart and Vascular Institute for collaborating on this work. 


\section{References}

1. Bandiera S, Ruberg S, Girard M, Cagnard N, Hanein S, Chretien D, Munnich A, Lyonnet S, and Henrion-Caude A. Nuclear outsourcing of RNA interference components to human mitochondria. PLoS One 6: e20746, 2011.

2. Barrey E, Saint-Auret G, Bonnamy B, Damas D, Boyer O, and Gidrol X. PremicroRNA and mature microRNA in human mitochondria. PLoS One 6: e20220, 2011.

3. Baseler WA, Dabkowski ER, Jagannathan R, Thapa D, Nichols CE, Shepherd DL, Croston TL, Powell M, Razunguzwa TT, Lewis SE, Schnell DM, and Hollander JM. Reversal of mitochondrial proteomic loss in Type 1 diabetic heart with overexpression of phospholipid hydroperoxide glutathione peroxidase. Am J Physiol Regul Integr Comp Physiol 304: R553-565, 2013.

4. Baseler WA, Dabkowski ER, Williamson CL, Croston TL, Thapa D, Powell MJ, Razunguzwa TT, and Hollander JM. Proteomic alterations of distinct mitochondrial subpopulations in the type 1 diabetic heart: contribution of protein import dysfunction. $A m J$ Physiol Regul Integr Comp Physiol 300: R186-200, 2011.

5. Biswas S, Thomas AA, Chen S, Aref-Eshghi E, Feng B, Gonder J, Sadikovic B, and Chakrabarti S. MALAT1: An Epigenetic Regulator of Inflammation in Diabetic Retinopathy. Sci Rep 8: 6526, 2018.

6. Bradford MM. A rapid and sensitive method for the quantitation of microgram quantities of protein utilizing the principle of protein-dye binding. Anal Biochem 72: 248-254, 1976.

7. Briani F, Carzaniga T, and Deho G. Regulation and functions of bacterial PNPase. Wiley Interdiscip Rev RNA 7: 241-258, 2016.

8. Calvo SE and Mootha VK. The mitochondrial proteome and human disease. Annu Rev Genomics Hum Genet 11:25-44, 2010.

9. Carden T, Singh B, Mooga V, Bajpai P, and Singh KK. Epigenetic modification of miR663 controls mitochondria-to-nucleus retrograde signaling and tumor progression. $J$ Biol Chem 292: 20694-20706, 2017.

10. Charif $D$ and Lobry JR. SeqinR 1.0-2: a contributed package to the $\{R\}$ project for statistical computing devoted to biological sequences retrieval and analysis. In: Structural approaches to sequence evolution: Molecules, networks, populations, edited by Bastolla U, Porto M, Roman HE and Vendruscolo M. New York: Springer Verlag, 2007, p. 207-232.

11. Chen J, Ke S, Zhong L, Wu J, Tseng A, Morpurgo B, Golovko A, Wang G, Cai JJ, Ma X, Li D, and Tian Y. Long noncoding RNA MALAT1 regulates generation of reactive oxygen species and the insulin responses in male mice. Biochem Pharmacol 152: 94-103, 2018.

12. Claycomb WC, Lanson NA, Jr., Stallworth BS, Egeland DB, Delcarpio JB, Bahinski A, and Izzo NJ, Jr. HL-1 cells: a cardiac muscle cell line that contracts and retains phenotypic characteristics of the adult cardiomyocyte. Proc Natl Acad Sci U S A 95: 2979-2984, 1998.

13. Croston TL, Shepherd DL, Thapa D, Nichols CE, Lewis SE, Dabkowski ER, Jagannathan R, Baseler WA, and Hollander JM. Evaluation of the cardiolipin biosynthetic pathway and its interactions in the diabetic heart. Life Sci 93: 313-322, 2013.

14. Croston TL, Thapa D, Holden AA, Tveter KJ, Lewis SE, Shepherd DL, Nichols CE, Long DM, Olfert IM, Jagannathan R, and Hollander JM. Functional deficiencies of subsarcolemmal mitochondria in the type 2 diabetic human heart. Am J Physiol Heart Circ Physiol 307: H54-65, 2014. 
15. Dabkowski ER, Baseler WA, Williamson CL, Powell M, Razunguzwa TT, Frisbee JC, and Hollander JM. Mitochondrial dysfunction in the type 2 diabetic heart is associated with alterations in spatially distinct mitochondrial proteomes. Am J Physiol Heart Circ Physiol 299: H529-540, 2010.

16. Dasgupta N, Peng Y, Tan Z, Ciraolo G, Wang D, and Li R. miRNAs in mtDNA-less cell mitochondria. Cell Death Discov 1: 15004, 2015.

17. Evans JS, Murphy MA, Holden ZA, and Cushman SA. Modeling species distribution and change using Random Forests. In: Predictive species and habitat modeling in landscape ecology: concepts and applications, edited by Drew CA, Wiersma YF and Huettmann F. New York: Springer, 2011, p. 139-159.

18. Gao S, Tian X, Chang H, Sun Y, Wu Z, Cheng Z, Dong P, Zhao Q, Ruan J, and Bu W. Two novel lncRNAs discovered in human mitochondrial DNA using PacBio full-length transcriptome data. Mitochondrion 38: 41-47, 2018.

19. Gupta RA, Shah N, Wang KC, Kim J, Horlings HM, Wong DJ, Tsai MC, Hung T, Argani P, Rinn JL, Wang Y, Brzoska P, Kong B, Li R, West RB, van de Vijver MJ, Sukumar S, and Chang HY. Long non-coding RNA HOTAIR reprograms chromatin state to promote cancer metastasis. Nature 464: 1071-1076, 2010.

20. Han S, Liang Y, Ma Q, Xu Y, Zhang Y, Du W, Wang C, and Li Y. LncFinder: an integrated platform for long non-coding RNA identification utilizing sequence intrinsic composition, structural information and physicochemical property. Brief Bioinform, 2018.

21. Hathaway QA, Durr AJ, Shepherd DL, Pinti MV, Brandebura AN, Nichols CE, Kunovac A, Goldsmith WT, Friend SA, Abukabda AB, Fink GK, Nurkiewicz TR, and Hollander JM. miRNA-378a as a key regulator of cardiovascular health following engineered nanomaterial inhalation exposure. Nanotoxicology: 1-20, 2019.

22. Hathaway QA, Nichols CE, Shepherd DL, Stapleton PA, McLaughlin SL, Stricker JC, Rellick SL, Pinti MV, Abukabda AB, McBride CR, Yi J, Stine SM, Nurkiewicz TR, and Hollander JM. Maternal-engineered nanomaterial exposure disrupts progeny cardiac function and bioenergetics. Am J Physiol Heart Circ Physiol 312: H446-H458, 2017.

23. Hathaway QA, Pinti MV, Durr AJ, Waris S, Shepherd DL, and Hollander JM. Regulating microRNA expression: at the heart of diabetes mellitus and the mitochondrion. Am $J$ Physiol Heart Circ Physiol 314: H293-H310, 2018.

24. Hayes RL, Noel JK, Mohanty U, Whitford PC, Hennelly SP, Onuchic JN, and Sanbonmatsu KY. Magnesium fluctuations modulate RNA dynamics in the SAM-I riboswitch. $J$ Am Chem Soc 134: 12043-12053, 2012.

25. Hui MP, Foley PL, and Belasco JG. Messenger RNA degradation in bacterial cells. Annu Rev Genet 48: 537-559, 2014.

26. Jagannathan R, Thapa D, Nichols CE, Shepherd DL, Stricker JC, Croston TL, Baseler WA, Lewis SE, Martinez I, and Hollander JM. Translational Regulation of the Mitochondrial Genome Following Redistribution of Mitochondrial MicroRNA in the Diabetic Heart. Circ Cardiovasc Genet 8: 785-802, 2015.

27. Kim D, Langmead B, and Salzberg SL. HISAT: a fast spliced aligner with low memory requirements. Nat Methods 12: 357-360, 2015.

28. Kren BT, Wong PY, Sarver A, Zhang X, Zeng Y, and Steer CJ. MicroRNAs identified in highly purified liver-derived mitochondria may play a role in apoptosis. RNA Biol 6: 65-72, 2009. 
29. Langmead B, Trapnell C, Pop M, and Salzberg SL. Ultrafast and memory-efficient alignment of short DNA sequences to the human genome. Genome Biol 10: R25, 2009.

30. Liaw A and Wiener M. Classification and Regression by randomForest. $R$ News 2: 1822, 2002.

31. Liu JY, Yao J, Li XM, Song YC, Wang XQ, Li YJ, Yan B, and Jiang Q. Pathogenic role of lncRNA-MALAT1 in endothelial cell dysfunction in diabetes mellitus. Cell Death Dis 5: e1506, 2014.

32. Livak KJ and Schmittgen TD. Analysis of relative gene expression data using real-time quantitative PCR and the 2(-Delta Delta C(T)) Method. Methods 25: 402-408, 2001.

33. Long Y, Wang X, Youmans DT, and Cech TR. How do lncRNAs regulate transcription? Sci Adv 3: eaao2110, 2017.

34. Lorenz R, Bernhart SH, Honer Zu Siederdissen C, Tafer H, Flamm C, Stadler PF, and Hofacker IL. ViennaRNA Package 2.0. Algorithms Mol Biol 6: 26, 2011.

35. Love MI, Huber W, and Anders S. Moderated estimation of fold change and dispersion for RNA-seq data with DESeq2. Genome Biol 15: 550, 2014.

36. Macgregor-Das AM and Das S. A microRNA's journey to the center of the mitochondria. Am J Physiol Heart Circ Physiol 315: H206-H215, 2018.

37. Martin M. Cutadapt Removes Adapter Sequences From High-Throughput Sequencing Reads. EMBnetjournal 17, 2011.

38. Nichols CE, Shepherd DL, Knuckles TL, Thapa D, Stricker JC, Stapleton PA, Minarchick VC, Erdely A, Zeidler-Erdely PC, Alway SE, Nurkiewicz TR, and Hollander JM. Cardiac and mitochondrial dysfunction following acute pulmonary exposure to mountaintop removal mining particulate matter. Am J Physiol Heart Circ Physiol 309: H2017-2030, 2015.

39. Palmer JW, Tandler B, and Hoppel CL. Biochemical properties of subsarcolemmal and interfibrillar mitochondria isolated from rat cardiac muscle. J Biol Chem 252: 8731-8739, 1977.

40. Paraskevopoulou MD, Vlachos IS, Karagkouni D, Georgakilas G, Kanellos I, Vergoulis T, Zagganas K, Tsanakas P, Floros E, Dalamagas T, and Hatzigeorgiou AG. DIANA-LncBase v2: indexing microRNA targets on non-coding transcripts. Nucleic Acids Res 44: D231-238, 2016.

41. Pertea M, Kim D, Pertea GM, Leek JT, and Salzberg SL. Transcript-level expression analysis of RNA-seq experiments with HISAT, StringTie and Ballgown. Nat Protoc 11: 16501667, 2016.

42. Pobre V and Arraiano CM. Next generation sequencing analysis reveals that the ribonucleases RNase II, RNase R and PNPase affect bacterial motility and biofilm formation in $\mathrm{E}$. coli. BMC Genomics 16: 72, 2015.

43. Rackham O, Shearwood AM, Mercer TR, Davies SM, Mattick JS, and Filipovska A. Long noncoding RNAs are generated from the mitochondrial genome and regulated by nuclearencoded proteins. RNA 17: 2085-2093, 2011.

44. Salani B, Ravera S, Amaro A, Salis A, Passalacqua M, Millo E, Damonte G, Marini C, Pfeffer U, Sambuceti G, Cordera R, and Maggi D. IGF1 regulates PKM2 function through Akt phosphorylation. Cell Cycle 14: 1559-1567, 2015.

45. Saramago M, Barria C, Dos Santos RF, Silva IJ, Pobre V, Domingues S, Andrade JM, Viegas SC, and Arraiano CM. The role of RNases in the regulation of small RNAs. Curr Opin Microbiol 18: 105-115, 2014.

46. Scheele C, Petrovic N, Faghihi MA, Lassmann T, Fredriksson K, Rooyackers $\mathbf{O}$, Wahlestedt C, Good L, and Timmons JA. The human PINK1 locus is regulated in vivo by a 
non-coding natural antisense RNA during modulation of mitochondrial function. BMC Genomics 8: 74, 2007.

47. Shepherd DL, Hathaway QA, Nichols CE, Durr AJ, Pinti MV, Hughes KM, Kunovac A, Stine SM, and Hollander JM. Mitochondrial proteome disruption in the diabetic heart through targeted epigenetic regulation at the mitochondrial heat shock protein 70 (mtHsp70) nuclear locus. J Mol Cell Cardiol 119: 104-115, 2018.

48. Shepherd DL, Hathaway QA, Pinti MV, Nichols CE, Durr AJ, Sreekumar S, Hughes KM, Stine SM, Martinez I, and Hollander JM. Exploring the mitochondrial microRNA import pathway through Polynucleotide Phosphorylase (PNPase). J Mol Cell Cardiol 110: 15-25, 2017.

49. Shi Z, Yang WZ, Lin-Chao S, Chak KF, and Yuan HS. Crystal structure of Escherichia coli PNPase: central channel residues are involved in processive RNA degradation. RNA 14: 23612371, 2008.

50. Shimada E, Ahsan FM, Nili M, Huang D, Atamdede S, TeSlaa T, Case D, Yu X, Gregory BD, Perrin BJ, Koehler CM, and Teitell MA. PNPase knockout results in mtDNA loss and an altered metabolic gene expression program. PLoS One 13: e0200925, 2018.

51. Smith MR, Fernandes J, Go YM, and Jones DP. Redox dynamics of manganese as a mitochondrial life-death switch. Biochem Biophys Res Commun 482: 388-398, 2017.

52. Song J, Wu X, Liu F, Li M, Sun Y, Wang Y, Wang C, Zhu K, Jia X, Wang B, and Ma $\mathbf{X}$. Long non-coding RNA PVT1 promotes glycolysis and tumor progression by regulating miR497/HK2 axis in osteosarcoma. Biochem Biophys Res Commun 490: 217-224, 2017.

53. Sripada L, Tomar D, Prajapati P, Singh R, Singh AK, and Singh R. Systematic analysis of small RNAs associated with human mitochondria by deep sequencing: detailed analysis of mitochondrial associated miRNA. PLoS One 7: e44873, 2012.

54. Stone CM, Butt LE, Bufton JC, Lourenco DC, Gowers DM, Pickford AR, Cox PA, Vincent HA, and Callaghan AJ. Inhibition of homologous phosphorolytic ribonucleases by citrate may represent an evolutionarily conserved communicative link between RNA degradation and central metabolism. Nucleic Acids Res 45: 4655-4666, 2017.

55. Stothard P. The sequence manipulation suite: JavaScript programs for analyzing and formatting protein and DNA sequences. Biotechniques 28: 1102, 1104, 2000.

56. Taanman JW. The mitochondrial genome: structure, transcription, translation and replication. Biochim Biophys Acta 1410: 103-123, 1999.

57. Thapa D, Nichols CE, Lewis SE, Shepherd DL, Jagannathan R, Croston TL, Tveter KJ, Holden AA, Baseler WA, and Hollander JM. Transgenic overexpression of mitofilin attenuates diabetes mellitus-associated cardiac and mitochondria dysfunction. J Mol Cell Cardiol 79: 212-223, 2015.

58. Thomson DW and Dinger ME. Endogenous microRNA sponges: evidence and controversy. Nat Rev Genet 17: 272-283, 2016.

59. Untergasser A, Nijveen H, Rao X, Bisseling T, Geurts R, and Leunissen JA. Primer3Plus, an enhanced web interface to Primer3. Nucleic Acids Res 35: W71-74, 2007.

60. Wang G, Chen HW, Oktay Y, Zhang J, Allen EL, Smith GM, Fan KC, Hong JS, French SW, McCaffery JM, Lightowlers RN, Morse HC, 3rd, Koehler CM, and Teitell MA. PNPASE regulates RNA import into mitochondria. Cell 142: 456-467, 2010.

61. Wang G, Shimada E, Zhang J, Hong JS, Smith GM, Teitell MA, and Koehler CM. Correcting human mitochondrial mutations with targeted RNA import. Proc Natl Acad Sci US A 109: 4840-4845, 2012. 
62. Wang K, Long B, Zhou LY, Liu F, Zhou QY, Liu CY, Fan YY, and Li PF. CARL lncRNA inhibits anoxia-induced mitochondrial fission and apoptosis in cardiomyocytes by impairing miR-539-dependent PHB2 downregulation. Nat Commun 5: 3596, 2014.

63. Wickham H. ggplot2: Elegant Graphics for Data Analysis. Use R: 1-212, 2009.

64. Wilusz JE, Sunwoo H, and Spector DL. Long noncoding RNAs: functional surprises from the RNA world. Genes Dev 23: 1494-1504, 2009.

65. Xing YH and Chen LL. Processing and roles of snoRNA-ended long noncoding RNAs. Crit Rev Biochem Mol Biol 53: 596-606, 2018.

66. Yamanaka R, Tabata S, Shindo Y, Hotta K, Suzuki K, Soga T, and Oka K. Mitochondrial $\operatorname{Mg}(2+)$ homeostasis decides cellular energy metabolism and vulnerability to stress. Sci Rep 6: 30027, 2016.

67. Yao RW, Wang Y, and Chen LL. Cellular functions of long noncoding RNAs. Nat Cell Biol 21: 542-551, 2019.

68. Zangrossi S, Briani F, Ghisotti D, Regonesi ME, Tortora P, and Deho G. Transcriptional and post-transcriptional control of polynucleotide phosphorylase during cold acclimation in Escherichia coli. Mol Microbiol 36: 1470-1480, 2000.

69. Zhang X, Zuo X, Yang B, Li Z, Xue Y, Zhou Y, Huang J, Zhao X, Zhou J, Yan Y, Zhang H, Guo P, Sun H, Guo L, Zhang Y, and Fu XD. MicroRNA directly enhances mitochondrial translation during muscle differentiation. Cell 158: 607-619, 2014. 
Figure 4.1
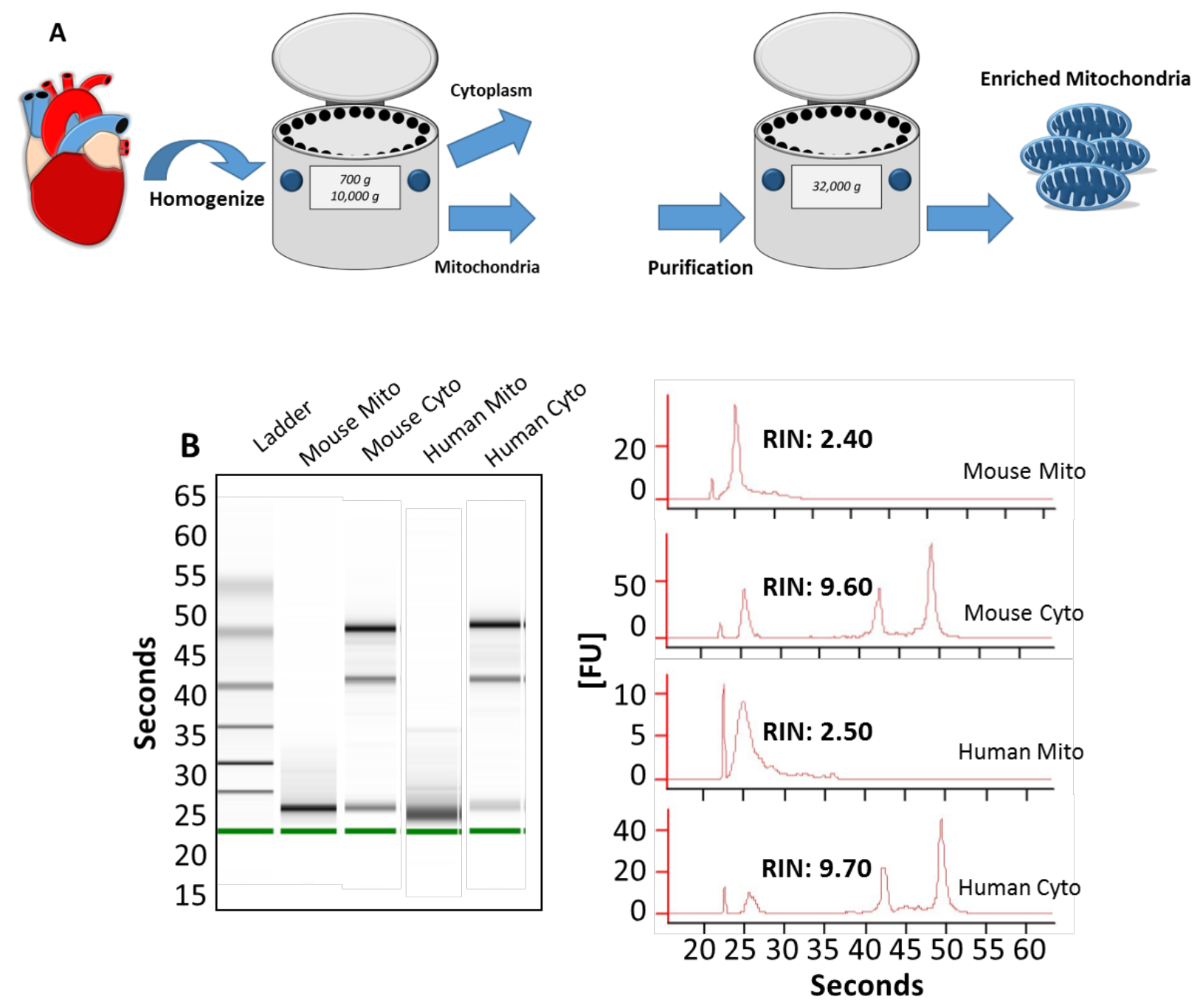

C
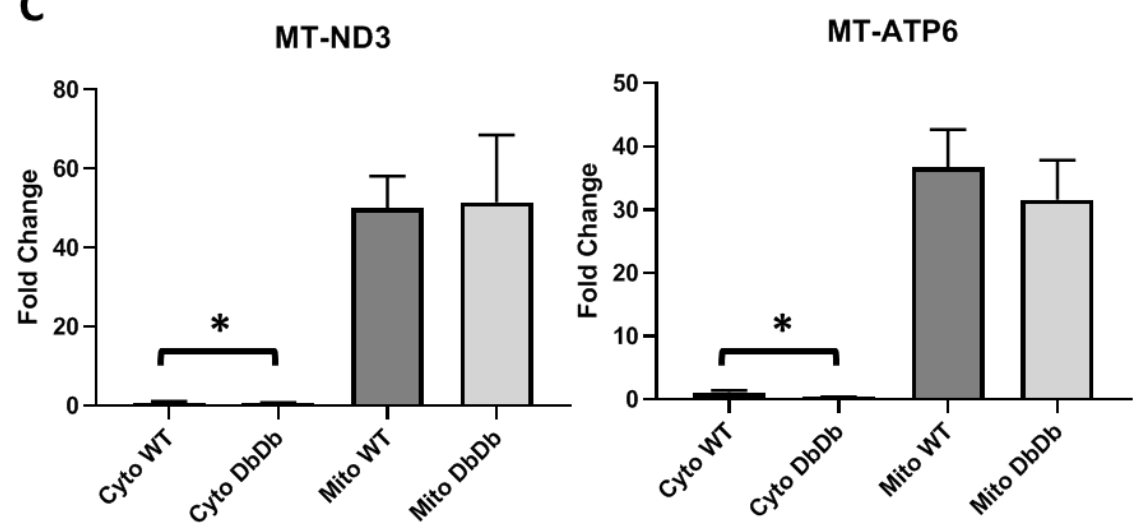
Figure 4.1: Enrichment of mitochondria from cardiac tissue. (A) From human atrial appendages and whole mouse hearts, samples are homogenized and differentially centrifuged to produce subcellular fractionations; mitochondria are further sucrose-gradient purified to remove cytoplasmic contamination. (B) Purity of cytoplasmic and mitochondrial RNA is assessed through the Bioanalyzer (Agilent Technologies), with a low RNA Integrity Number (RIN) indicating limited or no presence of cytoplasmic ribosomal RNA. (C) Real-time PCR of mitochondrial transcribed genes (MT-ND3 and MT-ATP6) from mouse cytoplasmic and mitochondrial isolated RNA. Differences between groups were considered statistically different if $P \leq 0.05$, denoted by *. All data are presented as the mean \pm standard error of the mean (SEM). MT-ND3 = mitochondrially encoded NADH:ubiquinone oxidoreductase core subunit 3, MT-ATP6 = mitochondrially mncoded ATP synthase membrane subunit 6, T2DM = type 2 diabetes mellitus, $\mathrm{ND}=$ non-diabetic, $\mathrm{DbDb}=\mathrm{FVB} / \mathrm{NJ}$ Lepr $^{d b_{-}{ }^{+/+}}$mice, $\mathrm{WT}=\mathrm{FVB} / \mathrm{NJ}$ wild type mice, Mito $=$ mitochondrial, Cyto $=$ cytoplasmic. 
Figure 4.2

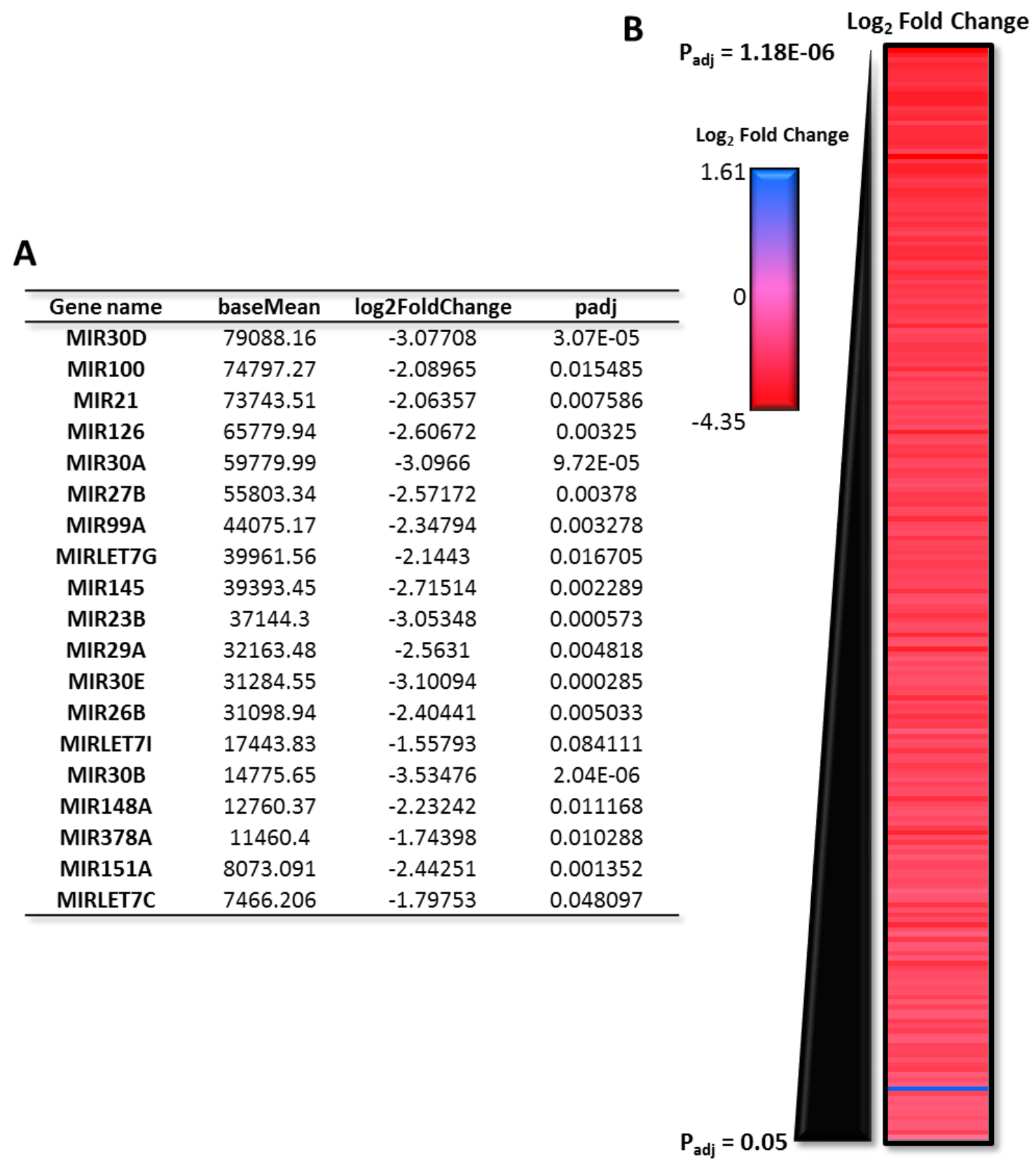


Figure 4.2

C
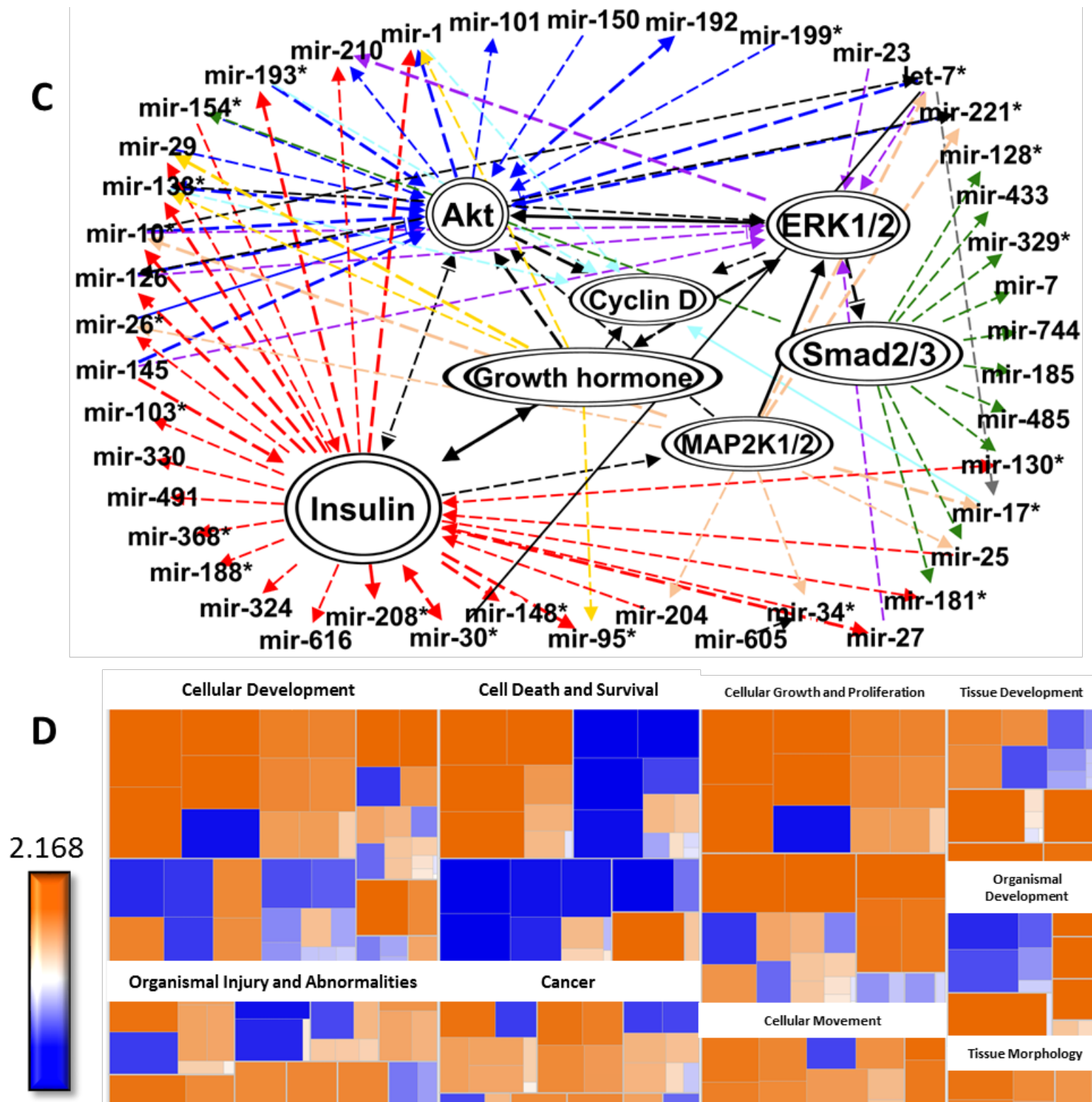

Organismal Injury and Abnormalities Cancer

2.195

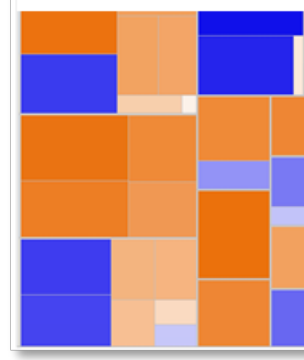

Cellular Movemen

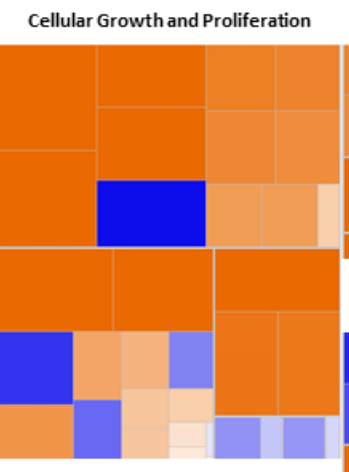

Tissue Development
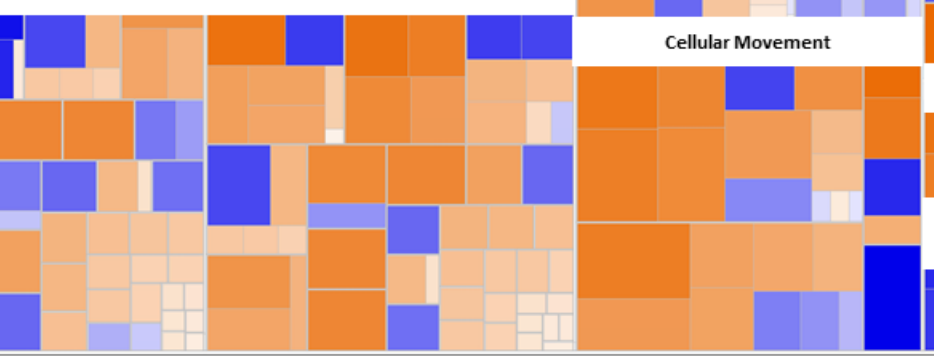

Tissue Morphology 
Figure 4.2: MiRNA profiles in human atrial mitochondria. (A) The top 20 most highly expressed nuclear-encoded miRNAs found in mitochondria of human atrial tissue from type 2 diabetic $(n=7)$ and non-diabetic $(n=7)$ patients. (B) Heat map illustrating the downregulation (red) and upregulation (blue) of all significantly impacted miRNAs following diabetic onset. (C) Ingenuity Pathway Analysis (IPA) of molecules revealing the highest number of suggested targeting interactions by miRNAs found to be differentially regulated in the mitochondrion. (D) IPA heat map revealing cellular pathways most likely impacted by the miRNAs found to be differentially affected in patients with diabetes mellitus. All statistically significant values are considered $P_{a d j}<0.05$ or $-\log _{10} \mathrm{P}_{\text {adj }}=1.30$. Z-scores are considered statistically significant if the value of the pathway affected is $\geq|2|$. 


\section{Figure 4.3}

\section{A $\mathrm{NS} \odot \mathrm{Log} 2 \mathrm{FC} \odot \mathrm{P} \odot \mathrm{P}$ \& Log2 FC}

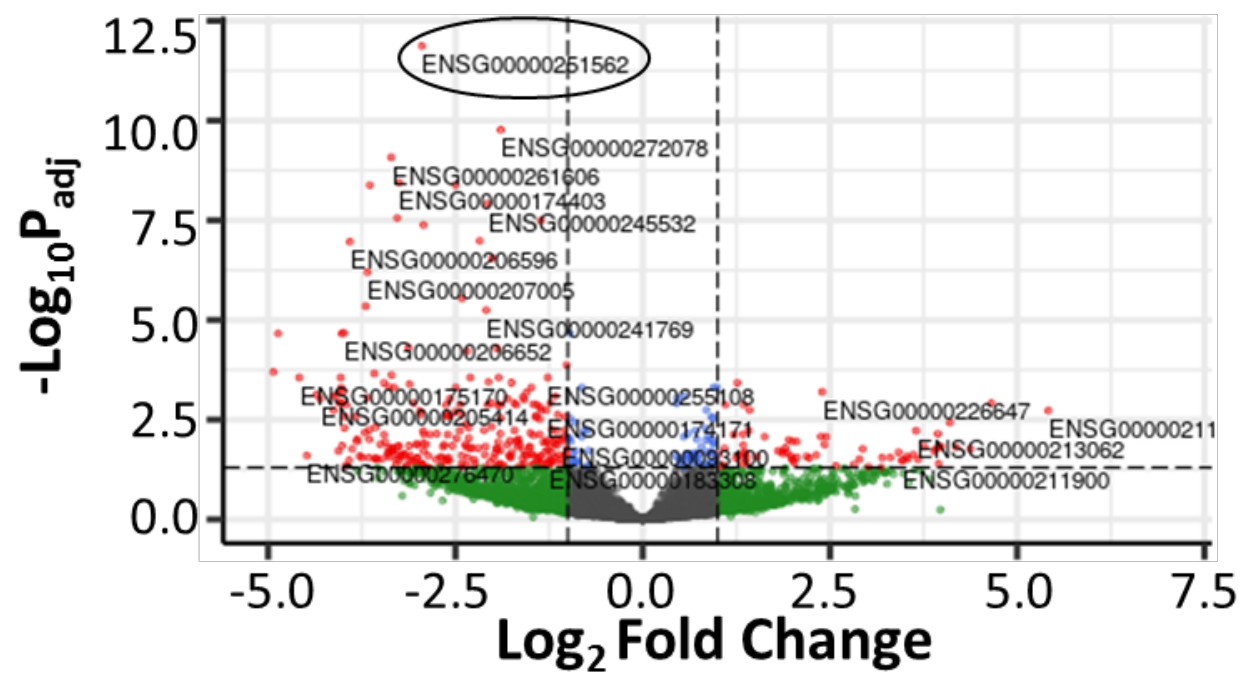

- NS $\bullet$ Log2 FC $P$ P P \& Log2 FC

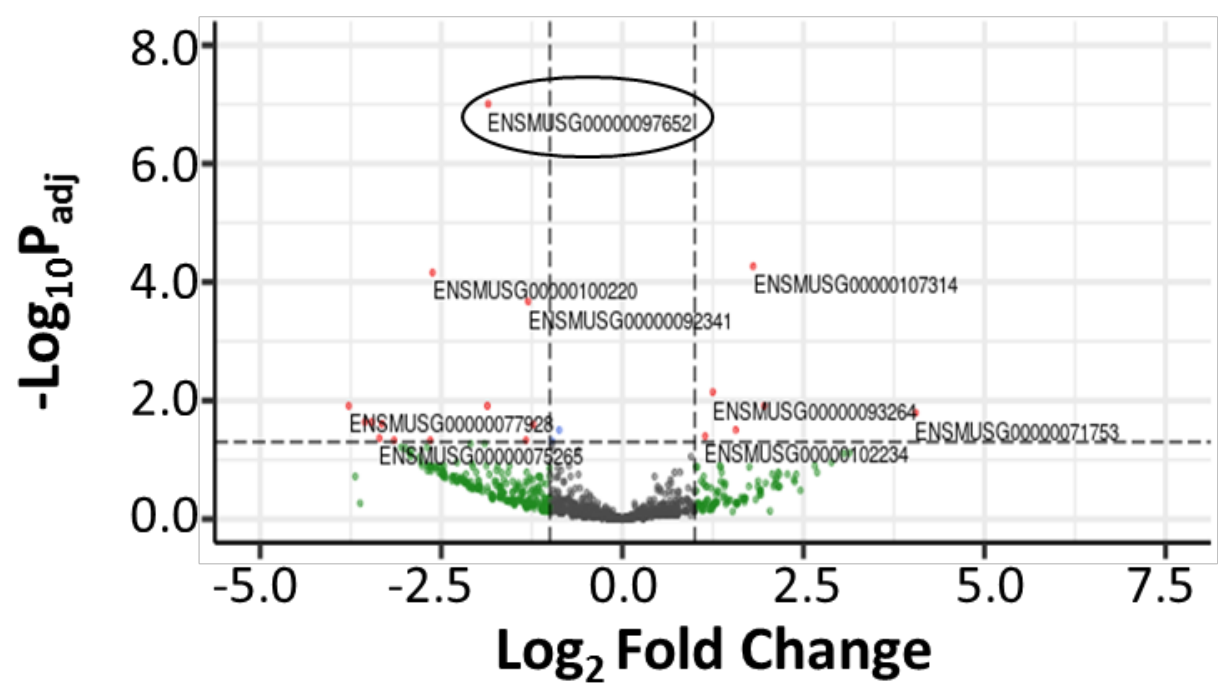




\section{Figure 4.3}
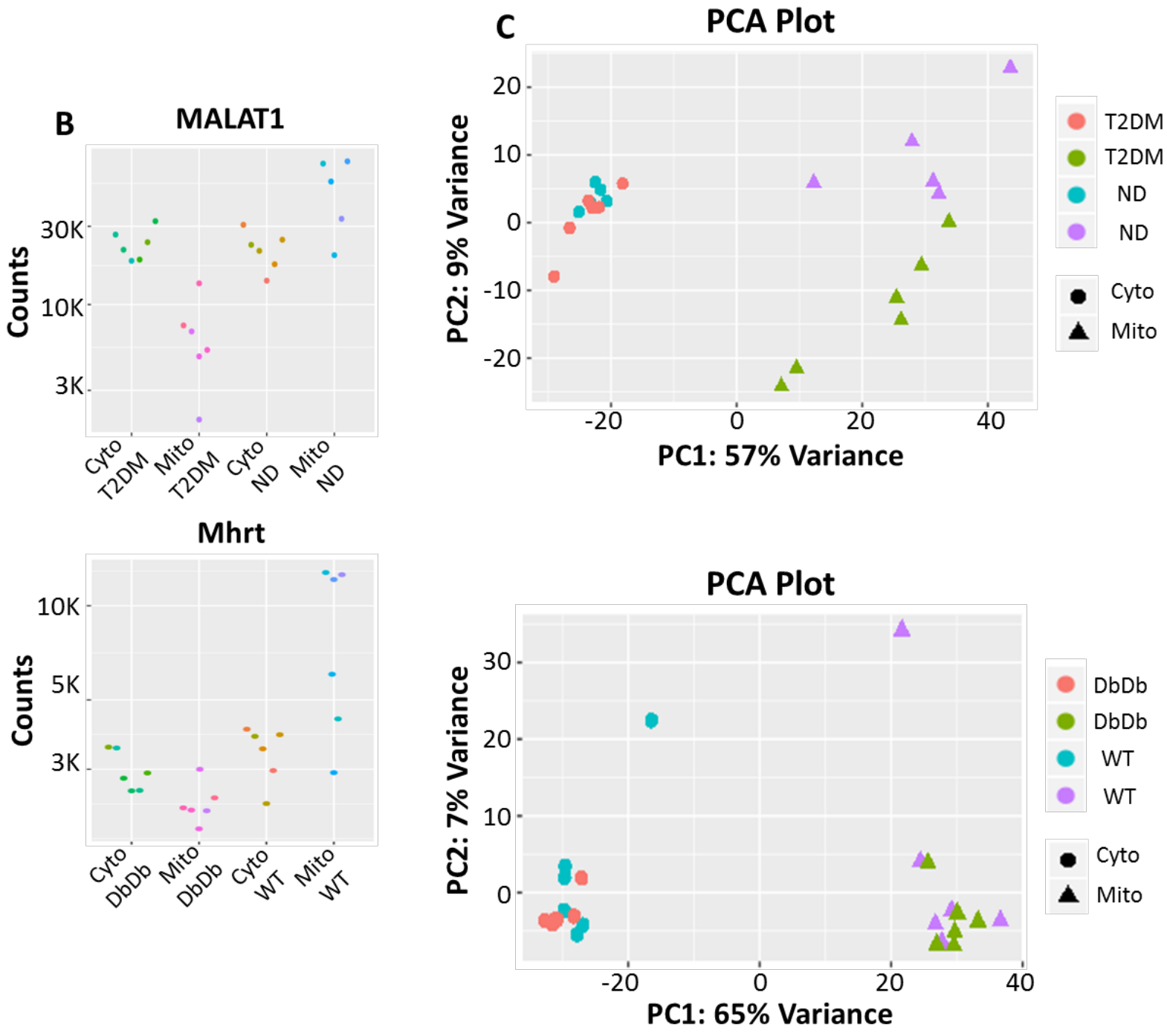


\section{Figure 4.3: Characterization of long-non coding RNA (IncRNA) in the mitochondrion.}

Human atrial tissue $(n=6)$ and mouse whole heart $(n=6)$ were assessed for lncRNA in the mitochondrion. (A) Volcano plots for human (top) and mouse (bottom) demonstrate the total lncRNAs differentially expressed in diabetic mitochondria, with the top gene (lowest $P_{\text {adj }}$ value)

circled. (B) The top most differentially regulated gene is depicted for human (top) and mouse (bottom) with the raw number of counts per each sample displayed. (C) Principle Component Analysis (PCA) for RNA in human (top) and mouse (bottom) of diabetic and non-diabetic samples for the cytoplasmic and mitochondrial portions. All statistically significant values are considered $P_{a d j}<0.05$ or $-\log _{10} \mathrm{P}_{\text {adj }}=1.30$. MALAT1 $=$ metastasis associated lung adenocarcinoma transcript 1, Mhrt $=$ myosin heavy chain associated RNA transcript, T2DM = type 2 diabetes mellitus, ND $=$ non-diabetic, $\mathrm{DbDb}=\mathrm{FVB} / \mathrm{NJ}$ Lepr $^{d b_{-}{ }^{+/+}}$mice, $\mathrm{WT}=\mathrm{FVB} / \mathrm{NJ}$ wild type mice, Mito = mitochondrial, Cyto $=$ cytoplasmic. 


\section{Figure 4.4}
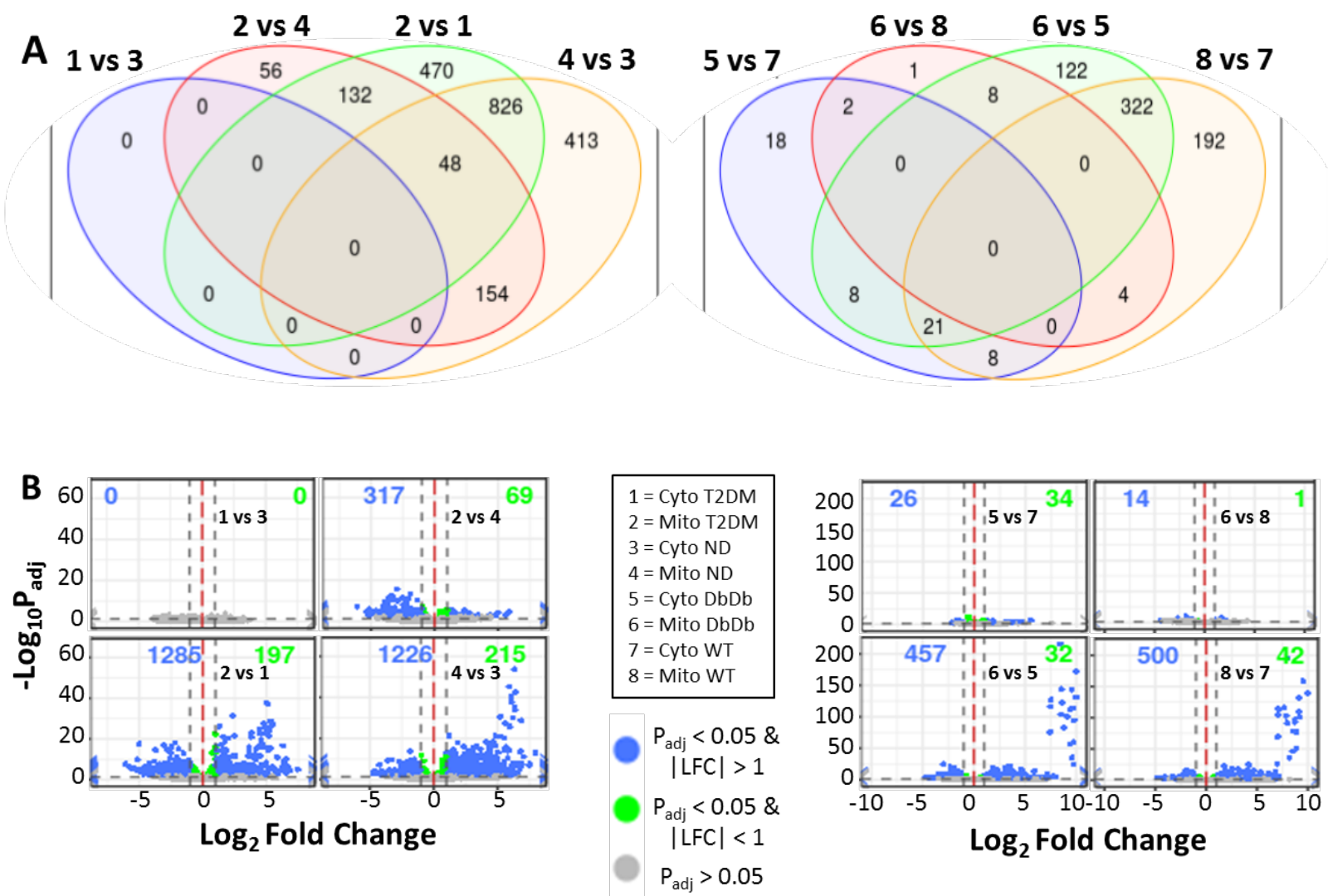

\begin{tabular}{lcccc}
$\mathbf{C}$ \\
\hline \multicolumn{7}{l}{ Mean Counts } & Fold Change & $\mathbf{P}_{\text {adj }}$ & Gene name & Gene type \\
\hline 3191 & 1.50 & 0.022 & AL138963.3 & antisense \\
1596 & 1.36 & 0.026 & VIM-AS1 & antisense \\
1586 & 1.37 & 0.001 & AL133415.1 & antisense \\
1423 & -3.24 & 0.0004 & AL132855.1 & antisense \\
847 & 1.49 & 0.031 & AP003352.1 & antisense \\
$\mathbf{2 4 7 6 7}$ & $\mathbf{- 7 . 2 5}$ & $\mathbf{1 . 3 5 E}-\mathbf{1 2}$ & MALAT1 & lincRNA \\
$\mathbf{5 5 5 0}$ & $\mathbf{- 4 . 0 8}$ & $\mathbf{1 . 2 2 E}-\mathbf{0 8}$ & NEAT1 & lincRNA \\
3592 & 1.37 & 0.0009 & NORAD & lincRNA \\
387 & -2.30 & 0.015 & MIR29B2CHG & lincRNA \\
194 & -1.92 & 0.011 & AL591543.1 & lincRNA \\
\hline
\end{tabular}

\begin{tabular}{ccccc}
\hline Mean Counts & Fold Change & $\mathbf{P}_{\text {adj }}$ & Gene name & Gene type \\
\hline $\mathbf{4 3 0 2}$ & $\mathbf{- 3 . 5 1}$ & $\mathbf{9 . 8 E}-\mathbf{0 8}$ & Mhrt & antisense \\
1636 & -1.81 & 0.031 & Gm49130 & antisense \\
$\mathbf{9 7}$ & $\mathbf{- 1 . 8 3}$ & $\mathbf{0 . 0 2 0}$ & Kcnq1ot1 & antisense \\
66 & -2.30 & 0.025 & 1700095B10Rik & antisense \\
51 & -5.46 & $6.92 \mathrm{E}-05$ & Gm29331 & antisense \\
$\mathbf{9 2 0 0}$ & $\mathbf{- 2 . 4 2}$ & $\mathbf{0 . 0 0 0 2}$ & Malat1 & lincRNA \\
$\mathbf{9 8 6}$ & $\mathbf{- 1 . 6 5}$ & $\mathbf{0 . 0 1 3}$ & Neat1 & lincRNA \\
36 & -3.35 & 0.012 & AU020206 & lincRNA \\
5 & 9.36 & 0.016 & C230004F18Rik & lincRNA \\
5 & -7.11 & 0.023 & 5430431A17Rik & lincRNA \\
\hline
\end{tabular}


Figure 4.4
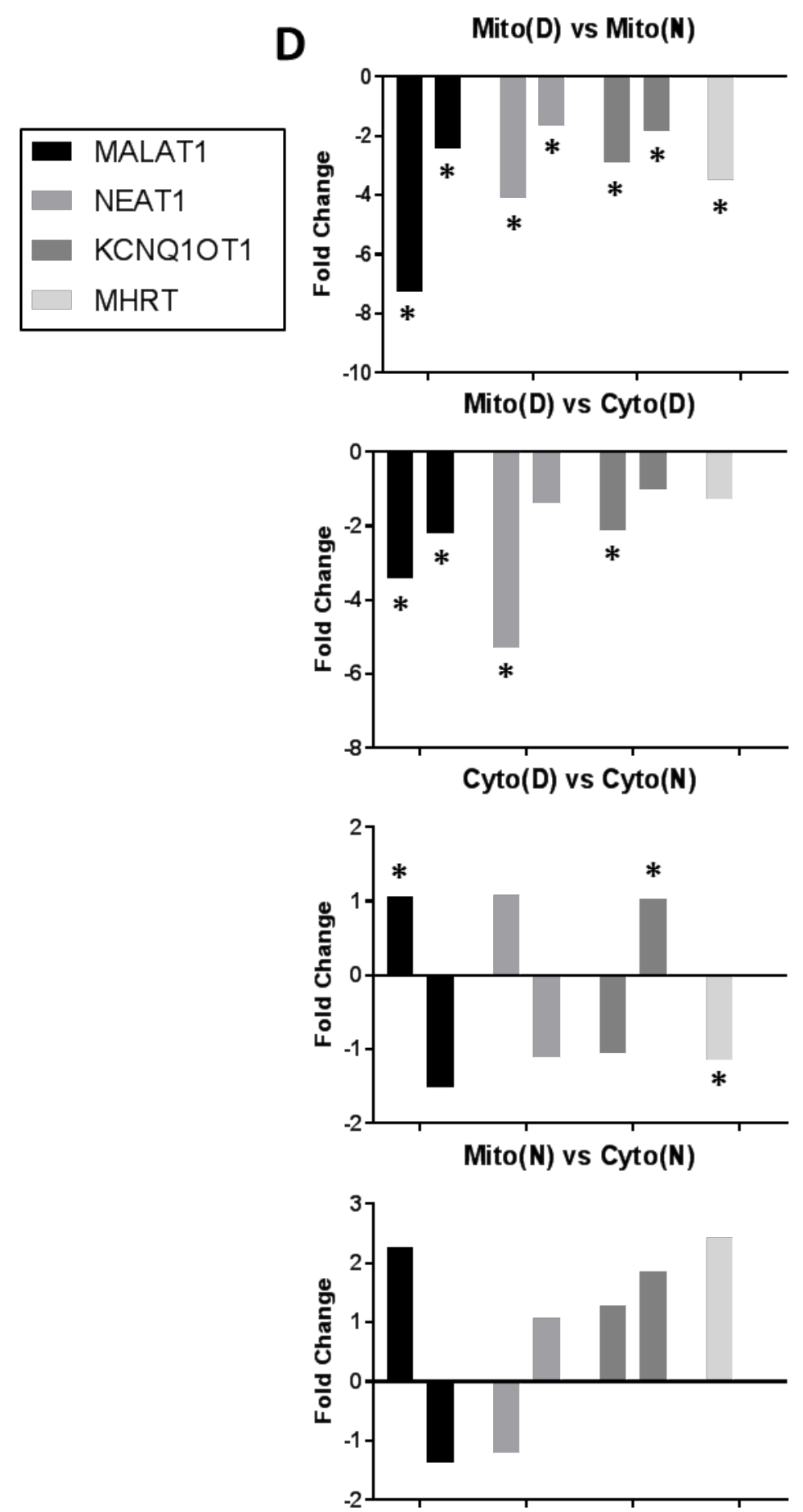
Figure 4.4: Expression profiles of long non-coding RNA (IncRNA) in the mitochondrial regulatory network. Human atrial tissue $(n=6)$ and mouse whole heart $(n=6)$ were assessed for lncRNA in the mitochondrion. (A) Venn diagrams comparing the differential expression of genes between diabetic and non-diabetic cytoplasmic and mitochondrial fractions in human (left) and mouse (right). (B) Illustration of genes in human (left) and mouse (right) that are significantly up or downregulated, including if the $\log _{2}$ Fold change is greater or less than one. (C) Tables containing the 5 top differentially regulated antisense and lincRNA transcripts in human (left) and mouse (right). Genes were chosen that had the highest mean count number as well as having a $P_{a d j}$ $<0.05$. (D) Examining expression profiles of MALAT1, NEAT1, KCNQ1OT1, and Mhrt in comparisons of diabetic vs. non-diabetic mitochondria (top), diabetic mitochondria vs. cytoplasm (top middle), diabetic vs. non-diabetic cytoplasm (bottom middle), and non-diabetic mitochondria vs. cytoplasm (bottom). Data are presented as grouped bars, with the human (left) and mouse (right) depicted, with the exception of Mhrt which is only present in mice with no human homologue. All statistically significant values are considered $P_{a d j}<0.05$ or $-\log _{10} \mathrm{P}_{\text {adj }}=1.30$. LincRNA $=$ long intergenic non-coding RNA, MALAT1 $=$ metastasis associated lung adenocarcinoma transcript 1 , NEAT 1 = nuclear paraspeckle assembly transcript 1, KCNQ1OT1 = KCNQ1 Opposite Strand/Antisense Transcript 1, Mhrt = myosin heavy chain associated RNA transcript, $\mathrm{T} 2 \mathrm{DM}=$ type 2 diabetes mellitus, $\mathrm{ND}=$ non-diabetic, $\mathrm{DbDb}=\mathrm{FVB} / \mathrm{NJ} L e p r^{d b}{ }_{-}^{+/+}$mice, $\mathrm{WT}=\mathrm{FVB} / \mathrm{NJ}$ wild type mice, Mito $=$ mitochondrial, Cyto $=$ cytoplasmic, $\mathrm{D}=$ diabetic, $\mathrm{N}=$ nondiabetic. 


\section{Figure 4.5}

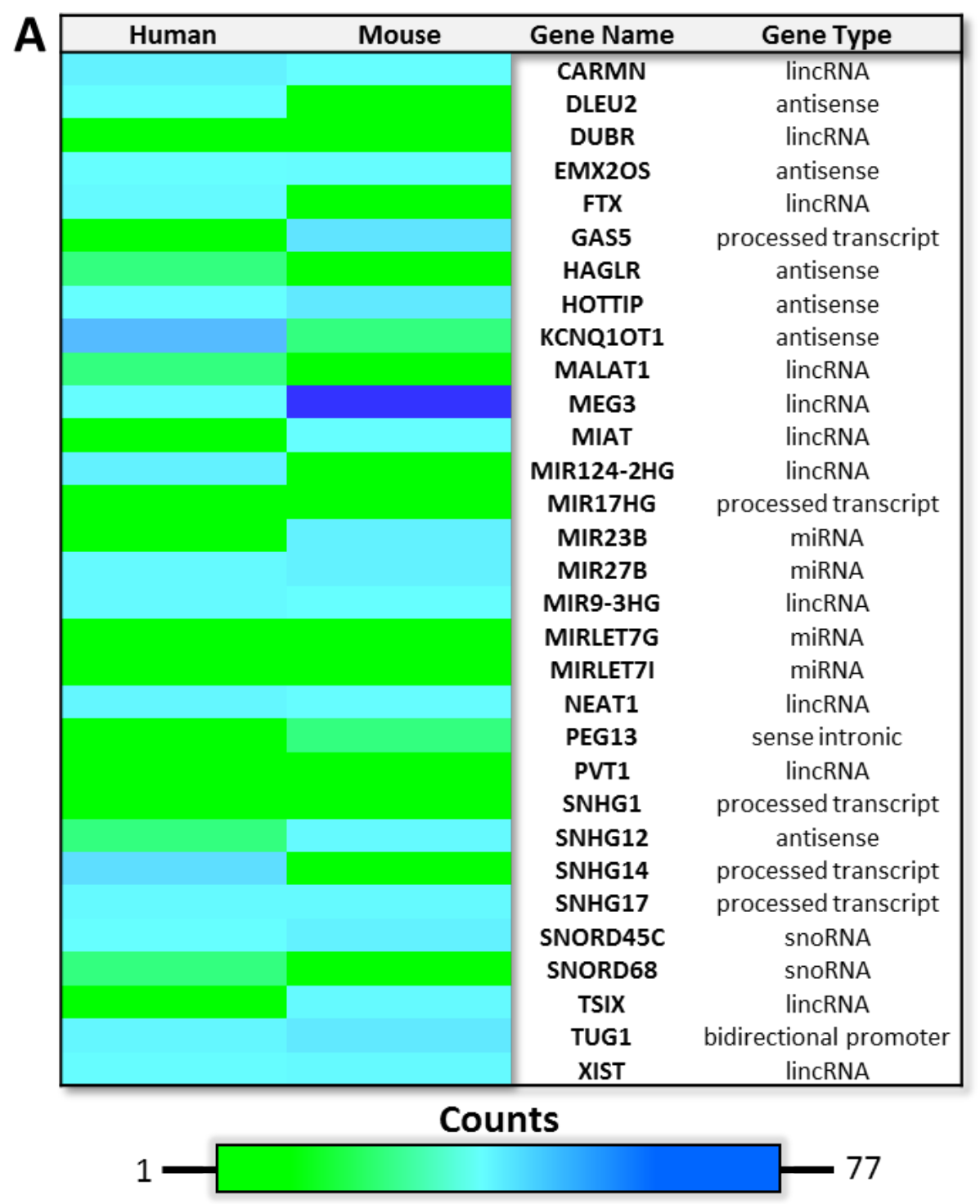

B Human

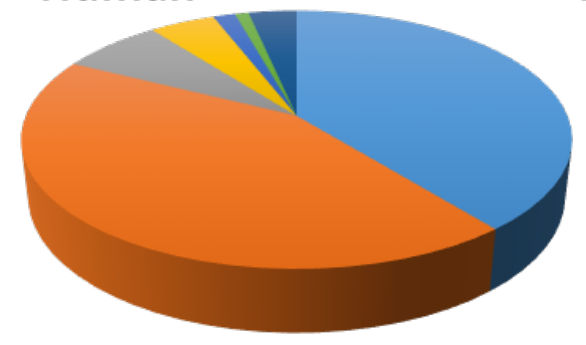

Mouse

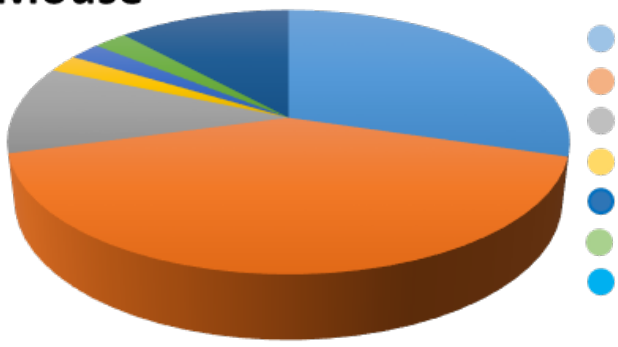

Antisense

LincRNA

Processed Transcript

Sense Intronic

MiRNA

Bidirectional Promoter Other 
Figure 4.5
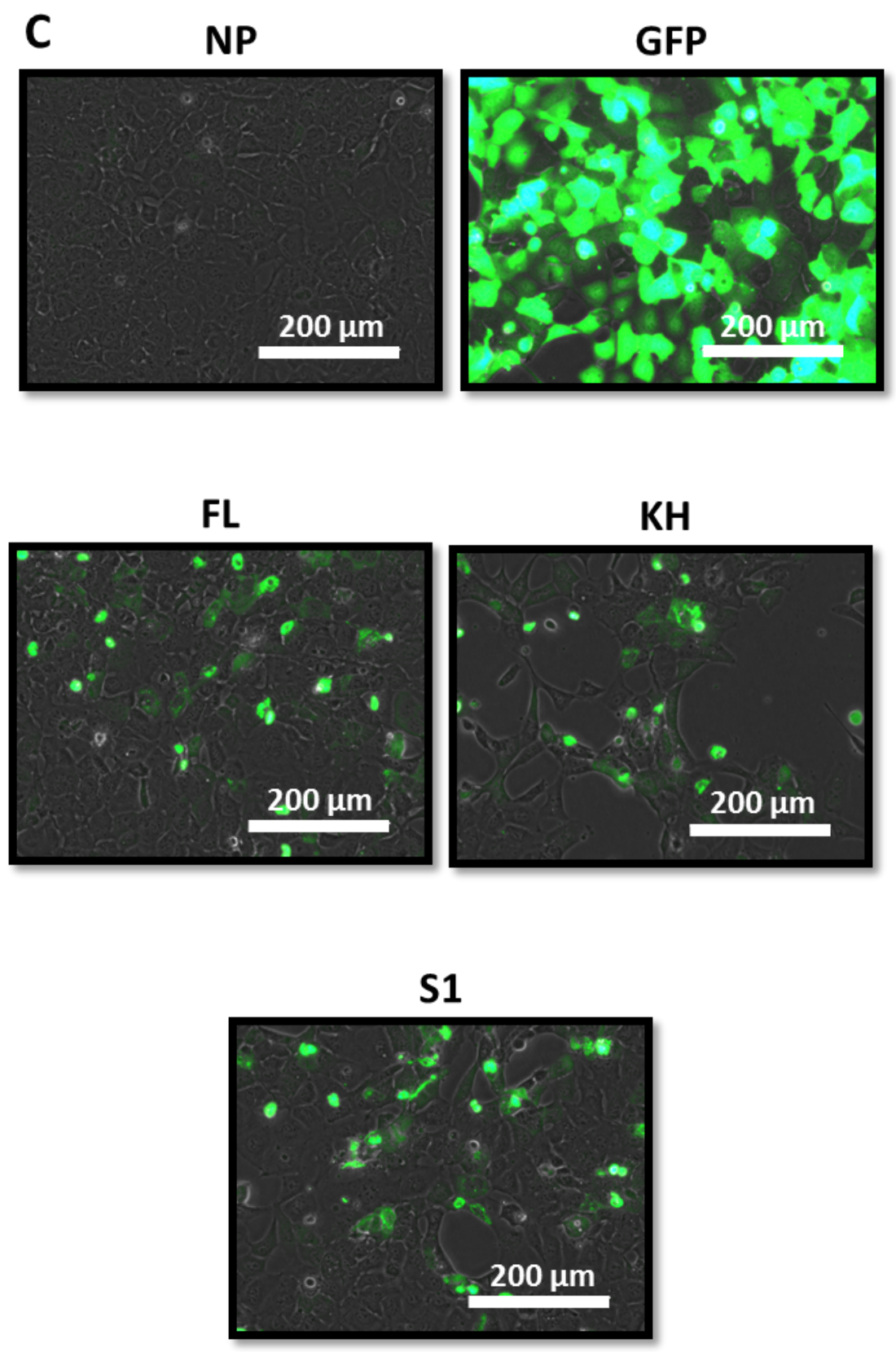
Figure 4.5
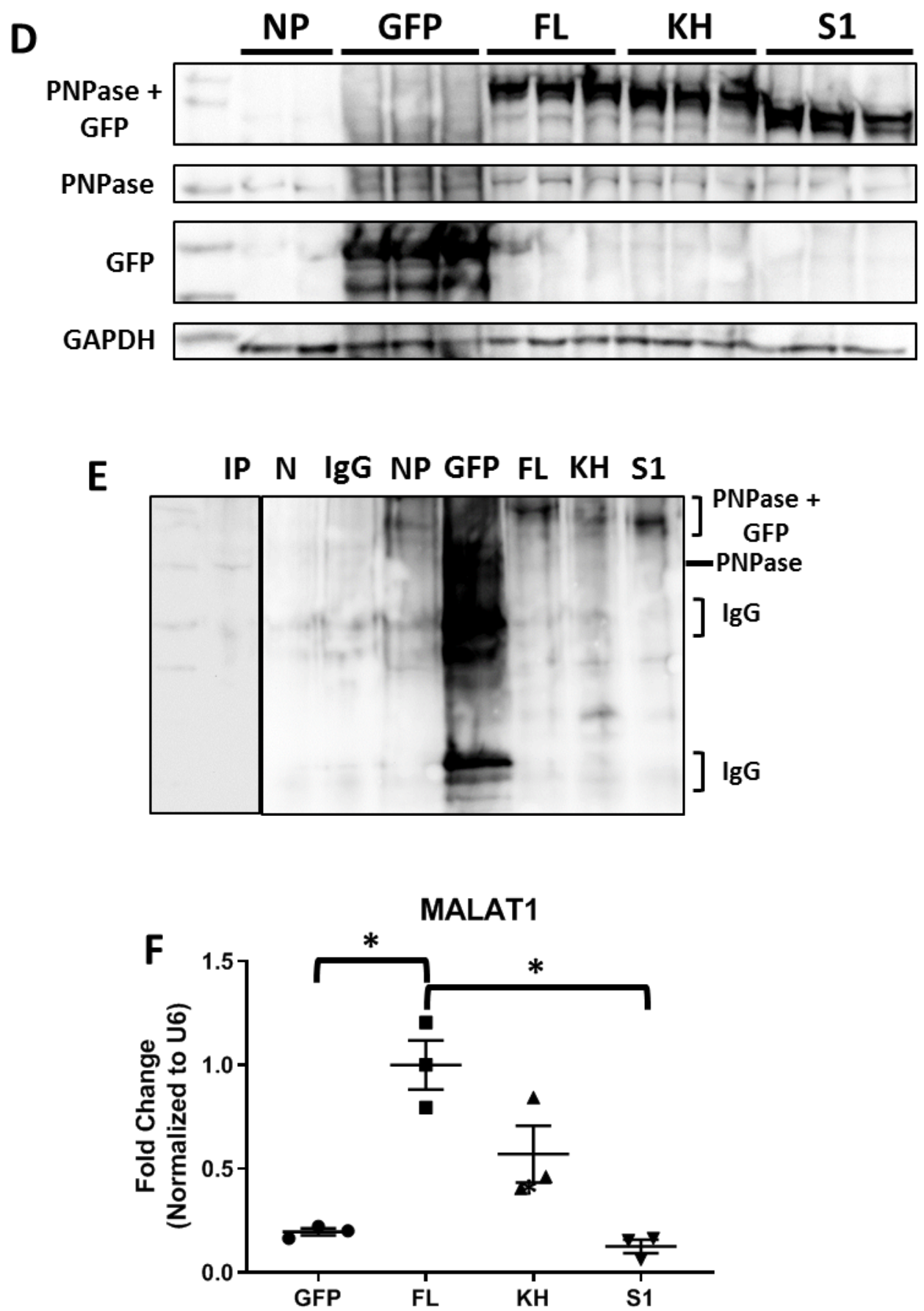
Figure 4.5: High-throughput sequencing crosslinking immunoprecipitation (HITS-CLIP)

for PNPase. (A) Heat map of ncRNA found in the PNPase CLIP for human atrial tissue type 2 diabetic $(\mathrm{n}=5)$ and non-diabetic $(\mathrm{n}=5)$ and whole heart from $\mathrm{Db} / \mathrm{Db}(\mathrm{n}=5)$ and wild type $(\mathrm{n}=$ 5) mice, with high (dark blue) and low (green) count values illustrated. Samples in each group were pooled, in order to achieve sufficient RNA for sequencing, with only the genes found in both human and mouse analyses displayed. (B) NcRNA species richness in human and mouse PNPase CLIP. (C) Representative images for HL-1 cells in each group ( $\mathrm{n}=10$ per group) showing fluorescence induced by pcDNA3.1+N-eGFP overexpression. (D) Immunoblotting depicting the overexpression in HL-1 cells, as well as shift in size, of the constructs. (E) A representative image of SDS-PAGE following crosslinking immunoprecipitation (CLIP) of HL-1 cells to retrieve RNA specifically bound to PNPase. (F) Real-time PCR on isolated RNA to assess binding affinity of lncRNA MALAT1 ( $\mathrm{n}=3$ each group) to PNPase variants. All statistically significant values are considered $P_{a d j}<0.05$ or $-\log _{10} \mathrm{P}_{\text {adj }}=1.30$. Differences between groups were considered statistically different if $P \leq 0.05$, denoted by *. All data are presented as the mean \pm standard error of the mean $(\mathrm{SEM}) . \mathrm{T} 2 \mathrm{DM}=$ type 2 diabetes mellitus, $\mathrm{ND}=$ non-diabetic, $\mathrm{DbDb}=\mathrm{FVB} / \mathrm{NJ}$ Lepr $^{d b_{-}{ }^{+/+}}$mice, $\mathrm{WT}=\mathrm{FVB} / \mathrm{NJ}$ wild type mice, $\mathrm{PNPase}=$ polynucleotide phosphorylase, $\mathrm{NP}=$ no plasmid control, GFP = pcDNA3.1+N-eGFP backbone only, FL = full length PNPase open reading frame $(\mathrm{ORF})$ in pcDNA3.1+N-eGFP, $\mathrm{KH}=$ exon 23 removed from full length PNPase in pcDNA3.1+N-eGFP, S1 = C-terminus removed from full length PNPase in in pcDNA3.1+NeGFP, MALAT1 = metastasis associated lung adenocarcinoma transcript 1 . 
Figure 4.6

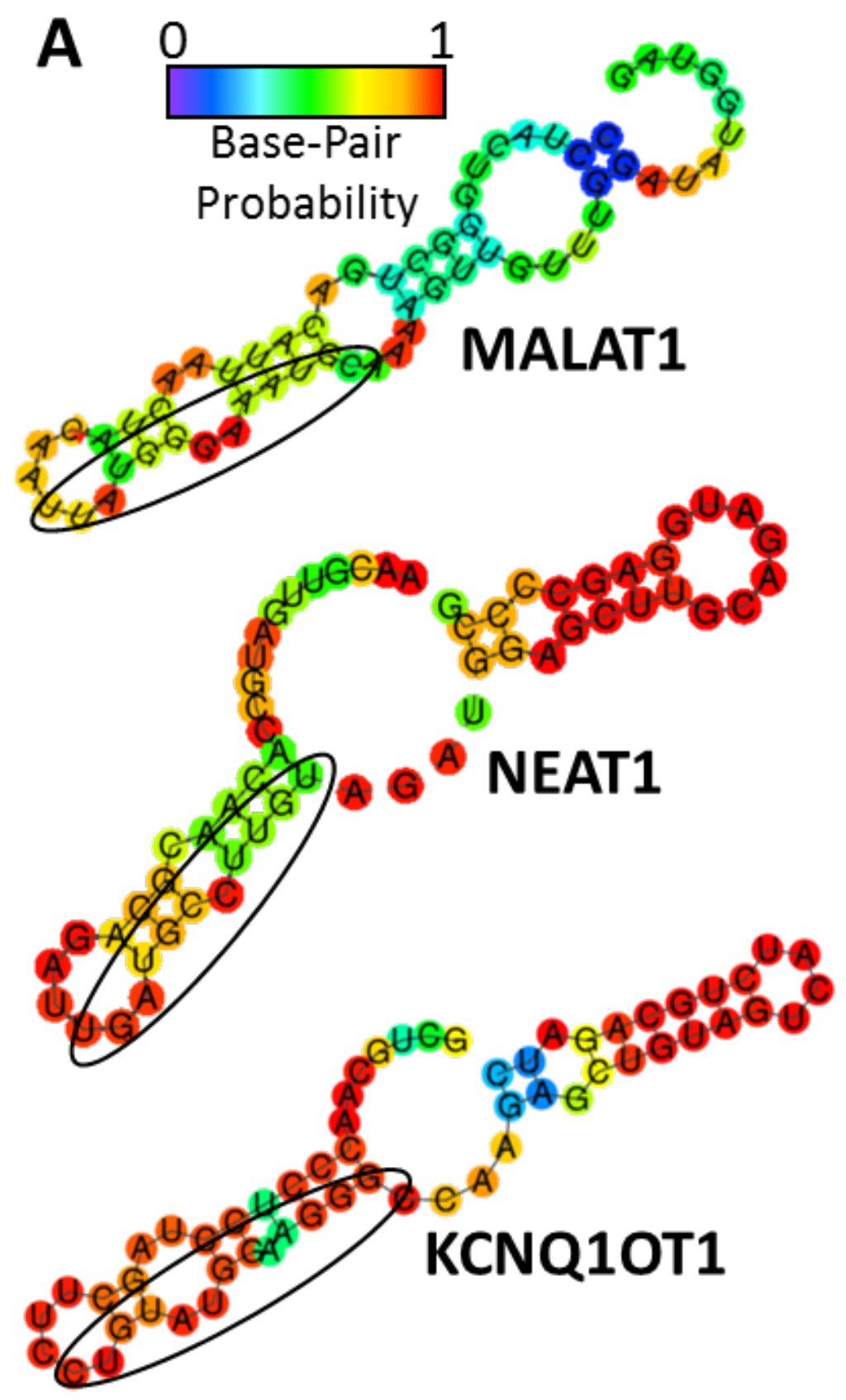


Figure 4.6
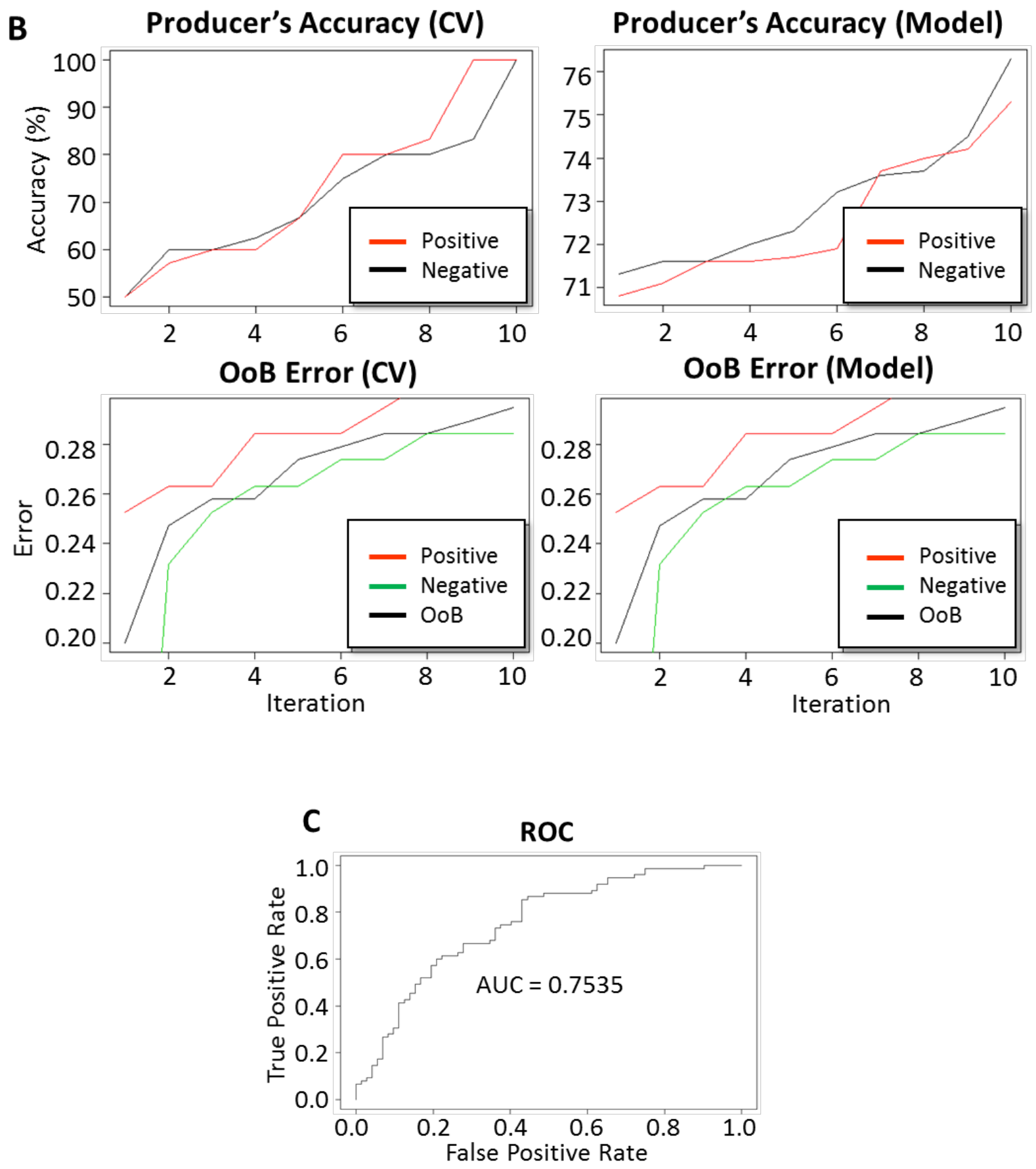
Figure 4.6
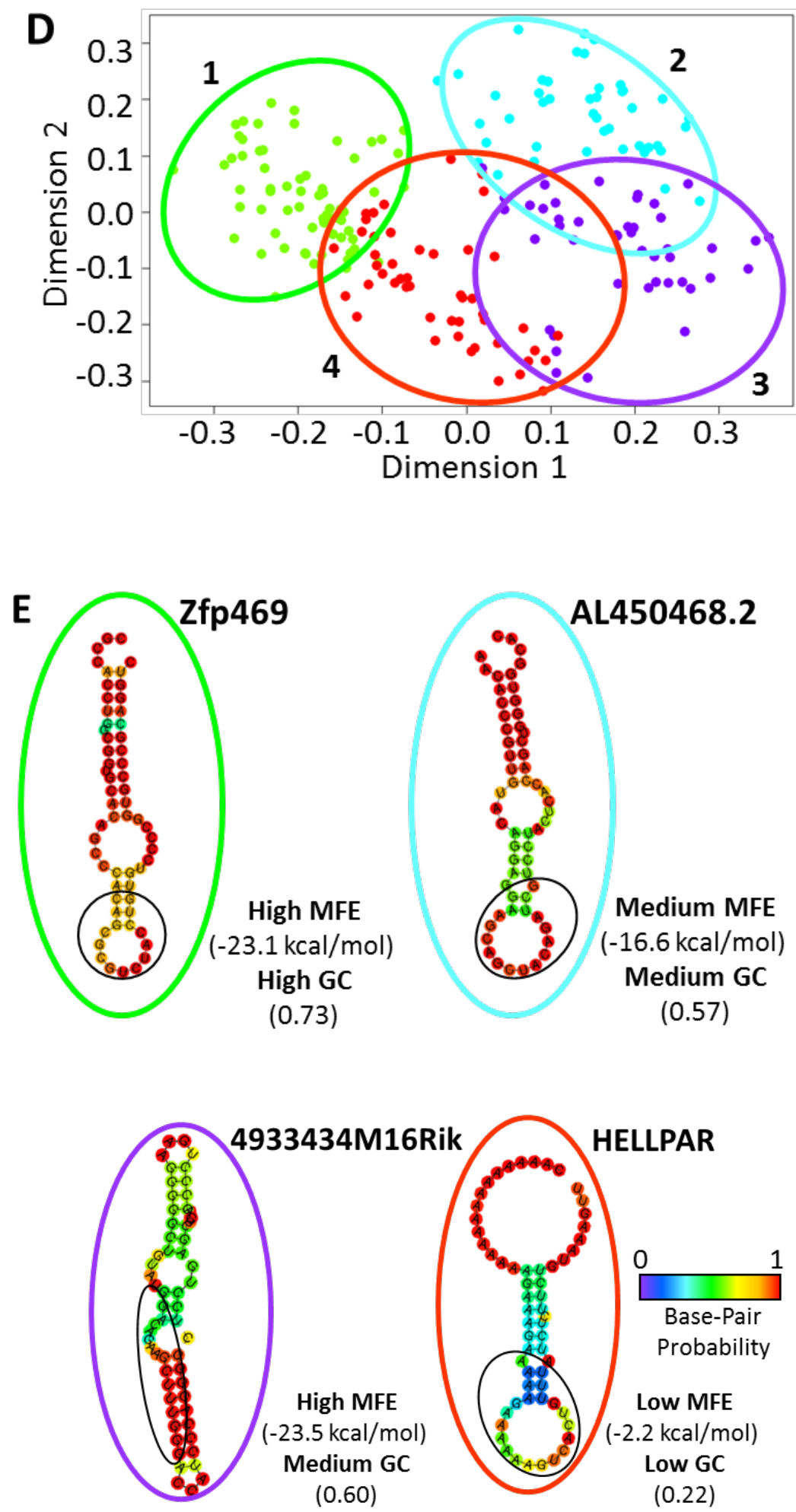
Figure 4.6: Mitochondrial RNA targeting sequence. (A) RNAfold of a 60 base-pair region of MALAT1, NEAT1, and KCNQT1OT, surrounding the reads (circled) found to bind to PNPase. (B) Supervised machine learning, through Classification and Regression Trees (CART), indicating the Producer's accuracy and Out of Bag $(\mathrm{OoB})$ error for both the model and 10-fold cross validation of positively identified ncRNAs and randomly generated sequences. (C) The average generated area under the curve (AUC) for prediction of ncRNA found in the PNPase CLIP. (D) Unsupervised learning to discover clustering $(\mathrm{n}=4)$ patterns of ncRNAs bound to PNPase. $(\mathbf{E})$ A representative ncRNA, sharing attributes of its respective cluster were selected and RNAfold was implemented to reveal structural and compositional differences. MALAT1 $=$ metastasis associated lung adenocarcinoma transcript 1 , NEAT1 = nuclear paraspeckle assembly transcript 1 , KCNQ1OT1 = KCNQ1 Opposite Strand/Antisense Transcript 1, CV = 10-fold cross validation, Positive $=50$ human and 50 mouse ncRNAs determined to be bound to PNPase, Negative $=100$ randomly generated sequences, Zfp469 = zinc finger protein 469, HELLPAR = HELLP associated long non-coding RNA, MFE = minimum free energy, $\mathrm{GC}=\mathrm{GC}$ content. 
Figure 4.7

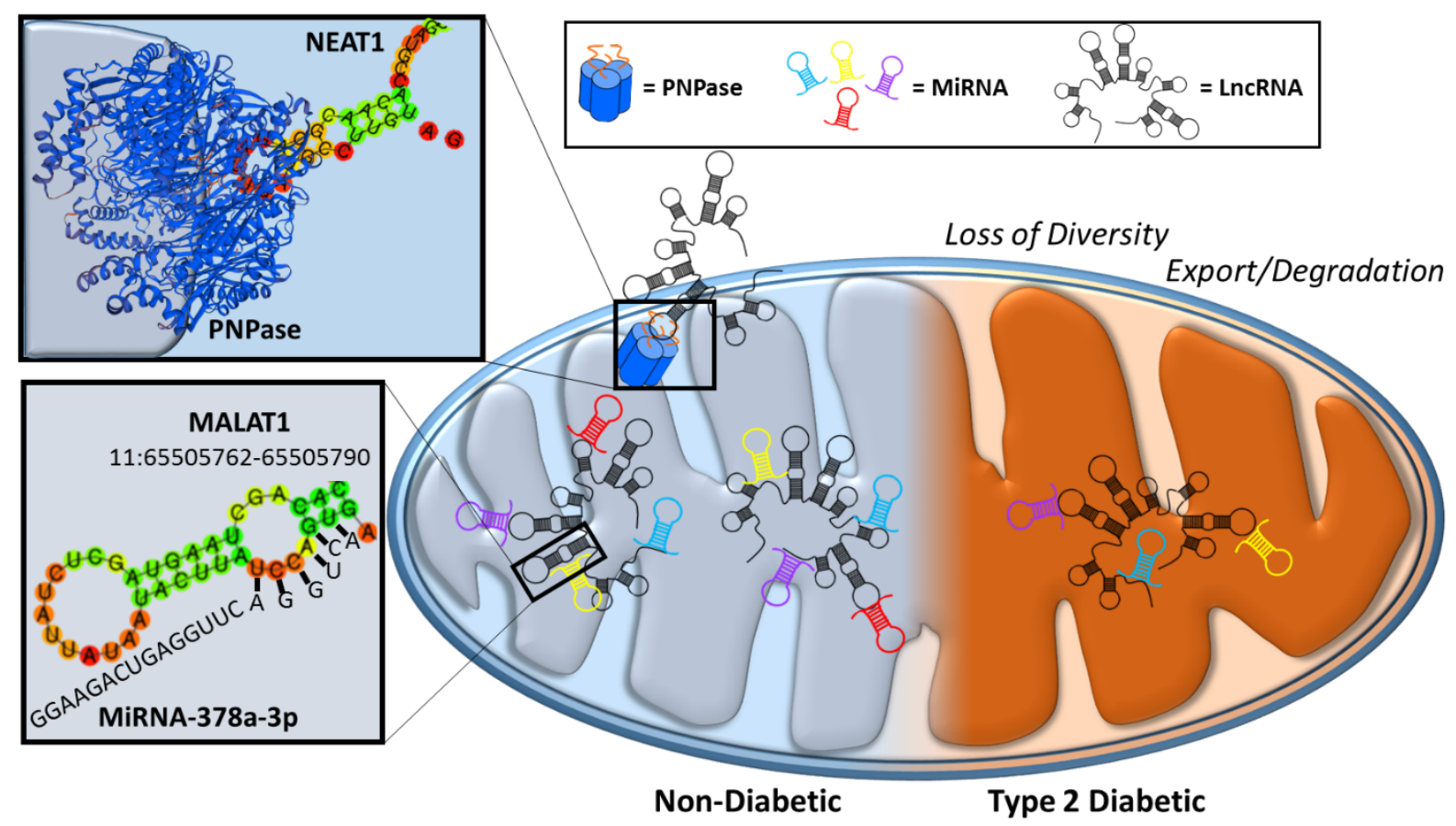


Figure 4.7: Illustrative overview mitochondrial regulatory network. PNPase has the capacity to bind lncRNA through stem-loop motifs, which may help to facilitate import of lncRNA into the mitochondrion. Within the mitochondrion, lncRNA could have the capacity to bind and sequester other ncRNA, to prevent regulation in other areas of the cell. Ultimately, the ncRNA diversity is significantly diminished in disease states (diabetes mellitus) likely impacting the cellular response to further insult. 
Table S4.1

\begin{tabular}{|c|c|c|c|}
\hline Assay & $\begin{array}{l}\text { Primer } \\
\text { Name }\end{array}$ & Forward $\left(5^{\prime}-3^{\prime}\right)$ & Reverse $\left(5^{\prime}-3^{\prime}\right)$ \\
\hline \multirow{3}{*}{$\begin{array}{l}\text { Figure 1: } \\
\text { Mitochondri } \\
\text { al Purity }\end{array}$} & MT- & AATGCGGATTCGACCCTAC & GAATTGCTCATGGTAGTGGAAG \\
\hline & ND3 & & \\
\hline & $\begin{array}{l}\text { MT- } \\
\text { ATP6 }\end{array}$ & GATTCCCAATCGTTGTAGCC & GGAAAGAATGGAGACGGTTG \\
\hline \multirow{3}{*}{$\begin{array}{l}\text { Figure S2: } \\
\text { LncRNA } \\
\text { mitochondria } \\
\text { I validation }\end{array}$} & Malat1 & TGGCAAGTAACTCCCAATCC & AGCATCACATCATCCCAGTG \\
\hline & -201 & & \\
\hline & $\begin{array}{c}\text { Neat1- } \\
201\end{array}$ & ATTGGCCAGAAGACAACAGG & TCTCATAGCAAGCGCAAGAC \\
\hline Figure 6: & Malat1 & GAATTGCGTCATTTAAAGCCTAG & GTTTCATCCTACCАСТCCCAАTTA \\
\hline PNPase HL-1 & & TT & AT \\
\hline CLIP & & & \\
\hline
\end{tabular}


Table S4.1: Primers designed for experiments. Primers were designed through Primer3 and NCBI Primer-BLAST. 
Table S4.2

\begin{tabular}{|c|c|c|c|c|c|}
\hline & $\begin{array}{c}\text { All } \\
\text { Positives }\end{array}$ & Cluster 1 & Cluster 2 & Cluster 3 & Cluster 4 \\
\hline \multicolumn{6}{|c|}{ Classification and Regression Cross-Validation (CV) } \\
\hline User's Accuracy (Positives) & 70.00 & 100.00 & 0 & 0 & 0 \\
\hline $\begin{array}{l}\text { User's Accuracy } \\
\text { (Negatives) }\end{array}$ & 80.00 & 100.00 & 100.00 & 100.00 & 100.00 \\
\hline $\begin{array}{l}\text { Producer's Accuracy } \\
\text { (Positives) }\end{array}$ & 70.85 & NA & NA & NA & NA \\
\hline $\begin{array}{l}\text { Producer's Accuracy } \\
\text { (Negatives) }\end{array}$ & 73.35 & 100.00 & 83.30 & 83.30 & 83.30 \\
\hline CV Kappa & 0.44 & 0.67 & 0.30 & 0.25 & 0.32 \\
\hline CV OoB Error & 0.28 & 0.09 & 0.15 & 0.18 & 0.18 \\
\hline CV Error Variance & $7.88 \mathrm{E}-4$ & $1.06 \mathrm{E}-3$ & $2.07 \mathrm{E}-4$ & $1.04 \mathrm{E}-4$ & $3.59 \mathrm{E}-3$ \\
\hline \multicolumn{6}{|c|}{ Classification and Regression Model } \\
\hline User's Accuracy (Positives) & 71.60 & 63.45 & 23.80 & 20.80 & 32.00 \\
\hline $\begin{array}{l}\text { User's Accuracy } \\
\text { (Negatives) }\end{array}$ & 72.60 & 97.9 & 98.90 & 97.90 & 95.25 \\
\hline $\begin{array}{l}\text { Producer's Accuracy } \\
\text { (Positives) }\end{array}$ & 72.75 & 88.90 & 80.00 & 71.40 & 62.55 \\
\hline $\begin{array}{l}\text { Producer's Accuracy } \\
\text { (Negatives) }\end{array}$ & 71.80 & 90.7 & 85.50 & 83.00 & 84.10 \\
\hline Model Kappa & 0.45 & 0.67 & 0.32 & 0.25 & 0.32 \\
\hline Model OoB Error & 0.28 & 0.09 & 0.15 & 0.18 & 0.18 \\
\hline Model Error Variance & $2.39 \mathrm{E}-4$ & $8.27 \mathrm{E}-5$ & $1.85 \mathrm{E}-4$ & $1.01 \mathrm{E}-4$ & $9.57 \mathrm{E}-5$ \\
\hline \multicolumn{6}{|c|}{ Support Vector Machines Cross-Validation (CV) } \\
\hline Sensitivity & 0.76 & 0.67 & 0.23 & 0.38 & 0.38 \\
\hline Specificity & 0.67 & 0.93 & 0.96 & 0.94 & 0.89 \\
\hline Accuracy & 0.72 & 0.88 & 0.83 & 0.83 & 0.79 \\
\hline F-Measure & 0.73 & NA & NA & NA & NA \\
\hline Kappa & 0.43 & 0.60 & 0.23 & 0.37 & 0.27 \\
\hline
\end{tabular}


Table S4.2: Machine Learning performance for Classification and Regression Trees (CART) and Support Vector Machines (SVM). Performance was determined for entire dataset (100 positives and 100 negatives) as well as for each cluster determined through unsupervised learning ( 25 positives and 100 negatives). Positives $=50$ human and 50 mouse ncRNAs determined to be bound to PNPase, or 25 genes found in each of the four clusters, Negatives $=100$ randomly generated sequences, $\mathrm{CV}=10$-fold cross validation, $\mathrm{OoB}=$ out of bag. 
Figure S4.1

Start Termination Mitochondrial Targeting Sequence KH Domain S1 Domain

\section{Pnpt1 - FL Protein (ORF)}

ATGGCGGCCTGCAGGCTGTGCTGCTTGTGCCCGTGCCTCCGACCGCTGGGCTGCGGCCCCCTCGGCCGGCCCGGG CGGAATCGGGCACTCAGCTATTTGCAGATGCGAGCGTTGTGGAGCAGCACCGGATCCAGAGCGGTGACTGTAGA CCTGGGACACAGAAAATTAGAAATATCCTCTGGGAAACTGGCAAGATTTGCTGATGGCTGTGCTGTGATACAGTC AGGCGATACTGCAGTAATGGTCACGGCAGTCAGCAAAACAAAAGCTTCGCCTTCCCAATTCATGCCGTTGGTGGT GGACTACCGACAGAAGGCTGCTGCAGCAGGCAGAATCCCCACAAACTACCTTAGAAGGGAGATTGGCTCCTCTGA CAGAGAGGTTCTTACAAGTCGAGTAATAGATCGTTCAATTCGCCCTCTCTTTCCAGCCGGCTATTTTTATGATACTC AGGTTCTCTGTAATCTGCTAGCAGTAGATGGCATCAATGAACCTGATATCCTAGCAGTTAATGGTGCTTCTGTAGC CCTCTCCTTATCAGATATCCCTTGGAATGGACCTGTTGGGGCAGTACGAATAGGAATGATTGATGGAGAATGTGTC GTTAACCCAACAAGGAGAGAAATGTCTTCTAGCACTTTAAATTTAGTAGTTGCCGGAGCACCTAAAAGCCAAATTG TTATGTTGGAAGCCTCTGCAGAAAATATTCTACAGCAGGACTTTTGCCATGCTATCAAAGTTGGGGTGAAGTATAC ACAGCAGATAATTCAGGGCATCCAGCAGTTGGTAAAAGAAATCGGTGTTGCCAAGAGGACACCGCAGAAGATATT TACTCCTTCTGCAGAGATTGTGAAGTACACGAAGATAATTGCCATGGAGAAACTCTATGCGGTTTTTACAGATTAT GAACATGATAAAGTTTCCAGGGATGAAGCTGTTAACAAGATAAGATTAGATACAGAGGAGCATCTAAAGGAAAA ATTTCCAGAGGTTGACCAATTTGAAATAATAGAATCCTTCAACATTGTTGCAAAGGAGGTTTTCCGAAGTATTATTT TGAATGAATACAAAAGGTGTGATGGAAGAGATCTGACTTCACTTAGGAATATAAGTTGTGAGGTCGATATGTTTA AAACACTTCATGGATCAGCATTATTTCAGAGAGGACAAACACAGGTACTCTGTACTGTTACATTTGATTCATTAGAA TCCAGTATTAAGTCAGATCAAATTATAACAGCTATAAATGGGGTAAAAGATAAAAATTTCATGCTGCACTATGAGT TCCCTCCTTATGCAACCAATGAAACCGGCAAAGTTACTGGTGTGAATCGAAGAGAACTTGGACATGGTGCTCTTGC CGAGAAAGCTTTGTGTCCTGTTATTCCCAAAGATTTTCCTTTTACCATAAGAGTTACATCTGAAGTCCTCGAATCAA ATGGGTCATCTTCTATGGCATCTGCATGTGGTGGAAGTTTGGCATTAATGGATGCAGGGGTCCCAATTTCATCTGC TGTTGCAGGTGTAGCAGTGGGATTGGTTACCAAAACCAATCCAGAGAAAGGTGAAATAGAAGACTACCGTTTGCT AACAGATATTCTGGGAATTGAAGATTATAATGGTGACATGGATTTCAAAATAGCCGGTACAAATAAAGGAATAAC TGCATTACAGGCTGATATTAAGTTACCTGGAGTACCAATTAAAATTATAATGGAAGCCATCCAACAAGCGTCAGTG GCAAAGAAGGAGATACTGCAGATAATGAACAAAACGATTTCAAAACCTCGAGCATCAAGAAAAGAAAATGGACC AGTTGTAGAAACAGTAAAGGTTCCATTATCAAAACGAGCAAAATTCGTTGGGCCTGGTGGATATCACTTAAAAAA ACTCCAGGCTGAGACAGGTGTAACAATTAGTCAGGTTGATGAAGAAACCTTCTCCATATTTGCACCAACACCTACT GCAATGCATGAAGCAAGAGATTTCATTACAGAAATTTGCAGAGATGATCAAGAGCAACAATTAGAATTTGGAGCA GTTTATACCGCGACAATAACTGAAATCAGAGACACTGGAGTGATGGTAAAACTGTATCCAAACATGACTGCAGTG CTGCTTCATAATTCACAACTTGACCAACGAAAGATTAAACATCCCACTGCCCTAGGACTAGAGGTTGGCCAAGAAA TTCAGGTCAAATACTTTGGCCGTGATCCAGCTGATGGAAGAATGAGGCTTTCTCGTAAAGTACTTCAGTCTCCAGC TACAACTGCTCTCAAAACTCTAAATGATAGAAGCAGCATTGTAATGGGAGAGCCTGTCTCACAGTCATCTAACTCT AACCCTTGA 
Figure S4.1

\section{Start Termination Mitochondrial Targeting Sequence KH Domain S1 Domain}

Pnpt1 - KH Domain KO, Removal of Exon 23 - 1-608 and 636-783 (conversion of E to G at 607)

ATGGCGGCCTGCAGGCTGTGCTGCTTGTGCCCGTGCCTCCGACCGCTGGGCTGCGGCCCCCTCGGCCGGCCCGGG CGGAATCGGGCACTCAGCTATTTGCAGATGCGAGCGTTGTGGAGCAGCACCGGATCCAGAGCGGTGACTGTAGA CCTGGGACACAGAAAATTAGAAATATCCTCTGGGAAACTGGCAAGATTTGCTGATGGCTGTGCTGTGATACAGTC AGGCGATACTGCAGTAATGGTCACGGCAGTCAGCAAAACAAAAGCTTCGCCTTCCCAATTCATGCCGTTGGTGGT GGACTACCGACAGAAGGCTGCTGCAGCAGGCAGAATCCCCACAAACTACCTTAGAAGGGAGATTGGCTCCTCTGA CAGAGAGGTTCTTACAAGTCGAGTAATAGATCGTTCAATTCGCCCTCTCTTTCCAGCCGGCTATTTTTATGATACTC AGGTTCTCTGTAATCTGCTAGCAGTAGATGGCATCAATGAACCTGATATCCTAGCAGTTAATGGTGCTTCTGTAGC CCTCTCCTTATCAGATATCCCTTGGAATGGACCTGTTGGGGCAGTACGAATAGGAATGATTGATGGAGAATGTGTC GTTAACCCAACAAGGAGAGAAATGTCTTCTAGCACTTTAAATTTAGTAGTTGCCGGAGCACCTAAAAGCCAAATTG TTATGTTGGAAGCCTCTGCAGAAAATATTCTACAGCAGGACTTTTGCCATGCTATCAAAGTTGGGGTGAAGTATAC ACAGCAGATAATTCAGGGCATCCAGCAGTTGGTAAAAGAAATCGGTGTTGCCAAGAGGACACCGCAGAAGATATT TACTCCTTCTGCAGAGATTGTGAAGTACACGAAGATAATTGCCATGGAGAAACTCTATGCGGTTTTTACAGATTAT GAACATGATAAAGTTTCCAGGGATGAAGCTGTTAACAAGATAAGATTAGATACAGAGGAGCATCTAAAGGAAAA ATTTCCAGAGGTTGACCAATTTGAAATAATAGAATCCTTCAACATTGTTGCAAAGGAGGTTTTCCGAAGTATTATTT TGAATGAATACAAAAGGTGTGATGGAAGAGATCTGACTTCACTTAGGAATATAAGTTGTGAGGTCGATATGTTTA AAACACTTCATGGATCAGCATTATTTCAGAGAGGACAAACACAGGTACTCTGTACTGTTACATTTGATTCATTAGAA TCCAGTATTAAGTCAGATCAAATTATAACAGCTATAAATGGGGTAAAAGATAAAAATTTCATGCTGCACTATGAGT TCCCTCCTTATGCAACCAATGAAACCGGCAAAGTTACTGGTGTGAATCGAAGAGAACTTGGACATGGTGCTCTTGC CGAGAAAGCTTTGTGTCCTGTTATTCCCAAAGATTTTCCTTTTACCATAAGAGTTACATCTGAAGTCCTCGAATCAA ATGGGTCATCTTCTATGGCATCTGCATGTGGTGGAAGTTTGGCATTAATGGATGCAGGGGTCCCAATTTCATCTGC TGTTGCAGGTGTAGCAGTGGGATTGGTTACCAAAACCAATCCAGAGAAAGGTGAAATAGAAGACTACCGTTTGCT AACAGATATTCTGGGAATTGAAGATTATAATGGTGACATGGATTTCAAAATAGCCGGTACAAATAAAGGAATAAC TGCATTACAGGCTGATATTAAGTTACCTGGAGTACCAATTAAAATTATAATGGAAGCCATCCAACAAGCGTCAGTG GCAAAGAAGGAGATACTGCAGATAATGAACAAAACGATTTCAAAACCTCGAGCATCAAGAAAAGAAAATGGACC AGTTGTAGGTGTAACAATTAGTCAGGTTGATGAAGAAACCTTCTCCATATTTGCACCAACACCTACTGCAATGCAT GAAGCAAGAGATTTCATTACAGAAATTTGCAGAGATGATCAAGAGCAACAATTAGAATTTGGAGCAGTTTATACC GCGACAATAACTGAAATCAGAGACACTGGAGTGATGGTAAAACTGTATCCAAACATGACTGCAGTGCTGCTTCAT AATTCACAACTTGACCAACGAAAGATTAAACATCCCACTGCCCTAGGACTAGAGGTTGGCCAAGAAATTCAGGTCA AATACTTTGGCCGTGATCCAGCTGATGGAAGAATGAGGCTTTCTCGTAAAGTACTTCAGTCTCCAGCTACAACTGC TCTCAAAACTCTAAATGATAGAAGCAGCATTGTAATGGGAGAGCCTGTCTCACAGTCATCTAACTCTAACCCTTGA 
Figure S4.1

\section{Start Termination Mitochondrial Targeting Sequence KH Domain S1 Domain \\ Pnpt1 - S1 Domain KO - 1-669 AA}

ATGGCGGCCTGCAGGCTGTGCTGCTTGTGCCCGTGCCTCCGACCGCTGGGCTGCGGCCCCCTCGGCCGGCCCGGG CGGAATCGGGCACTCAGCTATTTGCAGATGCGAGCGTTGTGGAGCAGCACCGGATCCAGAGCGGTGACTGTAGA CCTGGGACACAGAAAATTAGAAATATCCTCTGGGAAACTGGCAAGATTTGCTGATGGCTGTGCTGTGATACAGTC AGGCGATACTGCAGTAATGGTCACGGCAGTCAGCAAAACAAAAGCTTCGCCTTCCCAATTCATGCCGTTGGTGGT GGACTACCGACAGAAGGCTGCTGCAGCAGGCAGAATCCCCACAAACTACCTTAGAAGGGAGATTGGCTCCTCTGA CAGAGAGGTTCTTACAAGTCGAGTAATAGATCGTTCAATTCGCCCTCTCTTTCCAGCCGGCTATTTTTATGATACTC AGGTTCTCTGTAATCTGCTAGCAGTAGATGGCATCAATGAACCTGATATCCTAGCAGTTAATGGTGCTTCTGTAGC CCTCTCCTTATCAGATATCCCTTGGAATGGACCTGTTGGGGCAGTACGAATAGGAATGATTGATGGAGAATGTGTC GTTAACCCAACAAGGAGAGAAATGTCTTCTAGCACTTTAAATTTAGTAGTTGCCGGAGCACCTAAAAGCCAAATTG TTATGTTGGAAGCCTCTGCAGAAAATATTCTACAGCAGGACTTTTGCCATGCTATCAAAGTTGGGGTGAAGTATAC ACAGCAGATAATTCAGGGCATCCAGCAGTTGGTAAAAGAAATCGGTGTTGCCAAGAGGACACCGCAGAAGATATT TACTCCTTCTGCAGAGATTGTGAAGTACACGAAGATAATTGCCATGGAGAAACTCTATGCGGTTTTTTACAGATTAT GAACATGATAAAGTTTCCAGGGATGAAGCTGTTAACAAGATAAGATTAGATACAGAGGAGCATCTAAAGGAAAA ATTTCCAGAGGTTGACCAATTTGAAATAATAGAATCCTTCAACATTGTTGCAAAGGAGGTTTTCCGAAGTATTATTT TGAATGAATACAAAAGGTGTGATGGAAGAGATCTGACTTCACTTAGGAATATAAGTTGTGAGGTCGATATGTTTA AAACACTTCATGGATCAGCATTATTTCAGAGAGGACAAACACAGGTACTCTGTACTGTTACATTTGATTCATTAGAA TCCAGTATTAAGTCAGATCAAATTATAACAGCTATAAATGGGGTAAAAGATAAAAATTTCATGCTGCACTATGAGT TCCCTCCTTATGCAACCAATGAAACCGGCAAAGTTACTGGTGTGAATCGAAGAGAACTTGGACATGGTGCTCTTGC CGAGAAAGCTTTGTGTCCTGTTATTCCCAAAGATTTTCCTTTTACCATAAGAGTTACATCTGAAGTCCTCGAATCAA ATGGGTCATCTTCTATGGCATCTGCATGTGGTGGAAGTTTGGCATTAATGGATGCAGGGGTCCCAATTTCATCTGC TGTTGCAGGTGTAGCAGTGGGATTGGTTACCAAAACCAATCCAGAGAAAGGTGAAATAGAAGACTACCGTTTGCT AACAGATATTCTGGGAATTGAAGATTATAATGGTGACATGGATTTCAAAATAGCCGGTACAAATAAAGGAATAAC TGCATTACAGGCTGATATTAAGTTACCTGGAGTACCAATTAAAATTATAATGGAAGCCATCCAACAAGCGTCAGTG GCAAAGAAGGAGATACTGCAGATAATGAACAAAACGATTTCAAAACCTCGAGCATCAAGAAAAGAAAATGGACC AGTTGTAGAAACAGTAAAGGTTCCATTATCAAAACGAGCAAAATTCGTTGGGCCTGGTGGATATCACTTAAAAAA ACTCCAGGCTGAGACAGGTGTAACAATTAGTCAGGTTGATGAAGAAACCTTCTCCATATTTGCACCAACACCTACT GCAATGCATGAAGCAAGAGATTTCATTACAGAAATTTGCAGATGA 
Figure S4.1: Constructs designed for transfection into HL-1 cells. Sequences were inserted into a pcDNA3.1+N-eGFP vector and transfected into cells. FL = full length PNPase open reading frame $(\mathrm{ORF})$ in pcDNA3.1+N-eGFP, $\mathrm{KH}=$ exon 23 removed from full length PNPase in pcDNA3.1+N-eGFP, S1 = C-terminus removed from full length PNPase in in pcDNA3.1+NeGFP. 
Figure S4.2

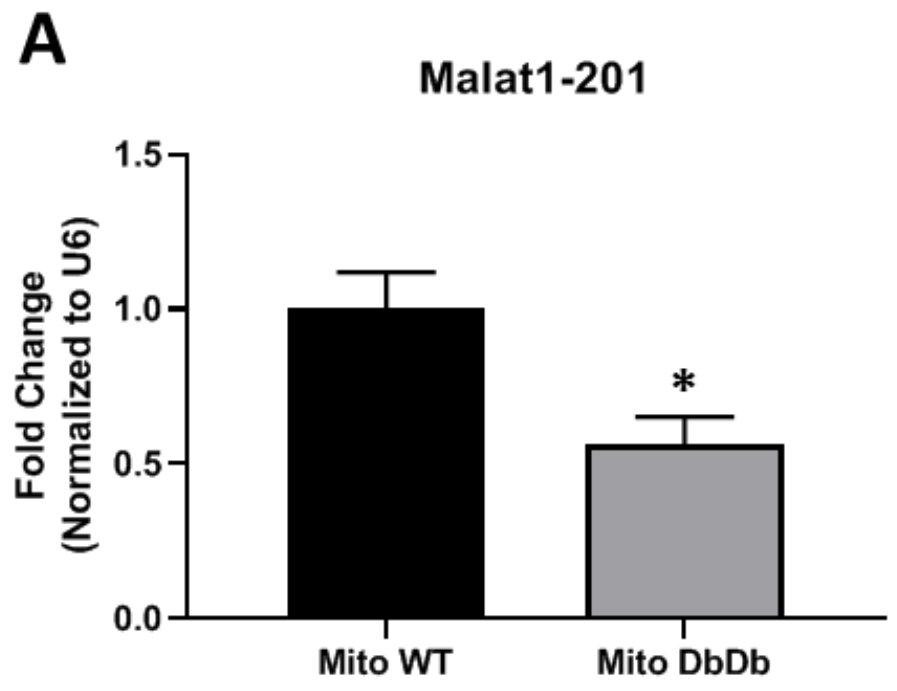

Neat1-201

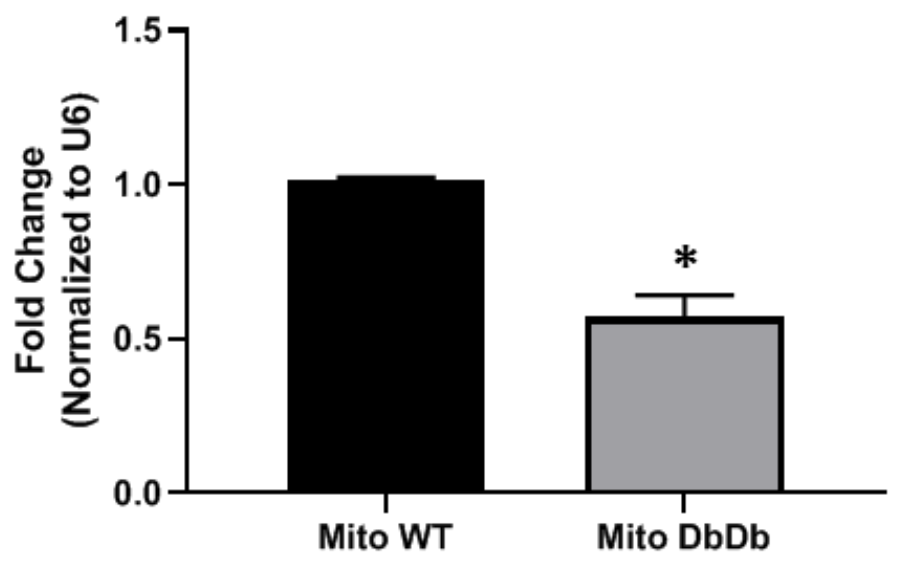


Figure S4.2

B

Human

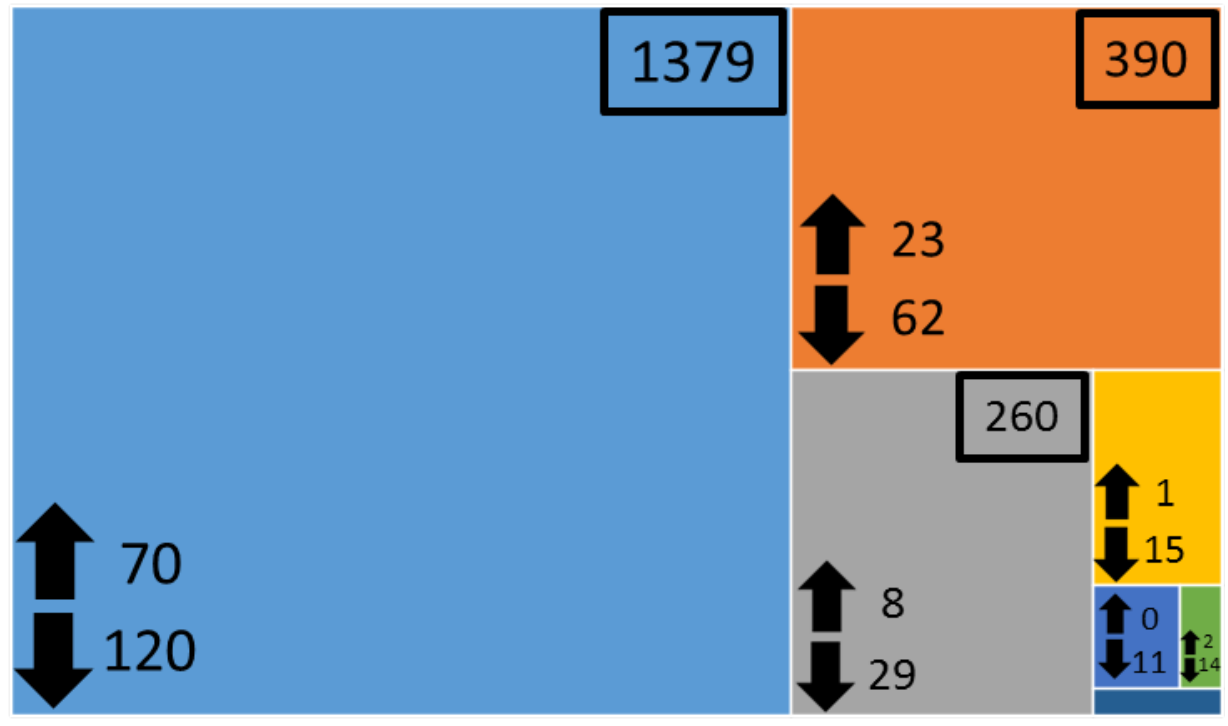

Mouse

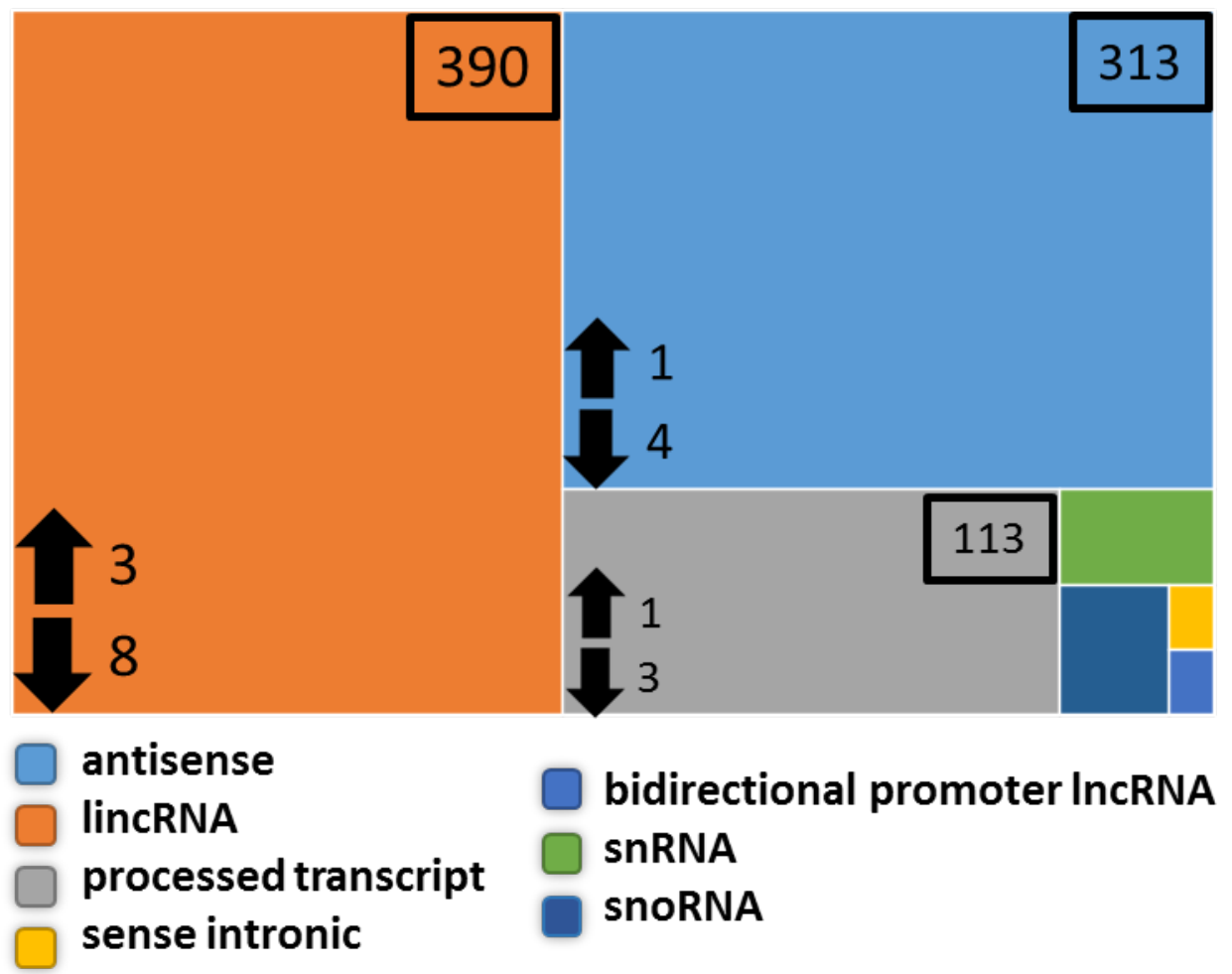


Figure S4.2
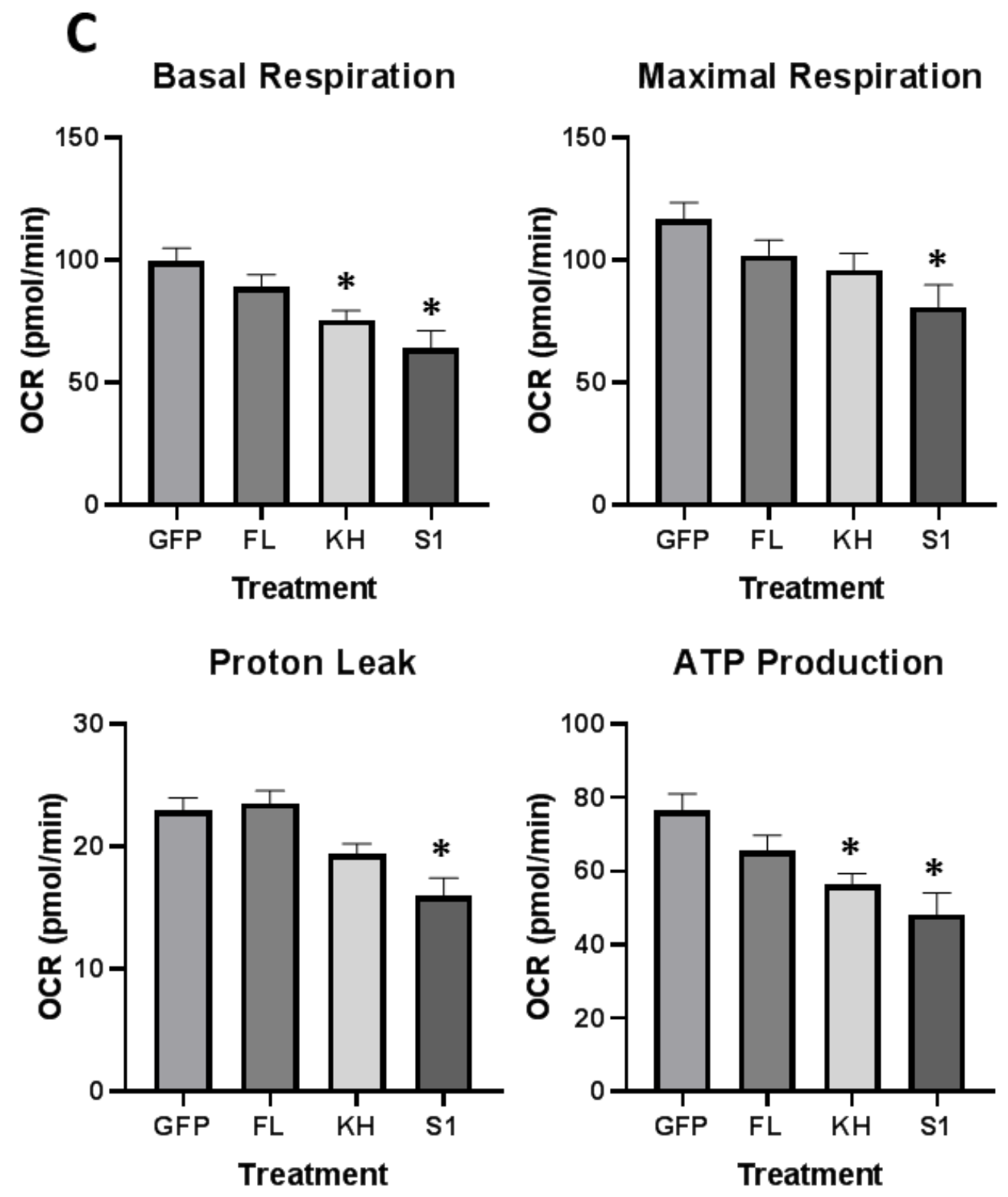
Figure S4.2: Mitochondrial and PNPase CLIP sequencing. (A) $\mathrm{In} \mathrm{Db} / \mathrm{Db}(\mathrm{n}=4)$ and wild type $(\mathrm{n}=4)$ mice, Malat1-201 and Neat1-201 were evaluated for expression through qPCR. (B) Tree map for human (top) and mouse (bottom) of ncRNAs found in the mitochondria ( $>10$ averaged counts, boxed number indicates number of genes), along with arrows indicating genes within each category shown to be significantly up or downregulated following differential expression analysis. (C) Mitochondrial respiratory capacity of HL-1 cells following transfection ( $\mathrm{n}=10$ per group) of PNPase variants. Differences between groups were considered statistically different if $P \leq 0.05$, denoted by *. All data are presented as the mean \pm standard error of the mean (SEM). $\mathrm{DbDb}=$ $\mathrm{FVB} / \mathrm{NJ}$ Lepr $^{\mathrm{d} b_{-}+/+}$mice, $\mathrm{WT}=\mathrm{FVB} / \mathrm{NJ}$ wild type mice, MALAT1 $=$ metastasis associated lung adenocarcinoma transcript 1 , NEAT1 $=$ nuclear paraspeckle assembly transcript 1 , GFP $=$ pcDNA3.1+N-eGFP backbone only, FL = full length PNPase open reading frame (ORF) in pcDNA3.1+N-eGFP, $\mathrm{KH}=$ exon 23 removed from full length PNPase in pcDNA3.1+N-eGFP, S1 $=\mathrm{C}$-terminus removed from full length $\mathrm{PNPase}$ in in pcDNA3.1+N-eGFP, lincRNA $=$ long intergenic non-coding RNA, snRNA = small nuclear RNA, snoRNA = small nucleolar RNA, OCR $=$ oxygen consumption rate. 
Figure S4.3

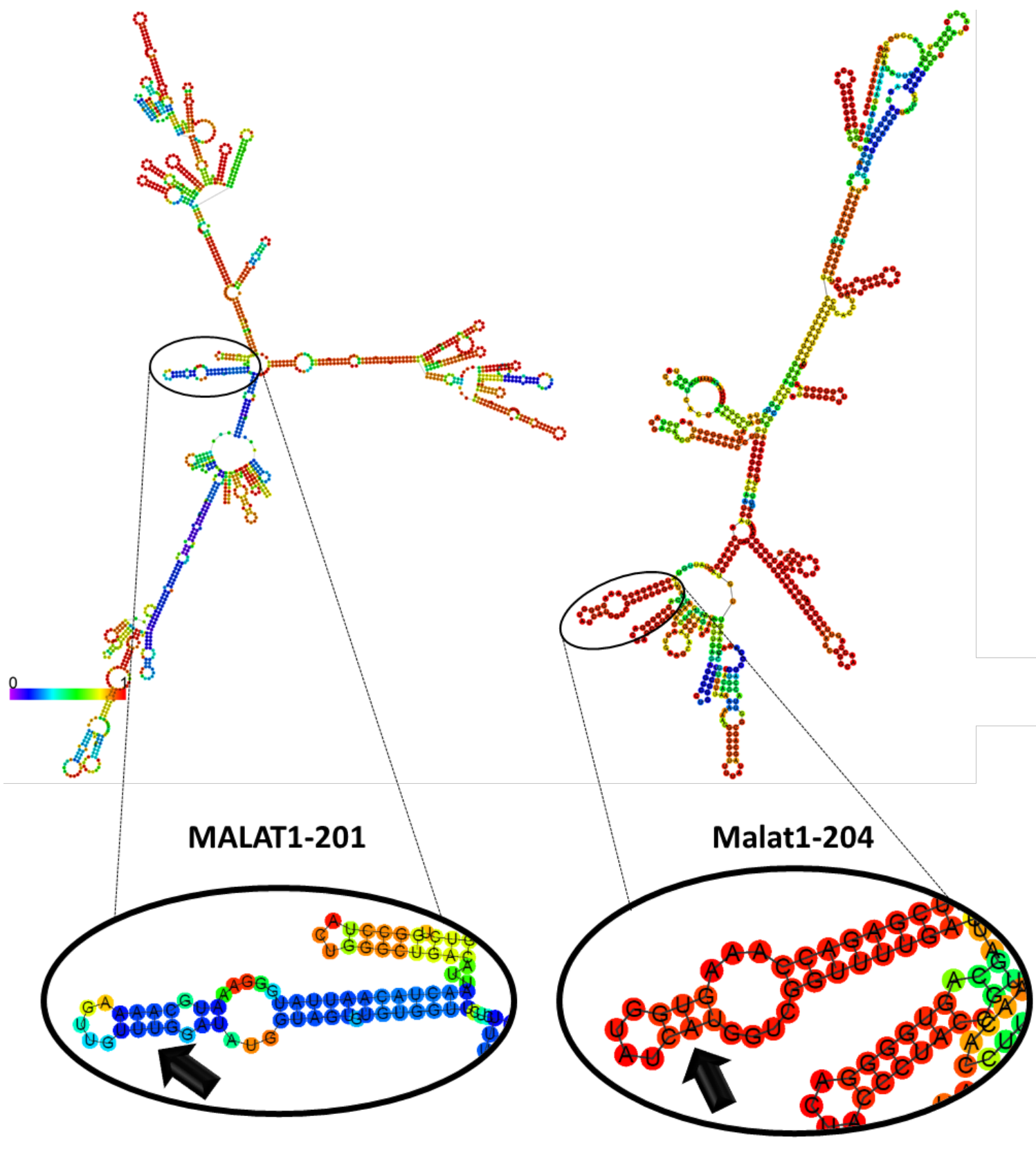


Figure S4.3
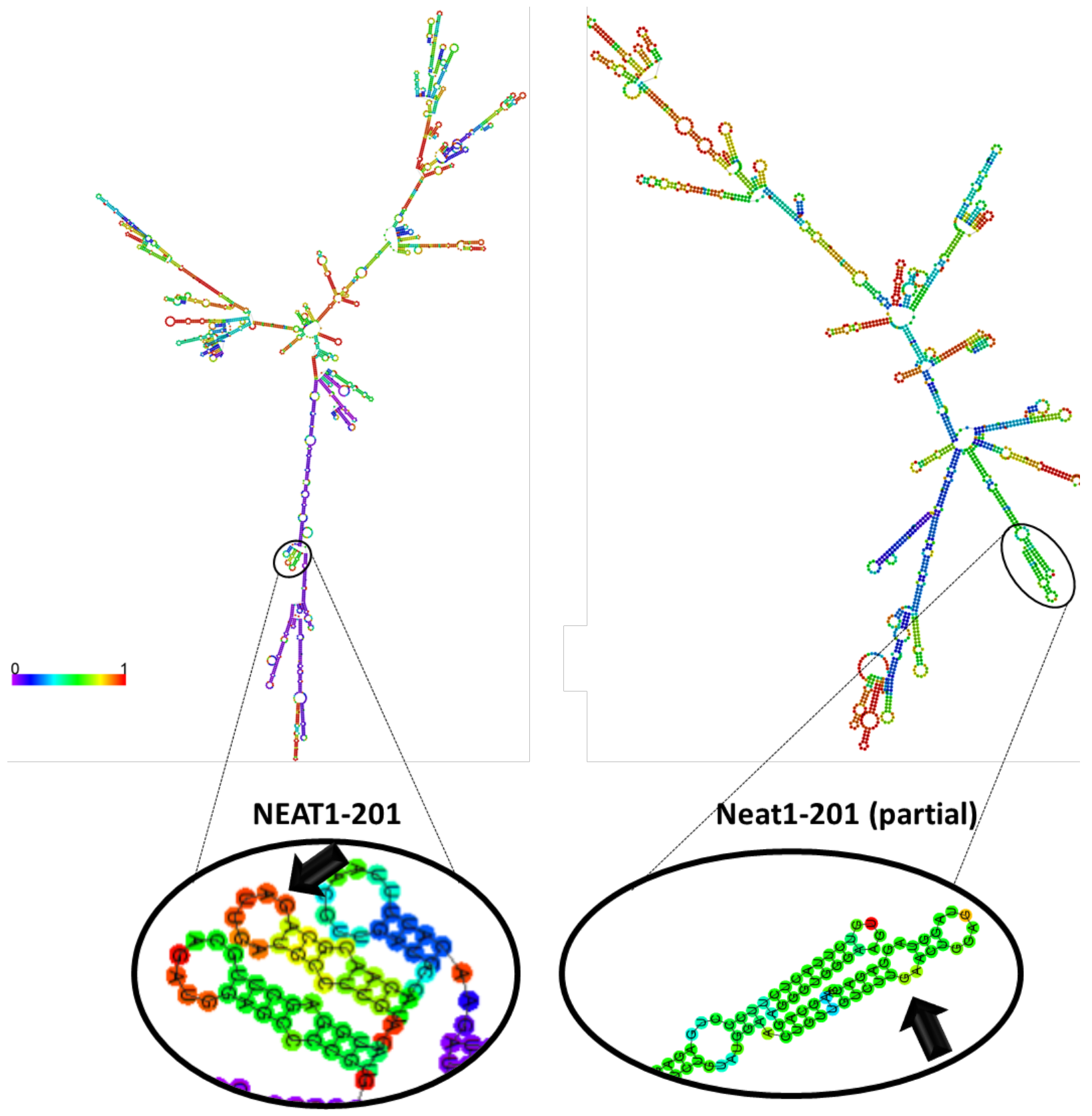
Figure S4.3: RNAfold of full length IncRNA. RNAfold was implemented for human (MALAT1201, NEAT1-201) and mouse (Malat1-204, Neat1-201 (partial)) lncRNA. Circled regions provide a zoomed in area where the reads from the PNPase CLIP (arrow indicating) are found. MALAT1 $=$ metastasis associated lung adenocarcinoma transcript 1 , NEAT $1=$ nuclear paraspeckle assembly transcript 1 . 
Figure S4.4
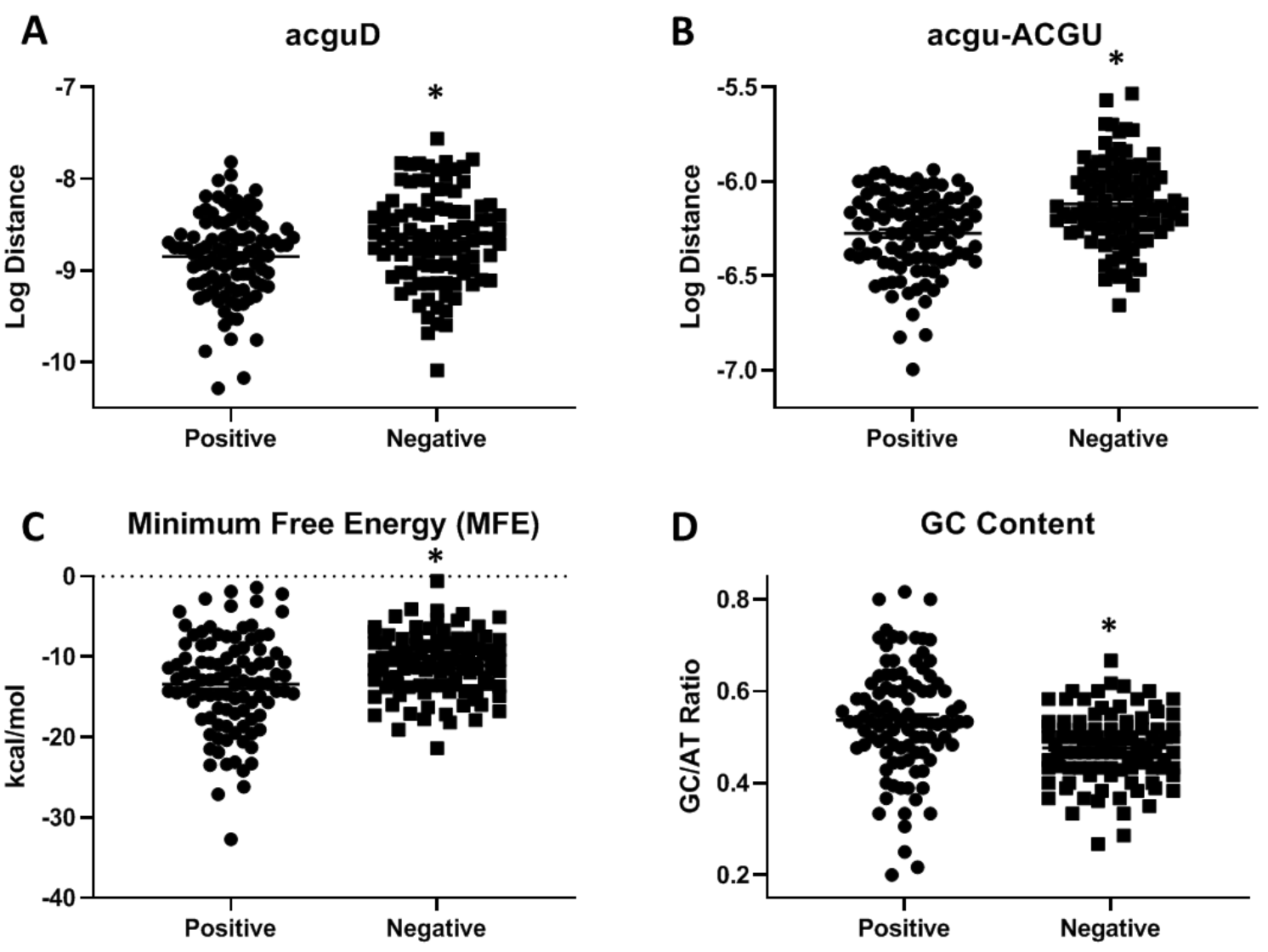
Figure S4.4: LncRNA sequence and secondary structure parameters differentially expressed between positive and negative groups. (A) The log distance of nucleotides found in unpaired configurations of dot-bracket secondary structure notation, or acguD, between groups. (B) The log distance of unpaired nucleotides (acgu) subtracted by paired nucleotides (ACGU) in the RNA secondary structure, or acgu-ACGU, between groups. (C) Minimum free energy of folding and (D) GC content between positive and negative groups. Differences between groups were considered statistically different if $P \leq 0.05$, denoted by *. All data are presented as the mean \pm standard error of the mean (SEM). Positive $=50$ human and 50 mouse ncRNAs determined to be bound to PNPase, Negative $=100$ randomly generated sequences. 


\section{Chapter 5: General Discussion}

The culmination of the work presented within this dissertation seeks to elucidate the role of the mitochondrion as a target, and effector, of epigenetic and non-coding RNA regulatory pathways in the cell. The research outlined highlights the role of engineered nanomaterials (ENMs) and diabetic insult as examples of extrinsic and intrinsic stimulation, respectively, in order to measure the mitochondrial response. Likewise, ENMs and diabetes mellitus are used as surrogates, allowing an understanding of how severity and length of insult can transiently or permanently alter the epigenetic and regulatory RNA response. Through acute (ENMs) or chronic (diabetes mellitus) activation of these pathways, a more holistic image is produced concerning the innate responses of the cell. Ultimately, the long-term goal of this research performed and data generated is to provide diagnostic or therapeutic value, providing mechanisms for better predicating mitochondrial dysfunction in any pathological state, not only in type 2 diabetes or inhalation exposure to toxicants.

My central hypothesis is that "changes in the epigenome through DNA methylation and histone modifications alter the ncRNA transcriptome subsequently changing the import dynamics of microRNA (miRNA), long non-coding RNA (lncRNA), and other ncRNA into the mitochondrion and affecting mitochondrial function through RNA-RNA and RNA-protein interactions; ultimately, producing a feedback loop where mitochondrial substrate availability shapes nuclear epigenetic machinery." The central hypothesis stated is strongly associated with the concept of nuclear influence on mitochondrial function (anterograde signaling) and the mitochondrial influence on metabolites distributed to the nucleus (retrograde) (39). While at the most fundamental level this could include direct interactions, such as reduced transcription of electron transport components needed by the mitochondrion to produce ATP, which continues to lead to a decrease in transcription of the genes by ATP dependent enzymes. In a more dynamic view, nuclear and mitochondrial signaling could influence microRNAs (miRNAs), other noncoding RNAs (ncRNAs), and epigenetic factors that could lead to indirect targeting of transcript and proteins in a systemic fashion $(18,58)$. We are still a long way from understanding which signaling pathway, changes in nuclear transcription or alterations to substrate utilization in the mitochondrion, precedes the other in the initiation of positive feedback loops between the two 
organelles. The research provided in this dissertation helps to further understand the processes of nuclear and mitochondrial adaptation to insult, and further entreat exploration into novel interaction networks within the cell.

In Specific Aim 1, we employed a transgenic mouse model with knockout expression for a ncRNA (miRNA-378a). This was the first time a ncRNA transgenic mouse had been used in the evaluation of ENM exposure paradigms, and the cardiovascular consequences seen provided significant insight into modes of ncRNA expression in the heart following exposure. While miRNA-378a has been shown to have a clear link to metabolism $(17,26,34)$ and the miRNA-378a knockout murine model has been implemented in diabetes/obesity research (6), the research presented in Specific Aim 1 highlights how perturbation to the nuclear mitochondrial interaction network can occur. ENM exposure was shown to not only decrease metabolic function through miRNA-378a inhibiting fatty acid metabolism genes, but the data suggest a restructuring of mitochondrial fission/fusion dynamics. While miRNA-378a may only be elevated for a few days following inhalation exposure to ENMs, changes to the number of mitochondria and mitochondrial ultrastructure suggests more latent effects, which could contribute to alterations in the nucleus.

While not explored in Specific Aim 1, epigenomic mechanisms could be one of the governing cellular dynamics producing the maladaptive response to ENM inhalation exposure. Epigenomic remodeling could occur in a multiplicity of ways which could disrupt miRNA-378a expression, but the two most probable sources would be 1) manipulation of transcription machinery and 2) regulation of the PGC-1 $\beta$ gene. A rise in miRNA-378a expression in young adult mice following ENM exposure could be linked to changes in the epigenetic regulation, transcription, and ultimate alteration of transcription factor activation/repression of the genome. In Specific Aim 1, Ppara, a transcription factor known to activate genes involved in fatty acid and lipid metabolism (62), was found to be increased in miRNA-378a knockout animals. Likewise, ENM inhalation exposure may cause a larger, dysregulated transcription factor profile in the heart, which could include targets directly increasing the expression of PGC-1 $\beta$, such as by Ppar $(29)$. Specifically, as documented in osteoblasts (25), increasing iron uptake by the cell transferrin receptor 1 (TfR1) promoted the transcriptional activation of PGC-1 $\beta$, which reveals the potential for environmental stimulation to alter PGC-1 $\beta$ expression. 
In the altered cellular state of cardiac tissue following ENM inhalation exposure, it may be the case that the epigenomic environment, either through histone alterations or DNA methylation, of promoter and enhancer regions in genomic sites encoding for transcription factors can lead to decreased/increased expression of that specific protein. These broad, genome-wide epigenomic changes are likely occurring to transcription factors in the experimental model outlined in Specific Aim 1, but the use of both broad-scale proteomic and DNA methylation analyses would be needed to verify which transcription factor networks are perturbed and the effects of miRNA expression, such as miRNA-378a. Another mechanism which could be controlling miRNA-378a expression following ENM inhalation exposure could be the direct epigenetic control of the PGC- $1 \beta$ promotor loci. Methylation of the PGC-1 $\beta$ promoter region has been previously reported, with detrimental health consequences associated with increased methylation (33). Through modification to the methylation status of the PGC-1 $\beta$ promoter region, ENM inhalation exposure could modify the expression profiles of PGC-1 $\beta$ and subsequently miRNA-378a.

As a proxy for understanding ncRNA dynamics, ENM exposure provides an acute, environmental stimulation that allows for tracking a very specific response. Unlike diabetes mellitus, which is generally chronic and has many systemic comorbidities, inhalation exposure can allow for tracking the persistence of specific epigenetic or regulatory networks across generational gaps $(21,52)$. Supporting Specific Aim 1, Chapter 6 outlines material pertinent to the assessment of ENM exposure on changes to cellular regulatory networks. In the heart, following maternal ENM inhalation exposure, mitochondrial and cardiovascular function are significantly perturbed in the fetal progeny and this continues, to a lesser extent, into adulthood. To investigate this, we examined both transcriptomic and epigenomic reprogramming in the heart of fetal progeny. Interestingly, the most significantly regulated genes through epigenetic alterations were nuclearencoded mitochondrial proteins involved in oxidative phosphorylation.

Increased histone modifications, specifically of the histone 3 lysine 4 tri-methylation mark (H3K4me3), suggests that these genes should become more transcriptionally activated through the relaxation of the chromatin ultrastructure (22), but the transcriptomic profile in Chapter 6 suggest that the actual expression of these genes are not becoming altered. Following gestational ENM inhalation exposure, the fetal progeny exhibit obvious epigenomic alterations that, while not immediately revealing transcriptional changes, could become preserved during development and 
ultimately make the transcription of those genes more susceptible to transcription factor binding and other stimulation in the future. While the transcriptomic analysis only surveyed mRNA within the heart, gene and protein ontology suggested that miRNA-145 was likely downregulated. MiRNA-145 is commonly downregulated in cancers $(9,42)$ and plays a role in unregulated cell growth. And while a more thorough analysis of ncRNA interaction with the epigenome following exposure is needed, the results obtained in the three studies $(20,21,52)$ suggest that dysregulation of miRNAs could be a common response that can alter cell survival (miRNA-145) or metabolism (miRNA-378a), having lasting consequences.

Specific Aim 2 engages the idea of mitochondrial and nuclear dynamics from a diagnostic perspective. While glycosylated hemoglobin (HbA1c) and/or fasting blood glucose remain primary diagnostic identifiers (16), there persists a major gap in understanding of the linear or noncorrelative nature of increased glucose in the blood stream on mitochondrial function. Mitochondrial dysfunction during diabetic insult is well known $(46,50)$, but the question of how mitochondrial function tracts with "severity" of diabetes, as assessed through changes in HbA1c, is not clear. In Specific Aim 2, we wanted to understand if a specific set of molecular markers coincided with progression of diabetes, as determined by HbAlc, and how those markers may be used in a clinical setting for diagnoses. We found that global DNA methylation (5-methylcytosine) was the best predictor of diabetic status. And interestingly, in patients who had been diagnosed as diabetic, even if they had since their diagnosis controlled their diabetes and reduced HbA1c to normal levels, still exhibited higher global methylation. HbAlc is a convenient and easy diagnostic tool, but the cellular damage induced by hyperglycemic conditions may not be easily reversible by lifestyle interventions (as indicated by DNA methylation levels) $(14,43)$.

The work in Specific Aim 2 also helps to elucidate how new bioinformatic and artificial intelligence tools can be applied to better characterize data. The interaction network between the nucleus and mitochondrion is very multifaceted. While ATP production and metabolism may be primary function of the mitochondrion, other functions including ROS production, calcium regulation, apoptotic responses, inflammation, and production of other metabolites make interpreting how a specific molecule or pathway is truly influencing the disease or pathogenic state (51). Machine learning, deep learning, and algorithms capable of building on data to predict outcomes introduces a new level to biological analyses. Cardiology is among the major fields 
utilizing machine learning algorithms (28), though this has been mainly employed in the assessment of imaging from non-invasive procedures, such as echocardiography and tomography $(30,47)$. As addressed in Specific Aim 2, what is lacking is a practical application to molecular datasets. In order to truly define the networks existing between the mitochondrion and nucleus, integration of data at a large scale is needed. In diabetes research, investigators are beginning to examine how using collected metadata for commonly assessed parameters, including BMI, age, sex, cardiovascular function, $\mathrm{HbA1c}$, and others can predict diabetic onset, progression, and future outcomes $(10,31)$. While this is certainly helpful, until machine learning is applied at the granularity of the cell, including DNA, RNA and protein modifications, it will be difficult to truly create a spectrum of disease progression and achieve true personalized medicine.

So where is the field when it comes to understanding "big data" and the applications of tools to derive more specific conclusions? Described in a 2013 by Berger et al., high-throughput data was complex, though the cost-to-data generation was decreasing, a fundamental understanding of how to even analyze large datasets was still needed. In the year 2019, analyzing large complex datasets is not the limitation to scientific output, it is understanding how to combine and integrate multiple, heterogeneous datasets $(23,44)$. As costs associated with acquiring omicsbased assessments continue to decrease (37), the accumulation of more and more datasets will rise. As we come to a critical mass of data collection, it is likely that the days of standalone highthroughput datasets is at an end. Through the use of the cloud, or in a shared workspace accessible by people all over the world, data can be collected, uploaded, and integrated into more useful interpretations. While the basic sciences likely will not see these changes for years to come, healthcare systems are currently moving toward this approach $(1,57,64)$. In the future, it is likely that through uploading sequencing datasets to a common repository online, such as a machine learning platform for all data generated around epigenetic marks in diabetes, a more universal understanding of disease pathogenesis can be ascertained.

In understanding how to develop diagnostic standards for molecular data, a major obstacle will be the definition of "normative" values within a population. While standards are well established for characterizing broad diseases such as diabetes mellitus (2) and heart disease (24), "normal" values for transcriptomic and epigenomic profiles need to be established in order to detail the nuances of disease progression. For example, while an HbAlc level of 6.8 may classify an 
individual as a type 2 diabetic, the expression profile of gene $\mathrm{X}, \mathrm{Y}$, and $\mathrm{Z}$ has only direct relation to a cohort that it is immediately compared to, with the absolute expression profile for most genes not having significant influence on disease diagnosis. Only recently, through the advent of more powerful statistical modelling and approaches such as co-expression analysis (54), have more concentric groupings of genes involved in major pathologies begun to be clustered. These approaches will be necessary to expand outside of the confines of transcriptome data and begin to compare epigenetic and epitranscriptomic profiles from individual-to-individual. Ultimately, the production and culmination of broad-scale molecular data will start shaping our definitions on molecular diagnoses, but reporting biases of high-throughput data (45) highlight the need for more standardized approaches when sequencing, uploading, and reporting omics data.

Presented in Specific Aim 3 of this dissertation is an old concept surrounding mitochondrial biology that is now present in a new light. In the first discoveries of ncRNAs in the mitochondrion, specifically miRNAs, it was thought that the mitochondrion may act as a "sink," holding miRNAs to interact with mRNA or to release when needed $(4,32)$. This theory has faded away as an interest for miRNA-directed mediation of the mitochondrial genome was pursued $(15,38)$. While evidence has been found of miRNA-directed regulation of mitochondrial transcripts, it is unlikely that this form of regulation is their primary function. A study by Dasgupta et al, reported that in mitochondrial DNA deficient cells, miRNAs are still found to be imported (13). Our group, in this report as well as previous datasets (26), has reported the presence of miRNAs in the mitochondrion, but most of which appear to have no direct mapping correlate to mitochondrial DNA or transcribed products. What could this mean about the larger mitochondrial regulatory network? As summarized by Kren et al., “...to provide an alternate mechanism for control of cellular function" (32).

The diverse nature of miRNAs, lncRNAs, and other ncRNAs found in/or in association with the mitochondrion suggests a larger regulatory role. The regulatory network of ncRNAs that interacts with the mitochondrion is likely another piece in the larger story of nuclear and mitochondrial signaling mechanics. In Specific Aim 3, the data suggests a significant drop in diversity of ncRNA during diabetic disease progression. While canonical mitochondrial miRNA theory has suggested that a rise in miRNAs within the mitochondrion during diabetic onset (26) and heart failure $(11,12)$ negatively affects the translation of the mitochondrial genome, the current 
data would suggest that a loss of miRNAs may be detrimental. The data within this dissertation help to elucidate the makeup of the ncRNA regulatory network, but also give clarity to the interactions during disease. We suggest that through a loss in ncRNAs, the robustness of response to cellular insults is decreased, impairing the capacity to inhibit specific mRNAs related to apoptosis and cellular stress (32).

With a depletion of total ncRNA within the mitochondrion, that also means a decreased diversity of lncRNAs. While Kren et al. suggested that miRNAs may be within the mitochondrion to regulate a sequestered population of mRNA (32), what if miRNAs were sequestered not by the mitochondrion, but by lncRNAs? In a review assessing the stoichiometric concentrations of ncRNAs and the competing endogenous RNA theory (53), it is suggested that for most lncRNAs, their abundance in the cellular milieu is generally too low to pose a significant threat in the sequestration of miRNAs. While studies have shown popular lncRNAs, such as MALAT1 (3, 8, 40), sponging miRNAs in the nucleus or cytoplasm, most lncRNAs are not transcribed in concentrations dense enough to truly impact miRNA activity (53). Though, if the lncRNA was localized to a cellular compartment, such as the mitochondrion, this could change its stoichiometric ratios. The mitochondrion may use the import of lncRNAs in order to sequester miRNAs to be used later in cellular defense. Through a database recording immunoprecipitation experiments validating lncRNA sponging (41), three of the lncRNAs found to be important in the study (MALAT1, NEAT1, and KCNQ1OT1) were found to bind to most of the miRNAs reported within the mitochondrion.

The work included in this dissertation only begins to assess sponging theory in the mitochondrion. More information is needed on the reason and mechanism for these ncRNAs disappearing during disease onset. One explanation could be that during diabetic insult, increases in ROS, changes in calcium handling, and subsequent increased permeability through the mitochondrial permeability transition pore (MPTP) may lead to increased release of sequestered miRNAs $(7,27)$. While during basal conditions the mitochondrion is able to import and regulate the flux of ncRNAs, changes in metabolism, such as in diabetes, could reduce the selectivity of the mitochondrial regulatory network. Though changes in membrane permeability might explain the efflux of ncRNA in the diabetic state, there still remains the concern of the import and export mechanisms involved. One popular theory is the transport of miRNAs into the mitochondrion 
through Ago2, which has been reported to be within the mitochondrion (35). Through a mechanism involving capping by GW182, to avoid degradation (60), Ago2 can transport into the mitochondrial matrix bringing along other miRNAs (63).

Ago2 has been a popular choice for miRNA import due to the miRNA processing and capacity and RISC formation (48). But, even if Ago2 is responsible for importing every miRNA into the mitochondrion, what mechanism could import larger ncRNAs, such as lncRNA and/or antisense transcripts? PNPase, while only shown to interact with tRNA, ribozymes, and other smaller ncRNA $(49,55,56)$ its regulation of larger RNAs has not been reported in mammalian systems. In Specific Aim 3, our data suggest that the RNA bound to PNPase are predominately large ncRNAs, with only a few miRNAs shown to have any binding to PNPase. With the CLIP data, as well as data suggesting PNPase has increased expression in diabetes, surmising a role for the protein is difficult; IncRNAs are binding PNPase, the expression of PNPase is elevated in diabetes, and total lncRNA biodiversity declines in diabetic mitochondrion. One logical conclusion to reach is that PNPase has an assortment of functions, ranging from import, export, and/or degradation of RNA. Because the protein is a known ribonuclease in bacterial systems $(5,19)$, it is plausible that its nuclease capacity is evolutionarily conserved. The mitochondrial ncRNA import pathway remains as elusive as it did in 2006 when miRNAs were first discovered in the mitochondrion (36), but the studies presented in Specific Aim 3 give a new perspective on the role the KH and S1 RNA binding domains of PNPase may have on mitochondrial RNA import.

As the work presented in Specific Aim 3 provide the first perspective of lncRNA and other ncRNAs occupying the mitochondrial space, future investigations should seek to understand the true purpose of this ncRNA regulatory network. While sequestering is proposed between miRNA/lncRNA a plethora of other possibilities could exist. These roles of lncRNA could include the use of the mitochondrion to provide further processing through small nucleolar RNA (snoRNA) (59), a location to avoid RNA degradation through proteins such as XRN1 (61), and the possible site for novel RNA/protein interactions to promote/disrupt mitochondrial health and function. Further, Specific Aim 3 reveals that snoRNA, small nuclear (snRNA), and other ncRNAs exist in the mitochondrion, adding to the complexity of the regulatory network and complicating the spectrum of associations that could be occurring. The ability to manipulate this multifaceted mitochondrial network may provide new and profound insights into the understanding of disease 
progression and the targeting of therapeutics; potentially, functional manipulation of the PNPase $\mathrm{KH}$ and S1 RNA binding domains may allow for the control of specific ncRNA species to be imported into the mitochondrion.

\section{Limitations}

While the included studies provide insight into mitochondrial biology and cardiovascular function following insult, weakness and limitations to the research should be addressed. Specific Aim 1 examines the effects of miRNA-378a on modifying the metabolic response following ENM exposure, but it is likely that other miRNAs play significant roles in the exposure. Though the transgenic model in Specific Aim 1 could only address one miRNA, future experimentations need to evaluate the complete miRNA and ncRNA transcriptomic response to ENM; this includes investigations applying co-expression analysis, ontology software, and other computer-assisted methodologies to describe the interaction networks of ncRNA found in the heart following insult. Specific Aim 2, an archetype for future applications of machine learning to the level of DNA and RNA, suffers from lack of sample size and patient heterogeneity.

With a sample size of fifty patients, CART analysis provided the best overall predicative accuracies, but this may not hold true with larger sample sizes. Though Specific Aim 2 serves as a guideline for future application, until further investigations are made on larger sample sizes with patient-matched sequencing datasets, the determination of the exact algorithms appropriate for application to a variety of disease-states remains unclear. Specific Aim 3 provides the most novel insights within the body of the dissertation, elucidating new, mitochondrial regulatory pathways. With the breadth of novel work, further validation of the targets found in the study are needed. While MALAT1 is confirmed through qPCR, additional experiments, such as CLIP of MALAT1 and RNA pull downs, are truly needed to explain and validate the novel biology.

\section{Conclusions}

The inner workings of the mitochondrial and nuclear network have much to be unveiled. My hope is that the work presented in this dissertation has contributed to the understanding of the anterograde and retrograde signaling of the cardiac cell; specifically, in the assessment of acute (ENM) and chronic (diabetes mellitus) exposures on the epigenetic and ncRNA framework. Machine learning at the DNA level hints at the possibility of precision medicine-based diagnostic 
modalities in the future, while PNPase remains a potential target for therapeutic intervention. Allin-all, the mitochondrion, its regulatory network, and the nuclear epigenome remain critical areas of research and need for innovative scientific approaches (Figure 5.1).

\section{Figure 5.1: Overview of Dissertation Aims}

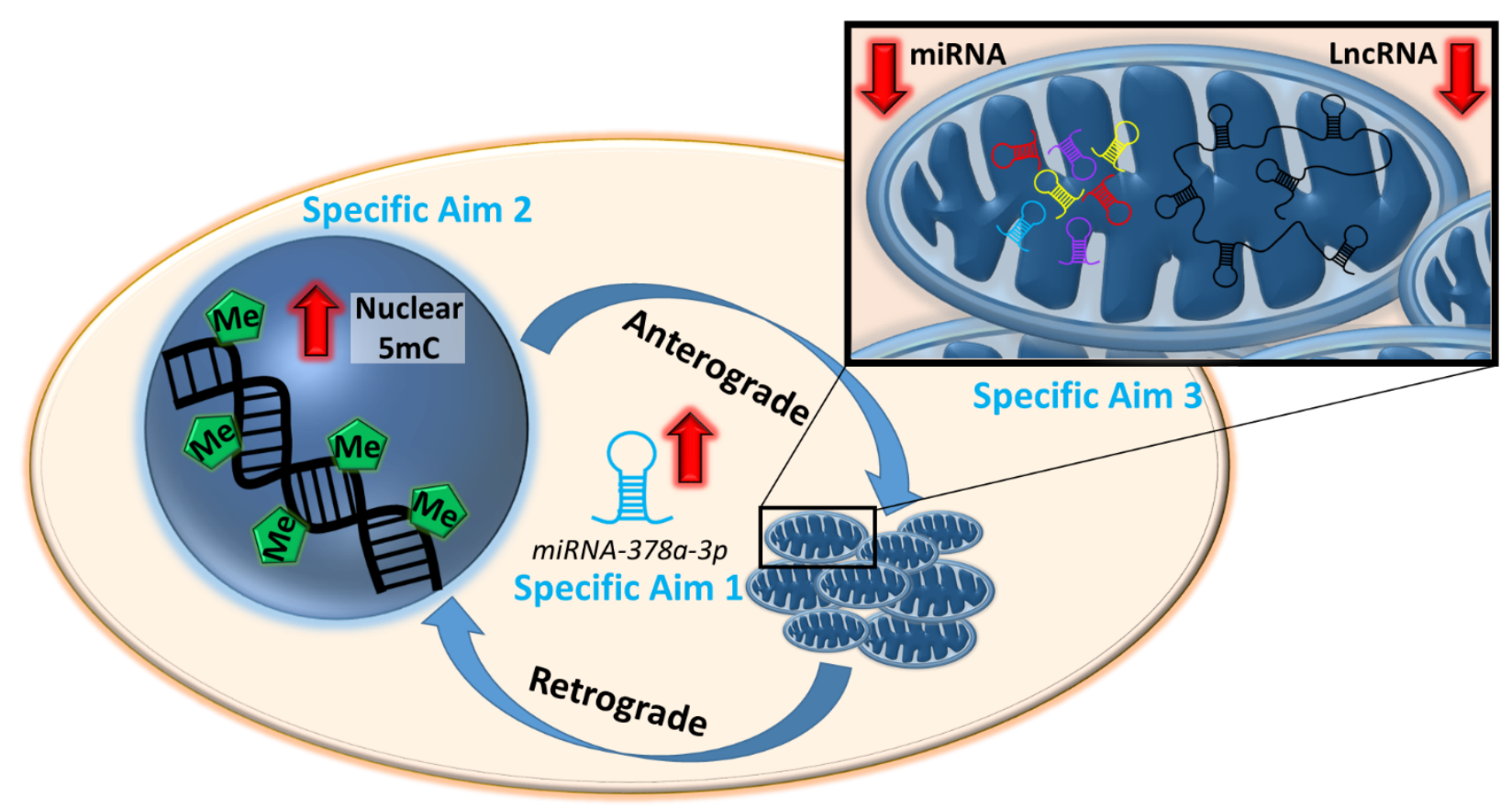

Figure 5.1: Disruption to the cellular and mitochondrial networks during insult through ENMs (Specific Aim 1) and diabetes mellitus (Specific Aim $2 \& 3$ ). 


\section{References}

1. Abdelaziz A, Elhoseny M, Salama AS, and Riad AM. A machine learning model for improving healthcare services on cloud computing environment. Measurement 119: 117-128, 2018.

2. American Diabetes A. Diagnosis and classification of diabetes mellitus. Diabetes Care 37 Suppl 1: S81-90, 2014.

3. Amodio N, Raimondi L, Juli G, Stamato MA, Caracciolo D, Tagliaferri P, and Tassone P. MALAT1: a druggable long non-coding RNA for targeted anti-cancer approaches. $J$ Hematol Oncol 11: 63, 2018.

4. Borralho PM, Rodrigues CMP, and Steer CJ. Mitochondrial MicroRNAs and Their Potential Role in Cell Function. Current Pathobiology Reports 2: 123-132, 2014.

5. Cameron TA, Matz LM, and De Lay NR. Polynucleotide phosphorylase: Not merely an RNase but a pivotal post-transcriptional regulator. PLoS Genet 14: e1007654, 2018.

6. Carrer M, Liu N, Grueter CE, Williams AH, Frisard MI, Hulver MW, Bassel-Duby R, and Olson EN. Control of mitochondrial metabolism and systemic energy homeostasis by microRNAs 378 and 378*. Proc Natl Acad Sci U S A 109: 15330-15335, 2012.

7. Chaudhuri AD, Choi DC, Kabaria S, Tran A, and Junn E. MicroRNA-7 Regulates the Function of Mitochondrial Permeability Transition Pore by Targeting VDAC1 Expression. J Biol Chem 291: 6483-6493, 2016.

8. Cremer S, Michalik KM, Fischer A, Pfisterer L, Jae N, Winter C, Boon RA, MuhlyReinholz M, John D, Uchida S, Weber C, Poller W, Gunther S, Braun T, Li DY, Maegdefessel L, Perisic Matic L, Hedin U, Soehnlein O, Zeiher A, and Dimmeler S. Hematopoietic Deficiency of the Long Noncoding RNA MALAT1 Promotes Atherosclerosis and Plaque Inflammation. Circulation 139: 1320-1334, 2019.

9. Cui SY, Wang R, and Chen LB. MicroRNA-145: a potent tumour suppressor that regulates multiple cellular pathways. J Cell Mol Med 18: 1913-1926, 2014.

10. Dagliati A, Marini S, Sacchi L, Cogni G, Teliti M, Tibollo V, De Cata P, Chiovato L, and Bellazzi R. Machine Learning Methods to Predict Diabetes Complications. J Diabetes Sci Technol 12: 295-302, 2018.

11. Das S, Bedja D, Campbell N, Dunkerly B, Chenna V, Maitra A, and Steenbergen C. miR-181c regulates the mitochondrial genome, bioenergetics, and propensity for heart failure in vivo. PLoS One 9: e96820, 2014.

12. Das S, Ferlito M, Kent OA, Fox-Talbot K, Wang R, Liu D, Raghavachari N, Yang Y, Wheelan SJ, Murphy E, and Steenbergen C. Nuclear miRNA regulates the mitochondrial genome in the heart. Circ Res 110: 1596-1603, 2012.

13. Dasgupta N, Peng Y, Tan Z, Ciraolo G, Wang D, and Li R. miRNAs in mtDNA-less cell mitochondria. Cell Death Discov 1: 15004, 2015.

14. DiNardo AR, Nishiguchi T, Mace EM, Rajapakshe K, Mtetwa G, Kay A, Maphalala G, Secor WE, Mejia R, Orange JS, Coarfa C, Bhalla KN, Graviss EA, Mandalakas AM, and Makedonas G. Schistosomiasis Induces Persistent DNA Methylation and Tuberculosis-Specific Immune Changes. J Immunol 201: 124-133, 2018.

15. Duarte FV, Palmeira CM, and Rolo AP. The Role of microRNAs in Mitochondria: Small Players Acting Wide. Genes (Basel) 5: 865-886, 2014.

16. Edelman D, Olsen MK, Dudley TK, Harris AC, and Oddone EZ. Utility of hemoglobin A1c in predicting diabetes risk. J Gen Intern Med 19: 1175-1180, 2004. 
17. Gerin I, Bommer GT, McCoin CS, Sousa KM, Krishnan V, and MacDougald OA. Roles for miRNA-378/378* in adipocyte gene expression and lipogenesis. Am J Physiol Endocrinol Metab 299: E198-206, 2010.

18. Guha M and Avadhani NG. Mitochondrial retrograde signaling at the crossroads of tumor bioenergetics, genetics and epigenetics. Mitochondrion 13: 577-591, 2013.

19. Hajnsdorf E, Steier O, Coscoy L, Teysset L, and Regnier P. Roles of RNase E, RNase II and PNPase in the degradation of the rpsO transcripts of Escherichia coli: stabilizing function of RNase II and evidence for efficient degradation in an ams pnp rnb mutant. EMBO J 13: 33683377, 1994.

20. Hathaway QA, Durr AJ, Shepherd DL, Pinti MV, Brandebura AN, Nichols CE, Kunovac A, Goldsmith WT, Friend SA, Abukabda AB, Fink GK, Nurkiewicz TR, and Hollander JM. miRNA-378a as a key regulator of cardiovascular health following engineered nanomaterial inhalation exposure. Nanotoxicology: 1-20, 2019.

21. Hathaway QA, Nichols CE, Shepherd DL, Stapleton PA, McLaughlin SL, Stricker JC, Rellick SL, Pinti MV, Abukabda AB, McBride CR, Yi J, Stine SM, Nurkiewicz TR, and Hollander JM. Maternal-engineered nanomaterial exposure disrupts progeny cardiac function and bioenergetics. Am J Physiol Heart Circ Physiol 312: H446-H458, 2017.

22. Huang X, Gao X, Li W, Jiang S, Li R, Hong H, Zhao C, Zhou P, Chen H, Bo X, and Li H. Stable H3K4me3 is associated with transcription initiation during early embryo development. Bioinformatics, 2019.

23. Hulsen T, Jamuar SS, Moody AR, Karnes JH, Varga O, Hedensted S, Spreafico R, Hafler DA, and McKinney EF. From Big Data to Precision Medicine. Front Med (Lausanne) 6: 34, 2019.

24. Hunt SA, American College of C, and American Heart Association Task Force on Practice G. ACC/AHA 2005 guideline update for the diagnosis and management of chronic heart failure in the adult: a report of the American College of Cardiology/American Heart Association Task Force on Practice Guidelines (Writing Committee to Update the 2001 Guidelines for the Evaluation and Management of Heart Failure). J Am Coll Cardiol 46: e1-82, 2005.

25. Ishii KA, Fumoto T, Iwai K, Takeshita S, Ito M, Shimohata N, Aburatani H, Taketani S, Lelliott CJ, Vidal-Puig A, and Ikeda K. Coordination of PGC-1beta and iron uptake in mitochondrial biogenesis and osteoclast activation. Nat Med 15: 259-266, 2009.

26. Jagannathan R, Thapa D, Nichols CE, Shepherd DL, Stricker JC, Croston TL, Baseler WA, Lewis SE, Martinez I, and Hollander JM. Translational Regulation of the Mitochondrial Genome Following Redistribution of Mitochondrial MicroRNA in the Diabetic Heart. Circ Cardiovasc Genet 8: 785-802, 2015.

27. Jaquenod De Giusti C, Roman B, and Das S. The Influence of MicroRNAs on Mitochondrial Calcium. Front Physiol 9: 1291, 2018.

28. Jiang F, Jiang Y, Zhi H, Dong Y, Li H, Ma S, Wang Y, Dong Q, Shen H, and Wang Y. Artificial intelligence in healthcare: past, present and future. Stroke Vasc Neurol 2: 230-243, 2017.

29. John E, Wienecke-Baldacchino A, Liivrand M, Heinaniemi M, Carlberg C, and Sinkkonen L. Dataset integration identifies transcriptional regulation of microRNA genes by PPARgamma in differentiating mouse 3T3-L1 adipocytes. Nucleic Acids Res 40: 4446-4460, 2012.

30. Johnson KW, Torres Soto J, Glicksberg BS, Shameer K, Miotto R, Ali M, Ashley E, and Dudley JT. Artificial Intelligence in Cardiology. J Am Coll Cardiol 71: 2668-2679, 2018. 
31. Kavakiotis I, Tsave O, Salifoglou A, Maglaveras N, Vlahavas I, and Chouvarda I. Machine Learning and Data Mining Methods in Diabetes Research. Comput Struct Biotechnol J 15: 104-116, 2017.

32. Kren BT, Wong PY, Sarver A, Zhang X, Zeng Y, and Steer CJ. MicroRNAs identified in highly purified liver-derived mitochondria may play a role in apoptosis. RNA Biol 6: 65-72, 2009.

33. Kresovich JK, Joyce BT, Gao T, Zheng Y, Zhang Z, Achenbach CJ, Murphy RL, Just AC, Shen J, Yang H, Vokonas P, Schwartz J, Baccarelli AA, and Hou L. Promoter methylation of PGC1A and PGC1B predicts cancer incidence in a veteran cohort. Epigenomics 10: 733-743, 2018.

34. Krist B, Florczyk U, Pietraszek-Gremplewicz K, Jozkowicz A, and Dulak J. The Role of miR-378a in Metabolism, Angiogenesis, and Muscle Biology. Int J Endocrinol 2015: 281756, 2015.

35. Latronico MV and Condorelli G. The might of microRNA in mitochondria. Circ Res 110: 1540-1542, 2012.

36. Lung B, Zemann A, Madej MJ, Schuelke M, Techritz S, Ruf S, Bock R, and Huttenhofer A. Identification of small non-coding RNAs from mitochondria and chloroplasts. Nucleic Acids Res 34: 3842-3852, 2006.

37. Luo J, Wu M, Gopukumar D, and Zhao Y. Big Data Application in Biomedical Research and Health Care: A Literature Review. Biomed Inform Insights 8: 1-10, 2016.

38. Macgregor-Das AM and Das S. A microRNA's journey to the center of the mitochondria. Am J Physiol Heart Circ Physiol 315: H206-H215, 2018.

39. Ng S, De Clercq I, Van Aken O, Law SR, Ivanova A, Willems P, Giraud E, Van Breusegem F, and Whelan $\mathbf{J}$. Anterograde and retrograde regulation of nuclear genes encoding mitochondrial proteins during growth, development, and stress. Mol Plant 7: 1075-1093, 2014.

40. Pan Y, Tong S, Cui R, Fan J, Liu C, Lin Y, Tang J, Xie H, Lin P, Zheng T, and Yu X. Long Non-Coding MALAT1 Functions as a Competing Endogenous RNA to Regulate Vimentin Expression by Sponging miR-30a-5p in Hepatocellular Carcinoma. Cell Physiol Biochem 50: 108120, 2018.

41. Paraskevopoulou MD, Vlachos IS, Karagkouni D, Georgakilas G, Kanellos I, Vergoulis T, Zagganas K, Tsanakas P, Floros E, Dalamagas T, and Hatzigeorgiou AG. DIANA-LncBase v2: indexing microRNA targets on non-coding transcripts. Nucleic Acids Res 44: D231-238, 2016.

42. Pashaei E, Guzel E, Ozgurses ME, Demirel G, Aydin N, and Ozen M. A MetaAnalysis: Identification of Common Mir-145 Target Genes that have Similar Behavior in Different GEO Datasets. PLoS One 11: e0161491, 2016.

43. Perez RF, Santamarina P, Tejedor JR, Urdinguio RG, Alvarez-Pitti J, Redon P, Fernandez AF, Fraga MF, and Lurbe E. Longitudinal genome-wide DNA methylation analysis uncovers persistent early-life DNA methylation changes. J Transl Med 17: 15, 2019.

44. Ristevski B and Chen M. Big Data Analytics in Medicine and Healthcare. J Integr Bioinform 15, 2018.

45. Rodriguez-Esteban $\mathbf{R}$ and Jiang $\mathbf{X}$. Differential gene expression in disease: a comparison between high-throughput studies and the literature. BMC Med Genomics 10: 59, 2017.

46. Rolo AP and Palmeira CM. Diabetes and mitochondrial function: role of hyperglycemia and oxidative stress. Toxicol Appl Pharmacol 212: 167-178, 2006. 
47. Shameer K, Johnson KW, Glicksberg BS, Dudley JT, and Sengupta PP. Machine learning in cardiovascular medicine: are we there yet? Heart 104: 1156-1164, 2018.

48. Shen J and Hung MC. Signaling-mediated regulation of MicroRNA processing. Cancer Res 75: 783-791, 2015.

49. Shepherd DL, Hathaway QA, Pinti MV, Nichols CE, Durr AJ, Sreekumar S, Hughes KM, Stine SM, Martinez I, and Hollander JM. Exploring the mitochondrial microRNA import pathway through Polynucleotide Phosphorylase (PNPase). J Mol Cell Cardiol 110: 15-25, 2017.

50. Sivitz WI and Yorek MA. Mitochondrial dysfunction in diabetes: from molecular mechanisms to functional significance and therapeutic opportunities. Antioxid Redox Signal 12: 537-577, 2010.

51. Spinelli JB and Haigis MC. The multifaceted contributions of mitochondria to cellular metabolism. Nat Cell Biol 20: 745-754, 2018.

52. Stapleton PA, Hathaway QA, Nichols CE, Abukabda AB, Pinti MV, Shepherd DL, McBride CR, Yi J, Castranova VC, Hollander JM, and Nurkiewicz TR. Maternal engineered nanomaterial inhalation during gestation alters the fetal transcriptome. Part Fibre Toxicol 15: 3, 2018.

53. Thomson DW and Dinger ME. Endogenous microRNA sponges: evidence and controversy. Nat Rev Genet 17: 272-283, 2016.

54. van Dam S, Vosa U, van der Graaf A, Franke L, and de Magalhaes JP. Gene coexpression analysis for functional classification and gene-disease predictions. Brief Bioinform 19: 575-592, 2018.

55. Wang G, Chen HW, Oktay Y, Zhang J, Allen EL, Smith GM, Fan KC, Hong JS, French SW, McCaffery JM, Lightowlers RN, Morse HC, 3rd, Koehler CM, and Teitell MA. PNPASE regulates RNA import into mitochondria. Cell 142: 456-467, 2010.

56. Wang G, Shimada E, Zhang J, Hong JS, Smith GM, Teitell MA, and Koehler CM. Correcting human mitochondrial mutations with targeted RNA import. Proc Natl Acad Sci U S A 109: 4840-4845, 2012.

57. Wang XL, Gui Q, Liu BW, Jin ZP, and Chen Y. Enabling Smart Personalized Healthcare: A Hybrid Mobile-Cloud Approach for ECG Telemonitoring. Ieee J Biomed Health 18: 739-745, 2014.

58. Weinhouse C. Mitochondrial-epigenetic crosstalk in environmental toxicology. Toxicology 391: 5-17, 2017.

59. Xing YH and Chen LL. Processing and roles of snoRNA-ended long noncoding RNAs. Crit Rev Biochem Mol Biol 53: 596-606, 2018.

60. Yao B, La LB, Chen YC, Chang LJ, and Chan EK. Defining a new role of GW182 in maintaining miRNA stability. EMBO Rep 13: 1102-1108, 2012.

61. Yoon JH, Kim J, and Gorospe M. Long noncoding RNA turnover. Biochimie 117: 1521, 2015.

62. Yoon M. The role of PPARalpha in lipid metabolism and obesity: focusing on the effects of estrogen on PPARalpha actions. Pharmacol Res 60: 151-159, 2009.

63. Zhang X, Zuo X, Yang B, Li Z, Xue Y, Zhou Y, Huang J, Zhao X, Zhou J, Yan Y, Zhang H, Guo P, Sun H, Guo L, Zhang Y, and Fu XD. MicroRNA directly enhances mitochondrial translation during muscle differentiation. Cell 158: 607-619, 2014.

64. Zhang Y, Qiu MK, Tsai CW, Hassan MM, and Alamri A. Health-CPS: Healthcare Cyber-Physical System Assisted by Cloud and Big Data. Ieee Syst J 11: 88-95, 2017. 


\title{
Chapter 6: Related Material to Specific Aims
}

\author{
Section 1.1: Supporting Documentation for Specific Aim 1
}

\section{Maternal Engineered Nanomaterial Exposure Disrupts Progeny Cardiac Function and Bioenergetics}

As published in Am J Physiol Heart Circ Physiol. 2017 Mar 1;312(3):H446-H458. doi: 10.1152/ajpheart.00634.2016. Epub 2016 Dec 23

Quincy A. Hathaway ${ }^{1,4}$, Cody E. Nichols ${ }^{1}$, Danielle M. Shepherd ${ }^{1,4}$, Phoebe A. Stapleton ${ }^{2}$, Sarah L. McLaughlin ${ }^{3}$, Janelle C. Stricker ${ }^{1}$ Stephanie L. Rellick ${ }^{2}$, Mark V. Pinti ${ }^{1}$, Alaeddin B. Abukabda $^{2}$, Carroll R. McBride ${ }^{2}$, Jinghai Yi $^{2}$, Seth M. Stine ${ }^{1,4}$, Timothy R. Nurkiewicz ${ }^{2,4}$, John M. Hollander ${ }^{1,4}$

West Virginia University School of Medicine, ${ }^{1}$ Division of Exercise Physiology; ${ }^{2}$ Department of Physiology and Pharmacology, ${ }^{3}$ Department of Cancer Cell Biology, Morgantown, ${ }^{4}$ Mitochondria, Metabolism \& Bioenergetics Working Group, WV 26506

Running Title: Gestational Nano- $\mathrm{TiO}_{2}$ and Cardiac Dysfunction

Key Words: Nanomaterials, Cardiac dysfunction, Pregnancy, Mitochondria 


\begin{abstract}
Nanomaterial production is expanding as new industrial and consumer applications are introduced. Nevertheless, the impacts of exposure to these compounds are not fully realized. The current study was designed to determine whether gestational nano- $\mathrm{TiO}_{2}$ exposure impacts cardiac and metabolic function of developing progeny. Pregnant Sprague Dawley rats were exposed to nano- $\mathrm{TiO}_{2}$ aerosols ( $\sim 10 \mathrm{mg} / \mathrm{m}^{3}, 130-150 \mathrm{~nm}$ count median aerodynamic diameter) for 7-8 non-consecutive days beginning at gestational day 5-6. Physiological and bioenergetic effects on heart function and cardiomyocytes across three time points: fetal (gestational day 20), neonatal (4-10 days), and young adult (6-12 weeks) were evaluated. Functional analysis utilizing echocardiography, speckletracking based strain, and cardiomyocyte contractility coupled with mitochondrial energetics revealed effects of nano- $\mathrm{TiO}_{2}$ exposure. Maternal exposed progeny demonstrated a decrease in $\mathrm{E}$ and A wave velocities with a 15\% higher E/A ratio than controls. Myocytes isolated from exposed animals exhibited $\sim 30 \%$ decrease in total contractility, departure velocity, and area of contraction. Bioenergetic analysis revealed a significant increase in proton leak across all ages, accompanied by decreases in metabolic function including basal respiration, maximal respiration, and spare capacity. Finally, electron transport chain complex I and IV activities were negatively impacted in the exposed group, which may be linked to a metabolic shift. Molecular data suggests that an increase in fatty acid metabolism, uncoupling, and cellular stress proteins may be associated with functional deficits of the heart. In conclusion, gestational nano- $\mathrm{TiO}_{2}$ exposure significantly impairs the functional capabilities of the heart through cardiomyocyte impairment, which is associated with mitochondrial dysfunction.
\end{abstract}




\section{New and Noteworthy}

Cardiac function is evaluated, for the first time, in progeny following maternal nanomaterial inhalation. The findings indicate that exposure to nano-sized titanium dioxide (nano- $\mathrm{TiO}_{2}$ ) during gestation negatively impacts cardiac function and mitochondrial respiration and bioenergetics. We conclude that maternal nano- $\mathrm{TiO}_{2}$ inhalation contributes to adverse cardiovascular health effects, lasting into adulthood. 


\section{Introduction}

Growing production, distribution, and use of engineered nanomaterials (ENMs) necessitates more thorough evaluations of the physiological effects that exposure to these ENMs induce. One of the most widely used ENM in the world is titanium dioxide (nano- $\mathrm{TiO}_{2}$ ); in 2006, 40,000 metric tons of nano- $\mathrm{TiO}_{2}$ were estimated to be produced domestically, with 2015 projections reaching as high as 260,000 metric tons (43). Specifically, it is well-accepted that inhaled ENMs can interact in one of three ways: an acute inflammatory response, direct translocation, and through the autonomous nervous system in the lungs (19); the link between pulmonary exposure and extrapulmonary effects provides a picturesque example of the complications that may arise when trying to decipher a prominent mechanism for nano- $\mathrm{TiO}_{2}$ toxicity.

In the heart, it has been shown that through pulmonary exposure $(15,36,57)$, intravenous administration (30), and orally (16), ENMs can affect cardiac function, though, this is not without disagreement $(8,20)$. Nano- $\mathrm{TiO}_{2}$ accumulation in cardiac tissue has been demonstrated to increase edema and apoptosis during embryonic development $(37,64)$. In adult exposures, nano- $\mathrm{TiO}_{2}$ causes a decrease in bioenergetics, increase in inflammatory signaling, and DNA damage $(15,16$, 67). While measures of direct interaction between an organism and nano- $\mathrm{TiO}_{2}$ have revealed much about cardiac dysregulation, very little has been shown regarding the mechanisms affecting cardiac tissue of gestational exposed progeny (22). Recent studies have demonstrated a role for diesel exhaust particulate matter air pollution in causing negative cardiac implications in progeny from maternal exposures $(18,58,59)$, but no study has focused specifically on the longitudinal effects of a single, characterized ENM on cardiac function.

Though many extrapulmonary effects are well recognized, in vivo cardiac functional impacts are less commonly identified as toxicological end-points, making investigation into the effects essential for expanding our knowledge. Studies have examined the effects of directly placing $\mathrm{TiO}_{2}$ nanoparticles on cardiomyocytes in vitro. These studies have concluded that high enough concentrations of nano- $\mathrm{TiO}_{2}(>10 \mu \mathrm{g} / \mathrm{mL})$ can directly affect the functionality of cardiomyocytes, including measures of shortening, re-lengthening, and amplitude of contraction $(26,46)$; decreased functionality remained consisted across varying frequencies of contractions. Previous work has examined the cardiac bioenergetics from young adult Sprague Dawley rats exposed during gestation to nano- $\mathrm{TiO}_{2}$; this study demonstrated that state 3 and 4 respiration were 
significantly altered (54), providing the rationale for defining the specific impacts on cardiomyocyte bioenergetics more closely.

Recent literature has examined how ENM exposure can affect the in utero environment. Maternal nano- $\mathrm{TiO}_{2}$ inhalation disrupts uterine microvascular function, and creates a hostile gestational environment for development (51), further, intravenous injection of carbon nanotubes has been shown to translocate to the placenta, resulting in fetal deformities and lower progeny yields $(7,23,42)$. With known physiological impacts to the developing fetus following ENM exposure, the need for an initial understanding of the cardiac consequences resulting from changes within the in utero environment is crucial. Ultimately this understanding could have profound effects, including: ENM exposure standards for pregnant women, defining kinetics of nanoparticles across the placental barrier, and outlining mechanisms affecting cardiac/mitochondrial development in exposed progeny. To determine how nano- $\mathrm{TiO}_{2}$ could affect progeny, we subjected pregnant Sprague Dawley rats to pulmonary exposure of nano- $\mathrm{TiO}_{2}$ throughout gestation. These exposures allowed for measurement across three critical developmental points: fetal, neonatal, and young adult. To date, no study has examined cardiomyocyte function following gestational exposure to ENMs. We hypothesized that maternal nano- $\mathrm{TiO}_{2}$ exposure would negatively impact cardiac function and cardiomyocyte bioenergetics as compared to air exposed progeny. Our results provide evidence that gestational exposure to nano- $\mathrm{TiO}_{2}$ significantly affects the heart and cardiomyocyte function of progeny. Further, changes in mitochondrial respiratory capacity, coupled with decreasing mitochondrial complex activity, provide a potential mechanism for the observed functional deficits. 


\section{Methods}

\section{Animal Model}

The experiments in this study conformed to the NIH Guide for the Care and Use of Laboratory Animals ( $8^{\text {th }}$ Edition) and were approved by the West Virginia University Animal Care and Use Committee. Sprague-Dawley rats (250-275 g female; 300-325 g male) were purchased from Hilltop Laboratories (Scottsdale, PA). Rats were housed at the West Virginia University Health Sciences Center with food and water provided ad libitum. Rats were acclimated for at least 72hours prior to mating, as previously described (53). At gestational day $\sim 6(5.85$, mean of exposure times), pregnant rats were exposed to nano- $\mathrm{TiO}_{2}$ as described below.

\section{Engineered Nanomaterial}

Nano- $\mathrm{TiO}_{2} \mathrm{P} 25$ powder, containing anatase $(80 \%)$ and rutile $(20 \%) \mathrm{TiO}_{2}$, was purchased from Evonik (Aeroxide $\mathrm{TiO}_{2}$, Parsippany, NJ). Primary particle size was $21 \mathrm{~nm}$ with a surface area of $48.08 \mathrm{~m}^{2} / \mathrm{g}(35,44,45)$. Aerosolization by drying, sieving, and storing are described previously for nano- $\mathrm{TiO}_{2}(27,35)$.

\section{Inhalation Exposure}

The design and use of the nanoparticle exposure system has been previously described (65). Briefly, aerosol size was measured through both electrical low pressure impactor (ELPI) (136.47 $\pm 1.44 \mathrm{~nm}$ ) (Figure 6.1.1A) and scanning particle mobility sizer (SPMS) $(134.80 \pm 1.24 \mathrm{~nm})$ (Figure 6.1.1B). Aerosol concentration of engineered $\mathrm{TiO}_{2}$ was determined to be $(10.35 \pm 0.13$ $\mathrm{mg} / \mathrm{m}^{3}$ ) (Figure $6 \cdot 1.1 \mathrm{C}$ ). Average aerosol concentration was measured over a total of $\sim 8$ exposures $\left(7.79 \pm 0.26\right.$ days) to each of the 13 animals in the gestational nano- $\mathrm{TiO}_{2}$ exposure group. The first exposure to nano- $\mathrm{TiO}_{2}$ was given on gestational day $\sim 6$, with the last exposure given 24 hours prior to birth. Exposures consisted of 5 hours per day treatments, with a calculated lung daily deposition of $43.8 \pm 1.2 \mu \mathrm{g}$. Control animals were exposed to filtered air only. Calculation of total exposure volume has been previously described $(29,35,41,52)$. To calculate the dose required to match the appropriate lung deposition paradigm for Sprague Dawley rats, the formula $\mathrm{D}=\mathrm{F} \times \mathrm{V}$ 
$\times \mathrm{C} \times \mathrm{T}$ was used, where $\mathrm{F}$ is the deposition fraction $(10 \%), \mathrm{V}$ is the minute ventilation based on body weight, $\mathrm{C}$ equals the mass concentration $\left(\mathrm{mg} / \mathrm{m}^{3}\right)$ and $\mathrm{T}$ equals the exposure duration (minutes).

\section{Echocardiography - B-mode, M-mode, and Doppler Imaging}

Nano- $\mathrm{TiO}_{2}$ exposed and control filtered air young adult (6-12 weeks) Sprague Dawley rats were used in analysis and, subsequently, for cardiomyocyte isolation. Anesthetization was performed similarly to James et al. (25); by slowly reducing the total isoflurane given, measurements were taken as the animal awakens, reducing the anesthetic-induced effect on cardiac function. B-mode, M-mode echocardiograph, as well as Doppler imaging procedures have been described previously in mice (48), but with some adaptations: including the use of a lower frequency transducer (13-24 $\mathrm{MHz}$ instead of $32-55 \mathrm{MHz}$ ) and 3\% isoflurane induction with sustained isoflurane at $1.5 \%$ or higher. Ultrasound images were taken using the Vevo 2100 Imaging System (Visual Sonics, Toronto, Canada). Briefly, ultrasound slightly left of the sternum, at the mid-papillary layer, were used for long-axis B-mode imaging. Rotation $90^{\circ}$, perpendicular to the animal, allowed for shortaxis B-mode images. B-mode procurement provided detailed information such as area and ejection volume of the left ventricle (LV). Gating midway between the short-axis B-mode images allowed for acquisition of M-mode parameters. In M-mode, measures to determine the left ventricular thickness (interventricular septal, inner, and posterior wall) were conducted on adjacent endsystolic and end-diastolic peaks in relation to LV trace analysis. Doppler echocardiography measured mitral valve function, $\mathrm{E}$ and $\mathrm{A}$ wave velocity, deceleration time, E/A ratio, mitral valve area, etc. All ultrasound procedures were carried out by one imaging specialist, taken at a frame rate of 109-284 frames/s. M-mode and Doppler echocardiography were examined using averaged values from at least three replicate analyses from each animal. All measures were completed by one analyst.

\section{Stress-Strain - Speckle Tracking}

B-mode images, in both the short and long axis, were used for speckle-tracking based strain analyses. Peak strain and strain rates were measured across radial, longitudinal, and 
circumferential dimensions. Speckle-tracking based strain analysis has been described previously in mice (48), with caveats for rat analyses mentioned above. Briefly, during each cardiac cycle, strain was measured as a function of total deformation length divided by the original length of a segment $(3,10,38)$. Displacement length, velocity, strain, and strain rate were measured in the endocardium over a minimum of three cycles. As previously described, short-axis images in the $\mathrm{LV}$ were split into anterior free (AF), lateral (L), posterior $(\mathrm{P})$, inferior free $(\mathrm{IF})$, posterior septum (PS), and anterior septum (AS), while long-axis was split into anterior base (AB), anterior mid $(\mathrm{AM})$, anterior apex $(\mathrm{AA})$, posterior apex $(\mathrm{PA})$, posterior mid $(\mathrm{PM})$, and posterior base $(\mathrm{PB})$ as previously described (48).

\section{Cardiomyocyte Isolation}

\section{Fetal/Neonatal}

For the fetal time point, pregnant dams were euthanized and pups were removed at gestational day 20 from the exposed and control rat uterus. For the neonatal time point, rats were sacrificed at 410 days post-birth Animals at both time points were kept on ice during dissection. During cardiomyocyte isolation, pregnant rats were anesthetized using 5\% isoflurane, with maintenance isoflurane levels sustained at $2.5 \%$. Hearts were removed through a midsagittal cut in the thoracic cavity. Hearts were collected in groups of 4-8, per litter, and atrial and ventricular appendages trimmed. Digestive solution included $2 \mathrm{mg}$ Pancreatin (Sigma Adrich, St. Louis, MO) and $2 \mathrm{mg}$ of Collagen Type II (Worthington Biochemical, Lakewood, NJ), mixed with $2 \mathrm{~mL}$ of physiological $1 \mathrm{X}$ ADS buffer $\left(0.1 \mathrm{M} \mathrm{NaCl}_{1}, 1.2 \mathrm{mM} \mathrm{NaH}_{2} \mathrm{PO}_{4}, 0.8 \mathrm{mM} \mathrm{MgSO}_{4}, 5.4 \mathrm{mM} \mathrm{KCl}\right.$, and $5 \mathrm{mM}$ glucose at $\mathrm{pH}$ 7.4) per heart digested. Digestive solution was used to initially wash the hearts (15 minutes and discarded) and to begin separating single cells from the tissue (3-4 washes at 22 minutes each which were saved). After each saved digestion, the supernatant was removed from the tissue debris and centrifuged at 1000 RPMs for 7 minutes. The supernatant was discarded and $2 \mathrm{~mL}$ of newborn calf serum (NCS) was added to the cell suspension and transferred to a $37^{\circ} \mathrm{C}$ incubator.

In a $15 \mathrm{~mL}$ conical tube, a percoll gradient was made for separation of cardiomyocytes from other cells of the heart. The percoll gradient (density $=1.130 \mathrm{~g} / \mathrm{mL}$ ) was made of two layers: clear (top) and red (bottom) percoll solutions. $4 \mathrm{~mL}$ of top percoll (density $=1.059 \mathrm{~g} / \mathrm{mL}, 90 \mathrm{~mL}$ 
percoll stock $+110 \mathrm{~mL} 1 \mathrm{X}$ Ads buffer) were first added, followed by pipetting bottom percoll (density $=1.082 \mathrm{~g} / \mathrm{mL}, 130 \mathrm{~mL}$ percoll stock $+70 \mathrm{~mL} 1 \mathrm{X}$ ADS buffer with phenyl red) below the first solution. After all cell collection steps, cells were centrifuged at 1000 RPMs and re-suspended in $2 \mathrm{~mL}$ of $1 \mathrm{X}$ ADS buffer, per 5 hearts, which was added to the top of the percoll gradient. Cells, on top of the percoll gradient were centrifuged at 3000 RPMs for 30 minutes, with 9-minute acceleration and deceleration time. Cells were then extracted from the middle layer, washed with NCS, and placed in plating media (with fetal bovine serum (FBS)). Two hours after placing in plating media, cells were exchanged to maintenance media (no FBS) and media changed every two days. Cells were counted using a hemocytometer.

\section{Adult}

Young adult rats were anesthetized using $5 \%$ isoflurane. The isolation procedure was adapted from $\mathrm{Xu}$ and Colecraft (61). Briefly, hearts were removed, rinsed in physiological salt solution (PSS), and suspended on a Langendorff perfusion system, with retrograde flow through the aorta. Enzymatic digestion with $20 \mathrm{mg}$ Collagen Type II (Worthington Biochemical, Lakewood, NJ), 10 mg Neutral Protease (Worthington Biochemical, Lakewood, NJ), 20 mg Carnitine (Sigma Aldrich, St. Louis, MO), 25 mg 2,3-Butanedione monoxime (BDM) (Sigma Aldrich, St. Louis, MO), 31 mg Taurine (Sigma Aldrich, St. Louis, MO), 20 mg Glutamine (Sigma Aldrich, St. Louis, MO), and $1.25 \mu \mathrm{L}$ of $1 \mathrm{M} \mathrm{CaCl}_{2}$ in $50 \mathrm{~mL}$ of Buffer A. After digestion (total calcium concentration added $0.6 \mathrm{mM}$ ), gravimetric separation of cells was performed with continual calcium loading during the three wash steps. Cells were counted using hemocytometer.

\section{Cardiomyocyte Contractility}

A video-based edge detection system (Ionoptix, Westwood, MA) was used to assess contractility of cardiomyocytes. Phase-contrast microscopy coupled with Ionoptix software, were used to track cardiomyocyte dimensions. Cells were placed in contractility buffer (50.55 mg BDM, $8.82 \mathrm{mg}$ $\mathrm{CaCl}_{2}, 119.5 \mathrm{mg}$ HEPES, $10.16 \mathrm{mg} \mathrm{MgCl}$, $394.47 \mathrm{mg} \mathrm{NaCl}, 90.075 \mathrm{mg}$ glucose, $14.91 \mathrm{mg} \mathrm{KCl}$, and $2.276 \mathrm{mg} \mathrm{NaH} \mathrm{NO}_{4}$ ) and then diluted 10:1 with contractility buffer from the original cell suspension. Field stimulation at $0.5 \mathrm{~Hz}$ at 10 volts was used for all measures. Dynamic measures 
taken included departure velocity, peak height, peak height $\%$ to baseline, return velocity, 10 and 90\% time to shortening, 10 and 90\% time to relengthening, area of departure, area of return, and retention of baseline. Cells included into the study matched the specific criteria: 1) chosen based on the best morphology when compared to others within the field (striations, no blebbing) and 2) cells sustain consistent contractions for at least 60 seconds. For reference, Ye et al. provides more detailed parameters of the contractile apparatus (63)

\section{Cardiomyocyte Mitochondrial Bioenergetics}

Isolated cardiomyocytes were plated on XF96 V3 cell culture microplates, using the Seahorse XF96 for analysis (Agilent Technologies, Santa Clara, CA). Measurements were taken 24-48 hours after plating cardiomyocytes. Concentrations of fetal and neonatal cells ranged from 35,000 85,000 per well. Adult cardiomyocytes, due to slight variation in cell viability and time before analysis for each sample, were not normalized based on cell number, but rather on basal respiration between groups. Replicates were generally performed in sets of 5-6. Yoshida et al provides a comprehensive overview of the Seahorse applications (66). Briefly, oxygen consumption rate (OCR), extracellular acidification rate (ECAR), and proton production rate (PPR) were measured using oligomycin, carbonyl cyanide-p-trifluoromethoxyphenylhydrazone (FCCP), antimycin A, and rotenone. Measures of mitochondrial respiration included ATP production, proton leak, basal respiration, maximal respiration, and spare capacity.

\section{Electron Transport Chain (ETC) Complex Activities}

ETC complexes I, III, IV $(11,12)$ and V (ATP synthase) activities were measured as previously described (9), with minor modifications. Briefly, cardiomyocytes from young adult animals were treated with RIPA Buffer (Life Technologies, Grand Island, NY). A Bradford assay was carried out on each sample to provide a basis for normalization to protein content. Activity was measured in complex I (reduction of decyclubiquinone), complex III (reduction of cytochrome $c$ ), complex IV (oxidation of reduced cytochrome $c$ ), and complex $\mathrm{V}$ (pyruvate kinase and phosphoenolpyruvate and ATP production). 


\section{ETC Complex Protein Expression}

Blue native polyacrylamide gel electrophoresis (BN-PAGE) was performed as previously described (56), with standardization to protein content through the Bradford method (4). Briefly, young adult cardiomyocytes were digested with RIPA Buffer (Life Technologies, Grand Island, $\mathrm{NY}$ ) and $40 \mu \mathrm{g}$ of protein were added to $1 \%$ digitonin on ice. Coomassie G-250 was added and samples were run on 4-16\% NativePAGE gels. Gels were placed in GelCode ${ }^{\circledR}$ Blue Stain Reagent (Thermo Fisher, Rockford, IL) to fix bands for representation, followed by washes until the desired background staining was removed. Densitometry was measured using Image J Software (National Institutes of Health, Bethesda, MD). Complexes were identified using a $480 \mathrm{KDa}$ ladder with criteria for identifying the molecular weight of each complex matching Wittig et al. (60).

\section{Quantitative PCR}

RNA was isolated from fetal progeny hearts using the Vantage ${ }^{\mathrm{TM}}$ Total RNA Purification Kit (Origene), following the manufacturer's instructions. RNA was then converted to cDNA for qPCR analysis using the cDNA Synthesis Kit for miRNA (product number: HP100042, Origene), following the manufacturer's instructions. cDNA was used to assess three markers: PPAR $\gamma$ (product number: Rn00440945_m1, Thermo Fisher, Rockford, IL), CPT1A (product number: Rn00580702_m1, Thermo Fisher, Rockford, IL), and UCP2 (product number: Rn01754856_m1, Thermo Fisher, Rockford, IL). Samples were standardized to GAPDH (product number: Rn01775763_g1, Thermo Fisher, Rockford, IL). Experiments were conducted on the Applied Biosystems 7900HT Fast Real-Time PCR system (Applied Biosystems, Foster City, CA), in a total reaction volume of $20 \mu \mathrm{L}$ with TaqMan ${ }^{\circledR} 2$ X Universal PCR Master Mix (Applied Biosystems, Foster City, CA).

\section{Western Blot Analyses}

12-well, 4-12\% gradient gels were used in SDS-PAGE, as previously described [3, 4, 6, 11, 12, $28,29]$ with modifications. Briefly, cardiomyocytes from the young adult group were used in all 
protein quantification, with the Bradford method being employed to standardize protein concentration using bovine serum albumin (4). Primary antibodies used in the study included: antiANT (product number: sc-9300, Santa Cruz Biotechnology Inc., Dallas, TX), anti-APAF-1 (product number: ab2000, Abcam Inc., Cambridge, MA), anti-BAX (product number: ab32503, Abcam Inc., Cambridge, MA), anti-BCL-2 (product number: sc-7382, Santa Cruz Biotechnology Inc., Dallas, TX), anti-H3K4me3 (product number: G.532.8, Thermo Fisher, Rockford, IL), antiH3K27me3 (product number: G.299.10, Thermo Fisher, Rockford, IL), anti-GAPDH (product number: ab8245, Abcam Inc., Cambridge, MA), anti-HDAC6 (product number: ab61058, Abcam Inc., Cambridge, MA), anti-OPA1 (product number: ab42364, Abcam Inc., Cambridge, MA), antiPDH (product no. 2784; Cell Signaling Technology, Danvers, MA), anti-PDH E1-alpha subunit (phospho S293) (product number: ab177461, Abcam Inc., Cambridge, MA), anti-PPAR $\alpha$ (product number: ab2779, Abcam Inc., Cambridge, MA), anti-UCP3 (product number: ab3477, Abcam Inc., Cambridge, MA), anti-VDAC (product no. 4866; Cell Signaling Technology, Danvers, MA). Secondary antibodies used included: goat anti-mouse IgG horseradish peroxidase (HRP) conjugate (product no. 31430; Pierce Biotechnology, Rockford, IL), goat anti-rabbit IgG HRP conjugate (product No. 10004301; Cayman Chemical, Ann Arbor, MI), and rabbit anti-goat IgG horseradish

peroxidase (HRP) conjugate (product no. 31402; Pierce Biotechnology, Rockford, IL). Standardization of protein levels for each blot were performed with both Ponceau S Solution (Sigma Adrich, St. Louis, MO) and the anti-GAPDH antibody. Pierce Enhanced Chemiluminescence Western Blotting substrate (Pierce Biotechnology; Rockford, IL) was used to detect signal per manufacturer's instructions. G:Box Bioimaging system (Syngene, Frederick, MD) was used to detect signals and data were captured using GeneSnap/GeneTools software (Syngene, Frederick, MD). Densitometry was analyzed using Image J Software (National Institutes of Health, Bethesda, MD). All values were expressed as optical density with arbitrary units (AU).

\section{Statistics}

A two-tailed student's $t$-test was used to determine statistical significance between control and gestational nano- $\mathrm{TiO}_{2}$ exposed groups. Differences between control and exposed groups were considered statistically trending $(P=0.075-0.055$, denoted by $\dagger)$, or statistically different $(P \leq$ 
0.05 , denoted by $*$, and $P \leq 0.01$, denoted by **) unless explicitly stated otherwise. All data are presented as means \pm standard error $(\mathrm{SEM})$. 


\section{Results}

\section{Echocardiography and Speckle-Tracking Based Strain Analysis}

Young adult animals (6-12 weeks) were assessed using a variety of ultrasound techniques to evaluate in vivo cardiac function. M-Mode, measuring end-systolic and end-diastolic diameters and volumes, and B-Mode, measuring the short and long axis diameter of the left ventricle (LV), are provided in Table 6.1.1 and Table 6.1.2, respectively. M-mode was found to have significant changes in the total mass of the LV between groups $(P=0.033)$ and a trending difference in stroke volume $(P=0.072)$. LV thickness was measured across end-diastole and end-systole for changes in interventricular septal, inner, and posterior wall thickness. Interventricular end-systole septal thickness showed a significant decrease $(P=0.015)$ in the experimental group (Table 6.1.3). Bmode showed no changes between groups. Doppler echocardiography revealed differences in LV filling between the control and gestational nano- $\mathrm{TiO}_{2}$ exposed group (Figure 6.1.2); this included differences in the velocities of both the $\mathrm{E}$ wave $(P=0.025)$ (Figure 6.1.2A) and the $\mathrm{A}$ wave $(P=$ 0.040) (Figure 6.1.2B), with the experimental group showing a significant decrease in both velocities.

Also, even though both the $\mathrm{E}$ and $\mathrm{A}$ wave velocities were altered, the $\mathrm{E} / \mathrm{A}$ ratio between groups was found to be higher in the experimental group $(P=0.052)$. Control animals exhibited a normal $\mathrm{E} / \mathrm{A}$ ratio (1.505) while the experimental group exhibited a higher ratio (1.813) (Figure 6.1.2C). This is represented by Figure 6.1.2D which depicts a control (left) and gestational exposed (right) animal with $\mathrm{E} / \mathrm{A}$ ratios similar to that of the average for each group. Other parameters were also measured, including mitral valve area (Figure 6.1.2E), and were not found to be significant. For the stress-strain analysis, only two parameters appeared to be altered during contraction of the LV; the long axis systolic displacement and strain (Figure 6.1.2F and 6.1.2G) showed a significant $(P$ $=0.049)$ and trending $(P=0.065)$ difference, respectively. These results demonstrate that maternal exposure can produce adverse effects in cardiac systolic and diastolic function, while also interfering with cardiac development.

\section{Cardiomyocyte Contractility}


To further delineate the cardiac outcomes of progeny predisposed to gestational nano- $-\mathrm{TiO}_{2}$, cardiomyocyte contractile function was assessed. Cells were isolated from young adult rats in the control and experimental groups. Cardiomyocyte contractile function was decreased in the gestational exposed compared to the control (Figure 6.1.3). An assessment of decreasing function was demonstrated through changes in departure velocity, peak height, baseline $\%$ to peak height, and area under the contractile curve. Departure velocity was significantly decreased in the young adult group $(P=0.0008)$ after gestational exposure (Figure 6.1.3A). In addition, the peak height of contraction was significantly decreased $(P=0.0001)$ (Figure 6.1.3B), with no differences observed in the resting baseline $(P=0.73)$ (Figure 6.1.3C). The exposed group also exhibited a decreased baseline $\%$ to peak height $(P=0.0003)$ (Figure 6.1.3D). Lastly, total area of the contraction was shown to be significantly reduced in the exposed group compared to the control, both in area of departure $(P=0.0001)$ and area of return $(P=0.002)$ (Figures 6.1.3E and 6.1.3F). Changes in return velocity, time to $90 \%$ peak, and time to $90 \%$ baseline, were not significant. Two initial transients, one from both the control and experimental contractile data, are overlaid for a visual representation of differences in the contractile curves (Figure 6.1.3G); the departure velocity and peak height measurements are similar to the averages for each group. All measures were performed on the first contraction after stimulation, referred to further as the first transient. Second transient, or the second contractile peak, measures displayed a similar trend (data not shown). At least three cardiomyocytes were used per control or exposed litter. Attenuated responsiveness during stimulation in the maternal exposure model establish modifications to cardiomyocytes function, which remain into adulthood.

\section{Mitochondrial Bioenergetics}

To determine whether changes in contractility resulted from changes in cellular energetics that drive contractile function, the Seahorse XF96 was implemented to determine the effects of gestational nano- $\mathrm{TiO}_{2}$ exposure on the bioenergetics of the growing progeny. Data from the Seahorse XF96 was compiled at three time points: fetal (gestational day 28), neonatal (4-10 days post birth), and young adult (6-12 weeks). The results indicated mitochondrial respiration dysfunction, with varying degrees of impairment across developmental stages (Figure 6.1.4). Fetal cardiomyocytes, seeded at a 65,000 cell density, showed a decrease in basal respiration $(P=$ 
0.0001) (Figure 6.1.4A) and an increase in proton leak $(P=0.003)$ in the experimental group. When cardiomyocytes were extracted from the neonatal hearts and seeded at a 55,000 cell density, effects on basal respiration diminished, but significant decreases in maximal respiration $(P=$ 0.0031) (Figure 6.1.4B) and spare capacity $(P=0.003)$ were observed within the nano- $\mathrm{TiO}_{2}$ exposed groups. Also, proton leak continued to show a significant increase in the exposed group $(P=0.044)$. Because of slight variations in the recovery percentage of viable adult cardiomyocytes and limited cell viability in culture, normalization of the young adult group was processed differently than the fetal and neonatal groups.

Due to changes in basal respiration diminishing in the neonatal group, this parameter was used to normalize the young adult age group. Basal respiration normalization resulted in a significant increase in the proton leak in the experimental group $(P=0.0072)$ (Figure 6.1.4C). Normalization to both maximal respiration and spare capacity also showed a significant increase in proton leak in the experimental group (data not shown). A depiction of the oxygen consumption rate (OCR), extracellular acidification rate (ECAR), and proposed bioenergetics capacity for neonatal cardiomyocytes in the control and experimental groups are depicted in Figure 6.1.4D to provide an example of the overall procedure. This experiment validates the sustained, longitudinal effects of gestational exposure to ENMs, highlighting changes occurring in the bioenergetics of the cardiomyocytes.

\section{ETC Complex I, III, IV, and V Activities and Expression}

To further target the molecular effects of gestational nano- $\mathrm{TiO}_{2}$ exposure on bioenergetics, mitochondrial complex activities was measured on young adult cardiomyocytes. Trends were shown in both complex I $(P=0.067)$ (Figure 6.1.5A) and complex IV $(P=0.056)$ (Figure 6.1.5B), with as much as a $20 \%$ decrease in activity in the gestational exposed group compared to the controls. Complex III and complex V (ATP Synthase) showed no significant differences in activity between groups (Figures 6.1.5C and 6.1.5D). Though trending differences, complex activity changes indicate potential lasting effects to the quantity, or function, of the mitochondrial electron transport chain constituents. To determine if a reduction in complex activity was due to a decrease in the expression level of the complexes, Blue Native PAGE was performed. No significant changes were found in any of the major ETC complex expressions (Table 6.1.4). 


\section{Metabolism and Cell Stress}

In order to understand some of the molecular changes resulting from gestational nano- $\mathrm{TiO}_{2}$ exposure to progeny, qPCR and Western blot analysis were used to probe changing mRNA and protein levels, respectively. At the fetal time point, mRNA of proteins contributing to proton leak and fatty acid metabolism were examined. For proton leak, qPCR of uncoupling protein 2 (UCP2) revealed increased $(P=0.006)$ mRNA levels in the experimental group compared to the control (Figure 6.1.6A), with qPCR of fatty acid metabolism constituents peroxisome proliferatoractivated receptor gamma $(\mathrm{PPAR} \gamma)(P=0.039)$ and carnitine palmitoyltransferase I (CPT1A) $(P$ $=0.023$ ) exhibiting a similar increase (Figure 6.1.6B and 6.1.6C). Because molecular markers involving proton leak and fatty acid metabolism were changed in progeny at the fetal level of development, the next step was to evaluate protein expression in the young adult cardiomyocytes.

Subsequent experiments to measure changes in metabolism involved assessing pyruvate dehydrogenase $(\mathrm{PDH})$, which was found to be trending toward reduced expression $(P=0.061)$ in the experimental group (Figure 6.1.6D), with activity of $\mathrm{PDH}$, evaluated through phosphorylation at serine 293 (p-S293), found to be statistically insignificant. When comparing the fluorescence ratio of PDH p-S293 to $\mathrm{PDH}$, the amount of p-S293 per PDH expression was higher in the experimental group, but not significantly $(P=0.082)$. Peroxisome proliferator-activated receptor alpha $(\mathrm{PPAR} \alpha)($ Figure 6.1.6E) was found to be significantly increased $(P=0.017)$. As an epigenetic evaluation of the proteome, histone 3 lysine 4 tri-methylation (H3K4me3) and histone 3 lysine 27 tri-methylation ( $\mathrm{H} 3 \mathrm{~K} 7 \mathrm{me} 3)$ were used to assess global histone activation/deactivation patterns in the cells, respectively. $\mathrm{H} 3 \mathrm{~K} 4 \mathrm{me} 3$ expression remained similar between groups (data not shown) while H3K27me3 (Figure 6.1.6F) showed a significant, increased expression $(P=$ $0.045)$ in the experimental animals. Signals for cell stress were also evaluated. Bcl-2-like protein 4 (BAX), B-cell lymphoma 2 (BCL2), and optic atrophy 1 (OPA1) were found to be similarly expressed between groups (data not shown), while apoptotic protease activating factor 1 (APAF1) (Figure 6.1.6G) was found to be significantly increased $(P=0.037)$ in the experimental group. Lastly, proton leak was further evaluated through voltage-dependent anion channel (VDAC), adenine nucleotide translocase (ANT), and uncoupling protein 3 (UCP3), showing no significant alterations between groups (data not shown). Changes in the epigenetic profile, metabolism, and 
mitochondrial stress of the experimental progeny demonstrate the implications of initial, as well as prolonged, effects of gestational nano- $\mathrm{TiO}_{2}$ exposure. 


\section{Discussion}

Nanotechnology holds the potential to significantly improve the lives of people around the world through a wide range of applications, including tumor abolition (2) or delivery of medication (31); but, the risks associated with nanomaterial use must first be properly identified. The most widely manufactured nanomaterial in the world is $\mathrm{TiO}_{2}$, with up to $80 \%$ of its uses being involved in cosmetics and other personal care products (40). The ratio of nano- $\mathrm{TiO}_{2} / \mathrm{TiO}_{2}$ production continues to increase, with some predictions speculating total conversion of the market to nano$\mathrm{TiO}_{2}$ by 2025 (43). With the growing interest in nano- $\mathrm{TiO}_{2}$ applications in the consumer market, nanotoxicological work is needed to fully define the extent to which this ENM contributes to human health and safety. Nano- $\mathrm{TiO}_{2}$ has demonstrated the potential to cause adverse health outcomes after exposure; what remains poorly understood, is how nano- $\mathrm{TiO}_{2}$ can interact through indirect, gestational exposure on growing progeny. More specifically, it is unclear as to how nano$\mathrm{TiO}_{2}$ impacts cardiac development and function.

The results of our study provide evidence that gestational nano- $\mathrm{TiO}_{2}$ exposure can significantly affect the cardiac function of progeny, leading to a decreased functional capacity in both the whole heart and the cardiomyocyte. A mechanism for this dysfunction was illustrated through changes to cardiomyocyte bioenergetics after gestational exposure, which was then validated more specifically through mitochondrial complex activity, suggesting a role of nano- $\mathrm{TiO}_{2}$ in decreasing cardiac function by the disruption of mitochondrial oxidative phosphorylation and respiration rates. The dose of nano- $\mathrm{TiO}_{2}$ administered was calculated as a conservative estimate of ENM occupational exposure projected as $2.3-8.5$ years of human exposure in a manufacturing setting. This calculation represents the maximum estimated dose deposited in the lungs. Because variables such as age, mass, clearance, and health states are not part of the calculation; we consider this dose very relevant to occupational settings. The exposures were also carried out over multiple, nonconsecutive days to more appropriately simulate human exposures. Low nanomaterial lung deposition, extended time-courses for dosing, and the use of an inhalation facility all support study observations that have a much higher potential of being attributable to true, physiological outcomes in humans and possessing repeatability across studies.

In vivo measurements, including M-Mode, B-Mode, Doppler echocardiography, and speckle-tracking based strain analysis were taken to assess changes in contraction mechanics of 
the LV and the flow of blood through the mitral valve. In systole, radial strain and displacement of the LV walls were decreased in the experimental group, demonstrating initial signs of dysfunction in LV contraction mechanics. Decreasing radial strain and displacement during contraction of the LV is coupled with more overt measures of LV systolic function presented in the study, including a decreased interventricular end-systolic septal thickness and decreased total mass of the LV in the experimental group (with total mass falling into the lower percentile of normative values for Sprague Dawley rats (39)). From this data, we conclude that gestational nano$\mathrm{TiO}_{2}$ exposure weakens the contractile properties of the heart, potentially leading to increased risk factors of heart disease in older age. This is also supported by the fact that a decreased stroke volume is also seen to be trending in the experimental group. By decreasing the size of the LV, the thickness of its contractile walls, and measures of systolic radial strain and displacement, the function of the heart becomes more susceptible to perturbation and may indicate early predictive signs of heart complications.

In diastole, a decrease in $\mathrm{E}$ and $\mathrm{A}$ wave velocity and an increase in total $\mathrm{E} / \mathrm{A}$ ratio was observed in the experimental group. Studies have suggested that E/A ratios greater than 1.5 can indicate diastolic dysfunction $(1,21)$, potentially leading to restrictive filling. Further, the total decrease in both the $\mathrm{E}$ and $\mathrm{A}$ wave velocities seems to indicate that diastole is not only dysregulated through atrial pumping abnormalities (restrictive filling) but potentially through asymmetrical, long axis filling (33). Changes in systolic and diastolic parameters demonstrate that the heart of gestational nano- $\mathrm{TiO}_{2}$ exposed progeny develops a maladaptive phenotype. These findings raised questions as to the link between the observed cardiac dysfunction and the potential contribution of the cellular contractile unit, the cardiomyocyte.

In cardiomyocytes, changes in the departure velocity, baseline $\%$ to peak height, and both the area of departure and return under the curve were detected (Figure 6.1.3F). Though, no changes were observed in other common parameters of cardiomyocyte contractility (time to peak $90 \%$ and time to baseline 90\%). The changes found with cardiomyocyte contractility in the experimental group further support the hypothesis that gestational nano- $\mathrm{TiO}_{2}$ exposure weakens the contractile properties of the heart. A reduced baseline \% to peak height and length of contraction in cardiomyocytes could be responsible for the attenuation in systolic radial strain and displacement, with reduced contractility also supporting the presentation of restrictive, diastolic filling. The 
decreased area of contraction in the experimental group (ultimately leading to a diminished cardiac output) could also be linked to the decreasing stroke volume.

Changes in cardiomyocyte contractility have been observed in the presence of nano- $\mathrm{TiO}_{2}$, but these experiments have been through direct incubation with the nanoparticle. Exceeding 10 $\mu \mathrm{g} / \mathrm{mL}$ nano- $\mathrm{TiO}_{2}$ concentrations, changes in the departure velocity, return velocity, and peak shortening were observed in the cardiomyocytes $(26,46)$. Differences between the two approaches (direct application and gestational inhalation exposure) are that return velocity parameters remained unchanged in the present study. The speed of the return velocity is indicative of the effectiveness of calcium shuttling in the cytoplasm (5). Primarily, calcium return to the endoplasmic reticulum from the cytoplasm is accomplished through SERCa pumps and $\mathrm{Na}^{+} / \mathrm{Ca}^{2+}$ transporters. Because return velocity remained constant across both groups, this provides an initial indication that cardiomyocyte dysfunction may not be the result of calcium handling, but rather a mechanism of bioenergetics and mitochondrial function.

Examining fetal/neonatal cardiomyocytes for changes in bioenergetics provided information on the longitudinal effects that gestational nano- $\mathrm{TiO}_{2}$ exposure may produce on the development of cardiomyocytes; illuminating time points during development which may be more fragile to perturbation. During fetal exposure to nano- $\mathrm{TiO}_{2}$, substrate unavailability/increased cytoplasmic oxidation could provide the reasoning for decreased basal respiration of cells and ultimately compromised bioenergetics, resulting from either direct or indirect effects of nano- $\mathrm{TiO}_{2}$ exposure. As observed, this decrease in basal respiration was also coupled with increased proton leak. Proton leak is a factor controlled by both environment (mitochondrial membrane potential and $\mathrm{pH})$ as well as protein constituents of the mitochondrion $(14,47)$. What is significant about these findings is that, following normal conditions, increases in basal respiration should coincide with increases in proton leak. But, as indicated in the results, a significant decrease in basal respiration was followed by an increase in proton leak.

Examining the data from the neonatal cardiomyocytes, it becomes evident that basal respiration is restored; depicting that at some point between the fetal and neonatal time point, basal respiration levels in the exposed group recovers, matching those found in the controls. This may be an indication that disturbances of basal respiration in the fetal cardiomyocytes were due to environmental conditions (likely through limitations of substrate availability $(5,55)$ promoted by 
nano- $\mathrm{TiO}_{2}$ interactions with the mother or placenta). Restoration of substrate supplies, no longer limited by nano- $\mathrm{TiO}_{2}$ interactions, restored basal respiration to that of controls in the neonatal group. Though, the neonatal group exhibited a decreased maximal respiration and spare respiratory capacity, with a similar pattern of increased proton leak in the experimental group. We conclude that changes in maximal respiration and spare capacity may reflect persistent effects of nano- $\mathrm{TiO}_{2}$ exposure, limiting mitochondrial health and function as the animal matures.

Normalization to basal respiration for the young adult group was performed for two reasons: 1) no significant change in basal respiration were observed in the neonatal group and 2) cell number for adult cardiomyocytes will vary depending on preparation and time before recording oxygen consumption. After normalizing to basal respiration, as well as maximal respiration and spare capacity, proton leak was seen to be increased in the exposed group over the control. With proton leak increasing over all three time points, unbalancing of the proton motive force, proton shuttling protein dysfunction/underexpression, and/or increased total reactive oxygen species could all be mechanisms contributing to the process.

To more specifically pinpoint the mechanisms behind changes in the respiration rates of cardiomyocytes from progeny exposed to nano- $\mathrm{TiO}_{2}$, ETC complex activities were examined. Complex I, III, IV, and V activities was measured in the young adult group, to determine if changes in mitochondrial ETC complex activities provide support for the longitudinal, detrimental effects of gestational nano- $\mathrm{TiO}_{2}$ on energy production. Our data revealed no changes in complex III and complex V (ATP Synthase), but strong trends for a decrease in complex I and complex IV as indicated by an average of $20 \%$ decrease in activity for both. Because overall complex expression is not changing, a decrease in ETC complex activities, whether through transcriptional, translational, or post-translational mechanisms, demonstrates that mitochondrial function, disturbed at both the fetal and neonatal time points, may still have an impact later in life.

Molecular changes at both the mRNA and protein level suggest that alterations in the metabolic profile of the heart becomes disrupted after gestational nano- $\mathrm{TiO}_{2}$ exposure, with variations also seen in the epigenetic profile and mitochondrial stability. Research suggests that increasing levels of fatty acid oxidation in the heart, with decreasing glucose metabolism, can be indicative of the initial stages of insulin-insensitivity, hyperglycemia, and/or heart complications $(6,17,28)$. In this study, PPAR $\alpha, \operatorname{PPAR} \gamma$, and CPT1A are seen to increase in the experimental group while PDH, 
though trending, is reduced; these molecular changes, coupled with the observed diastolic dysfunction, are supportive of the hypothesis that increasing utilization of fatty acids could be coupled to a decrease in diastolic function resulting from an insult (32). Increases in PPAR $\alpha$ protein expression may also be linked to proton leak and ROS production.

PPAR $\alpha$ acts as a transcriptional regulator of UCPs by activating expression of the proteins (17, 62). This study found increasing mRNA levels of UCP2 in gestational nano- $\mathrm{TiO}_{2}$ exposed progeny together with increased proton leak. Because increasing fatty acid metabolism leads to higher levels of incomplete $\beta$-oxidation, changes in ATP utilization, and changing initial substrates for the ETC, ROS production is likely to be increased $(34,68)$. With an increase in ROS, a greater proton leak could help to ameliorate damaging effects. The molecular data also reveals increasing APAF-1 expression in the experimental group, potentially pointing to decreased cell viability due to changing metabolic profiles. Because BAX and BCL2 levels were similar between groups (data not shown), APAF-1 may be activated independently of the canonical apoptotic pathway, which has been shown to be possible in certain tissue types (24).

\section{Limitations/Future Directions:}

This study focused specifically on the changing bioenergetics of gestational nano- $\mathrm{TiO}_{2}$ exposed cardiac tissue and cardiomyocytes, but future experimentation could examine alterations in calcium handling. Data presented in this paper, through molecular markers of metabolism and complex activity, suggest that bioenergetics plays a role in contractile dysfunction and reduced diastolic function; though, measures of complex activity are likely underpowered due to variability in progeny birth and cohort sizes. Fluctuations in calcium availability or shuttling may also contribute to some of the phenotype, but would need further exploration. The study was limited by not directly assessing mitochondrial membrane potential and measures of ROS. This study demonstrated that UCP2 could be contributing to proton leak and should indicate a lower mitochondrial membrane potential but, without direct measures of mitochondrial membrane potential, it is unclear if molecular changes resulted in functional changes, which we acknowledge as a limitation. With changing levels of proton leak, examining ROS production is also important in understanding if proton leak is a compensatory mechanism for preserving function, or a result of mitochondrial disease and dysregulation. 
Molecularly, changes in constituents involved in fatty acid metabolism suggests an increase of $\beta$ oxidation in the gestational nano- $\mathrm{TiO}_{2}$ exposed group. To validate these conclusions, analysis of mitochondrial respiration in the presence of fatty acids, instead of glucose metabolites, would have been insightful. Lastly, a more in-depth understanding of epigenetic changes could provide greater insight into how function is regulated in gestational nano- $\mathrm{TiO}_{2}$ exposed progeny. This study showed that transcriptional activation marks on histones (H3K4me3) were not changed between groups (data not shown), but global transcriptional repression on histones (H3K27me3) was upregulated in the experimental group, suggesting decreased global gene expression. Further evaluating epigenetic consequences through whole genome bisulfite sequencing or chromatin immunoprecipitation sequencing could greatly enhance our understanding of nano- $\mathrm{TiO}_{2}$ effects on growing progeny.

\section{Conclusions}

In summary, gestational nano- $\mathrm{TiO}_{2}$ exposure impacts cardiac function of the progeny. Manifestations of cardiac impairment in the maturing adult can be seen functionally through systolic and diastolic abnormalities and cardiomyocyte contractile attenuation. Mechanisms driving these deficiencies were found to include changes in electron transport of the mitochondria, including decreasing basal and maximal respiration, decreases in complex I and IV activities, and increased proton leak. Our study demonstrates that exposure to nano- $\mathrm{TiO}_{2}$ indirectly, either through translocation across the placental barrier, change of the in utero environment, or through other mechanisms of interaction with the nanomaterial, induces significant cardiac deficiencies in development and adulthood.

This study has begun to define the molecular and physiological health outcomes related to progeny development after maternal ENM exposures, building upon previous work that has examined uterine and developing progeny microvascular function $(51,54)$. These studies may provide valuable information regarding the effects of ENMs during gestational exposure, but insight into how the progeny heart is adversely affected has yet to be fully elucidated; though, it is likely that inflammatory signaling in the placenta could be linked to improper development of the progeny $(13,49)$, without even the need for direct translocation of the nanomaterial (50). The purpose of this paper is to better characterize the effects resulting from changes that can alter the 
uterine environment, providing insight into the possible mediators involved in nanomaterial exposures. The physiological changes observed in this paper through nano- $\mathrm{TiO}_{2}$ also begin to raise questions regarding other engineered metal oxides which may share similar physiochemical characteristics and pathologies. Though nanomaterials continue to be a vital part of our industrial society, the effects of prenatal nanomaterial exposure are important to consider as their use continues to increase. 


\section{Acknowledgements}

This work was supported by a National Science Foundation IGERT: Research and Education in Nanotoxicology at West Virginia University Fellowship [1144676] awarded to QAH. This work was supported by the National Institutes of Health from the National Heart, Lung and Blood Institute [R56 HL128485] awarded to JMH. This work was supported by the National Institutes of Health from the National Institute of Environmental Safety and Health [R01 ES015022] awarded to TRN. This work was supported by an American Heart Association Predoctoral Fellowship (AHA 13PRE16850066) awarded to CEN. This work was supported by the National Institutes of Health from the National Institute of Environmental Safety and Health [K99 ES024783] awarded to PAS. Small animal imaging and image analysis were performed in the West Virginia University Animal Models \& Imaging Facility (AMIF), which has been supported by the WVU Cancer Institute and NIH grants P20 RR016440, P30 GM103488 and S10 RR026378. Seahorse data acquisition was supported by the West Virginia Stroke CoBRE [P20 GM109098]. 


\section{References}

1. Bella JN, Palmieri V, Roman MJ, Liu JE, Welty TK, Lee ET, Fabsitz RR, Howard BV, and Devereux RB. Mitral ratio of peak early to late diastolic filling velocity as a predictor of mortality in middle-aged and elderly adults: the Strong Heart Study. Circulation 105: 1928-1933, 2002.

2. Bertrand N, Wu J, Xu X, Kamaly N, and Farokhzad OC. Cancer nanotechnology: the impact of passive and active targeting in the era of modern cancer biology. Adv Drug Deliv Rev 66: 2-25, 2014.

3. Blessberger $\mathbf{H}$ and Binder $\mathbf{T}$. Two dimensional speckle tracking echocardiography: clinical applications. Heart 96: 2032-2040, 2010.

4. Bradford MM. A rapid and sensitive method for the quantitation of microgram quantities of protein utilizing the principle of protein-dye binding. Anal Biochem 72: 248-254, 1976.

5. Brand MD and Nicholls DG. Assessing mitochondrial dysfunction in cells. Biochem $\mathrm{J}$ 435: 297-312, 2011.

6. Buchanan J, Mazumder PK, Hu P, Chakrabarti G, Roberts MW, Yun UJ, Cooksey RC, Litwin SE, and Abel ED. Reduced cardiac efficiency and altered substrate metabolism precedes the onset of hyperglycemia and contractile dysfunction in two mouse models of insulin resistance and obesity. Endocrinology 146: 5341-5349, 2005.

7. Campagnolo L, Massimiani M, Palmieri G, Bernardini R, Sacchetti C, Bergamaschi A, Vecchione L, Magrini A, Bottini M, and Pietroiusti A. Biodistribution and toxicity of pegylated single wall carbon nanotubes in pregnant mice. Part Fibre Toxicol 10: 21, 2013.

8. Chen J, Dong X, Zhao J, and Tang G. In vivo acute toxicity of titanium dioxide nanoparticles to mice after intraperitioneal injection. J Appl Toxicol 29: 330-337, 2009.

9. Croston TL, Shepherd DL, Thapa D, Nichols CE, Lewis SE, Dabkowski ER, Jagannathan R, Baseler WA, and Hollander JM. Evaluation of the cardiolipin biosynthetic pathway and its interactions in the diabetic heart. Life Sci 93: 313-322, 2013.

10. D'Hooge J, Heimdal A, Jamal F, Kukulski T, Bijnens B, Rademakers F, Hatle L, Suetens P, and Sutherland GR. Regional strain and strain rate measurements by cardiac ultrasound: principles, implementation and limitations. Eur J Echocardiogr 1: 154-170, 2000.

11. Dabkowski ER, Williamson CL, Bukowski VC, Chapman RS, Leonard SS, Peer CJ, Callery PS, and Hollander JM. Diabetic cardiomyopathy-associated dysfunction in spatially distinct mitochondrial subpopulations. Am J Physiol Heart Circ Physiol 296: H359-369, 2009.

12. Davies SM, Poljak A, Duncan MW, Smythe GA, and Murphy MP. Measurements of protein carbonyls, ortho- and meta-tyrosine and oxidative phosphorylation complex activity in mitochondria from young and old rats. Free Radic Biol Med 31: 181-190, 2001.

13. de Melo JO, Soto SF, Katayama IA, Wenceslau CF, Pires AG, Veras MM, Furukawa LN, de Castro I, Saldiva PH, and Heimann JC. Inhalation of fine particulate matter during pregnancy increased IL-4 cytokine levels in the fetal portion of the placenta. Toxicol Lett 232: 475480, 2015.

14. Divakaruni AS and Brand MD. The regulation and physiology of mitochondrial proton leak. Physiology (Bethesda) 26: 192-205, 2011.

15. Duan Y, Liu H, Zhao J, Liu C, Li Z, Yan J, Ma L, Liu J, Xie Y, Ruan J, and Hong F. The effects of nano-anatase $\mathrm{TiO}(2)$ on the activation of lactate dehydrogenase from rat heart. Biol Trace Elem Res 130: 162-171, 2009. 
16. Faddah LM, Abdel Baky NA, Al-Rasheed NM, and Al-Rasheed NM. Biochemical responses of nanosize titanium dioxide in the heart of rats following administration of idepenone and quercetin. Afr J Pharm Pharmacol 7: 2639-2651, 2013.

17. Finck BN, Lehman JJ, Leone TC, Welch MJ, Bennett MJ, Kovacs A, Han X, Gross RW, Kozak R, Lopaschuk GD, and Kelly DP. The cardiac phenotype induced by PPARalpha overexpression mimics that caused by diabetes mellitus. J Clin Invest 109: 121-130, 2002.

18. Gorr MW, Velten M, Nelin TD, Youtz DJ, Sun Q, and Wold LE. Early life exposure to air pollution induces adult cardiac dysfunction. Am J Physiol Heart Circ Physiol 307: H13531360, 2014.

19. Gwinn MR and Vallyathan V. Nanoparticles: health effects--pros and cons. Environ Health Perspect 114: 1818-1825, 2006.

20. Han W, Wang Y, and Zheng $\mathbf{Y}$. In vivo biocompatibility studies of nano TiO2 materials Adv Mat Res 79-82: 389-392, 2009.

21. Haney S, Sur D, and Xu Z. Diastolic heart failure: a review and primary care perspective. J Am Board Fam Pract 18: 189-198, 2005.

22. Hougaard KS, Campagnolo L, Chavatte-Palmer P, Tarrade A, Rousseau-Ralliard D, Valentino S, Park MV, de Jong WH, Wolterink G, Piersma AH, Ross BL, Hutchison GR, Hansen JS, Vogel U, Jackson P, Slama R, Pietroiusti A, and Cassee FR. A perspective on the developmental toxicity of inhaled nanoparticles. Reprod Toxicol 56: 118-140, 2015.

23. Huang X, Zhang F, Sun X, Choi KY, Niu G, Zhang G, Guo J, Lee S, and Chen X. The genotype-dependent influence of functionalized multiwalled carbon nanotubes on fetal development. Biomaterials 35: 856-865, 2014.

24. Imao T and Nagata S. Apaf-1- and Caspase-8-independent apoptosis. Cell Death Differ 20: 343-352, 2013.

25. James JF, Hewett TE, and Robbins J. Cardiac physiology in transgenic mice. Circ Res 82: 407-415, 1998.

26. Jawad H, Boccaccini AR, Ali NN, and Harding SE. Assessment of cellular toxicity of TiO2 nanoparticles for cardiac tissue engineering applications. Nanotoxicology 5: 372-380, 2011.

27. Knuckles TL, Yi J, Frazer DG, Leonard HD, Chen BT, Castranova V, and Nurkiewicz TR. Nanoparticle inhalation alters systemic arteriolar vasoreactivity through sympathetic and cyclooxygenase-mediated pathways. Nanotoxicology 6: 724-735, 2012.

28. Labbe SM, Grenier-Larouche T, Noll C, Phoenix S, Guerin B, Turcotte EE, and Carpentier AC. Increased myocardial uptake of dietary fatty acids linked to cardiac dysfunction in glucose-intolerant humans. Diabetes 61: 2701-2710, 2012.

29. Leavens TL, Parkinson CU, James RA, House D, Elswick B, and Dorman DC. Respiration in Sprague-Dawley rats during pregnancy. Inhal Toxicol 18: 305-312, 2006.

30. Liu H, Ma L, Zhao J, Liu J, Yan J, Ruan J, and Hong F. Biochemical toxicity of nanoanatase TiO2 particles in mice. Biol Trace Elem Res 129: 170-180, 2009.

31. Mei L, Zhang Z, Zhao L, Huang L, Yang XL, Tang J, and Feng SS. Pharmaceutical nanotechnology for oral delivery of anticancer drugs. Adv Drug Deliv Rev 65: 880-890, 2013.

32. Mori J, Basu R, McLean BA, Das SK, Zhang L, Patel VB, Wagg CS, Kassiri Z, Lopaschuk GD, and Oudit GY. Agonist-induced hypertrophy and diastolic dysfunction are associated with selective reduction in glucose oxidation: a metabolic contribution to heart failure with normal ejection fraction. Circ Heart Fail 5: 493-503, 2012.

33. Mottram PM and Marwick TH. Assessment of diastolic function: what the general cardiologist needs to know. Heart 91: 681-695, 2005. 
34. Muoio DM and Koves TR. Skeletal muscle adaptation to fatty acid depends on coordinated actions of the PPARs and PGC1 alpha: implications for metabolic disease. Appl Physiol Nutr Metab 32: 874-883, 2007.

35. Nurkiewicz TR, Porter DW, Hubbs AF, Cumpston JL, Chen BT, Frazer DG, and Castranova V. Nanoparticle inhalation augments particle-dependent systemic microvascular dysfunction. Part Fibre Toxicol 5: 1, 2008.

36. Oberdorster G, Sharp Z, Atudorei V, Elder A, Gelein R, Lunts A, Kreyling W, and Cox C. Extrapulmonary translocation of ultrafine carbon particles following whole-body inhalation exposure of rats. J Toxicol Environ Health A 65: 1531-1543, 2002.

37. Park H and Yeo M. Comparison of gene expression changes induced by exposure to Ag, $\mathrm{Cu}-\mathrm{TiO} 2$, and TiO2 nanoparticles in zebrafish embryos. Mol Cell Toxicol 9: 129-139, 2013.

38. Pavlopoulos $\mathbf{H}$ and Nihoyannopoulos $\mathbf{P}$. Strain and strain rate deformation parameters: from tissue Doppler to 2D speckle tracking. Int J Cardiovasc Imaging 24: 479-491, 2008.

39. Pawlush DG, Moore RL, Musch TI, and Davidson WR, Jr. Echocardiographic evaluation of size, function, and mass of normal and hypertrophied rat ventricles. $J$ Appl Physiol (1985) 74: 2598-2605, 1993.

40. Piccinno F, Gottschalk F, Seeger S, and Nowack B. Industrial production quantities and uses of ten engineered nanomaterials in Europe and the world. J Nanopart Res 14: 1-11, 2012.

41. Porter DW, Hubbs AF, Chen BT, McKinney W, Mercer RR, Wolfarth MG, Battelli L, Wu N, Sriram K, Leonard S, Andrew M, Willard P, Tsuruoka S, Endo M, Tsukada T, Munekane F, Frazer DG, and Castranova V. Acute pulmonary dose-responses to inhaled multiwalled carbon nanotubes. Nanotoxicology 7: 1179-1194, 2013.

42. Qi W, Bi J, Zhang X, Wang J, Wang J, Liu P, Li Z, and Wu W. Damaging effects of multi-walled carbon nanotubes on pregnant mice with different pregnancy times. Sci Rep 4: 4352, 2014.

43. Robichaud CO, Uyar AE, Darby MR, Zucker LG, and Wiesner MR. Estimates of upper bounds and trends in nano-TiO2 production as a basis for exposure assessment. Environ Sci Technol 43: 4227-4233, 2009.

44. Sager TM and Castranova V. Surface area of particle administered versus mass in determining the pulmonary toxicity of ultrafine and fine carbon black: comparison to ultrafine titanium dioxide. Part Fibre Toxicol 6: 15, 2009.

45. Sager TM, Kommineni C, and Castranova V. Pulmonary response to intratracheal instillation of ultrafine versus fine titanium dioxide: role of particle surface area. Part Fibre Toxicol 5: 17, 2008.

46. Savi M, Rossi S, Bocchi L, Gennaccaro L, Cacciani F, Perotti A, Amidani D, Alinovi R, Goldoni M, Aliatis I, Lottici PP, Bersani D, Campanini M, Pinelli S, Petyx M, Frati C, Gervasi A, Urbanek K, Quaini F, Buschini A, Stilli D, Rivetti C, Macchi E, Mutti A, Miragoli M, and Zaniboni M. Titanium dioxide nanoparticles promote arrhythmias via a direct interaction with rat cardiac tissue. Part Fibre Toxicol 11: 63, 2014.

47. Shah U, Bien H, and Entcheva E. Microtopographical effects of natural scaffolding on cardiomyocyte function and arrhythmogenesis. Acta Biomater 6: 3029-3034, 2010.

48. Shepherd DL, Nichols CE, Croston TL, McLaughlin SL, Petrone AB, Lewis SE, Thapa D, Long DM, Dick GM, and Hollander JM. Early detection of cardiac dysfunction in the type 1 diabetic heart using speckle-tracking based strain imaging. J Mol Cell Cardiol 90: 7483, 2016. 
49. Shirasuna K, Usui F, Karasawa T, Kimura H, Kawashima A, Mizukami H, Ohkuchi A, Nishimura S, Sagara J, Noda T, Ozawa K, Taniguchi S, and Takahashi M. Nanosilicainduced placental inflammation and pregnancy complications: Different roles of the inflammasome components NLRP3 and ASC. Nanotoxicology 9: 554-567, 2015.

50. Sood A, Salih S, Roh D, Lacharme-Lora L, Parry M, Hardiman B, Keehan R, Grummer R, Winterhager E, Gokhale PJ, Andrews PW, Abbott C, Forbes K, Westwood M, Aplin JD, Ingham E, Papageorgiou I, Berry M, Liu J, Dick AD, Garland RJ, Williams N, Singh R, Simon AK, Lewis M, Ham J, Roger L, Baird DM, Crompton LA, Caldwell MA, Swalwell H, Birch-Machin M, Lopez-Castejon G, Randall A, Lin H, Suleiman MS, Evans WH, Newson R, and Case CP. Signalling of DNA damage and cytokines across cell barriers exposed to nanoparticles depends on barrier thickness. Nat Nanotechnol 6: 824-833, 2011.

51. Stapleton PA, McBride CR, Yi J, and Nurkiewicz TR. Uterine microvascular sensitivity to nanomaterial inhalation: An in vivo assessment. Toxicol Appl Pharmacol 288: 420-428, 2015.

52. Stapleton PA, Minarchick VC, Cumpston AM, McKinney W, Chen BT, Sager TM, Frazer DG, Mercer RR, Scabilloni J, Andrew ME, Castranova V, and Nurkiewicz TR. Impairment of coronary arteriolar endothelium-dependent dilation after multi-walled carbon nanotube inhalation: a time-course study. Int J Mol Sci 13: 13781-13803, 2012.

53. Stapleton PA, Minarchick VC, Yi J, Engels K, McBride CR, and Nurkiewicz TR. Maternal engineered nanomaterial exposure and fetal microvascular function: does the Barker hypothesis apply? Am J Obstet Gynecol 209: 227 e221-211, 2013.

54. Stapleton PA, Nichols CE, Yi J, McBride CR, Minarchick VC, Shepherd DL, Hollander JM, and Nurkiewicz TR. Microvascular and mitochondrial dysfunction in the female F1 generation after gestational TiO2 nanoparticle exposure. Nanotoxicology 9: 941-951, 2015.

55. Takeda K, Suzuki K, Ishihara A, Kubo-Irie M, Fujimoto R, Tabata M, and Sugamata M. Nanoparticles Transferred from Pregnant Mice to Their Offspring Can Damage the Genital and Cranial Nerve Systems. $j$ Health Sci 55: 95-102, 2009.

56. Thapa D, Nichols CE, Lewis SE, Shepherd DL, Jagannathan R, Croston TL, Tveter KJ, Holden AA, Baseler WA, and Hollander JM. Transgenic overexpression of mitofilin attenuates diabetes mellitus-associated cardiac and mitochondria dysfunction. J Mol Cell Cardiol 79: 212-223, 2015.

57. Wang JX, Fan YB, Gao Y, Hu QH, and Wang TC. TiO2 nanoparticles translocation and potential toxicological effect in rats after intraarticular injection. Biomaterials 30: 4590-4600, 2009.

58. Weldy CS, Liu Y, Chang YC, Medvedev IO, Fox JR, Larson TV, Chien WM, and Chin MT. In utero and early life exposure to diesel exhaust air pollution increases adult susceptibility to heart failure in mice. Part Fibre Toxicol 10: 59, 2013.

59. Weldy CS, Liu Y, Liggitt HD, and Chin MT. In utero exposure to diesel exhaust air pollution promotes adverse intrauterine conditions, resulting in weight gain, altered blood pressure, and increased susceptibility to heart failure in adult mice. PLoS One 9: e88582, 2014.

60. Wittig I, Braun HP, and Schagger H. Blue native PAGE. Nat Protoc 1: 418-428, 2006.

61. Xu X and Colecraft HM. Primary culture of adult rat heart myocytes. J Vis Exp, 2009.

62. Yang $\mathbf{Q}$ and Li Y. Roles of PPARs on regulating myocardial energy and lipid homeostasis. J Mol Med (Berl) 85: 697-706, 2007.

63. Ye G, Metreveli NS, Donthi RV, Xia S, Xu M, Carlson EC, and Epstein PN. Catalase protects cardiomyocyte function in models of type 1 and type 2 diabetes. Diabetes 53: 1336-1343, 2004. 
64. Yeo M and Kim H. Gene expression in zebrafish embryos following exposure to $\mathrm{TiO} 2$ nanoparticles. Mol Cell Toxicol 6: 97-104, 2010.

65. Yi J, Chen BT, Schwegler-Berry D, Frazer D, Castranova V, McBride C, Knuckles TL, Stapleton PA, Minarchick VC, and Nurkiewicz TR. Whole-body nanoparticle aerosol inhalation exposures. J Vis Exp: e50263, 2013.

66. Yoshida S, Tsutsumi S, Muhlebach G, Sourbier C, Lee MJ, Lee S, Vartholomaiou E, Tatokoro M, Beebe K, Miyajima N, Mohney RP, Chen Y, Hasumi H, Xu W, Fukushima H, Nakamura K, Koga F, Kihara K, Trepel J, Picard D, and Neckers L. Molecular chaperone TRAP1 regulates a metabolic switch between mitochondrial respiration and aerobic glycolysis. Proc Natl Acad Sci U S A 110: E1604-1612, 2013.

67. Yu X, Hong F, and Zhang YQ. Cardiac inflammation involving in PKCepsilon or ERK1/2-activated NF-kappaB signalling pathway in mice following exposure to titanium dioxide nanoparticles. J Hazard Mater 313: 68-77, 2016.

68. Zhang L, Keung W, Samokhvalov V, Wang W, and Lopaschuk GD. Role of fatty acid uptake and fatty acid beta-oxidation in mediating insulin resistance in heart and skeletal muscle. Biochim Biophys Acta 1801: 1-22, 2010. 
Table 6.1.1

\section{TABLE 1}

\begin{tabular}{|cccc|}
\hline Calculation & Units & $\begin{array}{c}\text { Control Average and } \\
\text { SEM }\end{array}$ & $\begin{array}{c}\text { Exposed Average } \\
\text { and SEM }\end{array}$ \\
\hline Area;d (LV Trace) & $\mathrm{mm}^{2}$ & $60.39 \pm 3.87$ & $56.39 \pm 2.49$ \\
\hline Area;s (LV Trace) & $\mathrm{mm}^{2}$ & $25.64 \pm 2.69$ & $25.59 \pm 1.97$ \\
\hline CO (LV Trace) & $\mathrm{mL} / \mathrm{min}$ & $196.01 \pm 18.54$ & $177.18 \pm 12.10$ \\
\hline EF (LV Trace) & $\%$ & $77.29 \pm 2.69$ & $75.70 \pm 2.27$ \\
\hline FS (LV Trace) & $\%$ & $20.64 \pm 2.07$ & $16.26 \pm 1.64$ \\
\hline SV (LV Trace) & $\mu \mathrm{L}$ & $200.21 \pm 18.21$ & $179.19 \pm 12.07$ \\
\hline V;d (LV Trace) & $\mu \mathrm{L}$ & $262.54 \pm 24.54$ & $238.86 \pm 16.45$ \\
\hline V;s (LV Trace) & $\mu \mathrm{L}$ & $62.33 \pm 9.68$ & $59.67 \pm 7.022$ \\
\hline
\end{tabular}


Table 6.1.1: B-Mode echocardiography for control versus gestational $\mathrm{TiO}_{2}$ exposed animals at the young adult (6-12 weeks) stage. Average and SEM for each measure. Area;d = diastolic area, Area $; \mathrm{s}=$ systolic area, $\mathrm{CO}=$ cardiac output, $\mathrm{EF}=$ ejection fraction, $\mathrm{FS}=$ fractional shortening, $\mathrm{SV}$ $=$ stroke volume, $\mathrm{V} ; \mathrm{d}=$ volume at diastole, $\mathrm{V} ; \mathrm{s}=$ volume at systole. $\dagger(P=0.09-0.055),{ }^{*}(P \leq$ $0.05), * *(P \leq 0.01)$. 
Table 6.1.2

\section{TABLE 2}

\begin{tabular}{|cccc|}
\hline Calculation & Units & $\begin{array}{c}\text { Control Average and } \\
\text { SEM }\end{array}$ & $\begin{array}{c}\text { Exposed Average and } \\
\text { SEM }\end{array}$ \\
\hline CO (LV Trace) & $\mathrm{mL} / \mathrm{min}$ & $70.86 \pm 1.79$ & $65.14 \pm 3.05$ \\
\hline Diameter;d (LV Trace) & $\mathrm{mm}$ & $6.72 \pm 0.12$ & $6.38 \pm 0.16$ \\
\hline Diameter;s (LV Trace) & $\mathrm{mm}$ & $2.90 \pm 0.14$ & $2.83 \pm 0.11$ \\
\hline EF & $\%$ & $79.41 \pm 1.48$ & $80.07 \pm 1.05$ \\
\hline EF (LV Trace) & $\%$ & $85.56 \pm 1.23$ & $84.96 \pm 0.72$ \\
\hline FS & $\%$ & $49.54 \pm 1.54$ & $56.84 \pm 1.09$ \\
\hline FS (LV Trace) & $\%$ & $57.33 \pm 1.63$ & $\mathbf{6 9 6 . 8 9 ^ { * } \pm 4 0 . 9 3}$ \\
\hline LV MasS (LV Trace) & $\mathrm{mg}$ & $\mathbf{8 4 1 . 8 2 ^ { * } \pm 5 1 . 3 4}$ & $\mathbf{5 5 7 . 5 1 ^ { * } \pm 3 2 . 7 5}$ \\
\hline LV Mass Corr (LV Trace) & $\mathrm{mg}$ & $\mathbf{6 7 3 . 4 5} \pm 41.07$ & $195.27 \pm 11.32$ \\
\hline LV Vol;d & $\mu \mathrm{L}$ & $214.37 \pm 10.19$ & $38.90 \pm 3.11$ \\
\hline LV Vol;S & $\mu \mathrm{L}$ & $45.78 \pm 5.05$ & $\mathbf{1 7 8 . 3 1 \dagger \pm 9 . 1 9}$ \\
\hline SV (LV Trace) & $\mu \mathrm{L}$ & $\mathbf{2 0 0 . 0 9 \dagger \pm 6 . 9 5}$ & $211.59 \pm 11.45$ \\
\hline V;d (LV Trace) & $\mu \mathrm{L}$ & $236.10 \pm 9.63$ & $33.28 \pm 2.73$ \\
\hline V;S (LV Trace) & $\mu \mathrm{L}$ & $36.01 \pm 4.02$ & \\
\hline
\end{tabular}


Table 6.1.2: $M-M o d e$ echocardiography for control versus gestational $\mathrm{TiO}_{2}$ exposed animals at the young adult (6-12 weeks) stage. Average and SEM for each measure $\mathrm{CO}=$ cardiac output, Diameter; $\mathrm{d}=$ diameter at diastole, Diameter; $\mathrm{s}=$ diameter at systole, $\mathrm{EF}=$ ejection fraction, $\mathrm{FS}=$ fractional shortening, LV Mass = left ventricular mass, LV Vol; $=$ left ventricular volume at diastole, $\mathrm{LV}$ Vol; $\mathrm{s}=$ left ventricular volume at systole, $\mathrm{SV}=$ stroke volume, $\mathrm{V} ; \mathrm{d}=$ volume at diastole, $\mathrm{V} ; \mathrm{s}=$ volume at systole. $\dagger(P=0.09-0.055), *(P \leq 0.05), * *(P \leq 0.01)$. 
Table 6.1.3

\section{TABLE 3}

\begin{tabular}{|cccc|}
\hline Measurement & Units & $\begin{array}{c}\text { Control Average } \\
\text { and SEM }\end{array}$ & $\begin{array}{c}\text { Exposed Average } \\
\text { and SEM }\end{array}$ \\
\hline IVS;d & $\mathrm{mm}$ & $1.92 \pm 0.06$ & $1.77 \pm 0.06$ \\
\hline IVS;s & $\mathrm{mm}$ & $\mathbf{3 . 2 1 ^ { * } \pm 0 . 0 6}$ & $\mathbf{2 . 9 1 ^ { * } \pm 0 . 0 7}$ \\
\hline LVID;d & $\mathrm{mm}$ & $6.45 \pm 0.13$ & $6.14 \pm 0.17$ \\
\hline LVID;s & $\mathrm{mm}$ & $3.28 \pm 0.15$ & $3.07 \pm 0.10$ \\
\hline LVPW;d & $\mathrm{mm}$ & $1.88 \pm 0.10$ & $1.77 \pm 0.07$ \\
\hline LVPW;s & $\mathrm{mm}$ & $2.87 \pm 0.09$ & $2.69 \pm 0.08$ \\
\hline
\end{tabular}


Table 6.1.3: Left ventricle thickness, using M-Mode echocardiography for control versus gestational $\mathrm{TiO}_{2}$ exposed animals at the young adult (6-12 weeks) stage. Average and SEM for each measure IVS; $d=$ Interventricular end-diastolic septal thickness, IVS; $=$ Interventricular endsystolic septal thickness, LVID; $d=$ left ventricular end-diastolic internal thickness, LVID;s = left ventricular end-systolic thickness, LVPW; $\mathrm{d}=$ left ventricular end-diastolic posterior wall thickness, LVPW;s = left ventricular end-systolic posterior wall thickness. $\dagger(P=0.09-0.055)$, * $(P \leq 0.05), * *(P \leq 0.01)$. 
Table 6.1.4

\section{TABLE 4}

\begin{tabular}{|cccc|}
\hline $\begin{array}{c}\text { Electron Transport } \\
\text { Chain }\end{array}$ & $\begin{array}{c}\text { Optical } \\
\text { Density }\end{array}$ & $\begin{array}{c}\text { Control Average } \\
\text { and SEM }\end{array}$ & $\begin{array}{c}\text { Exposed Average } \\
\text { and SEM }\end{array}$ \\
\hline Complex I & AU & $8.69 \pm 1.66$ & $8.61 \pm 2.26$ \\
\hline Complex II & AU & $7.02 \pm 1.07$ & $6.58 \pm 1.98$ \\
\hline Complex III & AU & $9.80 \pm 1.10$ & $10.2 \pm 2.45$ \\
\hline Complex IV & AU & $10.6 \pm 0.27$ & $9.83 \pm 1.82$ \\
\hline Complex V & AU & $8.59 \pm 0.76$ & $8.66 \pm 2.00$ \\
\hline
\end{tabular}


Table 6.1.4: Blue native polyacrylamide gel electrophoresis (BN-PAGE) for control $(n=3)$ versus gestational $\mathrm{TiO}_{2}$ exposed $(\mathrm{n}=4)$ animals at the young adult $(6-12$ weeks) stage. Complex I $(P=$ 0.94), Complex II $(P=0.74)$, Complex III $(P=0.82)$, Complex IV $(P=0.5)$, and Complex V $(P$ $=0.96)$ were evaluated and found to be statistically insignificant. $\dagger(P=0.09-0.055),{ }^{*}(P \leq 0.05)$, $* *(P \leq 0.01)$. 
Figure 6.1.1
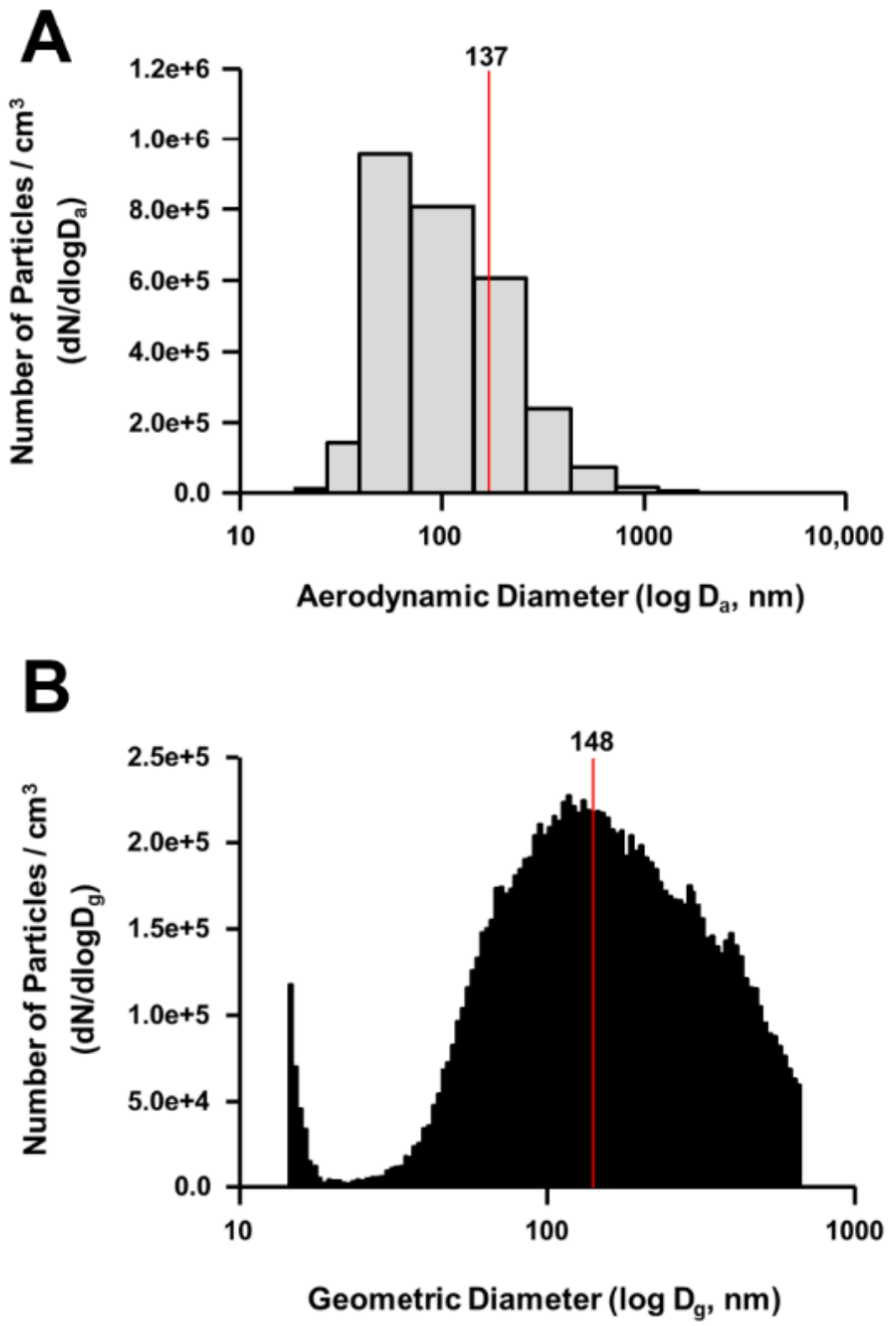
Figure 6.1.1

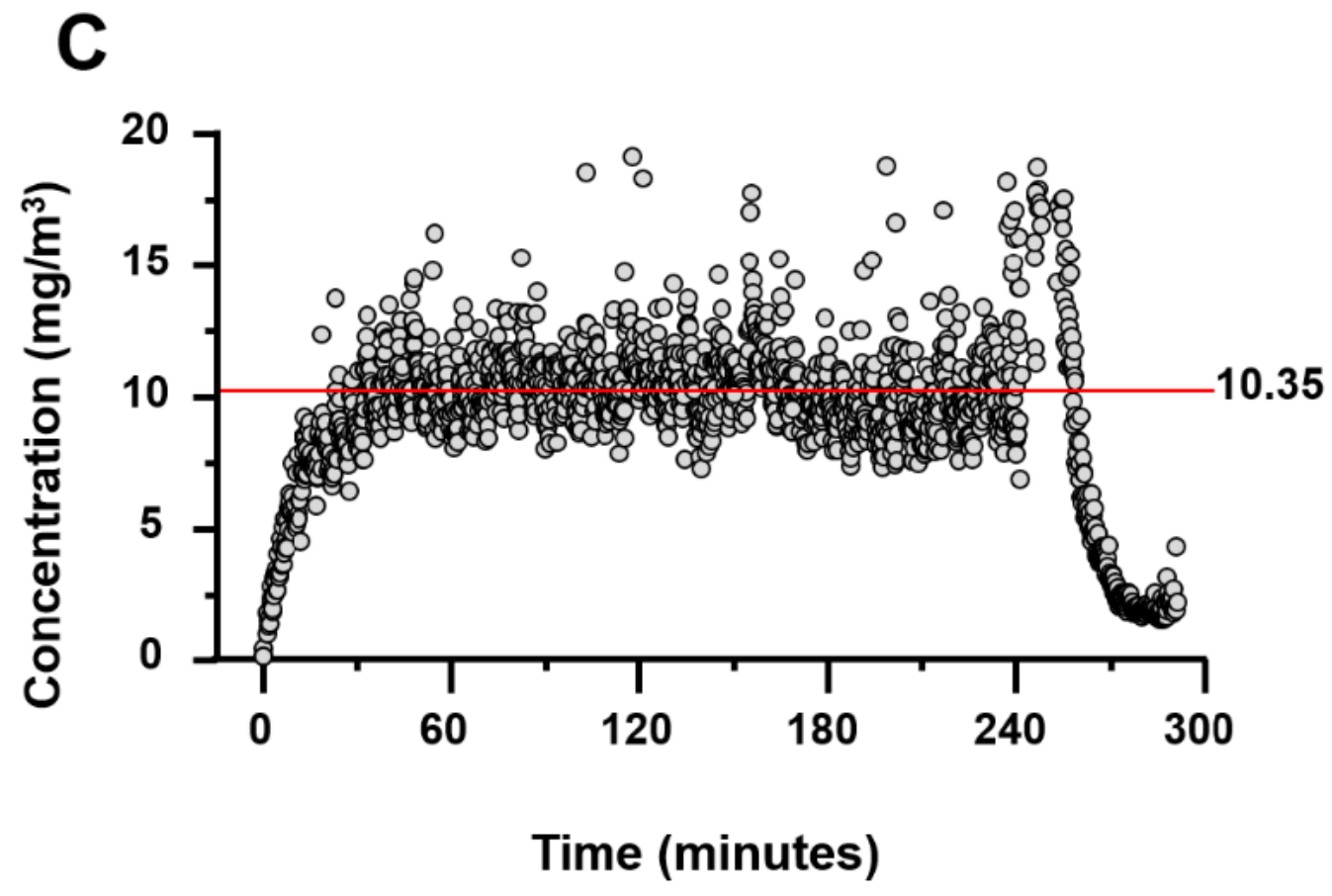


Figure 6.1.1: Characterization of nano- $\mathrm{TiO}_{2}$ used in aerosols exposure paradigm. Total daily deposition $=43.8 \pm 1.2 \mu \mathrm{g}$. (A) Electrical Low Pressure Impactor (ELPI) size distribution (aerodynamic diameter) of nano- $\mathrm{TiO}_{2}$ particles for $\mathrm{n}=89$ individual measurements $(136.465 \pm$ $1.436 \mathrm{~nm}$ ). (B) Scanning Mobility Particle Sizer (SMPS) size distribution (mobility diameter) of nano- $\mathrm{TiO}_{2}$ particles for $\mathrm{n}=89$ individual measurements $(134.802 \pm 1.241 \mathrm{~nm})$. (C) Aerosol concentration of engineered nano- $\mathrm{TiO}_{2}\left(10.352 \pm 0.134 \mathrm{mg} / \mathrm{m}^{3}\right)$ during exposures. 
Figure 6.1.2

$\boldsymbol{A}$
900
800
700
600
ڤึ 500
है 400
300
200
100
0

\section{E Wave}

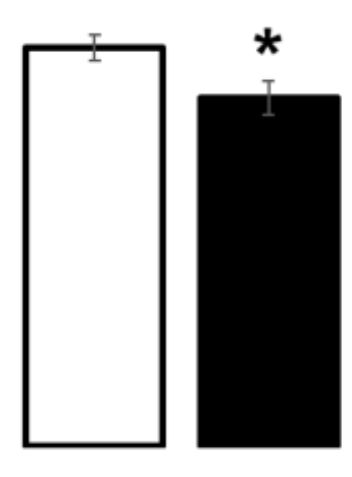

B

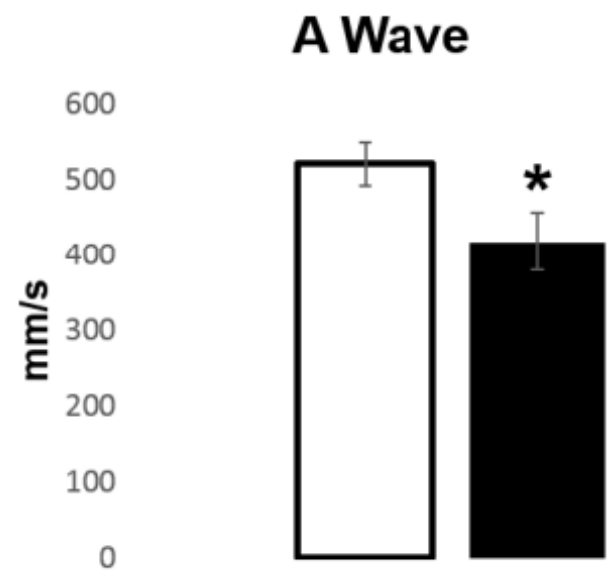

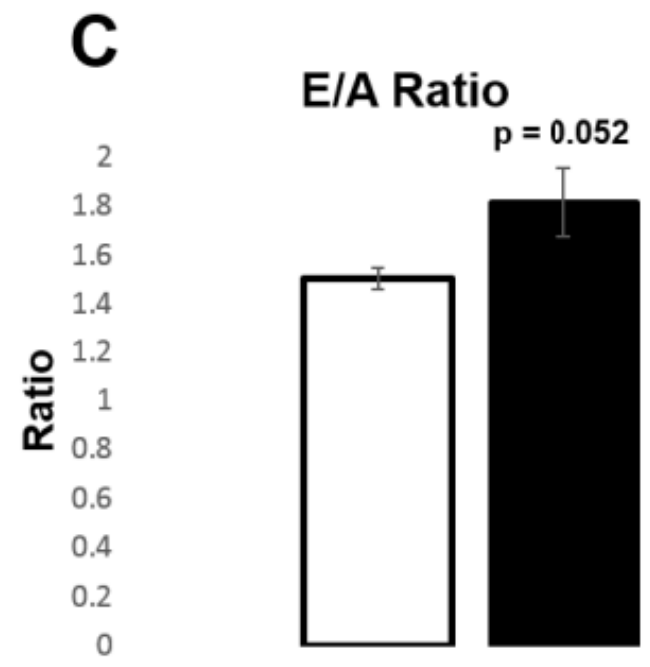


Figure 6.1.2

E

Mitral Valve Area

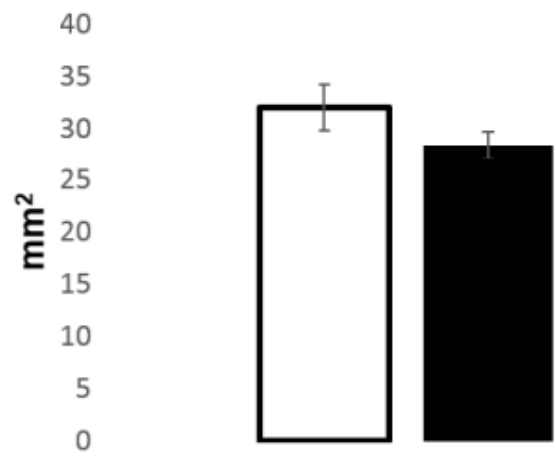

$\mathbf{F}$

Systolic Radial

Displacement - Long Axis

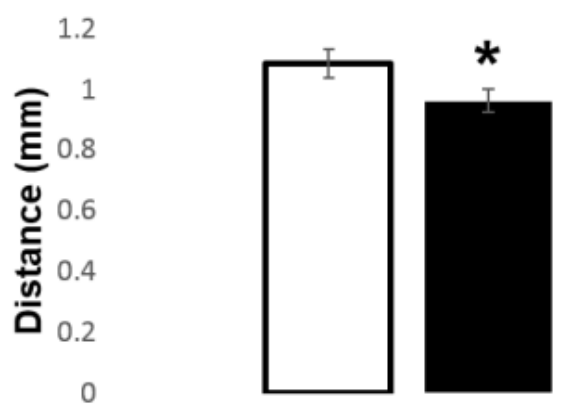

G

\section{Systolic Radial Strain -}

\section{Long Axis}

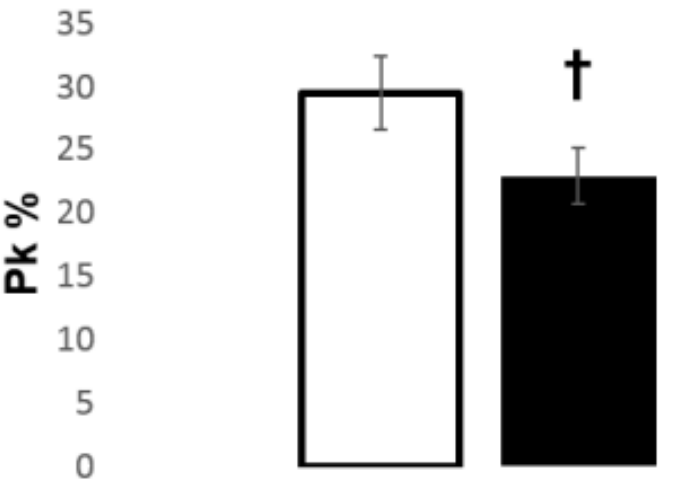


Figure 6.1.2: Echocardiography and speckle-tracking analysis, for control (white, $n=17$ ) versus gestational $\mathrm{TiO}_{2}$ exposed (black, $\left.\mathrm{n}=20\right)$ animals at the young adult $(6-12$ weeks) stage. (A) Velocity of E wave $(P=0.025),(\mathrm{B})$ velocity of A wave $(P=0.04)$, and $(\mathrm{C}) \mathrm{E} / \mathrm{A}$ ratio $(P=0.052)$. (D) Representative image of Doppler echocardiography for control (left) and exposed (right) animals. Images were chosen to represent the average E/A ratio for control $(1.506 \pm 0.043)$ and exposed $(1.813 \pm 0.140)$ animals during ultrasound. Other parameters of echocardiography were found to be insignificantly different $(\mathrm{E})$ mitral valve area $(P=0.16)$. Stress-strain analysis of systolic long axis $(\mathrm{F})$ radial displacement $(P=0.05)$ and $(\mathrm{G})$ radial strain $(P=0.065) . \dagger(P=0.075$ $-0.055), *(P \leq 0.05), * *(P \leq 0.01)$. 
Figure 6.1.3
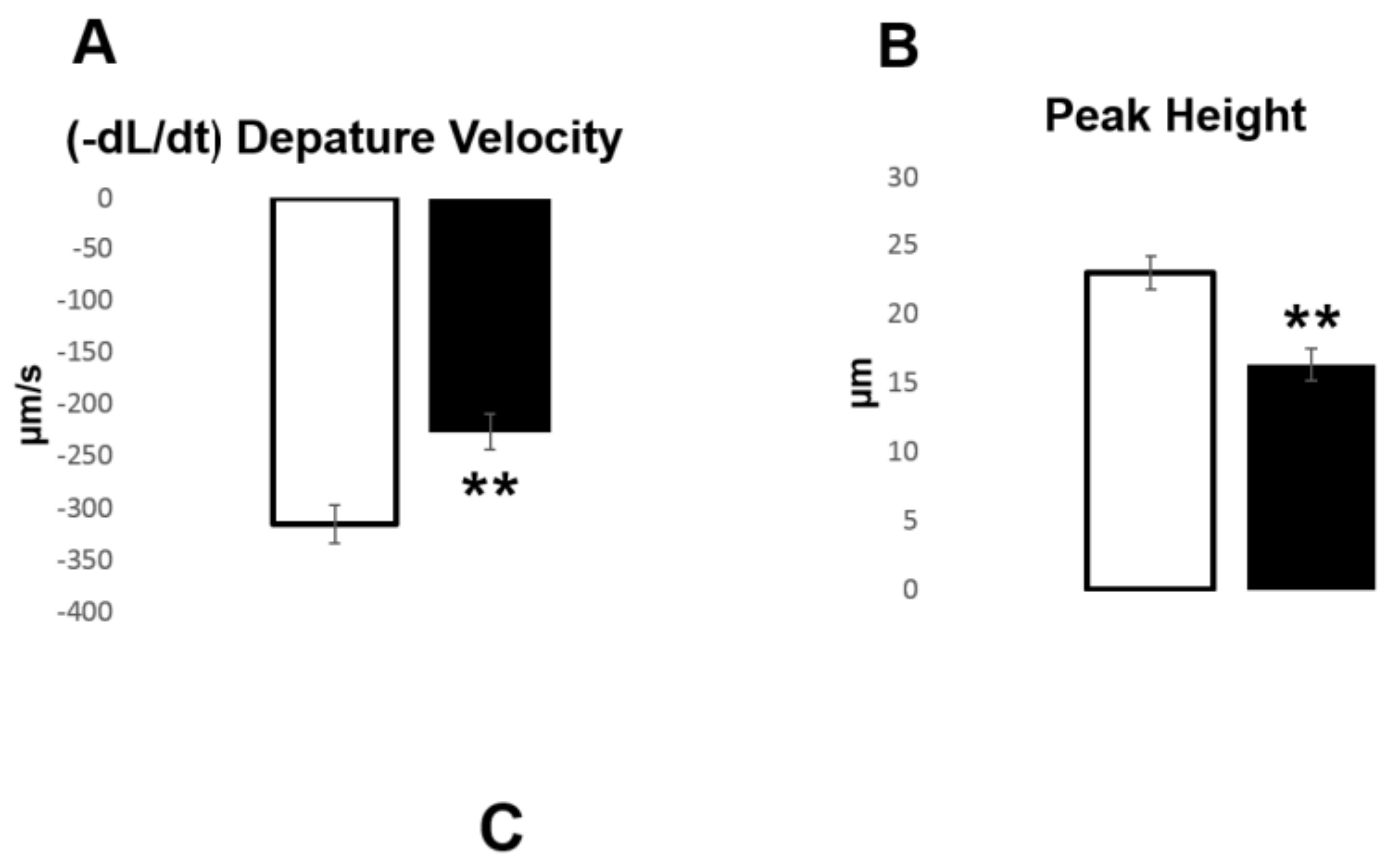

\section{Baseline}

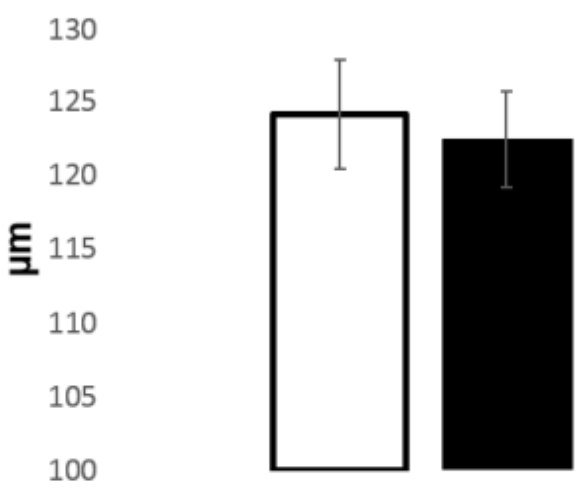


Figure 6.1.3

D

Percent Peak Shortening

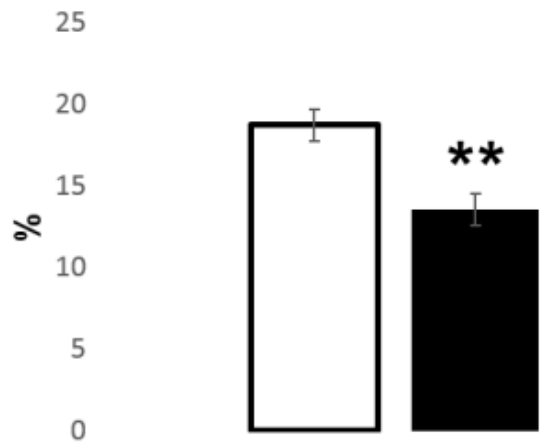

E

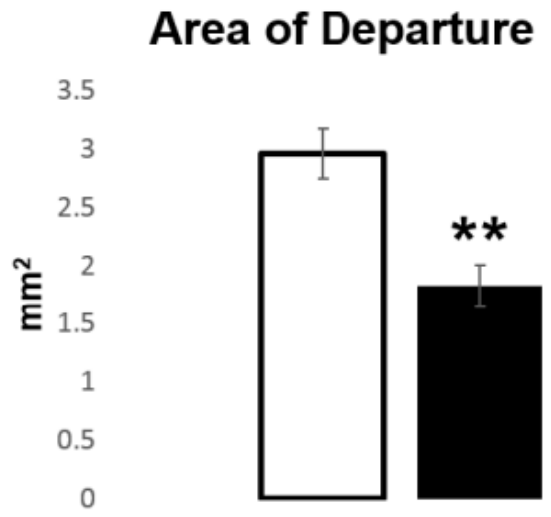

$\mathbf{F}$

Area of Return

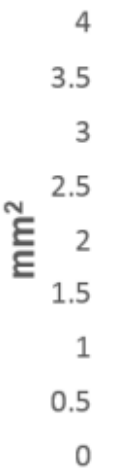

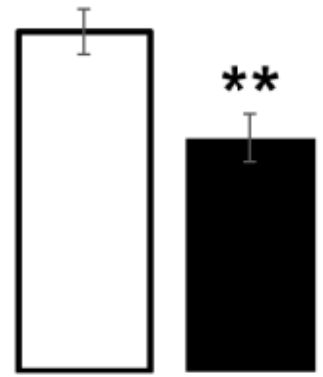


Figure 6.1.3

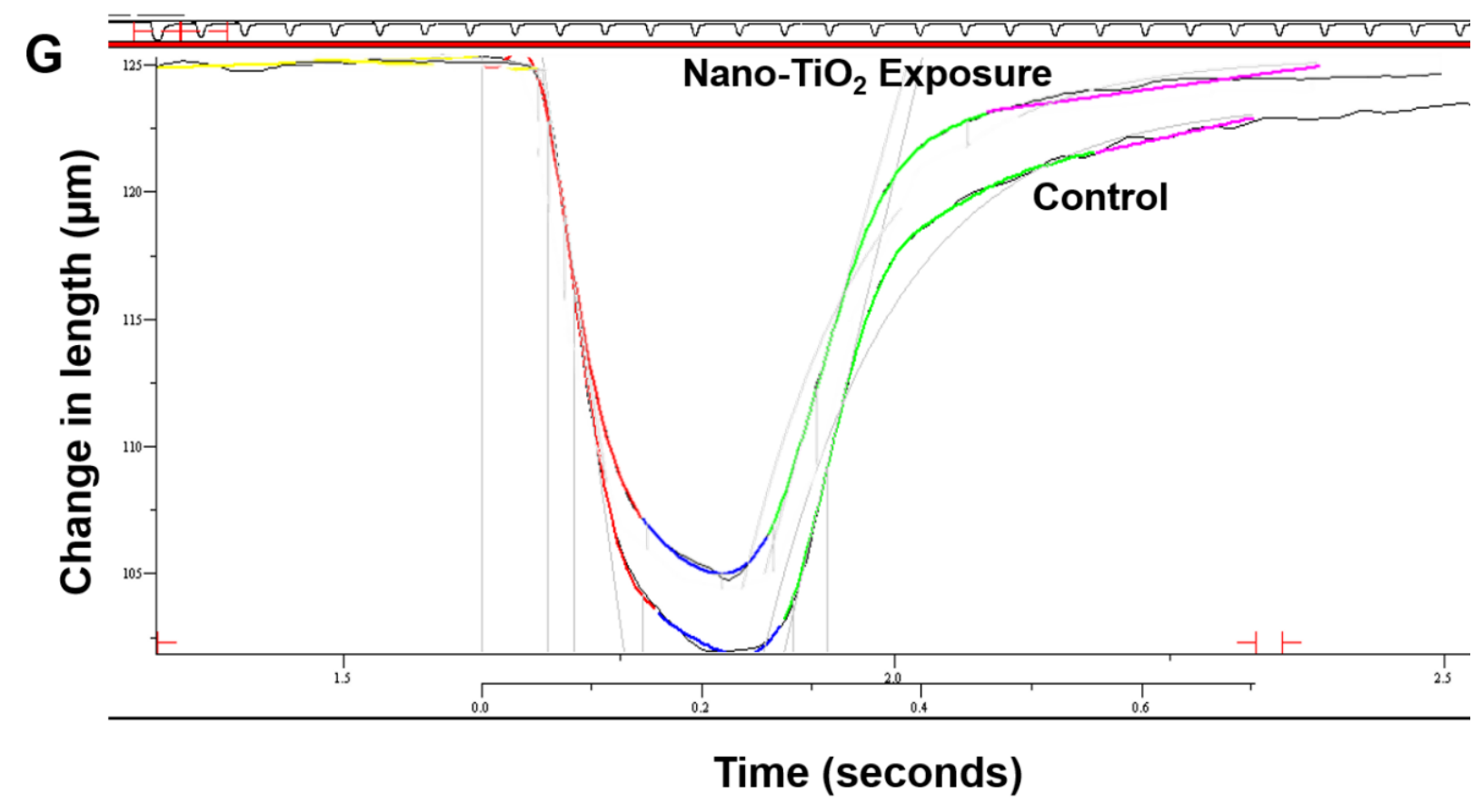


Figure 6.1.3: All first transient contractions (measured as the first contraction after stimulation) at 10 volts and $0.5 \mathrm{~Hz}$, control (white, $\mathrm{n}=39$ cardiomyocytes from $\mathrm{n}=8$ animals) versus gestational $\mathrm{TiO}_{2}$ exposed (black, $\mathrm{n}=42$ cardiomyocytes from $\mathrm{n}=9$ animals) cardiomyocytes at the young adult (6-12 weeks) stage. (A) departure velocity $(P=0.0008),(\mathrm{B})$ peak height $(P=$ 0.0001), (C) baseline ( $P=0.73)$, (D) baseline \% to peak height $(P=0.0003)$, (E) area of departure $(P=0.0001)$, and $(\mathrm{F})$ area of return $(P=0.0018) .(\mathrm{G})$ Illustration of contractile properties from the control and exposed cardiomyocytes. Specific contractions were chosen to match the average departure velocity and peak height found in the controls $(-315.446 \pm 18.611 \mu \mathrm{m} / \mathrm{s}, 22.920 \pm 1.174$ $\mu \mathrm{m})$ and the exposed $(-227.054 \pm 17.268 \mu \mathrm{m} / \mathrm{s}, 16.275 \pm 1.158 \mu \mathrm{m})$ groups. $\uparrow(P=0.075-0.055)$, $*(P \leq 0.05), * *(P \leq 0.01)$. 
Figure 6.1.4
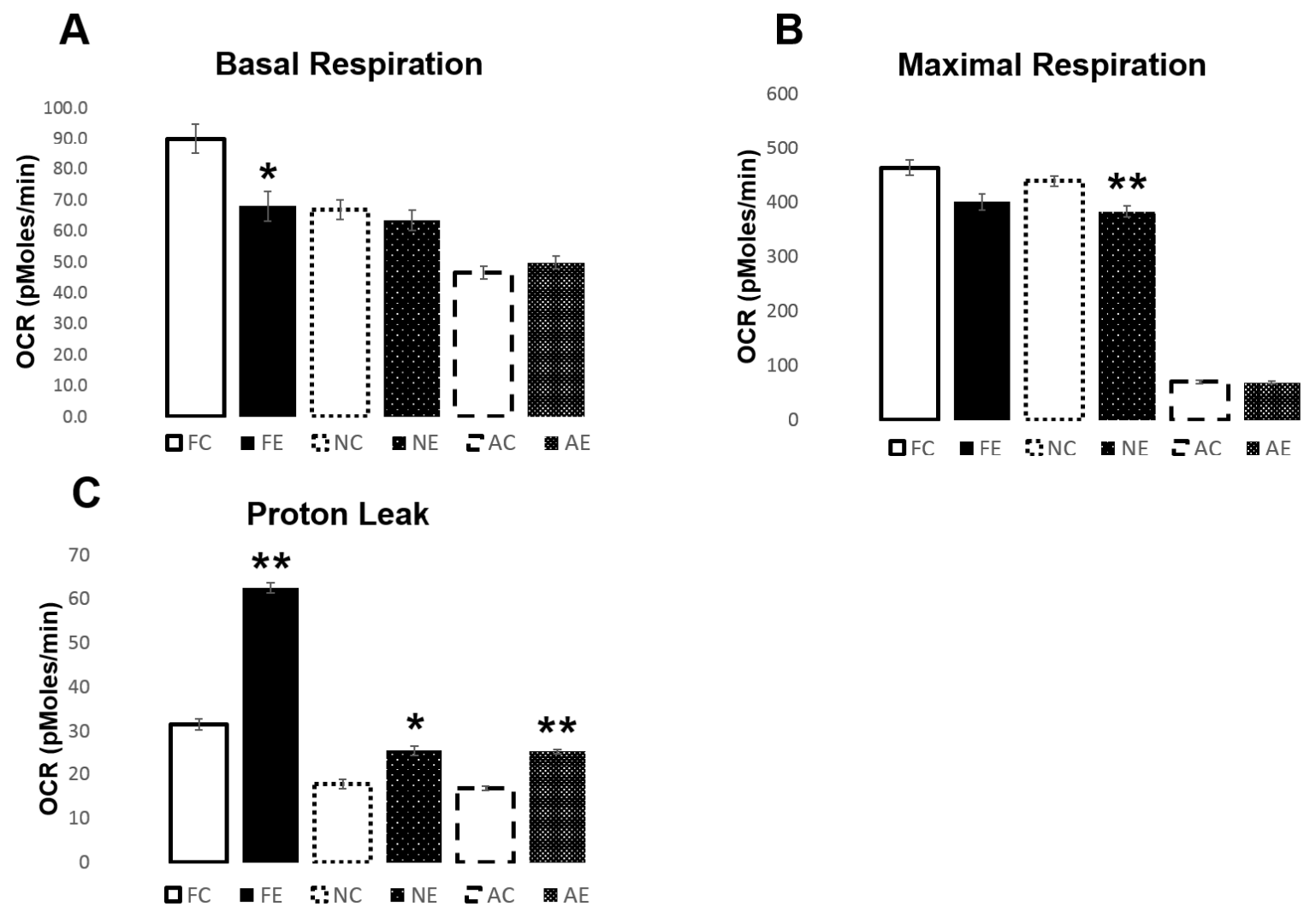
Figure 6.1.4
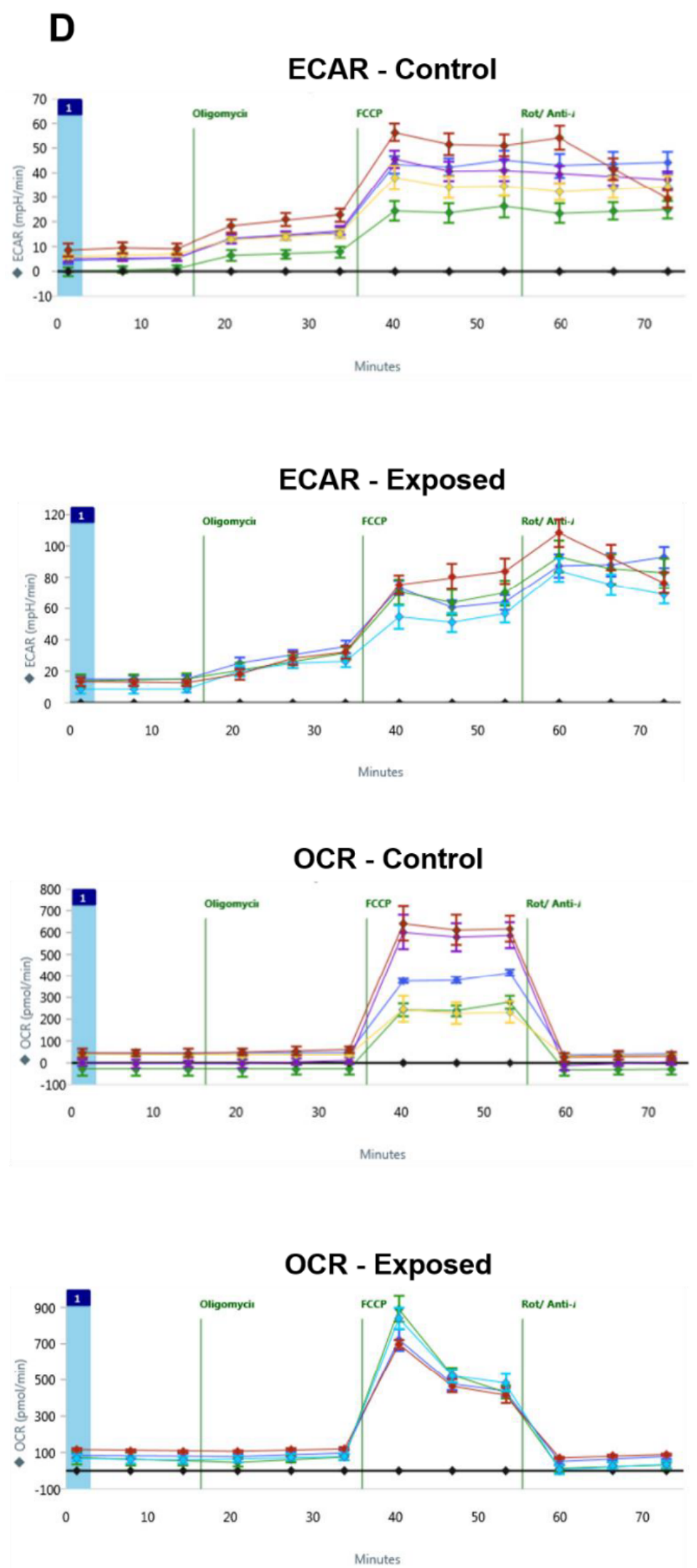
Figure 6.1.4: Seahorse $\mathrm{XFe} 96$ measurement for oxygen consumption rate (OCR) at pMoles/min, for controls (white) versus gestational $\mathrm{TiO}_{2}$ exposed (black) cardiomyocytes at the fetal (gestational day 28), neonatal (4-10 days), and young adult (6-12 weeks) stage. For all measures, and average of 65,000 cells were used for fetal measurements, 55,000 cells for neonatal, and the young adult group was normalized to basal respiration between the control and exposed groups. (A) Basal respiration rates, with a change observed between the fetal groups $(P=0.013)$. (B) Proton leak across all three groups, changing in the fetal $(P=0.0003)$, neonatal $(P=0.045)$, and young adult $(P=0.0072)$ groups. (C) Maximal respiration across all three groups, with changes in the neonatal group $(P=0.003)$. (D) Neonatal cardiomyocyte oxygen consumption rate $(\mathrm{OCR})$ and extracellular acidification rate (ECAR) in the control and experimental animals. Also an illustration of how values from the Seahorse analysis (basal respiration, maximal respiration, spare capacity, proton leak, etc.) are obtained using inhibitor agents such as oligomycin, FCCP, antimycin $\mathrm{A}$, and rotenone. Fetal $\mathrm{n}=5$ control and $\mathrm{n}=3$ exposed, neonatal $\mathrm{n}=4$ control and $\mathrm{n}=$ 5 exposed, and young adult $\mathrm{n}=5$ control and $\mathrm{n}=5$ exposed animals used for analysis. Figure 6.1.4C: $\mathrm{FC}=$ fetal control, $\mathrm{FE}=$ fetal exposed, $\mathrm{NC}=$ neonatal control, $\mathrm{NE}=$ neonatal exposed, $\mathrm{AC}$ $=$ adult control, $\mathrm{AE}=$ adult exposed. $\uparrow(P=0.075-0.055), *(P \leq 0.05), * *(P \leq 0.01)$. 
Figure 6.1.5

A

Complex I Activity

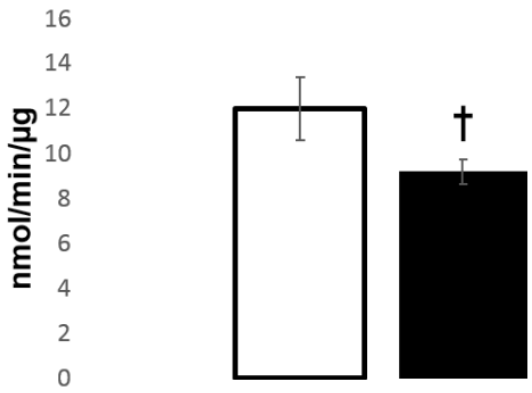

B

Complex IV Activity

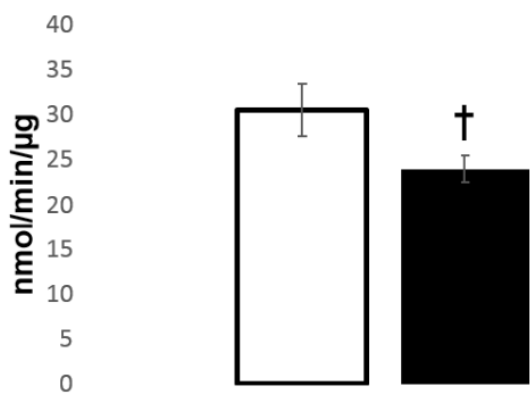

C

Complex III Activity

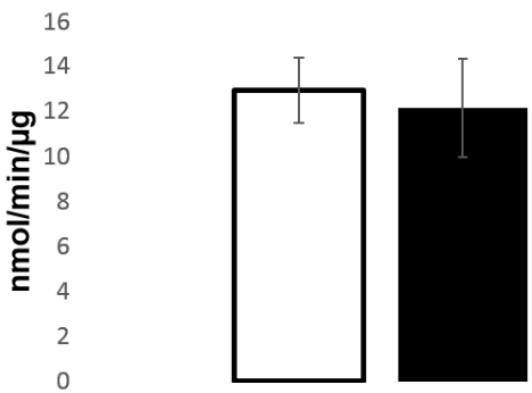

D

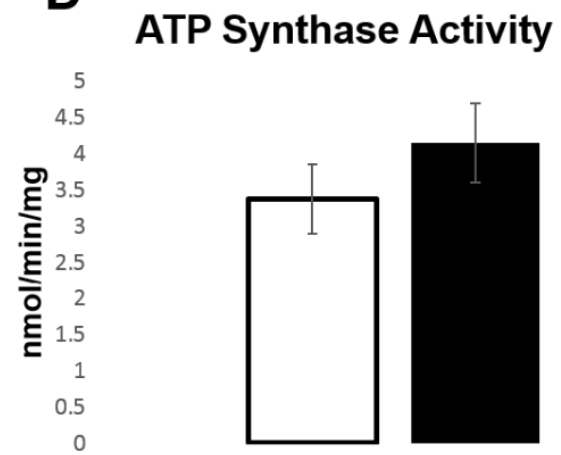


Figure 6.1.5: Electron transport chain complex activity, control (white, $n=7$ ) versus gestational $\mathrm{TiO}_{2}$ exposed (black, $\mathrm{n}=8$ ) cardiomyocytes at the young adult (6-12 weeks) stage. (A) Complex I activity $(P=0.067),(\mathrm{B})$ Complex III activity, $(\mathrm{C})$ Complex IV activity $(P=0.056)$, and (D) ATP Synthase activity. Activity was measured through the mass of cardiomyocyte cell lysates, not isolated mitochondria. $\dagger(P=0.075-0.055), *(P \leq 0.05), * *(P \leq 0.01)$. 
Figure 6.1.6

A

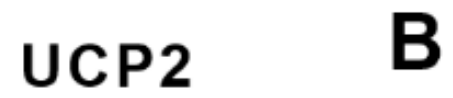

PPARG
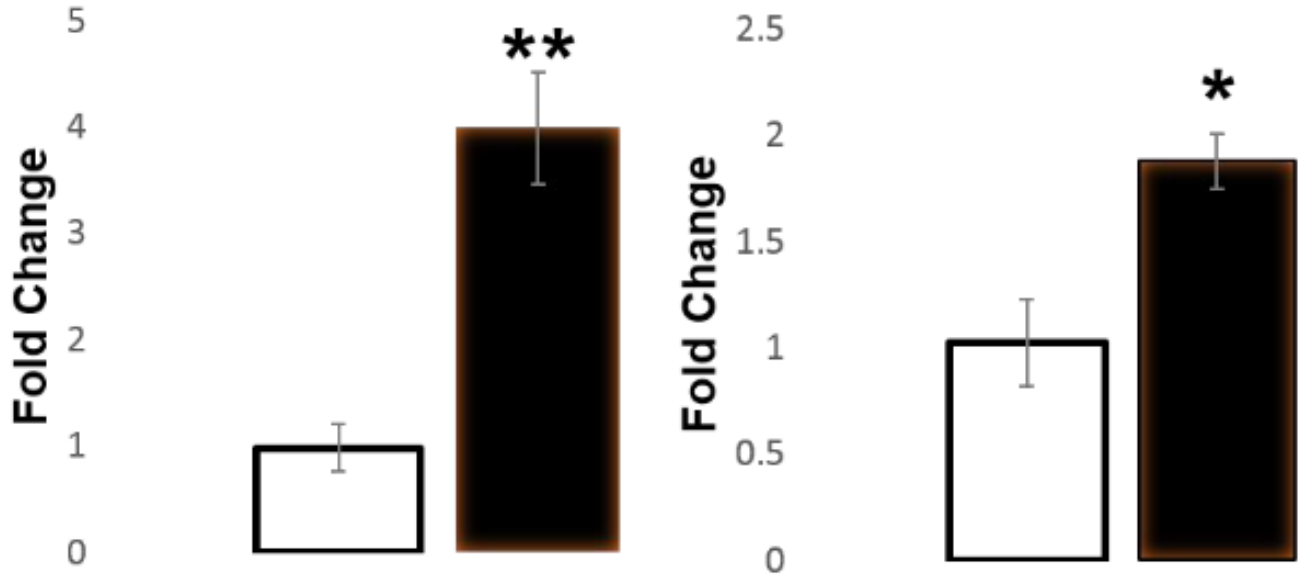

C

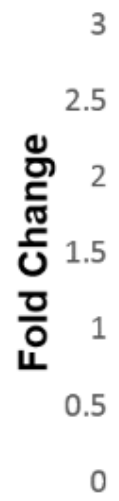

CPT1A

Con Ex
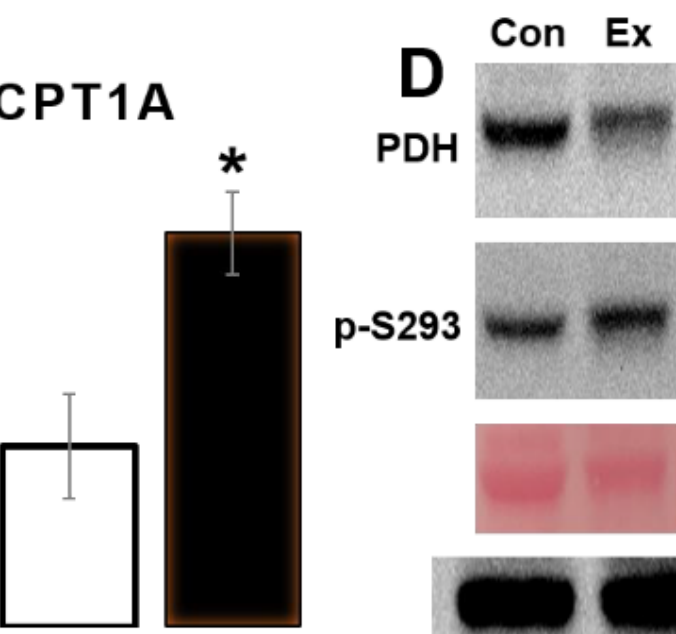

PDH

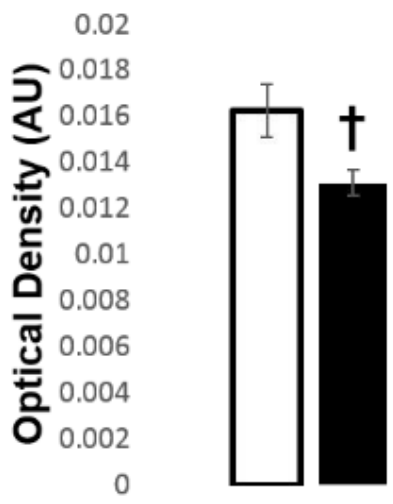


Figure 6.1.6
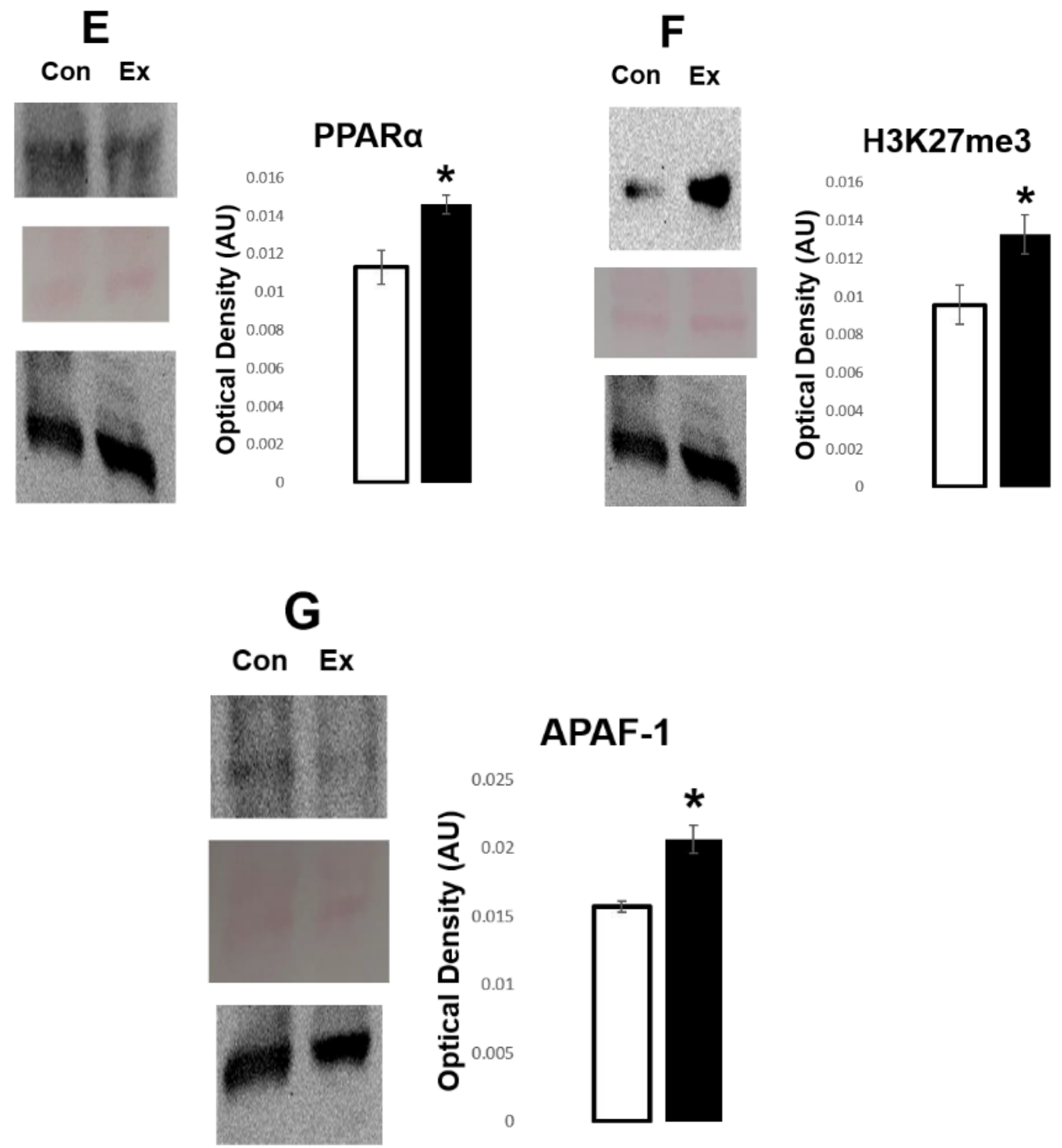
Figure 6.1.6: qPCR and western blot data from fetal and young adult progeny. Fetal heart tissue was analyzed using qPCR for mRNA levels while young adult cardiomyocytes were evaluated using western blotting for protein content. Control $(n=5)$ versus exposed $(n=5)$ mRNA fold change levels for (A) UCP2, (B) PPAR $\gamma$, and (C) CPT1A. mRNA levels were normalized to GAPDH. Control $(n=4)$ versus exposed $(n=4)$ optical density for (D) PDH and PDH p-S293, (E) PPAR $\alpha$, (F) H3K27me3, and (G) APAF-1. Blots are organized as follows: (Top) sample, (Middle) Ponceau S staining, and (Bottom) GAPDH. One representative image from both the control (left, labeled "con") and gestational exposed (right, labeled “ex") groups were chosen to display. $\dagger(P=0.075-0.055), *(P \leq 0.05), * *(P \leq 0.01) . \mathrm{UCP} 2=$ uncoupling protein 2, PPAR $\gamma$ = Peroxisome proliferator-activated receptor gamma, CPT1A = Carnitine palmitoyltransferase I, $\mathrm{PDH}=$ pyruvate dehydrogenase, PDH p-S293 = pyruvate dehydrogenase E1-alpha subunit (phospho S293), PPAR $\alpha=$ Peroxisome proliferator-activated receptor alpha, H3K27me3 = histone 3 lysine 27 tri-methylation, APAF-1 = Apoptotic protease activating factor 1 . 


\section{Section 1.2: Supporting Documentation for Specific Aim 1 \\ Maternal Engineered Nanomaterial Inhalation During Gestation Alters the Fetal Transcriptome}

As published in Part Fibre Toxicol. 2018 Jan 10;15(1):3. doi: 10.1186/s12989-017-0239-8.

*PA Stapleton ${ }^{1,2}$, QA Hathaway ${ }^{3,4,6}$, CE Nichols ${ }^{5}$, AB Abukabda ${ }^{6,7}$, MV Pinti ${ }^{3,4}$,

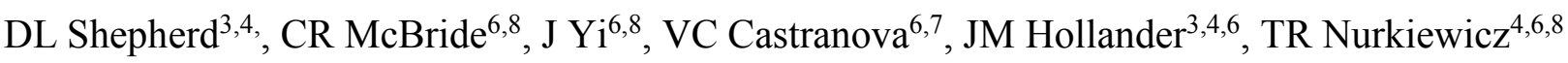

${ }^{*}$ Co-First Authors

${ }^{1}$ Department of Pharmacology and Toxicology, Ernest Mario School of Pharmacy, Rutgers University, Piscataway, NJ ; ${ }^{2}$ Environmental and Occupational Health Sciences Institute, Piscataway, NJ; ${ }^{3}$ Division of

Exercise Physiology, West Virginia University School of Medicine, Morgantown WV; ${ }^{4}$ Mitochondria, Metabolism \& Bioenergetics Working Group, West Virginia University School of Medicine, Morgantown, West Virginia; ${ }^{5}$ Immunity, Inflammation, and Disease Laboratory, National Institute of Environmental Health Sciences, Research Triangle Park, North Carolina; ${ }^{6}$ Toxicology Working Group, West Virginia University School of Medicine, Morgantown WV; ${ }^{7}$ Department of Pharmaceutical Sciences, West Virginia University School of Pharmacy; ${ }^{8}$ Department of Physiology, Pharmacology, and Neuroscience, West Virginia University School of Medicine, Morgantown WV

The authors report no conflict of interest.

Keywords: nanotechnology, toxicology, nanomaterial, inhalation, epigenetics, maternal, fetal 


\begin{abstract}
Background: The integration of engineered nanomaterials (ENM) is well-established and widespread in clinical, commercial, and domestic applications. Cardiovascular dysfunctions have been reported in adult populations after exposure to a variety of ENM. As the diversity of these exposures continues to increase, the fetal ramifications of maternal exposures have yet to be determined. We, and others, have explored the consequences of ENM inhalation during gestation and identified many cardiovascular and metabolic outcomes in the F1 generation. The purpose of these studies was to identify genetic alterations in the F1 generation of Sprague-Dawley rats that result from maternal ENM inhalation during gestation. Pregnant dams were exposed to nanotitanium dioxide (nano- $\mathrm{TiO}_{2}$ ) aerosols $\left(10 \pm 0.5 \mathrm{mg} / \mathrm{m}^{3}\right)$ for $7-8$ days (calculated, cumulative lung deposition $=217 \pm 1 \mu \mathrm{g}$ ) and on GD (gestational day) 20 fetal hearts were isolated. DNA was extracted and immunoprecipitated with modified chromatin marks histone 3 lysine 4 trimethylation (H3K4me3) and histone 3 lysine 27 tri-methylation (H3K27me3). Following chromatin immunoprecipitation (ChIP), DNA fragments were sequenced. RNA from fetal hearts was purified and prepared for RNA sequencing and transcriptomic analysis. Ingenuity Pathway Analysis (IPA) was then used to identify pathways most modified by gestational ENM exposure.

Results: The results of the sequencing experiments provide initial evidence that significant epigenetic and transcriptomic changes occur in the cardiac tissue of maternal nano- $\mathrm{TiO}_{2}$ exposed progeny. The most notable alterations in major biologic systems included immune adaptation and organismal growth. Changes in normal physiology were linked with other tissues, including liver and kidneys.
\end{abstract}

Conclusions: These results are the first evidence that maternal ENM inhalation impacts the fetal epigenome. 


\section{Introduction}

The Barker Hypothesis [1], Developmental Origins of Health and Disease (DOHaD) [2], and fetal programming [3], all explore the relationship between the health of the gestational environment and fetal development and how this predisposes to future disease or sensitivities. Maternal prenatal health challenges such as nutrient deficiency, undernourishment, gestational diabetes and hypertension have been linked to an elevated risk for postnatal cardiovascular diseases [4]. Recently, maternal environmental toxicant exposures have become of prominent interest in relation to the impact of exposure on the fetal milieu and subsequent progeny health [5]. We have reported that maternal ENM inhalation impairs the ability of uterine arterioles to properly dilate, and this impacts litter health in the form of pup weight, number and gender distribution; as well as impaired microvascular function [21]. While these studies have focused on the maternal development of a hostile gestational environment and subsequent reduction in fetal nutrients, fetal epigenetic modifications may also occur. Conceptually, this relationship is not novel, but applications of environmental toxicants to the maternal-fetal models are. For example, bisphenol A [6] and air pollution [7] have been shown to negatively impact fetal outcomes. However, the impact of maternal ENM on fetal health and/or epigenetic modification are poorly understood.

Despite the ubiquitous inclusion of engineered nanomaterials in widespread applications, and their projected proliferation in human endeavors, the consequences of maternal ENM inhalation on the developing fetus and their impacts on future health are at best, vague, yet they are increasingly becoming a health concern. The prevalence of ENM covers an immense spectrum: surface coatings and additives in common consumer products (electronics, food, cosmetics), additives in industrial processes (advanced building materials, synthetic fuels), and components of clinical applications (diagnostics, drug delivery, implantable devices). It is widely recognized that throughout the ENM life cycle, the greatest risk for human exposure and subsequent health consequences begins with ENM inhalation, and is typically followed by systemic injuries. We have reported that pulmonary and systemic microvascular inflammation [29, 32] follow ENM inhalation exposure. Consistent with this, other systemic morbidities known to follow pulmonary ENM exposures include: inflammation/apoptosis [8,9], macrovascular and microvascular dysfunction [10], atherogenesis [11], and organ level ischemia [12]. The developing fetus is equally a systemic target of numerous anthropogenic toxicants [13]. 
The impact of gestational ENM exposures on maternal and fetal health have been increasingly studied in the past decade. Adverse impact of ENM exposures on maternal health [14] and pregnancy $[15,16]$ have been reported in animal models. Teratogenic and embryo-lethal effects associated with ENM exposure have been shown [17]. The outcomes from several studies also highlight post-natal behavioral deficits [18, 19], cardiovascular [20, 21], renal [15], immune [22], reproductive [23, 24], pulmonary, and metabolic [20] [25] abnormalities.

Epigenetics, or the transient control of genes through DNA methylation or histone modification, is a recent area of intense focus by governmental agencies recognizing mechanistic links between environmental toxicants and gene expression [26]. These adverse maternal and fetal outcomes strongly reflect the potential risk of ENM exposure during pregnancy that may be linked. However, given the inherent physiological dependencies and complexities of developing and maintaining a healthy pregnancy, linking the mechanisms of pulmonary exposure and gestational effects remains very challenging. Given the magnitude of and the complexity these transgenerational effects, the most effective approach may be to initiate studies from the fetal epigenome and/or transcriptome. This is largely because fetal epigenetic outcomes resulting from maternal ENM exposure consequences may be caused by the creation of a hostile gestational environment [27], and/or the direct impact of ENM interacting with the developing embryo [13]. Because either of these possibilities would compromise health, the purpose of these studies was to identify epigenetic changes in cardiac gene expression within the maternally exposed F1 generations. We hypothesized that because maternal ENM inhalation lead to uterine microvascular dysfunction [21], this contributes to a hostile gestational environment, and altered fetal gene expression results. To test this, pregnant dams were intermittently exposed to nano- $\mathrm{TiO}_{2}$ aerosols during gestational days 5-19, and their litters were studied on GD 20. 


\section{Materials and Methods}

\section{Animal Model}

Sprague Dawley rats were purchased from Hilltop Laboratories (250-275 g female; 300$325 \mathrm{~g}$ male). All experiments were approved by the West Virginia University Animal Care and Use Committee and experiments adhered to the National Institutes of Health $(\mathrm{NIH})$ Guide for the Care and Use of Laboratory Animals (8th Ed.). Rats were provided food and water ad libitum and housed in an AAALAC approved animal facility at the West Virginia University Health Sciences Center. Before mating, rats were acclimated for a minimum of 72 hours, as previously described [20]. Pregnancy was verified by identification of the vaginal plug, after which, rats were randomly placed into one of two nano- $\mathrm{TiO}_{2}$ exposure groups. These two exposure groups were virtually identical and were created to generate a discrete tissue bank for RNA sequencing, or ChIP sequencing.

\section{Engineered Nanomaterial}

Nano- $\mathrm{TiO}_{2}$ P25 powder was purchased from Evonik (Aeroxide TiO2, Parsippany, NJ), containing anatase $(80 \%)$ and rutile $(20 \%) \mathrm{TiO}_{2}$. Nano- $\mathrm{TiO}_{2}$ was prepared by drying, sieving, and storing, as previously described $[28,29]$. Nano- $\mathrm{TiO}_{2}$ aerosols were created with our aerosol generator (US Patent No. 8,881,997) [30]. Particle characteristics have been determined including the primary particle size $(21 \mathrm{~nm})$, the specific surface area $\left(48.08 \mathrm{~m}^{2} / \mathrm{g}\right)[29,31]$, and the Zeta potential $(-56.6 \mathrm{mV})[32]$.

\section{Nano-TiO2 Inhalation Exposures}

The nano-particle aerosol generator (US Patent No. 8,881,997) and whole-body inhalation exposure system used for the current study have been described extensively in previous studies $[29,31]$. This collective exposure system consists of a vibrating fluidized bed, a Venturi vacuum pump, cyclone separator, impactor and mixing device, an animal housing chamber, and real-time monitoring devices with feedback control. Nano- $\mathrm{TiO}_{2}$ was aerosolized via a high velocity air 
stream passing through the vibrating fluidized bed and into the Venturi vacuum pump. The generated aerosols then entered the cyclone separated, which is designed to remove agglomerates $>400 \mathrm{~nm}$ at an input flow rate of $60 \mathrm{1} / \mathrm{min}$ of clean dry air before entering the exposure chamber.

Size distribution, mean aerodynamic diameter, and relative mass concentration of the aerosols were monitored in real time (Electrical Low Pressure Impactor (ELPI), Dekati, Tempere, Finland) while the particle size distribution was also measured in real-time with a Scanning Mobility Particle Sizer device (SMPS; TSI Inc., St. Paul, MN). These measurements were verified throughout a given exposure by collecting nanoparticle samples on filters, and making hourly gravimetric measurements with a microbalance. This approach was also used to collect samples for transmission electron microscopy.

Inhalation exposures were initiated on GD $5.78 \pm 0.11$ and lasted for $7.79 \pm 0.26$ days of gestation. Exposure days were not consecutive to decrease animal stress. Once the steady state nano- $\mathrm{TiO}_{2}$ aerosol concentration was achieved, exposure duration was adjusted to produce a daily calculated lung deposition of $31 \pm 1.1 \mu \mathrm{g}$ per day, and the cumulative, calculated dose was therefore $217 \pm 1.0 \mu \mathrm{g}$. Lung deposition was calculated based on previously described mouse methodology, and normalized to rat weight and to pregnant rat minute ventilation using the equation: $\mathrm{D}=\mathrm{F} \cdot \mathrm{V} \cdot \mathrm{C} \cdot \mathrm{T}$, where $\mathrm{F}$ is the deposition fraction $(14 \%), \mathrm{V}$ is the minute ventilation based on body weight, $\mathrm{C}$ equals the mass concentration $\left(\mathrm{mg} / \mathrm{m}^{3}\right)$, and $\mathrm{T}$ equals the exposure duration (minutes) [29, 33]. The target concentration was $10 \mathrm{mg} / \mathrm{m}^{3}$ and the duration was 4-6 hours/exposure (depending on the steady state concentration, as this was used to calculate the lung burden). The last exposure was conducted 24 hours prior to sacrifice and experimentation. Control animals were exposed to HEPA filtered air only.

\section{Chromatin Immunoprecipitation (ChIP) Sequencing}

\section{Isolation:}

Cardiac tissue was isolated from GD 20 pups in both the nano- $\mathrm{TiO}_{2}$ exposure and control groups. Each litter was considered an $n=1$, with cardiac tissue from 5-6 pups within each litter being pooled together to collect enough tissue ( $25 \mathrm{mg})$. Chromatin Immunoprecipitation (ChIP) was carried out using the MAGnify ${ }^{\text {TM }}$ Chromatin Immunoprecipitation System (Thermo Fisher, Rockford, IL) per manufacturer's instructions. Briefly, hearts were homogenized and treated with 
$37 \%$ formaldehyde, which was prepared fresh. Cross-linking was stopped with $1.25 \mathrm{M}$ glycine. Samples were pelleted through centrifugation and washed in D-PBS before sonication. Using a Sonicator Ultrasonic Processor XL2015 (Misonix Sonicator, Farmingdale, NY) chromatin was sheared to a size of 500-700 base pairs, determined using gel electrophoresis (Figure 6.2.1A). Chromatin was then isolated through ultracentrifugation $(20,000 \mathrm{~g})$ and diluted to $\sim 60 \mathrm{uL}$ of chromatin per immunoprecipitation reaction. Samples from both the control and nano- $\mathrm{TiO}_{2}$ cohorts were incubated with histone 3 lysine 4 tri-methylation (H3K4me3, product number: G.532.8, Thermo Fisher, Rockford, IL) or histone 3 lysine 27 tri-methylation (H3K27me3, product number: G.299.10, Thermo Fisher, Rockford, IL) antibody bound beads. These are two of the most prominently studied and classically applied for activation/repression analysis of gene activity. After incubation, samples were treated to reverse cross-linking solution and Proteinase $\mathrm{K}$ to remove bound proteins. DNA was then eluted from beads, using heat, and quantified using a Qubit (Thermo Fisher, Rockford, IL). The TruSeq ChIP Library Preparation Kit (Illumina, Inc., San Diego, CA) was implemented to build the libraries.

\section{ChIP Bioinformatics:}

Samples were processed using the Illumina MiSeq (Illumina, Inc., San Diego, CA) at the West Virginia University Genomics Core, ran as paired-end reads. Fastq files were assessed for quality using FastQC (Babraham Bioinformatics) (Figure 6.2.1B), where it was determined that partial trimming was needed. Trimming of fastq files was accomplished through Trimmomatic [34] (Figure 6.2.1C). Reads were then mapped to the rat genome (rn6) using the default parameters in bowtie2. To perform differential binding analysis on reads while distinguishing peaks, diffReps was used [35]. Bedtools functions were used to delineate upstream promoter regions of genes (bedtools slop) and evaluate the promoter/gene overlay (bedtools intersect). Genes were defined to include 1000 bases upstream from the start of the gene, indicative of our selected "promoter region."

\section{RNA Sequencing}

Isolation:

Cardiac tissue was procured through the same methods as listed above in the ChIP Sequencing section. RNA was then isolated from heart tissue using the Vantage ${ }^{\mathrm{TM}}$ Total RNA Purification Kit (Origene, Rockville, MD) per manufacturer's instructions. Briefly, tissue was 
homogenized and lysis buffer was added to the sample. Sample RNA was spin-column purified and measured for RNA concentration using the Qubit (Thermo Fisher, Rockford, IL). Library preparation was performed using TruSeq RNA Library Prep Kit v2 (Illumina, Inc., San Diego, CA). Quality of RNA was determined using the Agilent 2100 BioAnalyzer (Agilent Technologies, Santa Clara, CA); degradation of cytosolic ribosomal RNAs (28S and 18S) are used as a measure of the total RNA Integrity Number (RIN) (Figure 6.2.2A-B).

RNA Bioinformatics:

Samples were processed using the Illumina HiSeq (illumina, Inc., San Diego, CA) at Marshall University. Samples were run as paired-end reads. Paired-end, fastq files were aligned with HISAT2 [36] to the rat genome (rn6) without trimming. Samtools 1.2 [37] was used for the conversion of SAM to BAM format. Counts data was prepared using Subread 1.5.2 [38], specifically featureCounts [39]. Differential expression analysis was accomplished using DESeq2 [40] in R.

\section{Ingenuity Pathway Analysis (IPA)}

Protein ontology and pathway analysis were completed through QIAGEN's IPA (www.qiagen.com/ingenuity) software. Core analyses and comparative analyses were run on individual and combined ChIP and RNA data sets, respectively. Z-scores are representative of fold change between groups.

\section{RNA IPA Protein Ontology}

The intensity of the color, moving toward blue or red, indicates the degree to which a specific pathway is being decreased or increased, respectively. The change in color, reflective of the $\mathrm{z}$-score, is a quantitative measure of confidence (defined as the cumulative $P$-value of molecules in a specific pathway). This measure of confidence, defined on a color scale, indicates the propensity of all the molecules within that pathway to move in a certain direction, toward either increasing or decreasing the likelihood of developing the listed pathology or condition.

\section{Quantitative PCR}

As described above, RNA was isolated from fetal heart tissue. Using the First-strand cDNA Synthesis kit for miRNA (Origene, Rockville, MD, Catalog \#: HP100042), per manufacturer's 
instructions, RNA was converted to cDNA. The cDNA was used for differential quantification of mRNA transcripts Fibroblast Growth Factor Receptor 1 (Fgfr1), Interleukin-18 (Il-18), and Transforming Growth Factor Beta Receptor 2 (Tgfbr2). ChIP-qPCR was used to assess the Tgfbr2 promoter loci. As described above, chromatin was immunoprecipitated with H3K4me3. DNA was then probed at multiple locations along the Tgfbr2 promoter region in order to construct a histone peak profile. Primer design for both the mRNA and ChIP-qPCR are provided (Table S6.2.4). MRNA was normalized to Beta-Actin ( $\beta$-Actin), while immunoprecipitated DNA was normalized to its respective input control. Experiments were performed on the Applied Biosystems 7900HT Fast Real-Time PCR system (Applied Biosystems, Foster City, CA), using 2X SYBR Green Master Mix. Quantification was achieved using the 2- $\Delta \Delta \mathrm{CT}$ method.

\section{Statistics}

All measures of significance between the control and maternal nano- $\mathrm{TiO}_{2}$ exposure groups for the sequencing data are presented as adjusted $P$-values. Adjusted $P$-values are a composition of standard, unadjusted $P$-values and the stringency of the False Discovery Rate (FDR). Differential expression analysis through DESeq2 implements the Wald Test, using multiple testing against the null hypothesis that $P$-values are uniformly distributed across a data set, known as the Benjamini-Hochberg procedure. The FDR for this study was set at 0.05 . Z-score significance is

determined as greater than the absolute value of 2 . The z-score is computed as $z=\frac{x}{\sigma_{x}}=\frac{\sum_{i} x_{i}}{\sqrt{n}}=$ $\frac{N_{+}-N_{-}}{\sqrt{N}}$, where $\mathrm{N}_{+}=$the number of molecules following a consistent trend, $\mathrm{N}_{-}=$the number of molecules following an inconsistent trend, and $\mathrm{N}=$ the number of interactions within a given pathway. In this way, the z-score, using only values with a significant change $(P \leq 0.05)$ can infer direction of a specific pathway while accounting for relationship and data bias and properly weighting the statistical findings (https://www.qiagenbioinformatics.com/products/ingenuitypathway-analysis/). A consistency score is the non-statistical assignment of confidence to a specific pathway. Where appropriate, a Student's t-test was used with all data presented as \pm the standard error mean (SEM). Significance is determined as $P \leq 0.05$. 


\section{Results}

\section{Animal and Nano-TiO2 Aerosol Characteristics}

Animal number, age, body weight, and exposure conditions are provided (Table 6.2.1). Separate, but similar, inhalation exposures were used for the ChIP and RNA sequencing experiments. No statistical differences were noted between nano- $\mathrm{TiO}_{2}$ exposure in Experimental Group 1 (ChiP Seq) and Experimental Group 2 (RNA Seq). No statistical differences were noted in either progeny weight or total number of pups between maternal nano- $\mathrm{TiO}_{2}$ exposed or control groups. Representative nano- $\mathrm{TiO}_{2}$ aerosol characterization data are presented in Figure 6.2.3. The target particle concentration was $10 \mathrm{mg} / \mathrm{m}^{3}$ (Figure 6.2.3A). The real-time nano- $\mathrm{TiO}_{2}$ mobility diameter was $129 \mathrm{~nm}$ (Figure 6.2.3B), and the aerodynamic diameter was $143 \mathrm{~nm}$ (Figure 6.2.3C). Nanoparticles were collected on filters, and a representative transmission electron microscopy image is presented in Figure 6.2.3D.

\section{ChIP Sequencing}

\section{ChIP Sample Metrics:}

To better understand the quality and sample dispersion within our cohort for the ChIP sequencing experiment, a series of statistical models were used. To assess the distribution of subpeaks present within the forward and reverse strands of the H3K4me3 and H3K27me3 immunoprecipitations, the average fragment length was determined for each event using the $\mathrm{R}$ package csaw [41]. The cross-correlation graph measures the delay distance, or number of base pairs, which separate distinctive subpeaks, also evaluating the consistency of fragment lengths within the data set (Figure 6.2.4A and 6.2.4B). Multi-dimensional scaling (MDS) plots were used to evaluate individual library homology between both the H3K4me3 and H3K27me3 groups with the R package edgeR [42]. Log fold change (LogFC) determined the differences between libraries (control, red and maternal nano- $\mathrm{TiO}_{2}$ exposed, blue) within the MDS plots (Figure 6.2.4C and 6.2.4D). To visualize read coverage, the R packages ChIPpeakAnno and Gviz were installed [43]. Complex, differential binding was assessed for both the H3K4me3 (Figure 6.2.4E) and H3K27me3 (Figure 6.2.4F) binding loci. Together, these results suggest that the immunoprecipitation and chromatin fragmentation were successful, and that differential binding is observed between groups.

\section{ChIP IPA Protein Ontology}


Differential Binding data for both the H3K4me3 and H3K27me3 marks were uploaded and analyzed in QIAGEN's IPA; all changes are shown as maternal nano- $\mathrm{TiO}_{2}$ exposed condition relative to the control. Diseases and biological functions (z-score $\geq 2$ ) for H3K4me3 and H3K27me3 are provided in Table S6.2.1 and S6.2.2, respectively. Of the diseases and biological functions listed, one of the most prominent pathways for H3K4me3 involved infectious disease (Figure 6.2.5A). The heat map reveals how changes in molecular signaling could provide an increase susceptibility to infection in maternal nano- $\mathrm{TiO}_{2}$ exposed offspring. The top canonical pathways ( $\mathrm{z}$-score $\geq 2$ ) altered during maternal nano- $\mathrm{TiO}_{2}$ exposure are presented (Figure 6.2.5B). In general, the canonical pathways altered after exposure involve regulation of growth and cell cycle/apoptosis signaling.

For $\mathrm{H} 3 \mathrm{~K} 27 \mathrm{me}$, the top 10 canonical pathways which are altered are provided (Figure 6.2.5C). For the promoter regions associated with $\mathrm{H} 3 \mathrm{~K} 27 \mathrm{me} 3$, the majority of signaling changes involve cancer and immunity. A heat map for the toxicological functions of the data representing $\mathrm{H} 3 \mathrm{~K} 4 \mathrm{me} 3$ is also presented (Figure 6.2.5D). The size and distribution of each major category is proportional to the z-score, which revealed three major organs affected: the heart, kidney and liver. Toxicological pathways associated with the heart, including congenital heart anomaly, heart failure, cardiac hypertrophy (not shown), and cardiac dysfunction (not shown), were found to be significantly decreased in the maternal nano- $\mathrm{TiO}_{2}$ exposed group. Conversely, toxicological pathways associated with the liver and kidney including, renal necrosis and cell death, liver necrosis and cell death, renal damage, and liver damage (not shown) were found to be increased. Also, an increase in red blood cells, and subsequently the hematocrit, were observed. Increases in $\mathrm{H} 3 \mathrm{~K} 4 \mathrm{me} 3$ at promoter regions for infection capacity and growth signaling as well as loci involving kidney and liver dysfunction, suggests epigenetic regulation which could significantly alter an organism's susceptibility to disease and potential pre-disposition to future insult. The lack of changes shown for H3K27me3 may suggest an alternative repressive mark implemented as the bivalent companion of $\mathrm{H} 3 \mathrm{~K} 4 \mathrm{me} 3$.

\section{RNA Sequencing}

RNA Sample Metrics:

The raw and normalized counts from the RNA sequencing experiment were subjected to a variety of statistical modelling, using the DESeq2 package in $\mathrm{R}$ [44], in order to better understand 
sample parameters. To visualize the variance of the normalized counts data means, the rlog function was used (Figure 6.2.6A). For low-count genes, transformation using rlog, a log2 scale which normalizes data in reference to the library size, helps to better visualize variance-means. Figure 6.2.6A shows limited outliers within the data set for the control vs. control, but increasing variance in the control vs. maternal nano- $\mathrm{TiO}_{2}$ exposed. Sample-to-sample distance was measured using the PoiClaClu package in R. Sample dissimilarity is depicted as a heat map (Figure 6.2.6B), calculated from the original, not normalized count data. The heat map shows general dissimilarity between the maternal nano- $\mathrm{TiO}_{2}$ exposed and control groups, with the exception of one of the control samples. Another measure implemented for determining sample distance was a multidimensional scaling (MDS) plot based on the rlog-normalized counts (Figure 6.2.6C). Again, the plot shows a general dissimilarity between the maternal nano- $\mathrm{TiO}_{2}$ exposed and control cohorts. After performing differential expression analysis with DESeq2, we examined the gene with the lowest associated $P$-value (Figure 6.2.6D). The plot illustrates the similar expression of the gene within each group, while showing the disparities across groups. In Figure 6.2.6E, a MA-plot is used to illustrate the number of genes (red) that fall below the $P$-value of 0.05 . The statistical models used to assess the RNA sequencing samples indicate that normalized count values between groups are similar and that sample homology is close within groups, but not across groups.

\section{RNA IPA Protein Ontology:}

After differential expression analysis processing in $\mathrm{R}$, data was uploaded and analyzed in QIAGEN's IPA; all changes are shown as maternal nano- $\mathrm{TiO}_{2}$ exposed condition relative to the control. Diseases and biological functions (z-score $\geq 2$ ) for the RNA are provided in Table S6.2.3. Again, a prominent pathway that was found to be increased in the maternal nano- $\mathrm{TiO}_{2}$ exposed animals involved infectious diseases (Figure 6.2.7A). Both the open promoter conformation (H3K4me3) and the RNA transcript expression reveal an increased propensity for infection. The top canonical pathways ( $\mathrm{z}$-score $\geq 3.45$ ) altered during maternal nano- $\mathrm{TiO}_{2}$ exposure are presented (Figure 6.2.7B). The canonical pathways altered primarily involve inflammatory signaling and organismal development. Examining what factors could be causing differential regulation after maternal nano- $\mathrm{TiO}_{2}$ exposure, we wanted to evaluate molecular regulator effects. The top molecule (consistency score $\geq 10.453$ ) suggested to play a role in differential regulation of pathways was microRNA-145 (Figure 6.2.7C). 
In Figure 6.2.7C, it reveals how decreased expression of microRNA-145 can lead to increased expression of pathways involving cell growth and proliferation. A heat map for the toxicological functions of the data representing the RNA is also shown (Figure 6.2.7D). The size and distribution of each major category is proportional to the z-score and, again consistent with the H3K4me3 mark, three major organs were shown to be affected: the heart, kidney and liver. Toxicological pathways associated with the heart, including congenital heart anomaly, cardiac hypoplasia, heart failure, cardiac fibrosis, and cardiac damage, were found to be significantly decreased in the maternal nano- $\mathrm{TiO}_{2}$ exposed group. Alternatively, toxicological pathways associated with the liver and kidney including, renal necrosis and cell death, liver hyperplasia/hyperproliferation, renal proliferation, renal damage, and renal autophagy were found to be increased. As reported for the H3K4me3 promoter regions, increased RNA transcription of genes involving red blood production are shown. Similar to the epigenetic modification H3K4me3, the differential expression of transcripts follows a similar pattern of increased infection and growth of the organism, with increased molecular markers of dysfunction in the liver and kidney.

\section{Epigenetic Regulation of Transcription}

In order to examine how changes between the H3K4me3 mark and RNA transcript data aligned, we performed a comparative analysis through QIAGEN's IPA, all changes are shown as maternal nano-TiO2 exposed condition relative to the control. The top canonical pathways (z-score 24.5) for both the transcript and ChIP data are shown (Figure 6.2.8A). The combined data sets illustrate the common pathways involving both inflammation and organismal growth signaling. For toxicological functions, the molecular profile for cardiac dysfunction is significantly decreased compared to the controls, while kidney dysfunction is increased (Figure 6.2.8B). A heat map for the cumulative diseases and biological functions is shown (Figure 6.2.8C). The heat map depicts two major molecular changes that could impact the phenotype: increased survival and increased susceptibility to infection. In Figure 6.2.8D, canonical pathways are sorted by $P$-value, depicting pathways with large sets of molecules having significantly altered expression levels. Although, the mitochondrial dysfunction and oxidative phosphorylation pathways do not have significant $z-$ scores and a very small contribution of changes coming from the transcript data, Figure 6.2.8B demonstrates the epigenetic changes occurring at these loci to a large segment of genes. Figure 6.2.8E illustrates the NF-кB (Nuclear Factor kappa-light-chain-enhancer of activated B cells) 
signaling pathway for the RNA (right) and H3K4me3 (left) sequencing experiments. The comparative analysis suggests that maternal nano- $\mathrm{TiO}_{2}$ exposure can cause significant changes to how the development of the progeny takes place, changing the epigenetic landscape, which can directly affect transcript abundance.

\section{Molecular Validation of Sequencing}

To further confirm the reliability of the sequencing data, we implemented qPCR to examine molecules involved in the NF-KB Pathway, which are not shown in the illustrative Figures 6.2.8DE. The mRNA levels of Fgfr1, Il-18, and Tgfbr2 are reported, and coincide with similar expression profiles seen in the sequencing data (Figure 6.2.9A). In Figure 6.2.9A, the data obtained from RNA sequencing (grey bars) are used as a reference to validate the expression profile of the maternal nano-TiO2 group when running qPCR. Likewise, we also wanted to use ChIP-qPCR to validate that histone modifications were also reliably reported, with the ChIP-Seq revealing epigenetic changes at the Tgfbr2 promoter region. We confirmed the H3K4me3 histone modifications for Tgfbr2, showing higher H3K4me3 association at its promoter region (Figure 6.2.9B). The increased magnitude of the histone peak of the maternal nano-TiO2 group, Figure 6.2.9B, suggests the increased abundance of $\mathrm{H} 3 \mathrm{~K} 4 \mathrm{me} 3$ and active transcription of the Tgfbr2 gene. Tgfbr2 provides an explicit example of how genes reported to be epigenetically altered (ChIP-Seq, through H3K4me3 localization at the Tgfbr2 promoter region) with subsequent changes in transcription (RNA-Seq, reporting increased expression of Tgfbr2 transcripts) can be further validated using other molecular techniques, such as qPCR. An overview of the experimental design is illustrated in Figure 6.2.9C. Briefly, the figure provides an example of suggested functional outcomes related to maternal nano-TiO2 exposure, with the link between the exposure paradigm and end function being fetal, epigenetic consequences. 


\section{Discussion}

The gene expression and epigenetic analyses performed in this study provide the first evidence that maternal ENM inhalation may result in significant pathway alterations in the fetus. The two most prominently impacted mechanisms are: inflammatory signaling, and cardiac-renalhepatic pathology/toxicity. The nano- $\mathrm{TiO}_{2}$ exposure paradigm used herein $\left(10 \mathrm{mg} / \mathrm{m}^{3}, 4-6\right.$ hours $)$ resulted in a calculated lung deposition of approximately $217 \mu \mathrm{g}$. This lung burden, achieved over 7 days of exposure in the second half of gestation, has been previously shown to impair uterine arteriolar reactivity by almost 50\% [25]. To estimate how this lung burden compares to what a human may experience, alveolar surface areas must be known [32]. The rat alveolar surface area is $0.4 \mathrm{~m}^{2} / \mathrm{lung}$. Therefore, the rat burden of $217 \mu \mathrm{g} / \mathrm{lung}$ would result in $542.5 \mu \mathrm{g} / \mathrm{m}^{2}$. Given that the human alveolar surface area is $102 \mathrm{~m}^{2}$, the equivalent human burden of this exposure paradigm would be $55.3 \mathrm{mg}$. The next logical question is how long would it take to achieve this burden in humans. In this regard, lung burden may be calculated as:

nano-TiO $\mathrm{O}_{2}$ aerosol concentration · minute ventilation · exposure duration · deposition fraction, with the following values:

$55.3 \mathrm{mg}=$ nano- $\mathrm{TiO}_{2}$ aerosol concentration $\cdot 7600 \mathrm{ml} / \mathrm{min} \cdot(8 \mathrm{hr} /$ day $\cdot 60 \mathrm{~min} / \mathrm{hr}) \cdot 14 \%$, and therefore:

$$
55.3 \mathrm{mg}=\text { nano- } \mathrm{TiO}_{2} \text { aerosol concentration } \cdot 0.51 \mathrm{~m}^{3} / \text { day. }
$$

The National Institute for Occupational Safety and Health (NIOSH) Recommended Exposure Limit (REL), or aerosol concentration for nano- $\mathrm{TiO}_{2}$ is $0.3 \mathrm{mg} / \mathrm{m}^{3}$ (DHHS, 2011). This would result in a lung burden of $0.15 \mathrm{mg} /$ day. Whereas, the Occupational Safety and Health Administration (OSHA) Permissible Exposure Limit is $5 \mathrm{mg} / \mathrm{m}^{3}$ (DHHS 2011). This would result in a lung burden of $2.55 \mathrm{mg} / \mathrm{day}$. Considering the NIOSH REL and OSHA PEL together, it would require 1.45 working years or 21.7 working days (respectively) for a human to achieve comparable lung burdens with the exposure paradigm used herein. Because the human gestational period is 9 months, we consider our exposure paradigm highly relevant to the worker population.

Contrary to the functional deficits seen in the young adult $[20,25]$ we found that both the transcriptomic and epigenetic data support increased cardiac function (Figures 6.2.5D and 6.2.7D). Though this seems paradoxical, we suggest that the interplay between the heart, liver, and kidneys is vital in understanding the pathology associated with maternal nano- $\mathrm{TiO}_{2}$ exposure. It is equally plausible that as hematocrit increases, viscosity of the blood also increases, requiring an elevation 
in contractile force or a drop in peripheral resistance. Alternatively, it is possible that disruptions in maternal-fetal perfusion balances occur. The pulmonary exposure of the mother is well described, but the secondary effect(s) on the developing progeny is/are likely to come through impacts on the maternal/fetal circulation. Maternal nutrients are delivered to the placenta via the arterial circuit, if blood flow is inadequate, then fetal compensation must occur to support proper nutrient delivery via the umbilical vein to the fetal portal circulation.

At the fetal stage, the heart plays a less significant role in energetics [46]. Whereas, the liver and kidneys play pivotal roles in blood conditioning at this stage of development, and these signaling pathways are altered by maternal ENM inhalation during gestation (Figure 6.2.5). We hypothesize that potential liver and kidney damage from either inflammation, direct ENM translocation or a combination may result in an increased hematocrit, and or maternal-fetal perfusion balance. Together, this may suggest that in maternal nano- $\mathrm{TiO}_{2}$ exposed progeny, the functional deficits seen later in development may be a result of this initial hepatic and renal insult, with subsequent cardiac overcompensation which may represent a protective mechanism. These findings correspond to reports of hepatic DNA damage in newborn murine offspring after maternal nano- $\mathrm{TiO}_{2}$ inhalation [47]. Impairments in renal function may have profound effects on tubuloglomerular feedback, the renin angiotensin system, and/or osmotic regulation. These impairments may collectively or individually directly influence cardiovascular health throughout prenatal and postnatal development.

MicroRNA (miRNA) are well known to be altered by transcriptomic and epigenetic regulators. When expressed, miRNA broadly regulate cellular function [48] and have been implicated in numerous epigenetic pathways [49]. In Figure 6.2.7C transcriptomic data is provided that reflects the most consistently altered regulator after maternal nano- $-\mathrm{TiO}_{2}$ inhalation. Decreased expression of miRNA-145 has been suggested to increase protein synthesis of targets directly involved in signaling events that promote organism growth and development. The role of altered miRNAs in progeny after maternal ENM inhalation is poorly understood, and may provide a better understanding of the relationship between ENM toxicities, epigenetics, and gene expression.

Figure 6.2.8C presents an overview of the two primary cell signaling pathways that are altered during gestational exposure: immunity and development. Parameters of organismal health and development are presented largely as molecular markers for cardiac signaling and function. The increased gene expression of molecular markers associated with infection and immunity may 
indicate the likelihood of autoimmune disorders associated with an overactive immune system. This is most evident when considering the inflammatory pathways indicated in Figure 6.2.8A and the target organ (kidney) indicated in Figure 6.2.8B reflected by an increased susceptibility as shown in Figure 6.2.8C. These molecular markers may also represent the consequence presented in Figure 6.2.8A of a proinflammatory environment; such an environment has been associated with chronic conditions including cardiovascular disease and cancer [50]. Pulmonary exposure to carbon black nanoparticles has also been identified to contribute to the development of immunotoxicity, particularly in lymphoid organs [22]. Interestingly, organismal death and morbidity/mortality appears to be decreased in maternal nano- $\mathrm{TiO}_{2}$ offspring, which may again seem counterintuitive. However, we speculate this may reflect a greater systemic response to compensate for the numerous other mechanisms disturbed by ENM inhalation during gestation.

To better identify the future consequences of ENM exposure, the significance of the pathways was represented as the change in $P$-value (Figure 6.2.8D). Mitochondrial dysfunction and oxidative phosphorylation appeared to have the greatest changes in methylation, indicating that future complications in these pathways may occur. Given their widespread involvement, this epigenetic predisposition may manifest in any tissue. In other words, the epigenetic changes associated with energetics may reflect significant alterations that occur during fetal development. It is important to indicate that these changes may not be manifested in functional transcriptomic or proteomic changes until postnatal development or even later into adulthood. If correct, this would be consistent with the Barker Hypothesis and DOHaD.

Maternal nano- $\mathrm{TiO}_{2}$ exposure is also associated with a pronounced effect on key inflammatory pathways in the exposed progeny. In Figure 6.2.8E, protein kinase B (AKT) signaling is decreased, potentially resulting in an impairment in calcium-independent nitric oxide signaling which would likely result in dysfunctional endothelium-dependent responses. Indeed, calcium dependent and independent mechanisms, as well as endothelial arteriolar dilation are significantly impaired at 3-4 weeks of age [51]. Furthermore, augmented NF- $\kappa B$ signaling via both alternate and canonical pathways [52] has been reported. Maternal nano- $\mathrm{TiO}_{2}$ exposure significantly activated the expression of the Lymphotoxin Beta Receptor (LTBR) gene, while suppressing the expression of the regulating enzyme Inhibitor of NF- $\kappa B$ Kinase Subunit Alpha $(\mathrm{IKK} \alpha)$ Figure 6.2.8E. This is important in the negative feedback of the NF- $\kappa B$ canonical signaling that limits inflammatory gene activation and suggests that more robust inflammatory responses are 
possible as evidenced in Figure 6.2.8A. Furthermore, NF- $\mathrm{BB}$ plays a central role in the development of inflammation through further regulation of genes encoding not only proinflammatory cytokines, but also adhesion molecules such as E-selectin, VCAM-1 (vascular cell adhesion molecule-1) and ICAM-1 (intercellular adhesion molecule-1), chemokines, and inducible nitric oxide synthase (iNOS) [53, 54]. Figure 6.2.8E also reflects a significant increase in interleukin-8 (IL-8) signaling, a major chemokine associated with neutrophil chemotaxis and degranulation secreted by macrophages and endothelial cells during acute inflammatory responses [55]. Considered jointly, uncontrolled activation of NF- $\kappa B$ and IL-8 pathways in maternally exposed progeny may predispose towards endothelial-dependent dysfunction and leukocyte adhesion.

\section{Conclusions}

The pathway analyses reported herein indicate dysfunction in many physiologic systems. As it is not possible to functionally verify each of these functional implications, the primary goal of the manuscript is to identify those systems as a priority for future study. Systemic impairments associated with acute and chronic nanomaterial exposures is an evolving field as nanotechnology continues to expand. Maternal and fetal outcomes following gestational exposures have recently been considered. While initial functional microvascular assessments have begun, little is known regarding epigenetic alterations within the F1 generation. The findings from this study describe epigenetic changes in the progeny of mothers exposed to nano- $\mathrm{TiO}_{2}$ aerosols during gestation. The evidence of the study is strengthened by the use of two separate cohorts to separately probe the transcriptomic and epigenetic alterations, suggesting that even in separate discrete experimental populations, changes to the epigenome and RNA transcript levels align and similar exposure paradigms yield consistent results. Changes in the RNA transcripts and histone modifications on DNA suggest that maternal nano- $\mathrm{TiO}_{2}$ progeny exhibit a propensity toward hepatic and renal disease, increased inflammatory signaling, and growth/survival while showing decreased cardiac dysfunction. What remains to be understood is if and/or how far these epigenetic changes persistent into adulthood, the dose-response relationships, and what stage of development is most sensitive to maternal ENM exposure. 


\section{Declarations}

Ethics approval and consent to participate: not applicable.

Consent for publication: the authors consent to publication.

Availability of data and material: The datasets supporting the conclusions of this article are included within the article and its Supplemental file.

Competing interests: The authors do not have any competing interests to disclose.

Funding: This work was supported by: NIH-R00-ES024783 (PAS), R01-ES015022 (TRN), R01 HL-128485 (JMH), AHA-13PRE16850066 (CEN), NSF-1003907 (TRN), DGE-1144676 (QAH, ABA, TRN), and AHA-17PRE33660333 (QAH).

Authors' contributions: PAS-study design, pregnancy model, inhalation exposures, tissue harvesting, manuscript preparation. QAH-transcriptomic analysis, manuscript preparation. CENtranscriptomic analysis, manuscript preparation. ABA- tissue harvesting, manuscript preparation. MVP-transcriptome preparation. DLS-transcriptome preparation. CRM-pregnancy model, inhalation exposures, tissue harvesting. JY-inhalation exposures. JMH-transcriptome analysis design, manuscript preparation. VCC- lung burden calculations, rodent to human comparisons, manuscript preparation. TRN- study design, pregnancy model, inhalation exposures, tissue harvesting, manuscript preparation.

Acknowledgements: We thank Kevin Engels of the West Virginia University Department of Physiology, Pharmacology and Neuroscience; and Sherri A. Friend of NIOSH for their expert technical assistance. 


\section{References}

1. de Boo, H.A. and J.E. Harding, The developmental origins of adult disease (Barker) hypothesis. Aust N Z J Obstet Gynaecol, 2006. 46(1): p. 4-14.

2. Heindel, J.J. and L.N. Vandenberg, Developmental origins of health and disease: $a$ paradigm for understanding disease cause and prevention. Curr Opin Pediatr, 2015. 27(2): p. 248-53.

3. Lane, R.H., Fetal programming, epigenetics, and adult onset disease. Clin Perinatol, 2014. 41(4): p. 815-31.

4. Alexander, B.T., J.H. Dasinger, and S. Intapad, Fetal programming and cardiovascular pathology. Compr Physiol, 2015. 5(2): p. 997-1025.

5. Grason, H.A. and D.P. Misra, Reducing Exposure to Environmental Toxicants Before Birth: Moving from Risk Perception to Risk Reduction. Public Health Reports, 2009. 124(5): p. 629-641.

6. He, M., et al., Exposure to bisphenol A enhanced lung eosinophilia in adult male mice. Allergy Asthma Clin Immunol, 2016. 12: p. 16.

7. Dadvand, P., et al., Maternal exposure to particulate air pollution and term birth weight: a multi-country evaluation of effect and heterogeneity. Environ Health Perspect, 2013. 121(3): p. 267-373.

8. Perez-Hernandez, M., et al., Multiparametric analysis of anti-proliferative and apoptotic effects of gold nanoprisms on mouse and human primary and transformed cells, biodistribution and toxicity in vivo. Part Fibre Toxicol, 2017. 14(1): p. 41.

9. Adamcakova-Dodd, A., et al., Effects of prenatal inhalation exposure to copper nanoparticles on murine dams and offspring. Part Fibre Toxicol, 2015. 12: p. 30.

10. Abukabda, A.B., P.A. Stapleton, and T.R. Nurkiewicz, Metal Nanomaterial Toxicity Variations Within the Vascular System. Curr Environ Health Rep, 2016. 3(4): p. 379-391.

11. Yan, Z., et al., Zinc oxide nanoparticle-induced atherosclerotic alterations in vitro and in vivo. Int J Nanomedicine, 2017. 12: p. 4433-4442.

12. Holland, N.A., et al., Impact of pulmonary exposure to gold core silver nanoparticles of different size and capping agents on cardiovascular injury. Part Fibre Toxicol, 2016. 13(1): p. 48.

13. Hougaard, K.S., et al., A perspective on the developmental toxicity of inhaled nanoparticles. Reprod Toxicol, 2015. 56: p. 118-40.

14. Jackson, P., et al., Exposure of pregnant mice to carbon black by intratracheal instillation: toxicogenomic effects in dams and offspring. Mutat Res, 2012. 745(1-2): p. 7383.

15. Blum, J.L., et al., Effects of Maternal Exposure to Cadmium Oxide Nanoparticles During Pregnancy on Maternal and Offspring Kidney Injury Markers Using a Murine Model. J Toxicol Environ Health A, 2015. 78(12): p. 711-24.

16. Campagnolo, L., et al., Silver nanoparticles inhaled during pregnancy reach and affect the placenta and the foetus. Nanotoxicology, 2017. 11(5): p. 687-698.

17. Ema, M., et al., Reproductive and developmental toxicity of carbon-based nanomaterials: A literature review. Nanotoxicology, 2016. 10(4): p. 391-412.

18. Hougaard, K.S., et al., Effects of prenatal exposure to surface-coated nanosized titanium dioxide (UV-Titan). A study in mice. Part Fibre Toxicol, 2010. 7: p. 16. 
19. Engler-Chiurazzi, E.B., et al., Impacts of prenatal nanomaterial exposure on male adult Sprague-Dawley rat behavior and cognition. J Toxicol Environ Health A, 2016. 79(11): p. 447-52.

20. Hathaway, Q.A., et al., Maternal-engineered nanomaterial exposure disrupts progeny cardiac function and bioenergetics. Am J Physiol Heart Circ Physiol, 2017. 312(3): p. H446-H458.

21. Stapleton, P.A., et al., Maternal engineered nanomaterial exposure and fetal microvascular function: does the Barker hypothesis apply? Am J Obstet Gynecol, 2013. 209(3): p. 227 e1-11.

22. El-Sayed, Y.S., et al., Carbon black nanoparticle exposure during middle and late fetal development induces immune activation in male offspring mice. Toxicology, 2015. 327: p. 53-61.

23. Kyjovska, Z.O., et al., Daily sperm production: application in studies of prenatal exposure to nanoparticles in mice. Reprod Toxicol, 2013. 36: p. 88-97.

24. Hougaard, K.S., et al., Effects of lung exposure to carbon nanotubes on female fertility and pregnancy. A study in mice. Reprod Toxicol, 2013. 41: p. 86-97.

25. Stapleton, P.A., et al., Microvascular and mitochondrial dysfunction in the female F1 generation after gestational TiO2 nanoparticle exposure. Nanotoxicology, 2015. 9(8): p. 941-51.

26. Hu, X., et al., Toxicity Evaluation of Exposure to an Atmospheric Mixture of Polychlorinated Biphenyls by Nose-Only and Whole-Body Inhalation Regimens. Environ Sci Technol, 2015. 49(19): p. 11875-83.

27. Stapleton, P.A., Gestational nanomaterial exposures: microvascular implications during pregnancy, fetal development and adulthood. J Physiol, 2016. 594(8): p. 2161-73.

28. Knuckles, T.L., et al., Nanoparticle inhalation alters systemic arteriolar vasoreactivity through sympathetic and cyclooxygenase-mediated pathways. Nanotoxicology, 2012. 6(7): p. 724-35.

29. Nurkiewicz, T.R., et al., Nanoparticle inhalation augments particle-dependent systemic microvascular dysfunction. Part Fibre Toxicol, 2008. 5: p. 1.

30. Yi, J., et al., Whole-body nanoparticle aerosol inhalation exposures. J Vis Exp, 2013(75): p. e50263.

31. Sager, T.M., C. Kommineni, and V. Castranova, Pulmonary response to intratracheal instillation of ultrafine versus fine titanium dioxide: role of particle surface area. Part Fibre Toxicol, 2008. 5: p. 17.

32. Nurkiewicz, T.R., et al., Pulmonary particulate matter and systemic microvascular dysfunction. Res Rep Health Eff Inst, 2011(164): p. 3-48.

33. Stapleton, P.A., et al., Impairment of coronary arteriolar endothelium-dependent dilation after multi-walled carbon nanotube inhalation: a time-course study. Int J Mol Sci, 2012. 13(11): p. 13781-803.

34. Bolger, A.M., M. Lohse, and B. Usadel, Trimmomatic: a flexible trimmer for Illumina sequence data. Bioinformatics, 2014. 30(15): p. 2114-20.

35. Shen, L., et al., diffReps: detecting differential chromatin modification sites from ChIPseq data with biological replicates. PLoS One, 2013. 8(6): p. e65598.

36. Kim, D., B. Langmead, and S.L. Salzberg, HISAT: a fast spliced aligner with low memory requirements. Nat Methods, 2015. 12(4): p. 357-60. 
37. Li, H., et al., The Sequence Alignment/Map format and SAMtools. Bioinformatics, 2009. 25(16): p. 2078-9.

38. Liao, Y., G.K. Smyth, and W. Shi, The Subread aligner: fast, accurate and scalable read mapping by seed-and-vote. Nucleic Acids Res, 2013. 41(10): p. e108.

39. Liao, Y., G.K. Smyth, and W. Shi, featureCounts: an efficient general purpose program for assigning sequence reads to genomic features. Bioinformatics, 2014. 30(7): p. 923-30.

40. Lun, A.T., Y. Chen, and G.K. Smyth, It's DE-licious: A Recipe for Differential Expression Analyses of RNA-seq Experiments Using Quasi-Likelihood Methods in edgeR. Methods Mol Biol, 2016. 1418: p. 391-416.

41. Lun, A.T. and G.K. Smyth, csaw: a Bioconductor package for differential binding analysis of ChIP-seq data using sliding windows. Nucleic Acids Res, 2016. 44(5): p. e45.

42. Robinson, M.D., D.J. McCarthy, and G.K. Smyth, edgeR: a Bioconductor package for differential expression analysis of digital gene expression data. Bioinformatics, 2010. 26(1): p. 139-40.

43. Hahne, F. and R. Ivanek, Visualizing Genomic Data Using Gviz and Bioconductor. Methods Mol Biol, 2016. 1418: p. 335-51.

44. Love, M.I., W. Huber, and S. Anders, Moderated estimation of fold change and dispersion for RNA-seq data with DESeq2. Genome Biol, 2014. 15(12): p. 550.

45. Nichols, C.E., et al., Cardiac and mitochondrial dysfunction following acute pulmonary exposure to mountaintop removal mining particulate matter. Am J Physiol Heart Circ Physiol, 2015. 309(12): p. H2017-30.

46. Taegtmeyer, H., S. Sen, and D. Vela, Return to the fetal gene program: a suggested metabolic link to gene expression in the heart. Ann N Y Acad Sci, 2010. 1188: p. 191-8.

47. Jackson, P., et al., Pulmonary exposure to carbon black by inhalation or instillation in pregnant mice: effects on liver DNA strand breaks in dams and offspring. Nanotoxicology, 2012. 6(5): p. 486-500.

48. Shukla, G.C., J. Singh, and S. Barik, MicroRNAs: Processing, Maturation, Target Recognition and Regulatory Functions. Mol Cell Pharmacol, 2011. 3(3): p. 83-92.

49. Bartel, D.P., MicroRNAs: target recognition and regulatory functions. Cell, 2009. 136(2): p. 215-33.

50. Singh-Manoux, A., et al., Association between inflammatory biomarkers and all-cause, cardiovascular and cancer-related mortality. Canadian Medical Association Journal, 2017. 189(10): p. E384-E390.

51. Nurkiewicz, T.R. and M.A. Boegehold, Calcium-independent release of endothelial nitric oxide in the arteriolar network: onset during rapid juvenile growth. Microcirculation, 2004. 11(6): p. 453-62.

52. $\quad \mathrm{Ze}, \mathbf{Y}$., et al., TiO2 nanoparticles induced hippocampal neuroinflammation in mice. PLoS One, 2014. 9(3): p. e92230.

53. Imai, Y., et al., Interaction between cytokines and inflammatory cells in islet dysfunction, insulin resistance and vascular disease. Diabetes Obes Metab, 2013. 15 Suppl 3: p. 11729.

54. Sprague, A.H. and R.A. Khalil, Inflammatory cytokines in vascular dysfunction and vascular disease. Biochem Pharmacol, 2009. 78(6): p. 539-52.

55. Harada, A., et al., Essential involvement of interleukin-8 (IL-8) in acute inflammation. J Leukoc Biol, 1994. 56(5): p. 559-64. 
Table 6.2.1

\begin{tabular}{|c|c|c|c|c|c|c|c|c|c|}
\hline $\begin{array}{l}\text { Exposure } \\
\text { Group }\end{array}$ & Technique & $\begin{array}{l}\text { Number of } \\
\text { Animals } \\
\text { (N) }\end{array}$ & $\begin{array}{c}\text { Age } \\
\text { (days) }\end{array}$ & $\begin{array}{c}\text { Body } \\
\text { Weight } \\
\text { (grams) }\end{array}$ & Litter Size & $\begin{array}{c}\text { Pup } \\
\text { Weight } \\
\text { (grams) }\end{array}$ & $\begin{array}{c}\text { Aerosol } \\
\text { Concentration } \\
\left(\mathrm{mg} / \mathrm{m}^{3}\right)\end{array}$ & $\begin{array}{l}\text { Electrical } \\
\text { Low- } \\
\text { Pressure } \\
\text { Impactor } \\
\text { (nm) }\end{array}$ & $\begin{array}{c}\text { Scanning } \\
\text { Particle } \\
\text { Mobility } \\
\text { Sizer } \\
\text { (nm) }\end{array}$ \\
\hline $\begin{array}{l}\text { Sham } \\
\text { Control }\end{array}$ & RNA Sequencing & 7 & $109 \pm 7$ & $402 \pm 8.84$ & $13 \pm 2$ & $4.06 \pm 0.16$ & 0 & 0 & 0 \\
\hline $\begin{array}{c}\text { Nano- } \\
\mathrm{TiO}_{2}\end{array}$ & RNA Sequencing & 4 & $113 \pm 2$ & $422 \pm 13.34$ & $14 \pm 1$ & $3.99 \pm 0.22$ & $10.35 \pm 0.13$ & $\begin{array}{c}136.80 \pm \\
1.44\end{array}$ & $\begin{array}{c}134.80 \pm \\
1.24\end{array}$ \\
\hline $\begin{array}{l}\text { Sham } \\
\text { Control }\end{array}$ & $\begin{array}{c}\text { ChIP } \\
\text { Sequencing }\end{array}$ & 5 & $104 \pm 2$ & $407 \pm 8.09$ & $12 \pm 2$ & $5.19 \pm 1.02$ & 0 & 0 & 0 \\
\hline $\begin{array}{c}\text { Nano- } \\
\mathrm{TiO}_{2}\end{array}$ & $\begin{array}{c}\text { ChIP } \\
\text { Sequencing }\end{array}$ & 6 & $98 \pm 1$ & $\begin{array}{l}376 \pm \\
19.99\end{array}$ & $9 \pm 5$ & $4.88 \pm 1.53$ & $10.5 \pm 0.5$ & $\begin{array}{c}143.75 \pm \\
2.32\end{array}$ & $\begin{array}{c}129.43 \pm \\
3.21\end{array}$ \\
\hline
\end{tabular}


Figure 6.2.1

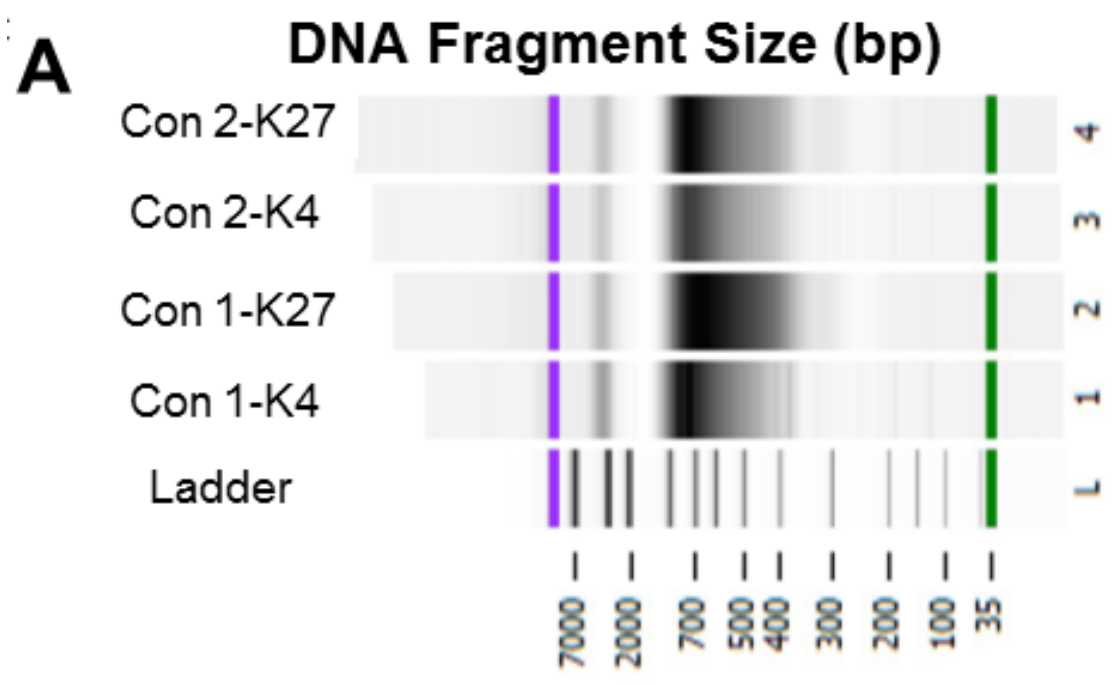

DNA Fragment Size (bp)

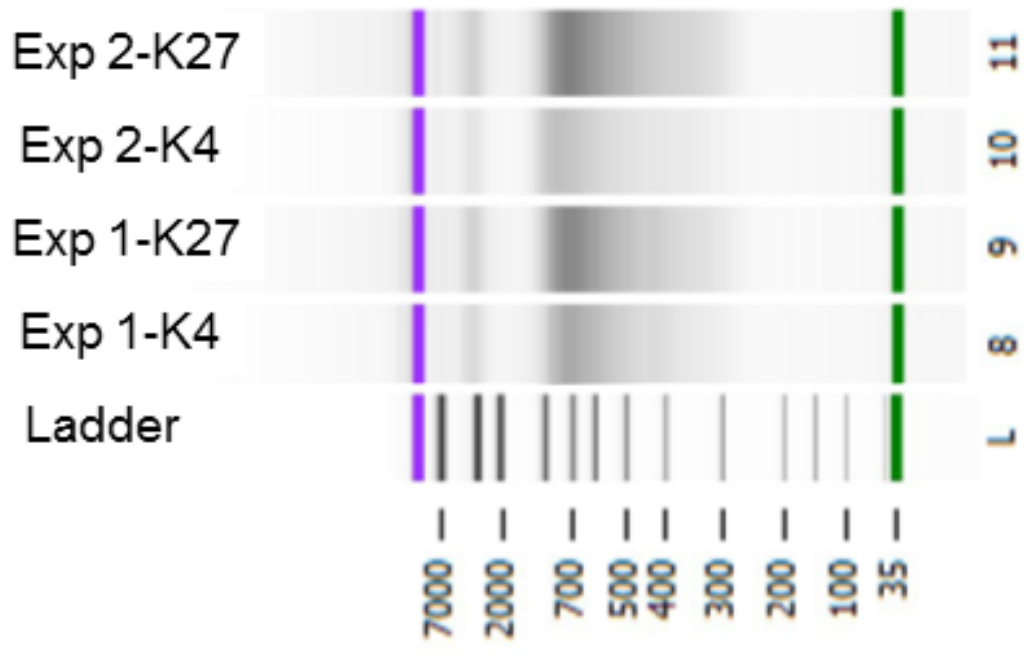


Figure 6.2.1
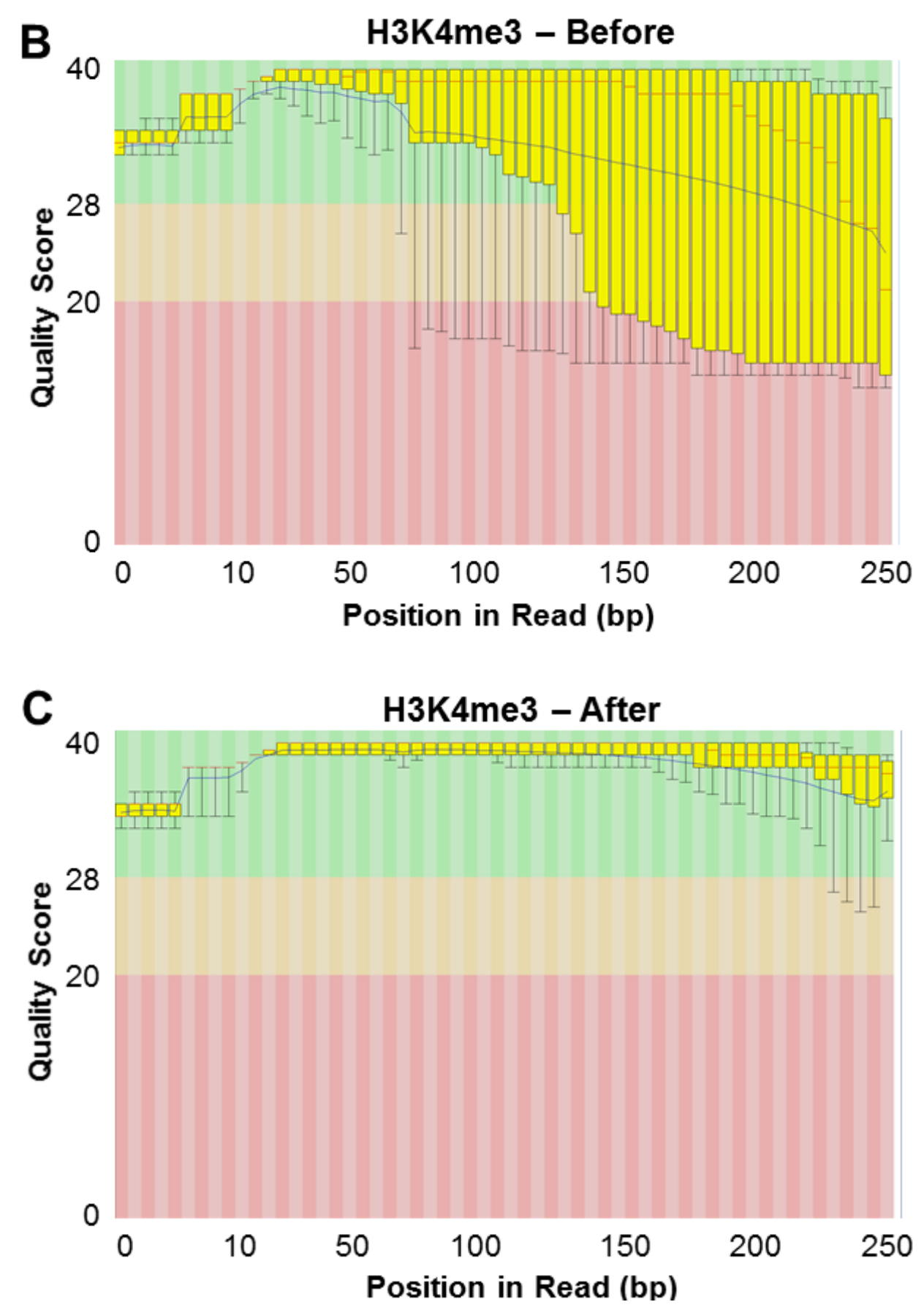
Figure 6.2.1: Evaluating DNA fragmentation and read quality for chromatin immunoprecipitation (ChIP) sequencing. (A) Using gel electrophoresis, DNA fragments were evaluated to determine size and distribution (average size of fragments $=654.3 \mathrm{bp}$ ). Two controls and two maternal nano$\mathrm{TiO}_{2}$ exposed representative samples are shown. Sample quality was assessed using FastQC for both forward and reverse reads (B) before and (C) after using Trimmomatic. Con $=$ control, Exp $=$ maternal nano- $\mathrm{TiO}_{2}$ exposed, $\mathrm{H} 3 \mathrm{~K} 4 \mathrm{me} 3$ and $\mathrm{K} 4$ = histone 3 lysine 4 tri-methylation, $\mathrm{K} 27$ = histone 3 lysine 27 tri-methylation. 
Figure 6.2.2

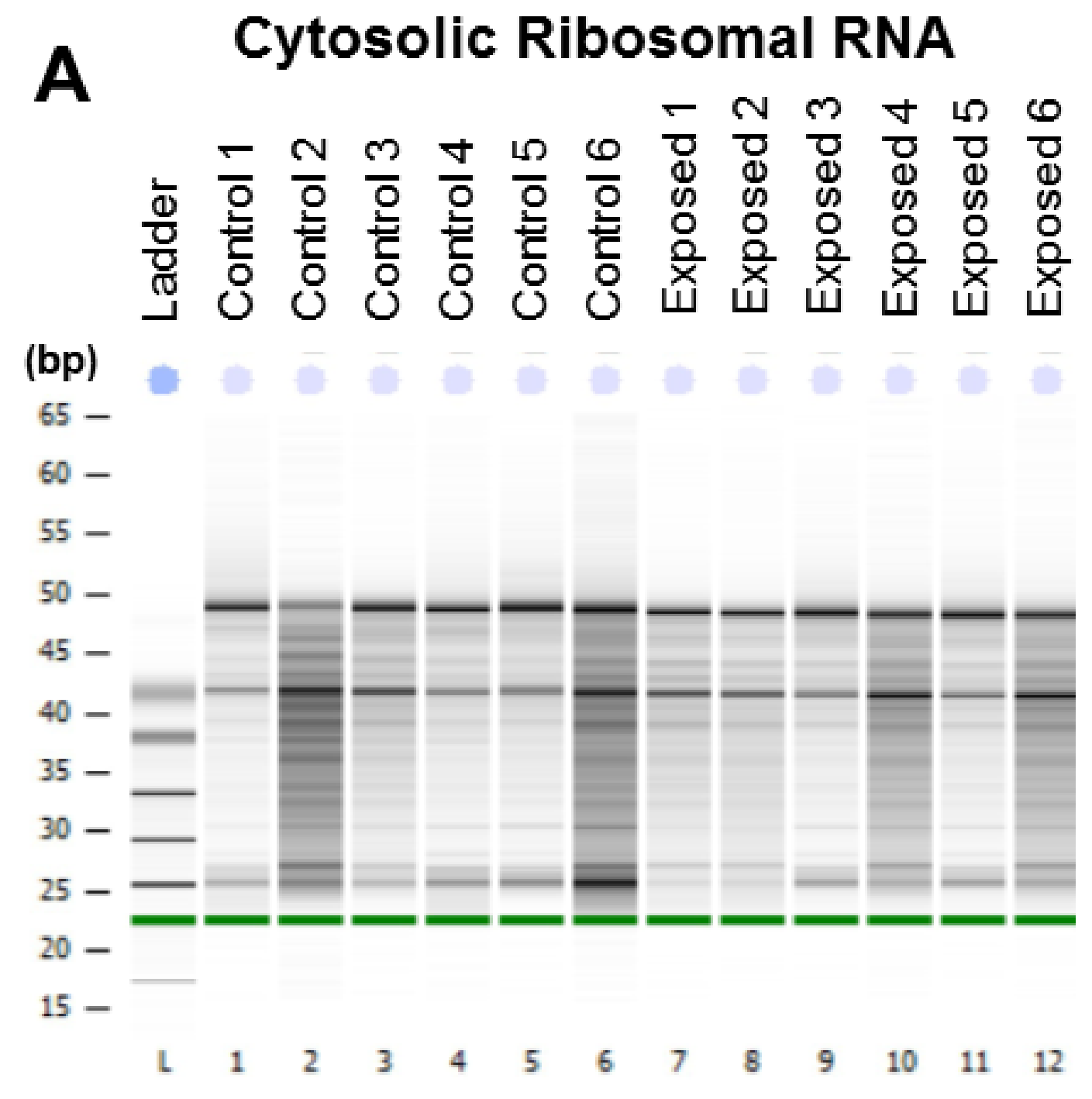


Figure 6.2.2

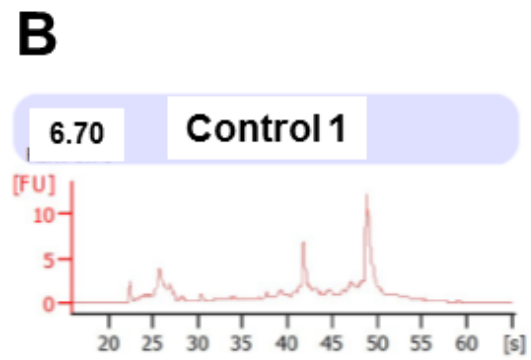

\section{RNA Integrity Number}
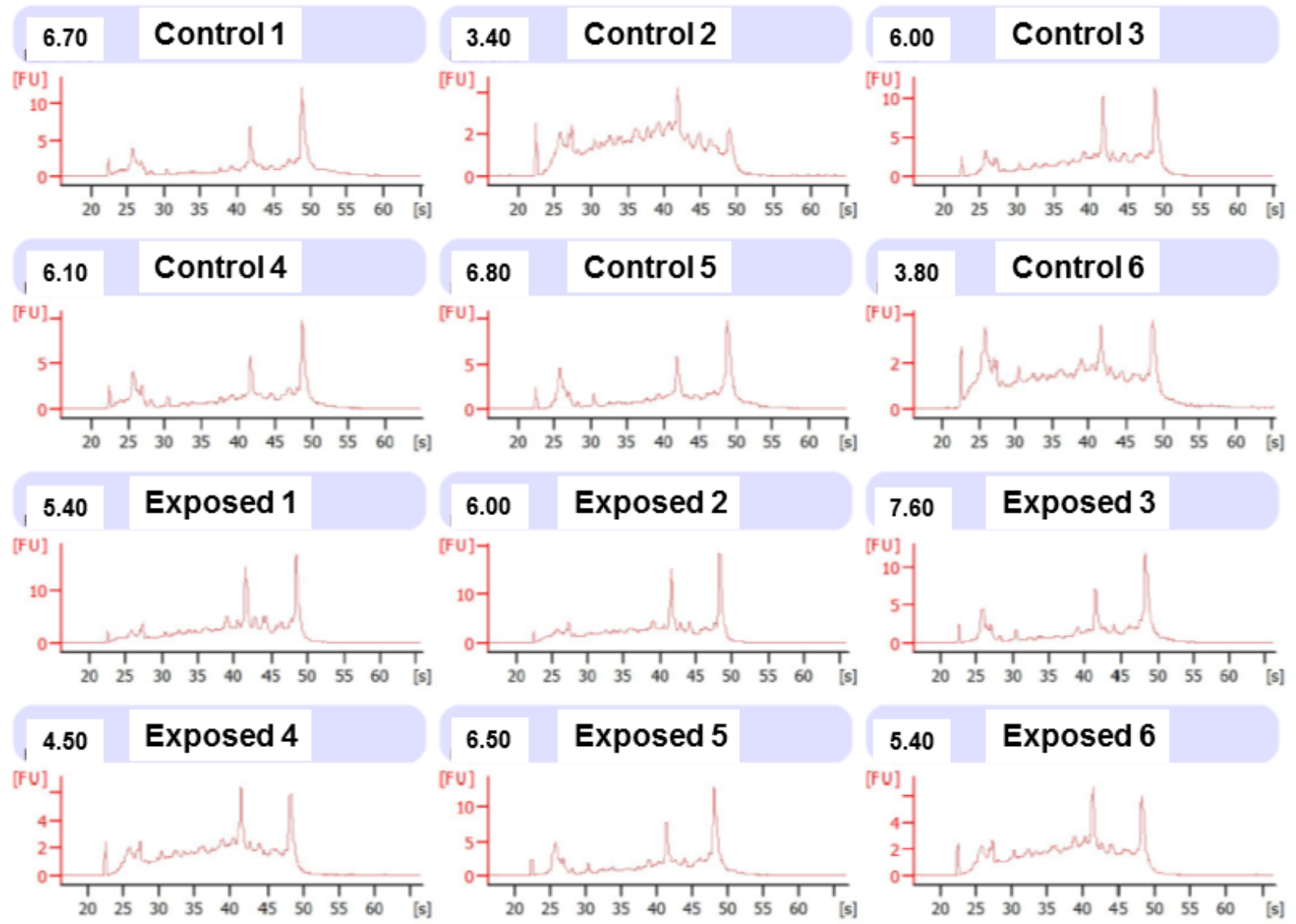
Figure 6.2.2: Assessing RNA quality for transcriptomic data. (A) Gel electrophoresis was implemented to visualize $28 \mathrm{~S}$ and $18 \mathrm{~S}$ ribosomal RNA quality. (B) Cytoplasmic, ribosomal RNA degradation was measured using the Agilent Bioanalyzer 2100. As determined by the RNA Integrity Number (RIN) (left of sample name) the five least degraded samples were chosen for the control $(\mathrm{RIN}=5.88 \pm 1.22)$ and exposed $(\mathrm{RIN}=6.18 \pm 0.92)$ groups. Exposed $=$ maternal nano$\mathrm{TiO}_{2}$ exposed. 
Figure 6.2.3
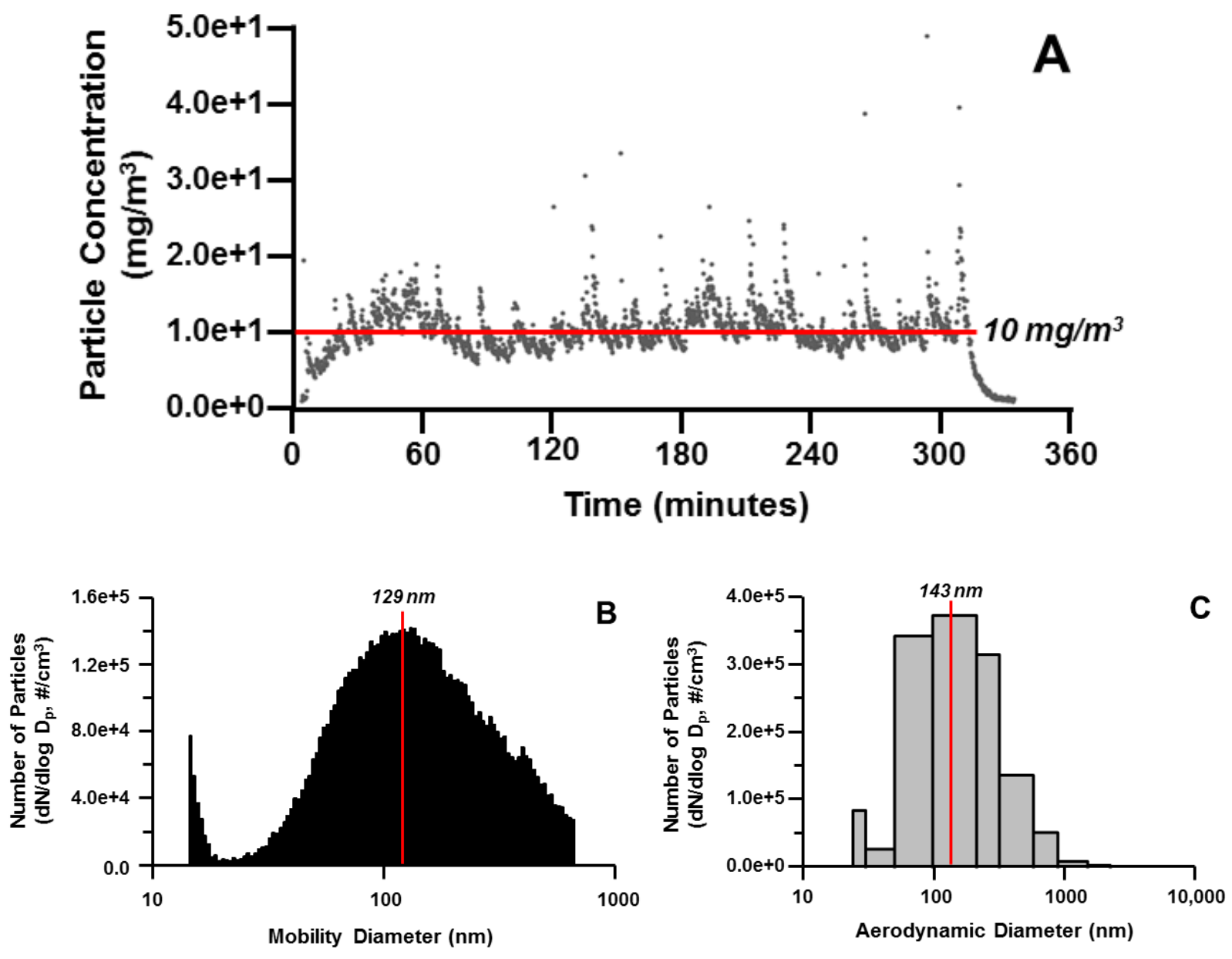
Figure 6.2.3: Maternal nano- $\mathrm{TiO}_{2}$ exposure particle characterization for RNA sequencing experiments. (A) Total aerosol concentration $\left(10 \mathrm{mg} / \mathrm{m}^{3}\right)$ of engineered nano- $\mathrm{TiO}_{2}$ during maternal exposures. (B) Nano- $\mathrm{TiO}_{2}$ size distribution (mobility diameter, $129.4 \mathrm{~nm}$ ) using a scanning mobility particle sizer (SMPS). (C) Nano- $\mathrm{TiO}_{2}$ size distribution (aerodynamic diameter, $143.3 \mathrm{~nm}$ ) using an electrical low-pressure impactor (ELPI). (D) Transmission electron microscopy image of aerosolized nano- $\mathrm{TiO}_{2}$ collected via a sampling filter during an exposure. 
Figure 6.2.4
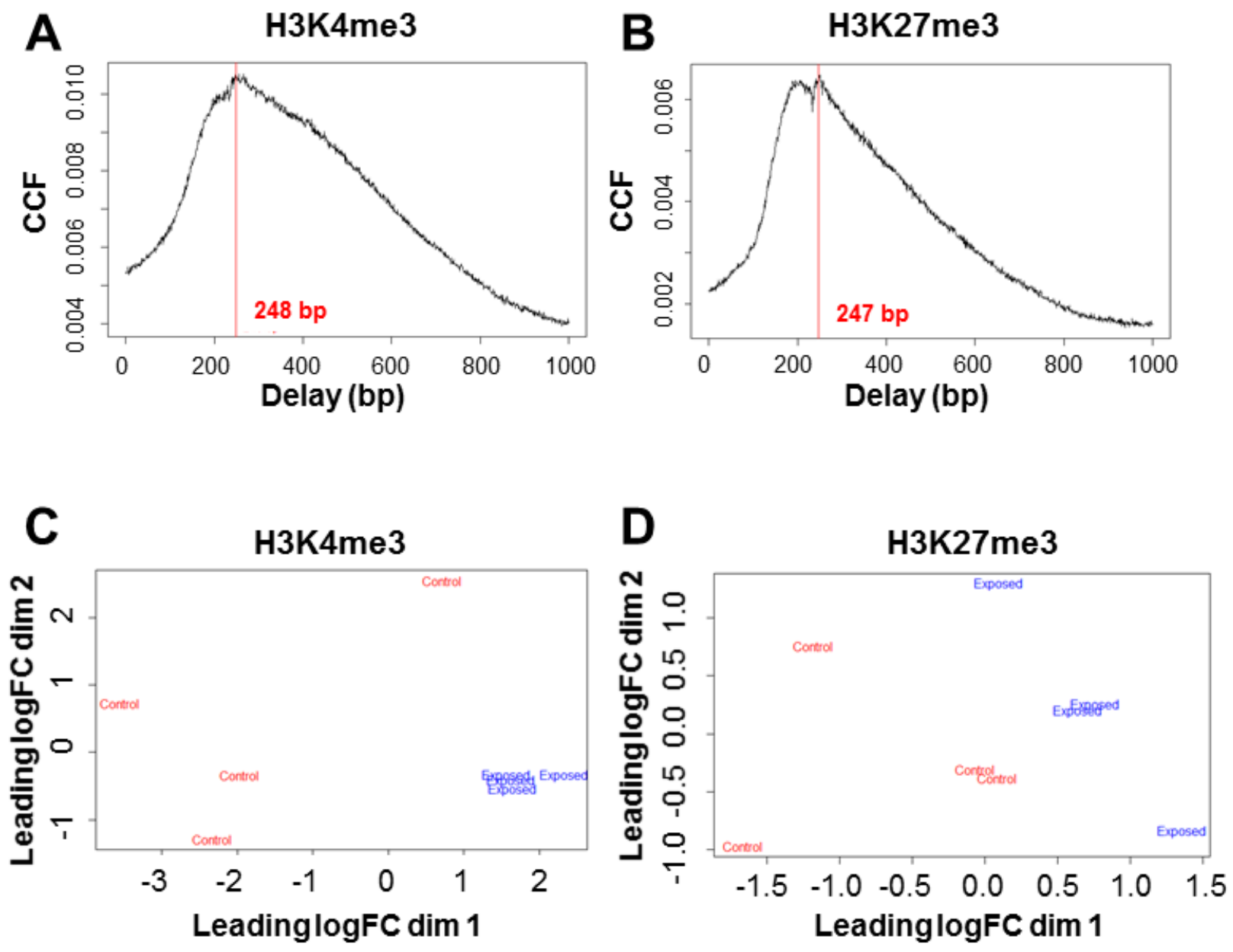
Figure 6.2.4

E

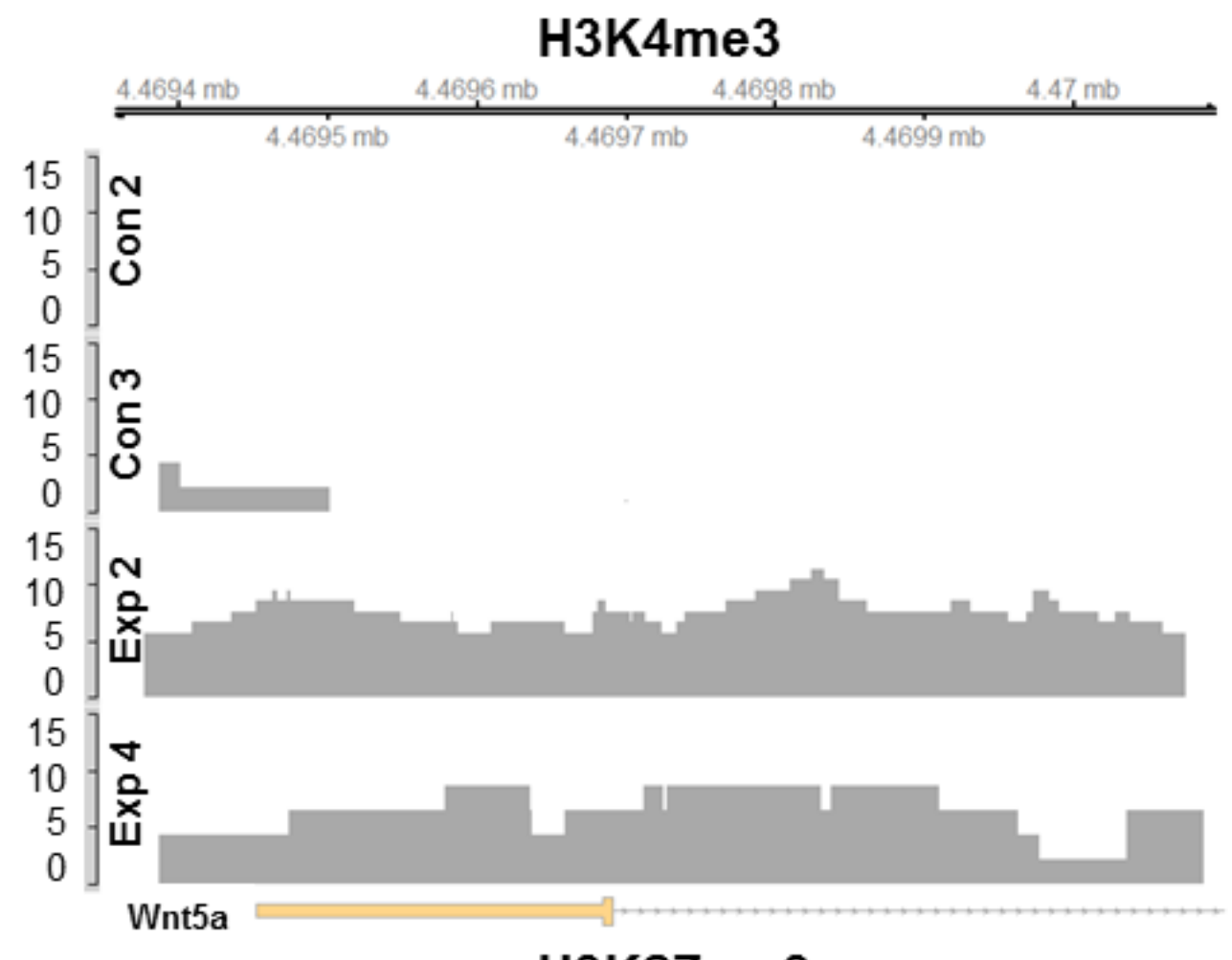

F

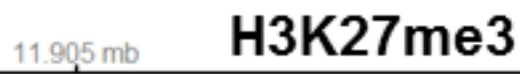

$11.907 \mathrm{mb}$

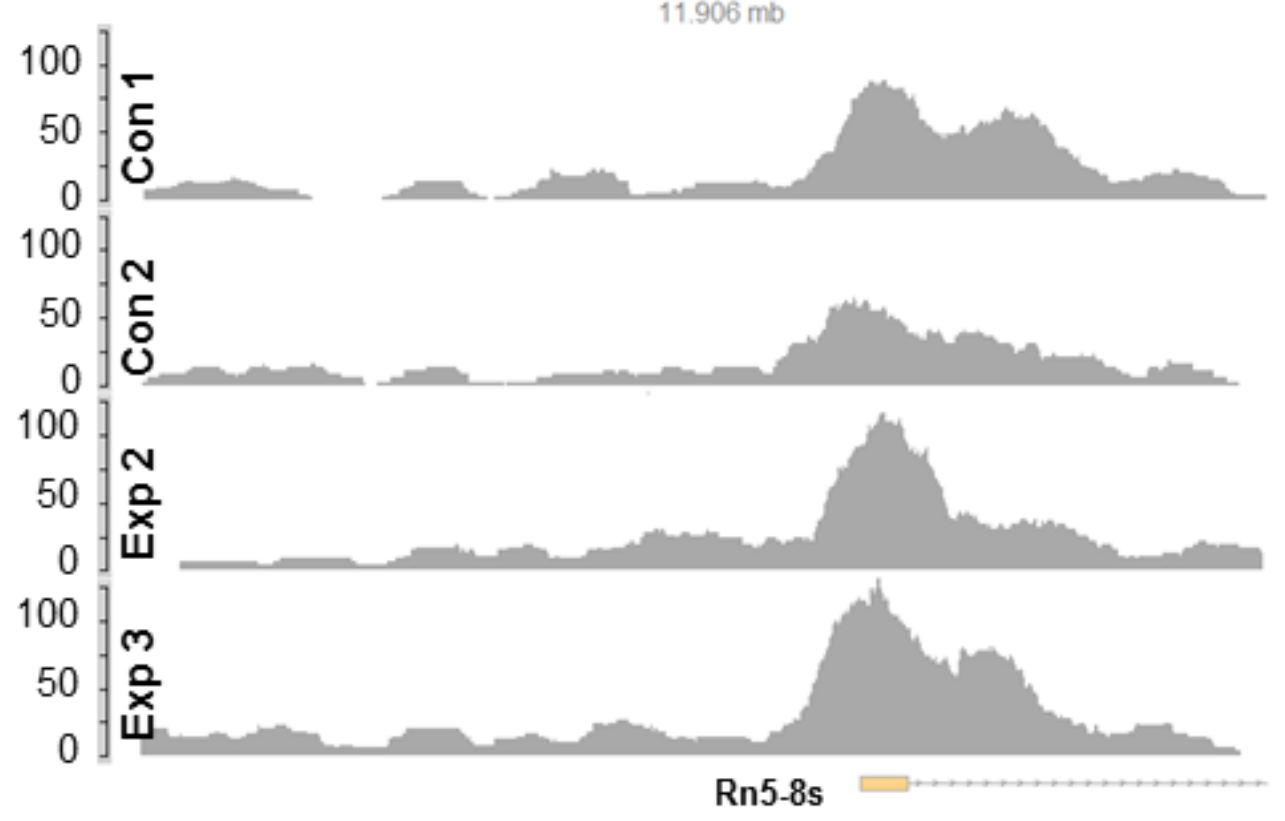


Figure 6.2.4: Chromatin immunoprecipitation (ChIP) sequencing fragment analysis and sample distribution. To measure the distance between subpeaks and find the maximum correlation, the cross-correlation function (CCF) was used to assess (A) H3K4me3 (248 bp) and (B) H3K27me3 (247 bp). Multi-dimensional scaling (MDS) plots indicate the log fold change (logFC) between samples within the (C) H3K4me3 and (D) H3K27me3 groups, describing sample-to-sample distances. Representative histone peaks are shown for differential binding regions $(P \leq 0.05)$ for both (E) H3K4me3 and (F) H3K27me3. Con = control, Exp = maternal nano-TiO2 exposed, H3K4me3 = histone 3 lysine 4 tri-methylation, H3K27me3 = histone 3 lysine 27 tri-methylation, Wnt5a $=$ Wnt Family Member 5A, Rn5-8s $=5.8 \mathrm{~S}$ ribosomal RNA for Rattus norvegicus. 
Figure 6.2.5

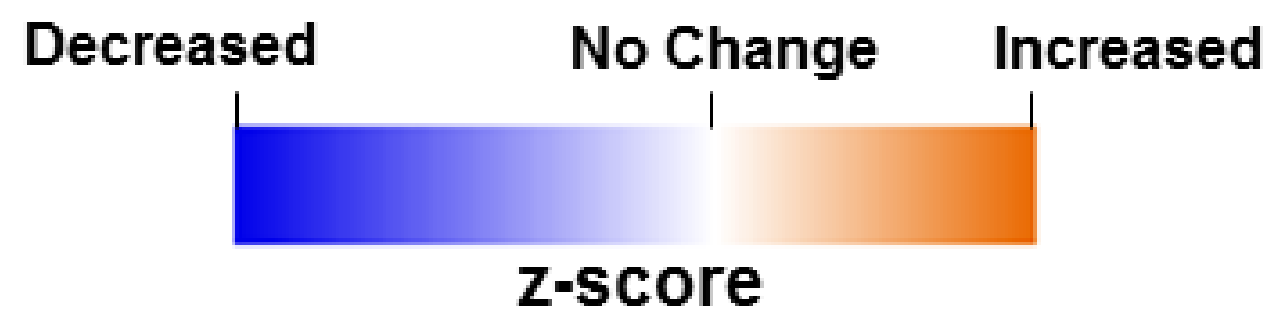

A

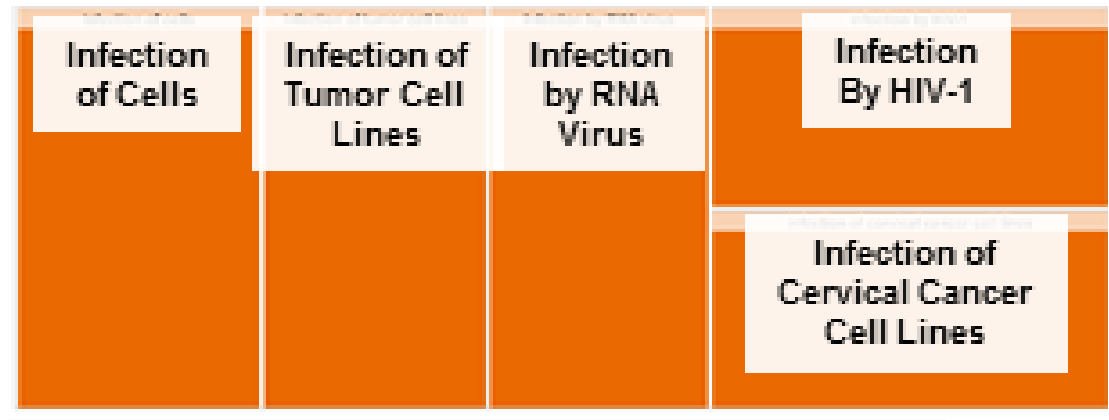

\begin{tabular}{|c||c|c|c|}
\hline $\begin{array}{c}\text { Infection of } \\
\text { Embryonic } \\
\text { Cell Lines }\end{array}$ & $\begin{array}{c}\text { Infection of } \\
\text { Kidney } \\
\text { Cell Lines }\end{array}$ & $\begin{array}{c}\text { Infection } \\
\text { by } \\
\text { Lenitvirus }\end{array}$ & $\begin{array}{c}\text { Viral } \\
\text { Infec- } \\
\text {-tion }\end{array}$ \\
\hline \begin{tabular}{c|c|c|} 
Infection of \\
Epithelial \\
Cell Lines
\end{tabular} & $\begin{array}{c}\text { Infection of } \\
\text { Rhabdomyo- } \\
\text {-sarcoma } \\
\text { Cell Lines }\end{array}$ & $\begin{array}{c}\text { Infection by } \\
\text { Picomaviridae }\end{array}$ \\
\hline
\end{tabular}




\section{Figure 6.2.5}
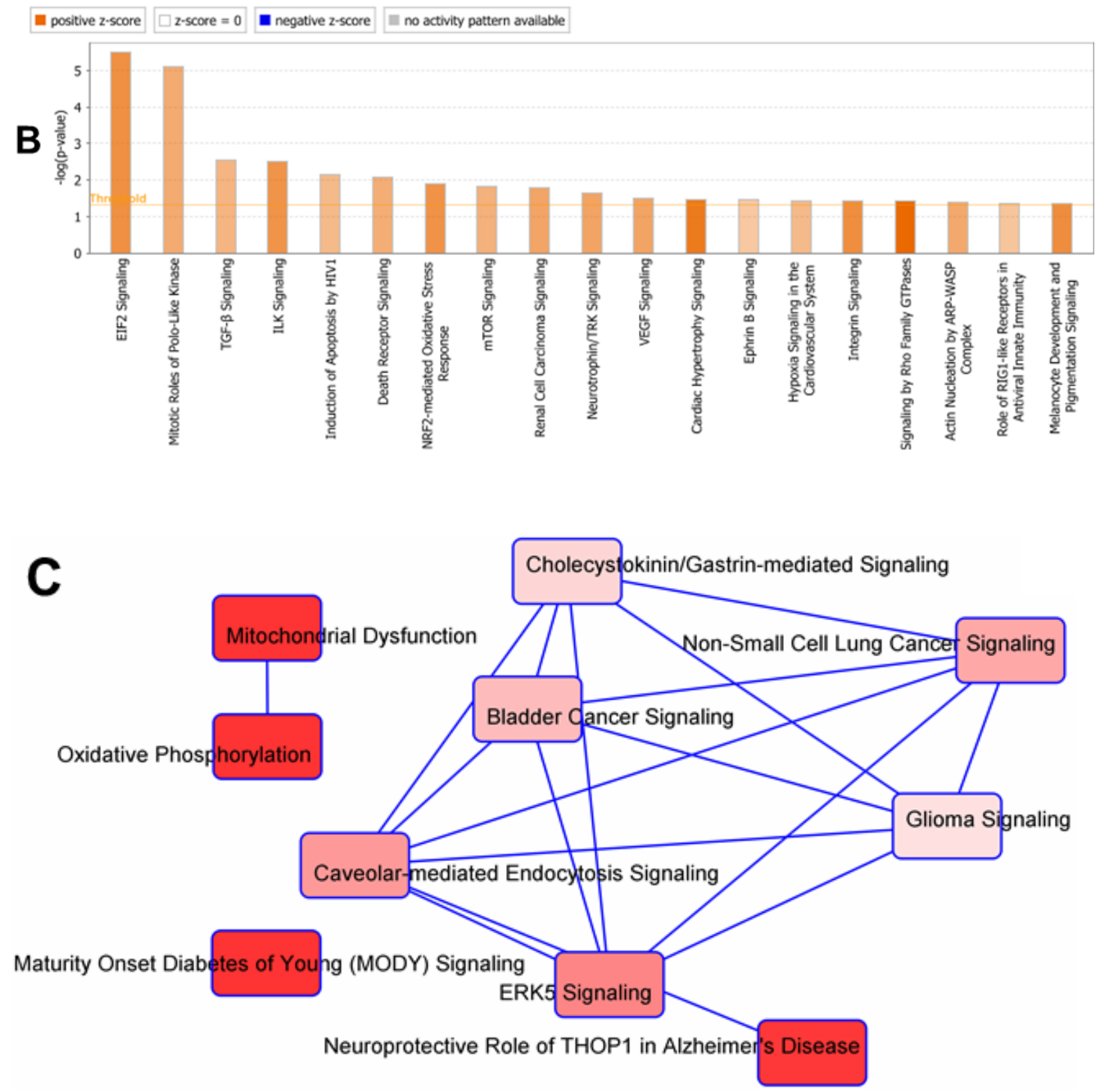
Figure 6.2.5

D
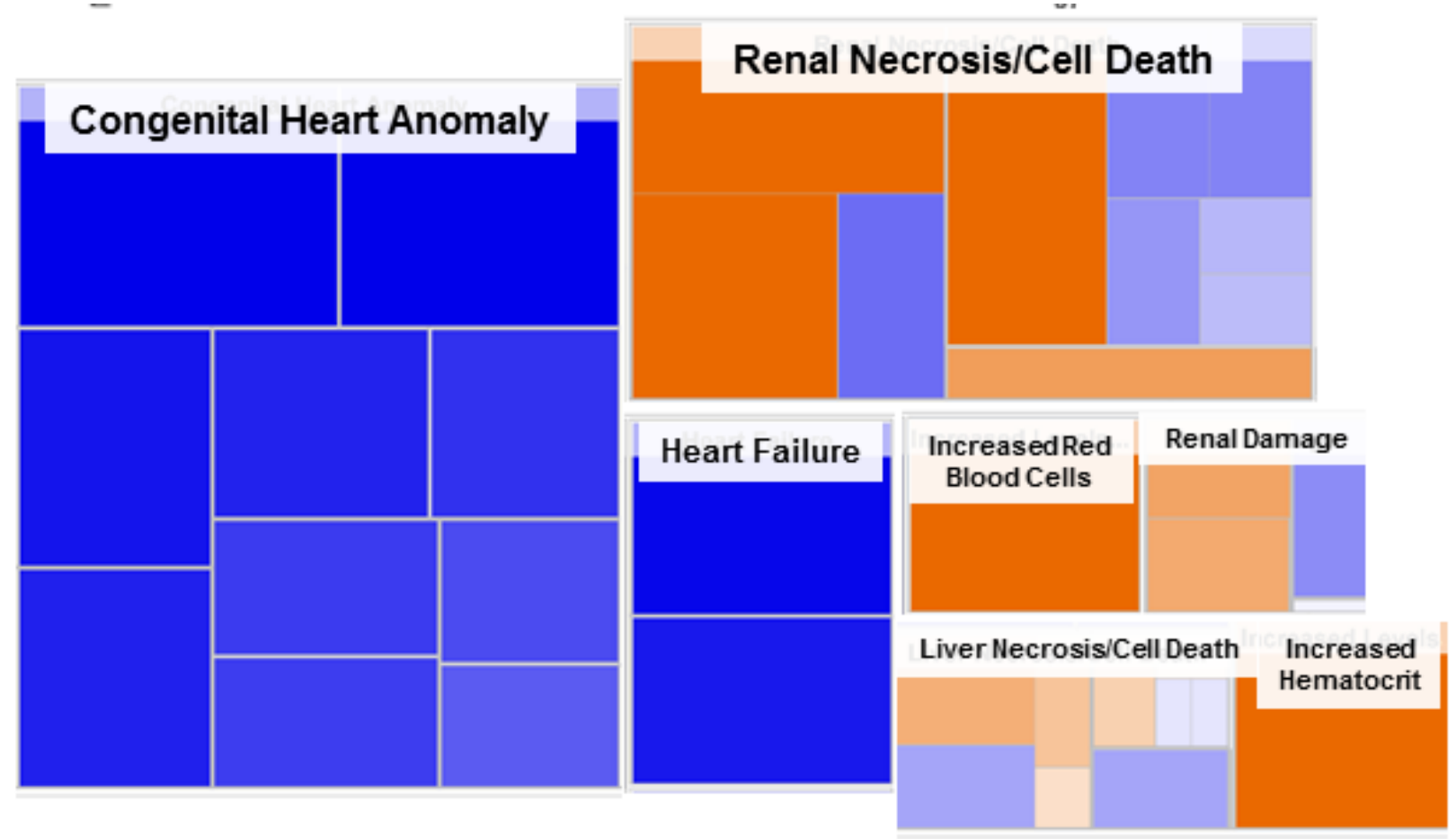
Figure 6.2.5: Assessment of disease and signaling pathways altered epigenetically during maternal nano- $\mathrm{TiO}_{2}$ exposure. (A) One of the primary disease pathways ( $\mathrm{z}$-score $=9.35 \pm 1.89$ ) altered epigenetically during exposure was the increased susceptibility to infection in the H3K4me3 group. Disease and toxicological pathways are constructed from specific, individual canonical signaling pathways. (B) Depicts the top canonical pathways for H3K4me3 (z-score $\geq \pm 2.0$ ) that are significantly $(P \leq 0.05)$ impacted, as indicated by the threshold line. (C) The top canonical pathways for H3K27me3 $(P \leq 0.05)$ are also shown following exposure (smaller $P$-values are associated with increasing red intensity for pathways). (D) Toxicological functions predicted for genes mapped to $\mathrm{H} 3 \mathrm{~K} 4 \mathrm{me} 3$ marks. 
Figure 6.2.6
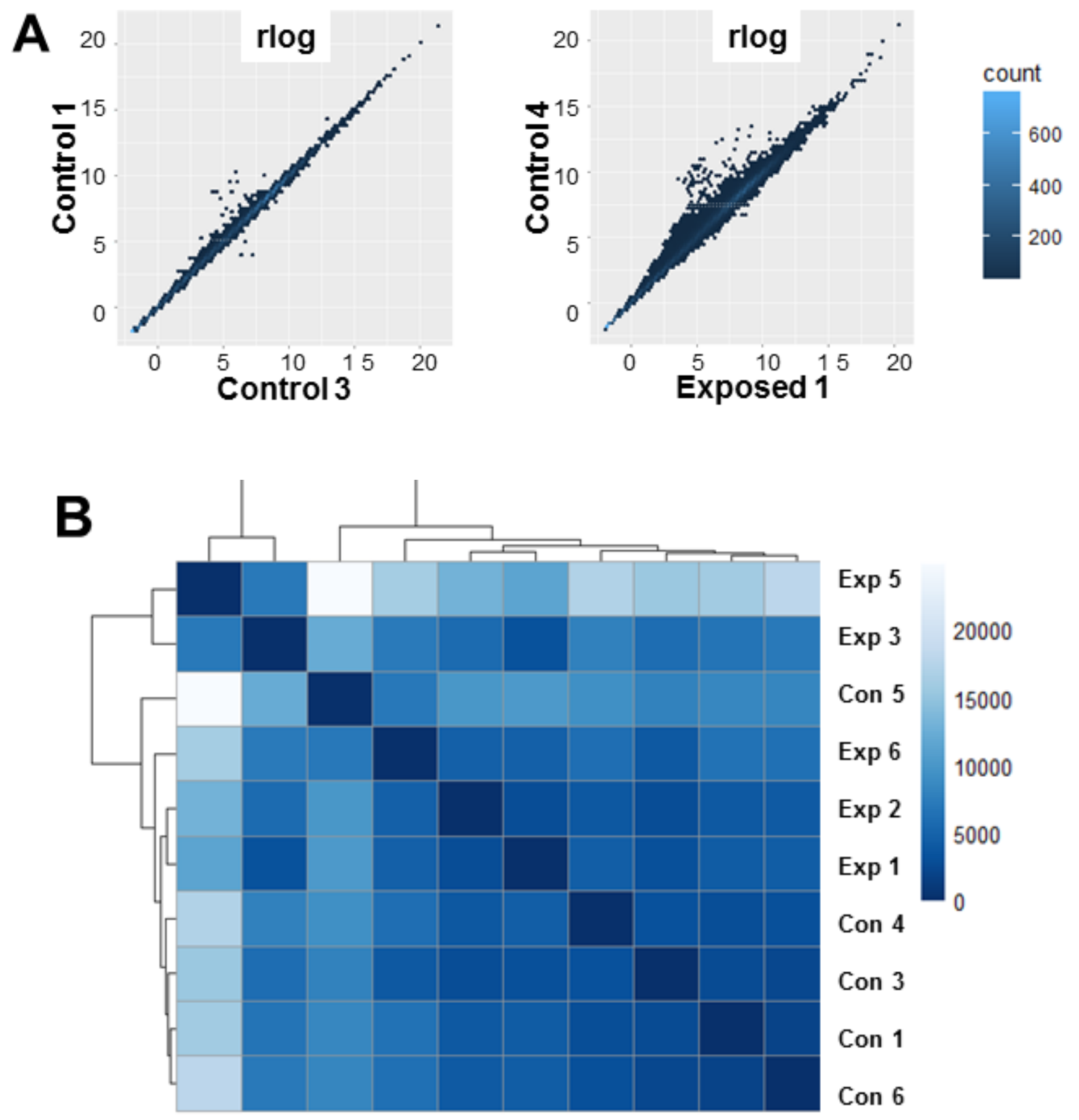
Figure 6.2.6
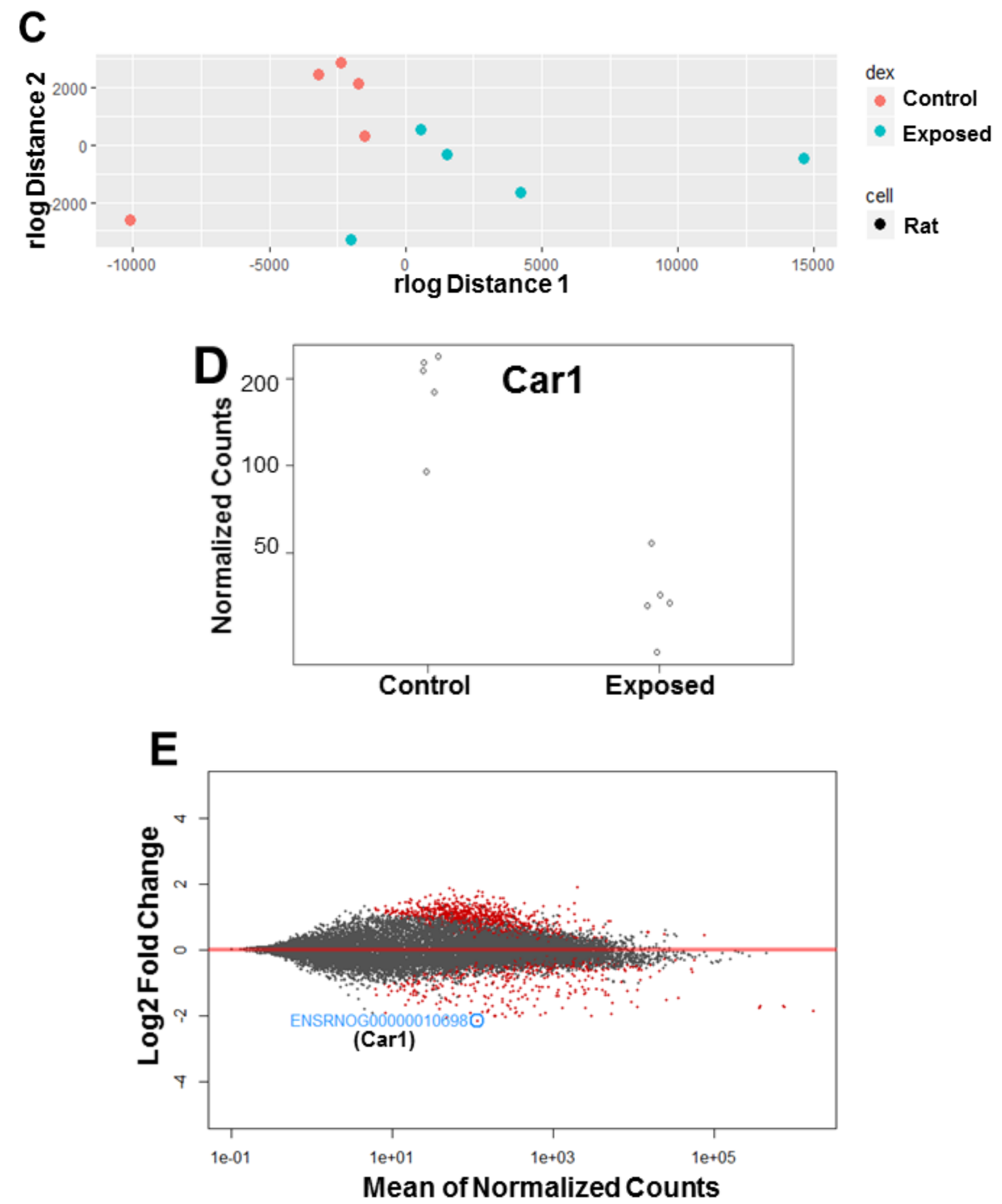
Figure 6.2.6: Sample-to-sample distribution and differential expression analysis for transcriptomic analysis. (A) Assessment of normalized counts between control vs. control (left) and control vs. maternal nano- $\mathrm{TiO}_{2}$ exposed (right) using a $\log 2$ transformed scale. (B) Measure of raw count matrices and (C) normalized count matrices to determine variance between samples. (D) The top differentially regulated gene between groups was determined through the normalized counts for each sample. (E) The MA-plot reveals the differentially expressed genes (red, $P \leq 0.05$ ) in comparison to genes with non-significant change between groups (grey). The top differentially regulated gene is highlighted (blue). Exposed and Exp $=$ maternal nano- $\mathrm{TiO}_{2}$ exposed, $\mathrm{Car} 1=$ carbonic anhydrase 1 . 
Figure 6.2.7
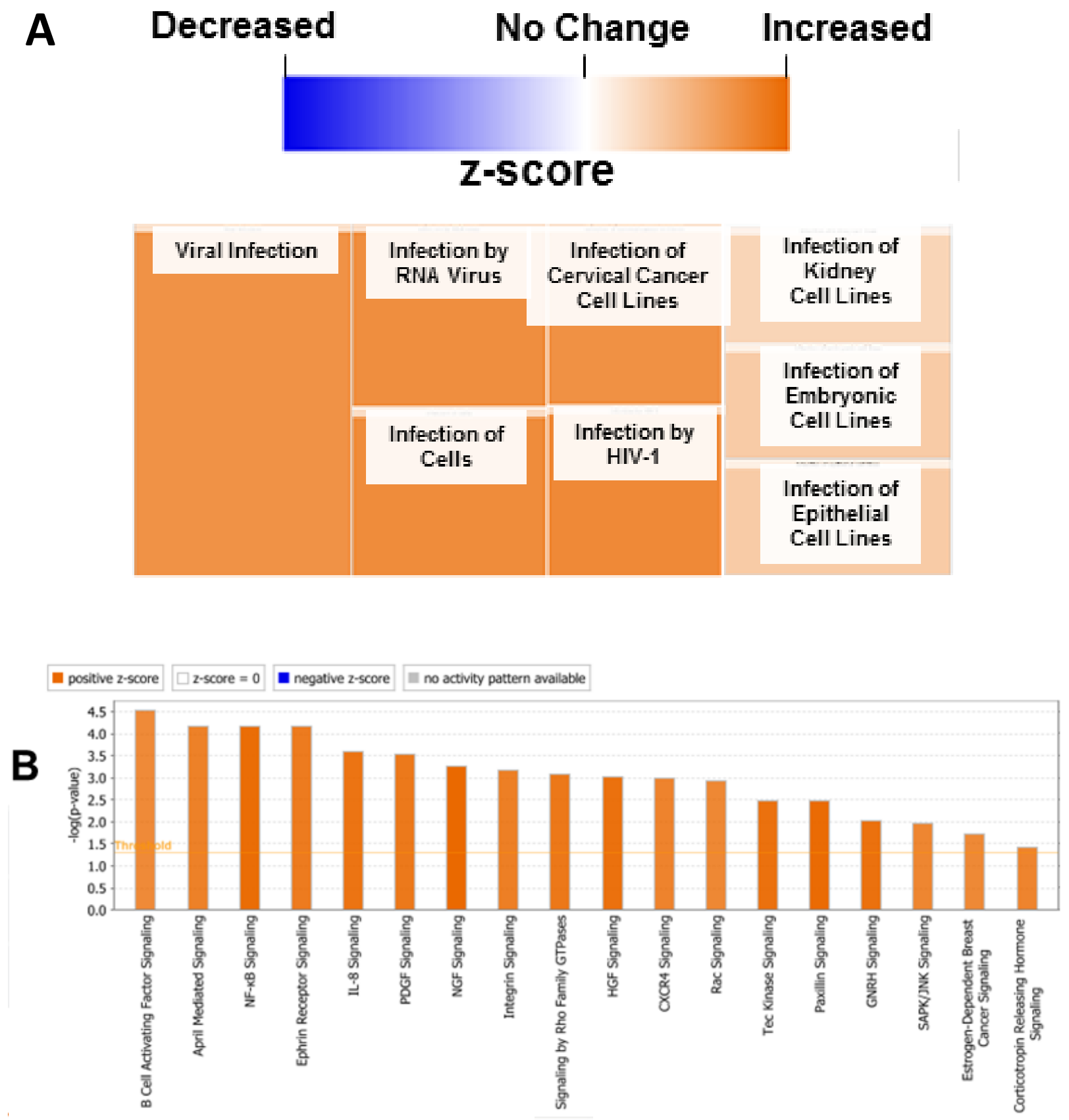
Figure 6.2.7

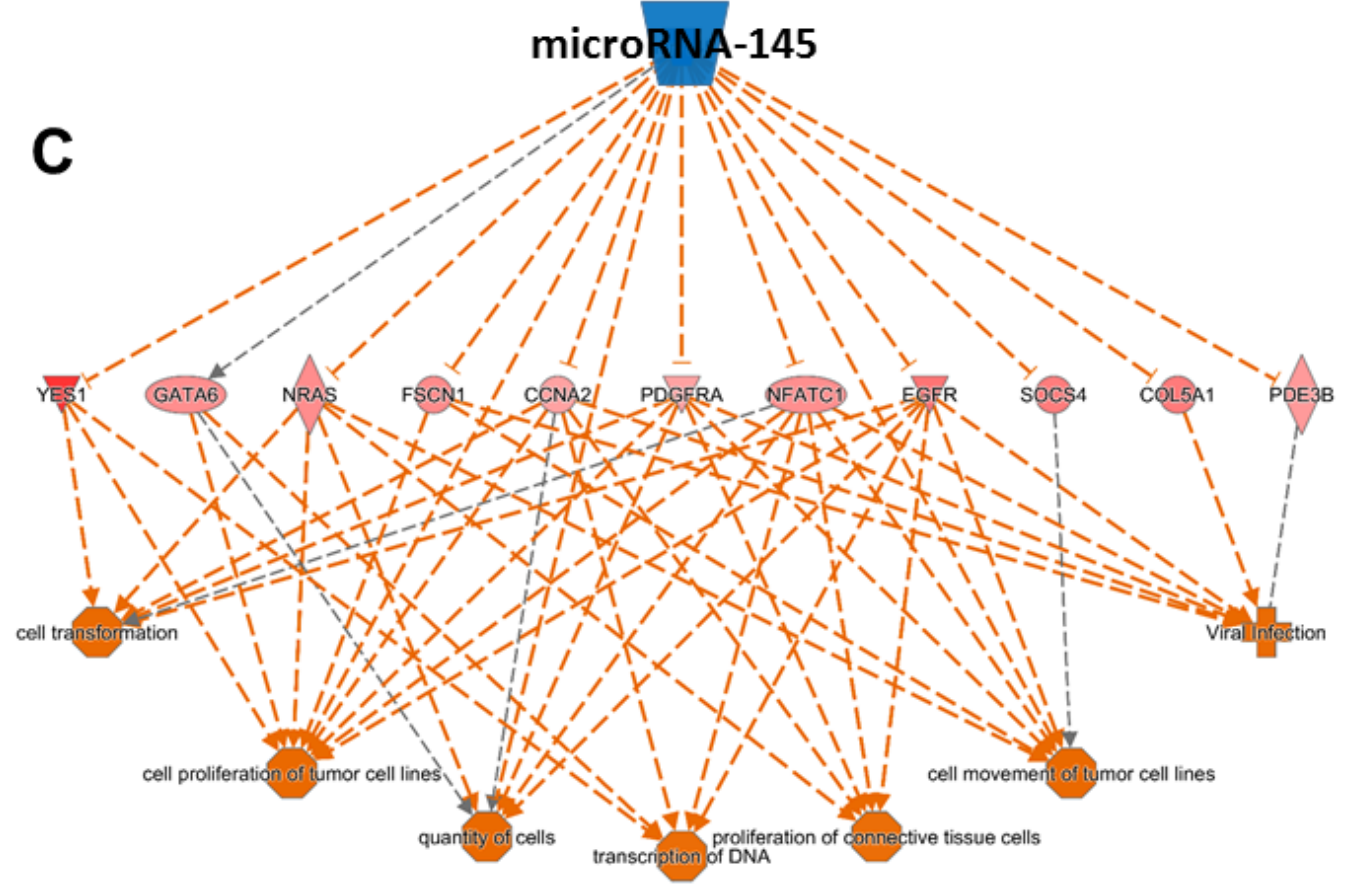

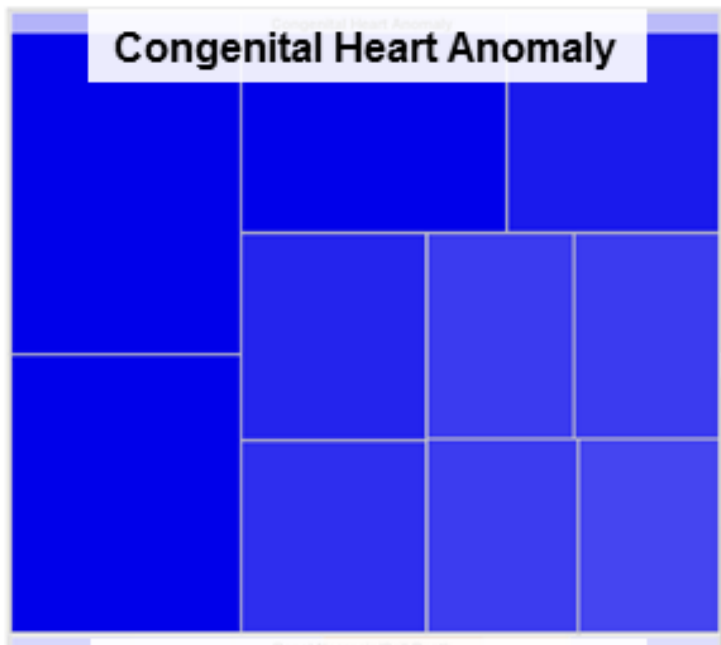

Renal Necrosis/Cell Death

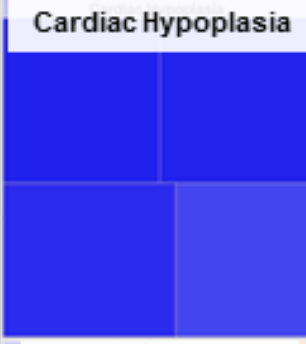

GlomerularInjury

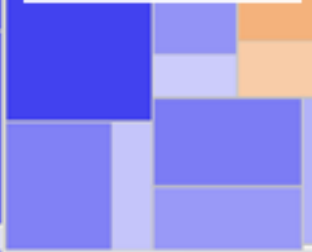

Liver Hyperplasia
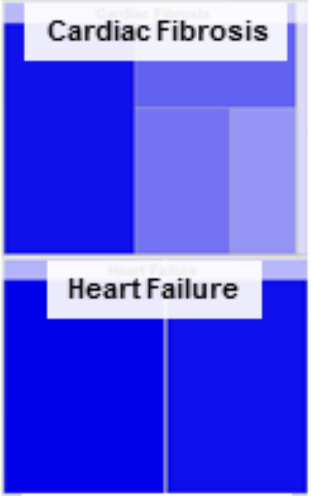

Cardiac Damage

Renal
Atrophy Red Blood Cell 5
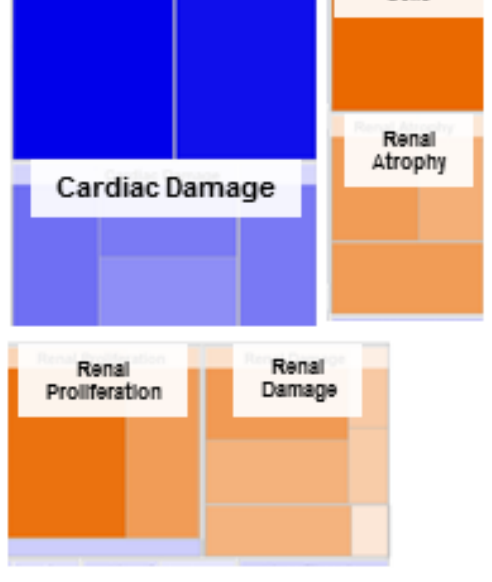
Figure 6.2.7: Assessment of disease and signaling pathways altered transcriptionally during maternal nano- $\mathrm{TiO}_{2}$ exposure. (A) Similar to the activation by $\mathrm{H} 3 \mathrm{~K} 4 \mathrm{me} 3$, transcriptional upregulation of genes associated with increased susceptibility to infection (z-score $=2.02 \pm 0.96)$ was found. (B) The top canonical pathways (z-score $\geq \pm 3.45)$ that are significantly $(P \leq 0.05)$ impacted transcriptionally, as indicated by the threshold line. The canonical pathways for the RNA sequencing reveal a significant increase in inflammatory and growth signaling. (C) The top regulator (consistency score $=10.453$ ) determined through pathway analysis of gene expression $($ arrows $=$ activation, bars $=$ repression). Increasing gene activation $($ red $)$ and suppression (blue) reveal targeting of multiple cell functions. (D) Toxicological functions predicted for transcript abundance in the RNA sequencing experiment. 


Signaling by Rho Family GTPases
IL-8 Signaling
Tec Kinase Signaling
NF-KB Signaling
GNRH Signaling
NGF Signaling
Paxillin Signaling
HGF Signaling
Integrin Signaling
Ephrin Receptor Signaling
Rac Signaling
CXCR4 Signaling
Melanocyte Development and Pigm...
ILK Signaling
PDGF Signaling

Decreased

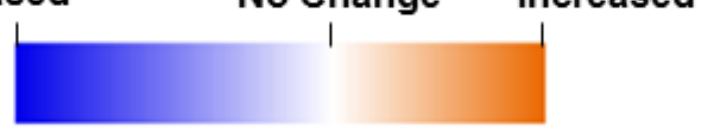

z-score

organismal death
morbidity or mortality
Growth Failure
infection of cells
infection by RNA virus
Viral Infection
HIV infection
infection by HIV-1
cell viability
infection of cervical cancer cell lines
cell viability of tumor cell lines

\section{congenital heart disease ventricular septal defect failure of heart congestive heart failure atrial septal defect familial congenital heart disease perimembranous ventricular septal ... fibrosis of heart cell death of kidney cell lines apoptosis of kidney cell lines hepatic steatosis hypoplasia of liver glomerulosclerosis

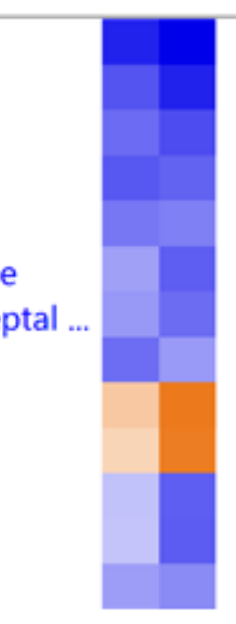

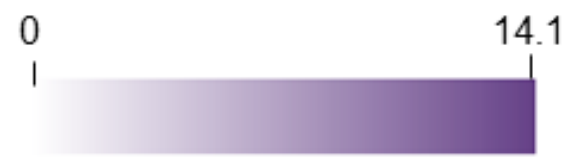

$-\log (p-v a l u e)$

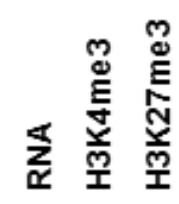

Mitochondrial Dysfunction

Oxidative Phosphorylation

EIF2 Signaling

Protein Ubiquitination Pathway

Molecular Mechanisms of Cancer

Regulation of eIF4 and p70S6K Sign...

Protein Kinase A Signaling

Sumoylation Pathway

Hypoxia Signaling in the Cardiovasc... 
Figure 6.2.8
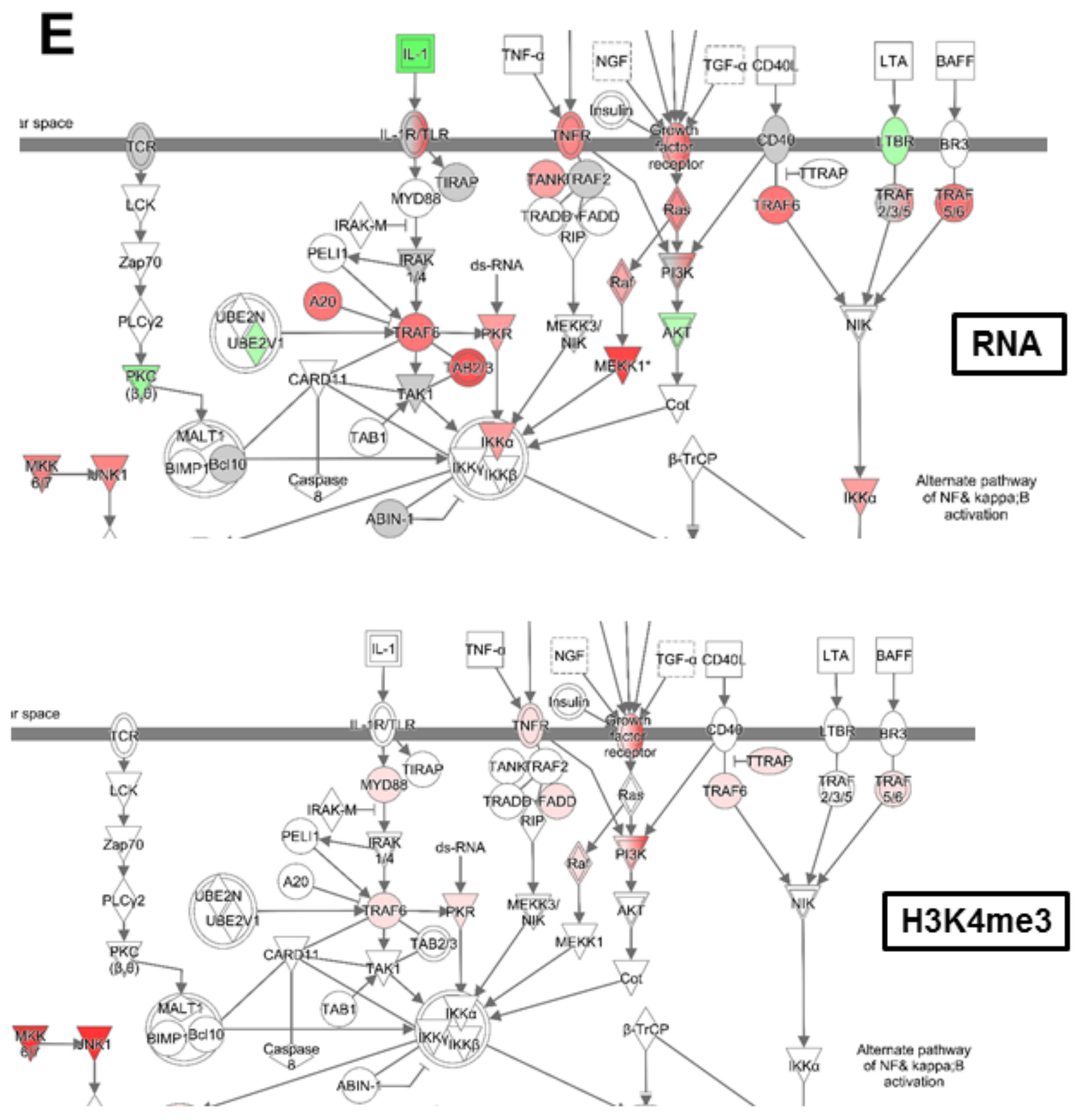
Figure 6.2.8: Comparison of epigenetic regulation (H3K4me3 and $\mathrm{H} 3 \mathrm{~K} 27 \mathrm{me} 3$ ) and transcriptional changes. (A) Top canonical pathways, ranked by z-score, which are changed between groups. (B) Top toxicological functions, ranked by z-score, which are changed between groups. (C) Top diseases and biological functions, ranked by z-score, which are changed between groups. (D) Top canonical pathways, ranked by cumulative $P$-value, which are changed between groups. (E) Example of one of the top canonical pathways altered during maternal nano- $\mathrm{TiO}_{2}$ exposure. NF-KB signaling changes transcriptionally (right) and epigenetically through H3K4me3 (left) $($ green $=$ decreased expression, red $=$ increased expression $) . \mathrm{NF}-\mathrm{kB}=$ nuclear factor kappalight-chain-enhancer of activated B cells. 
Figure 6.2.9
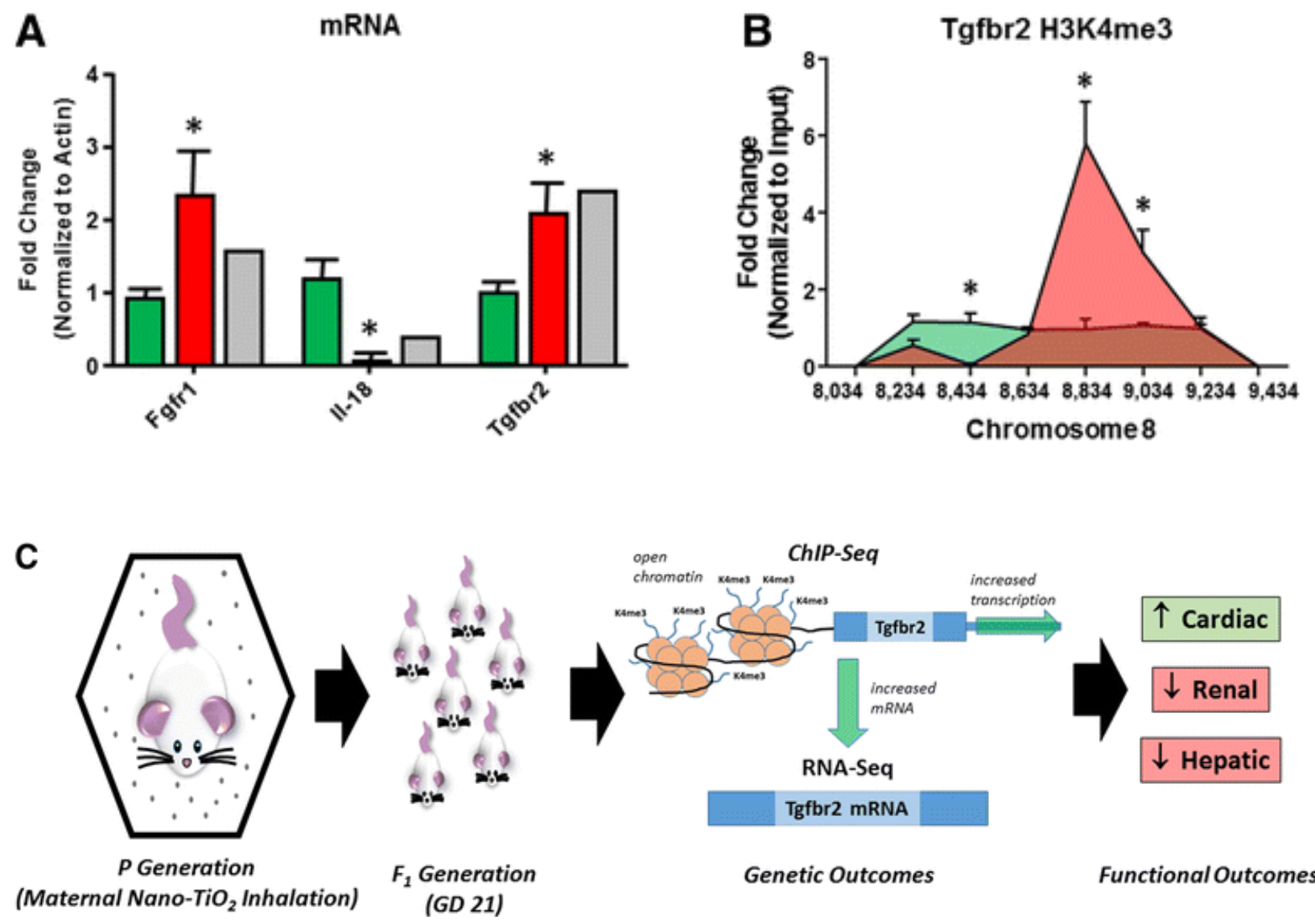
(GD 21) 
Figure 6.2.9: Validation of sequencing and model overview. (A) The mRNA of Fgfr1, Il-18, and Tgfbr2 were assessed in the sham (green, Sham-Control) and maternal nano- $\mathrm{TiO}_{2}$ (red, $\mathrm{Nano}^{-\mathrm{TiO}_{2}}$ Exposed) exposed progeny, reference to the RNA sequencing observed change (grey, Sequence). Expression was normalized to the $\beta$-Actin reporter gene. (B) Tgfbr2 was further characterized through ChIP-qPCR of H3K4me3 to measure the binding affinity of the modified histone at the Tgfbr2 promoter loci in the Sham-Control (green) and maternal nano- $\mathrm{TiO}_{2}$ (red) exposed progeny. Values were normalized to each sample's input control. Tick marks represent the chromosomal location of each qPCR measurement, ranging from 124,318,034 to $124,319,434$ on chromosome 8. (C) Schematic overview of the experimental model for nano- $\mathrm{TiO}_{2}$ maternal exposure and examination of the fetal progeny. As an example, the changes in Tgfbr2 are used to illustrate how epigenetic alterations through modification of chromatin can lead to increased expression of the mRNA transcript. Finally, the results of the study suggest that the gestational exposure paradigm impacts the heart, through increased function, while the liver and kidney have a detriment in function. Values are expressed as means \pm SE. $*=P \leq 0.05$. Fgfr $1=$ Fibroblast Growth Factor Receptor 1, Il-18 = Interleukin-18, Tgfbr2 = Transforming Growth Factor Beta Receptor 2, H3K4me3 = histone 3 lysine 4 tri-methylation, $\mathrm{ChIP}=$ Chromatin Immunoprecipitation. 
Table S6.2.1

H3K4me3 Disease and Biological Functions List

\begin{tabular}{|c|c|c|c|}
\hline Diseases or Functions Annotation & $P$-Value & $\begin{array}{l}\text { Predicted } \\
\text { Activation State }\end{array}$ & $\begin{array}{l}\text { Activation } z- \\
\text { score }\end{array}$ \\
\hline infection of cells & $\begin{array}{l}0.00000095 \\
5\end{array}$ & Increased & 11.372 \\
\hline infection by RNA virus & 0.00000358 & Increased & 11.184 \\
\hline Viral Infection & 0.00334 & Increased & 11.109 \\
\hline HIV infection & 0.000494 & Increased & 10.705 \\
\hline infection by HIV-1 & 0.0000125 & Increased & 10.623 \\
\hline infection by lentivirus & 0.000392 & Increased & 10.613 \\
\hline cell viability & 0.0094 & Increased & 9.722 \\
\hline infection of tumor cell lines & 0.00000277 & Increased & 9.561 \\
\hline infection of cervical cancer cell lines & 0.0000142 & Increased & 9.312 \\
\hline cell viability of tumor cell lines & 0.0000388 & Increased & 8.467 \\
\hline infection of kidney cell lines & 0.000339 & Increased & 7.151 \\
\hline infection of embryonic cell lines & 0.00018 & Increased & 7.151 \\
\hline infection of epithelial cell lines & 0.00018 & Increased & 7.151 \\
\hline $\begin{array}{l}\text { productive infection of cervical cancer } \\
\text { cell lines }\end{array}$ & 0.00461 & Increased & 6.321 \\
\hline $\begin{array}{l}\text { cell proliferation of breast cancer cell } \\
\text { lines }\end{array}$ & 0.0000852 & Increased & 5.014 \\
\hline cell transformation & 0.00024 & Increased & 5.01 \\
\hline cell proliferation of tumor cell lines & 0.00425 & Increased & 4.34 \\
\hline homologous recombination of cells & 0.00141 & Increased & 4.252 \\
\hline cell proliferation of fibroblasts & 0.00419 & Increased & 4.061 \\
\hline $\begin{array}{l}\text { cell viability of colorectal cancer cell } \\
\text { lines }\end{array}$ & 0.00106 & Increased & 4.03 \\
\hline
\end{tabular}




\begin{tabular}{llll} 
cytokinesis & 0.00193 & Increased & 3.855 \\
repair of DNA & $8.61 \mathrm{E}-09$ & Increased & 3.853 \\
metabolism of protein & 0.000352 & Increased & 3.587 \\
Clathrin mediated endocytosis & 0.00762 & Increased & 3.57 \\
development of cytoplasm & 0.00782 & Increased & 3.567 \\
transformation of fibroblast cell lines & 0.0133 & Increased & 3.548 \\
cell viability of myeloma cell lines & 0.00392 & Increased & 3.529 \\
recombination & 0.0101 & Increased & 3.475 \\
cell death of kidney cell lines & 0.000989 & Increased & 3.289 \\
formation of cytoskeleton & 0.0134 & Increased & 3.216 \\
apoptosis of kidney cell lines & 0.000769 & Increased & 3.21 \\
cell viability of breast cancer cell lines & 0.00257 & Increased & 3.181 \\
cytokinesis of tumor cell lines & 0.000305 & Increased & 3.148 \\
initiation of transcription & 0.0107 & Increased & 3.123 \\
excision repair & 0.00117 & Increased & 3.106 \\
proliferation of connective tissue cells & 0.00546 & Increased & 3.106 \\
interphase of fibroblasts & 0.00457 & Increased & 3.073 \\
interphase of connective tissue cells & 0.0121 & Increased & 3.066 \\
cell death of epithelial cell lines & 0.00656 & Increased & 2.992 \\
cell death of embryonic cell lines & 0.00184 & Increased & 2.99 \\
closure of embryonic tissue & 0.00665 & Increased & 2.97 \\
initiation of transcription of RNA & 0.0038 & Increased & 2.964 \\
apoptosis of embryonic cell lines & 0.00178 & Increased & 2.919 \\
apoptosis of epithelial cell lines & 0.011 & Increased & 2.87 \\
M phase & 0.000365 & Increased & 2.855 \\
growth of connective tissue & 0.013 & Increased & 2.852 \\
cytokinesis of cervical cancer cell lines & 0.000366 & Increased & 2.813 \\
\hline
\end{tabular}




\begin{tabular}{|c|c|c|c|}
\hline expression of RNA & 0.00861 & Increased & 2.801 \\
\hline transport of protein & 0.000134 & Increased & 2.743 \\
\hline entry into interphase & 0.00904 & Increased & 2.704 \\
\hline S phase & 0.00427 & Increased & 2.686 \\
\hline cell death of kidney cells & 0.00148 & Increased & 2.681 \\
\hline association of chromatin & 0.00108 & Increased & 2.606 \\
\hline association of chromosome components & 0.0071 & Increased & 2.606 \\
\hline modification of reactive oxygen species & 0.00717 & Increased & 2.502 \\
\hline proliferation of fibroblast cell lines & 0.000267 & Increased & 2.465 \\
\hline repair of cells & 0.00125 & Increased & 2.403 \\
\hline necrosis of kidney & 0.00153 & Increased & 2.377 \\
\hline initiation of expression of RNA & 0.00283 & Increased & 2.376 \\
\hline apoptosis of nervous tissue cell lines & 0.00718 & Increased & 2.359 \\
\hline M phase of cervical cancer cell lines & 0.00107 & Increased & 2.345 \\
\hline cell cycle progression & 0.000099 & Increased & 2.268 \\
\hline growth of yeast & 0.00836 & Increased & 2.266 \\
\hline $\begin{array}{l}\text { cell cycle progression of tumor cell } \\
\text { lines }\end{array}$ & 0.0105 & Increased & 2.25 \\
\hline checkpoint control & 0.000101 & Increased & 2.25 \\
\hline $\begin{array}{l}\text { infection of rhabdomyosarcoma cell } \\
\text { lines }\end{array}$ & 0.000352 & Increased & 2.236 \\
\hline modification of hydrogen peroxide & 0.00406 & Increased & 2.232 \\
\hline catabolism of protein & 0.00263 & Increased & 2.231 \\
\hline decay of RNA & 0.000808 & Increased & 2.213 \\
\hline closure of neural tube & 0.00866 & Increased & 2.213 \\
\hline maintenance of cells & 0.00741 & Increased & 2.209 \\
\hline processing of rRNA & 0.0068 & Increased & 2.176 \\
\hline ubiquitination & 0.0000226 & Increased & 2.15 \\
\hline
\end{tabular}




\begin{tabular}{|c|c|c|c|}
\hline molecular cleavage of DNA & 0.00265 & Increased & 2.114 \\
\hline condensation of chromatin & 0.0106 & Increased & 2.038 \\
\hline cell viability of melanoma cell lines & 0.00287 & Increased & 2.034 \\
\hline beta-oxidation of lipid & 0.014 & Increased & 2.028 \\
\hline cell death of double-positive thymocyte & 0.00535 & Increased & 2 \\
\hline hippocampal learning & 0.011 & Increased & 2 \\
\hline Diseases or Functions Annotation & p-Value & $\begin{array}{l}\text { Predicted } \\
\text { Activation State }\end{array}$ & $\begin{array}{l}\text { Activation z- } \\
\text { score }\end{array}$ \\
\hline organismal death & 0.000074 & Decreased & -20.188 \\
\hline morbidity or mortality & 0.000068 & Decreased & -19.932 \\
\hline Growth Failure & 0.00277 & Decreased & -11.033 \\
\hline incidence of malignant tumor & 0.00892 & Decreased & -6.336 \\
\hline cell death & 0.0000327 & Decreased & -6.293 \\
\hline cell death of osteosarcoma cells & $\begin{array}{l}0.00000046 \\
4\end{array}$ & Decreased & -6 \\
\hline Movement Disorders & 0.00954 & Decreased & -5.805 \\
\hline midline defect & 0.00194 & Decreased & -5.446 \\
\hline $\begin{array}{l}\text { congenital anomaly of cardiovascular } \\
\text { system }\end{array}$ & 0.00365 & Decreased & -5.027 \\
\hline congenital malformation of brain & 0.00639 & Decreased & -4.978 \\
\hline $\begin{array}{l}\text { congenital anomaly of central nervous } \\
\text { system }\end{array}$ & 0.00731 & Decreased & -4.978 \\
\hline congenital heart disease & 0.012 & Decreased & -4.875 \\
\hline incidence of lymphoma & 0.014 & Decreased & -4.829 \\
\hline neural tube defect & 0.00998 & Decreased & -4.788 \\
\hline kyphosis & 0.00517 & Decreased & -4.737 \\
\hline apoptosis & 0.000114 & Decreased & -4.721 \\
\hline exencephaly & 0.00568 & Decreased & -4.577 \\
\hline
\end{tabular}




\begin{tabular}{|c|c|c|c|}
\hline ventricular septal defect & 0.000324 & Decreased & -4.231 \\
\hline incidence of lung tumor & 0.0143 & Decreased & -4.042 \\
\hline necrosis & 0.0069 & Decreased & -3.81 \\
\hline non-melanoma solid tumor & 0.00000221 & Decreased & -3.115 \\
\hline familial cardiovascular disease & 0.0142 & Decreased & -3.087 \\
\hline damage of chromosomes & 0.00129 & Decreased & -2.874 \\
\hline neoplasia of epithelial tissue & 0.00000394 & Decreased & -2.827 \\
\hline $\begin{array}{l}\text { perimembranous ventricular septal } \\
\text { defect }\end{array}$ & 0.00492 & Decreased & -2.813 \\
\hline cancer & $1.42 \mathrm{E}-08$ & Decreased & -2.794 \\
\hline $\begin{array}{l}\text { Gastrointestinal Tract Cancer and } \\
\text { Tumors }\end{array}$ & 0.0101 & Decreased & -2.745 \\
\hline malignant solid tumor & $\begin{array}{l}0.00000020 \\
4\end{array}$ & Decreased & -2.697 \\
\hline breakage of chromosomes & 0.00146 & Decreased & -2.65 \\
\hline cell death of cancer cells & 0.0000006 & Decreased & -2.631 \\
\hline necrosis of malignant tumor & $\begin{array}{l}0.00000067 \\
6\end{array}$ & Decreased & -2.631 \\
\hline DNA damage response of cells & 0.0000451 & Decreased & -2.54 \\
\hline tumorigenesis of tissue & 0.00000215 & Decreased & -2.507 \\
\hline quantity of hydrogen peroxide & 0.00615 & Decreased & -2.483 \\
\hline digestive organ tumor & 0.0000111 & Decreased & -2.482 \\
\hline adenocarcinoma & 0.000662 & Decreased & -2.448 \\
\hline liver carcinoma & 0.00825 & Decreased & -2.34 \\
\hline hepatocellular carcinoma & 0.00872 & Decreased & -2.34 \\
\hline epithelial cancer & 0.0000026 & Decreased & -2.255 \\
\hline $\begin{array}{l}\text { entry into interphase of cervical cancer } \\
\text { cell lines }\end{array}$ & 0.00502 & Decreased & -2.236 \\
\hline damage of protein & 0.00000992 & Decreased & -2.219 \\
\hline
\end{tabular}




$\begin{array}{llll}\text { abdominal neoplasm } & 1.76 \mathrm{E}-08 & \text { Decreased } & -2.204 \\ \text { cell death of tumor cells } & 0.00000037 & \text { Decreased } & -2.195 \\ & 4 & & -2.195 \\ \text { necrosis of tumor } & 0.00000061 & \text { Decreased } & -2.137 \\ \begin{array}{l}\text { DNA damage response of tumor cell } \\ \text { lines }\end{array} & 0.00247 & \text { Decreased } & -2.084 \\ \begin{array}{l}\text { digestive system cancer } \\ \text { hepatobiliary system cancer }\end{array} & 0.0000244 & \text { Decreased } & -2.056 \\ \text { necrosis of brain cancer cell lines } & 0.0141 & \text { Decreased } & -2.042\end{array}$


Table S6.2.1: Listing of all disease and biological pathways with a positive or negative z-score greater than the absolute value of 2 for $\mathrm{H} 3 \mathrm{~K} 4 \mathrm{me} 3$. 
Table S6.2.2

H3K27me3 Disease and Biological Functions List

\begin{tabular}{|c|c|c|c|}
\hline Diseases or Functions Annotation & $P$-Value & $\begin{array}{l}\text { Predicted Activation } \\
\text { State }\end{array}$ & $\begin{array}{l}\text { Activation z- } \\
\text { score }\end{array}$ \\
\hline transcription of DNA & $8.38 \mathrm{E}-08$ & Increased & 2.656 \\
\hline $\begin{array}{l}\text { activation of DNA endogenous } \\
\text { promoter }\end{array}$ & $6.94 \mathrm{E}-09$ & Increased & 2.572 \\
\hline transcription of RNA & 0.0000023 & Increased & 2.483 \\
\hline Diseases or Functions Annotation & $P$-Value & $\begin{array}{l}\text { Predicted Activation } \\
\text { State }\end{array}$ & $\begin{array}{l}\text { Activation z- } \\
\text { score }\end{array}$ \\
\hline organismal death & 0.000264 & Decreased & -4.076 \\
\hline morbidity or mortality & 0.000106 & Decreased & -3.87 \\
\hline Growth Failure & 0.0000695 & Decreased & -3.132 \\
\hline weight loss & 0.00158 & Decreased & -2.236 \\
\hline
\end{tabular}


Table S6.2.2: Listing of all disease and biological pathways with a positive or negative z-score greater than the absolute value of 2 for $\mathrm{H} 3 \mathrm{~K} 27 \mathrm{me} 3$. 
Table S6.2.3

\section{RNA Transcript Disease and Biological Functions List}

\begin{tabular}{|c|c|c|c|}
\hline Diseases or Functions Annotation & $P$-Value & $\begin{array}{l}\text { Predicted Activation } \\
\text { State }\end{array}$ & $\begin{array}{l}\text { Activation z- } \\
\text { score }\end{array}$ \\
\hline size of body & 0.000000139 & Increased & 7.093 \\
\hline migration of tumor cell lines & $5.74 \mathrm{E}-08$ & Increased & 4.915 \\
\hline organization of cytoplasm & $6.32 \mathrm{E}-08$ & Increased & 4.865 \\
\hline organization of cytoskeleton & 0.0000006 & Increased & 4.865 \\
\hline microtubule dynamics & 0.00000924 & Increased & 4.513 \\
\hline cell movement of tumor cell lines & $7.56 \mathrm{E}-08$ & Increased & 4.504 \\
\hline cell death of osteosarcoma cells & $1.99 \mathrm{E}-09$ & Increased & 4.32 \\
\hline colony formation of cells & 0.0000099 & Increased & 3.903 \\
\hline development of body trunk & $1.01 \mathrm{E}-10$ & Increased & 3.896 \\
\hline cell proliferation of tumor cell lines & $2.08 \mathrm{E}-10$ & Increased & 3.77 \\
\hline cell movement & $9.27 \mathrm{E}-15$ & Increased & 3.71 \\
\hline $\begin{array}{l}\text { quantity of hematopoietic progenitor } \\
\text { cells }\end{array}$ & 0.000000765 & Increased & 3.694 \\
\hline quantity of cells & $5.04 \mathrm{E}-08$ & Increased & 3.634 \\
\hline migration of cells & $8 \mathrm{E}-14$ & Increased & 3.563 \\
\hline cell viability & $7.15 \mathrm{E}-12$ & Increased & 3.526 \\
\hline cell survival & $1.63 \mathrm{E}-12$ & Increased & 3.513 \\
\hline outgrowth of cells & 0.000041 & Increased & 3.435 \\
\hline colony formation & 0.00000244 & Increased & 3.394 \\
\hline cancer & $1.7 \mathrm{E}-41$ & Increased & 3.346 \\
\hline size of animal & $1.71 \mathrm{E}-08$ & Increased & 3.342 \\
\hline
\end{tabular}




\begin{tabular}{|c|c|c|c|}
\hline branching of cells & 0.0000051 & Increased & 3.193 \\
\hline quantity of erythroid precursor cells & 0.0000343 & Increased & 3.146 \\
\hline migration of endothelial cells & 2.65E-09 & Increased & 3.103 \\
\hline cell movement of endothelial cells & 1.27E-09 & Increased & 3 \\
\hline cell viability of tumor cell lines & 0.000000024 & Increased & 2.975 \\
\hline quantity of heavy metal & 0.000000869 & Increased & 2.954 \\
\hline synthesis of reactive oxygen species & 0.0000329 & Increased & 2.939 \\
\hline transport of molecule & 0.0000013 & Increased & 2.898 \\
\hline growth of neurites & 0.0000144 & Increased & 2.89 \\
\hline $\begin{array}{l}\text { cell proliferation of carcinoma cell } \\
\text { lines }\end{array}$ & 0.0000533 & Increased & 2.873 \\
\hline quantity of blood cells & $5.96 \mathrm{E}-10$ & Increased & 2.837 \\
\hline infection by HIV-1 & 0.0000122 & Increased & 2.82 \\
\hline cell transformation & 2.87E-08 & Increased & 2.819 \\
\hline HIV infection & 0.0000097 & Increased & 2.819 \\
\hline hydrolysis of nucleotide & 0.0000349 & Increased & 2.778 \\
\hline $\begin{array}{l}\text { activation of DNA endogenous } \\
\text { promoter }\end{array}$ & $1.13 \mathrm{E}-14$ & Increased & 2.776 \\
\hline development of head & 0.0000181 & Increased & 2.76 \\
\hline $\begin{array}{l}\text { movement of vascular endothelial } \\
\text { cells }\end{array}$ & 0.00000163 & Increased & 2.749 \\
\hline infection of cells & 0.00000573 & Increased & 2.74 \\
\hline $\begin{array}{l}\text { migration of vascular endothelial } \\
\text { cells }\end{array}$ & 0.00000714 & Increased & 2.699 \\
\hline transcription of RNA & $6.69 \mathrm{E}-19$ & Increased & 2.678 \\
\hline transport of metal & 0.0000371 & Increased & 2.662 \\
\hline
\end{tabular}




\begin{tabular}{|c|c|c|c|}
\hline infection by RNA virus & 0.00000199 & Increased & 2.626 \\
\hline invasion of cells & 0.000012 & Increased & 2.624 \\
\hline sprouting & $3.93 \mathrm{E}-08$ & Increased & 2.568 \\
\hline transcription & $5.36 \mathrm{E}-17$ & Increased & 2.559 \\
\hline $\begin{array}{l}\text { metabolism of reactive oxygen } \\
\text { species }\end{array}$ & 0.00000685 & Increased & 2.524 \\
\hline respiratory system development & 0.000000988 & Increased & 2.523 \\
\hline formation of lung & 0.0000032 & Increased & 2.514 \\
\hline infection of cervical cancer cell lines & 0.00000589 & Increased & 2.505 \\
\hline transport of heavy metal & 9.84E-09 & Increased & 2.491 \\
\hline development of body axis & 0.0000275 & Increased & 2.474 \\
\hline differentiation of embryonic cells & $8.82 \mathrm{E}-08$ & Increased & 2.468 \\
\hline advanced malignant tumor & 0.0000156 & Increased & 2.459 \\
\hline Viral Infection & $1.81 \mathrm{E}-13$ & Increased & 2.455 \\
\hline metastasis & 0.0000307 & Increased & 2.369 \\
\hline necrosis of malignant tumor & $2.54 \mathrm{E}-10$ & Increased & 2.307 \\
\hline cell death of cancer cells & $7.85 \mathrm{E}-10$ & Increased & 2.307 \\
\hline quantity of connective tissue & 0.00000002 & Increased & 2.265 \\
\hline quantity of red blood cells & $8.08 \mathrm{E}-08$ & Increased & 2.198 \\
\hline transcription of DNA & 7.81E-18 & Increased & 2.184 \\
\hline development of vasculature & $1.79 \mathrm{E}-18$ & Increased & 2.18 \\
\hline thrombocytosis & 0.0000028 & Increased & 2.178 \\
\hline angiogenesis & $1.06 \mathrm{E}-16$ & Increased & 2.164 \\
\hline spherocytosis & $3.06 \mathrm{E}-08$ & Increased & 2.159 \\
\hline
\end{tabular}




\begin{tabular}{|c|c|c|c|}
\hline $\begin{array}{l}\text { proliferation of connective tissue } \\
\text { cells }\end{array}$ & $4.28 \mathrm{E}-11$ & Increased & 2.136 \\
\hline necrosis of tumor & $9.88 \mathrm{E}-11$ & Increased & 2.121 \\
\hline cell death of tumor cells & $6.86 \mathrm{E}-10$ & Increased & 2.121 \\
\hline growth of connective tissue & $2.76 \mathrm{E}-10$ & Increased & 2.118 \\
\hline cell proliferation of fibroblasts & $4.96 \mathrm{E}-11$ & Increased & 2.116 \\
\hline expression of RNA & $1.9 \mathrm{E}-16$ & Increased & 2.085 \\
\hline transactivation & $2.22 \mathrm{E}-08$ & Increased & 2.016 \\
\hline transactivation of RNA & $5.26 \mathrm{E}-08$ & Increased & 2.002 \\
\hline Diseases or Functions Annotation & $P$-Value & $\begin{array}{l}\text { Predicted Activation } \\
\text { State }\end{array}$ & $\begin{array}{l}\text { Activation z- } \\
\text { score }\end{array}$ \\
\hline organismal death & $5.59 \mathrm{E}-27$ & Decreased & -10.051 \\
\hline morbidity or mortality & $4.69 \mathrm{E}-27$ & Decreased & -9.991 \\
\hline perinatal death & 0.000000536 & Decreased & -6.598 \\
\hline Bleeding & $1.41 \mathrm{E}-09$ & Decreased & -5.689 \\
\hline neonatal death & 0.00000164 & Decreased & -5.583 \\
\hline Growth Failure & 0.00000029 & Decreased & -5.542 \\
\hline Edema & 0.0000237 & Decreased & -5.48 \\
\hline $\begin{array}{l}\text { motor dysfunction or movement } \\
\text { disorder }\end{array}$ & 0.000000629 & Decreased & -4.374 \\
\hline Movement Disorders & 0.000000347 & Decreased & -3.935 \\
\hline death of perinatal stage organism & 0.0000385 & Decreased & -3.328 \\
\hline familial vascular disease & 0.0000265 & Decreased & -2.789 \\
\hline hypoalbuminemia & 0.0000374 & Decreased & -2.449 \\
\hline adhesion of cell-associated matrix & 0.0000211 & Decreased & -2.414 \\
\hline
\end{tabular}




\begin{tabular}{|c|c|c|c|}
\hline senescence of cells & 0.000000606 & Decreased & -2.314 \\
\hline adhesion of extracellular matrix & 0.0000059 & Decreased & -2.17 \\
\hline $\begin{array}{l}\text { congenital anomaly of central } \\
\text { nervous system }\end{array}$ & 0.0000163 & Decreased & -2.144 \\
\hline congenital encephalopathy & 0.0000293 & Decreased & -2.144 \\
\hline congenital malformation of brain & 0.0000402 & Decreased & -2.144 \\
\hline intestinal cancer & $3.74 \mathrm{E}-29$ & Decreased & -2.066 \\
\hline familial cardiovascular disease & 0.0000235 & Decreased & -2.035 \\
\hline apoptosis & $7.86 \mathrm{E}-19$ & Decreased & -2.012 \\
\hline diffuse lymphoma & 0.00000118 & Decreased & -2 \\
\hline
\end{tabular}


Table S6.2.3: Listing of all disease and biological pathways with a positive or negative z-score greater than the absolute value of 2 for RNA. 
Table S6.2.4

\begin{tabular}{|c|c|c|c|}
\hline \multicolumn{4}{|c|}{ Primer Sequences } \\
\hline Experiment & Primer Name & Orientation & Sequence \\
\hline \multirow{6}{*}{ mRNA-qPCR } & Fgfr1 & $\mathrm{F}$ & GAAAGCAACCGTACACGCA \\
\hline & Fgfr1 & $\mathrm{R}$ & CGGTTTGGTTTGGTGTTGTCT \\
\hline & Il-18 & $\mathrm{F}$ & TCAGACCACTTTGGCAGACTT \\
\hline & Il-18 & $\mathrm{R}$ & CACAGGCGGGTTTCTTTTGTC \\
\hline & Tgfbr2 & $\mathrm{F}$ & ATCCTAGTAAGAAGCGATCTAACC \\
\hline & Tgfbr2 & $\mathrm{R}$ & GTCTGCTTGAAGGACTCCATGT \\
\hline \multirow{16}{*}{ ChIP-qPCR } & Tgfbr2 8043 & & GTGTTTCACCCCAAATCCAC \\
\hline & Tgfbr2 8043 & & ATCACTGGCTTTCCATGACC \\
\hline & Tgfbr2 8243 & $\mathrm{~F}$ & GGACTGTCCTGCTCTTTTGC \\
\hline & Tgfbr2 8243 & $\mathrm{R}$ & CGGAAACGGGAAGTTTGTAG \\
\hline & Tgfbr2 8443 & $\mathrm{~F}$ & AGCGAGGAGCCCTAGTGAAC \\
\hline & Tgfbr2 8443 & $\mathrm{R}$ & GTTCCCAAGTCGGGTGAGT \\
\hline & Tgfbr2 8643 & $\mathrm{~F}$ & CCTTTATTCCTCGCCCTCTC \\
\hline & Tgfbr2 8643 & $\mathrm{R}$ & CTCCTGCGCAGCTCTCCT \\
\hline & Tgfbr2 8843 & $\mathrm{~F}$ & GGGAGGGAAACAGGAAACTC \\
\hline & Tgfbr2 8843 & $\mathrm{R}$ & AGGAGAGGACCGGAGCTG \\
\hline & Tgfbr2 9043 & $\mathrm{~F}$ & GGATTGGACCTTGAGGGACT \\
\hline & Tgfbr2 9043 & $\mathrm{R}$ & CGTGCGCTTAGTCACTTCTG \\
\hline & Tgfbr2 9243 & F & CAGGAGGGAGCTCTGAAATG \\
\hline & Tgfbr2 9243 & $\mathrm{R}$ & CGCATGCATGTCCATAAAAC \\
\hline & Tgfbr2 9443 & $\mathrm{~F}$ & GGATTGGAAGGACCATTTCC \\
\hline & Tgfbr2 9443 & $\mathrm{R}$ & CGATCACAGGGCCAACTATT \\
\hline
\end{tabular}


Table S6.2.4: Primer design for qPCR experiments. Primers for the mRNA-qPCR experiments were designed using Primer-BLAST (https://www.ncbi.nlm.nih.gov/tools/primer-blast/). MRNA primers were designed to produced amplicons $100-250 \mathrm{bp}$ in length. ChIP-qPCR primers were designed spanning a 1,400 bp loci in the promoter region of the Tgfbr2 gene. Primers were designed to measure chromatin H3K4me3 modifications every $200 \mathrm{bp}$. Primer sequences were constrained to 60-100 bp amplicon lengths, in order to appropriately span sheered chromatin. All primer designs were performed against the Rattus norvegicus July 2014 (RGSC 6.0/rn6) genome build. ChIP $=$ Chromatin Immunoprecipitation 


\author{
List of Abbreviations \\ ENM Engineered Nanomaterials \\ ChIP Chromatin Immunoprecipitation \\ GD Gestational Day \\ H3K4me3 3 lysine 4 tri-methylation \\ H3K27me3 3 lysine 27 tri-methylation \\ IPA Ingenuity Pathway Analysis \\ DOHad Developmental Origin of Health and Disease \\ LogFC Log Fold Change \\ IACUC Institutional Animal Care and Use Committee \\ RIN RNA Integrity Number \\ FDR False Discovery Rate \\ NF-кB Nuclear Factor kappa-light-chain-enhancer of activated B cells \\ AKT Protein Kinase B \\ VCAM-1 Vascular Cell Adhesion Molecule-1 \\ ICAM-1 Intercellular Adhesion Molecule-1 \\ LTBR Lymphotoxin Beta Receptor \\ IKK $\alpha \quad$ Inhibitor of NF-KB Kinase Subunit Alpha \\ IL-8 Interleukin 8 \\ NOS Nitric Oxide Synthase \\ Fgfr1 Fibroblast Growth Factor Receptor 1 \\ Il-18 Interleukin-18 \\ Tgfbr2 Transforming Growth Factor Beta Receptor 2
}


Car1 Carbonic anhydrase 1

qPCR quantitative Polymerase Chain Reaction

REL Recommended Exposure Limit

PEL Permissible Exposure Limit

Wnt5a Wnt Family Member 5A

Rn5-8s 5.8S ribosomal RNA for Rattus norvegicus 


\section{Section 2: Supporting Documentation for Specific Aim 2}

\section{Repository for Cardiovascular Studies}

Primary design and execution of IRB Surgical Tissue Banking protocol \# 1812394926A001 (Figure 6.3.1) 
Figure 6.3.1

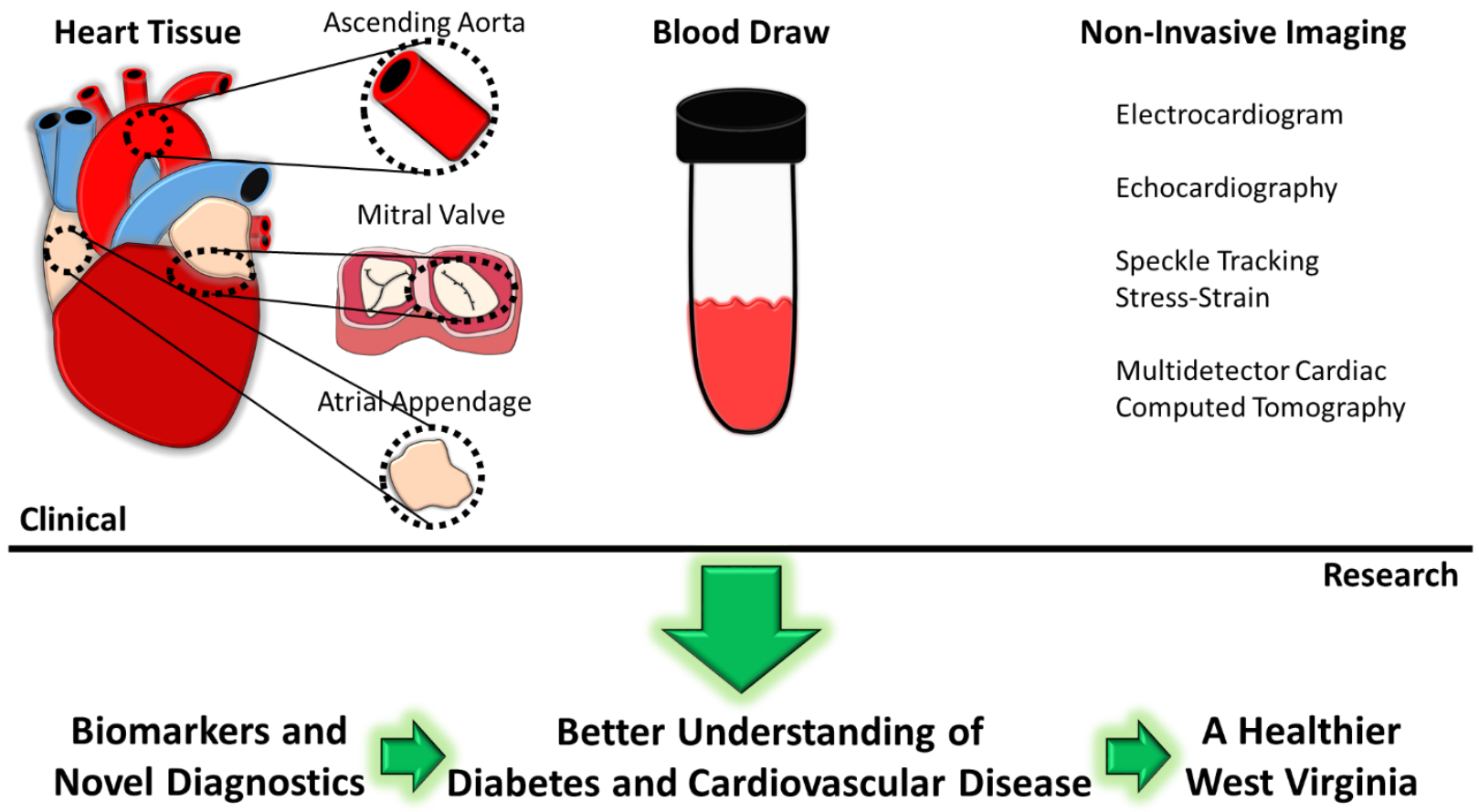


Figure 6.3.1: Samples collected include right atrial appendages, mitral valve tissue, ascending aorta, and other tissues routinely discarded during open heart and/or valvular surgeries. Blood (10 $\mathrm{mL}$ preoperatively and postoperatively from standard of care venipuncture), noninvasive cardiovascular imaging data, and/or other relevant medical records. 


\section{Section 3: Supporting Documentation for Specific Aim 3}

\section{Transgenic Animal Models}

Because we are interested in better understanding the in vivo consequences of PNPase manipulation to alterations in mitochondrial function and regulatory networks, we are begun the production of a transgenic murine line. Through a single-stranded ssDNA donor CRISPR/Cas9 approach $(3,4)$ we have been successful in targeting both the upstream (Figure 6.4.1A) and downstream (Figure 6.4.1B) sgRNA region in the introns surrounding Exon 23 of PNPT1. The single-stranded DNA oligonucleotide (ssDNA) (Figure 6.4.2) was created through Genewiz (South Plainfield, NJ) and the gRNA were designed through CHOPCHOP $(1,2)$ (Figure 6.4.3). Currently, we have found animals heterozygous for the homology direct repair ssDNA insert (Figure 6.4.4). Embryo microinjections were performed by the Genetically Engineered Murine Model (GEMM) Core at The University of Virginia. After the validation of the KH knockout, we plan to continue with the design and development of the S1 knockout mouse model.

\section{CRISPR/Cas9 Cell Models}

Using a CRISPR/Cas9 approach, we have designed gRNA (Figure 6.3.5), using CHOPCHOP, to be incorporated into plasmids purchased from Addgene (Watertown, MA) (pSpCas9(BB)-2A-GFP (PX458) was a gift from Feng Zhang (Addgene plasmid \# 48138 ; http://n2t.net/addgene:48138 ; RRID:Addgene_48138), pSpCas9(BB)-2A-Puro (PX459) V2.0 was a gift from Feng Zhang (Addgene plasmid \# 62988 ; http://n2t.net/addgene:62988 ; RRID:Addgene_62988)). The sgRNA was cloned using the guidelines from Ran et al. (5). 


\section{References}

1. Labun K, Guo X, Chavez A, Church G, Gagnon JA, and Valen E. Accurate analysis of genuine CRISPR editing events with ampliCan. Genome Res, 2019.

2. Labun K, Montague TG, Gagnon JA, Thyme SB, and Valen E. CHOPCHOP v2: a web tool for the next generation of CRISPR genome engineering. Nucleic Acids Res 44: W272-276, 2016.

3. Miura H, Quadros RM, Gurumurthy CB, and Ohtsuka M. Easi-CRISPR for creating knock-in and conditional knockout mouse models using long ssDNA donors. Nat Protoc 13: 195215, 2018.

4. Quadros RM, Miura H, Harms DW, Akatsuka H, Sato T, Aida T, Redder R, Richardson GP, Inagaki Y, Sakai D, Buckley SM, Seshacharyulu P, Batra SK, Behlke MA, Zeiner SA, Jacobi AM, Izu Y, Thoreson WB, Urness LD, Mansour SL, Ohtsuka M, and Gurumurthy CB. Easi-CRISPR: a robust method for one-step generation of mice carrying conditional and insertion alleles using long ssDNA donors and CRISPR ribonucleoproteins. Genome Biol 18: 92, 2017.

5. Ran FA, Hsu PD, Wright J, Agarwala V, Scott DA, and Zhang F. Genome engineering using the CRISPR-Cas9 system. Nat Protoc 8: 2281-2308, 2013. 
Figure 6.4.1

\section{A Upstream sgRNA site}

GCATGTGCCATATT GTAT GTCCCGGGTCCAACTTGTG

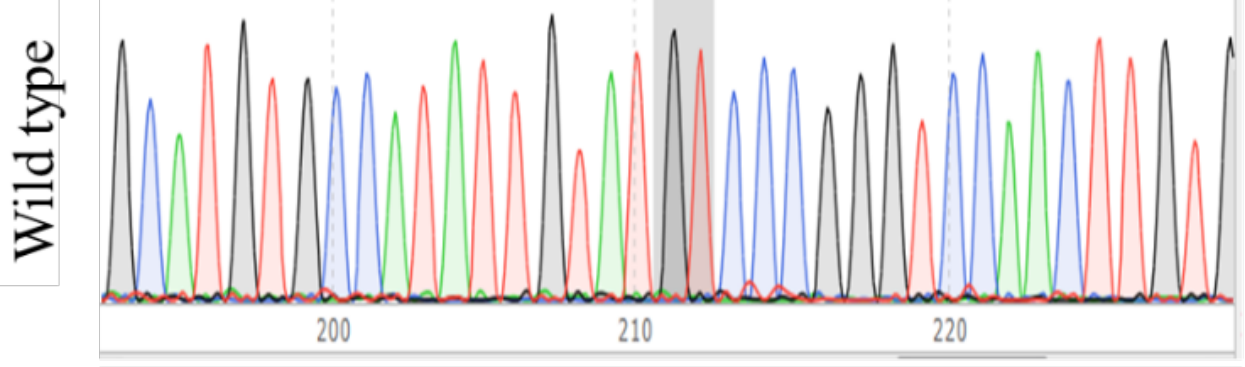

GCWTGT GSSAYWT KRT WMWAYYYKYSSRCCTTRWKWR

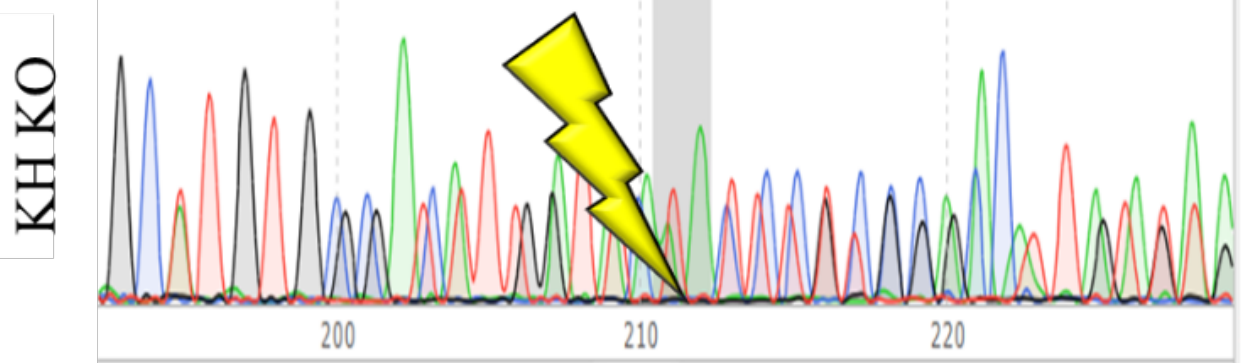

B

\section{Downstream sgRNA site}

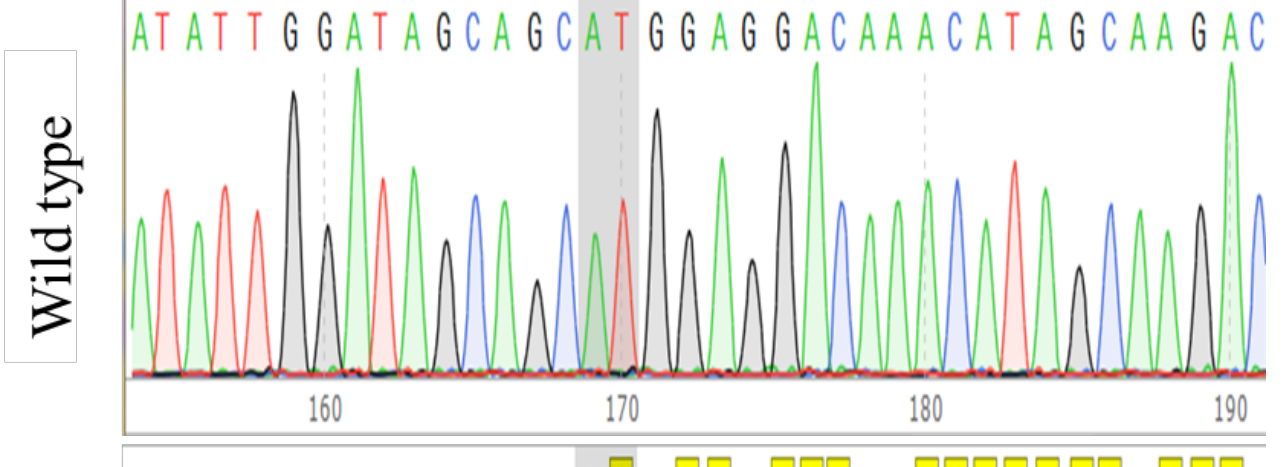

ATATT GGATAGCAGCAKGRRGRMAMMWWR SMARMC

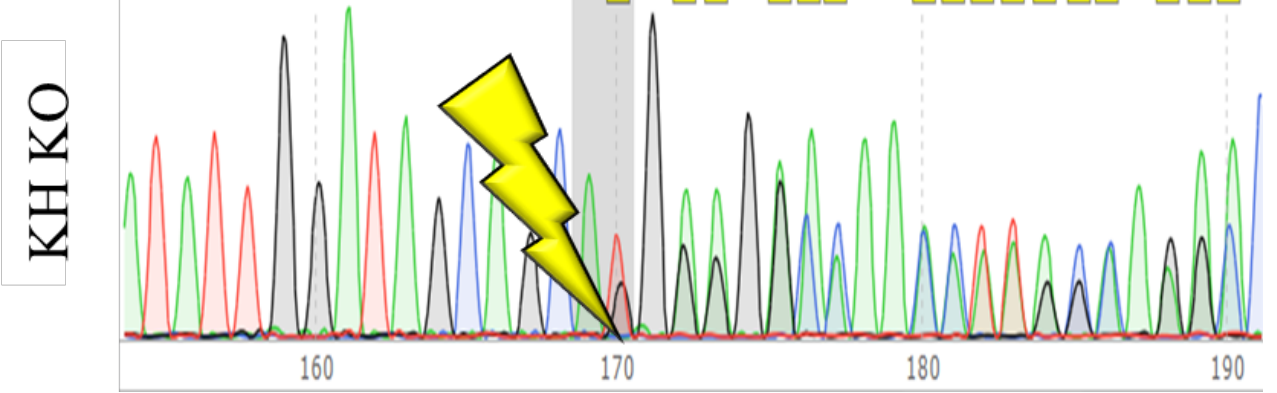


Figure 6.4.1: Sanger sequencing displaying regions surrounding PNPT1 exon 23 where the sgRNA were designed to target. (A) The upstream site (intron 22) showing both the wild type (top) and targeted (bottom) sites. (B) The upstream site (intron 23) showing both the wild type (top) and targeted (bottom) sites. Mutations, due to non-homologous end-joining, were produced in the resulting DNA strand. $\mathrm{KH} \mathrm{KO}=\mathrm{KH}$ RNA binding domain (exon 23, GxxG motif) removed from full length PNPase. 
Figure 6.4.2

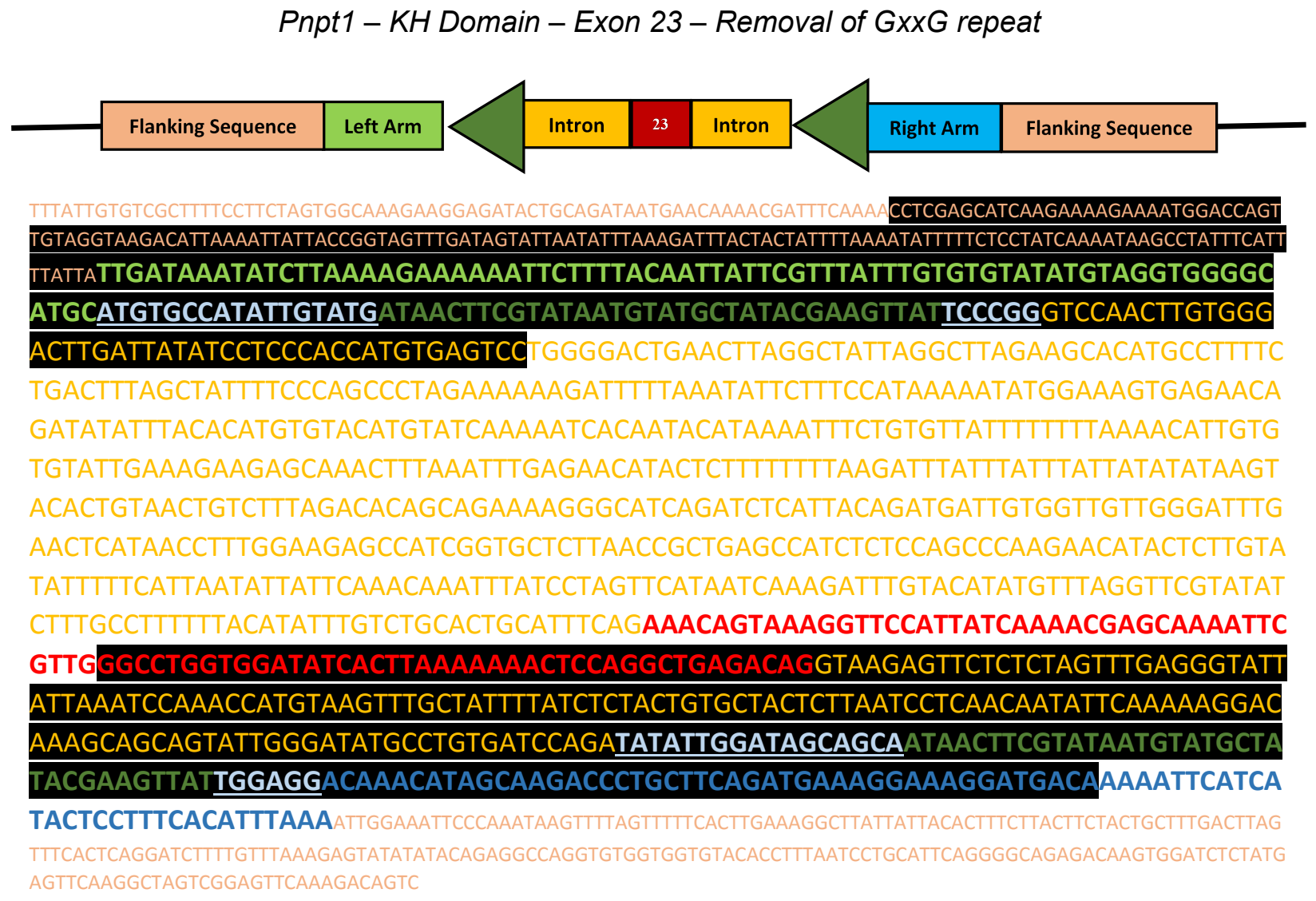

Amplicons for genotyping are highlighted in black. The underlined light blue region is the gRNA and separation where the loxP would theoretically be inserted. 
Figure 6.4.2: Bioinformatic approach for the creation of a conditional knockout animal. Through the homologous recombination of the single-stranded DNA (ssDNA), LoxP sites are inserted into the PNPT1 introns 22 and 23. Genotyping of animals was accomplished through designing primers that flanked the 5' and 3' LoxP sites, in order to determine if the sgRNA targeted the sequence and successful recombination occurred. 


\section{Figure 6.4.3}

Gene: Polynucleotide phosphorylase (Pnpt1 or PNPase)

Location: chr11:29,130,751-29,161,828 (UCSC Genome Browser GRCm38/mm10) Interest: Remove exon 23 of PNPase

\section{CHOP CHOP:}

Intron 22: chr11:29154899-29155713: $\quad$ ATGTGCCATATTGTATGTCCCGG

Intron 23: chr11:29155796-29156835: TATATTGGATAGCAGCATGGAGG

PAM Sequence $=$ RED 
Figure 6.4.3: Design of sgRNA primers for targeting introns 22 and 23 of PNPT1. Using CHOPCHOP, sgRNA with the highest ranking in each of the locations was chosen. UCSC Genome Browser GRCm38/mm10 was implemented to provide the genomic location, as well as SNPs that may occur in FVB/NJ mice. 
Figure 6.4.4

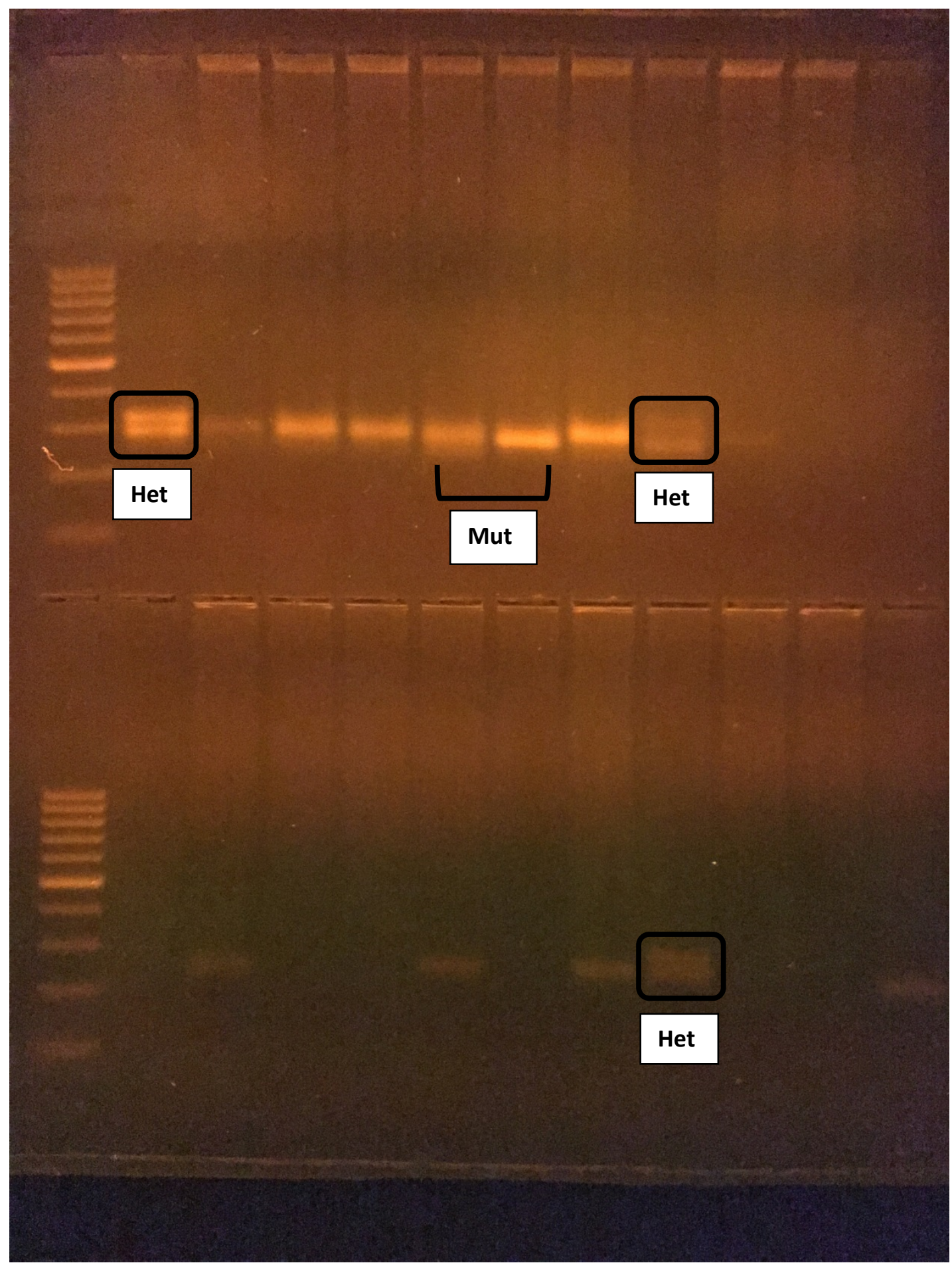


Figure 6.4.4: Genotyping for the KH RNA binding domain knockout using 2\% agarose gel electrophoresis. Amplification of the intronic insertation site of the single-stranded DNA (ssDNA) allowed for identification of animals with targeted DNA. The analysis revealed at least one animal containing heterozygous regions for both the upstream and downstream LoxP insertion site. Het $=$ heterozygous bands, Mut = mutations, likely the result from non-homologous end-joining. 
Figure 6.4.5

\begin{abstract}
Cloning Primers
FL Pnpt1 Ko 1 F: CACCGGCTGAGTGCCCGATTCCGCC

FL Pnpt1 KO 1 R: AAACCCGCCTTAGCCCGTGAGTCGC

FL Pnpt1 KO 2 F: CACCGgtTtTagGgGCAgTACGAat

FL Pnpt1 KO 2 R: AAACTAAGCATGACGGGGATTTTGC

RNA Pnpt1 KO 1 F: CACCGCGAGCAAAATTCGTTGGGCC

RNA Pnpt1 KO 1 R: AAACCCGGGTTGCTTAAAACGAGCC

RNA Pnpt1 KO 2 F: CACCGCTTACCTGACGCTTGTTGGA

RNA Pnpt1 KO 2 R: AAACAGGTTGTTCGCAGTCCATTCC
\end{abstract}

Sanger Sequencing Primers

hU6-F: GAGGGCCTATTTCCCATGATT Region: 1 .. 21

LKO.1 5': GACTATCATATGCTTACCGTRegion: 172 .. 191 
Figure 6.4.5: CRISPR/Cas9 approach for HL-1 cell line knockout. Using all-in-one Cas9/sgRNA plasmids, sgRNA was designed to target exon 1of PNPT1 (likely resulting in complete knockout of the protein as well as exon 23 (KH RNA binding domain, GxxG motif). In order to determine the proper cloning and orientation of Cas9/sgRNA plasmids, Sanger sequencing primers targeting the hU6 and LKO.1 5' region were designed for pSpCas9(BB)-2A-GFP (PX458) and pSpCas9(BB)-2A-Puro (PX459). FL = full length PNPase knockout targeting exon 1, KH $=\mathrm{KH}$ RNA binding domain knockout targeting exon 23. 


\section{Quincy A. Hathaway - Curriculum Vitae}

179 Hathaway Road, Jefferson, PA 15344 | 724-255-4637 | qahathaway@mix.wvu.edu

\section{Education}

MEDICAL DOCTOR | MAY 2023 | WEST VIRGINIA UNIVERSITY

- Medical student in the Class of 2023

DOCTOR OF PHILOSOPHY | MAY 2019 | WEST VIRGINIA UNIVERSITY

- GPA -4.0

- Graduate Degree: Applied and Molecular Pathophysiology (Division of Exercise Physiology)

BACHELOR OF SCIENCE | MAY 2015 | WAYNESBURG UNIVERSITY

- Valedictorian: GPA -4.0

- Major: B.S. Biology (Summa Cum Laude), B.S. Environmental Science (Summa Cum Laude)

- Minor: Chemistry

- Clubs/Organizations: ACS member, member of the Ecological Stewards and Biology clubs, and Habitat for Humanity Greene County Chapter member

HIGH SCHOOL DIPLOMA | JUNE 2011 | CARMICHAELS AREA HIGH SCHOOL

- Salutatorian: GPA - 3.98

- President of the Recycling Committee, Member of The National Honor Society, Newspaper Staff, Yearbook Staff, Envirothon Team, and Ralph K. Bell Bird Club Member

\section{Publications in Review}

Dysfunctional mitochondrial respiration can be targeted to induce death in drug resistant B-cell acute lymphoblastic leukemia. Nair RR, Piktel D, Thomas P, Hathaway QA, Rellick SL, Sarkar P, Martin K, Geldenhuys WJ, Hollander JM, Gibson GF. J Biol Chem. Submitted: December 14 2018.

\section{Accepted Publications}

ROS promote epigenetic remodeling and cardiac dysfunction in offspring following maternal engineered nanomaterial (ENM) exposure.

Kunovac A, Hathaway QA, Pinti MV, Goldsmith WT, Durr AJ, Fink GK, Nurkiewicz TR, Hollander JM.

Part Fibre Toxicol. 2019 Jun 18;16(1):24. doi: 10.1186/s12989-019-0310-8. PMID: 31215478

Machine-learning to stratify diabetic patients using novel cardiac biomarkers and integrative genomics. Hathaway QA, Roth SM, Pinti MV, Sprando DC, Kunovac A, Durr AJ, Cook CC, Fink GK, Cheuvront TB, Grossman JH, Aljahli GA, Taylor AD, Giromini AP, Allen JL, Hollander JM.

Cardiovasc Diabetol. 2019 Jun 11;18(1):78. doi: 10.1186/s12933-019-0879-0. PMID: 31185988 
miRNA-378a as a key regulator of cardiovascular health following engineered nanomaterial inhalation exposure.

Hathaway QA, Durr AJ, Shepherd DL, Pinti MV, Brandebura AN, Nichols CE, Kunovac A, Goldsmith WT, Friend SA, Abukabda AB, Fink GK, Nurkiewicz TR, Hollander JM.

Nanotoxicology. 2019 Jun;13(5):644-663. doi: 10.1080/17435390.2019.1570372. Epub 2019 Feb 1. PMID: 30704319

Mitochondrial Dysfunction in Type 2 Diabetes Mellitus: An Organ-Based Analysis. Pinti MV, Fink GK, Hathaway QA, Durr AJ, Kunovac A, Hollander JM.

Am J Physiol Endocrinol Metab. 2019 Feb 1;316(2):E268-E285. doi: 10.1152/ajpendo.00314.2018. Epub 2019 Jan 2. PMID: 30601700

Mitochondrial proteome disruption in the diabetic heart through targeted epigenetic regulation at the mitochondrial heat shock protein 70 (mtHsp70) nuclear locus.

Shepherd DL*, Hathaway QA*, Nichols CE, Durr AJ, Pinti MV, Hughes KM, Kunovac A, Stine SM, Hollander JM.

J Mol Cell Cardiol. 2018 Jun;119:104-115. doi: 10.1016/j.yjmcc.2018.04.016. Epub 2018 May 4. PMID: 29733819

Maternal engineered nanomaterial inhalation during gestation alters the fetal transcriptome. Stapleton PA*, Hathaway QA*, Nichols CE, Abukabda AB, Pinti MV, Shepherd DL, McBride CR, Yi J, Castranova VC, Hollander JM, Nurkiewicz TR.

Part Fibre Toxicol. 2018 Jan 10;15(1):3. doi: 10.1186/s12989-017-0239-8. PMID: 29321036

Reactive oxygen species damage drives cardiac and mitochondrial dysfunction following acute nanotitanium dioxide inhalation exposure.

Nichols CE, Shepherd DL, Hathaway QA, Durr AJ, Thapa D, Abukabda A, Yi J, Nurkiewicz TR, Hollander JM.

Nanotoxicology. 2018 Feb;12(1):32-48. doi: 10.1080/17435390.2017.1416202. Epub 2017 Dec 15. PMID: 29243970

Regulating microRNA expression: at the heart of diabetes mellitus and the mitochondrion. Hathaway QA, Pinti MV, Durr AJ, Waris S, Shepherd DL, Hollander JM.

Am J Physiol Heart Circ Physiol. 2018 Feb 1;314(2):H293-H310. doi: 10.1152/ajpheart.00520.2017. Epub 2017 Oct 6. Review. PMID: 28986361

Exploring the mitochondrial microRNA import pathway through Polynucleotide Phosphorylase (PNPase).

Shepherd DL*, Hathaway QA*, Pinti MV, Nichols CE, Durr AJ, Sreekumar S, Hughes KM, Stine SM, Martinez I, Hollander JM.

J Mol Cell Cardiol. 2017 Sep;110:15-25. doi: 10.1016/j.yjmcc.2017.06.012. Epub 2017 Jul 11. PMID: 28709769

Maternal-engineered nanomaterial exposure disrupts progeny cardiac function and bioenergetics. 
Hathaway QA, Nichols CE, Shepherd DL, Stapleton PA, McLaughlin SL, Stricker JC, Rellick SL, Pinti MV, Abukabda AB, McBride CR, Yi J, Stine SM, Nurkiewicz TR, Hollander JM.

Am J Physiol Heart Circ Physiol. 2017 Mar 1;312(3):H446-H458. doi: 10.1152/ajpheart.00634.2016. Epub 2016 Dec 23. PMID: 28011589

Role of microRNA in metabolic shift during heart failure.

Pinti MV, Hathaway QA, Hollander JM.

Am J Physiol Heart Circ Physiol. 2017 Jan 1;312(1):H33-H45. doi: 10.1152/ajpheart.00341.2016. Epub

2016 Oct 14. Review. PMID: 27742689

*These authors contributed equally

\section{Fellowship Awards}

\section{AMERICAN HEART ASSOCIATION - PREDOCTORAL FELLOWSHIP}

Project Title: "Mitochondrial microRNA Import and Regulation"

Awarded: July $1^{\text {st }}, 2017$ - July $1^{\text {st }}, 2019-$ ACCEPTED

\section{NATIONAL SCIENCE FOUNDATION- IGERT (INTEGRATIVE GRADUATE EDUCATION AND RESEARCH TRAINING)}

Research and Education in Nanotoxicology at West Virginia University

Project Title: "How pathological conditions within the heart shape the epigenetic landscape of the tissue, specifically in the control of factors important to bioenergetics"

Awarded: August $15^{\text {th }}, 2015$ - August $15^{\text {th }}, 2017$ - ACCEPTED

Awarded: August $15^{\text {th }}, 2017$ - August $15^{\text {th }}, 2018$ - DECLINED

\section{Research Awards}

\section{OUTSTANDING DOCTORAL STUDENT AWARD - EXERCISE PHYSIOLOGY}

Awarded: April $5^{\text {th }}, 2019$

Award Amount: N/A

\section{3-MINUTE THESIS FINALIST - WVU 2019}

Award Type: Selected as a finalist for the 3-minute thesis competition at WVU, presenting

"Mitochondria, at the Heart of Diabetes".

Awarded: April 5 $5^{\text {th }}, 2019$

Award Amount: \$50 


\section{TOXICOLOGY WORKING GROUP 2018 TRAVEL AWARD - SOT 2018}

Award Type: To present a poster on - "Engineered Nanomaterial Exposure: Effects on Epigenetic and MiRNA Regulation of Cardiac Mitochondria"

Awarded: February $19^{\text {th }}, 2018$

Award Amount: $\$ 700$

APS PG GROUP TRAINEE AWARD - EB 2018

Award Type: Oral presentation on - "Nuclear-Mitochondrial Crosstalk in the Heart during Diabetes Mellitus - The Impact on RNA in Mitochondrial Subpopulations"

Awarded: April $23^{\text {rd }}, 2018$ for $2^{\text {nd }}$ place

Award Amount: $\$ 200$

\section{AMERICAN HEART ASSOCIATION TRAVEL STIPEND}

Award Type: Funds for travel allocated from the Predoctoral fellowship entitled - "Mitochondrial microRNA Import and Regulation"

Awarded: July $1^{\text {st }}, 2017$

Award Amount: $\$ 2000$

\section{RISING STAR AWARD - APPLIED AND MOLECULAR PATHOPHYSIOLOGY (DIVISION OF EXERCISE} PHYSIOLOGY)

Award Type: One recipient per program, recognizing excellence in research for early career Graduate Research Assistants

Awarded: April 5 ${ }^{\text {th }}, 2017$

Award Amount: N/A

\section{Research Presentations}

\section{EXPERIMENTAL BIOLOGY (EB), SAN DIEGO, CA: 2018}

Oral and poster presentation, abstract entitled "Nuclear-Mitochondrial Crosstalk in the Heart during Diabetes Mellitus - The Impact on RNA in Mitochondrial Subpopulations" (April 23 ${ }^{\text {rd }}, 2018$ )

\section{WEST VIRGINIA UNIVERSITY VAN LIERE DAY, MORGANTOWN, WV 2018}

Poster presentation, abstract entitled "Non-coding RNA and the Mitochondrion - Function and Pathology in the Heart" (March 23 ${ }^{\text {rd }}, 2018$ )

\section{SOCIETY OF TOXICOLOGY (SOT), SAN ANTONIO, TX: 2018}

Poster presentation, abstract entitled "Engineered Nanomaterial Exposure: Effects on Epigenetic and MiRNA Regulation of Cardiac Mitochondria" (March 14 ${ }^{\text {th }}, 2018$ ) 
EXPERIMENTAL BIOLOGY (EB), CHICAGO, IL: 2017

Poster presentation, abstract entitled "Developing a Link between Cardiac Function and Maternal Engineered Nanomaterial Exposure in Growing Progeny" (April 24 ${ }^{\text {th }}, 2017$ )

WEST VIRGINIA UNIVERSITY TOXICOLOGY WORKING GROUP, MORGANTOWN, WV: 2017

Oral Presentation, talk entitled "Gestational Exposure to Nanomaterials: The Interplay between Bioenergetics and the Epigenome in Progeny Cardiac Tissue" (March 30 $\left.{ }^{\text {th }}, 2017\right) .10$-minutes talk.

WEST VIRGINIA UNIVERSITY VAN LIERE DAY, MORGANTOWN, WV: 2017

Poster presentation, abstract entitled "Polynucleotide Phosphorylase (PNPase) and Mitochondrial microRNA import" (March 24 $\left.{ }^{\text {th }}, 2017\right)$

WEST VIRGINIA UNIVERSITY METABOLISM, MITOCHONDRIAL FUNCTION, AND BIOENERGETICS (MMB) WORKING GROUP, MORGANTOWN, WV: 2016

Oral presentation, talk entitled "Mitochondrial Protein Regulation - CpG Methylation "(September $12^{\text {th }}$, 2016). 10-minute talk.

WEST VIRGINIA UNIVERSITY IGERT ORIENTATION SYMPOSIUM, MORGANTOWN, WV: 2016

Poster presentation, abstract entitled "Gestational Nano- $\mathrm{TiO}_{2}$ Exposure and Cardiac Implications" (August $11^{\text {th }}, 2016$ ).

\section{NATIONAL SCIENCE FOUNDATION-REU INTERNSHIP ON NANOTOXICOLOGY}

Poster presentation during the West Virginia University summer undergraduate research symposium (July 25 $5^{\text {th }}, 2014$ )

Poster presentation for the Waynesburg University undergraduate research symposium (April $24^{\text {th }}$, 2015)

\section{GREENE COUNTY CONSERVATION DISTRICT - 2014 MEETING}

Oral presentation, talk entitled "Environmental impacts of the Oil and Gas Industry on Local Streams and Rivers" (November 18th, 2014). 15-minute talk.

\section{Accepted Abstracts}

EXPERIMENTAL BIOLOGY (EB), ORLANDO, FL: 2019

Hathaway QA, Pinti MV, Roth SM, Sprando DC, Kunovac A, Durr AJ, Cook CC, Fink GK, Cheuvront TB, Grossman JH, Aljahli GA, Roberts HG, Salman M, Giromini AP, and Hollander JM. "Using Machine Learning to Predict the Development of Diabetes and Potential Biomarkers Linked to Cardiac Risk"

EXPERIMENTAL BIOLOGY (EB), ORLANDO, FL: 2019 
Kunovac A, Hathaway QA, Pinti MV, Durr AJ, Fink GK, Goldsmith WT, Nurkiewicz TR, and Hollander JM. "Elevated ROS and Epigenetic Remodeling Disrupt Cardiac Function in Offspring Following Maternal Engineered Nanomaterial (ENM) Exposure"

EXPERIMENTAL BIOLOGY (EB), ORLANDO, FL: 2019

Pinti MV, Hathaway QA, Kunovac A, Durr AJ, Cook CC, Roberts HG, Salman M, and Hollander JM.

"MicroRNA Changes in Diabetic Cardiac Mitochondria: What are they doing there?"

EXPERIMENTAL BIOLOGY (EB), ORLANDO, FL: 2019

Durr AJ, Hathaway QA, Pinti MV, Shepherd DL, Kunovac A, and Hollander JM. "Stress Strain SpeckleTacking Segmental Analysis Reveals Early Indications of Diastolic Dysfunction in a Type 2 Mouse Model of Diabetes Mellitus"

EXPERIMENTAL BIOLOGY (EB), SAN DIEGO, CA: 2018

Hathaway QA, Shepherd DL, Durr AJ, and Hollander JM. "Nuclear-Mitochondrial Crosstalk in the Heart during Diabetes Mellitus - The Impact on RNA in Mitochondrial Subpopulations"

EXPERIMENTAL BIOLOGY (EB), SAN DIEGO, CA: 2018

Durr AJ, Shepherd DL, Hathaway QA, and Hollander JM "Mitochondrial Bioenergetics in the Diabetic Heart and the Influence of Cortactin"

WEST VIRGINIA UNIVERSITY VAN LIERE DAY, MORGANTOWN, WV 2018

Hathaway QA, Pinti, MV, Durr AJ, Shepherd DL, and Hollander JM. "Non-coding RNA and the Mitochondrion - Function and Pathology in the Heart"

WEST VIRGINIA UNIVERSITY VAN LIERE DAY, MORGANTOWN, WV 2018

Durr AJ, Shepherd DL, Hathaway QA, and Hollander JM. “Cortactin's Influence on Mitochondrial Bioenergetics in the Diabetic Heart: A Novel Viewpoint"

WEST VIRGINIA UNIVERSITY VAN LIERE DAY, MORGANTOWN, WV 2018

Noble B, Hathaway QA, Durr AJ, and Shepherd DL. "Epigenetics in Diabetes"

SOCIETY OF TOXICOLOGY (SOT) 57 ${ }^{\text {TH }}$ ANNUAL MEETING, SAN ANTONIO, TX: 2018

Hathaway QA, Nichols CE, Shepherd DL, Durr AJ, Stapleton PA, Abukabda AB, Nurkiewicz TR, and Hollander JM. "Engineered Nanomaterial Exposure: Effects on Epigenetic and MiRNA Regulation of Cardiac Mitochondria"

TRANSLATIONAL RESEARCH CANCER CENTER CONSORTIUM $21^{\text {ST }}$ ANNUAL MEETING, SEVEN SPRINGS, PA 2018 
Nair RR, Piktel D, Thomas P, Hathaway QA, Geldenhuys WJ, Hollander JM, and Gibson LF. "Pyrvinium Pamoate mediates cell death by disrupting mitochondrial respiration and inhibiting beta-catenin signaling in B-cell acute lymphoblastic leukemia"

EXPERIMENTAL BIOLOGY (EB), CHICAGO, IL 2017

Hathaway QA, Nichols CE, Shepherd DL, Stapleton PA, McLaughlin SL, Stricker JC, Rellick SL, Pinti MV, Abukabda AB, McBride CR, Yi J, Stine SM, Nurkiewicz TR, and Hollander JM. "Developing a Link between Cardiac Function and Maternal Engineered Nanomaterial Exposure in Growing Progeny"

WEST VIRGINIA UNIVERSITY VAN LIERE DAY, MORGANTOWN, WV 2017

Hathaway QA, Shepherd DL, Pinti MV, Nichols CE, Sreekumar S, Hughes KM, Stine SM, Martinez I, and Hollander JM. "Polynucleotide Phosphorylase (PNPase) and Mitochondrial microRNA import"

SOCIETY OF TOXICOLOGY (SOT) 56 ${ }^{\text {TH }}$ ANNUAL MEETING, BALTIMORE, MD 2017

Stapleton PA, Abukabda AB, Nichols CE, Hathaway QA, Rellick SL, McBride CR, Yi J, Hollander JM, Nurkiewicz TR. "Temporal microvascular and mitochondrial implications of prenatal nano-TiO2 exposure"

\section{Patents}

\section{OMNI-CLIPZ | IGERT-WVU | MAY 2016 -MAY 2018}

- Omni-ClipZ provisional patent filed March 16, 2017: Application \#: 62472157. Filed under “Holster for Electronic Device". In summary, the device is a holster for insulin pumps, primarily used by Type I Diabetics. The innovation of the product is to include a more durable design as well as incorporating the tubing, which transmits the insulin to the host.

- I am one of four co-founders of the company

- Current Perspectives: conception of product design, patent searching identifying the legal boundaries of the product, preliminary budget and business model for the first 3 years of development, initial surveys gathered on the product design, market identified and channels for production/transportation of the product identified, materials characterized for use, and the first 3D-printed model build.

- Batch testing and additional surveys characterizing the product are currently underway 